\title{
Self Organizing Maps for the Extraction of Deep Inelastic Scattering Observables
}

\author{
by \\ Evan Askanazi \\ B.S., The Ohio State University, 2008
}

A thesis submitted in partial fulfillment for the

degree of Doctor of Philosophy

in the

University of Virginia

Physics Department

January 2016 
One of the fundamental goals of nuclear physics is understanding the structure of each type of subatomic particles that comprise matter and radiation. The two most basic types of components for these particles are quarks and gluons, which act to bind the quarks together in the form of a force called the strong interaction. [This is the short caption]This is the long caption The most fundamental composite particles in nuclear physics are hadrons, which can be composed of two or three quarks. Hadrons which consist of three quarks are Baryons; two notable types of Baryons are protons and neutrons which are the fundamental particles that comprise atomic nuclei and therefore are the fundamental building blocks of matter. Hadrons which consist of two quarks are mesons; this type of hadron forms from interactions in matter occurring at very high energies. Currently, nuclear scattering experiments are used to probe the structure of hadrons. The experiments consist of beams of leptons fired at designated target hadrons; leptons are a type of spin $\frac{1}{2}$ particle that like quarks and gluons has an unknown substructure. The leptons used in the scattering experiments of interest for this analysis are electrons and muons. Deep inelastic scattering (DIS) collisions are a critical example of scattering experiments that use leptons fired at high enough energies at the target hadrons to enable the user to determine the structure of these hadrons; the goal of these computations is to create theoretical models based on DIS data. The DIS between leptons and target hadrons can be probed using Quantum Chromo Dynamics, or QCD. QCD is a field theory used to describe and analyze strong interactions which occur among partons within the hadron. QCD provides a framework for separating the cross section of DIS into components that can be computed by expansions of the strong couplings and components that can only be computed by experiment, or the "soft" parts. Artificial neural networks (ANNs) provide a novel method for modeling the "soft" parts of DIS that eliminate user bias in making these models fit the experimental data. ANNs are sets of data organized into nodes, referred to as neurons, that take input data models and use layers of neurons containing computational algorithms to transform them into final sets of data neurons. Previous attempts to use ANNs to model DIS data have used supervised networks, where the final data set was used as a guidance step each time the ANN algorithm is used; this has led to success in eliminating bias in theoretical models but has not made it possible to visualize and classify these models. A new type of neural network, capable of dimensional reduction of data, without the supervising process of the previous networks is needed to effectively model functions describing nuclear scattering for a range of kinematics and to enable us to analyze the models formed during the ANN algorithm based on their behaviors and quality of fit to experimental data sets. The Self Organizing Map (SOM) is an ANN, using unsupervised learning, that was successfully used to create such desired, unbiased theoretical models of the Parton Distribution Functions, or PDFs. In addition, the SOM successfully showed the relationship between how well the generated models fit data sets and the models' 
behavior by making it possible to observe how the PDFs cluster on two dimensional maps. The SOM was particular useful in probing DIS models because this procedure made it possible to analyze various conditions placed upon the models, in terms of qualitative and quantitative analysis of the resulting cluster formation, and to determine errors in model formations based on these clusters. 


\section{Contents}

Abstract

List of Figures $\quad$ v

List of Tables $\quad x$

1 Introduction: Self Organizing Maps and their Intent . . . . . . . . 1

1.1 Introduction . . . . . . . . . . . . . . 1

$1.2 \quad$ Artificial Neural Networks . . . . . . . . . . . . . . . . . . 1

1.3 Deep Inelastic Scattering . . . . . . . . . . . . . . . . 7

1.4 Quark Parton Model . . . . . . . . . . . . . . . . . . . 20

1.5 Perturbative QCD . . . . . . . . . . . . . . . . 25

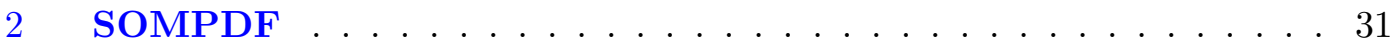

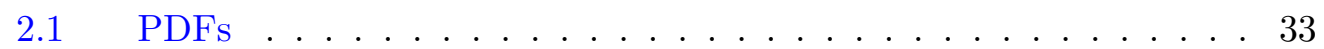

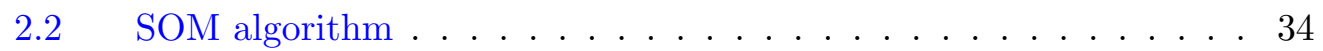

2.2 .1 Initialization . . . . . . . . . . . . . . 34

2.2 .2 Training . . . . . . . . . . . . . . . . . 35

$2.2 .3 \quad$ Mapping . . . . . . . . . . . . . 36

2.3 Representing PDFs as SOMs . . . . . . . . . . . . 36

2.4 Genetic Algorithm . . . . . . . . . . . . . . . . . 37

3 SOMPDF as a quantitative parametrization of DIS data . . . . 39

3.1 Main program SOMPDF . . . . . . . . . . . 40

$3.2 \quad$ Module Gpd Init . . . . . . . . . . . . . . . . . . . . . . . . 41

3.3 Module Gpd Gen . . . . . . . . . . . . . . . . . . . . . . . 42

3.4 Module Generators . . . . . . . . . . . . . . . . . . . . . 42

3.5 Module Gpd Utils . . . . . . . . . . . . . . . . . . . . . . . . . . . . . . . . . . . . .

3.6 Module Gpd Parameters . . . . . . . . . . . . . . . . . . . . 42

3.7 Module Gpd Functions . . . . . . . . . . . . . . . . . . . . . . . . . 42

3.8 Module Gpd Perturbs . . . . . . . . . . . . . . . . . . . . . . . . . . 43

3.9 Module Somkit . . . . . . . . . . . . . . . . . . . . . 43

3.10 Module Som Utils . . . . . . . . . . . . . . . . . . . . . 43

3.11 Module Gpd Eval . . . . . . . . . . . . . . . . . . . . . . . . . 43

3.12 Module Gpd Norm . . . . . . . . . . . . . . . . . . . . . . . . 44

3.13 MPI ........................... 44

3.14 SOMPDF flow Chart . . . . . . . . . . . . . . . . 47

3.15 New Initialization Method . . . . . . . . . . . . . . . . . . . . . . . . 48

3.16 Description of Structure Function Fit . . . . . . . . . . . 52

3.17 PQCD Evolution: Moments . . . . . . . . . . . . . . . . 53 
3.18 Map Features . . . . . . . . . . . . . . . . . . 69

3.19 Experimental Data . . . . . . . . . . . . . . . . 70

3.20 Error Analysis . . . . . . . . . . . . . . . . . 70

3.21 Lagrange Error . . . . . . . . . . . . . . . . . 76

4 SOMPDF PDF Fit Results . . . . . . . . . . . . . . 78

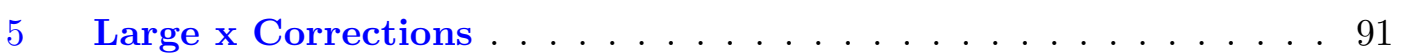

5.1 Target Mass Corrections . . . . . . . . . . . . . . . . . . 91

$5.2 \quad$ Large $\mathrm{x}$ Resummations . . . . . . . . . . . . . . . . . . . . . . 92

5.3 Averaging the Resonance Region with the Bernstein Moments . . 99

$5.4 \quad$ Large x Fit SOM . . . . . . . . . . . . . . . . . . . . 136

$5.5 \quad$ Large $\mathrm{x} \frac{d}{u}$ Cluster Analysis . . . . . . . . . . . . . . . . . . . 142

5.6 Large x quark and gluon Cluster Analysis . . . . . . . . . . . . 147

$5.7 \quad \frac{d}{u}$ Cluster Quantification . . . . . . . . . . . . . . . . . . . . . . . . . . . 151

$5.8 \quad \frac{d}{u}$ Dimensional Clusters and Error Extraction . . . . . . . . . . 153

6 Conclusion . . . . . . . . . . . . . . . . . . . . 167

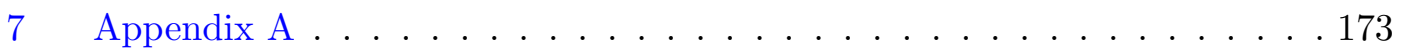




\section{List of Figures}

1.1 An example of a supervised neural network. . . . . . . . . . . . . 2

1.2 An example of an unsupervised neural network. . . . . . . . . . . . 6

1.3 A visualization of the SOM showing how data units in the neural network are positioned on a two dimensional map. . . . . . . . . . . . 7

1.4 A visualization a Deep Inelastic Scattering interaction . . . . . . . . . . 9

2.1 An example of an envelope formed for the first iteration for $Q^{2}=150 \mathrm{GeV}^{2} .38$

2.2 An example of an envelope formed for the $250^{\text {th }}$ iteration for $Q^{2}=150$

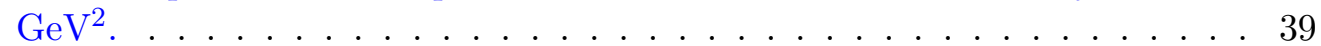

3.1 Structure function $F_{2}^{P}$ plotted vs $x$ at $Q^{2}$ values of $2.5,10,150$ and $800 \mathrm{GeV}^{2} 55$

3.2 Kinematic range of the DIS experimental data used in our analysis. . . . . 71

3.3 Kinematic range of the large $\mathrm{x}$ experimental data used in our analysis. . . 71

3.4 Illustration of the difference between the PDF uncertainty calculated with the Lagrange multipliers method . . . . . . . . . . . . . 75

3.5 Illustration of the behavior of the minimum fit value for the observable $F_{2}^{P}$ using the Lagrange Multiplier method. . . . . . . . . . . . 76

$4.1 \chi^{2}$ values for each PDF on a $6 \times 6$ map for the initial GA iterations. . . . 79

$4.2 \chi^{2}$ values for each PDF on a $6 \times 6$ map for the final GA iterations. . . . . 80

4.3 The SOM for the SOMPDF generated Structure Function, $F_{2}$, compared to the experimental values for a $6 \times 6$ map. . . . . . . . . . . . . 81

4.4 The SOMPDF fit results for number of iterations for a $6 \times 6$ map and a $1 \times 1$ map with the equivalent PDFs generated per cycle and used in training. . . . . . . . . . . . . . . . . . . . . 84

4.5 SOMPDF generated valence quark PDF $\left(u_{v}+d_{v}\right)$ at $Q^{2}=150 \mathrm{GeV}^{2}$ along with the following collaboration PDFs: CJ Mid [59] [60] NNPDF [1] ABM [61] CT10 [58]. . . . . . . . . . . . . . . . . . . . 85

4.6 SOMPDF generated difference between the up and down sea quark quark $\operatorname{PDF}(\bar{u}-\bar{d})$ at $Q^{2}=150 \mathrm{GeV}^{2}$ along with the following collaboration PDFs: CJ Mid [59] [60] NNPDF [1] ABM [61] CT10 [58]. . . . . . . . . . 85

4.7 SOMPDF generated up sea quark PDF $\bar{u}$ at $Q^{2}=150 \mathrm{GeV}^{2}$ along with the following collaboration PDFs: CJ Mid [59] [60] NNPDF [1] ABM [61]

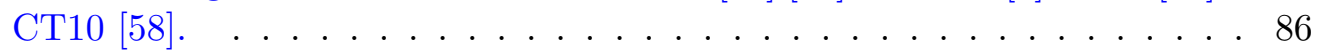

4.8 SOMPDF generated gluon PDF at $Q^{2}=150 \mathrm{GeV}^{2}$ along with the following collaboration PDFs: CJ Mid [59] [60] NNPDF [1] ABM [61] CT10

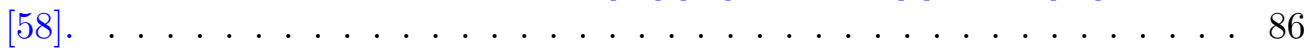

4.9 SOMPDF generated strange quark PDF at $Q^{2}=150 \mathrm{GeV}^{2}$ along with the following collaboration PDFs: CJ Mid [59] [60] NNPDF [1] ABM [61]

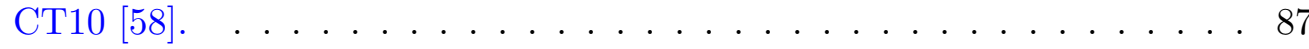


4.10 SOMPDF generated strange quark PDF at $Q^{2}=2.5 \mathrm{GeV}^{2}$ along with the following collaboration PDFs: CJ Mid [59] [60] NNPDF [1] ABM [61] CT10 [58].

4.11 SOMPDF generated valence quark $\operatorname{PDF}\left(u_{v}+d_{v}\right)$ at $Q^{2}=2.5 \mathrm{GeV}^{2}$ along with the following collaboration PDFs: CJ Mid [59] [60] NNPDF [1] ABM [61] CT10 [58].

4.12 SOMPDF generated up sea quark PDF $\bar{u}$ at $Q^{2}=2.5 \mathrm{GeV}^{2}$ along with the following collaboration PDFs: CJ Mid [59] [60] NNPDF [1] ABM [61] CT10 [58].

4.13 The pull of the SOMPDF generated Valence Quark PDF $\left(u_{v}+d_{v}\right)$ relative to collaboration PDFs is shown at $Q^{2}=150 \mathrm{GeV}^{2}$ alongside the relative error of the Valence Quark PDF. The collaboration PDFs are CJ Mid [59] $[60]$ NNPDF $[1]$ ABM $[61]$ and CT10 [58]. . . . . . . . . . . . .

4.14 The pull of the SOMPDF generated Valence Quark PDF $\left(u_{v}+d_{v}\right)$ relative to collaboration PDFs is shown at $Q^{2}=2.5 \mathrm{GeV}^{2}$ alongside the relative error of the Valence Quark PDF. The collaboration PDFs are CJ Mid [59] $[60]$ NNPDF [1] ABM [61] and CT10 [58]. . . . . . . . . . . . .

4.15 The pull of the SOMPDF generated gluon PDF relative to collaboration PDFs is shown at $Q^{2}=150 \mathrm{GeV}^{2}$ alongside the relative error of the gluon Quark PDF. The collaboration PDFs are CJ Mid [59] [60] NNPDF [1] ABM [61] and CT10 [58]. . . . . . . . . . . . . .

4.16 The pull of the SOMPDF generated gluon PDF relative to collaboration PDFs is shown at $Q^{2}=2.5 \mathrm{GeV}^{2}$ alongside the relative error of the gluon PDF. The collaboration PDFs are CJ Mid [59] [60] NNPDF [1] ABM [61] and CT10 [58]. . . . . . . . . . . . . . . . . . 90

$5.1 \quad F_{2}^{P}$ resonance moment integrands and corresponding Bernstein moment points for $Q^{2}=0.55 \mathrm{GeV}^{2}$.

$5.2 \quad F_{2}^{P}$ resonance moment integrands and corresponding Bernstein moment points for $Q^{2}=2.5 \mathrm{GeV}^{2} \ldots \ldots \ldots \ldots$. . . . . . . . . . . . . . . . .

$5.3 \quad F_{2}^{P}$ resonance moment integrands and corresponding Bernstein moment points for $Q^{2}=3.4 \mathrm{GeV}^{2} \ldots \ldots \ldots \ldots$. . . . . . . . . . . . . . . . . . . .

$5.4 \quad F_{2}^{P}$ resonance moment integrands and corresponding Bernstein moment points for $Q^{2}=8.2 \mathrm{GeV}^{2} \ldots \ldots \ldots \ldots$. . . . . . . . . . . . . . . . . .

5.5 $F_{2}^{P}$ values for the resonance region and Bernstein moments for $Q^{2}$ interval

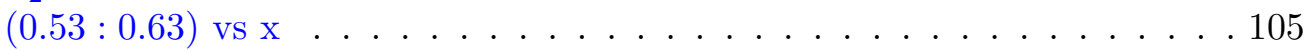

5.6 $\quad F_{2}^{P}$ values for the resonance region and Bernstein moments for $Q^{2}$ interval

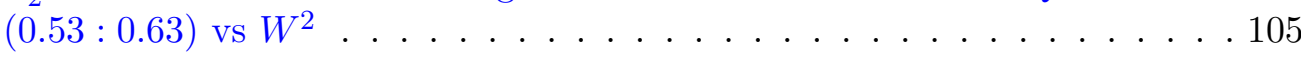

$5.7 F_{2}^{D}$ values for the resonance region and Bernstein moments for $Q^{2}$ interval $(0.53: 0.63)$ vs $x \ldots \ldots \ldots \ldots \ldots \ldots \ldots \ldots \ldots \ldots \ldots \ldots \ldots \ldots \ldots$

5.8 $F_{2}^{D}$ values for the resonance region and Bernstein moments for $Q^{2}$ interval $(0.53: 0.63)$ vs $W^{2} \ldots \ldots \ldots \ldots$. . . . . . . . . . . . . . . . . . . .

$5.9 \quad F_{2}^{P}$ values for the resonance region and Bernstein moments for $Q^{2}$ interval

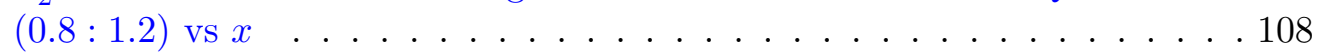

$5.10 F_{2}^{P}$ values for the resonance region and Bernstein moments for $Q^{2}$ interval $(0.8: 1.2)$ vs $W^{2} \ldots \ldots \ldots$. . . . . . . . . . . . . . . . . . . . . . . . . .

$5.11 F_{2}^{D}$ values for the resonance region and Bernstein moments for $Q^{2}$ interval $(0.8: 1.2)$ vs $x \ldots \ldots \ldots \ldots$

$5.12 F_{2}^{D}$ values for the resonance region and Bernstein moments for $Q^{2}$ interval $(0.8: 1.2)$ vs $W^{2} \ldots \ldots \ldots \ldots$ 
$5.13 F_{2}^{P}$ values for the resonance region and Bernstein moments for $Q^{2}$ interval

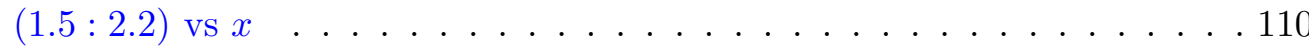

$5.14 F_{2}^{P}$ values for the resonance region and Bernstein moments for $Q^{2}$ interval $(1.5: 2.2)$ vs $W^{2} \ldots \ldots \ldots \ldots \ldots \ldots \ldots \ldots \ldots$

$5.15 F_{2}^{D}$ values for the resonance region and Bernstein moments for $Q^{2}$ interval

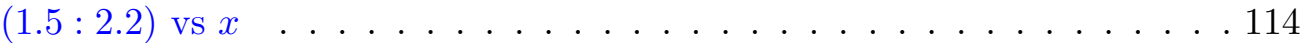

$5.16 F_{2}^{D}$ values for the resonance region and Bernstein moments for $Q^{2}$ interval

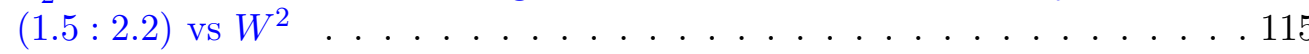

$5.17 F_{2}^{P}$ values for the resonance region and Bernstein moments for $Q^{2}$ interval

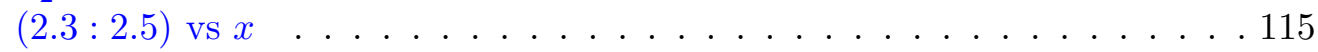

$5.18 F_{2}^{P}$ values for the resonance region and Bernstein moments for $Q^{2}$ interval

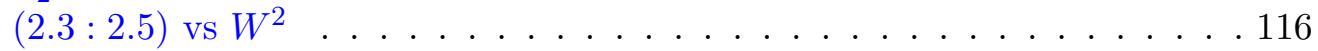

$5.19 F_{2}^{D}$ values for the resonance region and Bernstein moments for $Q^{2}$ interval

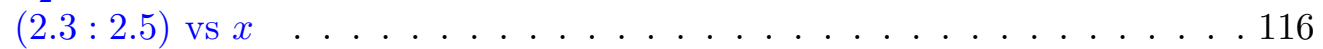

$5.20 F_{2}^{D}$ values for the resonance region and Bernstein moments for $Q^{2}$ interval $(2.3: 2.5)$ vs $W^{2} \ldots \ldots \ldots \ldots \ldots \ldots \ldots$

$5.21 F_{2}^{P}$ values for the resonance region and Bernstein moments for $Q^{2}$ interval

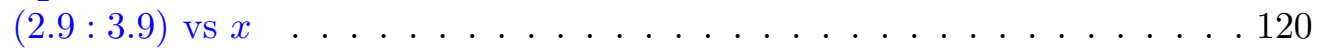

$5.22 F_{2}^{P}$ values for the resonance region and Bernstein moments for $Q^{2}$ interval $(2.9: 3.9)$ vs $W^{2} \ldots \ldots \ldots \ldots \ldots$

$5.23 F_{2}^{D}$ values for the resonance region and Bernstein moments for $Q^{2}$ interval

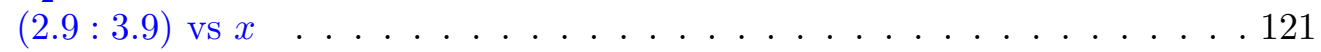

$5.24 F_{2}^{D}$ values for the resonance region and Bernstein moments for $Q^{2}$ interval

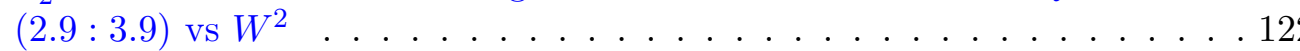

$5.25 F_{2}^{P}$ values for the resonance region and Bernstein moments for $Q^{2}$ interval $(5.3: 6.2)$ vs $x \ldots \ldots \ldots \ldots \ldots$

$5.26 F_{2}^{P}$ values for the resonance region and Bernstein moments for $Q^{2}$ interval

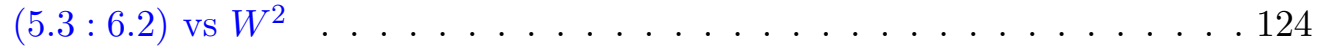

$5.27 F_{2}^{D}$ values for the resonance region and Bernstein moments for $Q^{2}$ interval

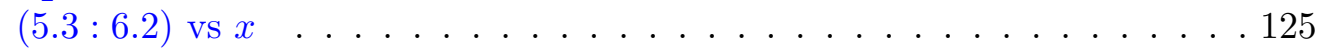

$5.28 F_{2}^{D}$ values for the resonance region and Bernstein moments for $Q^{2}$ interval $(5.3: 6.2)$ vs $W^{2} \ldots \ldots \ldots \ldots \ldots \ldots$

$5.29 F_{2}^{P}$ values for the resonance region and Bernstein moments for $Q^{2}$ interval

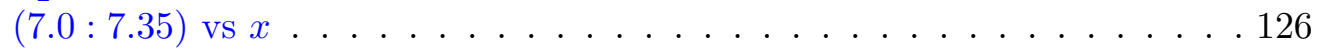

$5.30 F_{2}^{P}$ values for the resonance region and Bernstein moments for $Q^{2}$ interval

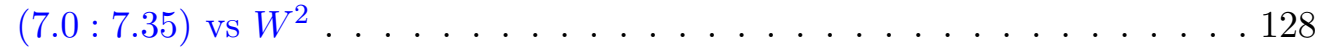

$5.31 F_{2}^{D}$ values for the resonance region and Bernstein moments for $Q^{2}$ interval $(7.0: 7.35)$ vs $x \ldots \ldots \ldots \ldots$

$5.32 F_{2}^{D}$ values for the resonance region and Bernstein moments for $Q^{2}$ interval $(7.0: 7.35)$ vs $W^{2} \ldots \ldots \ldots \ldots \ldots$

$5.33 F_{2}^{P}$ values for the resonance region and Bernstein moments for $Q^{2}$ interval $(7.8: 8.8)$ vs $x \ldots \ldots \ldots \ldots$

$5.34 F_{2}^{P}$ values for the resonance region and Bernstein moments for $Q^{2}$ interval $(7.8: 8.8)$ vs $W^{2} \ldots \ldots \ldots \ldots$. . . . . . . . . . . . . . . . . . . .

$5.35 F_{2}^{D}$ values for the resonance region and Bernstein moments for $Q^{2}$ interval

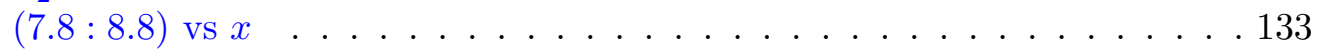

$5.36 F_{2}^{D}$ values for the resonance region and Bernstein moments for $Q^{2}$ interval

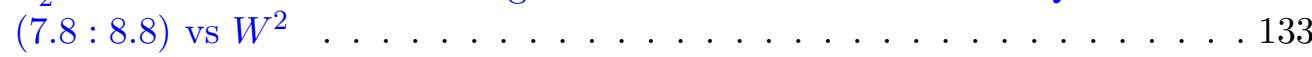


$5.37 F_{2}^{P}$ values for the resonance region and Bernstein moments for $Q^{2}$ interval

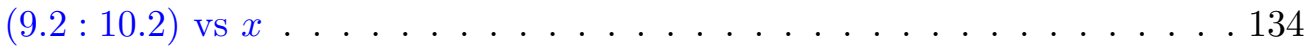

$5.38 F_{2}^{P}$ values for the resonance region and Bernstein moments for $Q^{2}$ interval $(9.2: 10.2)$ vs $W^{2} \ldots \ldots \ldots \ldots \ldots \ldots \ldots \ldots \ldots \ldots \ldots$

$5.39 F_{2}^{D}$ values for the resonance region and Bernstein moments for $Q^{2}$ interval

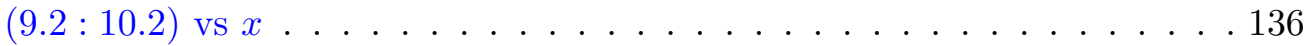

$5.40 F_{2}^{D}$ values for the resonance region and Bernstein moments for $Q^{2}$ interval $(9.2: 10.2)$ vs $W^{2} \ldots \ldots \ldots \ldots \ldots \ldots \ldots \ldots \ldots \ldots \ldots \ldots$

5.41 The $F_{2}^{P}$ values for the resonance region and Bernstein moments for 169 resonance points . . . . . . . . . . . . . . . 137

5.42 The initial generated envelope of $\frac{F_{2}^{D}}{F_{2}^{P}}$ theoretical curves for $Q^{2}=2.5 \mathrm{GeV}^{2}$ relative to the Bernstein moment points . . . . . . . . . . . . 138

5.43 Generated best fitting $\frac{d}{u}$ curve fitting generated $\frac{F_{2}^{D}}{F_{2}^{P}}$ values to the large $x$ data sets . . . . . . . . . . . . . . . . . . . 139

$5.44 \chi^{2}$ lego plot for a $6 \times 6$ map is shown without Target Mass Corrections and large $x$ Resummations added for $Q^{2}=2.5 \mathrm{GeV}^{2} \ldots$. . . . . . . . 140

$5.45 \chi^{2}$ lego plot for a $6 \times 6$ map is shown with Target Mass Corrections and large $x$ Resummations added for $Q^{2}=2.5 \mathrm{GeV}^{2} \ldots \ldots$. . . . . . 141

$5.46 \frac{d}{u}$ lego plot for a $6 \times 6$ map is shown without Target Mass Corrections and large $x$ Resummations added for $Q^{2}=2.5 \mathrm{GeV}^{2} \ldots \ldots$. . . . . . . 142

$5.47 \frac{d}{u}$ lego plot for a $6 \times 6$ map is shown with Target Mass Corrections and large $x$ Resummations added for $Q^{2}=2.5 \mathrm{GeV}^{2} \ldots \ldots$. . . . . . . 143

$5.48 \frac{d}{u}$ functions, without Target Mass Corrections and large $x$ Resummations added for $Q^{2}=2.5 \mathrm{GeV}^{2} \ldots \ldots \ldots \ldots$. . . . . . . . . . . . . . . . . . .

$5.49 \frac{d}{u}$ functions, with Target Mass Corrections and large $x$ Resummations added for $Q^{2}=2.5 \mathrm{GeV}^{2} \ldots \ldots \ldots \ldots$. . . . . . . . . . . . . . . . . . . . . .

5.50 Plots of $u_{v}$ PDFs without large $x$ Corrections added for $Q^{2}=2.5 \mathrm{GeV}^{2}$. .146

5.51 Plots of $d_{v}$ PDFs without large $x$ Corrections added for $Q^{2}=2.5 \mathrm{GeV}^{2}$. .147

5.52 Plots of $u$ PDFs without large $x$ Corrections added for $Q^{2}=2.5 \mathrm{GeV}^{2} \ldots 148$

5.53 Plots of $d$ PDFs without large $x$ Corrections added for $Q^{2}=2.5 \mathrm{GeV}^{2}$. . 149

5.54 Plots of gluon PDFs without large $x$ Corrections added for $Q^{2}=2.5 \mathrm{GeV}^{2} .150$

5.55 Plots of strange PDFs without large $x$ Corrections added for $Q^{2}=2.5$

$\mathrm{GeV}^{2} \ldots \ldots \ldots \ldots \ldots \ldots \ldots 151$

5.56 Plots of charm PDFs without large $x$ Corrections added for $Q^{2}=2.5 \mathrm{GeV}^{2} .152$

5.57 Plots of $u_{v}$ PDFs with large $x$ Corrections added for $Q^{2}=2.5 \mathrm{GeV}^{2} \ldots .153$

5.58 Plots of $d_{v}$ PDFs with large $x$ Corrections added for $Q^{2}=2.5 \mathrm{GeV}^{2}$. . . 154

5.59 Plots of $u$ PDFs with large $x$ Corrections added for $Q^{2}=2.5 \mathrm{GeV}^{2}$. . . 155

5.60 Plots of $d$ PDFs with large $x$ Corrections added for $Q^{2}=2.5 \mathrm{GeV}^{2}$. . . . 156

5.61 Plots of gluon PDFs with large $x$ Corrections added for $Q^{2}=2.5 \mathrm{GeV}^{2}$. .157

5.62 Plots of strange PDFs with large $x$ Corrections added for $Q^{2}=2.5 \mathrm{GeV}^{2} .158$

5.63 Plots of charm PDFs with large $x$ Corrections added for $Q^{2}=2.5 \mathrm{GeV}^{2}$. 159

5.64 The ratios $\frac{d}{u}$ for $Q^{2}=2.5 \mathrm{GeV}^{2}$, on a two dimensional plot, large $x$ corrections vs no large $x$ corrections. . . . . . . . . . . . . . 161

5.65 The ratios $\frac{d}{u}$ for $Q^{2}=2.5 \mathrm{GeV}^{2}$, on a two dimensional plot, large $x$ Resummations vs Target Mass Corrections. . . . . . . . . . . . . . . 162 
5.66 The ratios $\frac{d}{u}$ for $Q^{2}=2.5 \mathrm{GeV}^{2}$, on a two dimensional plot, large $x$ corrections vs no large $x$ corrections with the $25^{\text {th }}$ and $50^{\text {th }}$ iterations taken out. . . . . . . . . . . . . . . . . . . . 163

5.67 The ratios $\frac{d}{u}$ for $Q^{2}=2.5 \mathrm{GeV}^{2}$, on a two dimensional plot, large $x$ Resummations vs Target Mass Corrections with the $25^{\text {th }}$ and $50^{\text {th }}$ iterations taken out. . . . . . . . . . . . . . . . . . . . 164

5.68 The ratios $\frac{d}{u}$ for $Q^{2}=2.5 \mathrm{GeV}^{2}$, on a color coded two dimensional plot, large $x$ corrections vs no large $x$ corrections. . . . . . . . . . . 165

5.69 The ratios $\frac{d}{u}$ for $Q^{2}=2.5 \mathrm{GeV}^{2}$, on a color coded two dimensional plot, large $x$ Resummations vs Target Mass Corrections. . . . . . . . . . . . . 166

5.70 Theoretical $\frac{d}{u}$ curves, taken from a cluster of six PDF units with similarly $\chi^{2}$ and $\frac{d}{u}$ values without large $x$ corrections. . . . . . . . . . 167

5.71 Theoretical $\frac{d}{u}$ curves, taken from a cluster of six PDF units with similarly $\chi^{2}$ and $\frac{d}{u}$ values with large $x$ corrections. . . . . . . . . . . 168

$5.7236 \frac{d}{u}$ curves, taken from a cluster of six PDF units with similarly $\chi^{2}$ and $\frac{d}{u}$ values without large $x$ corrections. . . . . . . . . . . . 169

$5.7336 \frac{d}{u}$ curves, taken from a cluster of six PDF units with similarly $\chi^{2}$ and $\frac{d}{u}$ values with large $x$ corrections. . . . . . . . . . . . 170 


\section{List of Tables}

3.1 Code Module For Each Step . . . . . . . . . . . . . . . . . . . . 48

3.2 Parameter Set for $u_{v}$ PDF Equations . . . . . . . . . . . . . . . . 51

3.3 Parameter Set for $d_{v}$ PDF Equations . . . . . . . . . . . . . . . . 51

3.4 Parameter Set for gluon PDF Equations . . . . . . . . . . . . . . . 51

3.5 Parameter Set for $\bar{u}$ PDF Equations . . . . . . . . . . . . . . . . . . . 52

3.6 Parameter Set for $\bar{d}$ PDF Equations $\ldots \ldots \ldots \ldots . \ldots \ldots$

3.7 Parameter Set for $s$ PDF Equations $\ldots \ldots \ldots \ldots \ldots$

3.8 Free Parameters for the PDFs . . . . . . . . . . . . . . . . . . 54

3.9 Moment Values . . . . . . . . . . . . . . . . . . . . . . 6 . . . . . . . . . . . . . . .

3.10 Fit Results for Various Dimensions ． . . . . . . . . . . . . . . . 70

3.11 Scattering Data Kinematics . . . . . . . . . . . . . . . . 70

5.1 Functional Form Approximating the Bernstein Moment Curve for various

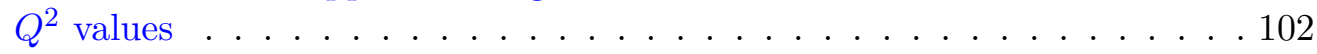

5.2 Integral values of Resonance Data Points with Errors, Bernstein Moments with Errors and Functional forms for various $Q^{2}$ values . . . . . . . . 102

5.3 Table of $Q^{2}=0.55 \mathrm{GeV}^{2} F_{2}^{P}$ Bernstein Moment Values. The Resonance Region here refers to $x$ values for which Resonance Data points exist, which for this $Q^{2}$ value is $(0.17,0.58) \ldots \ldots \ldots 6$. . . . . . . . 106

5.4 Table of $Q^{2}=0.55 \mathrm{GeV}^{2} F_{2}^{D}$ Bernstein Moment Values. The Resonance Region here refers to $x$ values for which Resonance Data points exist, which for this $Q^{2}$ value is $(0.17,0.58) \ldots \ldots \ldots \ldots$. . . . . . . . 106

5.5 Table of $Q^{2}=1 \quad \mathrm{GeV}^{2} F_{2}^{P}$ Bernstein Moment Values. The Resonance Region here refers to $x$ values for which Resonance Data points exist. . . 111

5.6 Table of $Q^{2}=1 \quad \mathrm{GeV}^{2} F_{2}^{D}$ Bernstein Moment Values. The Resonance Region here refers to $x$ values for which Resonance Data points exist. . . 111

5.7 Table of $Q^{2}=1.8 \mathrm{GeV}^{2} F_{2}^{P}$ Bernstein Moment Values. The Resonance Region here refers to $x$ values for which Resonance Data points exist. . . 112

5.8 Table of $Q^{2}=1.8 \mathrm{GeV}^{2} F_{2}^{D}$ Bernstein Moment Values. The Resonance Region here refers to $x$ values for which Resonance Data points exist. . . 112

5.9 Table of $Q^{2}=2.5 \quad \mathrm{GeV}^{2} F_{2}^{P}$ Bernstein Moment Values. The Resonance Region here refers to $x$ values for which Resonance Data points exist. . . 117

5.10 Table of $Q^{2}=2.5 \mathrm{GeV}^{2} F_{2}^{D}$ Bernstein Moment Values. The Resonance Region here refers to $x$ values for which Resonance Data points exist. . . 117

5.11 Table of $Q^{2}=3.4 \mathrm{GeV}^{2} F_{2}^{P}$ Bernstein Moment Values. The Resonance Region here refers to $x$ values for which Resonance Data points exist. . . 119

5.12 Table of $Q^{2}=3.4 \mathrm{GeV}^{2} F_{2}^{D}$ Bernstein Moment Values. The Resonance Region here refers to $x$ values for which Resonance Data points exist. . . 119 
5.13 Table of $Q^{2}=5.7 \mathrm{GeV}^{2} F_{2}^{P}$ Bernstein Moment Values. The Resonance Region here refers to $x$ values for which Resonance Data points exist. . . 123

5.14 Table of $Q^{2}=5.7 \mathrm{GeV}^{2} F_{2}^{D}$ Bernstein Moment Values. The Resonance Region here refers to $x$ values for which Resonance Data points exist. . . 123

5.15 Table of $Q^{2}=7 \mathrm{GeV}^{2} F_{2}^{P}$ Bernstein Moment Values. The Resonance Region here refers to $x$ values for which Resonance Data points exist. . 127

5.16 Table of $Q^{2}=7 \mathrm{GeV}^{2} F_{2}^{D}$ Bernstein Moment Values. The Resonance Region here refers to $x$ values for which Resonance Data points exist. . . 127

5.17 Table of $Q^{2}=8.2 \mathrm{GeV}^{2} F_{2}^{P}$ Bernstein Moment Values. The Resonance Region here refers to $x$ values for which Resonance Data points exist. . . 131

5.18 Table of $Q^{2}=8.2 \mathrm{GeV}^{2} F_{2}^{D}$ Bernstein Moment Values. The Resonance Region here refers to $x$ values for which Resonance Data points exist. . . 131

5.19 Table of $Q^{2}=9.6 \mathrm{GeV}^{2} F_{2}^{P}$ Bernstein Moment Values. The Resonance Region here refers to $x$ values for which Resonance Data points exist. . . 135

5.20 Table of $Q^{2}=9.6 \mathrm{GeV}^{2} F_{2}^{D}$ Bernstein Moment Values. The Resonance Region here refers to $x$ values for which Resonance Data points exist. . . 135 
I would like to give thanks to everyone who has supported me in my endeavors, worked with me and given me the tools I need to triumph in my journey towards the best possible dissertation. This includes my parents, who instilled in me the innate value of education and intellectual curiosity from the time I was old enough to read. They provided me with guidance, love and support from my preschool years, and earlier, all the way through to the end of my time at graduate school. My parents and others have provided consistent emotional support and advice that has been particularly helpful for me as I navigated the academic world. In high school and college, I also received training in research from Dr. Gujrati at the University of Akron and Dr. Michael Lisa at the Ohio State University. My quantum mechanics professor Dr. Robert Perry at the Ohio State University was also instrumental in ensuring I would be prepared for graduate school.

At the University of Virginia, numerous professors were especially helpful to me as I looked to gain as much knowledge and skills as possible during my tenure here. Dr. Sackett, Dr. Fowler and Dr. Lamacraft, as my course professors, were helpful to me as I looked to master the fundamentals of graduate level physics. Larry Suddarth and Dr. Craig Dukes devoted much of their time and energy to aiding me as I learned to teach college students the physics labs and the principles behind them. A special acknowledgment goes towards Dr. Dinko Pocanic for his assistance during my completion of my Master's Thesis.

For my $\mathrm{PhD}$, the help and support I received from Dr. Liuti and her colleague Dr. Day was immense. They worked with me on my ability to not only understand advanced nuclear physics concepts but also to be able to explain them to anyone inside or outside academia. Dr. Day provided financial support when it was needed out of his own initiative and regularly offered advice on how to organize, extract and present data. Dr. Liuti successfully recognized the communication and interaction skills I needed and ensured that went about developing them as much as possible. To that end, I would like to acknowledge the efforts of Deborah Berkeley, Jennifer Beard, Baozhen Xie and Dreama Johnson at the University of Virginia Learning Center for their tireless efforts as well. When it was time to solve problems that occured in our research endeavors and make our work presentable, she put in long hours working alongside me as we overcame any challenges that arose in this task. This research project would not have been possible to complete without the contributions of Katherine Holcomb in the form of the required 
code to perform the necessary algorithms, which could readily be modified, and Aurore Courtoy, who assisted in the computations of observables at critical kinematics. Therefore, I wish to give special thanks to Dr. Holcomb and Dr. Courtoy. Special recognition also goes to Dr. Nilanga Liyanage; he was willing to support my endeavors any way he could as Graduate Director and aid in overcoming any challenges that arose. Peter Arnold was of great help in ensuring that my ability to present the work I have performed was up to par as well. Lastly, I would like to acknowledge the work of fellow theoretical physics students Abha Rajan, Gabriel Wong, Shahin Iqbal and Ajinkya Kamat. They were an integral part of the physics community I worked with at University of Virginia. 


\section{Introduction: Self Organizing Maps and their Intent}

\section{$1.1 \quad$ Introduction}

Nuclear scattering has been probed for decades in attempts to analyze interactions among fundamental particles and the hadrons they comprise. In particular, developing models for the strong interactions among components of hadrons through inelastic scattering has been an ongoing process. Inelastic scattering cross sections, which provide us the scattering rates and the likelihood of a scattering event occurring for a given target hadron, have components for which numerous attempts to create theoretical models for have been made. These components are the structure functions of inelastic scattering. These structure functions in turn are made up of individual components called Parton Distribution Functions (PDFs). Articifial Neural Networks (ANNs) represent a novel method for creating theoretical models for determining the PDFs. Currently, the structure functions can only be determined though scattering experiments; numerous collaborations have looked to extract the PDFs from structure function data sets obtained from experimentation. Previous extraction attempts have relied on analytical parametric functional PDF forms in order to obtain their PDFs. This has led to inherent bias in the PDF extraction because these attempts required theoretical assumptions for determining the shape of the PDF curves. The Neural Network PDFs (NNPDFs) are a notable set of PDF parametrizations because they successfully used supervised learning in order to eliminate user's bias. The neural network the NNPDF collaboration used contained a set of 37 free parameters given by the ANN weights. These PDFs were then fitted to experimental data and the differences between the resulting structure functions and the structure function values taken from experimental measurements were minimized using a Genetic Algorithm (GA) approach [1]. The user's bias was eliminated because any information about the behavior of the PDFs was obtained directly from experimental data. However, because the process was dependent on the experimental data sets in order to extract PDF behavior, its use was particularly limited in kinematical regions where experimental data was not available.

The validity of the ANN has been analyzed in previous studies [2],[1],[3] by methods including a "convergence condition" or "stopping criterion". This refers to the point in the training phase of over-learning, or when the training steps produce only statistical 
fluctuations of data, thereby setting the length of the training phase. This supervised learning, however, is limited by its inability to extrapolate or predict the behavior of the functions in kinematic regions where no experimental data exist. This is particularly problematic when it comes to extrapolation and prediction of function behavior for newer sets of high energy experiments. These new experiments will include polarized and unpolarized semi-inclusive and exclusive high energy scatterings off of hadronic targets [4] [5]. The number of kinematics and observables measured over these kinematics will be larger and the kinematic coverage will be smaller than in previous high energy experiments. This has created a strong incentive to develop an ANN that has the same capabilities as the NNPDF to perform unbiased fits and can also make extrapolations and predictions on the scattering functions for which it creates models. These new sets of ANNs will use weighted combinations of previous attempts to create models for the PDFs in order to come up with proper initial functions than can be fed into the ANNs. The ANNs will then look to generate a unique set of models for these structure functions. These networks will then fit the theoretical models to the structure function data provided by the SLAC [6], BCDMS [7], NMC [8], Fermi Lab E665 [9], H1 [10], ZEUS [11] and Jefferson Lab experiments [12] in an attempt to come up with a reliable theoretical way to model the PDFs at all kinematical values.

The specific type of neural network used is the Self Organizing Map (SOM). This use of the SOM was influenced by previous attempts to use this network type to probe high energy physics [13]. This network will use a new type of parametrization for the PDFs called the SOMPDF method [14]. The SOM used will take variations of previously created theoretical models, perform semi random variations of them and take random combinations and create a resulting set of PDFs that will be entered into the SOM iterations. The fit between each PDF in the set and the full set of experimental data points will be used as the best fit on which the learning process will be based. The SOM relies on unsupervised learning, a competitive learning process that enables the network to extract the PDFs from the experimental structure function data without the structure function experimental data or any models of the data being used in a continuous feedback mechanism. The SOM is unique in its ability to visualize multidimensional data sets such as those presented by the structure functions and the PDFs. This network's ability to convert multidimensional data into two dimensional maps makes it especially advantageous in recognizing patterns in specific regions of these maps, or clusters, for 
PDFs in various kinematic ranges and for observables taken from these PDFs. This property of the SOM map can be used in tandem with the GA. This GA functions in the same manner as the NNPDF GA and so seeks to generate theoretical structure functions whose values for a set of kinematics are as close as possible to the corresponding experimental measurements of these functions.

The SOM and GA used together can enable us categorize the PDFs and their fits to experimental data in various cluster sizes within a two dimensional map. The NNPDFs also use a GA in order to make the neural network generated functions' fit to experimental data sets as ideal as possible by optimizing a function of the differences between the generated functions and the experimental sets. However, the NNPDF method does not allow for two dimensional observation of the clustering properties of the generated functions over kinematic ranges of interest nor enable us to see how incorporation of physics processes into the network affects the behaviors of the generated sets of functions during fitting. The first attempt to use the SOM to solve the case for unpolarized functions are in [15] [16] The remainder of Section 1 is dedicated to thorough explanations of the neural network methodology; Section 1.1 details the purpose and procedure behind ANNs as well as the different types of learning ANNs can utilize. Section 1.2 covers the physics processes that the ANNs will probe. Section 2 will be based on the methodology behind the SOM and how it will be utilized in conjunction with the GA to fit the PDFs to the data. Section 3 will describe how the SOMPDF code is designed and explain how the PDFs are initialized. Section 4 will show how the PDF errors will be determined. Section 5 will show the fit results of the SOM method. This section will also show how the SOMPDF network can enable us to visualize how the effects occurring when the constituent components of the hadron carry large momentum fractions during scattering affect the behavior of the generated functions and the possible ranges of observables. Clustering analysis and errors of the generated functions will also be analyzed. Section 6 will discuss the future possibilities resulting from the SOM extractions and analysis.

\section{$1.2 \quad$ Artificial Neural Networks}

ANNs are an information neural network based on the workings of an animals' central nervous system. The term neural network traditionally refers to models utilized in cognitive psychology, statistics and artificial intelligence (AI). These neural networks 
consist of sets of nodes that are classified as neurons because they can take sets of numerical input parameters and use learning algorithms to adapt them and because they can estimate non-linear functions of input data. These parameters can be referred to as adaptive weights; these weights are essentially the connection strengths between neurons in a generated network activated during the network's training. An ANN in its fundamental form has three layers; the first layer contains sets of of input neurons, the second layer, or the "hidden" layer, has a set of neurons which contain a process by which the input data are evolved and trained and the third layer of neurons contains a resulting set of output data. In a complex ANN the hidden layer can be a series of numerous layers, each of which takes the data neurons generated by the previous layer and transforms them into a new network of data neurons. A visualization of a supervised ANN is shown in Figure 1.1.

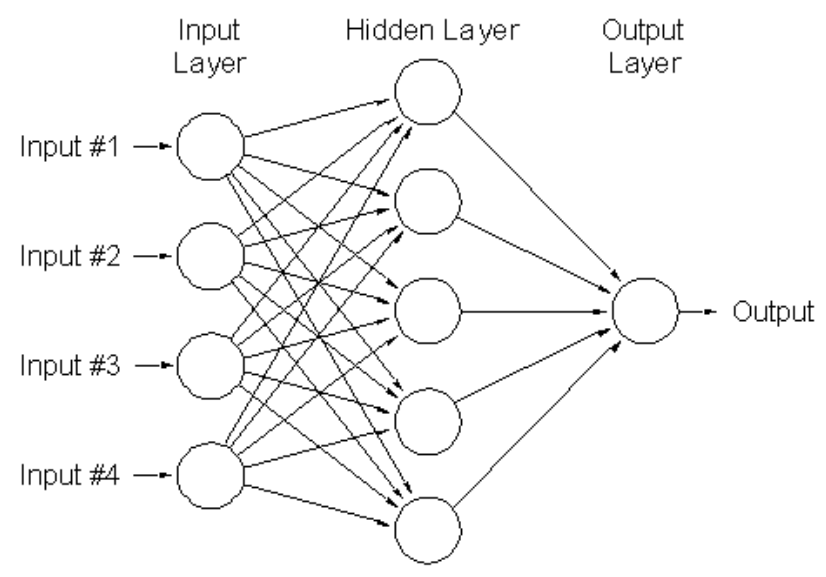

FiguRE 1.1: An example of a supervised neural network.

Although neural networks have an aforementioned traditional usage, in modern times there have been various important applications for them. The primary categories of ANN applications are function approximation, classification [17], data processing, robotics and control. Within these categories there exists a wide array of real life applications. They have been vital towards progress in understanding how biological neural systems work. The field of theoretical and computational neuroscience is centered around the analysis and computational modeling of neural networks found in various biological environments. The goal of this field is to create neural networks modeled after biological systems in order to gain a fundamental understanding of how biological systems operate. In order to do this, neuroscientists look to link together biological observables, biological neural networks and statistical learning and information theory. The types of neural network 
models used for this include models of short-term behavior of individual neurons, models of the formation of neural circuits from interactions among neurons and models how how behavior of neural networks can arise from abstract neural models of biological subsystems. These can be models of short term or long term plasticity of neural systems and their relationships towards learning and memory of systems ranging from those of individual neurons to complete neural networks. Researchers currently utilize ANNs in system identification and control, for example in controlling vehicle paths and predicting the trajectory of vehicles and other objects and pattern recognition, which has uses in radar systems analysis, face identification and object recognition. Additionally, ANNS have practical applications in directing manipulators, handwritten text and other speech recognitions, financial systems and control engineering. In medicine, they have also been notable for their use in radiology and in particular in their use in improving the accuracy and speed of various types of cancer diagnosis methods.

The ANNs have established themselves as an invaluable method for pattern recognition and data visualization since Warren McCullough and Walter Pitts [18] first designed in the 1940s. Donald Hebb, in developing his Hebbian Theory of how neurons in the brain connect to form engrams and adapt to chances, developed the first kind of unsupervised neural network [19]. In the past twenty years they have been widely used as computational tools in high energy, nuclear and computational physics analysis. In recent years there have been vast improvements on the capabilities of ANNs; the recurrent neural networks developed by the Jurgen Schmidhruber group [20], which have been internationally recognized for their abilities in pattern recognition, are a notable example.

Neural networks are, in essence, simply sets of models based on a function $f$ such that $f: X \rightarrow Y ; X$ and $Y$ are the input and output data maps in the networks. ANNs can also consist of distributions over $X$ or over $X$ and $Y$. An ANN has three parameters which it relies on to fit the input data sets. The first parameter is the interconnection pattern between different layers of neurons, the second parameter is the learning process for updating the weights of the interconnections between the layers and the third parameter is the activation function that transforms the data in the input neurons into the desired data in the output neurons. The function that maps input data to output data in neural networks can be written as a composition of other functions; a frequent description of this function is as a nonlinear weighted sum of functions that 
directly produce the values for the output neurons using the input values from the first neuron layer. These functions are referred to as the activation functions.

Neural networks are used in modeling in physics, robotics and other fields because they can utilize the principle of learning with regards to data sets and models. The possibility of networks to perform learning is in fact what has generated the most interest in their applications. Learning is a process in which pattern recognitions are used to find a set of models that can be used to solve a task while minimizing the cost function. The learning process computes the cost of using the models to accomplish the task; learning entails taking a set of functions $F$, which can be used to solve a given task, and finding a set of functions $f * \in F$ that form the optimal solution to the task. In order to achieve this, the learning procedure uses a cost function $C$ to attempt to find the optimal solution. The learning process looks to find the optimal solution $f *$ by ensuring that $C(f *) \leq C(f) \forall f \in F$. The cost function effectively computes how closely a model created to solve a given task compares to the most optimal model for that task; minimizing this function is a fundamental goal of learning. Because learning is designed to create models for a set of observables, the cost function must be a function of those observables as well. For a neural network with a very large number of data samples, machine learning becomes necessary. Machine learning in the late 1980s replaced AI networks based on systems using if-then rules; this network method is defined by its use of information contained in variable parameters of dynamical systems. In machine learning, the cost function is partially minimized every time it is computed from an input data sample taken from a distribution. Machine learning can be supervised or unsupervised. In supervised learning, pairs of data $x$ and $y$ are given with the goal of finding a function that can make the transformation $X \rightarrow Y$ for all data sets $Y$ and $Y$ that $x$ and $y$ could be taken from. In this learning type, the cost function is related to the mismatch between the data sets and the results obtained by attempting to map one set of data to another. In reinforcement learning, the data set $x$ is nor provided at the start of the network procedure but rather generated from the network's interactions with a specific environment over a period of time. In this learning type, after a given time $t$ the network performs an action $y_{t}$ with an associated cost $c_{t}$. The goal of this learning type is to select a policy for performing actions that leads to the slowest cost over an extended period $t_{t o t}$; the total cost can be the cumulative costs $c_{t}$ for all actions $y_{t}$ performed in time $t_{\text {tot }}$. In unsupervised learning, there is a set of data $x$ and a neural network that 
creates a final output $f$, dependent on both $x$ and $f$, without another set of data used to supervise the mapping. In this method, the cost function to be minimized is dependent on the observables that the ANN is trying to model and the parameters and variables of the ANN models themselves. A simple form of unsupervised learning involves the case where the data model is simply $f(x)=a$ for some constant $a$. By minimizing the cost function $C=E\left[(x-f(x))^{2}\right]$ we would obtain a value $a$ equal to the mean of data set $x$. The neural network used to generate DIS models will require a more complex cost function. ANNs can be categorized based on their capacity, or their ability to model a given function accurately, their computational power and their convergence. The convergence, however, can be difficult to categorize since it is dependent on a multitude of different factors. These include the number of local minima, which is dependent on the cost function, the number of variable parameters within the models generated and how far the initial models are from the desired observable to which we wish to fit them.

ANNs also allow for classification of data models based on their features and observables computed from these models and for two (or higher) dimensional pattern recognition among the output network of data values generated from the hidden layers. The patterns identified by the ANNs are referred to as clusters. Clustering is the facet of unsupervised learning that provides one of the primary motivations for using this particular network to probe DIS and its composite functions. In the case of models of structure functions and their component functions, the SOM can treat these models as data sets and identify clusters which form when the network generates an output map from an initial distribution of data models. These clusters can come from the properties of the models themselves and from their computed fits to experimental data sets. These properties of ANNs make them ideal for generating models based on inelastic scattering observables. An example of an unsupervised network is shown in Figure 1.2.

SOMs, as noted previously, are an unsupervised network that uses a two dimensional grid for mapping and fitting a multi dimensional set of input data. The map was first proposed as a type of neural network by Finnish researcher Tuevo Konohen [21]; its applications for problem solving in numerous physics, biological, mathematical and medical fields has made Konohen the most cited researcher in his nations' history. Researchers define the algorithm as a "map" because results using unsupervised learning are most often represented as 2D geometrical configurations. The ability of the SOM to project high dimensional input data onto lower dimensions representations while preserving the 


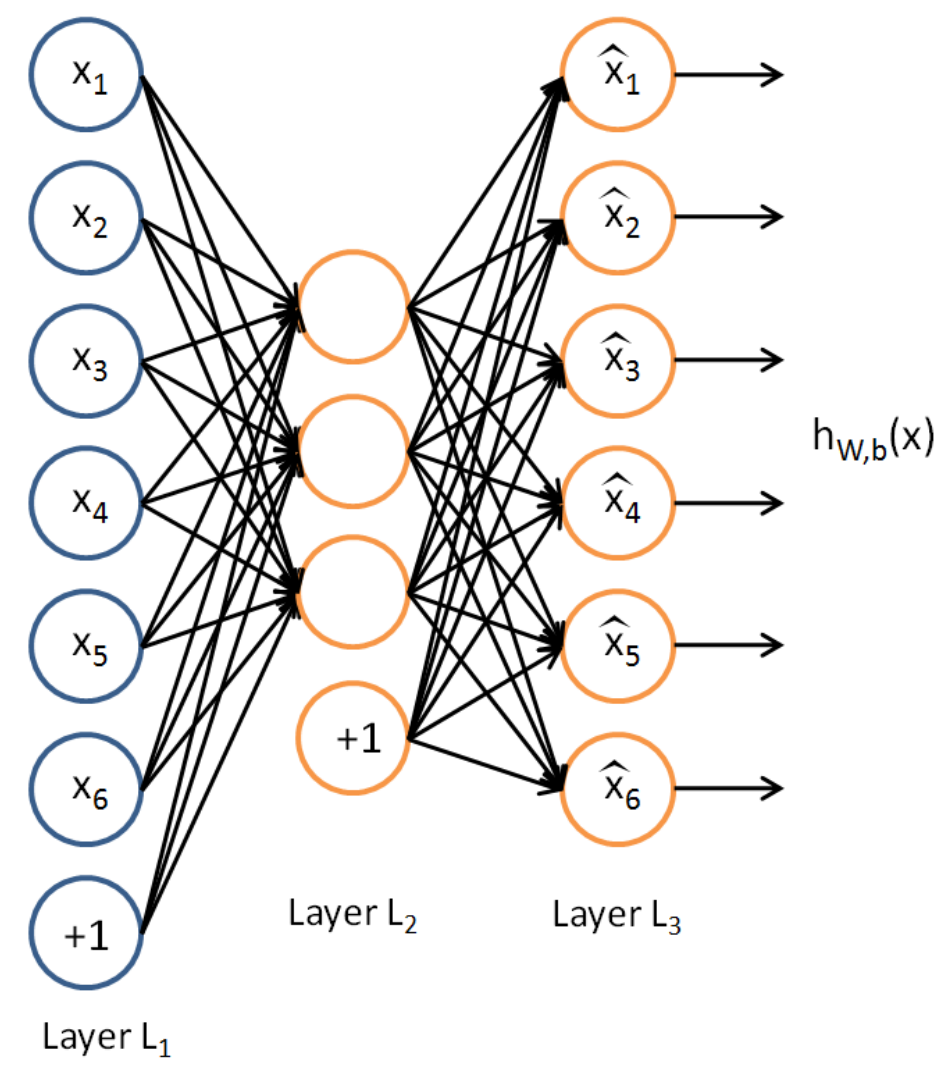

Figure 1.2: An example of an unsupervised neural network.

topological features present in the training data allows us to use this neural network in order to fit input models to final data sets generated from scattering experiments. This visualization allows us to isolate the individual properties of the input data functions we use and create more accurate models of them. It additionally allows us to observe how the cost functions of the input data sets adapt over time through the learning process and assists us in determining how to lower the cost function for a given number of iterations of the learning process. The SOM has the ability to preserve topological features of data units on a two dimensional map; the SOM when completed will feature sets of local neighborhoods of data units with essential common features preserved throughout the iterations of the SOM. This ensures that the SOM is one of the most useful networks in existence for visualizing complex, nonlinear sets of data units. A SOM network is shown in Figure 1.3.

The method by which the SOM works allows us to use experiments conducted for a given physics process to train input sets of data consisting of attempts to create functions of these processes. The SOM places a set of $n$ dimensional data vectors on an $N x N$ map, placing one data vector in each of the $N^{2}$ cells and uses a competitive learning process 


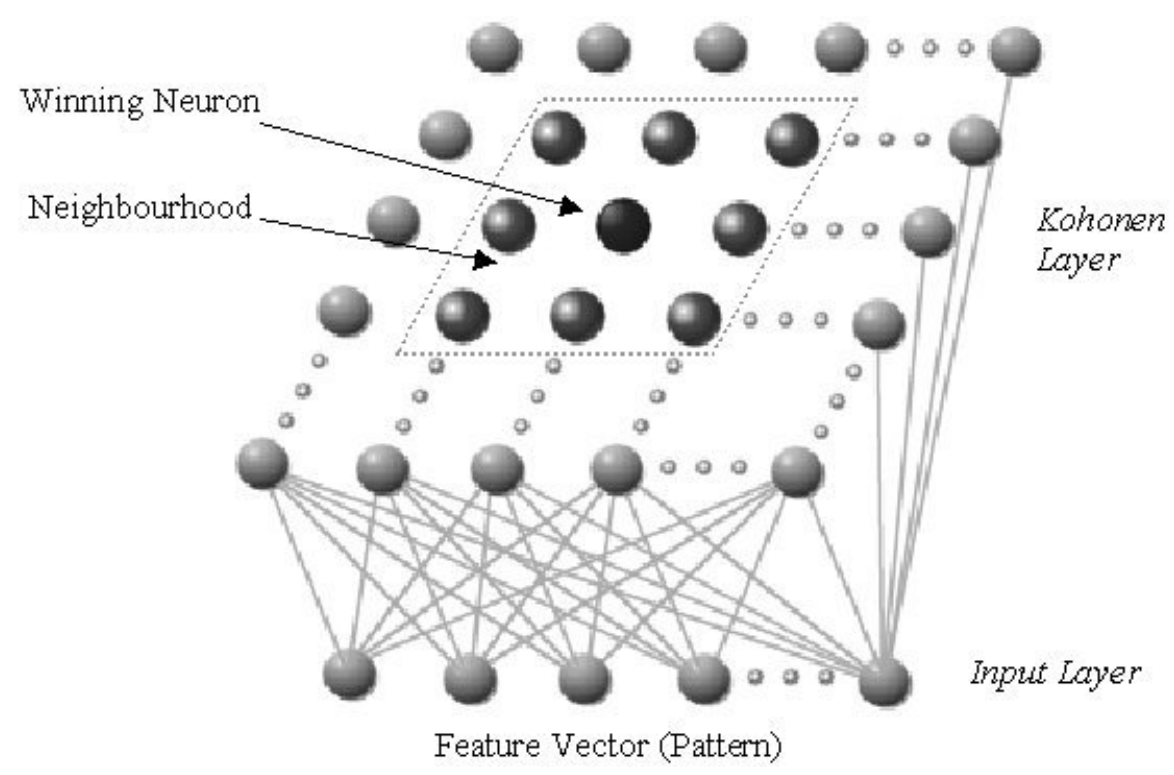

Figure 1.3: A visualization of the SOM showing how data units in the neural network are positioned on a two dimensional map.

to train the data. The competitive learning process has each of the data vectors on the map competing with each other to be the best fit for an outside set of training data, with the set of data vectors on the map undergoing evolution after each use of the competitive process to more closely fit the training data. Each data vector on the map will have a cost function, which for this map can also be called a discriminant function, and the aim is to find the map vector with the lowest cost function. We classify the map vector with the lowest cost function as the one having the best fit to the training data and declare it the winner in the competitive process. This process allows us to model functions that describe various processes in physics using only previous attempts to make theoretical models and known experimental results.

\subsection{Deep Inelastic Scattering}

The self organizing map is used to probe the cross section of Deep Inelastic Scattering (DIS). DIS is the inelastic scattering between a lepton and a hadron, which in the specific case of the SOMPDF experiments is a proton or a deuteron. A lepton is 'scattered', or deflected, off of a hadron at high energies, which give the leptons a short wavelength that allows the scattering process to probe the insides of the hadron. The ability of the process to probe the inside of the hadron this way is where the 'deep' part of deep inelastic scattering comes from. The first of such inelastic scattering experiments 
were performed at the Standford Linear Accelerator Center in 1968 using procedures similar to the Rutherford scattering experiments. Electrons were fired at proton and neutron targets and observations of the inelastic scattering that resulted led to several key conclusions. In particular, when high intensity electron beams were fired at nucleons, the rate of scattered electrons was far higher than expected [22]; Feynman concluded that this was because when the electrons collided with the nucleons, they were probing these targets at small enough distances to where the targets had to be treated as having a set of components with their own coupling forces. Thus, these experiments revealed that hadrons have internal structure; protons, neutrons and other baryons consist of three quarks and that the quarks within these hadrons consist of point-like charges with charge ratios later predicted by the Standard Model along with neutral point-like elements. These point-like elements are referred to as partons [23]. The scattering rates of the electron beams when fired at nucleon targets were a result of the scattering of electrons off of these partons, which each had momentum fraction $x$ of the target hadron. Conservation of momentum for these elastic collisions naturally required that $\sum_{x}=1$. This parton model described the distribution of constituent partons in terms of functions that could be called parton density functions. The parton theory was further developed by the classification of quarks as spin $\frac{1}{2}$ particles with six types and two possible charges for them; charges of $\frac{2}{3}$ for up, charm and top quarks and $\frac{-1}{3}$ for down, strange and bottom quarks. The up and down quarks were the key quarks that led to classification of nucleons according to the parton model; protons consisted of two up and one down quark and neutrons consisted of two down and one up quark. The quarks which determine the hadron type can be identified as valence quarks. The parton model also includes the existence of quark anti quark pairs which are referred to as "sea" quarks. In the DIS between electrons and hadrons, a virtual photon or other type of boson is also exchanged which allows us to further probe the structure of the hadron.

An example of a Deep Inelastic Collision is shown in Figure 1.4.

A lepton scatters off of a hadron, which originally has momentum $p$, with initial momentum $k$ and final momentum $k^{\prime}$, while the momentum $q$ of the virtual photon $\gamma^{\prime}$ produced in the exchange is given by $q=k-k^{\prime}$. The hadron in the scattering process breaks apart with each parton within the hadron carrying its own momentum fraction $x$ after the collision. $Q^{2}$ is defined as an explicitly positive quantity related to the virtual photon momentum by $-Q^{2}=q^{2}$. For $Q^{2}<10^{3} \mathrm{GeV}^{2}$, the electromagnetic interaction 


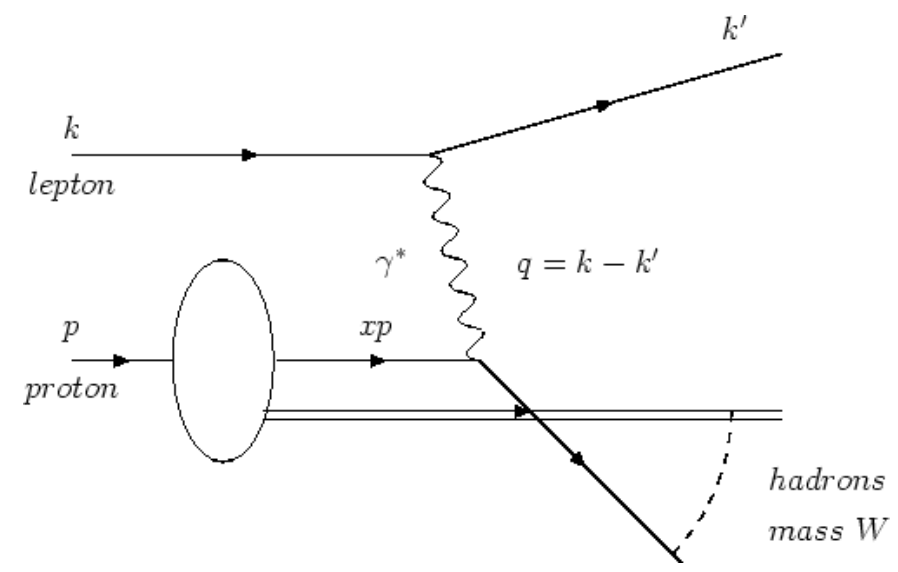

Figure 1.4: A visualization a Deep Inelastic Scattering interaction is shown. $\gamma^{*}$ is the virtual photon exchanged and $q$ is the momentum transfer as a result of the photon exchange.

dominates the cross section enabling us to focus on the photon exchange in evaluating it. In the scattering experiments, only the leptons with momentum $k^{\prime}$ are detected.

The scattering experiments begin with the electron beams fired towards a nucleus that contains the designated target set of hadrons. For low $Q^{2}$ values, defined such that $Q^{2}<<M^{2}$ where $M$ is the mass of the target hadron, the struck nucleons recoil as a whole when struck by the electron and so there is no probing of the constituent particles within the hadron. The recoil energy is $\nu=\frac{Q^{2}}{2 M_{A}}$ where $\nu=E-E^{\prime}$ is the energy transfer of the virtual photon in the exchange, $E$ and $E^{\prime}$ are the initial and final energies of the scattered electron and $M_{A}$ is the mass of the nucleus that contains the target hadron. The nucleus can also move to an excited state containing energy $\nu=\frac{Q^{2}}{2 M_{A}}-\frac{\left(M_{A *}^{2}-M_{A}^{2}\right)}{2 M_{A}}$. In the electron nucleus collisions, the variable $x_{A}=\frac{Q^{2}}{2 M_{a} \nu}$, which for elastic collisions goes to 1 , should be introduced to quantify the properties of the collisions. In higher energy limits, the resolving power of the probing from the collisions increases, the elastic scattering cross section decreases and the probability of the electron scattering off of an individual nucleon within the nucleus as opposed to the whole nucleus increases. As $Q^{2}$ increases towards a typical hadronic scale, which corresponds to $Q^{2}$ being above $0.5 \mathrm{GeV}^{2}$ the target hadron behaves increasingly less as a pointlike particle and the component particles of the nucleus are increasingly involved in the scattering. Therefore, it becomes more practical to introduce the kinematic $x=\frac{Q^{2}}{2 M \nu}$. The proton and neutron have magnetic moments that reflect their construction as well; these moments are described in terms of the constituent quarks within the proton uud and neutron $u d d$. The quark charges are $Q_{u}=\frac{2}{3}$ for the up quark and $Q_{d}=-\frac{1}{3}$ for the 
down quark. The magnetic moments for the up and down quarks are given in terms of the proton and neutron moments below in relation 1.1 and equation 1.2. $\mu_{0}=\frac{\hbar}{2 M c}$ is the proton's Dirac moment.

$$
\begin{aligned}
& \mu_{u}=\frac{1}{5}\left(4 \mu_{p}+\mu_{n}\right) \mu_{0} \\
& \mu_{d}=\frac{1}{5}\left(4 \mu_{n}+\mu_{p}\right) \mu_{0}
\end{aligned}
$$

Feynman's development of the picture of the parton model along with the results of the first inelastic scattering experiments [24] [25] led to a fundamental understanding of the scaling in $x$ of the structure functions proposed by Bjorken. In the context of Deep Inelastic Scattering (DIS), the point-like partons within the hadron interacted with the electrons and the photons exchanged during the electron scattering process. When the electron hadron inelastic scattering occurred, the virtual photons exchanged scattered inelastically off the partons with the DIS cross-section being the incoherent sum of the cross sections of the virtual photon scatterings. In the high momentum limit of the proton, the Bjorken scaling $x$ variable for a given quark is the fraction of the proton momentum contained in the parton during the inelastic scattering of the electron. The size of the elastic cross section of the elastically scattered parton is directly proportional to the probability $q_{i}(x)$ of finding a parton of type $i$ with momentum fraction $x$ in the proton.

The electron, muon, neutrino and anti neutrino scattering experiments made it possible to get a full picture of the quark and anti quark distributions inside the hadrons. The $u$ and $d$ valence quark probability distributions were shown to approach 0 in the large $x$ limit and the $x=0$ limit. The small $x$ region was shown to be dominated by sea quarks; the total momentum of the quarks was shown to only contain half the total momentum of the hadron target. Another critical factor in the quark gluon model is that in the small time frame over which the electron hadron scattering occurs, the quarks act as free particles without any fundamental interactions occurring between them. Therefore, in the limit $Q^{2} \rightarrow \infty$ the elastic scattering of the photons off of the partons has an increasingly small cross section. The $Q^{2} \rightarrow \infty$ limit thus corresponds to a set of scattering interactions over which all the partons in the hadron behave as free 
particles. The field theory of the parton model dictates that in the asymptotic limit of $Q^{2}$ all the partons act as free particles [26] [27] [28]. The principle of asymptotic freedom in the parton quark model, as a crucial part of the field theory behind the development of the quark gluon models, completed the picture. Asymptotic freedom predicts a weakening in the coupling of the strong interactions as the energy transfer in the interactions increases.

Feynman and Bjorken introduced the parton model, which was capable of successfully separating the long distance physics from the short distance physics in the processes within the parton, in the late 1960s [29] [30]. The short distance, high energy processes could be computed through perturbative expansions of the strong coupling and the lower energy, long distance processes which could only be computed by the creation of phenomenological models. These models are the Parton Distribution Functions, or PDFs. The parton model is understood to be the lowest order approximation of perturbative QCD. The separation of QCD determinations of scattering cross sections into the hard and soft parts requires the PDFs to be dependent on $x$ and $Q^{2}$. The data from HERA experiments, among others, has confirmed the PDF dependence on $Q^{2}$ [31]. The physical meaning of the $Q^{2}$ dependence comes from the production of additional partons from a given set of partons upon being probed by the exchanged vector photons. This $Q^{2}$ dependence is predicted by perturbative QCD and quantified by the PDF evolution equations.

The quark parton model indicates that gluons are a crucial component of the hadrons, however it only enters the quark parton model through their interactions with the sea quarks. As a result, since virtual photons do not scatter off of gluons as they do for quarks, the scattering cross sections cannot be used to determine gluon distributions they way they can for quark distributions. Only indirect measurements of the gluon distributions can be made. The gluons contribute to the total momentum of the hadron in the DIS; containing roughly fifty percent of the hadron's total momentum. The gluons also are more prevalent in the small $x$ region of the hadron. However, beyond this there is relatively little that is know about the gluons other than the expected normalized moments of their probability distributions over $x$. The electron proton scatterings and proton proton interactions will need to be more extensively probed at very small $x$ values, ideally in the range $x<10^{-4}$, where gluon gluon interactions dominate. This will be needed to obtain further information about the gluon distributions. 
Quantum Chromodynamics, or QCD, is used to probe and determine the strong interactions among the partons within the hadrons; in the inelastic scattering collisions probed, perturbative QCD is utilized since interactions among quarks and gluons within the hadron are analyzed. QCD is a type of quantum field theory, introduced in 1973, specifically centered around strong interactions in which its Lagrangian is invariant under transformations around the $S U(3)$ symmetry group [32] [33] [34]. Gell-Mann, Ne'eman and Zweig first brought forth a model in 1963, in an attempt to understand the effects of hadron production in nuclear scattering, where the hadrons were to be governed by this symmetry type. This led to quarks being introduced as the most fundamental element of QCD; the quarks and gluons constitute the elementary fields in this field theory type [35] [36] [37] [38]. Currently, perturbative QCD cannot be used to determine the full cross section of the collisions since color charged particles within the hadron cannot be isolated and observed. Therefore the cross section is divided into a hard part that can be determined through the use of perturbative QCD, and a "soft part" that currently can only be reliably found through experimentation. The soft part's dependence on $x$ and $Q^{2}$ is what allows us to separate the hadronic cross sections and composite Feynman amplitudes into these hard and soft parts. In our computations, ANNs are utilized in attempt to determine the cross section for DIS processes where electrons are scattered off of protons and neutrons. The classical Lagrangian that corresponds to the QCD field theory is given in equation 1.3.

$$
L_{\text {class }}=\underset{\text { quarks }}{\Sigma_{a}} \bar{\Psi}_{a}\left(i \gamma_{\mu} D^{\mu}-m\right)_{a b} \Psi_{b}-\frac{1}{4} \operatorname{Tr} G_{\mu \nu}^{A} G_{A}^{\mu \nu}
$$

In the QCD lagrangian, $\Psi_{a}$ are the quark vector fields, $A$ is the gluon vector field, $G_{A}^{\mu \nu}$ is the corresponding gluon field strength tensor and $D^{\mu}$ is the covariant derivative.

In QCD there is a basic coupling $g$ regarding the subatomic interactions is dimensionless. However, the quark gluon interactions within deep inelastic scattering contain loop corrections to the quark gluon coupling and so a new scale to the effective coupling is added. The coupling is now given below for $\alpha_{s}\left(Q^{2}\right)$ in Next to Leading Order (NLO) in equation 1.4 with $\Lambda$ as the associated scale for the $Q^{2}$ values and $\beta_{0}$ and $\beta_{1}$ as positive constants obtained from expansions of Beta functions whose values depend on the number of quark flavors included in the coupling. 


$$
\frac{\alpha_{s}}{4 \pi}=\frac{1}{\left(\beta_{0} \ln \frac{Q^{2}}{\Lambda^{2}}\right)}-\frac{\beta_{1}}{\beta_{0}} \frac{\ln \left(\ln \left(\frac{Q^{2}}{\Lambda^{2}}\right)\right)^{2}}{\left(\beta_{0} \ln \frac{Q^{2}}{\Lambda^{2}}\right)}
$$

Our knowledge of the quark distributions in the parton today is still only reliably obtained from DIS experiments. The starting point for the separation of the unpolarized cross section for the inelastic scattering interactions is given in expression 1.5. This expression for the cross section allows us to begin to extract the hadronic tensors and subsequently the structure functions due to parity conservation and electromagnetic current conservation. This results in invariance of the Feynman amplitude $T$.

$$
d \sigma=\frac{1}{\operatorname{flux}} \frac{d^{3} k^{\prime}}{2 k_{0}^{\prime}} \frac{1}{4} \underset{\sigma \lambda \lambda^{\prime}}{\sum_{1}}\left|T^{2}\right|
$$

$\lambda$ and $\lambda^{\prime}$ are the helicities of the lepton before and after it scatters and $\sigma$ is the spin of the proton. $k^{\prime}$ is given by $k^{\prime}=q-k$. The flux refers to the flux of the incoming neutron beam, defined to be flux $=\sqrt{\nu^{2}+Q^{2}}$ where $\nu$ in terms of the kinematics $M, W^{2}$ and $Q^{2}$ can be written as $\nu=\frac{1}{2 M}\left(W^{2}+Q^{2}-M^{2}\right) . k_{0}^{\prime}$ is the $0^{\text {th }}$ component among the 4 momentum components of $k^{\prime}$. The scattering amplitude $T$ is given in expression 1.6.

$$
T=e^{2} \bar{u}\left(k^{\prime}, \lambda^{\prime}\right) \gamma^{\mu} \mu(k, \lambda) \frac{1}{q^{2}}<X|| J_{\mu}^{e m} \mid p, \sigma>
$$

$X$ is the hadronic state after scattering and $J_{\mu}^{e m}$ is the electromagnetic current. The cross section can now be expressed in terms of the structure functions, allowing us to evaluate the structure functions in order to determine them. This is shown below in expression 1.7.

$$
k_{0}^{\prime} \frac{d \sigma}{d^{3} k^{\prime}}=\frac{2 M}{s-M^{2}} \frac{\alpha^{2}}{Q^{4}} l^{\mu \nu} W_{\mu \nu}
$$

$s$ in terms of the initial hadron and lepton momenta defined previously is $s=(p+k)^{2}$. The leptonic tensor is given below in equation 1.8. 


$$
\begin{array}{r}
l_{\mu \nu}\left(k, k^{\prime}\right)=\frac{1}{2} \operatorname{Tr}\left(k^{\prime} \gamma_{\mu} k \gamma_{\nu}\right)= \\
2\left(k_{\mu} k_{\nu}^{\prime}+k_{\mu}^{\prime} k_{\nu}-\frac{1}{2} Q^{2} g_{\mu \nu}\right)
\end{array}
$$

The Hadronic tensor is given in equation 1.9.

$$
W_{\mu \nu}(p, q)=\frac{1}{4 M} \sum_{\sigma} \int \frac{d^{4} \eta}{2 \pi} e^{i q \cdot \eta}<p, \sigma\left|T\left(J_{\mu}^{e m}(\eta) J_{\nu}^{e m}(0)\right)\right| p, \sigma>
$$

The leptonic and hadronic tensors can be combined as shown in equation 1.10.

$$
l^{\mu \nu} W_{\mu \nu}=2 W_{1} Q^{2}+\frac{1}{M^{2}} W_{2}\left[\left(s-M^{2}\right)\left(s-W^{2}-Q^{2}\right)-M^{2} Q^{2}\right]
$$

$W^{2}$ in terms of the other kinematics is given by $W^{2}=Q^{2}\left(\frac{1}{x}-1\right)+M^{2}$. The resulting cross section is given below in relation 1.11.

$$
\begin{array}{r}
\frac{d^{2} \sigma}{d Q^{2} d W^{2}}=\frac{2 \pi \alpha^{2} M}{s-M^{2} Q^{2}}\left[2 W_{1}\left(W^{2}, Q^{2}\right)\right. \\
\left.+W_{2}\left(W^{2}, Q^{2}\right)\left\{\frac{\left(s-M^{2}\right)\left(s-W^{2}-Q^{2}\right)}{M^{2} Q^{2}}-1\right\}\right]
\end{array}
$$

The Mott Cross Section is given in expression 1.12.

$$
{\frac{d \sigma}{d Q^{2}}}_{\text {MOTT }}=\frac{4 \pi \alpha^{2}}{Q^{4}}\left[\frac{\left(s-M^{2}\right)\left(s-W^{2}-Q^{2}\right)}{M^{2} Q^{2}}-1\right]
$$

The combined result of these cross sections is in expression 1.13.

$$
\begin{gathered}
\frac{d^{2} \sigma^{D I S}}{d Q^{2} d W^{2}}=\frac{1}{2 M}\left[2 W_{1}\left(W^{2}, Q^{2}\right)\left\{\frac{\left(s-M^{2}\right)\left(s-W^{2}-Q^{2}\right)}{M^{2} Q^{2}}-1\right\}^{-1}\right. \\
\left.+W_{2}\left(W^{2}, Q^{2}\right)\right] \frac{d \sigma}{d Q^{2}}{ }_{\text {MOTT }}
\end{gathered}
$$


The structure functions $W_{1}$ and $W_{2}$ can also be expressed terms of each other in the limit $Q^{2} \rightarrow \infty$ by the Callan Gross relation [39] in relation 1.14. Because the quark currents coupled to the photon consisted of spin $\frac{1}{2}$ particles, Callan and Gross [39] were able to relate the two scaling structure functions $F_{1}$ and $F_{2}$ using the relation in formula 1.15. Given the relationship between $W_{1}$ and $F_{1}$ shown in formula 1.16, this leads directly to equation 1.17 for relating $W_{2}$ to $F_{2}$.

$$
2 x M W_{1}=W_{2}
$$

$$
F_{2}(x)=2 x F_{1}(x)
$$

$$
M W_{1}=F_{1}
$$

$$
\frac{1}{2 M}\left(W^{2}+Q^{2}-M^{2}\right) W_{2}=F_{2}
$$

The equations for the scattering cross section use only Lorentz scalars; therefore they can be computed in any frame. As an example, the following kinematics, shown in equation 1.18 for the target nucleon rest frame, can be used in order to compute the cross section. The kinematic $y$ is given by $y=\frac{W^{2}+Q^{2}-M^{2}}{\left(s=M^{2}\right)}$ and is related to $x$ and $s$ by $Q^{2}=s x y$. At higher scattering energies $s>>M$ and the scaling of $x$ becomes closer to Bjorken scaling.

$$
\begin{array}{r}
E=\frac{\left(s-M^{2}\right)}{2 M}, E^{\prime}=\frac{\left(s-M^{2}\right)}{2 M}(1-y) \\
\left(\sin \frac{\theta}{2}\right)^{2}=\frac{M^{2} x y}{\left(s-M^{2}\right)(1-y)}, \frac{d \Omega d E^{\prime}}{d x d y}=\frac{2 \pi M y}{1-y}
\end{array}
$$

These terms enable us to rewrite the inelastic cross section as follows in expression 1.19. 


$$
\begin{array}{r}
\frac{d^{2} \sigma}{d \Omega d E^{\prime}}=\frac{\alpha^{2} M}{8 E^{2} E_{h}\left(\sin \frac{\theta}{2}\right)^{4}}\left[2 W_{1}\left(\sin \frac{\theta}{2}\right)^{2}\right. \\
+W_{2} \frac{4 E_{h}^{2}}{M^{2}}\left(\cos \frac{\theta}{2}\right)^{2}
\end{array}
$$

$E^{\prime}$ and $E$ are the initial and final energies of the scattered electron, $E_{h}$ is the target hadron energy and $\theta$ is the scattering angle. In terms of the computation of the PDFs and the PDF moments, to be used in normalizing the PDFs, the input variables $Q^{2}$, where $q^{2}=\left(k-k^{\prime}\right)^{2}=-Q^{2}$, and $x$ are utilized. These scattering energies, momentum transfers and momentum fractions can additionally be written in terms of each other through the relations $Q^{2}=4 E E^{\prime}\left(\sin \frac{\theta}{2}\right)^{2}$ and $x=\frac{Q^{2}}{2 M \nu}$. In the process of determining the valence quark PDFs, the variables $y=\frac{\nu}{E}$ and $z=\frac{x}{y}$ are also introduced, with integration being done over $z$ to compute the PDFs. $W^{2}$ is an invariant which can also be written as $W^{2}=p_{X}^{2}$ where $p_{X}^{2}$ is the momentum of the hadronic system in the inelastic scattering process.

The kinematics for the inelastic collision can also be written in terms of the the momentum $p$ of the target nucleon. The variable $\nu$ can be written, for example, in the form $\nu=\frac{p \cdot q}{M}=\frac{W^{2}+Q^{2}-M^{2}}{2 M}$. In terms of the momenta of the leptons and nucleons and hadronic systems, the kinematics of the momentum fractions are $x=\frac{-\left(k-k^{\prime}\right)^{2}}{2 P \cdot\left(k-k^{\prime}\right)}$ and $y=\frac{p \cdot\left(k-k^{\prime}\right)}{p \cdot k}$.

The flexibility of the inelastic cross section expressions finally allows us to use the HERA frame, or the lab frame of an electron with energy $E$ colliding with a proton (or other target nucleon) of energy $E_{p}$ with $E^{\prime}$ and $\theta$ referring to the same kinematics used in the other frames. In this frame, the inelastic cross section can be written in terms of the electron and proton kinematics as follows in expression 1.20 and expression 1.21.

$$
\begin{array}{r}
(1-y)=\frac{E}{E^{\prime}} \cos \left(\frac{\theta}{2}\right)^{2}, x y=\frac{E^{\prime}}{E_{p}} \sin \left(\frac{\theta}{2}\right)^{2} \\
\frac{d \Omega d E^{\prime}}{d x d y}=\frac{4 \pi E \sin \left(\frac{\theta}{2}\right)^{2}}{x}
\end{array}
$$




$$
d^{2} \sigma d \Omega d E^{\prime}=\frac{\alpha^{2} M}{8 E^{2} E_{p} \sin \left(\frac{\theta}{2}\right)^{4}}\left[2 W_{1} \sin \left(\frac{\theta}{2}\right)^{2}+W_{2} \frac{4 E_{p}^{2}}{M^{2}} \cos \left(\frac{\theta}{2}\right)^{2}\right]
$$

The first set of inelastic scattering experiments indicated how the structure functions $W_{1}$ and $\nu W_{2}$ are scaled according to the kinematic $x$. Bjorken predicted that in the high energy limit $\nu, Q^{2} \rightarrow \infty$ the structure functions approach the limits $M W_{1}\left(x, Q^{2}\right) \rightarrow$ $F_{1}(x)$ and $\nu W_{2}\left(x, Q^{2}\right) \rightarrow F_{2}(x)[40]$.

The elastic contributions to the cross sections can also be determined in terms of the elastic structure functions. These structure functions are computed chiefly in terms of the electromagnetic form factors $G_{M}^{2}$ and $G_{E}^{2}$. These elastic contributions $W_{1}^{e l}$ and $W_{2}^{e l}$ are below in expression 1.22 .

$$
\begin{gathered}
W_{1}^{e l}\left(W^{2}, Q^{2}\right)=\delta\left(W^{2}-M^{2}\right) \frac{Q^{2}}{2 M} G_{M}^{2}\left(Q^{2}\right) \\
W_{2}^{e l}\left(W^{2}, Q^{2}\right)=\delta\left(W^{2}-M^{2}\right) 2 M G\left(Q^{2}\right)
\end{gathered}
$$

The electromagnetic form factor $G\left(Q^{2}\right)$ can also be expressed in terms of the individual electric and magnetic form factors seen in expression 1.23.

$$
G\left(Q^{2}\right)=\left[G_{E}^{2}+\frac{Q^{2}}{4 M^{2}} G_{M}^{2}\right]\left(1+\frac{q^{2}}{2 m^{2}}\right)
$$

The elastic scattering cross section expression can now be completed, shown in expression 1.24 .

$$
\frac{d \sigma^{e l}}{d Q^{2}}=\frac{2 \pi \alpha^{2}}{\left(s-M^{2}\right)^{2}}\left[G_{M}^{2}\left(Q^{2}\right)+\frac{2\left(s-M^{2}\right)^{2}-s Q^{2}}{Q^{4}} G\left(Q^{2}\right)\right]
$$

The electromagnetic form factors approach $\frac{1}{Q^{2}}$ for larger values of $Q^{2}$; however, for $Q^{2}<5 \mathrm{GeV}^{2}$ the cross sections and structure functions of partonic, hadronic and parton hadron interactions must include the elastic components. 
The structure functions $W_{1}$ and $W_{2}$ can also be computed in terms of each other using their relationships to the cross sections for the transverse and longitudinal boson exchanges in DIS. This computation begins with the standard photon polarization vectors $e_{\nu}$ whose individual 4 momentum and transverse components meet conditions $e \cdot q=0$, $e_{T}^{2}=-1$ and $e_{0}^{2}=1$. The transverse and longitudinal scattering cross sections $\sigma_{T}$ and $\sigma_{0}$ can now be written as follows in terms of the electromagnetic flux shown in equation 1.25 .

$$
\begin{array}{r}
\sigma_{T}=\frac{4 \pi \alpha^{2} W_{1}}{\text { flux }} \\
\sigma_{T}+\sigma_{0}=4 \pi \alpha^{2}\left(1+\frac{\nu^{2}}{Q^{2}}\right) \frac{W_{2}}{\text { flux }}
\end{array}
$$

The structure functions $W_{1}$ and $W_{2}$ can now be expressed in terms of the longitudinal and transverse cross sections $\sigma_{0}$ and $\sigma_{T}$ as shown in relation 1.26.

$$
\begin{array}{r}
W_{1}\left(\nu, Q^{2}\right)=\frac{\sqrt{\nu^{2}+Q^{2}}}{4 \pi \alpha^{2}} \sigma_{T} \\
W_{2}\left(\nu, Q^{2}\right)=\frac{Q^{2}}{4 \pi \alpha^{2} \sqrt{\nu^{2}+Q^{2}}}\left(\sigma_{0}+\sigma_{T}\right)
\end{array}
$$

Given the ratio of longitudinal to transverse cross sections $R=\frac{\sigma_{0}}{\sigma_{T}}$ the structure functions can now be written in terms of each other as follows in expression 1.27 and equation 1.28 .

$$
\frac{W_{1}\left(\nu^{2}, Q^{2}\right)}{W_{2}\left(\nu^{2}, Q^{2}\right)}=(1+R)^{-1}\left(1+\frac{\nu^{2}}{Q^{2}}\right)
$$

The polarization vector $\epsilon$ of the virtual photon with a cross section proportional to $\sigma_{T}+\epsilon \sigma_{0}$ can be written below in formula 1.28.

$$
\epsilon^{-1}=1+2\left(1+\frac{\nu^{2}}{Q^{2}}\right)\left[\frac{\left(s-M^{2}\right)\left(s-2 M \nu-M^{2}\right)}{M^{2} Q^{2}}-1\right]^{-1}
$$

The total cross section can now be written as (equation 1.29) 


$$
\frac{d^{2} \sigma}{d Q^{2} d W^{2}}=\frac{\alpha}{\pi} \frac{M}{Q^{2}} \frac{\sqrt{\nu^{2}+Q^{2}}}{\left(s-M^{2}\right)^{2}} \frac{\sigma_{T}}{1-\epsilon}(1+\epsilon R)
$$

and since the longitudinal and transverse cross sections, representing scattering rates, must by their definition be positive, we obtain the following inequality (equation 1.30 ).

$$
0 \leq W_{1} \leq\left(1+\frac{\nu^{2}}{Q^{2}}\right) W_{2}
$$

In a Deep Inelastic Experiment, when the scattered particle is an electron or muon the kinematics above allow us to determine $Q^{2}, x$ and $y$ from the observables $E^{\prime}$ and $\theta$. In order to extract $W_{1}$ and $W_{2}$ from the differential cross section, this observable can be obtained for varying values of $\theta$ and $E$. This can be used to extract the value of $R$ for individual values of $\nu$ and $Q^{2}$ or the average value of $R$ can be computed for a full range of $x$ and $Q^{2}$ for an experimental data set. In collaborations that determine the structure function, an average $R$ value for the full range of kinematic variables is obtained and used to extract the structure functions from observable cross sections. This method is shown below in expression 1.31 .

$$
\nu W_{2}\left(x, Q^{2}\right)=\left[\frac{\frac{d^{2} \sigma}{d \Omega d E^{\prime}}}{\left(\frac{d \sigma}{d \Omega}\right)_{M o t t}}\right]\left[\frac{\nu \epsilon(1+R)}{1+\epsilon R}\right]
$$

A collaboration such as SLAC can therefore extract structure function values for a substantial $x$ and $Q^{2}$ range once the differential cross section is found for a given set of $E^{\prime}$ and $\Theta$ values .

The hadron structure functions can be separated in terms of the Parton Distribution Functions, or PDFs, and the charges on each of the parton types in the hadron. The PDFs define the probability that a parton type will be found with at a specific $x$ value for an input $Q^{2}$. Since QCD dictates that free quarks do not exist when a hadron is struck by an electron, it is necessary to know the values of the PDFs for all quarks within a hadron in a collision. 


\subsection{Quark Parton Model}

A fundamental understanding of the quark parton model is needed in order to evaluate the quark and gluon PDFs and to determine the scaling of the kinematics of which these PDFs are dependent. In this model, the partons within the hadrons probed by high energy leptons behave as collections of scattering components. The hadrons probed in high energy DIS reactions have a dependency not on the energy of all components in the scattering but on the specific kinematics discussed previously, for example $\Theta$ and $\nu$. This dependency is referred to as Bjorken scaling. In addition, the partons within the hadron are governed by asymptotic freedom. This refers to the principle that the strength of the coupling constant $\alpha_{s}\left(Q^{2}\right)$, which is given in Next to Leading Order in 1.4, weakens for the strong interactions at increasing energies. In the Deep Inelastic Scattering model, if we use the target rest frame with the virtual photon emitted along the $\mathrm{z}$ axis, we have $p=(M, 0,0,0)$ and $q=\left(\nu, 0,0,-\sqrt{\nu^{2}+Q^{2}}\right)$. In terms of these 4 momentum kinematics, the light cone variables $a^{+}=\frac{a^{0}+a^{3}}{\sqrt{2}}$ and $a^{-}=\frac{a^{0}-a^{3}}{\sqrt{2}}$ can be introduced, which give us the scalar product $a \cdot b=a^{+} b^{+}+a^{-} b^{-}-a_{T} \cdot b_{T}$ resulting in equation 1.32 .

$$
q^{+}=-\frac{M x}{\sqrt{2}}, q^{-}=\frac{(2 \nu+M x)}{\sqrt{2}}
$$

$q^{+}$and $q^{-}$represent the previously defined light cone variables for 4 momentum $q$. In the limit $Q^{2} \rightarrow \infty, q^{-} \rightarrow \infty$ and $q^{+}$is a constant. The space time interval centered around the points where the currents $J_{\mu}(\xi)$ and $J_{\mu}(0)$ affect the DIS are space time scales of interest. $\xi$ is the space time interval between the points at which the eletromagnetic currents $J_{\mu}(\xi)$ and $J_{\mu}(0)$ act on the scattering process. In the Bjorken limit, the space time intervals can be approximated as $\xi^{+} \rightarrow 0$ and $\xi^{-}<\frac{\sqrt{2}}{M x}$. Because all of the space time components in which the electromagnetic current act, except for $\xi^{-}$, approach 0 , the characterization of DIS is shown to be light cone dominated physics.

In order to derive the $Q^{2} \rightarrow \infty$ limits of the structure functions, we start with constructing the previously determined hadronic tensor $W_{\mu \nu}$ that describes the physics in the hadron parton model. This tensor is given below in expression 1.33. 


$$
W_{\mu \nu}(q, p)=\sum_{i} \sum_{s} \int d^{4} k f_{s}^{i}(p, k) w_{\mu \nu}^{i}(q, k) \delta\left[k+q^{2}\right] d x
$$

This tensor sums the individual tensors $w_{\mu \nu}^{i}(q, k)$, describing the interactions between quarks and virtual photons, and the structure functions $f_{s}^{i}(p, k)$ which are dependent on the scalar $p \cdot k$. The kinematics of the parton model can be used to show that the fraction of light cone momentum carried by quark $x_{i}=\frac{k^{+}}{p^{+}}$is the same as the Bjorken momentum fraction $x$. This is because we have the kinematic relations $k^{2}=2 k^{+} k^{-}-k_{T}^{2}=0$ and $k^{-}=\frac{k_{T}^{2}}{2 x_{i} p^{+}}$, leading to $k \cdot q=k^{+} q^{-}+k^{-} q^{+}=x_{i} p^{+} q^{-}=x_{i} p \cdot q=x_{i} M \nu$. Now, we note that $k+q^{2}=k^{2}+2 k \cdot q+q^{2}=2 x_{i} M \nu-Q^{2}=2 x_{i} M \nu-2 x M \nu=2 M \nu\left(x_{i}-x\right)$. From here, we see that the delta function becomes $\delta\left[k+q^{2}\right]=\frac{1}{2 M \nu} \delta\left[x_{i}-x\right]$. The new structure function tensor that comes from this change in variables is written below in expression 1.34 .

$$
\begin{array}{r}
W_{\mu \nu}(q, p)=\sum_{i} e_{i}^{2} \int \frac{d^{4} k}{2 M \nu}\left[f_{+}^{i}(p \cdot k)+f_{-}^{i}(p \cdot k)\right] \delta\left(x_{i}-x\right) \\
\times\left[2 k_{\mu} k_{\nu}+k_{\mu} q_{\nu}+q_{\mu} k_{\nu}-g_{\mu \nu} k \cdot q\right]
\end{array}
$$

This expression for the hadronic tensor can be used to determine how structure functions relate to each other in the Bjorken limit. In the condition $\nu=\mu=0$ the tensor is written below in expression 1.35 . 


$$
\begin{array}{r}
W_{00}=\sum_{i} e_{i}^{2} \int \frac{d^{4} k}{2 M \nu}\left[f_{+}^{i}(p \cdot k)+f_{-}^{i}(p \cdot k)\right] \delta\left(x_{i}-x\right) \\
\times\left[2 k_{0} k_{0}+k_{0} q_{0}+q_{0} k_{0}-g_{00} k \cdot q\right]= \\
\sum_{i} e_{i}^{2} \int \frac{d^{4} k}{2 M \nu}\left[f_{+}^{i}(p \cdot k)+f_{-}^{i}(p \cdot k)\right] \delta\left(x_{i}-x\right) \\
\times\left[2 k_{0} k_{0}+2 q_{0} k_{0}-g_{00} x_{i} M \nu\right]= \\
\sum_{i} e_{i}^{2} \int \frac{d^{4} k}{2 M \nu}\left[f_{+}^{i}(p \cdot k)+f_{-}^{i}(p \cdot k)\right] \delta\left(x_{i}-x\right) \\
\times\left[2 k_{0}\left(k_{0}+q_{0}\right)-x_{i} M \nu\right]= \\
\sum_{i} e_{i}^{2} \int \frac{d^{4} k}{2 M \nu}\left[f_{+}^{i}(p \cdot k)+f_{-}^{i}(p \cdot k)\right] \delta\left(x_{i}-x\right) \\
\times \nu\left(2 k_{0}-x M\right)
\end{array}
$$

The condition $\nu=\mu=0$ also gives the following relation (equation 1.36 ) for the corresponding structure function tensor.

$$
W_{00}=\frac{\nu^{2}}{M Q^{2}}\left[-M W_{1}+\frac{1}{2 x} W_{2}\right]
$$

These two expressions for the hadronic tensor $W_{00}$ can be reduced, since $W_{00} \rightarrow 0$ in the target rest frame, to the structure function relations $\nu W_{2}=2 x M W_{1}$ and $\nu W_{2} \rightarrow F_{2}(x)$, the latter the result of the Bjorken limit. In addition, the structure functions can now be expressed in terms of the individual quark distributions as well. This relation is described in 1.37 .

$$
F_{2}=\sum_{i} e_{i}^{2} x q_{i}(x)
$$

$q_{i}(x)$ in terms of the quark flavor distributions $f_{+}^{i}$ and $f_{-}^{i}$ is given in expression 1.38.

$$
q_{i}(x)=\frac{\pi}{4} \int d k_{T}^{2}\left[f_{+}^{i}(p \cdot k)+f_{-}^{i}(p \cdot k)\right]
$$


$e_{i}$ is the charge of each parton according to the Standard Model and $q_{i}(x)$ is the subsequent PDF for that parton. $f_{+}^{i}(p \cdot k)$ and $f_{-}^{i}(p \cdot k)$ are the quark functions for $+\frac{1}{2}$ and $-\frac{1}{2}$ helicities. The conventional interpretation of $q_{i}(x)$ is the probability of finding a quark type $i$ that function is associated with containing a fraction of parton momentum $x$ in the inelastic scattering interaction. The singlet and nonsinglet quark distributions must also be defined as well. The singlet distribution is $\Sigma(x)=\underline{\mathrm{i}} \Sigma\left(q_{i}(x)+\underline{\mathrm{i}} \bar{q}\right)$ and the nonsinglet distribution is $N S x=\underline{\mathrm{i}} \Sigma\left(q_{i}(x)-\underline{\mathrm{i}} \bar{q}\right)=u_{v}(x)+d_{v}(x)$, with $u_{v}(x)$ as the up valence quark distribution and $d_{v}(x)$ as the down valence quark distributions. In terms of the total up and down quark distributions $u(x)$ and $d(x)$, the valence quark distributions are $u_{v}(x)=u(x)-\bar{u}(x)$ and $d_{v}(x)=d(x)-\bar{d}(x)$. The total sea quark distribution is then $S(x)=2(\bar{u}(x)+\bar{d}(x)+\bar{s}(x)+\bar{c}(x))$.

The parton distributions in the quark model exhibit unique characteristics in the limit $x \rightarrow 1$, the elastic limit for the DIS. The structure function $W_{1}$, from which the other structure functions can be computed, takes the following form ( equation 1.39 ).

$$
W_{1}\left(\nu, Q^{2}\right)=\delta\left(W^{2}-M^{2}\right) \frac{Q^{2}}{2 M} G_{M}^{2}\left(Q^{2}\right)
$$

This form factor dominates the $Q^{2}$ behavior of the structure functions in the resonance region of the parton model. The effect of the form factors in the resonance region for the parton model, where $M^{2}<W^{2}<4 \mathrm{GeV}^{2}$, means that values for these form factors in these regions affect the $Q^{2}$ behavior of the structure functions at sufficiently small $Q^{2}$ values and for large $x$ values. In order to isolate these contributions, the structure functions can be rewritten in terms of the rescaled variable $x^{\prime}$ with $x^{\prime}=x\left[1+\frac{m_{0}^{2} x}{Q^{2}}\right]^{-1}$ with $m_{0} \approx 1.1 \mathrm{GeV}$. The structure function in this rewritten scaling kinematic is below in expression 1.40 .

$$
\int d x^{\prime} F_{1}\left(x^{\prime}\right) \propto \sum_{\text {res }} \frac{Q^{2}}{2 M} G_{M, r e s}^{2}\left(Q^{2}\right)
$$

We can approximate the corresponding structure function $F_{1}(x) \propto 1-x^{\prime n}$ and approximate the electromagnetic form factor as $G_{M}\left(Q^{2}\right) \propto Q^{N}$. This leads to $Q^{2}$ for this form factor for $N=2$. Then, in the resonance region we also have the approximation $(1-x) \propto \frac{1}{Q^{2}}$ from which we get the relations $n=2 N-1$ and so we get the resonance 
region approximations $F_{1}(x), F_{2}(x) \propto 1-x^{3}$ for the large $x$ limit. The $Q^{2}$ dependence of the form factors and subsequent structure functions, dictated by the exponents within the form factors, are dictated by the constituent rules for these exponents [41] [42] . These rules state that the exponents in these factors are dependent only on the number of elementary constituents that exist in a hadronic bound state. These rules are based on the assumption that within this state at least one of the components is involved in a hard scattering process. For the $F_{2}(x)$ behavior the large $x$ limit under these rules is $F_{2}(x) \propto 1-x^{2 n_{s}-1}$. In this estimate, $n_{s}=n_{H}-1$ where $n_{H}$ is the total number of types of quarks found within the hadron.

The PDFs currently obtained at large $x$ limits can be estimated from experimental results by noting the relationships between the different structure function types and the PDFs that comprise them. For example, in the large $x$ limit, the quark distributions are valence dominated and sea quarks are negligible. Therefore, the estimation of the up and down valence quark distributions from the experimentally determined structure functions can be determined as follows in equation 1.41.

$$
\frac{F_{2}^{n}}{F_{2}^{p}}=\frac{1+4 \frac{d_{v}(x)}{u_{v}(x)}}{4+\frac{d_{v}(x)}{u_{v}(x)}}
$$

If $S U(6)$ symmetry were exact, we would have for large $x \frac{d_{v}(x)}{u_{v}(x)} \rightarrow \frac{1}{2}$. However, the experimental data from the SLAC and EMC collaborations show that for large $x, \frac{F_{2}^{u n}}{F_{2}^{u p}} \rightarrow$ $\frac{1}{4}$ which suggests that the limit for $\frac{d_{v}(x)}{u_{v}(x)}$ for large $x$ is $(1-x)$.

$\alpha_{s}\left(Q^{2}\right)$ as a function of $Q^{2}$ is in Leading Order approximated by $\alpha_{s} \propto \frac{1}{\ln \left(\frac{Q^{2}}{\Lambda^{2}}\right)}$ and so for very large $Q^{2}$ values $\alpha_{s}$ becomes very small. This approximation is the focus of Perturbative QCD. For smaller $Q^{2}$ values $\alpha_{s}$ becomes larger and different methods need to be used to probe these strong interactions. Lattice QCD is a primary example of the techniques that are used. The SOMPDF code will use QCD computations to determine the moments for $Q^{2}$ values ranging from $1.0 \mathrm{GeV}^{2}$ to $30000 \mathrm{GeV}^{2}$. These $Q^{2}$ values are taken from experimental measurements of the structure functions at specific kinematics. 


\subsection{Perturbative QCD}

The perturbative regime of QCD treats quarks in the target hadron as being very weakly bound for lower scattering energies and approaching free point-like particles in their behavior for larger energies. This theory of asymptotic freedom should be derived from QCD field theories in order to fundamentally understand DIS.

QCD is symmetric relative to the colors of the quarks in question. Its Lagrangian is in expression 1.42 .

$$
L=\frac{1}{2} G_{\mu \nu}^{\alpha} G_{\alpha}^{\nu \mu}+\sum_{k}^{N_{f}} \bar{q}_{k}\left(D_{\mu}-m_{k}\right) q_{k}
$$

In the above Lagrangian, $q_{k}$ is the 4 -vector propagation of the quark $k$ in the target hadron, where $k$ is one of the $N_{f}$ types of quarks that are taken into account in the hadron. $G$ is the 4 vector propagation of gluons in the hadron. The gluon propagation vectors $G_{\mu \nu}^{\alpha}$ and $D_{\mu}$ can be written as follows in expression 1.43.

$$
\begin{array}{r}
G_{\mu \nu}^{\alpha}=\partial_{\mu} A_{\nu}^{\alpha}-\partial_{\nu} A_{\mu}^{\alpha}+g f_{a b c} A_{\mu}^{b} A_{\nu}^{c} \\
D_{\mu}=\partial_{\mu}-i g T^{\alpha} A_{\mu}^{\alpha}
\end{array}
$$

In the gluon vector component expressions, $A_{\mu}^{\alpha}$ is the $\mu_{t h}$ component of the $a_{t h}$ gluon propagation vector. The gluons in a hadron are confined by $S U(3)$ symmetry. Therefore, the matrices $T^{\alpha}$ describing the gluon 4 vectors must obey the relation $\left[T^{\alpha}, T^{\beta}\right]=i f_{a b c} T^{c}$. Gluon loops are associated with color factors that are needed to normalize the dimensionless quark gluon coupling factor $g$ and the vector fields associated with gluon gluon and quark quark interaction loops. $T_{2}(F)=\frac{1}{2}$ is the color factor normalizing $g, C_{2}(F)=\frac{4}{3}$ is the color factor associated with the quark quark interactions and $C_{2}(A)=3$ is the color factor for the gluon gluon interactions. The quark gluon coupling $g$ needs to modified to include all the higher order loop corrections to quark gluon interactions. Given quark gluon interactions with momentum $Q$, the gluon and quark loop corrections to the coupling result in the coupling being expressed in equation 1.44 as 


$$
\bar{g}=\bar{g}\left(Q^{2}\right)=g-\beta_{0} \frac{g^{3}}{32 \pi^{2}}\left[\ln \frac{Q^{2}}{\Lambda^{2}}+\kappa\right]+\frac{3}{2} \beta_{0}^{2} \frac{g^{5}}{\left(32 \pi^{2}\right)^{2}} \ln ^{2} \frac{Q^{2}}{\Lambda^{2}}
$$

This is a Green's function which is non convergent for $\Lambda^{2} \rightarrow \infty$. In order to solve the problem of divergence of this modified coupling, a scaling choice $\mu$, where $Q^{2}=\mu^{2}$ which can renormalize the coupling needs to be introduced. Then, when the coupling is fixed at this introduced scaling parameter the coupling at any other $Q^{2}$ value could be expressed in terms of this fixed coupling quantity. This leads to the modified coupling now being independent of the wavelength $\Lambda$; however, the dependence on the renormalization scale $\mu$ is the necessary trade-off to achieve this independence.

The Green's functions in the QCD can be renormalized from here using the modified group equation. To provide an example of this equation, we can start with a simply scalar field $\omega_{0}$ which represents a field theory with interactions quantified by coupling $g_{0}$. The renormalized field $\omega$ can be introduced in terms of the initially defined scaling parameter $\mu$ and the scale factor $Z$ as $\omega_{0}=Z_{\omega}\left(g_{0}, \frac{\Lambda}{\mu}\right) \omega$. These fields have Lagrangians that describe the same types of parton loop propagation's; therefore, the Green's functions for the QCD fields can be written as $\Gamma^{(n)}(p, g, \mu)=Z_{\omega}\left(g_{0}, \frac{\Lambda}{\mu}\right) \Gamma_{0}^{(n)}\left(p, g_{0}, \Lambda\right)$. This Green's function's dependence has been taken away from the momentum scaling value $\Lambda$ and in the process it has become necessary to use the renormalization scaling $\mu$. This normalization constraint $\mu d \Gamma / d \mu=0$ gives the following constraint in expression 1.45 on the renormalized Green's function in terms of how it must vary relative to the coupling and remormalization scaling.

$$
\begin{array}{r}
{\left[\mu \frac{\partial}{\partial \mu}+\beta(g) \frac{\partial}{\partial g}-n \gamma_{\omega}(g)\right] \Gamma^{(n)}(p, g, \mu)=0} \\
\gamma_{\omega}(g)=\mu \frac{\partial}{\partial \mu}\left[Z_{\omega}\left(g_{0}, \frac{\Lambda}{\mu}\right)\right] \\
\beta(g)=\mu \frac{\partial}{\partial \mu} g(\mu)
\end{array}
$$

For each type of coupling among quarks and gluons, there is typically a single $\beta$ field, which is needed to determine the behavior of the effective couplings within the target hadron in the high energy limit. The quark and vector propagation vectors are associated with anomalous dimensions $\gamma_{F}(g)$ and $\gamma_{A}(g)$. The RGE equation solutions can first 
be analyzed with a solution where the total propagation of the hadron components is zero, i.e. $\gamma_{\omega}(g)=0$. Let's also take the variable $t$, with $t$ being the solution to $t=\int \frac{d g}{\beta(\bar{g})}$. Changing the external momentum $p$ to the hadronic system to $p \rightarrow e^{t} p$ requires the renormalization scaling factor $\mu$ to change to $\mu \rightarrow e^{-t} \mu$. In this case, the coupling value $g$ must change to $g \rightarrow \bar{g}(\mu)$. The Green's function, as a result of the renormalization, changes to $\Gamma\left(e^{t} p, g, \mu\right)=\Gamma(p, \bar{g}(t), \mu)$. If the propagation vectors of the quarks and gluons are not conserved, the Green's functions changes by the factor $\Gamma\left(e^{t} p, g, \mu\right)=\Gamma(p, \bar{g}(t), \mu) \exp \left\{-n \int d t^{\prime} \gamma\left(\bar{g}\left(t^{\prime}\right)\right)\right\}$. The $\beta$ function can also be computed from an independent Green's function [43]; the result is shown in equation 1.46.

$$
\beta(g)-\gamma_{A}(g) g-2 \gamma_{F}(g) g=-\frac{g^{3}}{16 \pi^{2}}\left[\left(\frac{3}{2}+\frac{\eta}{2}\right) C_{2}(A)+2 \eta C_{2}(F)\right]
$$

resulting in $\beta(g)=-\beta_{0}\left(\frac{g^{3}}{16 \pi^{2}}\right)$ with $\beta_{0}$ defined as $\beta_{0}=11-\frac{2}{3} N_{f}$. The solution to the rescaled coupling factor is now $\frac{d \bar{g}(t)}{d t}=-\beta_{0} \frac{\bar{g}^{3}}{16 \pi^{2}}$.

The rescaled coupling, including all the quark and gluon interactions during DIS, is related to the strong coupling constant by $\alpha_{s}=\bar{g}\left(Q^{2}\right) / 4 \pi$. The strong coupling factor $\alpha_{s}$ can also be written as $\ln \frac{Q^{2}}{\mu^{2}}=-\int \frac{4 \pi}{\beta_{0}} \frac{d \alpha_{s}}{\alpha_{s}^{2}}$. The resulting expression for the strong coupling is now in formula 1.47. Both sides of 1.47 must be set to a constant in order to satisfy it. When this constant is set to $-\frac{\beta_{0}}{4 \pi} \ln \Lambda^{2}, 1.47$ produces the Leading Order term in 1.4.

$$
\frac{1}{\alpha_{s}\left(Q^{2}\right)}-\frac{\beta_{0}}{4 \pi} \ln Q^{2}=\frac{1}{\alpha_{s}\left(\mu^{2}\right)}-\frac{\beta_{0}}{4 \pi} \ln \mu^{2}
$$

The fact that $\beta_{0}>0$ results in the relation $\alpha_{s}\left(Q^{2}\right) \rightarrow 0$ for $Q^{2} \rightarrow \infty$. It is also dependent on the scaling factor $\Lambda$ which can only be determined from experimentation. For perturbative expansions in QCD to be effective $\alpha_{s}\left(Q^{2}\right.$ must also be sufficiently small relative to the loop expansion terms to the coupling so that Taylor series expansions are still applicable.

The inelastic scattering cross section needs to be factored into the parts where the principles of perturbative QCD apply and where they do not apply as noted in subsection 1.3. In order to do this, the components of the inelastic scattering governing the virtual photon exchange must be split into their subsequent perturbative and non perturbative 
parts. The factoring of the virtual Compton scattering amplitude in DIS is where the application of QCD becomes fully understood. The first part of the Compton scattering is in terms of interactions among the hadronic and leptonic components. Specifically, this part is the amplitude section describing long distance interactions involving low and high energy exchanges; for this part the techniques of perturbative QCD do not apply. The second part involves exchanges of high energy values and has singularities for large $Q^{2}$ values that can be handled by perturbative QCD; its computation is done by higher order series expansions of the coupling $\alpha_{s}$ which are increasingly accurate for $\alpha_{s} \rightarrow 0$. We start with the forward scattering amplitude of the virtual photon $T_{\mu, \nu}\left(q^{2}, \nu\right)$, making it scalar instead of a 4 vector for further simplification. The scalar amplitude is given in expression 1.48 by

$$
T\left(q^{2}, \nu\right)=i \sum_{\sigma} \int d^{4} \eta e^{i q \cdot \eta}<p, \sigma|T(J(\eta) J(0))| p, \sigma>
$$

This complex plot has a pole relative to $\nu$ at $\nu=\frac{-q^{2}}{2 M}$ and a cut of simple poles from $\nu=$ $\frac{\left(2 M m_{\pi}-q^{2}\right)}{2 M}$ to $\nu=\infty$ and a cut of poles resulting from the process $p \rightarrow \gamma X$. These cuts have discontinuities at the $\nu$ and $Q^{2}$ values where the hadronic tensors, reduced now to a hadronic scalar, and the Compton amplitudes are related by $\operatorname{Im} T\left(q^{2}, \nu\right)=2 \pi W\left(q^{2}, \nu\right)$ and $T\left(q^{2}, \nu\right)=4 \int \frac{d \nu^{\prime} \nu^{\prime}}{\nu^{\prime 2}-\nu^{2}} W\left(q^{2}, \nu\right)$. In order to obtain the structure functions from these tensors, we use a technique referred to as operator product expansion (OPE). It begins with writing out the time ordered products of the Compton scattering amplitude as follows in expression 1.49 .

$$
i T(J(\eta) J(0)) \rightarrow \sum_{\tau=2}^{\infty} \sum_{n=0}^{\infty} C_{\tau, n}\left(\eta^{2}, \mu^{2}\right) \eta^{\mu_{1}} \ldots \eta^{\mu_{n}} O_{\mu_{1} \ldots \mu_{n}}^{\tau}\left(\mu^{2}\right)
$$

$\mu$ is the renormalization scale as was used earlier in reevaluating the Green's functions, $O^{\tau}$ is a set of traceless operators, $\tau$ is the twist number for the structure functions and $C_{\tau, n}$ represents the Wilson coefficients in the structure function expansions. The OPE results in the Compton scattering amplitude being expressed as follows in equation 1.50.

$$
T\left(q^{2}, \nu\right) \rightarrow \sum_{\tau, n} \frac{\partial}{\partial_{\mu_{1}}} \ldots \frac{\partial}{\partial_{\mu_{n}}}\left[\int d^{4} \eta e^{i q \cdot \eta} C_{\tau, n}^{\prime}\left(\eta^{2}, \mu^{2}\right)\left(\frac{1}{\eta^{2}}\right)^{1-\frac{\tau}{2}}\right] \times p_{\mu_{1}} \ldots p_{\mu_{n}} \bar{O}_{n}^{\tau}\left(\mu^{2}\right)
$$


with ( equation 1.51 )

$$
C_{\tau, n}\left(\eta^{2}, \mu^{2}\right)=C_{\tau, n}^{\prime}\left(\eta^{2}, \mu^{2}\right)\left(\frac{1}{\eta^{2}}\right)^{1-\frac{\tau}{2}}
$$

and also ( equation 1.52 )

$$
<p\left|O_{\mu_{1} \ldots \mu_{n}}^{\tau}\left(\mu^{2}\right)\right| p>=\bar{O}_{n}^{\tau}\left(\mu^{2}\right) p_{\mu_{1} \ldots \mu_{n}}
$$

The Compton amplitude can now be written ( equation 1.53 ) as a straightforward expression based on the $Q^{2}$ dependence of the Wilson coefficient functions and the renormalization scale dependence of the accompanying operators.

$$
T\left(q^{2}, \nu\right)=\sum_{\tau, n} \bar{C}_{\tau, n}\left(Q^{2}, \mu^{2}\right) \bar{O}_{n}^{\tau}\left(\mu^{2}\right)\left(\frac{1}{x}\right)^{n}\left(\frac{1}{Q^{2}}\right)^{\frac{\tau}{2}-1}
$$

Due to the symmetry of the Compton scattering amplitude, odd terms for $n$ vanish. Therefore, the relation between the Compton scattering amplitude and the structure function tensors can be written below in expression 1.54.

$$
\int_{-1}^{1} d x x^{n-1} W\left(q^{2}, x\right)=\frac{1}{4} \sum_{\tau=2}^{\infty} \bar{C}_{\tau, n}\left(Q^{2}, \mu^{2}\right) \bar{O}_{n}^{\tau}\left(\mu^{2}\right)\left(\frac{1}{Q^{2}}\right)^{\frac{\tau}{2}-1}
$$

The same OPEs applied to the electromagnetic current ( equation 1.55 ) yield a similar result for $F_{1}$.

$$
\int_{-1}^{1} d x x^{n-1} F_{1}\left(q^{2}, x\right)=\frac{1}{4} \sum_{\tau=2}^{\infty} \bar{C}_{\tau=2, n}\left(Q^{2}, \mu^{2}\right) \bar{O}_{n}^{\tau=2}\left(\mu^{2}\right)
$$

Since $F_{2}$ is the next highest moment structure function, the expression for this function changes to $n-2$ in the structure function integral. This application of the OPEs shows that all of the $Q^{2}$ dependence in the structure functions is contained in the Wilson coefficients. Defining the functions $f\left(y, \mu^{2}\right)$ and $\sigma\left(z, Q^{2}, \mu^{2}\right)$ so that $\int d y y^{n-1} f\left(y, \mu^{2}\right)=$ $\bar{O}_{n}^{\tau=2}\left(\mu^{2}\right)$ and $\int d z z^{n-1} \sigma\left(z, Q^{2}, \mu^{2}\right)=\bar{C}_{\tau=2, n}\left(Q^{2}, \mu^{2}\right)$ make the expansion of the structure functions in terms of their Wilson Coefficient and tensor components clearer in the 
physical sense. This expansion in terms of the functions relevant to the parton physics are below in expression 1.56 .

$$
F_{1}\left(x \cdot Q^{2}\right)=\int_{x}^{1} \frac{d y}{y} f\left(y, \mu^{2}\right) \sigma\left(z, Q^{2}, \mu^{2}\right)
$$




\section{SOMPDF}

The goal of the neural network we use is to create reliable theoretical models for the PDFs which also produce structure functions which sufficiently fit the experimental data and allow for various types of clustering of these models. The quality of fit is defined using the observable $\chi^{2}$. The $\chi^{2}$ is evaluated according to equation 2.1 [44] [45].

$$
\chi^{2}=\Sigma_{i_{\text {exp }}} \chi_{i_{\text {exp }}}^{2}=\Sigma_{i_{\text {exp }}} \Sigma_{\text {jdata }}\left(\frac{D_{\text {jdata }}^{i_{\text {exp }}}-T_{j \text { jata }}^{i_{\text {exp }}}}{\sigma_{\text {jdata }}^{i_{\text {exp }}}}\right)^{2}
$$

For each $i, D_{j \text { data }}^{i_{\text {exp }}}$ is the structure function measurement from the data set, $T_{j d a t a}^{i_{\text {exp }}}$ is the theoretically determined structure function value for the same kinematic and $\sigma_{\text {jdata }}^{i_{\text {exp }}}$ is the uncertainty of each structure function measurement from an experimental data set at a given kinematic.

For our SOMPDF code, the self organizing process can be used to analyze and separate how the individual PDFs contribute to the $\chi^{2}$ values and how the $\chi^{2}$ values are affected by attempts to fit the structure functions at large $x$ vs small $x$. They can also potentially be used to separate the effects of large $x$ corrections, particularly from dividing out the $\chi^{2}$ components by another arbitrarily chosen parametrized structure function, using the Target Mass Corrections (TMC) and the large $x$ Resummations (LxR). The SOM places the PDFs that are rescaled upwards and downwards by a preset range of possible magnitudes, or 'wiggled', on an $n \times n$ grid and organizes them around local regions. The organization is completed by finding the best fitting PDFs and matching the PDFs within a surrounding radius to the best matching PDF. By adjusting PDFs in local regions towards the best fitting $\mathrm{PDF}$, the $\mathrm{SOM}$ process can allow us to determine how each of the individual PDFs is wiggled in order to produce the best possible generated PDF and how the PDFs are wiggled in the small $x$ and large $x$ regions to produce the best possible functions. It could also allow us to separate the effects of the large $x$ physics corrections, chiefly the TMC and the LxR, by seeing how adding these corrections results in the structure functions being wiggled to form the structure functions most comparable to experimentalal data. Other supervised networks, in contrast, fit the structure functions to final data sets by using the PDF distributions without analysis of the specific aspects of these distributions and without visualizing how they affect 
the overall fit. Self organizing maps allow for isolation and proper visualizations of the various components of the structure functions, corrections to the structure functions and their composite PDFs. The SOM can also be used to determine how the $\chi^{2}$ values from the generated PDFs are organized and thereby allow us to see how these $\chi^{2}$ values are decreased over a set number of iterations. A map of the PDFs and the structure functions for large and small $x$ could also be shown in order to determine how these plots cluster in local regions around the best fitting structure functions and component PDFs.

The basic SOM algorithm can be defined as a non linear extension of Principal Component Analysis (PCA) [46]. In PCA one applies an orthogonal transformation to convert a set of data that are possibly correlated into sets of values that are linearly uncorrelated, and which constitute the principal components. The first principal component exhibits the largest variance, i.e. it is a straight line that minimizes the sum of the squared distances from the data points (least squares approximation) of the data set by a line. The second principal components is by subtracting from the original data vectors their projections onto the first principal component and by finding a new straight line approximation. The procedure is applied to the following components recursively. PCA is useful for interpreting the behavior of high dimensional data because, by allowing one to represent the dominant data sets in a linear form, and by simultaneously discarding the sub-dominant components, PCA can reduce the number of dimensions of the problem. PCA, however, cannot account for nonlinear relationship among data. Furthermore, it has poor visualization properties in cases where more than two dimensions are important [47]. The essential feature that sets the SOM algorithm a part from PCA and similar data reduction methods is that the lines resulting from PCA can be effectively replaced by lower dimensional manifolds in the SOM method. Because of their flexibility, these can detect features of the data that the PCA would not. In this procedure SOM essentially identifies two dimensional clusters which form among the generated map of functions and observables computed from these functions. These clusters would not be identifiable using PCA. In addition, SOMs have enhanced visualization features to represent higher dimensional data, while visualization for more than four components becomes an impossible task for PCA [47].

Finally, from the theoretical point of view, SOMs are particularly relevant algorithms in systems theory, as they model the emergence of a collective ordering in a composite 
system through the competition of its constituents. We anticipate that the SOM method of neural network analysis will have future applications to complex nuclear and high energy data. [48].

\section{$2.1 \quad$ PDFs}

The structure functions that the SOMs will be fitting to experimental data are in equation 2.2 - equation 2.3 where $C_{i}$ are the coefficient functions, and $q_{i}(x)$ as defined in equation 1.38 are the quark flavor PDFs. As noted in the DIS section, PDFs, the functions $f_{i}\left(x, Q^{2}\right)$, with $i=q$ or $g$, comprise the structure functions of inelastic processes in QCD. Three attempts to make theoretical models for the PDFs are the GRV model, [49] [50], the MSTW model, [51] and the ABM model [52]. The SOMPDF procedure will use weighted mixings of semi-randomized variations of these PDF models as input PDFs for the SOM process.

$$
\begin{gathered}
F\left(x, Q^{2}\right)=\sum_{i} \int_{x}^{1} \frac{d y}{y} C_{i}\left(y, \alpha_{S}\right) f_{i}\left(x / y, Q^{2}\right), \\
\frac{\partial}{\partial \ln Q^{2}} f_{i}\left(x, Q^{2}\right)=\frac{\alpha_{S}}{2 \pi} \int_{x}^{1} \frac{d y}{y} P_{q q}\left(\frac{x}{y}, \alpha_{S}\right) f_{i}\left(y, Q^{2}\right)
\end{gathered}
$$

In the above expressions, $P_{q q}(z)$, the splitting function, is the probability for a quark in DIS scattering emitting a new quark that reduces its momentum by a fraction of $z$. The splitting functions can also be formed for other vertices. $P_{G q}(z)$ describes the probability for emission of a gluon in a quark gluon vertice. $P_{G \rightarrow q \bar{q}}(z)$ gives the probability of a quark and an antiquark being emitted from an annihilation vertex. $P_{G G}(z)$ applies to a three gluon vertex, providing the probability of a gluon being emitted from an initial gluon. This function is given in Leading Order in formula 2.4

$$
P_{q q}(z)=C_{2}(F) \frac{1+z^{2}}{1-z}
$$


PDFs are extracted from experimental data on deep inelastic lepton-nucleon scattering and from related hard scattering processes in a continuously increasing range of $x$ and $Q^{2}$ data values taken from the multiGeV region using fixed target measurements at Jefferson Lab and others [53][54][55] [56] . A summary of PDFs and uncertainties is given in [57]. LHC precision measurements of $W^{ \pm}, t \bar{t}$ will be extracted in the future. The continuously increasing experimental data set which needs to be included in the analysis while simultaneously attacking the various theoretical open questions in PQCD render global fits a challenging enterprise. Currently, numerous collaborations have determined the parametrization for the unpolarized PDFs. CT [58], CJ [59],[60],[49],[50] and ABM [61] use a parametric form for the PDFs with $4-5$ free parameters per parton distribution type, at an input scale, $Q_{o}^{2}$, which varies for the different fitting forms. NNPDF [1], [3],[62] utilize neural networks to create unbiased initial distributions. All parametrization except for CTEQ use the perturbative QCD evolution equations to Leading Order (LO), Next to Leading Order (NLO), and Next to Next to Leading Order (NNLO) in terms of $\alpha_{s}$.

\subsection{SOM algorithm}

The SOM is formed by a two dimensional grid of neurons, or nodes. The nodes are presented with a stimulus in the form of a parametrized vector of dimension $n$; this is called the input vector and it describes the set of data to be processed. Each element of the vector is presented to all nodes on the map with a synapse or weight, $w$. Each node corresponds to the weight vector $w$ containing $n$ weights (same dimension as the input vector).

The SOM algorithm consists of three stages: i) Initialization; ii) Training; iii) Mapping.

\subsubsection{Initialization}

The SOM learning process, as discussed previously, is defined as unsupervised. During the initialization procedure weight vectors of dimension $n$ are associated with each cell $i$ :

$$
V_{i}=\left[v_{i}^{(1)}, \ldots, v_{i}^{(n)}\right]
$$


$V_{i}$ are given spatial coordinates. This means that the geometry and topology of a $2 \mathrm{D}$ map that gets populated randomly is defined to be the $V_{i}$ values. Typically each of these vectors consist of a randomized value of the data unit type to be placed on the map. The data for these initial vectors are defined to be the map vectors.

\subsubsection{Training}

Next, an input vector is presented to the grid. The node whose map vector is most similar to the input vector is defined as the best matching unit or BMU. The weights, $w$, of the BMU and of the surrounding nodes form a neighborhood, $N$, of some specified radius $r$.

For the training, a set of input data

$$
\xi=\left[\xi_{i}^{(1)}, \ldots, \xi_{i}^{(n)}\right]
$$

(isomorphic to $V_{i}$ ) is then presented to $V_{i}$, or compared using a quantity called the similarity metric that is chosen to be

$$
L_{2}(x, y)=\sum_{i=1,2} \sqrt{x_{i}^{2}-y_{i}^{2}}
$$

This is the ordinary Euclidean norm for vectors $x$ and $y$.

As noted in Section 1.1, SOMs are based on unsupervised and competitive learning as opposed to previously used supervised networks. For the map, this means that the cells that are closest to the BMU activate each other in order to "learn" from $\xi$. In order to complete this learning, data units in the neighboring cells adjust their values according to the following relation ( 2.5$)$.

$$
V_{i}(n+1)=V_{i}(n)+h_{c i}(n)\left[\xi(n)-V_{i}(n)\right]
$$

where $n$ is the iteration number, and $h_{c i(n)}$ is the neighborhood function defining a radius on the map which decreases with both $n$, and the distance between the BMU and node $i$. In our case we use square maps of size $L_{M A P}$, and the value of $h_{c i}(n)$ is in equation 2.6. 


$$
h_{c i}(n)=1.5\left(\frac{n_{\text {train }}-n}{n_{\text {train }}}\right) L_{M A P}
$$

Here, $n_{\text {train }}$ is the number of iterations. At the end of a properly trained SOM, cells that are topologically close to each other will contain data which are similar to each other. In the final phase the data units to be used for analysis are positioned on the map and the cluster formation is completed. For this neural network type, the clustering properties are particularly important for analysis of the data contained in the maps.

Once the learning process is complete, the newly generated set of data will be associated with the location of its BMU.

\subsubsection{Mapping}

SOMs are built as two dimensional arrays whose cells get adjusted towards a specific set of input signals according to a given order. Since each map vector now represents a specific type of information, the SOM is an ideal tool to visualize $N$-dimensional data. It visualizes these data sets by projecting them onto an $n$-dimensional map, where $n<N$, with the data units clustered according to a designated common attribute.

\subsection{Representing PDFs as SOMs}

The envelopes of theoretical generated curves that are used in the SOM are formed from PDFs using semi randomly varied parameters for each of the parton PDFs that comprise them. The curves are referred to as an envelope of curves because the resulting structure functions from the PDFs are designed to encircle the experimental data, with generated curves forming above and below the data values. The details of this method are in Section 3. We select PDFs from the envelope to: i) form our training data, which are defined as the code PDF vectors; ii) place vectors on the map, which are defined as the map vectors. An iteration is defined as the process where the entire set of code vectors, or input PDFs, is presented to the map vectors, the most closely matching the input PDFs being declared the "winning" PDFs. The SOMPDF code generates map vectors of PDFs. The map PDFs are grouped around the winning PDF according to the neighborhood radius function. The parameters in the PDFs that comprise the SOM are used to regulate their $x$ and $Q^{2}$ dependence. Wiggling these parameters up and down 
semi-randomly by a given scaling factor creates a set of generated PDFs which comprise the $\xi$ vectors which can be used in the Self Organizing process. The $n$ dimensions of the $\xi$ vector in this case are the differences between the PDFs at large and small $x$ values for an array of $Q^{2}$ values, with the $Q^{2}$ values taken from the available quantities given by the experimental data. This means that the experimental data sets for the structure function are read into the SOMPDF code and all the resulting $Q^{2}$ ranges from these data sets represent the $Q^{2}$ values for which the differences amongst the map PDFs will be determined. These differences between these PDFs represent the quantity $\left[\xi(n)-V_{i}(n)\right]$ in the adjustment function equation 2.5 for the neighboring PDFs surrounding the best matching PDF. The map PDF with the smallest differences relative to the code training PDFs represents the BMU for the PDF map.

\subsection{Genetic Algorithm}

Finally, we use a GA whereupon the new map PDFs or the input PDFs are analyzed relative to known experimental data for the $F_{2}$ values and PDFs with the lowest $\chi^{2}$ values are used as seeds for the next set of input PDFs. This process is repeated for 250 iterations; over the course of these iterations the $\chi^{2}$ values eventually asymptotically approach a given value, which is referred to as the saturation value. The number of PDFs depends on the size of the map; for an $n \times n$ map there are $n^{2}$ PDFs for each envelope. The PDF maps will be done using two different types of maps. The first type will use a $6 \times 6$ map and the second type will use a $1 \times 1$ map with 36 PDFs. These two different methods of using the SOMPDF code will allow us to isolate the efficiency and other possible effects of using the Self Organizing process.

In the genetic algorithm, the PDFs are generated from an previous attempt to parametrize them and then they are wiggled upwards or downwards to create a set of PDFs for each cell in the $n \times n$ map. Of the generated PDFs, those with the best fit, defined here as the PDFs whose values most closely match those of the experimental data structure function values, are chosen for each cell. From here, the PDF from all the $n^{2}$ number of PDFs with the closest fit to the experimental data are chosen as the best PDF. This PDF is then used as a seed vector from which the next set of PDFs are generated. This process is repeated a given number of times for each iteration and the SOMPDF code runs this process for a select number of iterations. Each iteration uses the GA along 
with the Self Organizing Process to evolve the PDFs from initial to final stages. The PDFs from each cell, from which the best fitting PDF is used, form an envelope of PDFs and a subsequent envelope of structure function values. An example of the initial and final PDFs are shown in Figure 2.1 and Figure 2.2. We aim to initialize the formation of this PDF envelope so that it sufficiently encircles the experimental structure function values from above and below these values. This is done to eliminate any biases in evolving the best fitting PDFs by creating a sufficiently randomized set of generated PDF curves. This formation is also done to fit the experimental data closely enough to ensure that future SOM and GA iterations produce the structure functions with the best possible fit to data. The need to eliminate bias in fitting the data arises because if the envelope of SOM generated PDFs does not encompass the data on both sides, when the PDF sets are trained and evolved with the SOP and the GA, the PDFs in future iterations will remain above or below the experimental data and the processes will not sufficiently be able to learn from $\chi^{2}$ values to form PDFs for subsequent iterations. A fit to the experimental data is no longer feasible if the initial envelopes do not encompass the data.

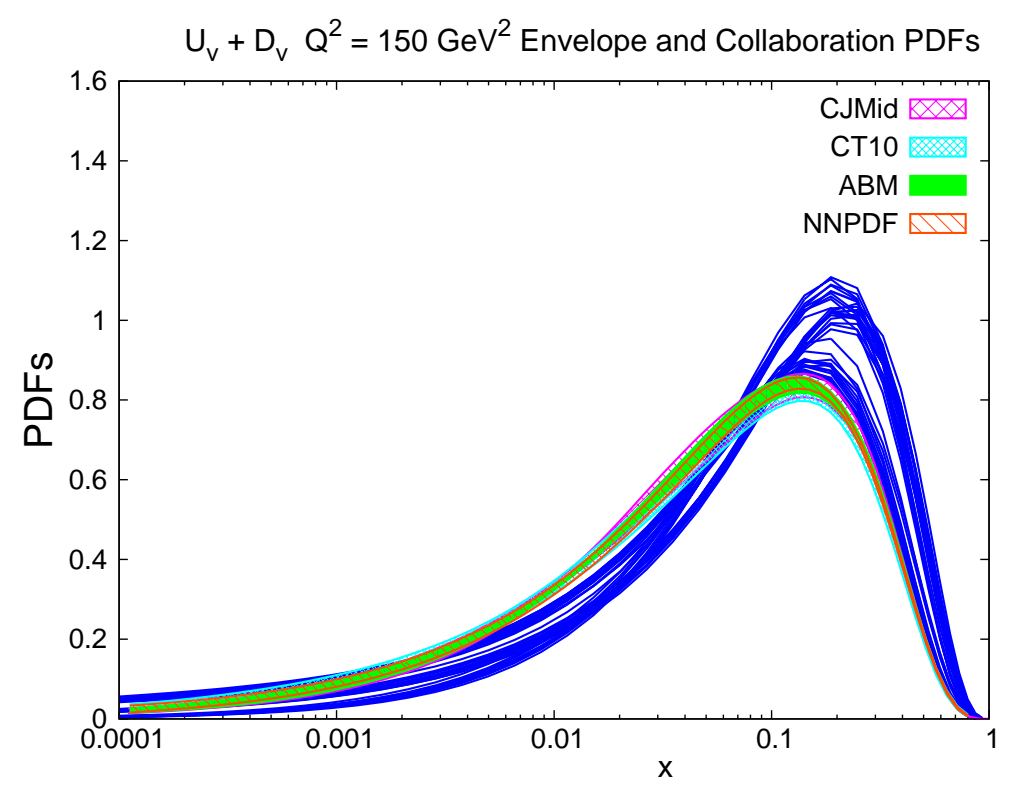

Figure 2.1: An example of an envelope formed for the first iteration, for $Q^{2}=150$ $\mathrm{GeV}^{2}$, for the valence quark PDFs relative to the valence quark PDFs determined by other collaborations. 


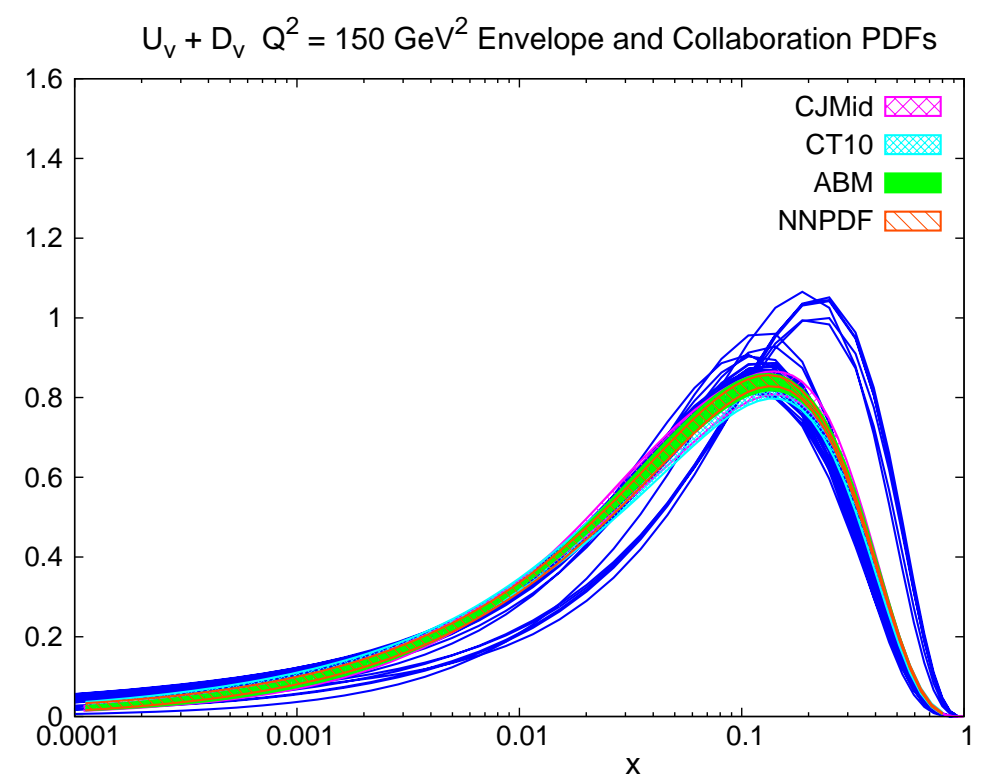

FiguRe 2.2: An example of an envelope formed for the $250^{\text {th }}$ iteration, for $Q^{2}=150$ $\mathrm{GeV}^{2}$, for the valence quark PDFs relative to the valence quark PDFs determined by other collaborations.

\section{SOMPDF as a quantitative parametrization of DIS data}

The previous two sections ANNs as a whole and the SOM, the specific type of ANN used in our procedure, were explained in detail along with the nuclear physics processes this network was designed to probe and how the SOM network would successfully probe them. The first section discussed the need to create, visualize and classify unbiased parametrized theoretical models of scattering cross section observables. From there, the case was made for the unsupervised SOM as an ideal network to achieve this. The nuclear and high energy physics that generated the observables we look to model were analyzed in order to to show the full scope of applications of the generated theoretical models. The second section provided a step by step of the workings of the SOM so that the process by which the theoretical models are generated could be fully understood.

In this Section we give a detailed report of the major changes that were applied to the initial approach in Ref.[63]. The initialization procedure is now based on a new criterion which we describe below. The new freedom of variation allowed by the updated initialization procedure enabled us to introduce also a new, more flexible criterion to take into account $Q^{2}$ evolution. Although our current attempts to apply the SOM to DIS are limited to PQCD at NLO, our new method allows us to naturally take into account 
other sources of $Q^{2}$ dependence. These sources include TMC,LxR and nuclear effects which all affect the large $x$ behavior of the structure functions.

Before proceeding with the detailed description it is important to underline the difference between the experimental data and the training data. This distinction forms the basis of the unsupervised learning process. The training data constitute an envelope of generated theoretical PDF curves which are built in the initialization procedure. These theoretical curves encompass the measured observable data values. The envelope of data sets forms the input data vectors for the SOM fitting process. The experimental scattering data sets are structure function observables to which the nuclear models formed from the SOM and GA are designed to be fitted.

\subsection{Main program SOMPDF}

The input SOM parameters are read in from the input file 'Sompdfparameters.txt' using the subroutines Setparamfile and Readparams.

The experimental data files are read in using the subroutine Getf2data and the total number of $x$ and $Q^{2}$ values to be used in PDF computations, based on the experimental data files, are obtained with the subroutine Uniquevals. The experimental data files may contain duplicate points for the same $x$ and $Q^{2}$ values. In order to ensure that these duplicate values do not get used in the structure function fitting procedure, the Uniquevals subroutine creates a set of points containing only one structure function value for each $x$ and $Q^{2}$ value. The subroutines SOMgridcreate and L2DistArr create the actual SOM that the map PDF vectors are placed on and enables us to determine the total number of map cell units on which the map vectors will be placed.

To start the training of the PDF envelopes there are the three previously mentioned three types of PDF sets declared that will be set up in later subroutines: GRV [49] [50], MSTW [51] and ABM[52]. The PDF envelopes that are used in the SOM training and the GA will be weighted combinations of these three PDF types. The Initweight and Initgpd subroutines construct the initial weight vectors and PDF envelopes, the ones to be used in the first iteration of the GA and SOM. GPDcreate sets up the PDF arrays, based on the number of $x$ and $Q^{2}$ values previously determined, in order to fill them with values computed from the three PDF sets used. The Mixvecs subroutine creates 
combinations of the three different types of PDFs used in the SOM/GA computations, filling the arrays set up in GPDcreate, and subsequently utilizes them to train the map vectors in the Self Organizing Process and for the first step of the GA. The subroutine Writemapvecs calls a series of subroutines in the Printutils module that prints out the initial envelope of PDF map vectors used in the first iteration of the SOM and GA methods. The Train subroutine is where the SOM procedure is utilized; the placement of training PDF vectors on the map, finding the best fitting PDF map vectors and adjusting the neighboring factors using a neighborhood radius dependent function are all done in this section. The next set of subroutines comprise the GA method for maximizing the fit of the theoretical generated PDFs to scattering data. The training PDF vectors are then normalized with the Processweights subroutine. The normalization is done by first completing the integral of all of the PDF types. From there, the normalization factors for each PDF type are computed such that when the PDF integrals are redone, the valence quark PDFs satisfy the Baryon number constraint, the sea quarks satisfy the Mellin moment normalizations and the quark and qluon PDFs satisfy the conservation of momentum constraint. The Baryon number, Mellin moment and conservation of momentum constraints are explained in detail in later subsections. The PDF vectors in this set with the lowest $\chi^{2}$ values, which designate the best fitting PDF vectors to the experimental data, are found using the Findbestvecs subroutine. From there, the Createseedgpds subroutine sets up PDF seed vectors using the best fitting vectors found in the Findbestvecs subroutine. These set vectors are then used to generate the PDF vectors for the next iteration of the SOM and GA. This process is repeated for a designated number of iterations and the best fitting PDF and the accompanying generated envelope of PDFs that contain the best fitting PDF are printed out in the Writebestmapvec and Writemapvecs subroutines.

\subsection{Module Gpd Init}

This module contains the Initweight and Initgpd subroutines that set up the array size for the PDFs and make calls to generate PDFs for use in the SOM. 


\subsection{Module Gpd Gen}

This module contains the Mixvecs and Processweights subroutines that generate the map and training PDFs for each iteration of the SOM and the GA.

\subsection{Module Generators}

This module contains the subroutines that produce semi randomly generated PDFs, for the SOM and for the training vectors, using the functions constructed in the Module Gpd Functions.

\subsection{Module Gpd Utils}

This Module reads in the experimental data files and sets up the allocatable arrays $x$ and $Q^{2}$ values as well as for the quark and gluon PDFs. The module uses the number of different $Q^{2}$ values contained in the data files read in to set up the array for $Q^{2}$ used to set up the PDFs. The module uses Bjorken scaling to set up the $x$ arrays. The $Q^{2}$ and $x$ arrays are then used to set up the PDF arrays.

\subsection{Module Gpd Parameters}

This module sets up groups of parameters, some of which are scaled by constants in order to ensure that a proper envelope forms, for the three PDF types, GRV, FMRST and ABM.

\subsection{Module Gpd Functions}

This Module computes three types of PDF sets using Bjorken $x$ values and the $Q^{2}$ values read in from the data sets: GRV, FMRST and ABM. The parameters that are semi randomly wiggled provide the $Q^{2}$ dependence for each PDF type. 


\subsection{Module Gpd Perturbs}

This Module wiggles a subset of the parameters in each PDF set semi randomly by a predetermined scaling factor. For the parameters set at a given value $P_{o}$ the module determines a new value for this parameter in the range $\left[P_{o}-\Delta, P_{o}+\Delta\right]$ where $\Delta$ is the scaling factor used for the parameter subset.

\subsection{Module Somkit}

This Module contains the Train subroutine where the actual SOP process is performed. The aforementioned subroutine takes given set of training PDF data vectors and finds the best matching unit, or bmu, on the SOM of PDF vectors relative to each data vector in the training set training PDF vectors. After the bmu is determined, the neighboring SOM PDF vectors are adjusted using the functional form $L e^{\frac{L}{2 R}}$ where $L$ is the distance between the bmu and a neighboring map vector and $R$ is the neighborhood radius for the SOM. The bmu among the SOM PDF vectors is also determined for the data vectors after the map training step is complete.

\subsection{Module Som Utils}

This module contains the SOMgridcreate subroutine that set up the size of the SOM and the neighborhood radius used in the SOM. It also contains the Getextrmmetric subroutine that find the PDFs in a generated set with the lowest $\chi^{2}$ value for use to generate a set of seed PDFs in the next GA iteration.

\subsection{Module Gpd Eval}

This Module takes in the PDFs formed from the semi random parameter wigglings in the gpd functions module and forms the proton and deuteron structure functions. It also calculates the $\chi^{2}$ value for each Bjorken $x$ and $Q^{2}$ value read in relative to the experimental data point closest to that $x$ and $Q^{2}$ value for the appropriate structure function. From here, the Module determines a total $\chi^{2}$ value for each PDF set in a generated envelope of PDFs to be used in the SOM and GA. 


\subsection{Module Gpd Norm}

This module performs the integrations of the PDFs over the Bjorken $x$ values for each $Q^{2}$ value read in and normalizes them to a set of conditions read in after the integration routine. The integrals of $u_{v}$ and $d_{v}$ are computed so that the normalization constant satisfying the conditions below can be met as specified by the Baryon number constraints.

$$
\begin{aligned}
& N_{u} \int U_{v} \mathrm{~d} x=2 \\
& N_{d} \int D_{v} \mathrm{~d} x=1
\end{aligned}
$$

The $\bar{u}, \bar{d}, s$ and $c$ quark PDFs are normalized according to their designated Mellin Moments, which will be provided in detail in later sections. These moments are determined from quark non singlet and singlet combinations. The Gluon moment is then normalized so that the momentum sum rule is satisfied.

$$
\int x U_{v}+x D_{v}+x 2 \bar{u}+x 2 \bar{d}+x s+x c+x G d x=1
$$

\subsection{MPI}

The SOMPDF code uses MPI in order to run a set of processes in parallel to ensure that the generation of the required map and code PDFs occurs in a timely manner. The map vector and a set of code vectors are generated for one process and then the code information is broadcast to the other parallel processes so that the map and code vectors can be generated in the same manner. The code is currently on the University of Virginia Rivanna coding system. The code currently runs 36 parallel processes, one for each map cell unit, and the Rivanna script for running the code with MPI is shown below. This script allocates 6 nodes with 6 processes for each node in order to obtain the full 36 processes.

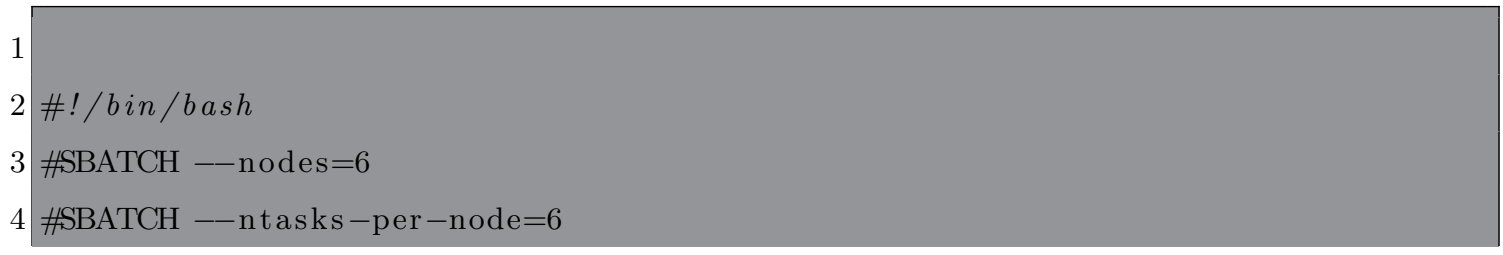




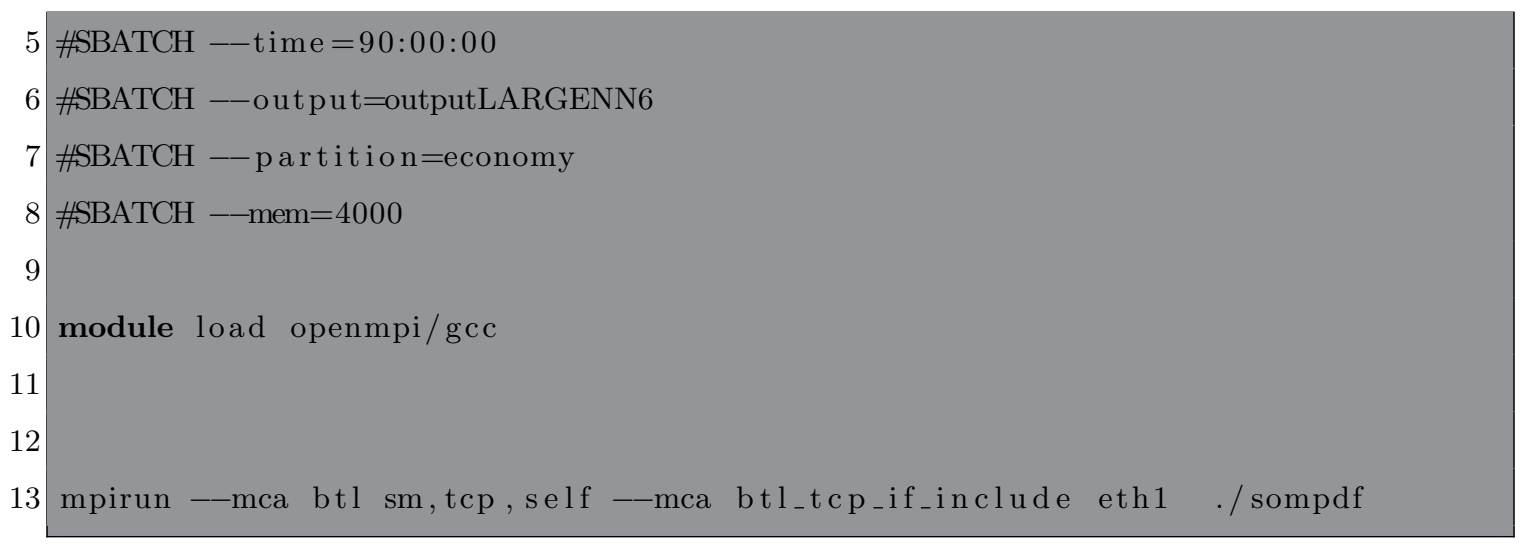

Listing 1: MPI code 


\subsection{SOMPDF flow Chart}

Descriptions of Code Steps

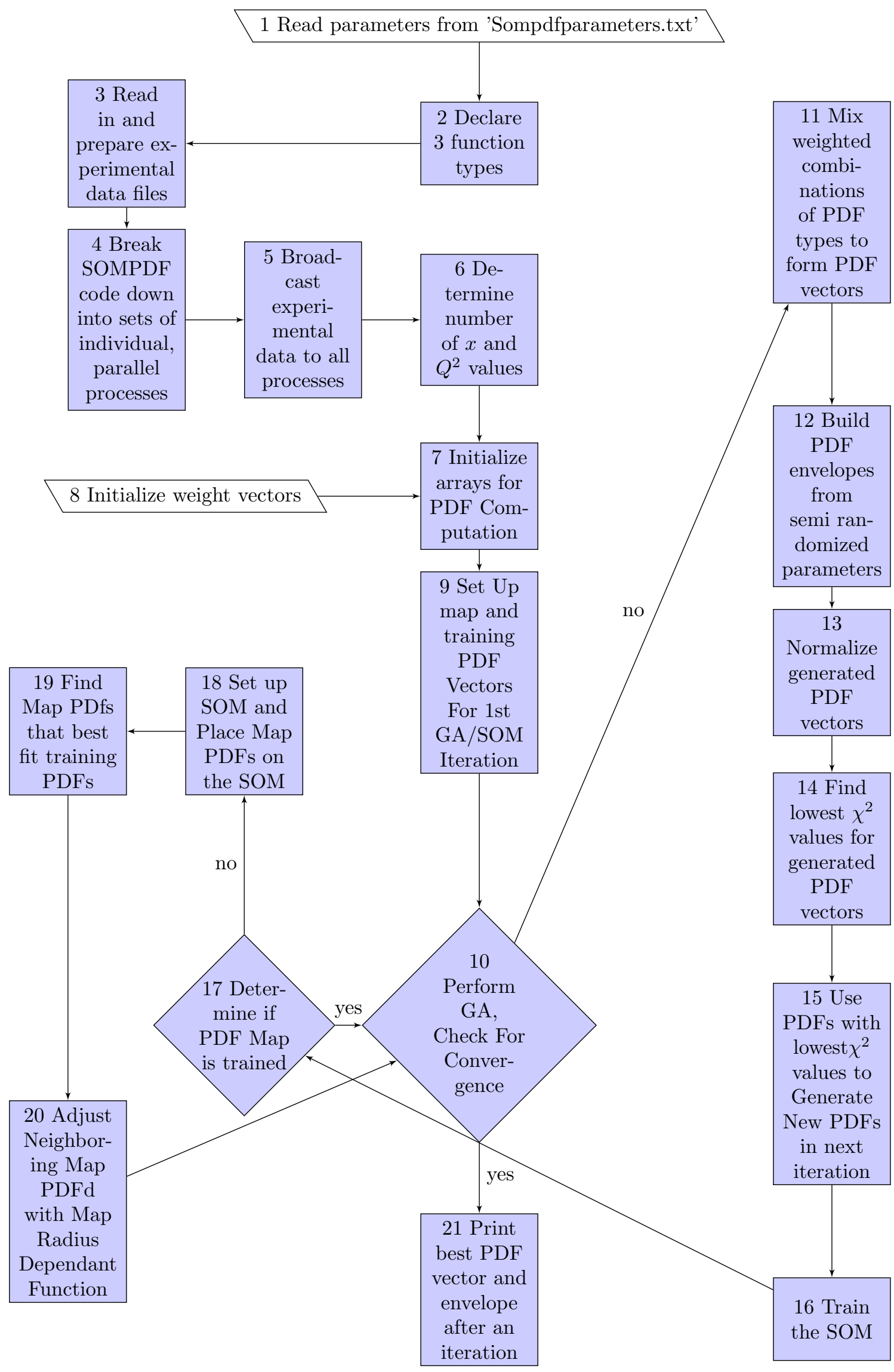


TABle 3.1: Code Module For Each Step

\begin{tabular}{|c|c|}
\hline \hline Step Number & Code Section \\
\hline \hline 1 & module fileutils.f90 \\
2 & subroutine setfunctionnames \\
3 & f2utils.f90 \\
4 & SLURM Script \\
5 & module mpivars.F90 \\
6 & module mathfuncs.f90 \\
7 & module gpdinit.F90 \\
8 & module gpdinit.F90 \\
9 & module gpdinit.F90 \\
10 & module gpdgen.F90 \\
11 & module gpdgen.F90 \\
12 & module gpdfunctions.f90 + gpdperturbs.f90 + gpdparameters.f90 \\
13 & module gpdnorm.f90 \\
14 & module gpdeval.f90andsomutils.f90 \\
15 & module somkit.f90 \\
16 & module somkit.f90 \\
17 & module somkit.f90 \\
18 & module somkit.f90 \\
19 & module somkit.f90 \\
20 & module somkit.f90 \\
21 & module printutils.f90 \\
\hline \hline
\end{tabular}

\subsection{New Initialization Method}

We start by describing the construction of the initial envelope for the SOM training. When we subsequently apply the GA, we construct new envelopes which contain sets of PDFs that are generated from each previous iteration, and that are selected based on their $\chi^{2}$ values so that, after a number of iterations we minimize it.

As discussed in subsection 2.4, the challenge one meets in forming an initial envelope is that on the one hand it must be constructed randomly in order to meet the criterion of unbiased formation, and on the other hand it must be adjusted enough to sufficiently follow the experimental data.

Our envelope is formed with randomly weighted combinations of three distinct parametric forms of PDFs, GRV, FMRST, and ABM, at an initial $Q^{2}=Q_{o}^{2}$. We then introduce $Q^{2}$ dependent parameters by using the procedure from previous parametrization [49] [50] [52] [51] . $Q^{2}$ dependent parameters were introduced for all three parametric forms we used. 
The observables we want to use in constructing the initial envelopes are shown in equation 3.4 and equation 3.5 .

$$
\begin{aligned}
& F_{2}^{P}=x \frac{4}{9}\left(u_{v}+2 \bar{u}\right)+\frac{1}{9}\left(d_{v}+2 \bar{d}\right)+\frac{2}{9} s+\frac{2}{9} c+\frac{2}{9} b \\
& F_{2}^{D}=x \frac{1}{9}\left(u_{v}+2 \bar{u}\right)+\frac{4}{9}\left(d_{v}+2 \bar{d}\right)+\frac{2}{9} s+\frac{2}{9} c+\frac{2}{9} b
\end{aligned}
$$

In forming the initial envelopes of these functions, there were a set of fundamental observations to take into account in varying the parameters. One was that certain parameters that were adjusted either did not have fundamental effects on the nature of the best fitting and envelope PDFs when varied or aqdjusted to a large enough extent as to make varying these parameters unproductive. For the remaining parameters, it was discovered, for a given parameter $P_{i}$, that varying that parameter by the factor $P_{i}+\Delta P$ where $\Delta P$ was a predetermined shifting factor, was ideal for creating envelopes enclosing the data.

In order to illustrate how the parameters were varied in order to form an envelope we take the quark distributions, starting with the valence distributions $u_{v}$ and $d_{v}$ in the GRV parametric form [49], which are shown in expression 3.6 and equation 3.7. The gluon and sea quark distributions are shown in expression 3.8 -equation 3.11 .

$$
\begin{aligned}
& u_{v}=A_{U}(t) x^{B_{1}^{U}(t)}(1-x)^{B_{2}^{U}(t)}\left(1+C_{1}^{U}(t) \sqrt{x}+C_{2}^{U}(t) x\right) \\
& d_{v}=A_{D}(t) x^{B_{1}^{D}(t)}(1-x)^{B_{2}^{D}(t)}\left(1+C_{1}^{D}(t) \sqrt{x}+C_{2}^{D}(t) x\right)
\end{aligned}
$$




$$
\begin{array}{r}
g=\left(x^{A L_{G}(t)}\left(A_{G}(t)+B_{G}(t) x+C_{G}(t) x^{2}\right)(-\ln x)^{B L_{G}(t)}\right. \\
+\left(\left(t^{A A_{G}(t)}\right) \exp \left(-E_{G}(t)\right)+\sqrt{\left.-\left(E P_{G}(t)\right)\left(t^{\beta_{D B}(t)}\right) \ln (x)\right)}\right) \\
(1-x)^{D_{G}(t)}
\end{array}
$$

$$
\begin{array}{r}
\bar{u}=\left(x^{A L_{U B}(t)}\left(A_{U B}(t)+B_{U B}(t) x+C_{U B}(t) x^{2}\right)(-\ln x)^{B L_{U B}(t)}\right. \\
\left.+\left(\left(t^{A A_{U B}(t)}\right) \exp \left(-E_{U B}(t)\right)+\sqrt{-\left(E P_{U B}(t)\right)\left(t^{\beta_{D B}(t)}\right) \ln (x)}\right)\right) \\
(1-x)^{D_{U B}(t)} \\
\bar{d}=\left(x^{A L_{D B}(t)}\left(A_{D B}(t)+B_{D B}(t) x+C_{D B}(t) x^{2}\right)(-\ln x)^{B L_{D B}(t)}\right. \\
\left.+\left(\left(t^{A A_{D B}(t)}\right) \exp \left(-E_{D B}(t)\right)+\sqrt{-\left(E P_{D B}(t)\right)\left(t^{\beta_{D B}(t)}\right) \ln (x)}\right)\right) \\
(1-x)^{D_{D B}(t)}
\end{array}
$$

$$
\begin{gathered}
\left(1+\left(A_{S}(t)\right)(\sqrt[2]{x})+B_{S}(t) x\right)\left((1-x)^{D_{S}(t)}\right) \\
\exp \left(-E_{S}(t)+\sqrt[2]{-E P_{S}(t)\left(t^{\beta_{S}(t)}\right) \ln (x)}\right)
\end{gathered}
$$

$$
\begin{gathered}
s=\left(\frac{\left(t-S_{S}(t)\right)^{A A_{S}(t)}}{(-\ln (x))^{A L_{S}(t)}}\right) \\
\left.+B_{S}(t) x\right)\left((1-x)^{D_{S}(t)}\right)
\end{gathered}
$$

For the GRV functions, the parameters used in determining the $Q^{2}$ dependence of the $u_{v}$ quark $\mathrm{PDF}$ are in 3.2. For the $d_{v}$ quark PDF the are in 3.3. For the gluon PDF they are in 3.4. For the $\bar{u}$ quark PDF they are in 3.5, for the $\bar{d}$ quark PDF they are in 3.6 and for the $s$ quark PDFs they are in 3.7. The free parameters used to set up the parton parameters in $3.2-3.7$ are initialized as shown in 3.8 .

with $t$ shown in formula 3.12 [64].

$$
t=\ln \frac{\ln Q^{2} / \Lambda^{2}}{\ln Q_{o}^{2} / \Lambda^{2}}
$$




\begin{tabular}{|c|c|}
\hline \hline$u_{v}$ parameter & Expression \\
\hline \hline$A_{U}(t)$ & $a_{u 11}+a_{u 12} t+a_{u 13}\left(t^{2}\right)+a_{u 14}\left(t^{3}\right)$ \\
$B_{1}^{U}(t)$ & 0.285 \\
$B_{2}^{U}(t)$ & $b_{u 21}+b_{u 22} t+b_{u 23}\left(t^{2}\right)+b_{u 24}\left(t^{3}\right)$ \\
$C_{1}^{U}(t)$ & $c_{u 11}+c_{u 12} t+c_{u 13}\left(t^{2}\right)+c_{u 14}\left(t^{3}\right)$ \\
$C_{2}^{U}(t)$ & $c_{u 21}+c_{u 22} t+c_{u 23}\left(t^{2}\right)+c_{u 24}\left(t^{3}\right)$ \\
\hline \hline
\end{tabular}

TABlE 3.2: Parameter Set for $u_{v}$ PDF Equations

\begin{tabular}{|c|c|}
\hline \hline$d_{v}$ parameter & Expression \\
\hline \hline$A_{D}(t)$ & $a_{d 11}+a_{d 12}(\sqrt[2]{t})+a_{d 13} t$ \\
$B_{1}^{D}(t)$ & $b_{d 11}+b_{d 12} t$ \\
$B_{2}^{U}(t)$ & $b_{d 21}+b_{d 22} t+b_{d 23}\left(t^{2}\right)+b_{d 24}\left(t^{3}\right)$ \\
$C_{1}^{D}(t)$ & $c_{d 11}+c_{d 12}(\sqrt[2]{t})+c_{d 13} t$ \\
$C_{2}^{D}(t)$ & $c_{d 21}+c_{d 22}(\sqrt[2]{t})+c_{d 23} t$ \\
\hline \hline
\end{tabular}

TABle 3.3: Parameter Set for $d_{v}$ PDF Equations

\begin{tabular}{|c|c|}
\hline \hline gluon parameter & Expression \\
\hline \hline$A L_{G L U O N}(t)$ & $a l_{g_{1}}+a l_{g_{2}} t$ \\
$B L_{G L U O N}(t)$ & $b l_{g_{1}}+b l_{g_{2}} t$ \\
$a_{G L U O N}(t)$ & $a_{g_{1}} t+a_{g_{2}}\left(t^{2}\right)$ \\
$b_{G L U O N}(t)$ & $b_{g_{1}}+b_{g_{2}} t$ \\
$c_{G L U O N}(t)$ & $c_{g_{1}}+c_{g_{2}} t$ \\
$d_{G L U O N}(t)$ & $d_{g_{1}}+d_{g_{2}} t$ \\
$E_{G L U O N}(t)$ & $e_{g_{1}}+e_{g_{2}} t$ \\
$E P_{\text {GLUON }}(t)$ & 2.466 \\
$A A_{\text {GLUON }}(t)$ & 1.128 \\
$\beta_{\text {GLUON }}(t)$ & 1.575 \\
\hline \hline
\end{tabular}

TABle 3.4: Parameter Set for gluon PDF Equations

The PDFs enter the observable, $F_{2}$, as was shown in equation 2.2 - equation 2.3. The quantitative analysis of the PDFs is shown in [49]. In equation 3.13, $C_{1}, C_{2}$ and $C_{3}$ are the uniform, randomized weighting factors that gauge how much each of the structure function types contributes to the formation of the function used in the GA. $f_{G R V}, f_{M S T W}$ and $f_{A B M}$ are the generated structure functions generated from the input models using semi randomized parameter variations.

$$
F_{i}^{e n v}=C_{1} f_{G R V}+C_{2} f_{M S T W}+C_{3} f_{A B M}
$$




\begin{tabular}{|c|c|}
\hline \hline $\bar{u}$ parameter & Expression \\
\hline \hline$A L_{\bar{u}_{B}}(t)$ & $a l_{\bar{u}_{1}}+a l_{\bar{u}_{2}} t$ \\
$A_{\bar{u}_{B}}(t)$ & $a_{\bar{u}_{1}}+a_{\bar{u}_{2}} t$ \\
$B_{\bar{u}_{B}}(t)$ & $b_{\bar{u}_{1}} t+b_{\bar{u}_{2}}\left(t^{2}\right)$ \\
$C_{\bar{u}_{B}}(t)$ & $c_{\bar{u}_{1}}+c_{\bar{u}_{2}} t$ \\
$D_{\bar{u}_{B}}(t)$ & $d_{\bar{u}_{1}}+d_{\bar{u}_{2}} t$ \\
$E_{\bar{u}_{B}}(t)$ & $e_{\bar{u}_{1}}+e_{\bar{u}_{2}} t$ \\
$E P_{\bar{u}_{B}}(t)$ & $e p_{\bar{u}_{1}}+e p_{\bar{u}_{2}} t$ \\
$b l_{\bar{u}_{B}}(t)$ & 0.0 \\
$A A_{\bar{u}_{B}}(t)$ & 0.594 \\
$\beta_{\bar{u}}(t)$ & 0.614 \\
\hline \multicolumn{2}{|c}{} \\
\hline
\end{tabular}

TABLE 3.5: Parameter Set for $\bar{u}$ PDF Equations

\begin{tabular}{|c|c|}
\hline \hline $\bar{d}$ parameter & Expression \\
\hline \hline$A L_{\bar{d}_{B}}(t)$ & $a l_{\bar{d}_{1}}+a l_{\bar{d}_{2}} t$ \\
$A_{\bar{d}_{B}}(t)$ & $a_{\bar{d}_{1}}+a_{\bar{d}_{2}} t$ \\
$B_{\bar{d}_{B}}(t)$ & $b_{\bar{d}_{1}} t+b_{\bar{d}_{2}}\left(t^{2}\right)$ \\
$C_{\bar{d}_{B}}(t)$ & $c_{\bar{d}_{1}}+c_{\bar{d}_{2}} t$ \\
$D_{\bar{d}_{B}}(t)$ & $d_{\bar{d}_{1}}+d_{\bar{d}_{2}} t$ \\
$E_{\bar{d}_{B}}(t)$ & $e_{\bar{d}_{1}}+e_{\bar{d}_{2}} t$ \\
$E P_{\bar{d}_{B}}(t)$ & $e p_{\bar{d}_{1}}+e p_{\bar{d}_{2}} t$ \\
$b l_{\bar{d}_{B}}(t)$ & 0.0 \\
$A A_{\bar{d}_{B}}(t)$ & 0.594 \\
$\beta_{\bar{d}}(t)$ & 0.614 \\
\hline \hline
\end{tabular}

TABLE 3.6: Parameter Set for $\bar{d}$ PDF Equations

Notice that whether the PDFs given above are able to fit quantitatively all, or a subset of the existing data is not important for constructing the envelope. The parametrization provide functional forms that are sufficiently close to the data so that by properly varying some of their parameters one can construct a bundle of curves whose envelope encompasses all of the available data. This step of our analysis can be challenging in that by using some of the baseline parametrization formulas it is nearly impossible to bracket newer data.

\subsection{Description of Structure Function Fit}

The structure functions shown in equation 2.2 - equation 2.3 can be used to determine the $\chi^{2}$ values for the PDFs in each of the $n^{2}$ sets and also to plot and analyze the behavior of the generated structure function values relative to the experimental data. 


\begin{tabular}{|c|c|}
\hline \hline$s$ parameter & Expression \\
\hline \hline$A L_{S_{B}}(t)$ & $a l_{s_{1}}+a l_{s_{2}} t$ \\
$E P_{S}(t)$ & 8.12 \\
$A_{S}(t)$ & $a_{s_{1}}+a_{s_{2}} t$ \\
$B_{S}(t)$ & $b_{s_{1}}+b_{s_{2}} t$ \\
$D_{S}(t)$ & $d_{s_{1}}+d_{s_{2}} t+d_{s_{3}} t^{2}$ \\
$E_{S}(t)$ & $e_{s_{1}}+e_{s_{2}} t$ \\
$S_{S}(t)$ & 0.0 \\
$A A_{S}(t)$ & 0.756 \\
$\beta_{S}(t)$ & 0.101 \\
\hline \hline
\end{tabular}

TABle 3.7: Parameter Set for $s$ PDF Equations

Once the given number of iterations is complete and the best fitting PDFs are chosen from the final evolved PDF set, we can evaluate not only if the $\chi^{2}$ value is sufficiently low but also of the structure function behavior matches that of the experimental data for the full $x$ range. These structure functions are plotted, for a $6 \times 6$ and a $10 \times 10$ map, alongside the experimentally generated structure function values in Figure 3.1. The results show that the SOMPDF generated structure functions were able to fit the experiments in terms of function behavior at large and small $x$. There were difficulties for the $6 \times 6$ map in fitting the SOMPDF structure function values to the experimental values for small as well as large $x$ and these difficulties were profoundly more apparent for the lowest two $Q^{2}$ values. The $10 \times 10$ map showed a marked improvement in fitting the generated structure functions to the experimental values and showed the use of the SOM in fitting the data. Increasing the map size therefore led to substantial improvements in the ability of the SOMPDF code to fit its theoretical models to the scattering data due to its use of the Self Organizing Process and increased number of theoretical input models to use for the GA. Ultimately, $6 \times 6$ maps were chosen for creating the final sets of generated theoretical PDF and structure function sets. This was because this size was sufficient to make effective use of the GA and the network's self organizing abilities and cluster formation properties while simultaneously maintaining a level of practicality.

\subsection{PQCD Evolution: Moments}

When the PDF sets are generated, for use in the SOM and the GA, they must be normalized in accordance to the principles of QCD; each time a PDF, whether for use as a map or training PDF, is generated from semi randomly generated combinations of the 


\begin{tabular}{|c|c|c|c||c|c|}
\hline \hline free parameter & Expression & free parameter & Expression & free parameter & Expression \\
\hline \hline$a_{u 11}$ & 0.330 & $b_{u 21}$ & 3.17 & $c_{u 11}$ & -2.28 \\
$a_{u 12}$ & 0.151 & $b_{u 21}$ & 1.17 & $c_{u 12}$ & 15.73 \\
$a_{u 13}$ & -0.059 & $b_{u 21}$ & -0.47 & $c_{u 13}$ & -4.58 \\
$a_{u 14}$ & 0.027 & $b_{u 21}$ & 0.09 & $c_{u 14}$ & 0.0 \\
$c_{u 21}$ & 56.7 & $a_{d 11}$ & 0.459 & $b_{d 12}$ & -0.031 \\
$c_{u 22}$ & -53.6 & $a_{d 12}$ & 0.315 & $b_{d 21}$ & 3.98 \\
$c_{u 23}$ & 11.21 & $a_{d 13}$ & 0.515 & $b_{d 22}$ & 1.04 \\
$c_{u 24}$ & 0.0 & $b_{d 11}$ & 0.624 & $b_{d 23}$ & -0.3 \\
$b_{d 24}$ & 0.0 & $c_{d 11}$ & 8.13 & $c_{d 12}$ & -6.77 \\
$c_{d 13}$ & 0.46 & $c_{d 21}$ & 6.59 & $c_{d 22}$ & -12.83 \\
$c_{d 23}$ & 5.65 & $a l_{g_{1}}$ & 0.323 & $a l_{g_{2}}$ & 1.653 \\
$b_{g_{1}}$ & 0.811 & $b_{g_{2}}$ & 2.044 & $a_{g_{1}}$ & 1.963 \\
$a_{g_{2}}$ & -0.519 & $b_{g_{1}}$ & 0.078 & $b_{g_{2}}$ & 0.624 \\
$c_{g_{1}}$ & 30.77 & $c_{g_{2}}$ & -24.19 & $d_{g_{1}}$ & 3.188 \\
$d_{g_{2}}$ & 0.720 & $e_{g_{1}}$ & -0.881 & $e_{g_{2}}$ & 2.687 \\
$a l_{s_{1}}$ & 2.942 & $a l_{s_{2}}$ & -1.016 & $a_{s_{1}}$ & -4.60 \\
$a_{s_{2}}$ & 1.167 & $b_{s_{1}}$ & 9.31 & $b_{s_{2}}$ & -1.324 \\
$d_{s_{1}}$ & 11.49 & $d_{s_{2}}$ & -1.198 & $d_{s_{3}}$ & 0.053 \\
$e_{s_{1}}$ & 2.630 & $a l_{\bar{u}_{1}}$ & 0.636 & $a l_{\bar{u}_{2}}$ & -0.084 \\
$e_{s_{2}}$ & 1.729 & $a_{\bar{u}_{1}}$ & 1.121 & $a_{\bar{u}_{2}}$ & -0.193 \\
$b_{\bar{u}_{1}}$ & 0.751 & $b_{\bar{u}_{2}}$ & -0.785 & $c_{\bar{u}_{1}}$ & 8.57 \\
$c_{\bar{u}_{2}}$ & -1.763 & $d_{\bar{u}_{1}}$ & 10.22 & $d_{\bar{u}_{2}}$ & 0.668 \\
$e_{\bar{u}_{1}}$ & 3.784 & $e_{\bar{u}_{2}}$ & 1.280 & $e p_{\bar{u}_{1}}$ & 1.808 \\
$e p_{\bar{u}_{2}}$ & 0.980 & $a l_{\bar{d}_{1}}$ & 0.636 & $a l_{\bar{d}_{2}}$ & -0.084 \\
$a_{\bar{d}_{1}}$ & 1.121 & $a_{\bar{d}_{2}}$ & -0.193 & $e p_{\bar{d}_{2}}$ & 0.980 \\
$b_{\bar{d}_{1}}$ & 0.751 & $b_{\bar{d}_{2}}$ & -0.785 & $c_{\bar{d}_{1}}$ & 8.57 \\
$c_{\bar{d}_{2}}$ & -1.763 & $d_{\bar{d}_{1}}$ & 10.22 & $d_{\bar{d}_{2}}$ & 0.668 \\
$e_{\bar{d}_{1}}$ & 3.784 & $e_{\bar{d}_{2}}$ & 1.280 & $e p_{\bar{d}_{1}}$ & 1.808 \\
\hline \hline
\end{tabular}

TABLE 3.8: Free Parameters for the PDFs

three PDF types, a normalization factor allowing the integrated PDFs to satisfy three requirements must be computed. These conditions are the momentum sum rule, Baryon conservation and the individual Mellin Moments. Perturbative evolution was taken into account by requiring that the various PDFs reproduce the momentum fractions evolution predicted by NLO pQCD. The PDF theoretical curves were generated through variations of the $Q^{2}$ dependent parameters that comprise the quark PDFs in order to fit them to data sets. Subsequently, it was not necessary to have all the quark and gluon PDF types be subjected to Perturbative QCD evolution.

The current work for the SOMPDF project has been centered around first normalizing the values of and the parton distribution functions $(\bar{u} x, \bar{d} x, s x \ldots)$ over $x$, where $x$ is the momentum fraction carried away by the partons during the deep inelastic collision 


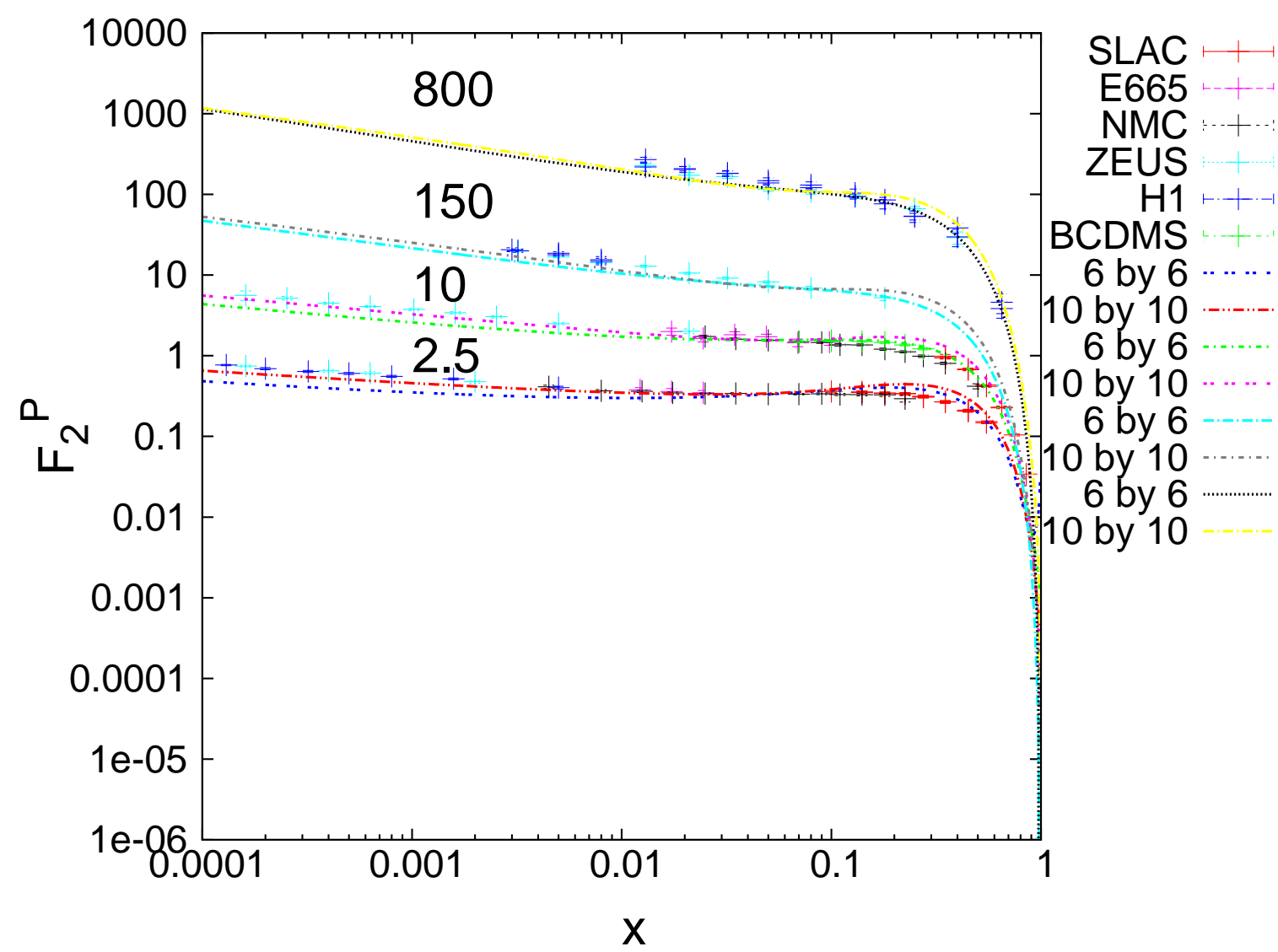

Figure 3.1: Structure function $F_{2}^{P}$ plotted vs $x$ at $Q^{2}$ values of $2.5,10,150$ and 800 $\mathrm{GeV}^{2}$

of the hadron with an electron, over the range of 0 to 1 . Each of the individual PDFs are normalized to values satisfying the conditions outlined in Section 3.12 by their own specified factors. These normalization factors are determined by the Mellin Moments for $Q^{2}$ evolution.

Prior to determining the normalization moments for the PDFs, the normalization rules for partons must be established; these rules will constrain the formation of the PDFs when the SOM and GA are used. This is because for a proton there must be two up quarks and one down quark, and the momentum of the partons in the proton must be conserved during the collision. Subsequently, the up and down quark PDFs are constrained by their Baryon numbers and the partons are all constrained by the momentum sum rule. The conditions for the PDF constraints are in relation 3.14-equation 3.16. 


$$
\begin{gathered}
\int u_{v} \mathrm{~d} x=2 \\
\int d_{v} \mathrm{~d} x=1 \\
\int x U_{v}+D_{v}+2 \bar{u}+2 \bar{d}+2 s+2 c+G d x=1
\end{gathered}
$$

The Mellin Moments are taken from non singlet, or NS, and singlet structure functions [65]. The NS combination of quarks is given through the sets of valence quarks; $u_{v}=u-\bar{u}$ and $d_{v}=d-\bar{d}$ are the NS quark combinations used in the Mellin Moment computations. The singlet quark combination is given in terms of the sum of quark combinations; the singlet distribution can be written as $u_{v}+d_{v}+2 \bar{u}+2 \bar{d}+2 s+2 c$ when strange and charm quarks are included. For NS PDF combinations, the $Q^{2}$ dependent Mellin Moments are in relation 3.17 [65].

$$
M_{n}^{N S}\left(Q^{2}\right)=M_{n}^{N S}\left(Q_{0}^{2}\right)\left(\frac{\alpha_{s}\left(\frac{Q^{2}}{\Lambda^{2}}\right)}{\alpha_{s}\left(\frac{Q_{0}^{2}}{\Lambda^{2}}\right)}\right)^{d_{n}^{N S}}
$$

In this NS evolution, $d_{n}^{N S}$ refers to the non singlet constants of asymptotic freedom given in Roberts [65].

The $Q^{2}$ dependence of the singlet quark combinations must be computed in terms of the mixing of quarks and gluons. Therefore, it requires two sets of operators as opposed to one set for the NS moments. The operators are ( equation 3.18 )

$$
O_{n}^{S}=\frac{i^{n-1}}{n !}\left[\bar{q} \gamma^{\mu_{1}} D^{\mu_{2}} \ldots+D^{\mu_{n}} q+\text { permutations }\right]
$$

and ( equation 3.19 )

$$
O_{n}^{G}=\frac{i^{n-2}}{n !} \operatorname{Tr}\left[D^{\mu_{2}} \ldots . .+D^{\mu_{n-1}} G_{\nu}^{\mu_{n}}+\text { permutations }\right]
$$


Given $i=Q, g$ we now have the singlet and gluon coefficient functions ( equation 3.20).

$$
\bar{C}_{n}^{i}\left(\frac{Q^{2}}{\mu^{2}}\right)=\sum_{j=q, G} \bar{C}_{n}^{j}\left(1, \bar{g}^{2}(t)\right) \exp \left[-\frac{\gamma_{0}^{n, j i}}{2 \beta_{0}} \ln t\right]
$$

with ( equation 3.21)

$$
\gamma^{n, i j}(g)=\gamma_{0}^{n, j i} \frac{g^{2}}{16 \pi^{2}}
$$

The dimensions $\gamma_{0}^{n, j i}$ are calculated form one loop gluon corrections [66] [67]. For the singlet dimensions, in LO $\gamma_{0}^{n, q q}=\gamma_{0}^{n, N S}$ and the anomalous gluon dimensions are given below in expression 3.22 .

$$
\begin{array}{r}
\gamma^{n, G G}=2 C_{2}(A)=\left[\frac{1}{3}-\frac{4}{n(n-1)}-\frac{4}{(n+1)(n+2)}+\sum_{j=2}^{n} \frac{1}{j}\right] \\
+\frac{8}{3} N_{f} T_{2}(F)
\end{array}
$$

The eigenvalues of the anomalous dimension matrix can be defined as $\gamma_{0}^{ \pm}$resulting in 3.23 .

$$
\gamma_{0}^{ \pm}=\frac{1}{2}\left\{\left(\gamma_{0}^{n, q q}-\gamma_{0}^{n, G G}\right) \pm\left[\left(\gamma_{0}^{n, q q}-\gamma_{0}^{n, G G}\right)^{2}+4 \gamma_{0}^{n, g G} \gamma_{0}^{G q}\right]^{\frac{1}{2}}\right\}
$$

The final expressions for the quark and singlet moments are written below in expression 3.24 .

$$
\begin{array}{r}
M_{n}^{S}\left(Q^{2}\right)=\left(\left(1-a_{n}\right) r_{n}^{+}+a_{n} r_{n}^{-}\right) M_{n}^{S}\left(Q_{0}^{2}\right)+b_{n}\left(r_{n}^{+}-r_{n}^{-}\right) M_{n}^{G}\left(Q_{0}^{2}\right) \\
M_{n}^{G}(Q)=a_{n} \frac{\left(1-a_{n}\right)\left(r_{n}^{+}-r_{n}^{-}\right)}{b_{n}} M_{n}^{S}\left(Q_{0}^{2}\right)+\left(\left(1-a_{n}\right) r_{n}^{-}+a_{n} r_{n}^{+}\right) M_{n}^{G}\left(Q_{0}^{2}\right)
\end{array}
$$

with 3.25

$$
a_{n}=\frac{\gamma_{0}^{n, q q}-\gamma_{0}^{n,+}}{\gamma_{0}^{n,-}-\gamma_{0}^{n,+}}, b_{n}=\frac{-\gamma_{0}^{n, q G}}{\gamma_{0}^{n,-}-\gamma_{0}^{n,+}}
$$


and 3.26

$$
r_{n}^{ \pm}=\left[{\frac{\alpha_{s}\left(Q^{2}\right.}{\alpha_{s}\left(Q_{0}^{2}\right.}}^{ \pm}\right], d_{n}^{ \pm}=\frac{\gamma_{0}^{n, \pm}}{2 \beta_{0}}
$$

The singlet moments evolve, shown in equation 3.27, relative to $Q_{0}^{2}$ over $Q^{2}$ the same way the NS quark moments do.

$$
M_{n}^{s}\left(Q^{2}\right)=\int d x x^{n-2} \Sigma\left(x, Q^{2}\right)
$$

The gluon moments are not physical quantities the way the non singlet and singlet quark moments are. However, the gluon moments need to be normalized such that the total moments of the quarks and gluons satisfy conservation of momentum independent of $Q^{2}$. To do this, we need to switch to the Gross and Wilzek [66] convention shown in equation 3.28 .

$$
\begin{array}{r}
\gamma_{0}^{n, q G}=-8 N_{f} T_{2}(F) \frac{n^{2}+n+2}{n(n+1(n+2)} \\
\gamma_{0}^{n, G q}=-4 C_{2}(F) \frac{n^{2}+n+2}{n\left(n^{2}-1\right)}
\end{array}
$$

For the second quark and gluon moments the result is shown in expression 3.29

$$
\begin{gathered}
\gamma_{0}^{2, q q}+\gamma_{0}^{2, G q}=0 \\
\gamma_{0}^{2, G G}+\gamma_{0}^{2, q G}=0
\end{gathered}
$$

The pair of singlet and gluon evolution moment equations now give the following result shown in expression 3.30 .

$$
M_{2}^{S}\left(Q^{2}\right)+M_{2}^{G}\left(Q^{2}\right)=M_{2}^{S}\left(Q_{0}^{2}\right)+M_{2}^{G}\left(Q_{0}^{2}\right)
$$

The $Q^{2}$ dependent evolution of the singlet quark PDF combination is in formula 3.31 . 


$$
\left[M_{2}^{S}\left(Q^{2}\right)-\frac{3 N f}{16+3 N f}\right]=\left[\left(\frac{\alpha_{s}\left(Q^{2}\right)}{\alpha_{s}\left(Q_{0}^{2}\right)}\right)^{d_{2}^{+}}\right]\left[M_{2}^{S}\left(Q_{0}^{2}\right)-\frac{3 N f}{16+3 N f}\right]
$$

$d_{2}^{+}$refers to the non singlet constants of asymptotic freedom given in Roberts [65]. In these singlet structure forms, the value of $Q_{0}^{2}$ is set to be $Q_{0}^{2}=0.55 \mathrm{GeV}^{2}$. The initial values of the Mellin Moments are set so that for the $\bar{u}$ and $\bar{d}$ moments, they are approximately equal to each other and roughly twice the moment value of the $s$ quark.

When determining the $Q^{2}$ dependent evolution of the PDFs, the strong coupling constant is also evolved past its Leading Order value. The $\alpha_{s}$ to NLO needs to be determined to account for the corrections to $\Lambda$ from the rescaling of the quark normalizations in the hadron absorbed to second order and higher corrections to the NS moment.

The two-loop correction to the coupling constant results in a new expression (equation 3.32 ) for $\alpha_{s}$ where the two loop term $\beta_{1}$ is given by $\beta_{1}=102-\frac{38}{3} N_{f}$.

$$
\frac{d}{d \ln Q^{2}}\left(\frac{\alpha_{s}\left(Q^{2}\right)}{4 \pi}\right)=\beta_{0}\left(\frac{\alpha_{s}\left(Q^{2}\right)}{4 \pi}\right)^{2}+\beta_{1}\left(\frac{\alpha_{s}\left(Q^{2}\right)}{4 \pi}\right)^{3}+\ldots
$$

The algebraic terms $a, b$ and $t$ can be introduced, where $a=\beta_{0} \alpha_{s}\left(Q^{2}\right) / 4 \pi, b=\beta_{1} / \beta_{0}{ }^{2}$ and $t=\ln \left(Q^{2} / \mu^{2}\right)$. The evolution term $\ln \frac{Q^{2}}{\Lambda^{2}}$ can be written in terms of the combined algebraic expressions in formula 3.33 .

$$
\frac{1}{a}-b \ln \left(\frac{1+b a}{a}\right)-\ln Q^{2}=\frac{1}{a\left(\mu^{2}\right)}-b \ln \left(\frac{1+b a}{a}\right)-\ln \mu^{2}
$$

Setting each side equal to $-\ln \Lambda^{2}$ gives us equation 3.34 for $\ln Q^{2}$ in terms of $\alpha_{s}$.

$$
\ln \frac{Q^{2}}{\Lambda^{2}}=\frac{4 \pi}{\beta_{0} \alpha_{s}}-\frac{\beta_{1}}{\beta_{0}^{2}} \ln \left[\frac{4 \pi}{\beta_{0} \alpha_{s}}+\frac{\beta_{1}}{\beta_{0}^{2}}\right]
$$

The NLO $\alpha_{s}$ is given in expression 3.35 as an approximation for small $\alpha_{s}$. For the computation of the moments the values of $\alpha_{s}\left(M_{z}\right)$ are allowed to vary within the range $\alpha_{s}\left(M_{z}\right)=0.1135-0.1195$, which is consistent with other PDF collaborations. This results in the computation of the theoretical models implicitly taking into account the correlations between $\alpha_{s}$ and PDF uncertainty. 


$$
\frac{\alpha_{s}}{4 \pi}=\frac{1}{\left(\beta_{0} \ln \frac{Q^{2}}{\Lambda^{2}}\right)}-\frac{\beta_{1}}{\beta_{0}} \frac{\ln \ln {\frac{Q^{2}}{\Lambda^{2}}}^{2}}{\left(\beta_{0} \ln \frac{Q^{2}}{\Lambda^{2}}\right)}
$$

In order to set up the Altarelli-Parisi equations [68], the total set of contributions to the $Q^{2}$ dependence of the non singlet quark, singlet quark and gluon structure functions must be computed. This begins with the fundamental expression for the quark density from which non singlet quark, singlet quark and gluon distribution functions can be obtained. This desired density can be given in expression 3.36 .

$$
d q(x)=\int d y q(y)\left[\frac{\alpha_{s}}{2 \pi} P_{q q}(z) d \ln L_{T}^{2} d z\right] \delta(z y-x)
$$

As $Q^{2}$ in the scattering gets larger, gluons are emitted in the quark quark annihilation vertex that governs parton splitting. This emission also governs the size of the proton transverse momentum $L_{T}^{2}$. When $Q^{2}$ increases, the quark density in the hadron target changes due to two factors. The first one is a second quark emerging with momentum fraction $x$ when the first quark, with momentum fraction $y>x$, emits a gluon. This is the source of the quark distribution $d q(x)$. In addition, the hadron will contain other quarks with momentum fraction $x$ that emit gluons which contain momentum fraction $x^{\prime}$ with $x^{\prime}<x$. The splitting function for both contributions is the same and results in a new expression, shown in expression 3.37 for the non singlet, or valence, quark distribution.

$$
\begin{array}{r}
\frac{d q^{N S}\left(x, Q^{2}\right)}{d \ln Q^{2}}=\frac{\alpha_{s}\left(Q^{2}\right)}{2 \pi} \\
\int d y d z q^{N S}\left(y, Q^{2}\right) P_{q q}(z)[\delta(z y-x)-\delta(y-x)]
\end{array}
$$

From here, we can work towards deriving the more conventional form of the AP equations. The first step is bringing the delta functions into the splitting functions to give us equation 3.38 .

$$
P_{q q}(z)=C_{2}(F)\left[\frac{1+z^{2}}{(1-z)_{+}}-\frac{3}{2} \delta(1-z)\right]
$$


$(1-z)_{+}$is defined in equation 3.39

$$
\int d z \frac{f(z)}{(1-z)_{+}}=\int d z \frac{f(z)-f(1)}{(1-z)}
$$

This definition comes from the standard definitions of the plus function given in equation 3.40 .

$$
(F(x))_{+}=\lim _{\beta \rightarrow 0}\left\{F(x) \theta(1-x-\beta)-\delta(1-x-\beta) \int_{0}^{1-\beta} F(y) d y\right\}
$$

This function must vanish quickly enough as $x \rightarrow 1$ for the + function to satisfy the following distribution integral (equation 3.41 ).

$$
\int_{0}^{1}(F(x))_{+} d x=0
$$

The quark gluon splitting term $P_{q q}(z)$ contains a + function of the form $\frac{f(z)}{(1-x)_{+}}$. This type of + function has a limit shown in equation 3.42

$$
\frac{f(z)}{(1-x)_{+}}=\lim _{\beta \rightarrow 0}\left\{\frac{f(z)}{(1-x)} \theta(1-x-\beta)-f(z) \delta(1-x-\beta) \ln (\beta)\right\}
$$

When a function $\frac{f(z)}{(1-x)_{+}}$is evaluated over the $(0: 1)$ range, the first term on the rhs of the expansion in equation 3.42 yields the integral $\int d z \frac{f(z)}{(1-z)}$ and the second term vanishes everywhere except $z=1$ yielding $\int d z \frac{f(1)}{(1-z)}$. The integral on the rhs of equation 3.39 vanishes at the endpoints, yielding, with integration by parts, equation 3.43.

$$
\int d z \frac{f(z)-f(1)}{(1-z)}=\int d z \ln (1-z) \frac{d f(z)}{d z}
$$

This allows us to write the non singlet $Q^{2}$ dependent moment equation in expression 3.44.

$$
\frac{d q^{N S}\left(Q^{2}\right)}{d \ln Q^{2}}=\frac{\alpha}{2 \pi} \int_{x}^{1} \frac{d y}{y} q^{N S}\left(y, Q^{2}\right) P_{q q}\left(\frac{x}{y}\right)
$$

This non singlet moment becomes, given $M^{N S}\left(Q^{2}\right)=\int d x x^{n-1} q^{N S}\left(x, q^{2}\right)$, equation 3.45 


$$
Q^{2} \frac{d M_{n}^{N S}\left(Q^{2}\right)}{d Q^{2}}=\frac{\alpha_{s}\left(Q^{2}\right)}{2 \pi} \int d z z^{n-1} P_{q q}(z)
$$

We can from here relate the splitting function to the anomalous dimensions for valence quark combinations (equation 3.46 ).

$$
\int d z z^{n-1} P_{q q}(z)=-\frac{1}{4} \gamma_{0}^{n, N S}
$$

The above is the valence moments for one quark type. Factoring in all the valence quark types and adding in the contributions from the emitted gluon loops gives us the relations for the quark and gluon distribution functions shown in expression 3.47 - equation 3.48 .

$$
\begin{array}{r}
\frac{d q^{i}\left(x, Q^{2}\right)}{d \ln Q^{2}}=\frac{\alpha_{s}\left(Q^{2}\right.}{2 \pi} \\
\times \int \frac{d y}{y}\left[\sum_{j} q^{j}\left(y, Q^{2}\right) P_{q^{i} q^{j}}\left(\frac{x}{y}\right)+G\left(y, Q^{2} P_{q^{i} G}\left(\frac{x}{y}\right)\right]\right. \\
\frac{d G\left(x, Q^{2}\right)}{d \ln Q^{2}}=\frac{\alpha_{s}\left(Q^{2}\right.}{2 \pi} \\
\times \int \frac{d y}{y}\left[\sum_{j} q^{j}\left(y, Q^{2}\right) P_{G q^{j}}\left(\frac{x}{y}\right)+G\left(y, Q^{2} P_{G G}\left(\frac{x}{y}\right)\right]\right.
\end{array}
$$

In the above quark and gluon distributions, $i$ and $j$ run over every quark type found within the target hadron. At this level, the quark gluon interactions cannot lead to changes in quark type, the probability of a quark emitting a gluon is independent of the type of quark and the emission of a gluon creates a quark anti quark pair whose probability of formation is also independent of that quark type. This gives us the relations $P_{q^{i} q^{j}}=\delta_{i j} P_{q q}, P_{G q^{j}}=P_{G q}$ and $P_{q^{i} G}=P_{q G}$.

From here, we can expand the splitting function $P_{q q}(z)$ from a specific quark type to all quark types included in the NS moments as $\int d z z^{n-1} P_{i j}(z)=-\frac{1}{4} \gamma_{0}^{n, i j}$. Then, we have for the quark and gluon AP equations, equation 3.49 


$$
\begin{gathered}
\frac{d q^{s}\left(x, Q^{2}\right)}{d \ln Q^{2}}=\frac{\alpha_{s}\left(Q^{2}\right.}{2 \pi} \int \frac{d z}{z} P_{q q}(z) q^{s}\left(\frac{x}{z}, Q^{2}\right)+N_{f} P_{q G}(z) G\left(\frac{x}{z}, Q^{2}\right) \\
\frac{d G\left(x, Q^{2}\right)}{d \ln Q^{2}}=\frac{\alpha_{s}\left(Q^{2}\right.}{2 \pi} \int \frac{d z}{z} P_{G q}(z) q^{s}\left(\frac{x}{z}, Q^{2}\right)+P_{G G}(z) G\left(\frac{x}{z}, Q^{2}\right)
\end{gathered}
$$

The splitting equations, since they describe the probability of quark and gluon emission from an initial set of quarks and gluons within the target hadron, are also governed by conservation of momentum. The quark gluon vertices and the combination of annihilation and three gluon vertices, which produce quark and gluon pairs, must each result in zero net momentum added to the hadron. This allows us to relate the splitting functions as shown in relation 3.50 .

$$
\begin{aligned}
\int d z z\left[P_{q q}(z)+P_{G q}(z)\right] & =0 \\
\int d z z\left[2 N_{f} P_{q G}(z)+P_{G G}(z)\right] & =0
\end{aligned}
$$

The splitting functions are also related, for $z<1$, by the expressions $P_{q q}(z)=P_{G q}(1-z)$, $P_{q Q}(z)=P_{q G}(1-z)$ and $P_{G G}(z)=P_{G G}(1-z)$. Then, the splitting functions for the quark gluon vertex for quark gluon interactions, the annihilation vertex and the gluon pair production vertex can be given as well, in relation 3.51 - equation 3.53.

$$
\begin{gathered}
P_{G q}(z)=C_{2}(F) \frac{1+(1-z)^{2}}{z} \\
P_{q Q}(z)=T_{2}(F)\left[z^{2}+(1-z)^{2}\right] \\
P_{G G}(z)=C_{2}(A)\left[\frac{z}{(1-z)_{+}}+\frac{1-z}{z}+z(1-z)\right]+\frac{1}{2 \beta_{0}} \delta(1-z)
\end{gathered}
$$

The full $Q^{2}$ dependence of the Mellin Moments for the PDFs can from these conditions be determined from integration of the AP Equations, given in expression 3.54 - equation 3.56 The AP equations describe the evolution of densities for every parton type and combination present in a hadron. 


$$
\begin{gathered}
\frac{d}{d \log Q} F_{g}(x, Q)=\frac{\alpha_{s}\left(\frac{Q^{2}}{\Lambda^{2}}\right)}{\pi} \int \frac{\mathrm{dz}}{\mathrm{z}}\left[P_{g \leftarrow q} \sum_{f}\left(F_{f}\left(\frac{x}{z}, Q\right)+F_{\bar{f}}\left(\frac{x}{z}, Q\right)\right)+P_{g \rightarrow q}(z) F_{g}\left(\frac{x}{z}, Q\right)\right] \\
\frac{d}{d \log Q} F_{f}(x, Q)=\frac{\alpha_{s}\left(\frac{Q^{2}}{\Lambda^{2}}\right)}{\pi} \int \frac{\mathrm{dz}}{\mathrm{z}}\left[P_{g \leftarrow q} F_{\bar{f}}\left(\frac{x}{z}, Q\right)+P_{g \rightarrow q} F_{g}\left(\frac{x}{z}, Q\right)\right] \\
\frac{d}{d \log Q} F_{\bar{f}}(x, Q)=\frac{\alpha_{s}\left(\frac{Q^{2}}{\Lambda^{2}}\right)}{\pi} \int \frac{\mathrm{dz}}{\mathrm{z}}\left[P_{g \leftarrow q} F_{f}\left(\frac{x}{z}, Q\right)+P_{g \rightarrow q} F_{g}\left(\frac{x}{z}, Q\right)\right]
\end{gathered}
$$

We can also express using the non singlet moment evolution in LO using the $(1-z)_{+}$ term and taking into account $z$ behavior at the endpoints. The $(1-z)_{+}$expansion term is evaluated in expression 3.57.

$$
\begin{array}{r}
\int_{x}^{1} d z f\left(\frac{x}{z}\right)(1-z)_{+}^{-1}=\int_{0}^{1} d z f\left(\frac{x}{z}\right)(1-z)_{+}^{-1} \\
-\int_{0}^{x} d z f\left(\frac{x}{z}\right)(1-z)_{+}^{-1}= \\
\int_{x}^{1} d z\left[f\left(\frac{x}{z}\right)-f(x)\right](1-z)^{-1} \\
-f(x) \int_{0}^{x} d z(1-z)^{-1}
\end{array}
$$

The non singlet structure function $F^{N S}$, where $F^{N S}\left(x, Q^{2}\right)=x q^{N S}\left(Q^{2}\right)$, can be expanded by noting that (equation 3.58) 


$$
\begin{array}{r}
\frac{d x q^{N S}\left(x, Q^{2}\right)}{d \ln Q^{2}}=\frac{\alpha_{s}\left(Q^{2}\right)}{2 \pi} \int_{x}^{1} \frac{d y}{y} y z q^{N S}\left(y, Q^{2}\right) P_{q q}\left(\frac{x}{y}\right)= \\
C_{2}(F)\left\{\frac{\alpha_{s}\left(Q^{2}\right)}{2 \pi} \int_{x}^{1} \frac{d y}{y} y z q^{N S}\left(y, Q^{2}\right)\right. \\
\left.\left[\frac{1+z^{2}}{(1-z)_{+}}-\frac{3}{2} \delta(1-z)\right]\right\}= \\
C_{2}(F) \frac{\alpha_{s}\left(Q^{2}\right)}{2 \pi} \int_{x}^{1} d y\left[\left(1+z^{2}\right) F^{N S}\left(\frac{x}{z}, Q^{2}\right)-\left.\left(1+z^{2}\right)\right|_{z=1} F^{N S}\left(x, Q^{2}\right)\right](1-z)^{-1}- \\
\left.F^{N S}\left(\frac{x}{z}, Q^{2}\right)\left(1+z^{2}\right)\right|_{z=1} \int_{x}^{1} d z(1-z)^{-1}+\frac{3}{2} F^{N S}\left(x, Q^{2}\right)
\end{array}
$$

Using these expansion terms and completing the integrals of the + function expansion terms in equation 3.58 at the $z=1$ endpoint gives us the expression for the non singlet structure functions in equation 3.59 .

$$
\begin{array}{r}
\frac{d F^{N S}\left(x, Q^{2}\right)}{d \ln Q^{2}}=\frac{\alpha_{s}\left(Q^{2}\right)}{2 \pi} C_{2}(F)\left\{\left[\frac{3}{2}+2 \ln (1-x)\right] F^{N S}\left(x, Q^{2}\right)+\right. \\
\left.\int_{x}^{1} \frac{d z}{1-z}\left[\left(1+z^{2}\right) F^{N S}\left(\frac{x}{z}, Q^{2}\right)-2 F^{N S}\left(x, Q^{2}\right)\right]\right\}
\end{array}
$$

The NS moments can be evolved to NLO by expanding the dimensionless terms $\gamma_{1}^{n, N S}$ [69] and $B_{n}^{N S}$ which are an integral part of the NLO expansions of the coefficient functions. They can be written in equation 3.60.

$$
\begin{array}{r}
\gamma^{n, N S}=\gamma_{0}^{n, N S}\left(\frac{\alpha_{s}}{4 \pi}\right)+\gamma_{1}^{n, N S}\left(\frac{\alpha_{s}}{4 \pi}\right)^{2}+\ldots \\
\bar{C}_{n}^{N S}\left(1, \bar{g}^{2}\right)=1+B_{n}^{N S}\left(\frac{\alpha_{s}}{4 \pi}\right)+\ldots
\end{array}
$$

The results for $B_{n}^{N S}$ in $x$ and $n$ space [70] are shown in expression 3.61 - equation 3.62. 


$$
\begin{gathered}
B_{2}^{N S}=C_{2}(F)\left[-\frac{3}{2} \frac{1+x^{2}}{(1-x)_{+}}+\frac{1}{2}(9+5 x)\right. \\
\left.-2 \ln x \frac{1+x^{2}}{1-x}+2\left(1+x^{2}\right)\left[\frac{\ln (1-x)}{1-x}\right]_{+}-\left(9+\frac{2}{3} \pi^{2}\right) \delta(1-x)\right] \\
B_{2}^{N S}=C_{2}(F)\left[3 \sum_{j=1}^{n} \frac{1}{j}-4 \sum_{j=1}^{n} \frac{1}{j^{2}}-\frac{2}{n(n+1)} \sum_{j=1}^{n} \frac{1}{j}\right. \\
+4 \sum_{s=1}^{n} \frac{1}{s} \sum_{j=1}^{s} \frac{1}{j}+\frac{3}{n}+\frac{4}{n+1}-\frac{2}{n^{2}}-9
\end{gathered}
$$

This allows us to express the NLO evolution of the non singlet moment in terms of the above dimensionless terms as shown in equation 3.63.

$$
\begin{aligned}
M_{n}^{N S}\left(Q^{2}\right) & =M_{n}^{N S}\left(Q_{0}^{2}\right)\left(\frac{\alpha_{s}\left(\frac{Q^{2}}{\Lambda^{2}}\right)}{\alpha_{s}\left(\frac{Q_{0}^{2}}{\Lambda^{2}}\right)}\right)^{d_{n}^{N S}} \\
\times & {\left[1+C_{n}^{N S}\left(\frac{a_{s}\left(Q^{2}\right)-a_{s}\left(Q_{0}^{2}\right)}{4 \pi}\right)\right] }
\end{aligned}
$$

The quantities $d_{n}$ and $C_{n}^{N S}$ can be written in terms of the other quantities as $d_{n}=\frac{\gamma_{0}^{n, N S}}{2 \beta_{0}}$ and $C_{n}^{N S}=B_{n}^{N S}+\frac{\gamma_{1}^{n, N S}}{2 \beta_{0}}-\frac{\beta_{1} \gamma_{0}^{n, N S}}{2 \beta_{0}^{2}}$.

For the NLO non singlet moment equations the resulting logarithmic derivative is now shown in relation 3.64.

$$
\begin{array}{r}
\frac{d F\left(x, Q^{2}\right)}{d \operatorname{In} Q^{2}}=\int_{x}^{1} \frac{\alpha_{s}\left(\frac{Q^{2}}{\Lambda^{2}}\right)}{2 \pi} P_{q q}^{(0)} F\left(\frac{x}{z}, Q^{2}\right) \mathrm{d} z \\
+\int_{x}^{1}\left(\frac{\alpha_{s}\left(\frac{Q^{2}}{\Lambda^{2}}\right)}{2 \pi}\right)^{2}\left[P_{q q}^{(1)}-\beta / 4 B_{n}^{N S}(z)\right] F\left(\frac{x}{z}, Q^{2}\right) \mathrm{d} z
\end{array}
$$

The splitting function can be integrated to the resulting form shown in expression 3.65.

$$
\int \mathrm{dz} z^{n-1} P_{i j}(z)=-\frac{1}{4} \gamma^{n, i j}-\left[\beta_{0} C_{n}^{N S}+\frac{\beta_{1} \gamma_{0}^{n, N S}}{2 \beta_{0}}\right]\left(\frac{\alpha_{s}}{4 \pi}\right)
$$


where ( equation 3.66)

$$
\gamma^{n, i j}=2 \beta_{o} d_{n}^{N S}
$$

The non singlet moment is now displayed in equation 3.67.

$$
\frac{d\left(\ln M_{n}^{N S}\left(Q^{2}\right)\right)}{d\left(\ln \left(Q^{2}\right)\right.}=-\frac{\gamma_{0}^{n, N S}}{2}\left(\frac{\alpha_{s}\left(\frac{Q^{2}}{\Lambda^{2}}\right)}{4 \pi}\right)-\left[\beta_{0} C_{n}^{N S}+\frac{\beta_{1} \gamma_{0}^{n, N S}}{2 \beta_{0}}\right]\left(\frac{\alpha_{s}\left(\frac{Q^{2}}{\Lambda^{2}}\right)}{4 \pi}\right)^{2}
$$

The singlet moments can be written using the terms in equation 3.68. In addition, the sea quark moments in terms of the NS and singlet moments determined previously are written in equation 3.73 - equation 3.75 (equation 3.73 is the strange quark moment, equation 3.74 is the up antiquark moment and equation 3.75 is the down antiquark moment). The moments for the SOMPDF generated theoretical PDFs and the collaboration $\mathrm{PDF}$ for $Q^{2}=2.5 \mathrm{GeV} 2 a n d \mathrm{Q}^{2}=150 \mathrm{GeV}^{2}$ are in 3.9 .

TABLE 3.9: Moment Values

\begin{tabular}{|c|c|c|c|}
\hline \hline Collaboration & $M_{2}^{\text {val }}$ & $M_{2}^{\text {sea }}$ & $M_{2}^{G}$ \\
\hline \multicolumn{4}{|c|}{$Q^{2}=2.5 \mathrm{GeV}^{2}$} \\
\hline ABM [52] & 0.4644 & 0.0849 & 0.4226 \\
CT10 [58] & 0.4482 & 0.0873 & 0.4263 \\
MSTW [51] & 0.4416 & 0.0900 & 0.4322 \\
NNPDF [1] & 0.4601 & 0.0790 & 0.4378 \\
SOMPDF 6 × 6 & 0.4269 & 0.086 & 0.3852 \\
\hline \multicolumn{4}{|c}{$Q^{2}=150 \mathrm{GeV}^{2}$} \\
\hline ABM [52] & 0.3407 & 0.0995 & 0.5098 \\
CT10 [58] & 0.3276 & 0.1010 & 0.4793 \\
MSTW [51] & 0.3177 & 0.1050 & 0.4844 \\
NNPDF [1] & 0.3340 & 0.0965 & 0.4861 \\
SOMPDF 6 $\times 6$ & 0.3248 & 0.0986 & 0.4601 \\
\hline \hline
\end{tabular}

$$
\begin{array}{r}
T 3=\left(u_{v}\left(Q_{0}^{2}\right)+2 \bar{u}\left(Q_{0}^{2}\right)\right)-\left(d_{v}\left(Q_{0}^{2}\right)+2 \bar{d}\left(Q_{0}^{2}\right)\right) \\
T 8=\left(u_{v}\left(Q_{0}^{2}\right)+2 \bar{u}\left(Q_{0}^{2}\right)\right)+\left(d_{v}\left(Q_{0}^{2}\right)+2 \bar{d}\left(Q_{0}^{2}\right)\right)-4 s\left(Q_{0}^{2}\right) \\
T 15=\left(u_{v}\left(Q_{0}^{2}\right)+\bar{u}\left(Q_{0}^{2}\right)\right)+\left(d_{v}\left(Q_{0}^{2}\right)+2 \bar{d}\left(Q_{0}^{2}\right)\right)+2 s\left(Q_{0}^{2}\right)-6 c\left(Q_{0}^{2}\right) \\
T 24=\left(u_{v}\left(Q_{0}^{2}\right)+2 \bar{u}\left(Q_{0}^{2}\right)\right)+\left(d_{v}\left(Q_{0}^{2}\right)+2 \bar{d}\left(Q_{0}^{2}\right)\right)+2 s\left(Q_{0}^{2}\right)+2 c\left(Q_{0}^{2}\right) \\
T 35=\left(u_{v}\left(Q_{0}^{2}\right)+2 \bar{u}\left(Q_{0}^{2}\right)\right)+\left(d_{v}\left(Q_{0}^{2}\right)+2 \bar{d}\left(Q_{0}^{2}\right)\right)+2 s\left(Q_{0}^{2}\right)+2 c\left(Q_{0}^{2}\right)
\end{array}
$$




$$
\begin{aligned}
& s=\frac{1}{2}\left(\frac{-T 8}{3} M_{n}^{N S}\left(Q^{2}\right)+\right. \\
& \frac{T 15}{12} M_{n}^{N S}\left(Q^{2}\right)+ \\
& \left(( \frac { N f } { 4 C f } ) \left(\text { gluon }_{1}+\right.\right. \\
& u_{v}\left(Q_{0}^{2}\right)+d_{v}\left(Q_{0}^{2}\right)+2 \bar{u}\left(Q_{0}^{2}\right)+ \\
& \left.2 \bar{d}\left(Q_{0}^{2}\right)+s\left(Q_{0}^{2}\right)+c\left(Q_{0}^{2}\right)\right) \\
& -\left(u_{v}\left(Q_{0}^{2}\right)+d_{v}\left(Q_{0}^{2}\right)+2 \bar{u}\left(Q_{0}^{2}\right)+2 \bar{d}\left(Q_{0}^{2}\right)+\right. \\
& \left.s\left(Q_{0}^{2}\right)+c\left(Q_{0}^{2}\right)-\text { gluon }_{1} \frac{N f}{4 C f}\right) \\
& \left.\left.\left(\frac{\alpha_{s}\left(Q^{2}\right)}{\alpha_{s}\left(Q_{0}^{2}\right)}\right)^{Y f}\right)\left(\frac{N f}{4 C f}+1\right)^{-1}\right)\left(\frac{T 24}{4}\right) \\
& \bar{u}=\frac{1}{4}\left(M_{n}^{N S}\left(Q^{2}\right) T 3+\frac{T 8}{3} M_{n}^{N S}\left(Q^{2}\right)+\right. \\
& \frac{T 15}{6} M_{n}^{N S}\left(Q^{2}\right)+ \\
& \left(( \frac { N f } { 4 C f } ) \left(\text { gluon }_{1}+\right.\right. \\
& u_{v}\left(Q_{0}^{2}\right)+d_{v}\left(Q_{0}^{2}\right)+2 \bar{u}\left(Q_{0}^{2}\right)+ \\
& \left.2 \bar{d}\left(Q_{0}^{2}\right)+s\left(Q_{0}^{2}\right)+c\left(Q_{0}^{2}\right)\right) \\
& -\left(u_{v}\left(Q_{0}^{2}\right)+d_{v}\left(Q_{0}^{2}\right)+2 \bar{u}\left(Q_{0}^{2}\right)+2 \bar{d}\left(Q_{0}^{2}\right)+\right. \\
& \left.s\left(Q_{0}^{2}\right)+c\left(Q_{0}^{2}\right)-\text { gluon }_{1} \frac{N f}{4 C f}\right) \\
& \left.\left.\left(\frac{\alpha_{s}\left(Q^{2}\right)}{\alpha_{s}\left(Q_{0}^{2}\right)}\right)^{Y f}\right)\left(\frac{N f}{4 C f}+1\right)^{-1}\right)\left(\frac{T 24}{2}\right) \\
& -2 M_{n}^{N S}\left(Q^{2}\right) u_{v}\left(Q_{0}^{2}\right)
\end{aligned}
$$




$$
\begin{array}{r}
\bar{d}=\frac{1}{4}\left(M_{n}^{N S}\left(Q^{2}\right) T 3+\frac{T 8}{3} M_{n}^{N S}\left(Q^{2}\right)+\right. \\
\frac{T 15}{6} M_{n}^{N S}\left(Q^{2}\right)+ \\
\left(( \frac { N f } { 4 C f } ) \left(\text { gluon }_{1}+\right.\right. \\
u_{v}\left(Q_{0}^{2}\right)+d_{v}\left(Q_{0}^{2}\right)+2 \bar{u}\left(Q_{0}^{2}\right)+ \\
\left.2 \bar{d}\left(Q_{0}^{2}\right)+s\left(Q_{0}^{2}\right)+c\left(Q_{0}^{2}\right)\right) \\
-\left(u_{v}\left(Q_{0}^{2}\right)+d_{v}\left(Q_{0}^{2}\right)+2 \bar{u}\left(Q_{0}^{2}\right)+2 \bar{d}\left(Q_{0}^{2}\right)+\right. \\
\left.s\left(Q_{0}^{2}\right)+c\left(Q_{0}^{2}\right)-g l u o n_{1} \frac{N f}{4 C f}\right) \\
\left.\left.\left(\frac{\alpha_{s}\left(Q^{2}\right)}{\alpha_{s}\left(Q_{0}^{2}\right)}\right)^{Y f}\right)\left(\frac{N f}{4 C f}+1\right)^{-1}\right)\left(\frac{T 24}{2}\right) \\
-2 M_{n}^{N S}\left(Q^{2}\right) d_{v}\left(Q_{0}^{2}\right)
\end{array}
$$

\subsection{Map Features}

The SOMPDF procedure uses a list of map parameters than can be adjusted each time the code is run. Below we list the values that represent the best possible choices in terms of speed of convergence and flexibility of results, and which as a result we used in the final SOMPDF run.

- number of PDF types to be used for mixing nfuncs $=1-3$;

- number of PDFs per cell $n_{\text {cell }}=2$;

- number of PDFs to be generated during each cycle in training, $n_{g e n}=4$;

- number of new PDFs to be generated each cycle, $n_{N E W}=10$;

- number of steps to be used in training each SOM, $n_{\text {step }}=5$;

- type of norm (e.g. $L_{1}$ or $\left.L_{2}\right)$ to use for calculating distances between map and code PDFs

- initial learning rate, $L_{R}^{0}$

- maximum number of iterations regardless of the fitting method, $N_{M A X}=200$ 
- slope parameters based on the number of previous $\chi^{2}$ values to look at when checking whether the $\chi^{2}$ curve had flattened out yet, $s_{\text {flat }}=2 \times 10^{-3}$.

The results for the $\chi^{2}$ for each map size attempted are in 3.10 .

\begin{tabular}{|c|c|}
\hline \hline Size & Minimum $\chi^{2}$ \\
\hline \hline $4 \times 4$ & 1.05202 \\
$5 \times 5$ & 1.0463 \\
$6 \times 6$ & 1.02279 \\
$7 \times 7$ & 1.0648 \\
\hline \hline
\end{tabular}

TABLE 3.10: Fit Results for Various Dimensions

\subsection{Experimental Data}

The experimental data that the SOMPDF code uses to fit the generated PDFs to comes from a set of numerous collaborations. The experimental data sets used come strictly from proton and deuteron DIS "highest quality" data along with collider data. The collaborations are, as noted previously, SLAC, BCDMS, E665, H1, ZEUS and NMC. Only DIS and collider data are used because it is required to determine the capability of the Self Organizing process in fitting parametrized curves to data for isolated processes.

The kinematic range of the DIS experimental data is in Figure 3.2 and the kinematic range of the large $\mathrm{x}$ data is in Figure 3.3. The corresponding table is in 3.12.

TABLE 3.11: Scattering Data Kinematics

\begin{tabular}{|c|c|c|c|c|}
\hline \hline Experiment & Measurement & Usable Points & x range & $Q^{2}$ range \\
\hline \hline BCDMS & $F_{2}^{P}$ & 350 & $7.0 \times 10^{-2}-0.75$ & $7.50-2.3 \times 10^{2}$ \\
H1 & $F_{2}^{P}$ & 364 & $1.0 \times 10^{-4}-0.65$ & $2.0-3.0 \times 10^{4}$ \\
ZEUS & $F_{2}^{P}$ & 240 & $1.0 \times 10^{-4}-0.65$ & $2.7-3.0 \times 10^{4}$ \\
NMC & $F_{2}^{D}$ & 145 & $8.0 \times 10^{-3}-0.5$ & $1.75-6.5 \times 10^{1}$ \\
SLAC & $F_{2}^{P}$ & 194 & $7.0 \times 10^{-2}-0.85$ & $1.12-29.2 \times 10^{1}$ \\
E66 & $F_{2}^{P}$ & 46 & $1.2 \times 10^{-2}-0.39$ & $1.0-6.5 \times 10^{1}$ \\
\hline
\end{tabular}

TABLE 3.12:

\subsection{Error Analysis}

For our error analysis we used the Statistical Error method and then, for the final set of results, the Lagrange multiplier method. We started with the Statistical Error method 


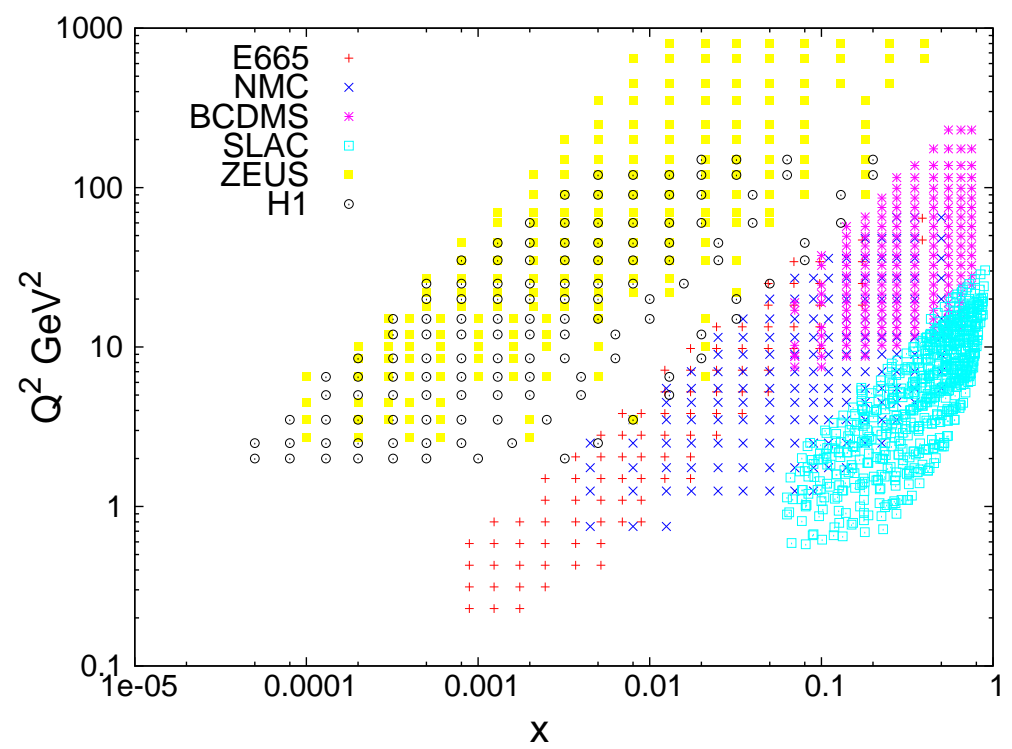

FiguRE 3.2: Kinematic range of the DIS experimental data used in our analysis.

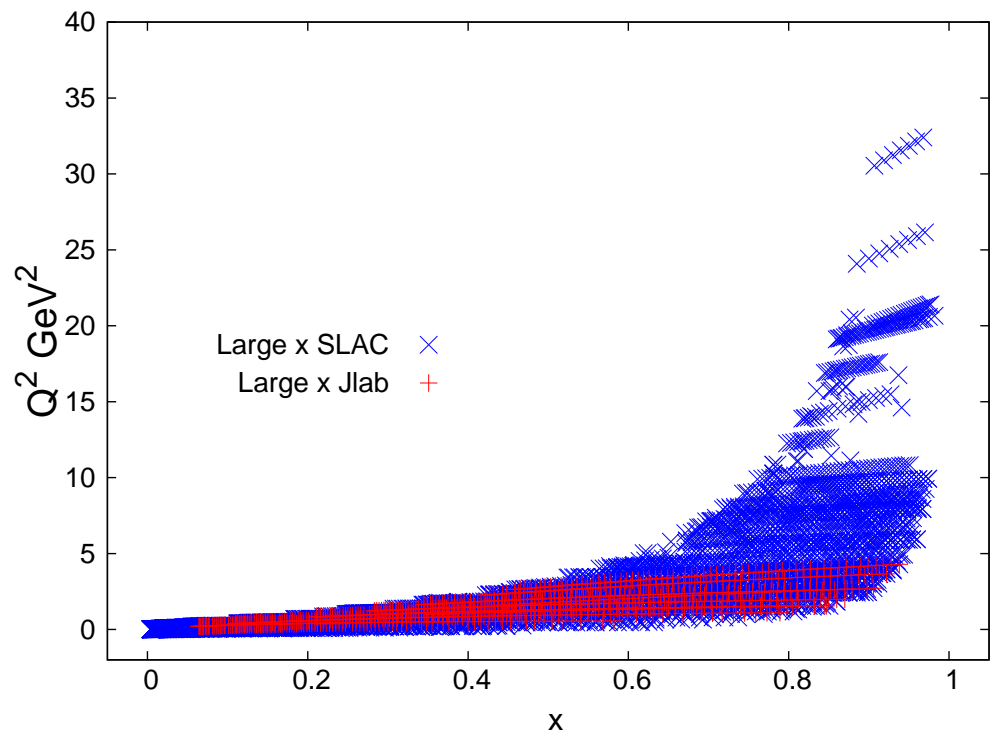

Figure 3.3: Kinematic range of the large $\mathrm{x}$ experimental data used in our analysis. 
so that we could determine the extent to which the PDF envelopes fluctuate and to determine how capable the Statistical Error method is at calculating the error band relative to the Lagrange Multiplier Method. The Statistical Error method also enabled us to determine the extent to which the Self Organizing Process and the GA generated a set PDF curves, at the end of the final iteration, that had significant fluctuations relative to one another. In the Statistical Error method, each of the $n^{2}$ PDFs generated in the final iteration of the SOMPDF code is treated as its own experiment. The standard deviation of these PDFs is used as the error band and the mean value of the PDFs is taken to be the central band.

The results of the Lagrange and Statistical Error band are shown in Figure 3.4. The Statistical Error Band is significantly larger than the Lagrange Error band. This result suggests that the curves that comprise the PDF envelope after the final iteration are not sufficiently precise for determining the PDF errors, using the Statistical Error method, as effectively as the Lagrange Multiplier method can. The central bands for the Statistical Error method and the Lagrange multiplier method are also distinctly different. This shows that the mean value of the PDF curves for the final iteration does not give the same measurement as the best fitting PDFs resulting from the GA. A fundamental issue of the PDF fitting procedure is that the best fit of the structure functions is done relative to a global data set that contains data points from a variety of experiments, each with their own level of precision. The number of parameters involved in the fitting procedure further complicate the error determination.

As a result of these complications, the Lagrange Multiplier method is more ideal for determining the error bands as shown by the generated error bands for each method. The reason that the Lagrange method for determining the error band more effectively incorporates the number of parameters and variation of experimental sets is because the Statistical method incorporates all generated PDF curves regardless of how well or poorly they fit and the Lagrange method does not. Consequently, the Statistical method incorporates the parameter variations that do not lead to effective fits and so becomes increasingly less reliable when the number of variable parameters used and the number of different experimental sets, each with their own precision level as noted above, increase in the computations. In contrast, the Lagrange Multiplier method is based on using variations of the best fitting PDFs relative to the free parameters. For varied free parameters, setting up Hessian matrixes of their derivates to determine PDF errors 
cannot be done so the Lagrange Error Method presents an alternative way to determine PDF errors based on the observables $\chi^{2}$ sand $F_{2}$. This method's use of the best fitting PDFs and their dependence on parameter wiggling significantly improves the precision of this error computation relative to the Standard Error method. It also makes it more ideal for taking into account the different precision levels of the various experimental sets used.

This method evaluates the variation of the $\chi^{2}$ along a specific direction defined by the maximum variation of a given physical variable. In our case the physical variables are the proton (deuteron) structure functions $F_{2}^{P(D)}$. However, at variance with previous analysis that used this method [44], we do not have at our disposal sets of individual parameters for each given PDF, that can be varied. In order to overcome this problem we devised a strategy that we describe below, based on another error method calculation [45], which uses SOMPDFs on appropriately rescaled data to determine the PDFs error. Because the Lagrange multiplier method does not rely on basic statistical variations, it requires an input value for the $\chi^{2}$ tolerance for the best fitting PDFs.

We apply the Lagrange Multiplier Method to PDF analysis where one uses a function $\psi$ determined by equation 3.76 for which the minimum for a given $\lambda, x$ and $Q^{2}$ can be found relative to the parameters $a$. The parameters $a$ are the wiggled $Q^{2}$ dependent parameters whose variations generate the desired envelope of theoretical PDF curves.

$$
\psi(\lambda, a)=\chi_{0}^{2}(a)+\lambda F_{2}^{P(D)}(a)
$$

where $\lambda$ is a series of Lagrange multipliers. $\chi_{0}^{2}(a)$ is the absolute minimum of $\psi$; this minimum is the standard $\chi^{2}$ used in 2.1, for a specific $x$ and $Q^{2}$, to determine the fit of the generated structure functions to experimental data. The designated minimum of $\chi^{2}$ is the minimum of $\chi^{2}$ for all $x$ and $Q^{2}$ values used in the SOMPDF procedure summed together. For each $\lambda$ value there is a singular minimum value of $\psi$ as a function of $a$. Subsequently, each minimum of $\psi$ has a resulting $F_{2}^{P(D)}$ and $\chi^{2}$ value that can be extracted. Then, when the minimum of $\psi$ is computed for a sufficient number of $\lambda$ values, $\chi^{2}$ as a function of $F_{2}^{P(D)}$ can be plotted. The extraction is completed by determining the parameters $a$ that resulted in the minimum value of $\psi$ and calculating the resulting $F_{2}^{P(D)}$ and $\chi^{2}$. 
The Lagrange Multiplier Method is based on using the $\chi^{2}$ values of the structure functions relative to experimental data at specific $Q^{2}$ and $x$ values in order to determine the errors on $F_{2}^{P(D)}$ for these $Q^{2}$ and $x$ values. This method depends on using the experimental data sets in order to find corresponding errors in $F_{2}$. The error is therefore calculated only in $x$ and $Q^{2}$ regions where experimental data points are defined. In the $x$ and $Q^{2}$ regions where experimental data points do not exist, the PDFs are not constrained by the data points and therefore the error bands are not constrained by the Lagrange error. This means that the Lagrange error method is dependent on having a sufficient number of experimental data points in order for it to work properly.

The method first depends on computing the $\chi^{2}$ as a function of $F_{2}^{P}$ or $F_{2}^{D}$, for a given $Q^{2}$ [ for example $Q^{2}=150 \mathrm{GeV}^{2}$ ] for the $x$ values that the experimental data cover for this input $Q^{2}$ value. This was done for $Q^{2}=150$ and $Q^{2}=2.5$ since those were the $Q^{2}$ values that were used to determine the error bands plotted for the PDFs. The $\lambda$ values were chosen to be multiples of 25 and the function determined for each $\lambda$ is $\psi$ as written in expression 3.76 for $\lambda=25,50,75 \ldots$ For each $\lambda$, the minimum value of $\psi$ is determined and this set of minimum values forms a parabola. This is because minimum values of $\psi$ for all the $\lambda$ values will create a parametric relationship between $\chi^{2}$ and $F_{2}^{P(D)}(a)$; the determining of the variations $\Delta \chi^{2}$ will then create the error $\Delta F_{2}^{P(D)}(a)$. The parabola is calculated around a confidence level of 10 percent around $\chi^{2}=1.0$ which was found to be the global minimum $\chi^{2}$ when all data sets were used. The difference in corresponding $F_{2}$ values for $\chi^{2}=1.0$ and $\chi^{2}$ at the 10 percent confidence level, which in this case is $\chi^{2}=1.0+0.1 * 1.0=1.1$, is the resulting Lagrange error on $F_{2}$. This resulted in very low Lagrange values for $F_{2}$ because the Lagrange method is using the $\chi^{2}$ values in relation to $F_{2}$ values for specific $x$ and $Q^{2}$ values and not using measurements on observables which are independent of $x$ and $Q^{2}$ or are taken over all of these values instead of one value at a time. In order for the Lagrange multiplier method to work properly, the errors in $\chi^{2}$ and $F_{2}$ must be completely independent of our Lagrange multiplier spacing choice. The parameters $a$ within the PDFs do not explicitly appear in the Lagrange error computations; however the size of the Lagrange error band will depend on the extent of the parameter variation and the freedom to wiggle the parameters in obtaining the best fitting PDFs.

We represent the $\chi^{2}$ obtained for our choice of interval and $\lambda$ values plotted as a function of $F_{2}^{P(D)}$ in Figure 3.5. As one can see, for each $\lambda$ value, there is a singular minimum 
value of $\chi^{2}$ (Eq.(??)) as a function of $F_{2}^{P(D)}$. The curve around this minimum follows a parabolic shape. The minima of $\psi$ were calculated for a sufficeint number of $\lambda$ values to ensure that the $\chi^{2}$ range of the resulting parabola, after the $\chi^{2}$ and $F_{2}$ values for each minima of $\psi$ were extracted, included the global minimum $\chi^{2}$ and the $\chi^{2}$ for a 10 percent confidence level. The differences between the corresponding $F_{2}^{P(D)}$ values for the global minimum $\chi^{2}$ and the $\chi^{2}$ at 10 percent confidence level were used to determine the Lagrange error in $F_{2}^{P(D)}$, denoted here by $\Delta F$.

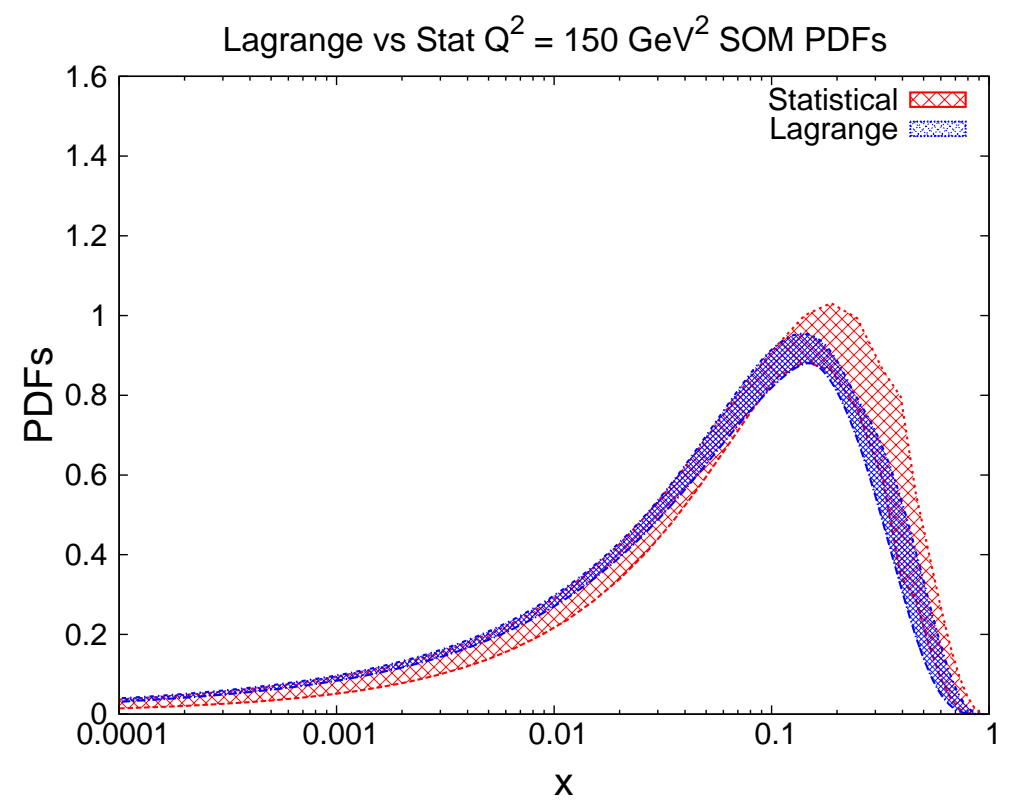

FiguRE 3.4: Illustration of the difference between the PDF uncertainty calculated with the Lagrange multipliers method and the statistical error analysis. Analogous results are obtained for other PDFs.

So far, we followed closely the analysis of Ref.[44]. In order to apply the Lagrange multiplier method to our SOM approach we proceeded as follows. We generate sets of "pseudo experimental data" by shifting $F_{2}^{e x p}$ for given $x$ and $Q^{2}$ values by $\pm \Delta F$, and we repeat the SOMPDF fit for each new data set. The new structure functions are defined by a corresponding set of new individual PDFs, $F_{2}^{e x p, N E W}$. The difference between the individual PDFs from the limiting upper and lower $F_{2}^{\exp , N E W}$ values define then the Lagrange error for each of the individual PDFs for the original $F_{2}^{(D)}$. 


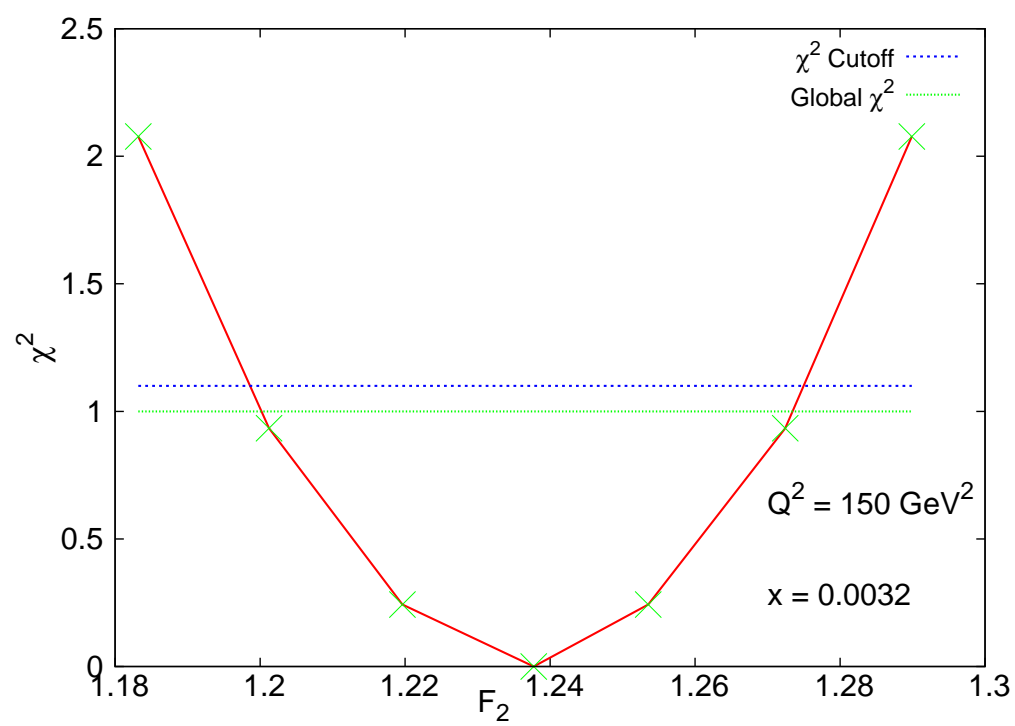

FIGURE 3.5: Illustration of the behavior of the minimum fit value for the observable $F_{2}^{P}$ using the Lagrange Multiplier method. The dots correspond to $\lambda=0,+-25,+-50,+-$ 75. The minimum fit value was evaluated here in one kinematical bin : $x=0.0032$, $Q^{2}=150 \mathrm{GeV}^{2}$, corresponding to equation 2.1 for that specific kinematic. Similar graphs are obtained for all the other $x$ and $Q^{2}$ bins.

\subsection{Lagrange Error}

The error bands for the individual PDFs are the result of using the Lagrange Multiplier method. In this method, the Lagrange error, $\delta F_{2}$ is determined and the $F_{2}$ structure functions are fitted to an experimental data set. The $F_{2}$ structure functions are then fitted, using the same self organizing process, to a new data set determined by taking each experimental data value $F_{2}^{E X P}$ and shifting it by $F_{2}^{E X P}+\delta F_{2}$ and then the process is repeated a third time fitting the structure function to a data set determined by shifting the $F_{2}^{E X P}$ values to $F_{2}^{E X P}-\delta F_{2}$. The differences between the PDFs determined from fitting $F_{2}$ to $F_{2}^{E X P}$ and the PDFs determined from fitting $F_{2}$ to $F_{2}^{E X P}+\delta F_{2}$ are taken and this forms the upper Lagrange error. The differences between the PDFs determined from fitting $F_{2}$ to $F_{2}^{E X P}$ and the PDFs determined from fitting $F_{2}$ to $F_{2}^{E X P}-\delta F_{2}$ are taken and this forms the lower Lagrange error. The Lagrange multiplier method relies on using the self organizing, unsupervised fitting methods for fitting the $F_{2}$ structure functions to all three of the different experimental data sets

The individual PDFs comprise the structure functions that are fitted as shown in expression 2.1. The PDF error bands therefore depend directly on the methods by which the 
structure functions they comprise are fitted. The lack of using the final experimental data sets as a reinforcement for fitting the PDFs resulted in the SOMPDF Lagrange error bands being more similar to each other for each of the PDFs than they were to the error bands determined by the other collaborations. 


\section{SOMPDF PDF Fit Results}

After the PDF envelope is formed, the Self Organizing map is initialized and run through the training process. The initial and final maps can be plotted so that their clustering properties can be observed. These clustering properties for the $6 \times 6$ are shown in Figure 4.1, 4.2 and 4.3. Figure 4.1 and Figure 4.2 show the clustering properties of the global $\chi^{2}$ values for the initial and final map. The global $\chi^{2}$ does not have any clustering patterns in the initial map. The neighborhood radius function used in the SOM, with its ability to preserve the topology of the best fitting theoretical PDFs, enables us to make note of multiple local minimal $\chi^{2}$ values that result from fitting generated sets of theoretical curves. In the final map, the lowest values of the global $\chi^{2}$, representing the best fits to the experimental data sets, are clustered in the lower and upper left hand corners and in the center of the map as well. The structure functions that are used to form these $\chi^{2}$ values can be positioned on a map so that the properties of these structure functions over small and large $x$ that contribute to this $\chi^{2}$ clustering can be analyzed. The actual curves are shown in Figure 4.3.

The Self Organizing Process is designed to minimize the global $\chi^{2}$ values of the generated PDFs and disentangle the components of the DIS and deeply virtual exclusive and semiinclusive processes. Specifically, the task is to identify pattern formation in the structure functions and composite PDFs in terms of local $\chi^{2}$ values and function behavior for a range kinematics. The map in Figure 4.3 reveals the generated theoretical SOMPDF curves along with the experimental data sets, for $Q^{2}=2.5 \mathrm{GeV}^{2}$, that correspond to the PDF $\chi^{2}$ map in Figure 4.2. Figure 4.2 and Figure 4.3 reveal that the PDFs' clustering properties are based on the quality of their fit to the data and on their behavior at small and large $x$. The Self Organizing Process depicted in Figure 4.3 shows how the PDFs, which are formed as a result of this process's evolution over various iterations, and their individual properties can be visualized. This visualization also demonstrates the ability of the Self Organizing process to determine how to make the structure functions fit the data ideally without outside supervision. The Self Organizing process has shown to be effective in clustering recognition for the fit attempt using proton and deuteron Structure Functions using DIS data sets. This process can also be used, as will be shown in Section 5, to isolate and disentangle the properties of the DIS and other processes when large $x$ physics corrections are factored in as well. 


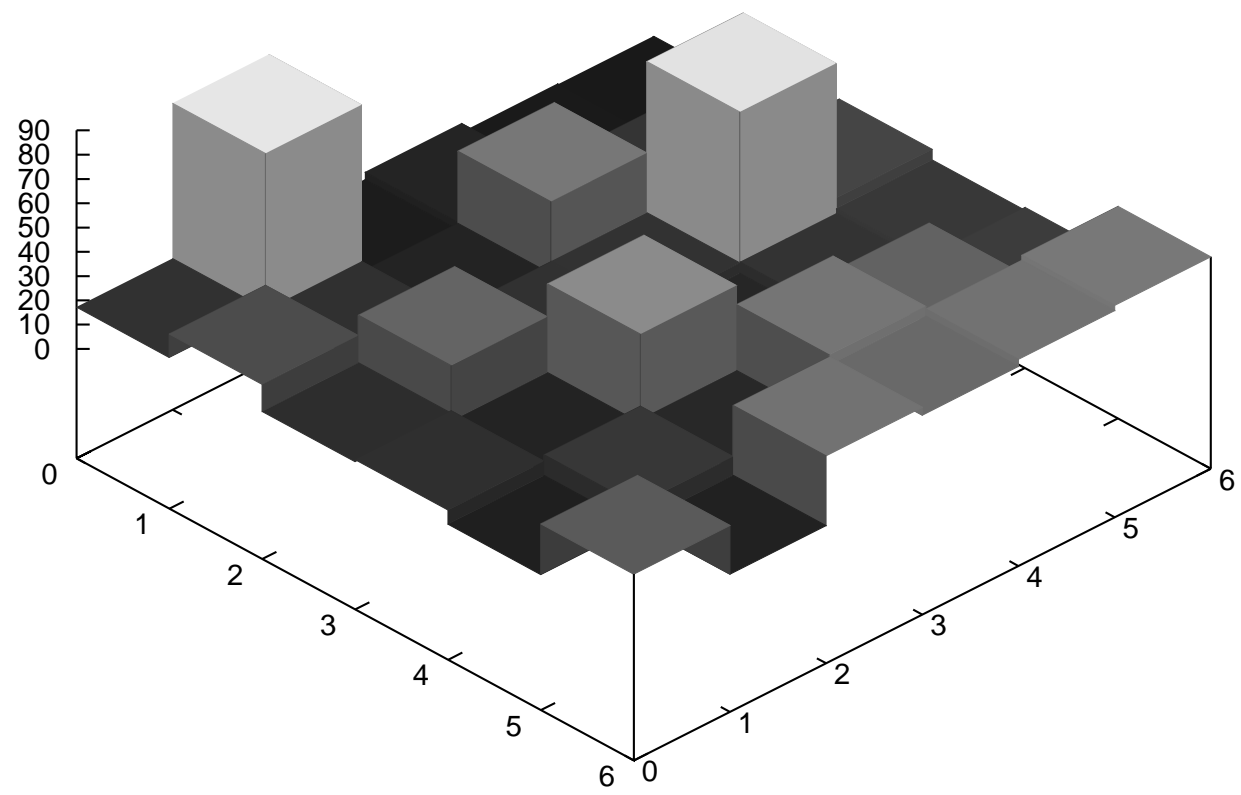

Figure 4.1: $\chi^{2}$ values from the fit on a $6 \times 6$ map for the initial GA iterations. The $\chi^{2}$ values are lower for darker squares.

The results of the $\chi^{2}$ values for the final iteration for the $6 \times 6$ and $1 \times 1$ map are in Figure 4.4. The $6 \times 6$ map has two PDFs used per map cell and four PDFs generated per cycle as per the original map conditions. The $1 \times 1$ map is designed to be the equivalent of the $6 \times 6$ map without self organizing features. Therefore, it uses one cell with 72 PDFs in the cell and 144 PDFs generated for that cell in a given cycle. The $6 \times 6$ map shows the effects of the GA and the Self Organizing Process while the $1 \times 1$ map shows the effects of using only the GA. The $6 \times 6$ map shows a somewhat faster rate of decrease in the $\chi^{2}$ values, revealing the effects the Self Organizing process has on the ability of the SOMPDF method to fit parametrized PDFs to experimental data sets. The Self Organizing process also results in a lower overall $\chi^{2}$ at the end of the final iteration. This effect is not particularly large. However, the local neighborhoods showing multiple $\chi^{2}$ minima have proven to be a critical asset in observing how the PDFs are computed as the neural network tries to fit them to data. Therefore, the Self Organizing process is 


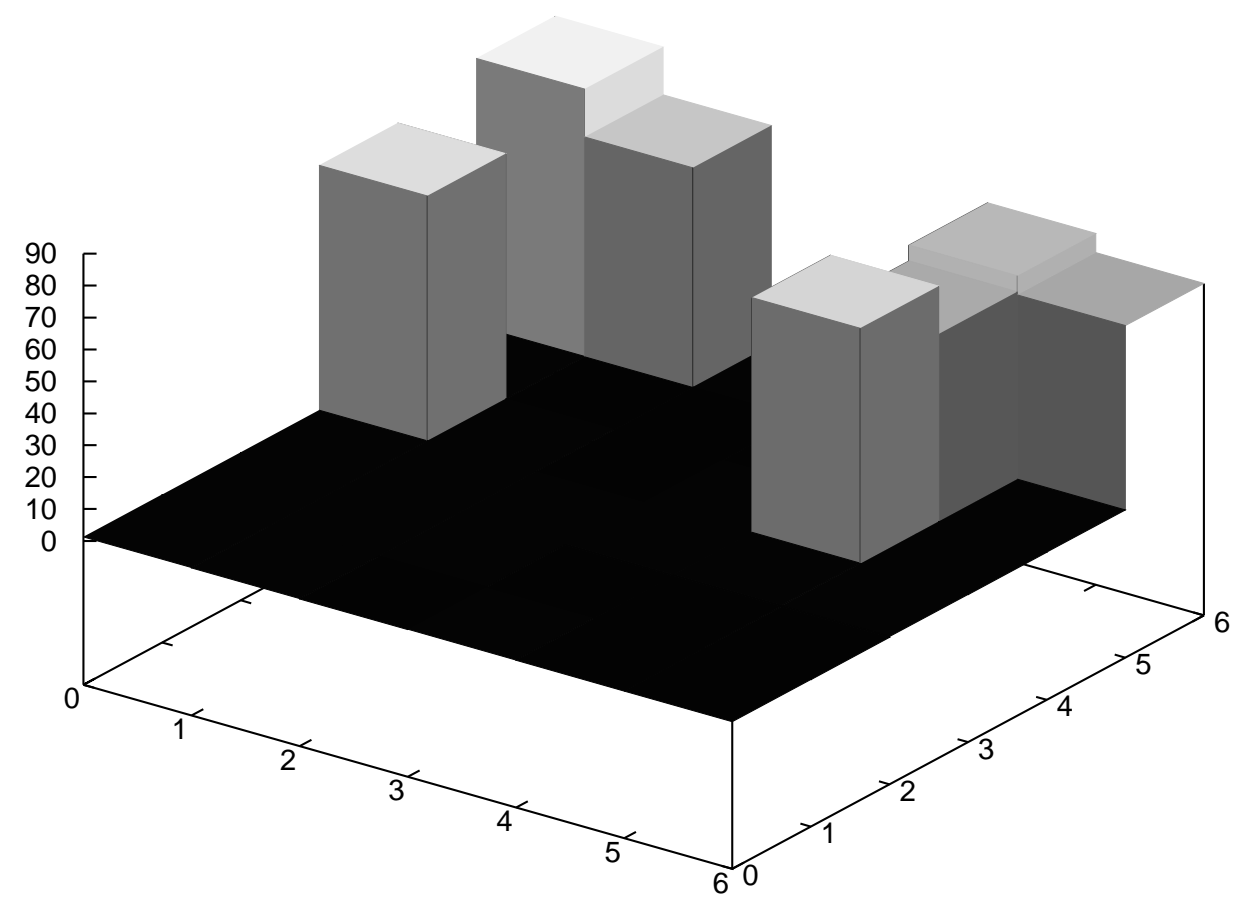

FIgURE 4.2: $\chi^{2}$ values from the fit on a $6 \times 6$ map for the final GA iterations. The $\chi^{2}$ values are lower for darker squares. The clustering properties of the $\chi^{2}$ values are clearly visible. In addition, the decrease of the values relative to those in Fig. 4.1 illustrates the effectiveness of the GA.

critical in allowing us to analyze the clustering properties of the structure functions and the PDFs that comprise them. In addition, the SOM shows nonlinear correlations among the generated functions that form during the fitting process as it reveals multiple local minima as opposed to a single local minima that forms from the GA. These nonlinear correlations are visualized through the two dimensional groupings of generated PDF curves with similar $\chi^{2}$ values. The heights of the data units can be contrasted to each other to see where on the map the non linear correlations occur. These groupings are formed by local neighborhood radii during the Self Organizing Procedure. Subsequently, multiple ways in which the procedure can be used to improve the fit can be visualized. Therefore, the $\chi^{2}$ measurement is not the sole factor in determining the success of the Self Organizing process. 

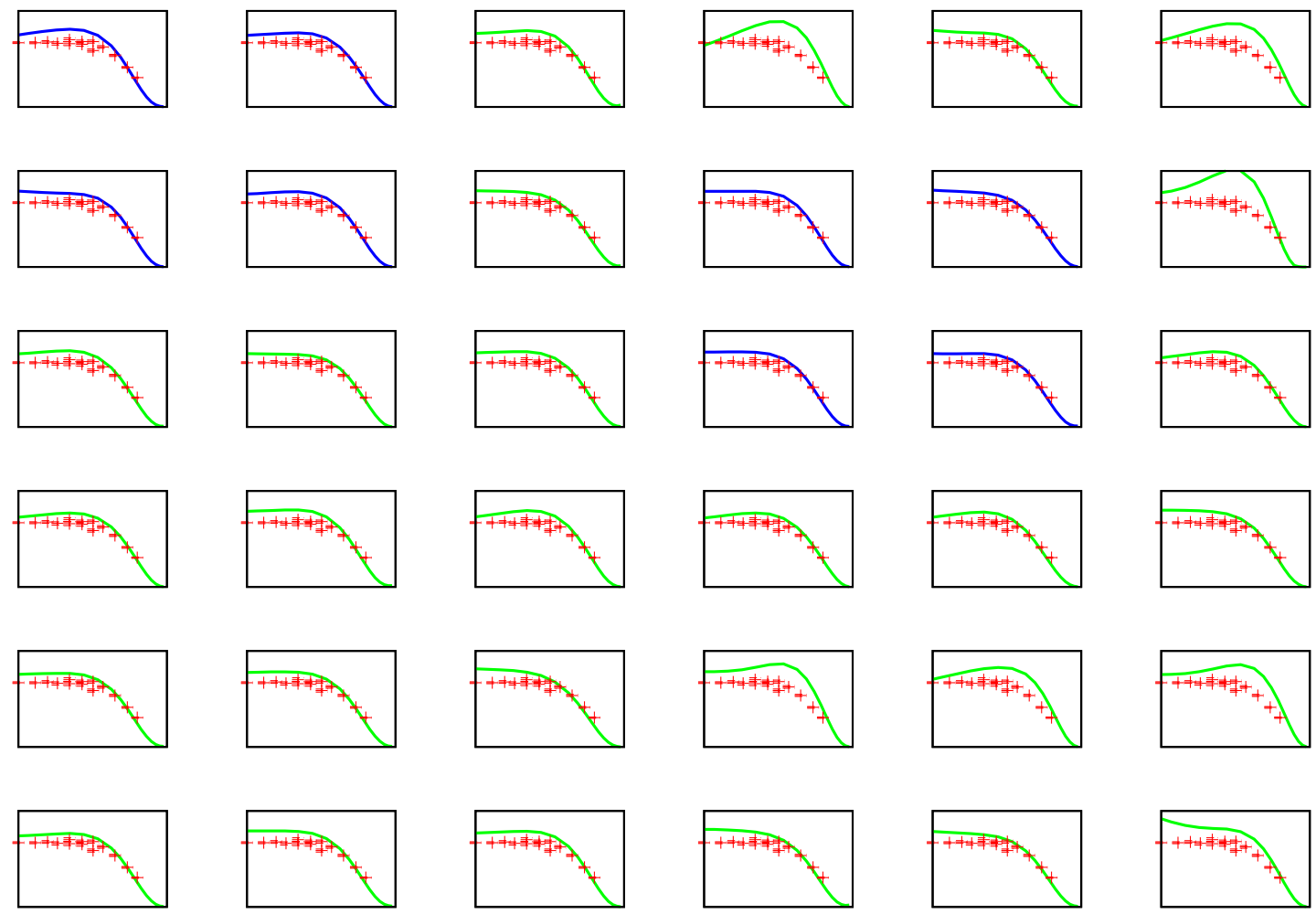

Figure 4.3: The SOM for a $6 \times 6$ map representing the SOMPDF generated structure function, $F_{2}$, for $Q^{2}=2.5 \mathrm{GeV}^{2}$, compared to the experimental values. The visualization properties of the map in forming the clusters structure at small and large $x$ ranges are also shown. The blue curves are clusters of structure functions which have both a low $\chi^{2}$ (dark squares in Fig. 4.2) and a selected particular behavior in $x$. The red points are corresponding the experimental structure function values. The Bjorken $x$ range (the $\mathrm{x}$ axis) is $(0.05: 1.0)$ and the Structure Function range (the y axis) is $(0: 0.5)$.

In Figure 4.5- 4.9, the results for PDFs using the SOMPDF fitting method are shown for $Q^{2}=150 \mathrm{GeV}^{2}$ and in Figure 4.10- 4.12 they are shown for $Q^{2}=2.5 \mathrm{GeV}^{2}$. The PDFs are shown along with the CTEQ, MSTW, ABM and NNPDF collaborations and with the Lagrange error bands for the SOMPDF and the four other collaboration PDFs. The PDF curves show a fit with the collaboration PDFs, within the errors of the SOM and Collaboration PDFs, for the quark PDFs, for the majority of possible $x$ values used in the data fitting. However, there was difficulty getting the valence quark PDFs to fit the collaboration PDFs for $Q^{2}=150 \mathrm{GeV}^{2}$ for $x$ in the range $(0.1: 0.3)$ and the $\bar{d}-\bar{u}$ and strange quarks at this same $Q^{2}$ value for small $x$. This was largely 
due to the lack of experimental constraints in forming the PDFs at low $x$. The DIS experimental data points in this $x$ range are not sufficient to restrict the resulting PDF behaviors, particularly if no previous theoretical assumptions about these behaviors are made. The lack of experimental restrictions at small $x$ also explains the relatively large error bands obtained by the collaborations for their strange quark PDF computations. The gluon quark PDFs were also not as accurate of a fit at $Q^{2}=150 \mathrm{GeV}^{2}$; however, that is largely because the values of the gluon PDFs are not factored into the structure functions and so are not part of the determination of the $\chi^{2}$ values.

To better study the error determination, we construct the "pull" graphs which show the ratio of our PDFs to various collaborations. In Figure 4.13- 4.16, the ratios of the SOMPDF curves to each of the four other collaboration curves are shown along with the relative Lagrange error on the SOMPDF curves. For the fits, the ratio of the valence quark PDFs to the collaboration PDFs for both $Q^{2}$ values used fell within the relative error range relative to one for the majority of the $x$ values, with the exception of the $0.2-1.0$ range and the $0.0001-0.001$ range for $Q^{2}=150 \mathrm{GeV}^{2}$ and the $0.0001-0.009$ and $0.02-0.05$ range for $Q^{2}=2.5 \mathrm{GeV}^{2}$. The gluon quark ratio for $Q^{2}=150 \mathrm{GeV}^{2}$ did not fall within the relative error range for all collaborations for $x$ values less than 0.008 or greater than 0.15 . For $Q^{2}=2.5 \mathrm{GeV}^{2}$ the gluon quark ratio did not fall within the error range for all collaborations for $x$ values less than 0.04 or greater than 0.2 . As a whole, however, the ratios were reasonable close to one for the majority of $x$ values.

There are multiple possible reasons for the fact that the SOMPDF generated PDFs did not fit the collaboration PDFs for all $x$ values and for the sizes of the error bands. There are many different free parameters that were semi randomly varied to create proper initial envelopes of generated curves and eliminate theoretical bias. The tradeoff was an increased difficulty in computing the error bands for each PDF type. The SOMPDF code tries to fit the PDFs to both small and large $x$ data. As a result of this, when the code scales the PDF parameters to fit the small $x$ data, it can decrease the quality of the fit to the large $x$ data and vice versa. Another possible factor is that there are an insufficient number of restrictions on the the quark PDF behavior for the full range of permissible $x$ and $Q^{2}$ values. Subsequently, simply fitting the structure functions to the experimental values for the structure functions is not by itself constraining enough. Finding the best fit values using the lowest possible $\chi^{2}$ results for all the possible $Q^{2}$ values could also lead to difficulties in fitting the PDFs for the full $Q^{2}$ and $x$ range. This 
is because the contributions of the PDFs at various $x$ values to the $\chi^{2}$ results could be partially dependent on the input $Q^{2}$ points used in the PDF computation. The valence quarks are also constricted by the Baryon number sum rule, allowing their quark PDFs less freedom of variance for all $x$ ranges, limiting the ability to make the SOMPDF valence quark PDFs fit those of the Collaboration PDFs and also restricting their error bands. There are also more available data points for lower $x$ ranges where sea quarks dominate using the DIS data; this results in the error bands for the strange quarks to more consistently correlate with uncertainties in the structure function data points for the low $x$ region. For the valence quark distributions, the lack of available DIS data points in the region $0.05 \leq x \leq 0.45$, resulting in fewer restrictions for these quark distributions in that $x$ range for the SOMPDF code, also explains why the quark model, for both large and small $Q^{2}$ values, is not as consistent with the collaborations' model as it is for lower $x$ values. In the SOMPDF code the $\chi^{2}$ values are computed using the relation shown in equation 4.1 .

$$
\chi^{2}=\sum_{i} \frac{\left[F_{2}{ }^{S O M P D F}\left(x_{i}, Q_{i}^{2}\right)-F_{2}^{E X P}\left(x_{i}, Q_{i}^{2}\right)\right]^{2}}{\left(\Delta F_{2}^{E X P}\right)^{2}}
$$

The $F_{2}$ values are proportionally much larger for smaller $x$ than for larger $x$. This means that the SOM PDFs that comprise the structure functions will not be as effected by the GA and the Self Organizing process at larger $x$. The reason for this is because the GA and the Self Organizing process use the $\chi^{2}$ values in order to evolve the PDFs from their starting point to the final iteration result and the magnitude of the $\chi^{2}$ values that determine the extent to which these processes evolve the PDFs are smaller at larger $x$. The lack of consistency for the gluon PDFs to the collaboration PDFs and the relatively weak gluon fit at lower $Q^{2}$ is due to the fact that the gluon PDFs, as noted previously, do not contribute to the structure functions and so the GA does not play a direct role in evolving the gluon PDFs they same way it does for the quark PDFs. The evolution of the gluon PDFs are more dependent on the self organizing process than the evolution of the quark PDFs are since the gluon PDFs do not evolve in accordance to the Self Organizing process and the GA in conjunction as the quark PDFs do. This also leads to more fluctuations in the gluon PDFs, including fluctuations across different $Q^{2}$ values. An additional factor in explaining the size of the error bands for the strange quarks is the total set of experimental data used by the SOMPDF code relative to the 
experimental data used by the collaborations the code PDFs are contrasted against. In the SOMPDF code and in the Alekhin [61] collaboration, only the Deep Inelastic scattering data sets are used. The NNPDF [1] collaboration uses DIS data along with HERA Charm structure function, Fixed Target Drell Yan production, Collider Vector Boson and Collider Jet Production data. The CTEQ [58] collaboration uses DIS data along with Drell Yan, Jet Production, $\gamma$ and Jet Production, W Asymmetry and Z Rapidity production data. The CJ collaboration [59] [60] uses DIS, Drell Yan and $W$ asymmetry data. The more limited data sets used for fitting the structure functions in the SOMPDF code and the Alekhin collaboration result in smaller error bands for the strange quarks relative to those of the other collaborations analyzed because the the increased amount of experimental data sets leads to an increased number of conditions for the fitting procedures which leads to an increase on sources of statistical and systematic uncertainties.

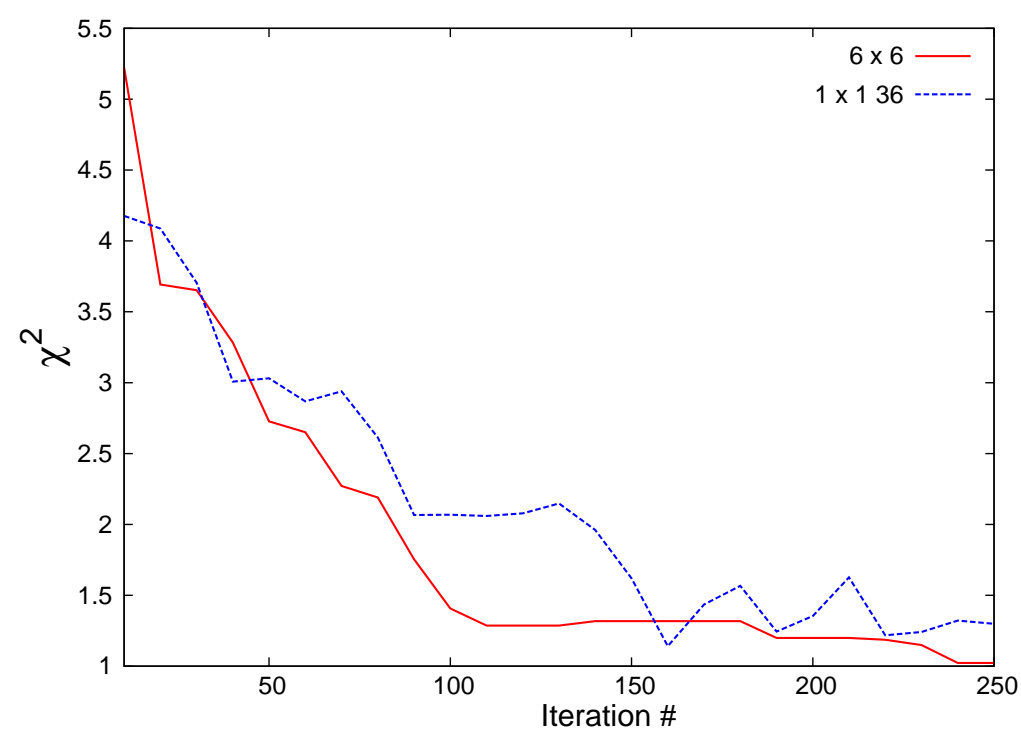

FIgure 4.4: The SOMPDF fit results for number of iterations for a $6 \times 6$ map and a $1 \times 1$ map with the equivalent PDFs generated per cycle and used in training. 


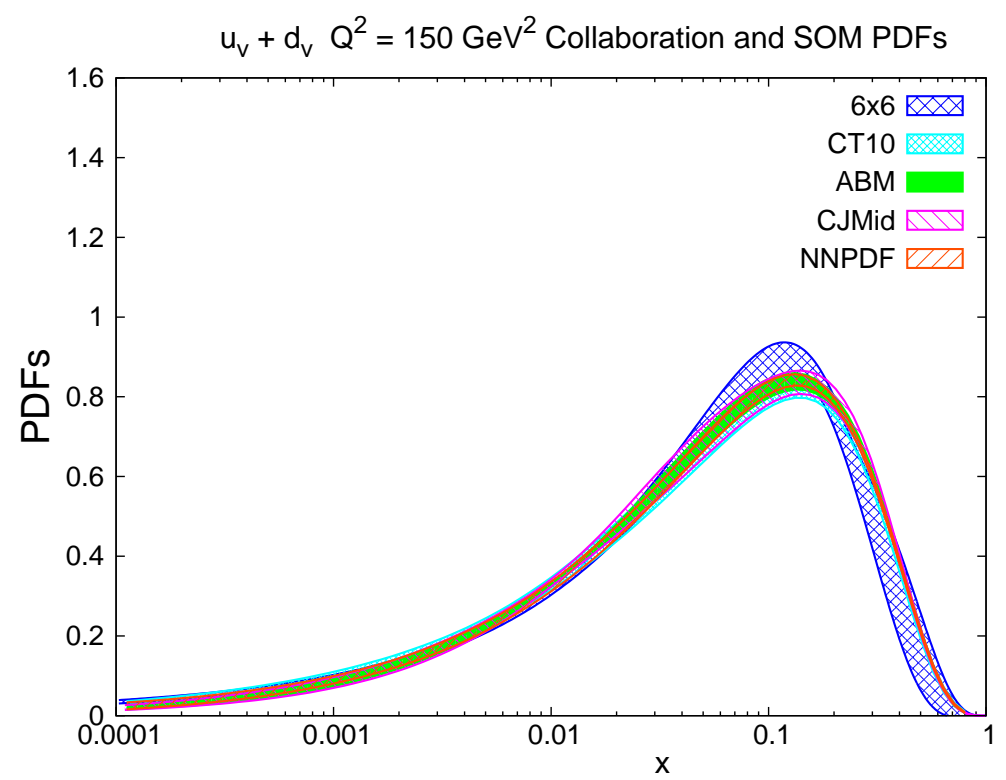

Figure 4.5: SOMPDF generated valence quark PDF $\left(u_{v}+d_{v}\right)$ at $Q^{2}=150 \mathrm{GeV}^{2}$ along with the following collaboration PDFs: CJ Mid [59] [60] NNPDF [1] ABM [61] CT10 [58].

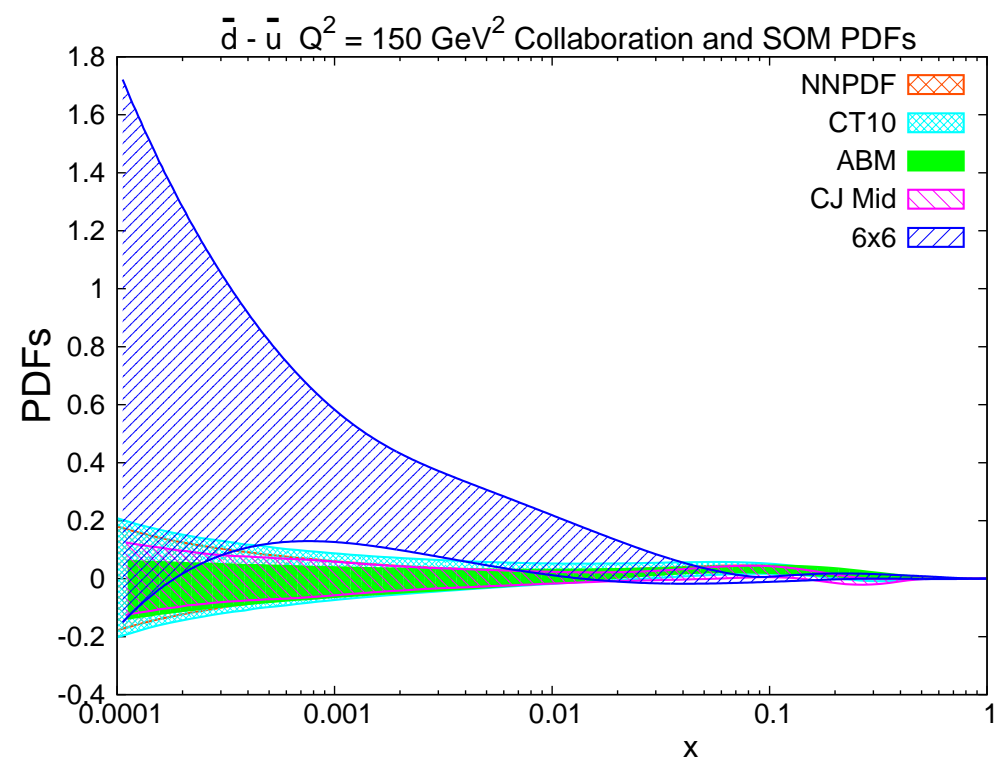

FIGURE 4.6: SOMPDF generated difference between the up and down sea quark quark $\operatorname{PDF}(\bar{u}-\bar{d})$ at $Q^{2}=150 \mathrm{GeV}^{2}$ along with the following collaboration PDFs: CJ Mid [59] [60] NNPDF [1] ABM [61] CT10 [58]. 


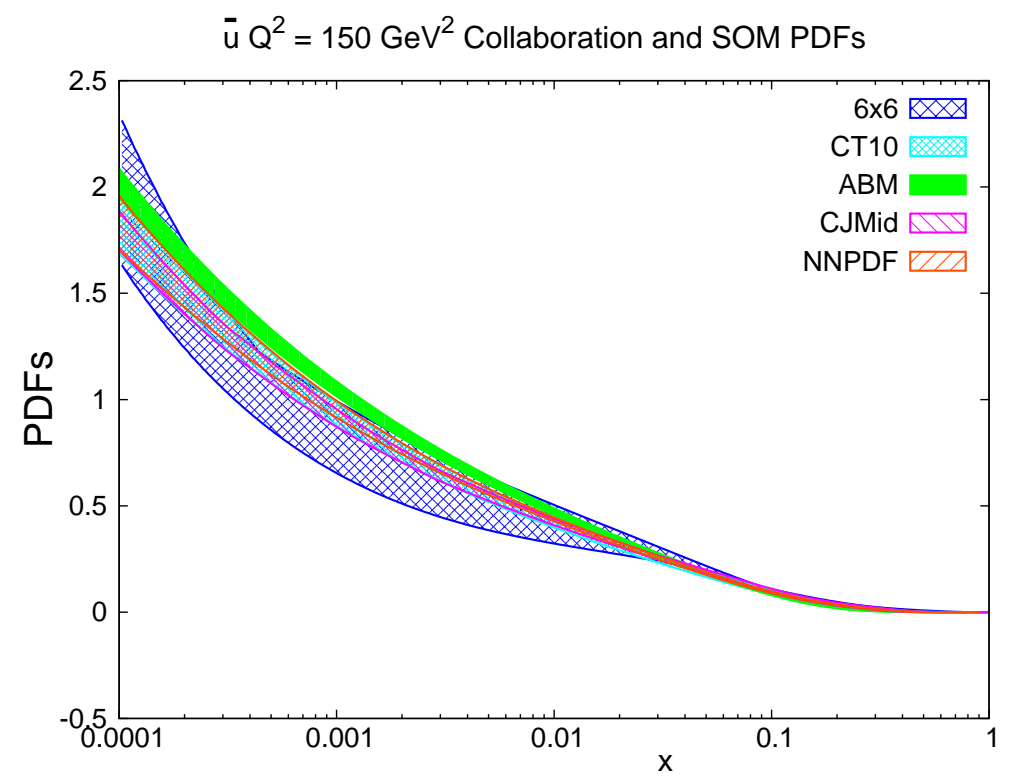

Figure 4.7: SOMPDF generated up sea quark PDF $\bar{u}$ at $Q^{2}=150 \mathrm{GeV}^{2}$ along with the following collaboration PDFs: CJ Mid [59] [60] NNPDF [1] ABM [61] CT10 [58].

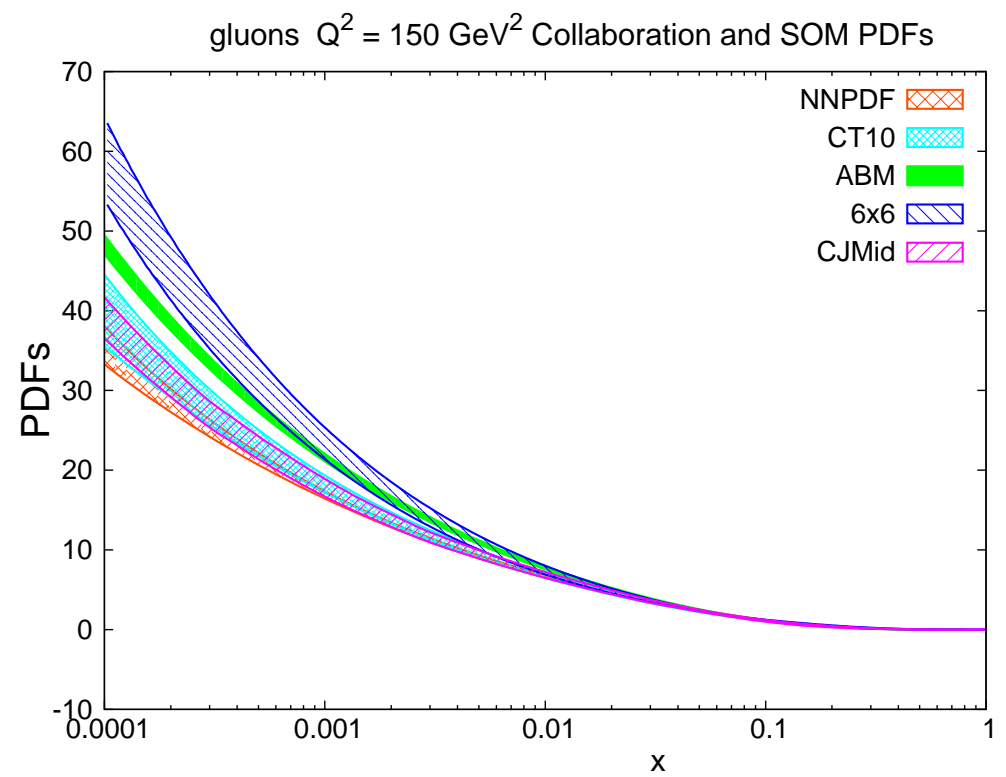

Figure 4.8: SOMPDF generated gluon PDF at $Q^{2}=150 \mathrm{GeV}^{2}$ along with the following collaboration PDFs: CJ Mid [59] [60] NNPDF [1] ABM [61] CT10 [58]. 


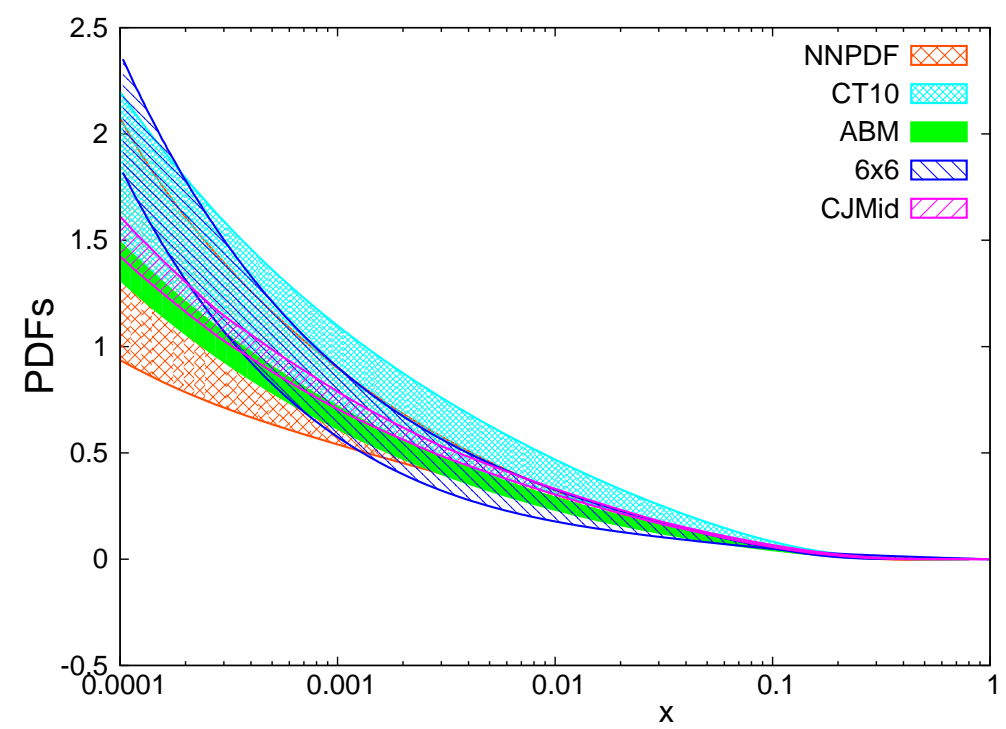

Figure 4.9: SOMPDF generated strange quark PDF at $Q^{2}=150 \mathrm{GeV}^{2}$ along with the following collaboration PDFs: CJ Mid [59] [60] NNPDF [1] ABM [61] CT10 [58].

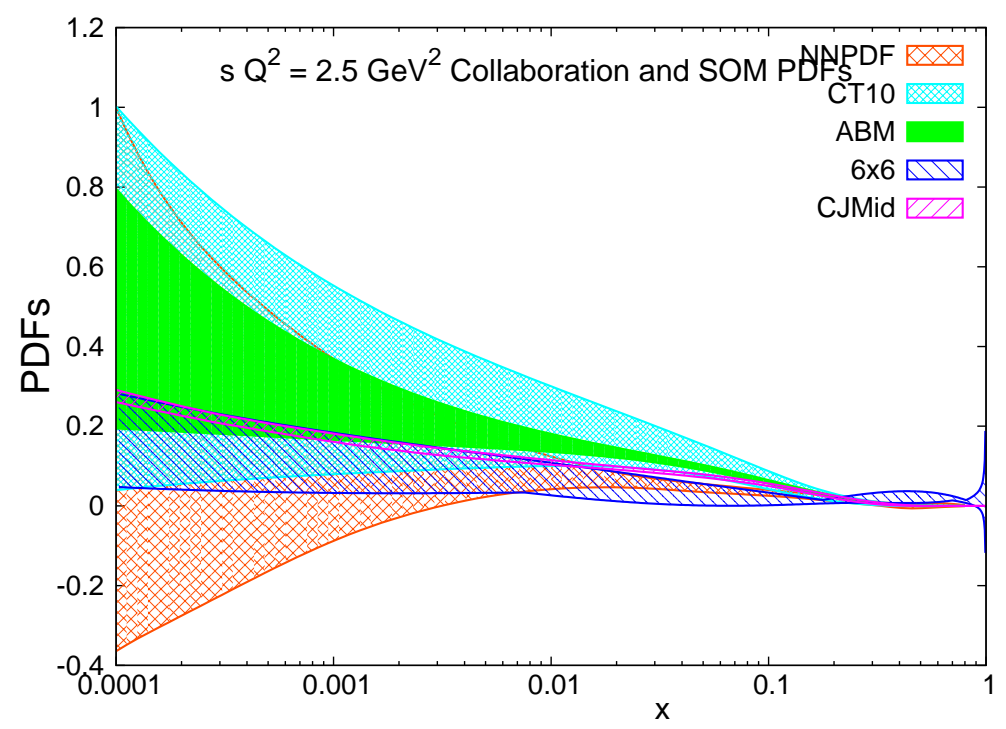

FiguRE 4.10: SOMPDF generated strange quark PDF at $Q^{2}=2.5 \mathrm{GeV}^{2}$ along with the following collaboration PDFs: CJ Mid [59] [60] NNPDF [1] ABM [61] CT10 [58]. 


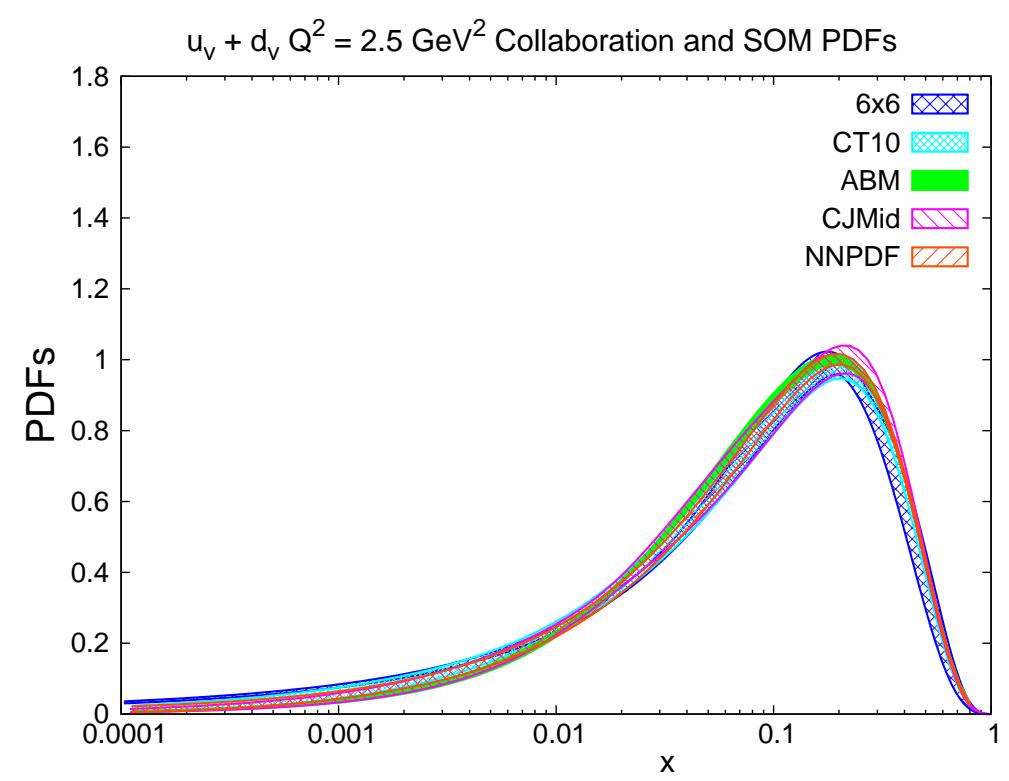

Figure 4.11: SOMPDF generated valence quark PDF $\left(u_{v}+d_{v}\right)$ at $Q^{2}=2.5 \mathrm{GeV}^{2}$ along with the following collaboration PDFs: CJ Mid [59] [60] NNPDF [1] ABM [61] CT10 [58].

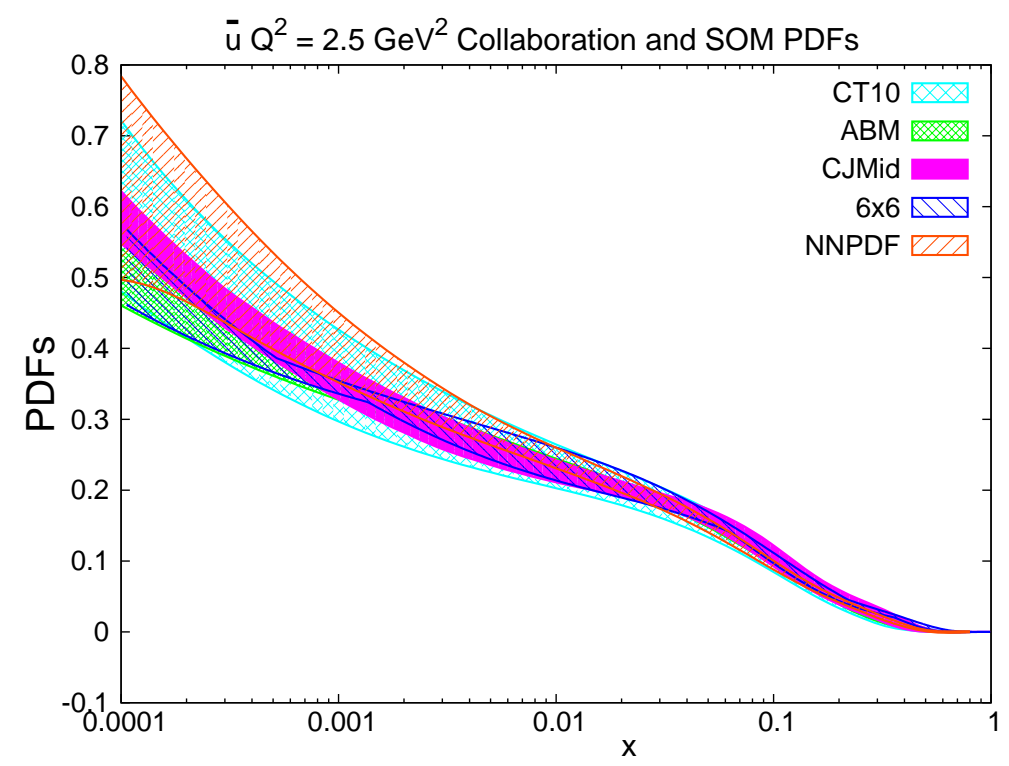

Figure 4.12: SOMPDF generated up sea quark PDF $\bar{u}$ at $Q^{2}=2.5 \mathrm{GeV}^{2}$ along with the following collaboration PDFs: CJ Mid [59] [60] NNPDF [1] ABM [61] CT10 [58]. 


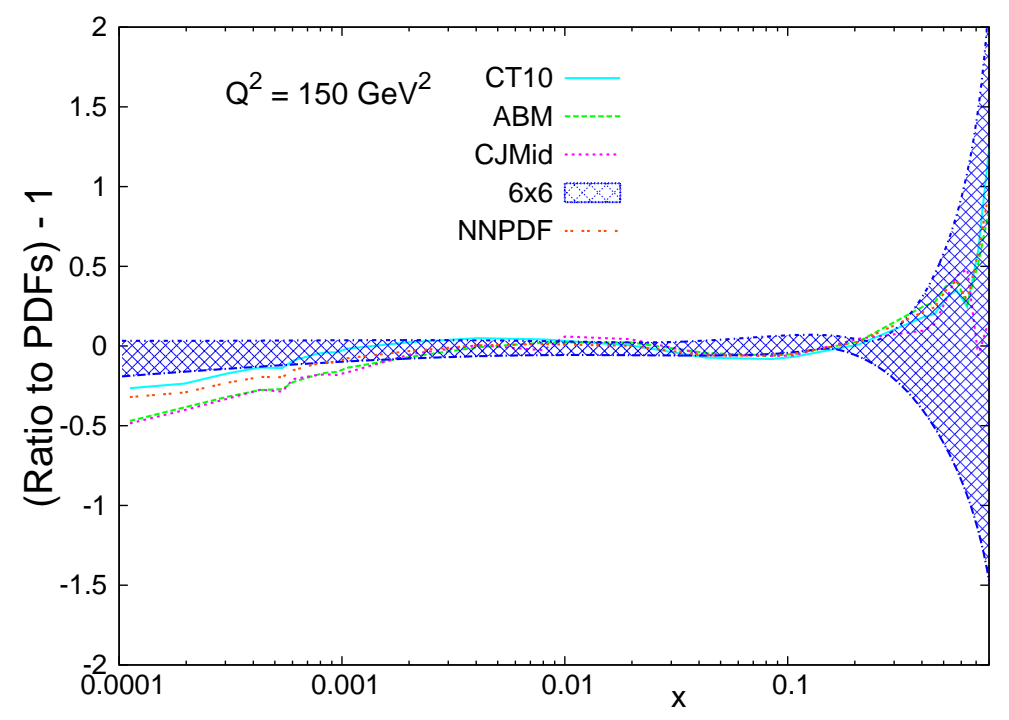

Figure 4.13: The pull of the SOMPDF generated Valence Quark PDF $\left(u_{v}+d_{v}\right)$ relative to collaboration PDFs is shown at $Q^{2}=150 \mathrm{GeV}^{2}$ alongside the relative error of the Valence Quark PDF. The collaboration PDFs are CJ Mid [59] [60] NNPDF [1]

$\mathrm{ABM}[61]$ and CT10 [58].

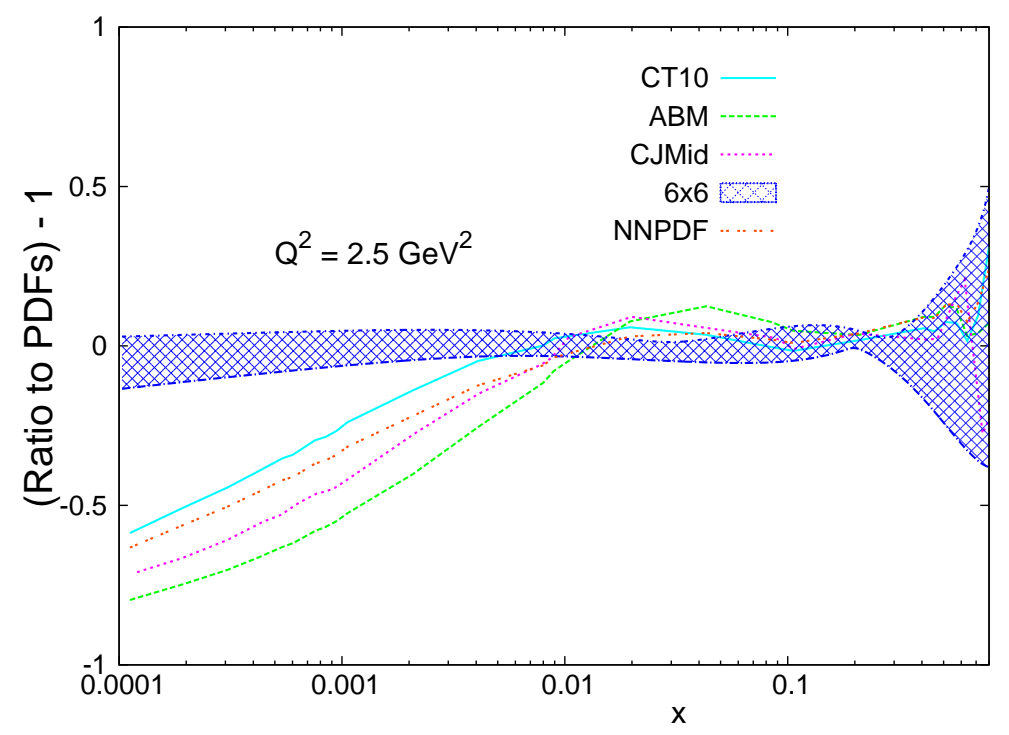

Figure 4.14: The pull of the SOMPDF generated Valence Quark PDF $\left(u_{v}+d_{v}\right)$ relative to collaboration PDFs is shown at $Q^{2}=2.5 \mathrm{GeV}^{2}$ alongside the relative error of the Valence Quark PDF. The collaboration PDFs are CJ Mid [59] [60] NNPDF [1] ABM [61] and CT10 [58]. 


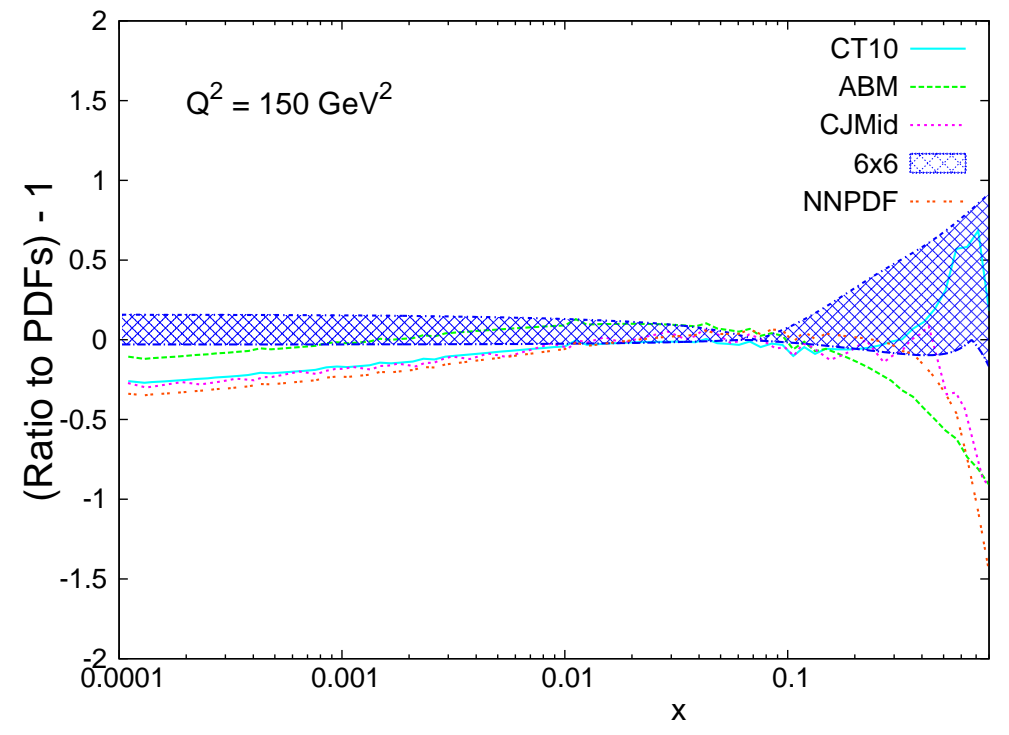

Figure 4.15: The pull of the SOMPDF generated gluon PDF relative to collaboration PDFs is shown at $Q^{2}=150 \mathrm{GeV}^{2}$ alongside the relative error of the gluon Quark PDF. The collaboration PDFs are CJ Mid [59] [60] NNPDF [1] ABM [61] and CT10 [58].

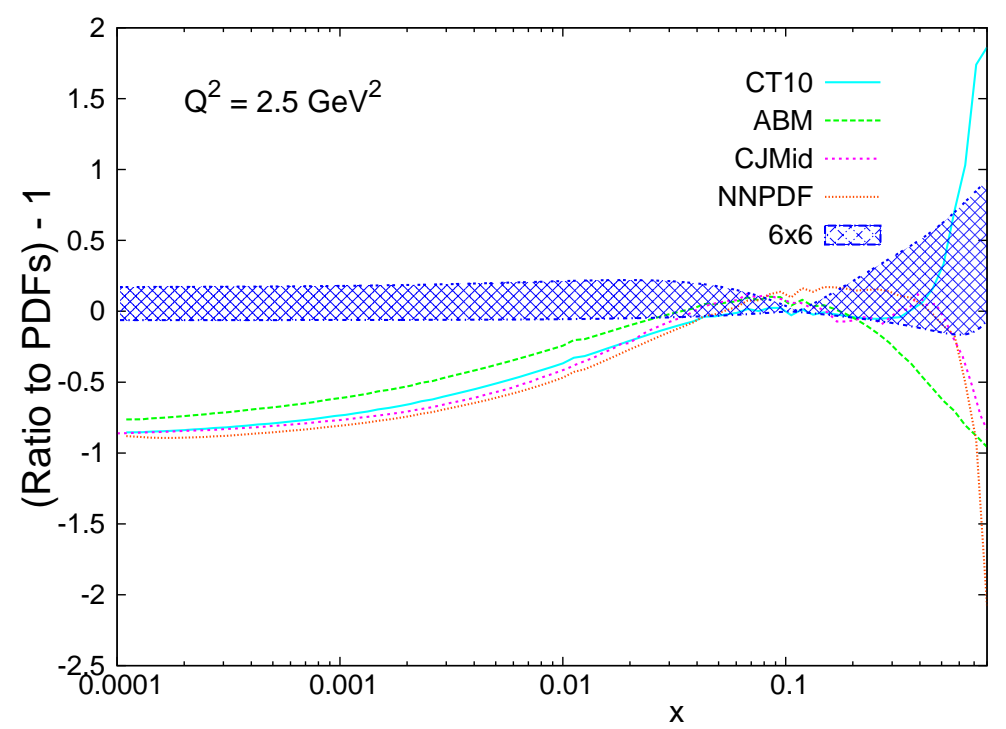

FIGURE 4.16: The pull of the SOMPDF generated gluon PDF relative to collaboration PDFs is shown at $Q^{2}=2.5 \mathrm{GeV}^{2}$ alongside the relative error of the gluon PDF. The collaboration PDFs are CJ Mid [59] [60] NNPDF [1] ABM [61] and CT10 [58]. 


\section{$5 \quad$ Large $\mathrm{x}$ Corrections}

\subsection{Target Mass Corrections}

The fundamental issue about the large $x$ data calculations was modifying the gpd evaluation code, designated here as the gpdeval.f90 module, so that it took into account TMC and LxR.

The previous moment calculations were done using the NS spin operator and using terms $\left(\frac{M^{2}}{Q^{2}}\right)^{n}$, which are negligible for $Q^{2} \gg M^{2}$ but not for $Q^{2}$ in the intermediate range closer to $M^{2}$. Nachtmann [71] accounted for this correction by writing the NS moment relative to the structure function as shown in expression 5.1. These corrections are applied specifically to the NS moments in 3.17. This is the Nachtmann moment; this new moment represents the correction to the previous computation of the moments accounting for TMC. Here, $r$ is given in expression 5.2 and $\zeta$ is given in equation 5.3. The relationship between the resulting Nachtmann moment $\mu^{n}\left(Q^{2}\right)$ and Cornwall Norton moment $M^{n}\left(Q^{2}\right)$, to NLO for the $n^{\text {th }}$ moment, is in expression 5.4. The TMC for the structure functions are [72] equation 5.5. In the computation of the TMC the $n=2$ moment was used.

$$
\begin{gathered}
M^{n}\left(Q^{2}\right)=\int_{x}^{1} \frac{\zeta^{n+1}}{x^{3}}\left(\frac{3+3(n+1) r+n(n+2) r^{2}}{(n+2)(n+3)}\right) \mathrm{d} x \\
r=\sqrt{\left(1+\frac{4 M^{2} x^{2}}{Q^{2}}\right)} \\
\zeta=\frac{2 x}{1+r} \\
\mu^{n}\left(Q^{2}\right)=M^{n}\left(Q^{2}\right)+\frac{n(n-1)}{n+2} \frac{M^{2}}{Q^{2}} M^{n+2}\left(Q^{2}\right) \\
F_{2}^{T M C}\left(x, Q^{2}\right)=\frac{x^{2}}{r^{3}} F_{2}\left(\zeta, Q^{2}\right)+\frac{6 x^{3} M^{2}}{Q^{2} r^{4}} \int_{\zeta}^{1} d \zeta^{\prime} F_{2}\left(\zeta^{\prime}, Q^{2}\right)
\end{gathered}
$$




\subsection{Large x Resummations}

The next step is combining the target mass corrections with the large $x$ Resummations. As noted previously in subsection 3.17 the $Q^{2}$ dependent parameters are wiggled during the SOM and GA processes which is how we account for Perturbative QCD evolution. However, there are still NLO corrections to the PDF computations consisting of $(\ln n)^{2}$ terms that result from the formation of virtual and real gluon emission mismatches. These real and virtual gluon emissions are the two types of gluon corrections to the deep inelastic scattering processes. For $x$ values sufficiently far away from 1, the real and virtual gluon emissions cancel each other out. However, in the limit $x \rightarrow 1.0$ there is less phase space for real gluon emissions and they no longer cancer out virtual gluon emissions. This results in terms of order $(\ln n)^{2}$ in the NLO corrections to the moments of the structure functions. The method of renormalizing the moments of the structure functions lacks the ability to properly resum these terms with powers of $(\ln n)$ with the power 2 or higher and so the large $x$ resummation is used to pick up the $(\ln n)^{2}$ terms so that they can be used in the renormalization of the moments. The gpdeval.f90 module is modified so that these Resummations are taken into account below. The large $x$ resummation starts with equation 5.6.

$$
\frac{d F\left(x, Q^{2}\right)}{d \ln Q^{2}}=\int_{x}^{1} \frac{\alpha_{s}\left(Q^{2}\right)}{2 \pi} P_{q q}^{(0)} F\left(\frac{x}{z}, Q^{2}\right) \mathrm{d} z
$$

The NLO corrections are formula 5.7.

$\frac{d F\left(x, Q^{2}\right)}{d \ln Q^{2}}=\int_{x}^{1} \frac{\alpha_{s}\left(Q^{2}\right)}{2 \pi} P_{q q}^{(0)} F\left(\frac{x}{z}, Q^{2}\right) \mathrm{d} z+\int_{x}^{1}\left(\frac{\alpha_{s}\left(Q^{2}\right)}{2 \pi}\right)^{2}\left[P_{q q}^{(1)}-\beta / 4 B_{n}^{N S}(z)\right] F\left(\frac{x}{z}, Q^{2}\right) \mathrm{d} z$

The large $x$ resummation technique uses the simple and eloquent solution of replacing $Q^{2}$ with $Q^{2} \frac{(1-z)}{z}$, which can be approximated to $Q^{2}(1-z)$ in the strong coupling $\alpha_{s}$ in the limit $z \rightarrow 1$. This replacement results in the $\alpha_{s}$ expansion given in equation 5.8.

$$
\frac{d F\left(x, Q^{2}\right)}{d \ln Q^{2}}=\int_{x}^{1} \frac{\alpha_{s}\left(Q^{2}(1-z)\right)}{2 \pi} P_{q q}^{(0)} F\left(\frac{x}{z}, Q^{2}\right) \mathrm{d} z
$$


The $\alpha_{s}\left(Q^{2}(1-z)\right)$ term can be Taylor expanded while keeping terms up to order $\left(\alpha_{s}\left(Q^{2}\right)\right)^{2}$. This expansion is given in relation 5.9.

$$
\alpha_{s}\left(Q^{2}(1-z)\right)=\alpha_{s}\left(Q^{2}\right)-\beta_{0} \ln (1-z)\left(\alpha_{s}{ }^{2}\left(Q^{2}\right)\right)
$$

The resulting non singlet structure function with this newly expanded $\alpha_{s}$ is expression 5.10 .

$$
\frac{d F\left(x, Q^{2}\right)}{d \ln Q^{2}}=\int_{x}^{1}\left(\frac{\alpha_{s}\left(Q^{2}\right)}{2 \pi} P_{q q}^{(0)}(z)-\left(\frac{\alpha_{s}\left(Q^{2}\right)}{2 \pi}\right)^{2}\left(\frac{\beta_{0}}{4}\right) 2 \ln (1-z)\left(P_{q q}^{(0)}\right)(z)\right) F\left(\frac{x}{z}, Q^{2}\right) \mathrm{d} z
$$

Switching on the NLO corrections to the non singlet structure functions, without using the large $x$ resummations, gives us, second order in $\alpha_{s}$, equation 5.11. In this expression, $P_{q q}^{(0)}$ is the Leading Order splitting function and $P_{q q}^{(1)}$ is the next to Leading Order splitting function. By observing the $z$ dependent components in the expansion of $B_{n}^{N S}$ in expression 3.61, and recalling the Leading Order splitting function formula in 2.4, we can verify that that the structure function in expression 5.10 picks up the resulting NLO expansion term expected in 5.11 from the $\ln (1-z)$ term in $B_{n}^{N S}$.

$$
\frac{d F\left(x, Q^{2}\right)}{d \ln Q^{2}}=\int_{x}^{1} \frac{\alpha_{s}\left(Q^{2}\right)}{2 \pi} P_{q q}^{(0)} F\left(\frac{x}{z}, Q^{2}\right)+\left(\frac{\alpha_{s}\left(Q^{2}\right)}{2 \pi}\right)^{2}\left[P_{q q}^{(1)}-\beta / 4 B_{n}^{N S}(z)\right] F\left(\frac{x}{z}, Q^{2}\right) \mathrm{d} z
$$

The terms from equation 5.10 can then be transformed from $z$ space to $N$ space using the Mellin transform relative to the $z$ variable. These Mellin transforms are given in expression 5.12 .

$$
\frac{d}{d \ln Q^{2}}\left(\frac{\alpha_{s}\left(Q^{2}\right)}{4 \pi}\right)=-\beta_{0}\left(\left(\frac{\alpha_{s}\left(Q^{2}\right)}{4 \pi}\right)^{2}\right)-\left[\beta_{0} \frac{B_{n}^{N S}}{d_{n}^{N S}}+\frac{\gamma_{1}^{n, N S}}{2 d_{n}^{N S}}\right]\left(\left(\frac{\alpha_{s}\left(Q^{2}\right)}{4 \pi}\right)^{3}\right)
$$

Similarly, we can take $B^{N S}$ terms, including those of order $(\ln n)^{2}$, in $x$ space in equation 3.61 for the $F_{2}$ structure function and transform them to $N$ space.

The individual mellin transforms of each term in $B^{N S}$ are in formula 5.13. 


$$
B_{2}^{N S}=C_{2}(F)\left[3 \sum_{j=1}^{n} \frac{1}{j}-4 \sum_{j=1}^{n} \frac{1}{j^{2}}-\frac{2}{n(n+1)} \sum_{j=1}^{n} \frac{1}{j}+4 \sum_{s=1}^{n} \frac{1}{s} \sum_{j=1}^{s} \frac{1}{j}+\frac{3}{n}+\frac{4}{n+1}-\frac{2}{n^{2}}-9\right.
$$

equation 5.13 is the NLO moment to order $(\ln n)^{2}$ once the large $x$ Resummations are applied.

The scaling for $\alpha_{s}\left(Q^{2}\right)$ can further be changed to $\alpha_{s}\left(Q^{2}\right) \rightarrow \alpha_{s}\left(Q^{2} \frac{1-z}{z}\right)$.

The expansion of $\alpha_{s}\left(Q^{2}\right)$ can then be completed in expression 5.14.

$$
\begin{array}{r}
\alpha_{s}\left(Q^{2} \frac{1-z}{z}\right)=\frac{4 \pi}{\beta_{0} \ln \left(\frac{Q^{2}}{\Lambda^{2}} \frac{1-z}{z}\right)} \\
=\frac{4 \pi}{\beta_{0}\left(\ln \left(\frac{Q^{2}}{\Lambda^{2}}\right)+\ln \left(\frac{1-z}{z}\right)\right)} \\
\frac{4 \pi}{\beta_{0} \ln \left(\frac{Q^{2}}{\Lambda^{2}}\right)}\left(\frac{1}{\left.1+\frac{\ln \left(\frac{1-z}{z}\right)}{\ln \left(\frac{Q^{2}}{\Lambda^{2}}\right)}\right)}\right.
\end{array}
$$

Given the mathematical approximation in 5.15, 5.14 can be reduced to an expansion around $\alpha_{s}\left(Q^{2}\right)$.

$$
\frac{1}{1+x} \approx 1-x
$$

Using the replacement $x \rightarrow \frac{\ln \left(\frac{1-z}{z}\right)}{\ln \left(\frac{Q^{2}}{\Lambda^{2}}\right)}$ in 5.15 , the resulting expansion of $\alpha_{s}\left(Q^{2} \frac{1-z}{z}\right)$ is 5.16 .

$$
\begin{array}{r}
\alpha_{s}\left(Q^{2} \frac{1-z}{z}\right)=\frac{4 \pi}{\beta_{0} \ln \left(\frac{Q^{2}}{\Lambda^{2}}\right)}\left(1-\frac{\ln \left(\frac{1-z}{z}\right)}{\ln \left(\frac{Q^{2}}{\Lambda^{2}}\right)}\right)= \\
\frac{4 \pi}{\beta_{0} \ln \left(\frac{Q^{2}}{\Lambda^{2}}\right)}-\ln \left(\frac{1-z}{z}\right) \frac{\beta_{0}}{4 \pi}\left(\frac{4 \pi}{\beta_{0} \ln \left(\frac{Q^{2}}{\Lambda^{2}}\right)}\right)^{2} \alpha_{s}\left(Q^{2}\right)-\frac{\beta_{0}}{2} \ln \left(\frac{1-z}{z}\right) \frac{1}{2 \pi}\left(\alpha_{s}\left(Q^{2}\right)\right)^{2}
\end{array}
$$

In this rescaling, when $z$ gets large enough perturbative expansions are not sufficient when evaluating the structure functions. When this rescaling is used, it is particularly 
useful to introduce a parameter $z_{\max }$ which can be used to split the structure function integral into two sections. The first section, where perturbative expansions are sufficient, is $x<z<z_{\max }$ and the second section, where perturbative expansions are not sufficient, is $z_{\max }<z<1$.

With $\alpha_{s}\left(Q^{2}\right)$ replaced with $\alpha_{s}\left(Q^{2} \frac{1-z}{z}\right)$ the non singlet Structure Function distribution can be rewritten as shown in 5.17 .

$$
\begin{array}{r}
\frac{d x q\left(x, Q^{2}\right)}{d \ln Q^{2}}=\int_{x}^{1} \frac{d y}{y}\left(\frac{\alpha_{s}}{2 \pi} P_{q q}^{(0)}(z)+\left(\frac{\alpha_{s}}{2 \pi}\right)^{2}\left[P_{q q}^{(1)}(z)-P_{q q}^{(1)}(z)-\frac{\beta_{0}}{2} \ln \left(\ln \frac{1-z}{z}\right) P_{q q}^{(0)}(z)\right]\right)(z) y q\left(y, Q^{2}\right)= \\
\int_{x}^{1} \frac{d y}{y}\left(\frac{\alpha_{s}}{2 \pi} P_{q q}^{(0)}(z)+\left(\frac{\alpha_{s}}{2 \pi}\right)^{2}\left[P_{q q}^{(1)}(z)-P_{q q}^{(1)}(z)-\frac{\beta_{0}}{4} B_{n}^{N S \ln z}(z)-\frac{\beta_{0}}{4} B_{n}^{N S \text { divergent }}(z)\right]\right)(z) y q\left(y, Q^{2}\right)
\end{array}
$$

The convergent and divergent components of $B_{n}^{N S}$ are equation 5.18 and equation 5.19 .

$$
\begin{gathered}
B_{\ln z}^{N S}(z)=-2 \ln (z) P_{q q}^{(0)}(z) \\
B_{\text {divergent }}^{N S}(z)=2 \ln (1-z) P_{q q}^{(0)}(z)
\end{gathered}
$$

The non singlet Altarelli-Parisi equations in 5.17 can also be converted to structure function form as 5.20. This expression comes from using a new scaling parameter $(\theta(z))$ in the coupling constant, which can be set to $(\theta(z))=W^{2}$, so that the non singlet expansion in NLO can be expressed in terms of the Wilson Coefficient $B_{2}^{N S}$. Because the coupling $\alpha_{s}$ is cut at the endpoints, they can be computed from the + function computation can then be computed separately from the $z$ integral of the non singlet terms. The coupling constant also undergoes the shift $\alpha_{s}\left(Q^{2} z\right) \rightarrow \alpha_{s}\left(Q^{2} \frac{1-z}{z}\right)$, with $W^{2}$ set to $W^{2}=Q^{2} \frac{1-z}{z}$. 


$$
\begin{array}{r}
F_{2}^{N S}(x)=q_{N S}(x)+\int_{x}^{1} d z \frac{\alpha_{s}(\ln (\theta(z)))}{4 \pi} B_{q}(z) q_{N S}\left(\frac{x}{z}\right)= \\
q_{N S}(x)+\int_{x}^{1} d z \frac{\alpha_{s}\left(W^{2}\right)}{4 \pi}\left[P_{q q}^{0}(z)\left(\ln (1-z)-\ln z-\frac{3}{2}\right)+\frac{1}{2}(9+5 z)\right] q_{N S}\left(\frac{x}{z}\right)= \\
q_{N S}(x)+\int_{x}^{1} d z \frac{\alpha_{s}\left(Q^{2}\right)+\delta \alpha_{s}\left(z, Q^{2}\right)}{4 \pi}\left[P_{q q}^{0}(z)\left(\ln (1-z)-\ln z-\frac{3}{2}\right)+\frac{1}{2}(9+5 z)\right] q_{N S}\left(\frac{x}{z}\right)
\end{array}
$$

The logarithmic terms in equation 5.20 can be expressed as equation 5.21.

$$
\frac{\alpha_{s}\left(Q^{2}\right)}{4 \pi} \ln (1-z)=\frac{1}{2} \int_{Q_{0}^{2}}^{Q^{2}} d \ln Q^{2} \frac{\alpha_{s}\left(W^{2}\right)-\alpha_{s}\left(Q^{2}\right)}{2 \pi}=\frac{1}{\beta_{0}} \ln \left(1+\frac{\ln (1-z)}{\ln Q^{2} / \Lambda^{2}}\right)
$$

The $\ln (1-z)$ expansion terms can be rewritten [73] in formula 5.22.

$$
\begin{array}{r}
\ln (1-z)=\frac{1}{\alpha_{s, L O}\left(Q^{2}\right)} \int_{Q_{0}^{2}}^{Q^{2}} d \ln Q^{2}\left[\alpha_{s, L O}\left(Q^{2}(1-z)\right)-\alpha_{s, L O}\left(Q^{2}\right)\right] \\
\rightarrow \frac{1}{\alpha_{s, L O}\left(Q^{2}\right) \beta_{0}} \ln \left(1+\frac{\ln (1-z)}{\ln Q^{2} / \Lambda^{2}}\right)
\end{array}
$$

This gives a new expression for the non singlet structure functions in equation 5.23.

$$
\begin{array}{r}
F_{2}^{N S}(x)=q_{N S}(x)+\int_{x}^{1} d z \frac{\alpha_{s}\left(Q^{2}\right)}{4 \pi}\left[P_{q q}^{0}(z)\left(-\ln z-\frac{3}{2}\right)+\frac{1}{2}(9+5 z)+\right. \\
\left.P_{q q}^{0}(z) \frac{4 \pi}{\beta_{0} \alpha_{s, L O}\left(Q^{2}\right)} \ln \left(1+\frac{\ln (1-z)}{\ln Q^{2} / \Lambda^{2}}\right)\right] q_{N S}\left(\frac{x}{z}\right)
\end{array}
$$

Once the NS structure function has been rewritten in this manner, the integrals should be split according to a given $z_{\max }$ endpoint using the + functions to split the integral into two ranges, one for $z<z_{\max }$ and the other for $z>z_{\max }$ in equation 5.24. 


$$
\int_{x}^{1} d z\left[\Theta\left(-z+z_{\max }\right) \ln \left(1+\frac{\ln (1-z)}{\ln Q^{2} / \Lambda^{2}}\right)\left[\frac{1}{1-z}\right]_{+}+\Theta\left(z-z_{\max }\right) \ln \left(1+\frac{\ln \left(1-z_{\max }\right)}{\ln Q^{2} / \Lambda^{2}}\right) \frac{1}{1-z}\right]
$$

Evaluating the step functions in expression 5.24 results in expression 5.25.

$$
\int_{x}^{z_{\max }} d z\left[\ln \left(1+\frac{\ln (1-z)}{\ln Q^{2} / \Lambda^{2}}\right)\left[\frac{1}{1-z}\right]_{+}+\int_{z_{\max }}^{1} d z \ln \left(1+\frac{\ln \left(1-z_{\max }\right)}{\ln Q^{2} / \Lambda^{2}}\right) \frac{1}{1-z}\right]
$$

A new set of splitting functions is equation 5.26.

$$
\begin{array}{r}
\int_{0}^{1} d z\left[\frac{f(z)}{1-z}\right]_{+}=\int_{a}^{1} d z\left[\frac{f(z)}{1-z}\right]_{+}+\int_{b}^{a} d z\left[\frac{f(z)}{1-z}\right]_{+}+\int_{0}^{b} d z\left[\frac{f(z)}{1-z}\right]_{+} \\
\int_{a}^{1} d z\left[\frac{f(z)}{1-z}\right]_{+}=\int_{0}^{1} d z\left[\frac{f(z)}{1-z}\right]_{+}-\int_{b}^{a} d z\left[\frac{f(z)}{1-z}\right]_{+}-\int_{0}^{b} d z\left[\frac{f(z)}{1-z}\right]_{+} \\
\int_{a}^{1} d z\left[\frac{f(z)}{1-z}\right]_{+} \int_{0}^{1} d z=\int_{0}^{1} d z \frac{f(z)-f(1)}{1-z}-\int_{0}^{a} d z \frac{f(z)}{1-z} \\
\int_{a}^{1} d z\left[\frac{f(z)}{1-z}\right]_{+} \int_{a}^{1} d z=\int_{a}^{1} d z \frac{f(z)-f(1)}{1-z}+\int_{0}^{a} d z \frac{f(z)-f(1)}{1-z}-\int_{0}^{a} d z \frac{f(z)}{1-z}
\end{array}
$$

A resulting form for $\int_{z_{\max }}^{1} d z\left[\frac{f(z)}{1-z}\right]_{+}$is equation 5.27 .

$$
\int_{z_{\max }}^{1} d z\left[\frac{f(z)}{1-z}\right]_{+}=\int_{z_{\max }}^{1} d z \frac{f(z)-f(1)}{1-z}-f(1) \int_{0}^{z_{\max }} \frac{d z}{1-z}
$$

This means equation 5.25 can be written using the expression in equation 5.28. 


$$
\begin{array}{r}
\int_{x}^{z_{\max }} d z \ln \left(1+\frac{\ln (1-z)}{\ln Q^{2} / \Lambda^{2}}\right) \frac{1+z^{2}}{1-z} \tilde{q}_{N S}\left(\frac{x}{z}\right) \\
+\left(\int_{z_{\max }}^{1}\left[\frac{1+z^{2}}{1-z}\right]_{+} \tilde{q}_{N S}\left(\frac{x}{z}\right)\right) \times \ln \left(1+\frac{\ln \left(1-z_{\max }\right.}{\ln Q^{2} / \Lambda^{2}}\right) \\
\int_{x}^{z_{\max }} d z \ln \left(1+\frac{\ln (1-z)}{\ln Q^{2} / \Lambda^{2}}\right) \frac{1+z^{2}}{1-z} \tilde{q}_{N S}\left(\frac{x}{z}\right) \\
+\int_{z_{\max }}^{1} d z \frac{\ln \left(1+z^{2}\right) \tilde{q}_{N S}\left(\frac{x}{z}\right)-2 \tilde{q}_{N S}(x)}{1-z} \times \ln \left(1+\frac{\ln \left(1-z_{\max }\right)}{\ln Q^{2} / \Lambda^{2}}\right) \\
-2 \tilde{q}_{N S}(x) \int_{0}^{z_{\max }} \frac{d z}{1-z} \times \ln \left(1+\frac{\ln \left(1-z_{\max }\right)}{\ln Q^{2} / \Lambda^{2}}\right) \\
\int_{x}^{z_{\max }} d z \ln \left(1+\frac{\ln (1-z)}{\ln Q^{2} / \Lambda^{2}}\right) \frac{1+z^{2}}{1-z} \tilde{q}_{N S}\left(\frac{x}{z}\right) \\
+\int_{z_{\max }}^{1} d z \frac{\left(1+z^{2}\right) \tilde{q}_{N S}\left(\frac{x}{z}\right)-2 \tilde{q}_{N S}(x)}{1-z} \times \ln \left(1+\frac{\ln \left(1-z_{\max }\right)}{\ln Q^{2} / \Lambda^{2}}\right) \\
-2 \tilde{q}_{N S}(x) \ln \left(1-z_{\max }\right) \times \ln \left(1+\frac{\ln \left(1-z_{\max }\right)}{\ln Q^{2} / \Lambda^{2}}\right)
\end{array}
$$

The endpoints of equation 5.28 are equation 5.29.

$$
\begin{array}{r}
P=r \int_{0}^{z_{\max }} d z \frac{1+z^{2}}{1-z} \ln \left(1+\frac{\ln (1-z)}{\ln Q^{2} / \Lambda^{2}}\right) \frac{1}{\alpha_{s, L O}\left(q^{2}\right)} \\
+\left(\int_{z_{\max }}^{1-\Lambda^{2} / Q^{2}} d z(-)(1+z)\right) \times \ln \left(1+\frac{\ln \left(1-z_{\max }\right)}{\ln Q^{2} / \Lambda^{2}}\right) \frac{1}{\alpha_{s, L O}\left(q^{2}\right)}
\end{array}
$$

The large $x$ corrections are implemented in the SOMPDF procedure by using the new expression for $\operatorname{In}(1-z)$ written equation 5.22 and performing the subsequent integrals over $x$ for the structure function $F_{2}^{N S}(x)$. The large $x$ experimental data sets and PDF computations are centered on the regions of $x$ and $Q^{2}$ that are defined in terms of $W^{2}$ such that $1.0 \leq W^{2} \leq 4.0$ with $W^{2}$ being the same kinematic defined in Chapter $1, W^{2}=Q^{2}\left(1-\frac{1}{x}\right)+M^{2}$, and with $M$ being the mass of the target hadron. The experimental structure functions at these $x$ and $Q^{2}$ values comprise the resonance data sets which the SOMPDF code needs to fit the structure functions to at large $x$. 


\subsection{Averaging the Resonance Region with the Bernstein Moments}

For large $x$ data values, the Deuteron Structure Function $F_{2}^{D}$ and the Proton Structure Function $F_{2}^{P}$ are taken from sets of resonance data sets for each of these structure function types. The generated theoretical structure functions are fitted to resonance data from [74] and [75]. The resonance region data, for $F_{2}^{D}$ and $F_{2}^{P}$, needs to be averaged over the resonance region so that the SOM and GA procedure can fit the generated theoretical structure functions to smooth curves of data sets. The Mellin Moment was the first attempt to average out the resonance region and form a continuous set of large $x$ data. However, these moment computations did not sufficiently factor in the behaviors at large $x$ where the resonance phenomena occurs. To address this, a new attempt was made use integrated moments that could form a continuous large $x$ data set with the non resonance data; this attempt was the Bernstein polynomials.

The $x$ values and the error values on the $x$ values are given by the corresponding Bernstein polynomials. The specific polynomials are given in relation 5.30- equation 5.32 for $x$ and equation 5.33 for the error in $x$.

$$
\begin{gathered}
p_{N, k}(x)=x^{k}(1-x)^{N-k} C_{N, k} \\
C_{N, k}=\frac{\Gamma(N+2)}{\Gamma(k+1) \Gamma(N-k+1)} \\
\bar{x}_{N, k}=\int_{0}^{1} \mathrm{~d} x x p_{N, k}(x)=\frac{k+1}{N+2} \\
(\Delta x)^{2}=\frac{N-k+1}{(N+2)^{2}(N+3)}
\end{gathered}
$$

In relation 5.30- equation 5.33, $x$ is the Bjorken $x$ value over which the Bernstein integral is being done, $F_{2}^{x}$ is the structure function for a specific $Q^{2}$ value at a given $x$ value, $N$ is the total number of Bernstein Moments used and $i$ is the $i^{\text {th }}$ Bernstein Moment being computed. The constant $C_{N, k}$ is a Gamma Function related constant that is given in relation 5.31. In the above equations, $N$ is the total number of Bernstein Moments being 
used in the Bernstein integrals and $i$ is the specific Bernstein Moment being calculated. For our computations, there are 16 Bernstein moments being used so $N$ is set at 15 and $i$ ranges from 0 to 15 ; in this case, $i=3$ as an example would be used to determine the 4th Bernstein integral moment in the computation of $x$ in expression 5.32 and the $4 t h$ Bernstein integral moment of the error on $x$ in expression 5.33 .

The Bernstein Moment is shown in relation 5.34. The Bernstein error on $F_{2}$ is calculated by using the Bernstein integrals with the error in $F_{2}$ in the same manner as the Bernstein errors on the $F_{2}$ values themselves are calculated. The Bernstein error is shown in relation 5.35. The Proton and Deuteron structure functions for which the Resonance Bernstein values and the extrapolated functions are plotted are in formula 5.36 - equation 5.38 .

$$
\begin{gathered}
F_{N, k}^{(e x p)}\left(Q^{2}\right)=\int_{0}^{1} \mathrm{~d} x p_{N, k}(x) F_{2}^{(e x p)}\left(x, Q^{2}\right) \\
\left(\Delta F_{N, k}^{(e r r)}\left(Q^{2}\right)\right)^{2}=\sum_{i}\left[p_{N, k}\left(x_{i}\right) F_{i}^{(e r r)}\left(x_{i}, Q^{2}\right) \Delta x_{i}\right]^{2}
\end{gathered}
$$

In table 5.1, a functional form is determined for the proton structure functions based on the Bernstein Moment values. In table 5.2, the integrals of the structure functions are shown when they are computed by integration of the resonance and extrapolation regions, the Bernstein points and the functional forms shown in table 5.1. These integral tables are computed as a check on the Bernstein integration method to demonstrate that this technique can be used with reasonable accuracy to compute the Bernstein Moments of resonance data points. The integrations from the resonance data, Bernstein Moments and the functional forms all show agreement with each other within $15 \%$ error; the reliability of the Bernstein technique is therefore verified. In the tables $5.3-5.20$, the Bernstein integrals for $F_{2}\left(x_{k}\right)$ were computed in the $x$ range $\left(x_{\min }, x_{\max }\right)$ where $x_{\min }$ and $x_{\max }$ are the minimum and maximum $x$ values of the resonance region in $x$ for each $Q^{2}$ value where resonance $F_{2}$ data points exist. In addition, the Bernstein integrals in the $x$ ranges $\left(0, x_{\text {min }}\right)$ are calculated using the extrapolated $F_{2}$ values computed from the CT10 PDFs in these regions. The CT10 PDF extrapolation is performed in the region $\left(0, x_{\text {min }}\right)$ with the PDF combinations $\sum_{i} e_{i}^{2} q_{i}\left(Q^{2}, x\right)$ where $e_{i}$ is the parton charge and $q_{i}\left(Q^{2}, x\right)$ is the $\mathrm{PDF}$ value for a given parton. 
In Figure 5.1 - 5.4, the resonance data figures and their Bernstein averages are shown along with a series of Bernstein integrands used to compute the averages. This is done by computing the value of $F_{2}$ using the combinations of PDFs for the proton and deuteron structure functions shown in expression 5.36 and equation 5.38, with the neutron structure function given in equation 5.37. For the deuteron structure function, the smearing factor $S_{p}$ is included in the computation of the deuteron structure function. The smearing factor comes from the deuteron structure functions being smeared with the momentum of the nucleons in the deuteron. The resulting binding, Fermi motion and off shell structure corrections result in the deuteron structure function being dependent on the smearing factors. These integrands are the Bernstein functions $p_{N, k}(x) F_{2}^{(e x p)}\left(x, Q^{2}\right)$ over the total range $\left(0, x_{\max }\right)$ calculated in order to find each of the $k$ Bernstein points for each $Q^{2}$ range. The figures indicate that the Bernstein integration moments pick up resonance peaks as the cover greater regions of $x$ where the resonance regions are located. The closer they align with the $x$ ranges of the resonance regions, the more they pick up the values of the resonance peaks. In Figure 5.5 - 5.40, the computed Bernstein averages along with the extrapolated curves are shown for all $Q^{2}$ values for $x$ and $W^{2}$ values inside and outside the resonance regions. In Figure 5.41 an example of a resonance curve in the in the $Q^{2}$ interval $(2.9: 3.9)\left(\mathrm{GeV}^{2}\right)$ to show how the resonance curve changes as a result of an increased value of $N$. In Figure 5.42 an envelope of $\frac{F_{2}^{D}}{F_{2}^{P}}$ curves for $Q^{2}=3.4 \mathrm{GeV}^{2}$ is shown to illustrate the ability of the SOMPDF procedure to create unbiased fits for large $x$ data as well as DIS data. In Figure 5.43 a generated curve for $Q^{2}=7 \mathrm{GeV}^{2}$ for $\frac{d}{u}$ for a $6 \times 6$ and a $1 \times 1$ map is displayed with the lagrange error.

$$
\begin{gathered}
\frac{F_{2}^{P}\left(Q^{2}, x\right)}{x}=\frac{4}{9}\left(u_{v}\left(Q^{2}, x\right)+2 \bar{u}\left(Q^{2}, x\right)\right)+\frac{1}{9}\left(d_{v}\left(Q^{2}, x\right)+2 \bar{d}\left(Q^{2}, x\right)\right)+\frac{1}{9} s\left(Q^{2}, x\right) \\
\frac{F_{2}^{N}\left(Q^{2}, x\right)}{x}=\frac{4}{9}\left(d_{v}\left(Q^{2}, x\right)+2 \bar{d}\left(Q^{2}, x\right)\right)+\frac{1}{9}\left(u_{v}\left(Q^{2}, x\right)+2 \bar{u}\left(Q^{2}, x\right)\right)+\frac{1}{9} s\left(Q^{2}, x\right) \\
\frac{F_{2}^{D}\left(Q^{2}, x\right)}{x}=\frac{1}{2} S_{p} F_{2}^{N}\left(Q^{2}, x\right)+S_{p} F_{2}^{P}\left(Q^{2}, x\right)
\end{gathered}
$$


TABle 5.1: Functional Form Approximating the Bernstein Moment Curve for various $Q^{2}$ values

\begin{tabular}{|c|c|c|c|c|}
\hline \hline$Q^{2} \mathrm{GeV}^{2}$ & Functional Form & $\mathrm{a}$ & $\mathrm{b}$ & $\mathrm{c}$ \\
\hline \hline 0.55 & $\left(a(1-x)^{4}+b x^{4}\right)$ & 0.777285 & 1.19712 & 0.0 \\
1.0 & $(a(1-x)-b x+c(1-x) x)$ & 0.46873 & 0.0774563 & 0.0283069 \\
1.8 & $\left(a(1-x)^{3}+b x^{2}\right)$ & 1.26009 & 0.0699836 & 0.0 \\
2.5 & $\left(a(1-x)^{0.5}-b x^{0.5}-c(1-x) x\right)$ & 0.7408 & 0.165159 & 0.841746 \\
3.4 & $(a(1-x)-b x-c(1-x) x)$ & 0.567944 & 0.00827568 & 0.406153 \\
5.7 & $\left(a(1-x)^{4}+b x^{3}\right)$ & 5.0532 & 0.0205239 & 0.0 \\
7.0 & $(a(1-x)-b x-c(1-x) x)$ & 0.369392 & 0.00113869 & 0.306584 \\
8.2 & $\left(a(1-x)^{5}+b x^{6}\right)$ & 20.1298 & 0.0155009 & 0.0 \\
9.6 & $\left(a(1-x)^{2} \cdot+b(1-x) x-c x^{2}\right)$ & 0.303746 & 0.0384961 & 0.000667997 \\
\hline \hline
\end{tabular}

TABLE 5.2: Integral values of Resonance Data Points with Errors, Bernstein Moments with Errors and Functional forms for various $Q^{2}$ values

\begin{tabular}{|c|c|c|c|c|c|}
\hline \hline$Q^{2} \mathrm{GeV}^{2}$ & Resonance & Bernstein & Functional & Resonance Unc & Bernstein Unc \\
\hline \hline 0.55 & 0.097286 & 0.1079 & 0.09735 & $4.2264 \mathrm{E}-04$ & $2.6210 \mathrm{E}-04$ \\
1.0 & 0.11494 & 0.120381 & 0.121119 & $2.7484 \mathrm{E}-04$ & $1.9697 \mathrm{E}-04$ \\
1.8 & $7.4188 \mathrm{E}-02$ & $6.7255 \mathrm{E}-02$ & $7.1065 \mathrm{E}-02$ & $2.0064 \mathrm{E}-04$ & $1.2309 \mathrm{E}-04$ \\
2.5 & $5.0253 \mathrm{E}-02$ & $5.0650 \mathrm{E}-02$ & $5.5433 \mathrm{E}-02$ & $2.2534 \mathrm{E}-04$ & $1.5350 \mathrm{E}-04$ \\
3.4 & $2.7969 \mathrm{E}-02$ & $2.4721 \mathrm{E}-02$ & $2.9112 \mathrm{E}-02$ & $1.1580 \mathrm{E}-04$ & $8.1337 \mathrm{E}-05$ \\
5.7 & $8.5448 \mathrm{E}-03$ & $8.0573 \mathrm{E}-03$ & $8.3974 \mathrm{E}-03$ & $2.1821 \mathrm{E}-04$ & $7.3200 \mathrm{E}-05$ \\
7.0 & $4.5377 \mathrm{E}-03$ & $3.8151 \mathrm{E}-03$ & $5.2264 \mathrm{E}-03$ & $2.01616 \mathrm{E}-04$ & $8.0562 \mathrm{E}-05$ \\
8.2 & $3.4128 \mathrm{E}-03$ & $3.4724 \mathrm{E}-03$ & $3.2301 \mathrm{E}-03$ & $3.2299 \mathrm{E}-04$ & $4.1881 \mathrm{E}-05$ \\
9.6 & $1.8364 \mathrm{E}-03$ & $1.4628 \mathrm{E}-03$ & $2.1553 \mathrm{E}-03$ & $2.8876 \mathrm{E}-04$ & $2.6195 \mathrm{E}-05$ \\
\hline \hline
\end{tabular}




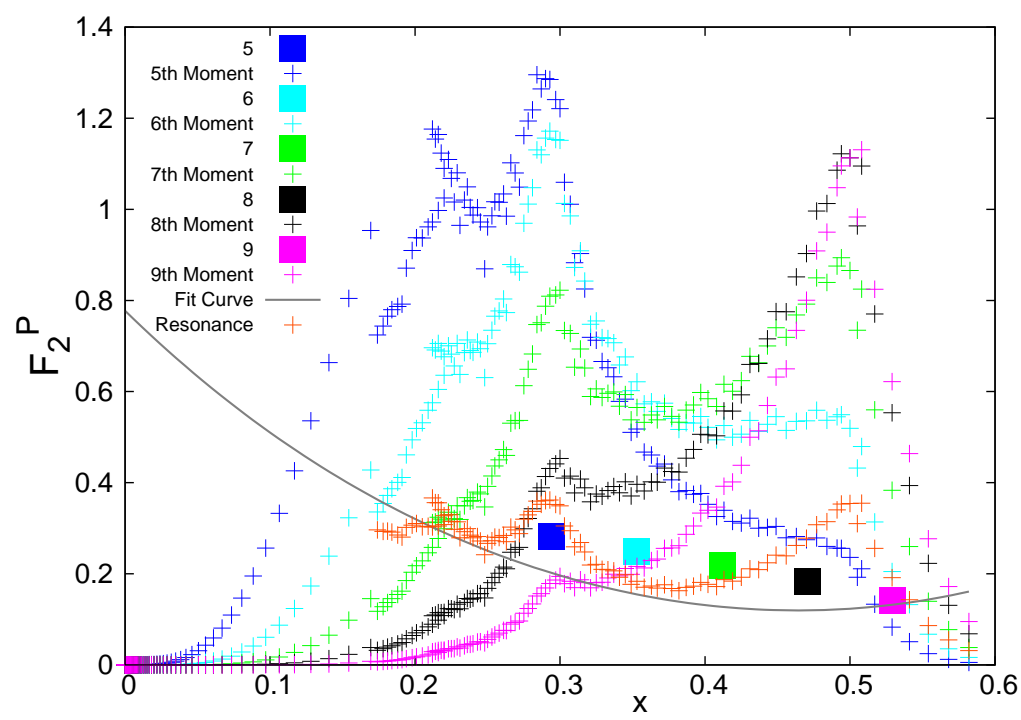

Figure 5.1: The $F_{2}^{P}$ resonance moment integrands and corresponding Bernstein moment points, for $Q^{2}=0.55 \mathrm{GeV}^{2}$, are shown for the $5^{\text {th }}, 6^{\text {th }}, 7^{\text {th }}, 8^{\text {th }}$ and $9^{\text {th }}$ moments. The filled squares are the Bernstein moment points and the crosses are the corresponding Bernstein moment resonance integrands.

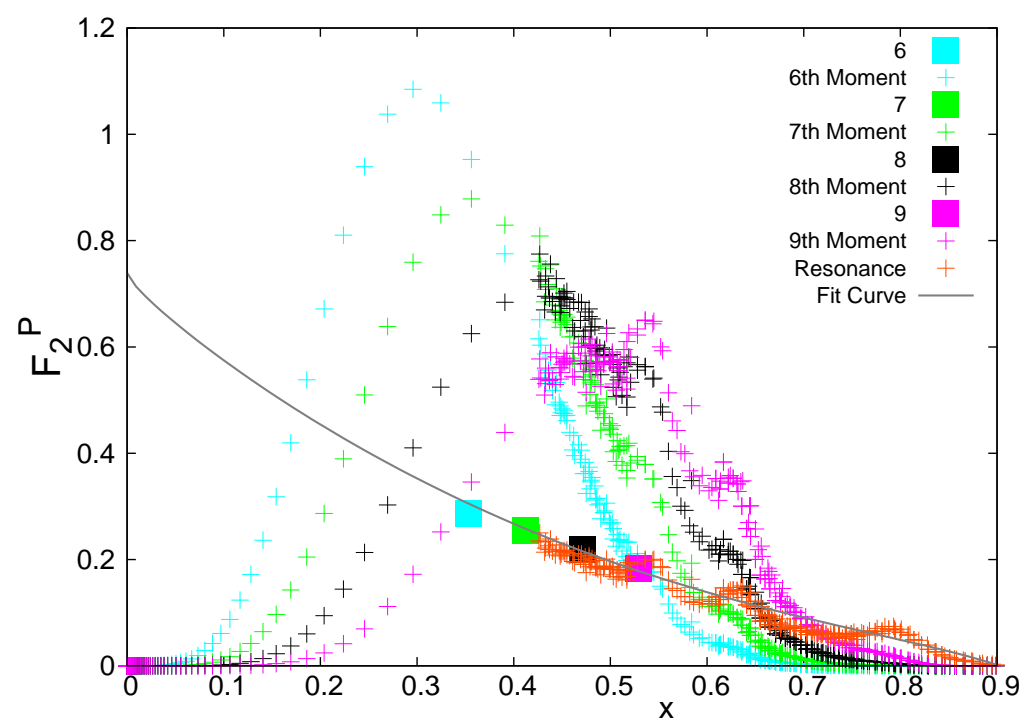

FIGURE 5.2: The $F_{2}^{P}$ resonance moment integrands and corresponding Bernstein moment points, for $Q^{2}=2.5 \mathrm{GeV}^{2}$, are shown for the $6^{t h}, 7^{\text {th }}, 8^{\text {th }}$ and $9^{\text {th }}$ moments. The filled squares are the Bernstein moment points and the crosses are the corresponding Bernstein moment resonance integrands 


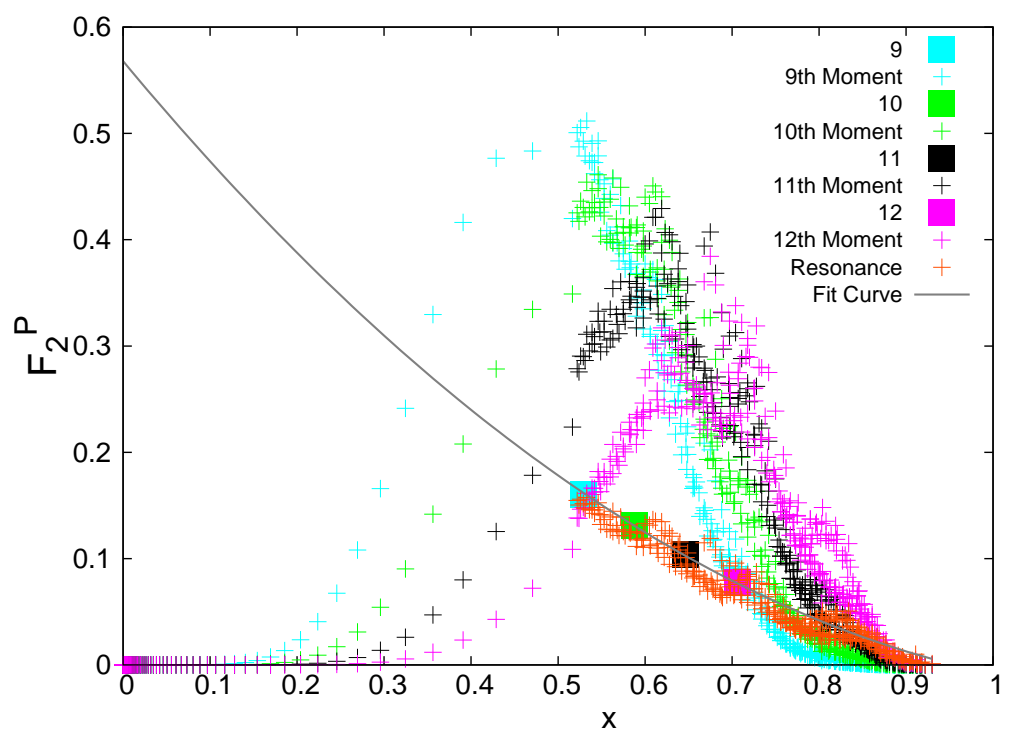

Figure 5.3: The $F_{2}^{P}$ resonance moment integrands and corresponding Bernstein moment points, for $Q^{2}=3.4 \mathrm{GeV}^{2}$, are shown for the $9^{\text {th }}, 10^{\text {th }}, 11^{\text {th }}$ and $12^{\text {th }}$ moments. The filled squares are the Bernstein moment points and the crosses are the corresponding Bernstein moment resonance integrands

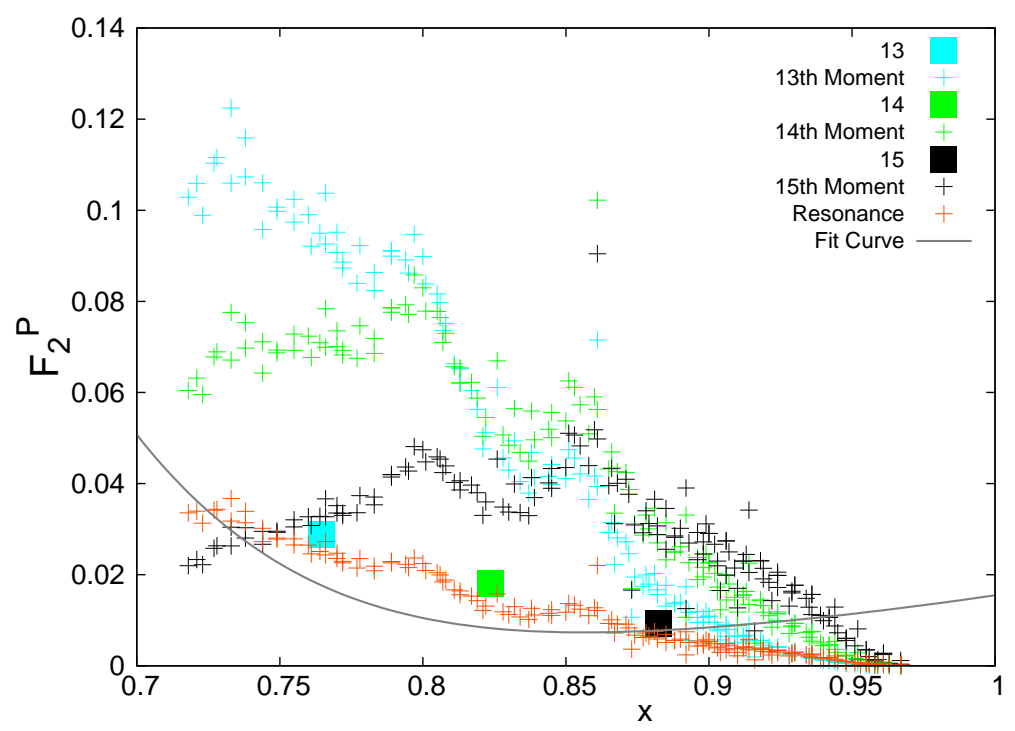

Figure 5.4: The $F_{2}^{P}$ resonance moment integrands and corresponding Bernstein moment points, for $Q^{2}=8.2 \mathrm{GeV}^{2}$, are shown for the $13^{\text {th }}, 14^{\text {th }}$ and $15^{\text {th }}$ moments. The filled squares are the Bernstein moment points and the crosses are the corresponding Bernstein moment resonance integrands 


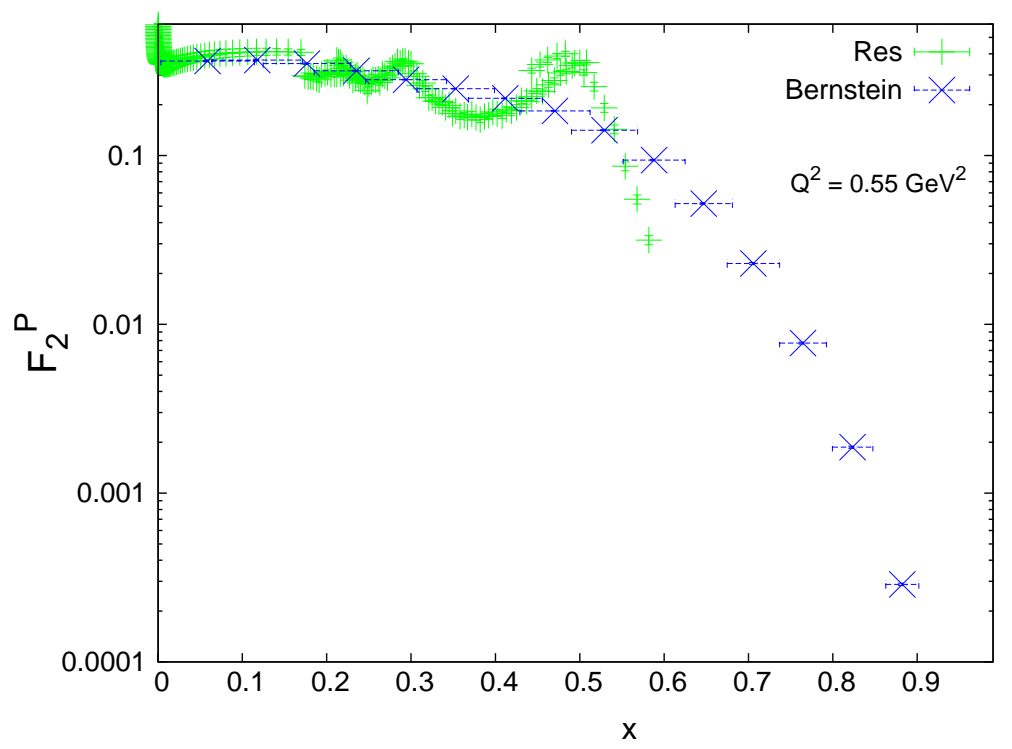

FiguRE 5.5: The $F_{2}^{P}$ values for the resonance region and the Bernstein moments are shown here. The green points are all of the $F_{2}^{P}$ resonance points in the $Q^{2}$ interval $(0.53: 0.63)$ and blue points are the Bernstein averages for those points in the $x$ range that constitutes the resonance region for $Q^{2}=0.55 \mathrm{GeV}^{2}$.

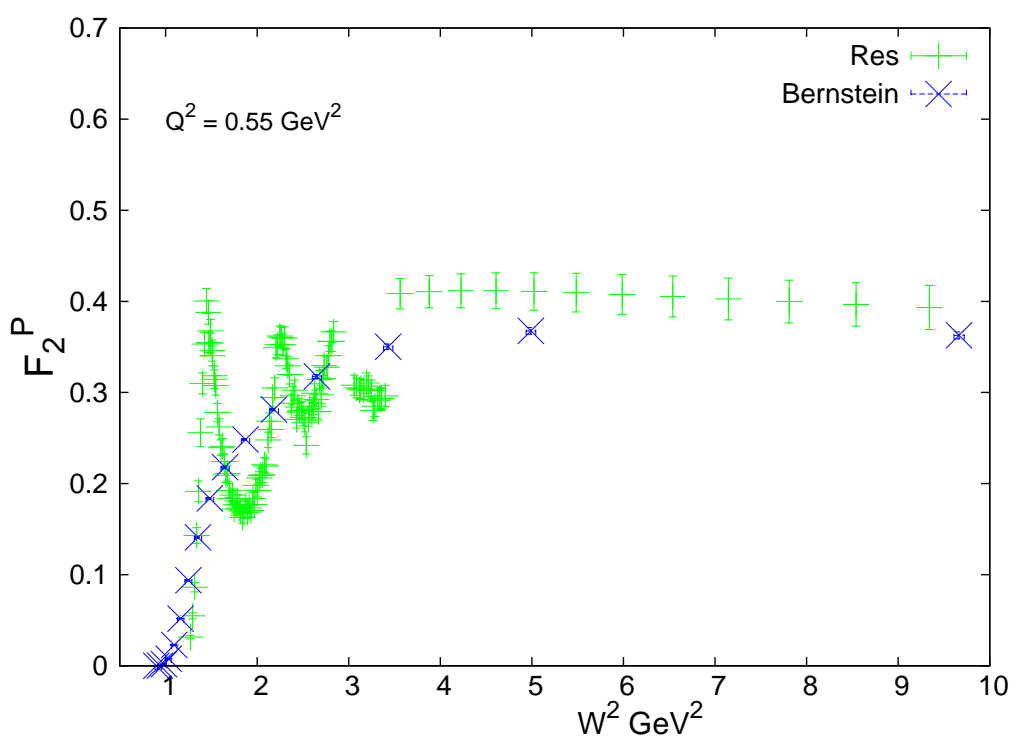

FIGURE 5.6: The $F_{2}^{P}$ values for the resonance region and the Bernstein moments are shown here. The green points are all of the $F_{2}^{P}$ resonance points in the $Q^{2}$ interval (0.53: 0.63) and blue points are the Bernstein averages for those points in the $W^{2}$ range that constitutes the resonance region for $Q^{2}=0.55 \mathrm{GeV}^{2}$. 
TABle 5.3: Table of $Q^{2}=0.55 \mathrm{GeV}^{2} F_{2}^{P}$ Bernstein Moment Values. The Resonance Region here refers to $x$ values for which Resonance Data points exist, which for this $Q^{2}$ value is $(0.17,0.58)$.

\begin{tabular}{|c|c|c|c|c|c|c|}
\hline \hline$k$ & $x$ & $\Delta x$ & $F_{N, k}^{(e x p)}\left(Q^{2}\right)$ & $\Delta F_{N, k}^{(e r r)}\left(Q^{2}\right)$ & Res & DIS \\
\hline \hline 0.00 & 0.0588 & 0.0555 & 0.3627 & $0.3908 \mathrm{E}-02$ & 0.0374 & 0.9592 \\
1.00 & 0.1176 & 0.0537 & 0.3676 & $0.3666 \mathrm{E}-02$ & 0.1605 & 0.8289 \\
2.00 & 0.1765 & 0.0519 & 0.3503 & $0.3017 \mathrm{E}-02$ & 0.3667 & 0.6168 \\
3.00 & 0.2353 & 0.0500 & 0.3176 & $0.2064 \mathrm{E}-02$ & 0.6007 & 0.3827 \\
4.00 & 0.2941 & 0.0480 & 0.2813 & $0.1289 \mathrm{E}-02$ & 0.7953 & 0.1929 \\
5.00 & 0.3529 & 0.0460 & 0.2484 & $0.9720 \mathrm{E}-03$ & 0.9161 & 0.0777 \\
6.00 & 0.4118 & 0.0438 & 0.2180 & $0.9930 \mathrm{E}-03$ & 0.9723 & 0.0252 \\
7.00 & 0.4706 & 0.0416 & 0.1837 & $0.1072 \mathrm{E}-02$ & 0.9924 & 0.0068 \\
8.00 & 0.5294 & 0.0392 & 0.1410 & $0.1032 \mathrm{E}-02$ & 0.9982 & 0.0016 \\
9.00 & 0.5882 & 0.0367 & $0.9387 \mathrm{E}-01$ & $0.8270 \mathrm{E}-03$ & 0.9996 & 0.0004 \\
10.0 & 0.6471 & 0.0340 & $0.5191 \mathrm{E}-01$ & $0.5330 \mathrm{E}-03$ & 0.9999 & 0.0001 \\
11.0 & 0.7059 & 0.0310 & $0.2294 \mathrm{E}-01$ & $0.2680 \mathrm{E}-03$ & 1.0000 & 0.0000 \\
12.0 & 0.7647 & 0.0277 & $0.7743 \mathrm{E}-02$ & $0.1010 \mathrm{E}-03$ & 1.0000 & 0.0000 \\
13.0 & 0.8235 & 0.0240 & $0.1871 \mathrm{E}-02$ & $0.2700 \mathrm{E}-04$ & 1.0000 & 0.0000 \\
14.0 & 0.8824 & 0.0196 & $0.2880 \mathrm{E}-03$ & $0.5000 \mathrm{E}-05$ & 1.0000 & 0.0000 \\
15.0 & 0.9412 & 0.0139 & $0.2100 \mathrm{E}-04$ & 0.000 & 1.0000 & 0.0000 \\
\hline \hline
\end{tabular}

TABle 5.4: Table of $Q^{2}=0.55 \mathrm{GeV}^{2} F_{2}^{D}$ Bernstein Moment Values. The Resonance Region here refers to $x$ values for which Resonance Data points exist, which for this $Q^{2}$ value is $(0.17,0.58)$.

\begin{tabular}{|c|c|c|c|c|c|c|}
\hline \hline$k$ & $x$ & $\Delta x$ & $F_{N, k}^{(e x p)}\left(Q^{2}\right)$ & $\Delta F_{N, k}^{(e r r)}\left(Q^{2}\right)$ & Res & DIS \\
\hline \hline 0.00 & 0.0588 & 0.0555 & 0.3468 & $0.3571 \mathrm{E}-02$ & 0.0362 & 0.9604 \\
1.00 & 0.1176 & 0.0537 & 0.3429 & $0.3355 \mathrm{E}-02$ & 0.1571 & 0.8318 \\
2.00 & 0.1765 & 0.0519 & 0.3210 & $0.2785 \mathrm{E}-02$ & 0.3612 & 0.6213 \\
3.00 & 0.2353 & 0.0500 & 0.2868 & $0.1879 \mathrm{E}-02$ & 0.5945 & 0.3876 \\
4.00 & 0.2941 & 0.0480 & 0.2514 & $0.1078 \mathrm{E}-02$ & 0.7909 & 0.1962 \\
5.00 & 0.3529 & 0.0460 & 0.2214 & $0.7030 \mathrm{E}-03$ & 0.9143 & 0.0789 \\
6.00 & 0.4118 & 0.0438 & 0.1960 & $0.7460 \mathrm{E}-03$ & 0.9720 & 0.0253 \\
7.00 & 0.4706 & 0.0416 & 0.1691 & $0.9100 \mathrm{E}-03$ & 0.9925 & 0.0067 \\
8.00 & 0.5294 & 0.0392 & 0.1357 & $0.9880 \mathrm{E}-03$ & 0.9983 & 0.0015 \\
9.00 & 0.5882 & 0.0367 & $0.9677 \mathrm{E}-01$ & $0.8910 \mathrm{E}-03$ & 0.9996 & 0.0003 \\
10.0 & 0.6471 & 0.0340 & $0.5894 \mathrm{E}-01$ & $0.6490 \mathrm{E}-03$ & 0.9999 & 0.0001 \\
11.0 & 0.7059 & 0.0310 & $0.2954 \mathrm{E}-01$ & $0.3730 \mathrm{E}-03$ & 1.0000 & 0.0000 \\
12.0 & 0.7647 & 0.0277 & $0.1164 \mathrm{E}-01$ & $0.1640 \mathrm{E}-03$ & 1.0000 & 0.0000 \\
13.0 & 0.8235 & 0.0240 & $0.3377 \mathrm{E}-02$ & $0.5200 \mathrm{E}-04$ & 1.0000 & 0.0000 \\
14.0 & 0.8824 & 0.0196 & $0.6400 \mathrm{E}-03$ & $0.1100 \mathrm{E}-04$ & 1.0000 & 0.0000 \\
15.0 & 0.9412 & 0.0139 & $0.5900 \mathrm{E}-04$ & $0.1000 \mathrm{E}-05$ & 1.0000 & 0.0000 \\
\hline \hline
\end{tabular}




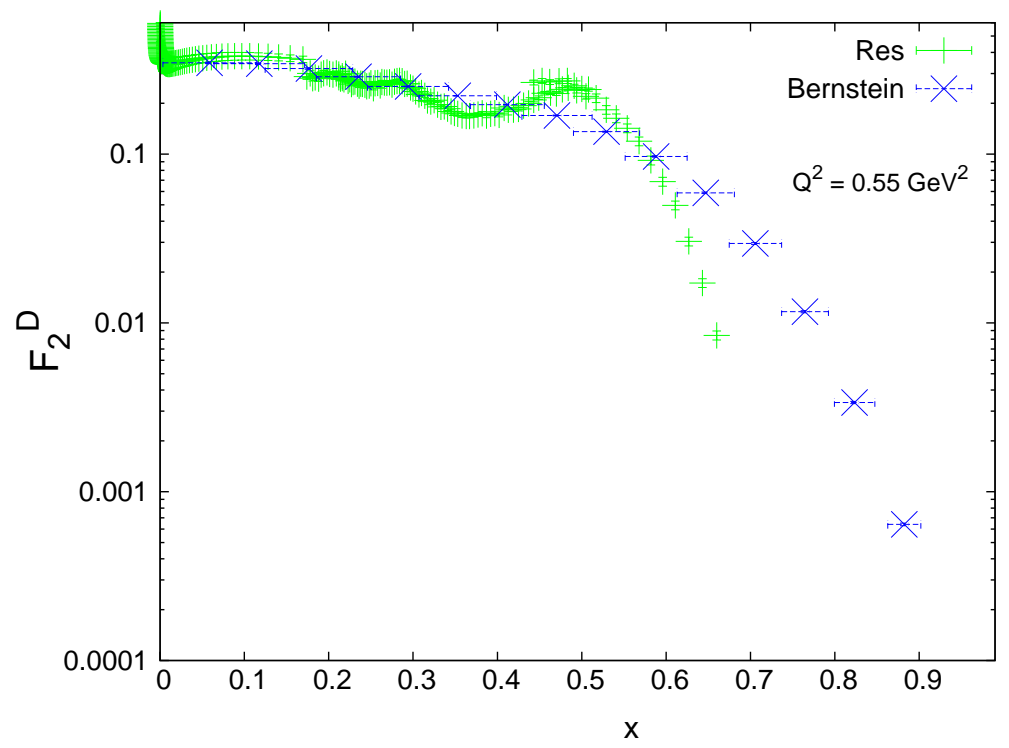

Figure 5.7: The $F_{2}^{D}$ values for the resonance region and the Bernstein moments are shown here. The green points are all of the $F_{2}^{D}$ resonance points in the $Q^{2}$ interval $(0.53: 0.63)$ and blue points are the Bernstein averages for those points in the $x$ range that constitutes the resonance region for $Q^{2}=0.55 \mathrm{GeV}^{2}$. 


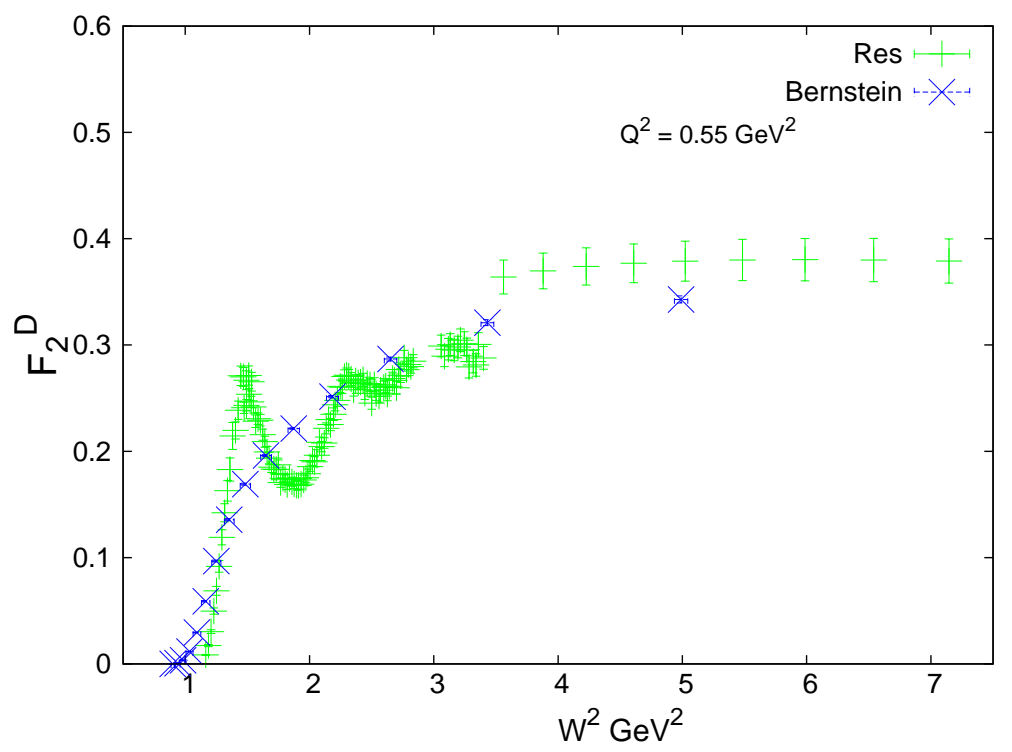

Figure 5.8: The $F_{2}^{D}$ values for the resonance region and the Bernstein moments are shown here. The green points are all of the $F_{2}^{D}$ resonance points in the $Q^{2}$ interval $(0.53: 0.63)$ and blue points are the Bernstein averages for those points in the $W^{2}$ range that constitutes the resonance region for $Q^{2}=0.55 \mathrm{GeV}^{2}$.

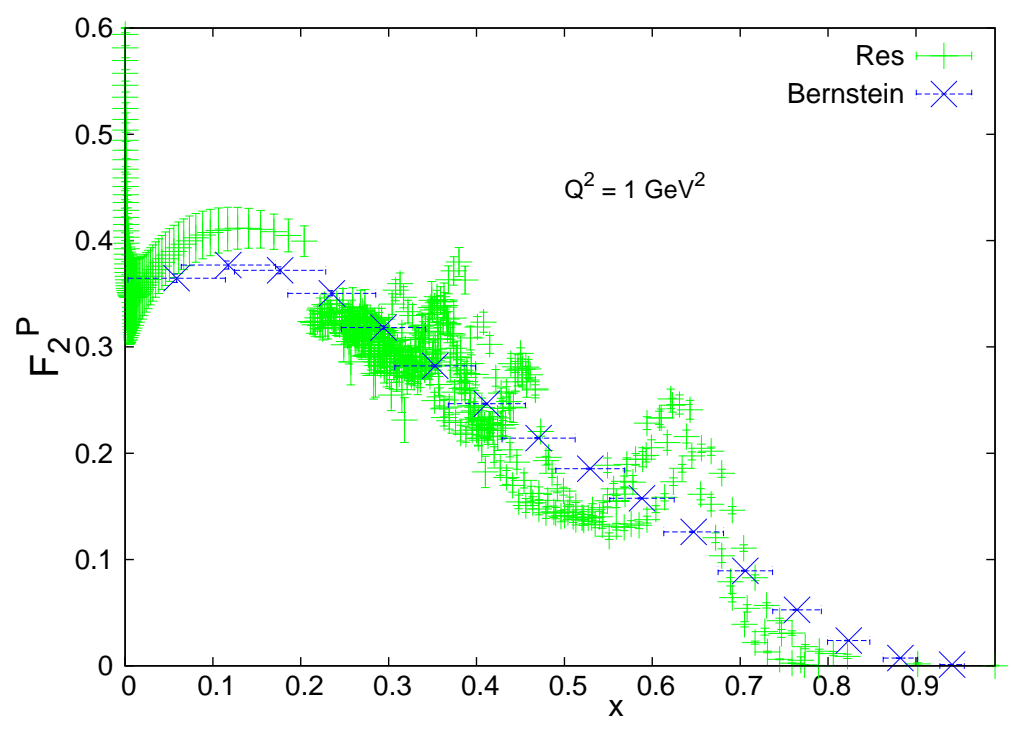

Figure 5.9: The $F_{2}^{P}$ values for the resonance region and the Bernstein moments are shown here. The green points are all of the $F_{2}^{P}$ resonance points in the $Q^{2}$ interval $(0.8: 1.2)$ and blue points are the Bernstein averages for those points in the $x$ range that constitutes the resonance region for $Q^{2}=1 \mathrm{GeV}^{2}$. 


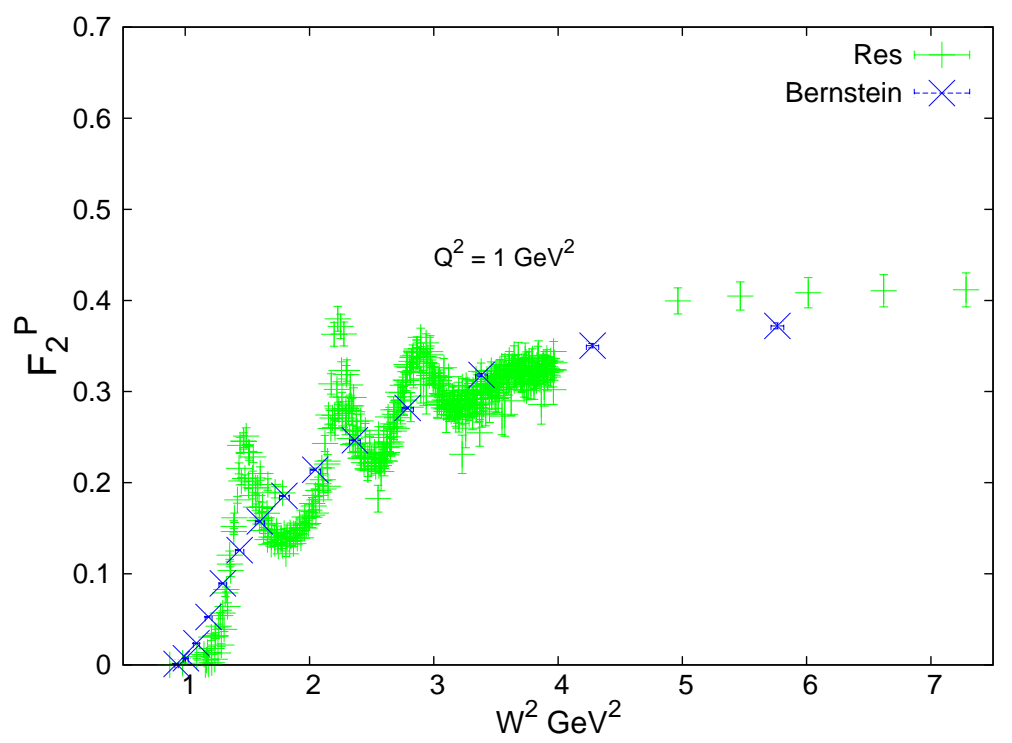

Figure 5.10: The $F_{2}^{P}$ values for the resonance region and the Bernstein moments are shown here. The green points are all of the $F_{2}^{P}$ resonance points in the $Q^{2}$ interval $(0.8: 1.2)$ and blue points are the Bernstein averages for those points in the $W^{2}$ range that constitutes the resonance region for $Q^{2}=1 \mathrm{GeV}^{2}$.

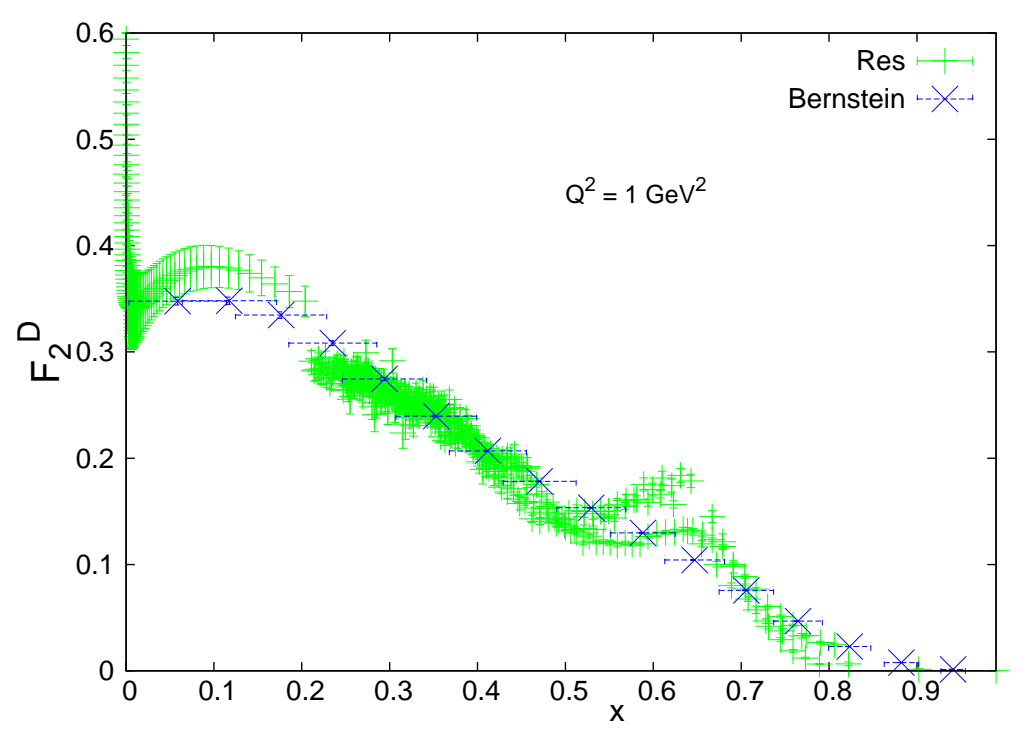

FiguRE 5.11: The $F_{2}^{D}$ values for the resonance region and the Bernstein moments are shown here. The green points are all of the $F_{2}^{D}$ resonance points in the $Q^{2}$ interval $(0.8: 1.2)$ and blue points are the Bernstein averages for those points in the $x$ range that constitutes the resonance region for $Q^{2}=1 \mathrm{GeV}^{2}$. 


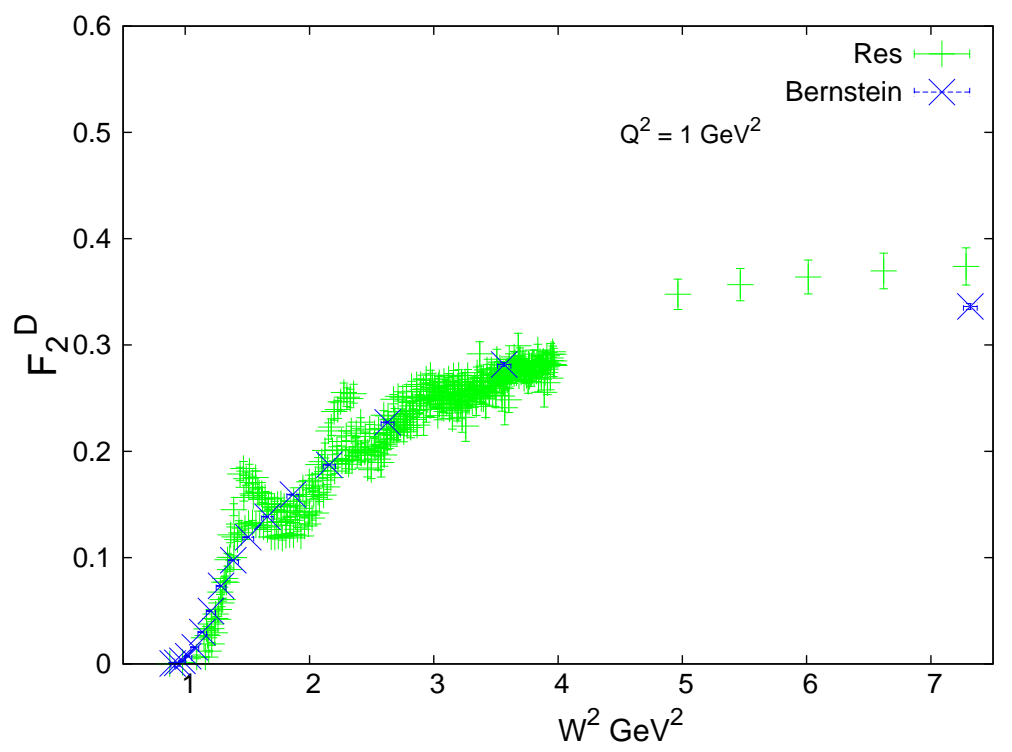

Figure 5.12: The $F_{2}^{D}$ values for the resonance region and the Bernstein moments are shown here. The green points are all of the $F_{2}^{D}$ resonance points in the $Q^{2}$ interval $(0.8: 1.2)$ and blue points are the Bernstein averages for those points in the $W^{2}$ range that constitutes the resonance region for $Q^{2}=1 \mathrm{GeV}^{2}$.

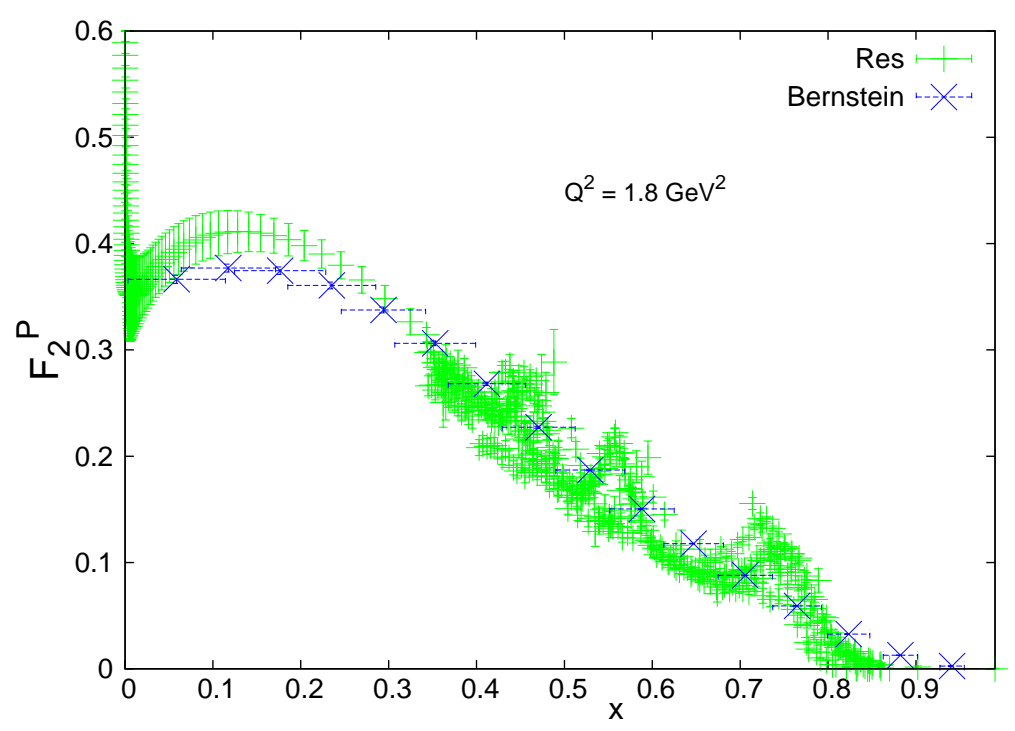

FIGURE 5.13: The $F_{2}^{P}$ values for the resonance region and the Bernstein moments are shown here. The green points are all of the $F_{2}^{P}$ resonance points in the $Q^{2}$ interval $(1.5: 2.2)$ and blue points are the Bernstein averages for those points in the $x$ range that constitutes the resonance region for $Q^{2}=1.8 \mathrm{GeV}^{2}$. 
TABle 5.5: Table of $Q^{2}=1 \quad \mathrm{GeV}^{2} F_{2}^{P}$ Bernstein Moment Values. The Resonance Region here refers to $x$ values for which Resonance Data points exist.

\begin{tabular}{|c|c|c|c|c|c|c|}
\hline \hline$k$ & $x$ & $\Delta x$ & $F_{N, k}^{(e x p)}\left(Q^{2}\right)$ & $\Delta F_{N, k}^{(e r r)}\left(Q^{2}\right)$ & Res & DIS \\
\hline \hline 0.00 & 0.0588 & 0.0555 & 0.3646 & $0.3914 \mathrm{E}-02$ & 0.0193 & 0.9778 \\
1.00 & 0.1176 & 0.0537 & 0.3770 & $0.3744 \mathrm{E}-02$ & 0.0979 & 0.8909 \\
2.00 & 0.1765 & 0.0519 & 0.3721 & $0.3276 \mathrm{E}-02$ & 0.2565 & 0.7222 \\
3.00 & 0.2353 & 0.0500 & 0.3504 & $0.2444 \mathrm{E}-02$ & 0.4694 & 0.5044 \\
4.00 & 0.2941 & 0.0480 & 0.3182 & $0.1543 \mathrm{E}-02$ & 0.6800 & 0.2969 \\
5.00 & 0.3529 & 0.0460 & 0.2821 & $0.9010 \mathrm{E}-03$ & 0.8398 & 0.1449 \\
6.00 & 0.4118 & 0.0438 & 0.2466 & $0.6230 \mathrm{E}-03$ & 0.9343 & 0.0579 \\
7.00 & 0.4706 & 0.0416 & 0.2142 & $0.5470 \mathrm{E}-03$ & 0.9781 & 0.0188 \\
8.00 & 0.5294 & 0.0392 & 0.1855 & $0.5380 \mathrm{E}-03$ & 0.9941 & 0.0049 \\
9.00 & 0.5882 & 0.0367 & 0.1576 & $0.5590 \mathrm{E}-03$ & 0.9987 & 0.0010 \\
10.0 & 0.6471 & 0.0340 & 0.1260 & $0.5580 \mathrm{E}-03$ & 0.9998 & 0.0002 \\
11.0 & 0.7059 & 0.0310 & $0.8944 \mathrm{E}-01$ & $0.4960 \mathrm{E}-03$ & 1.0000 & 0.0000 \\
12.0 & 0.7647 & 0.0277 & $0.5267 \mathrm{E}-01$ & $0.3790 \mathrm{E}-03$ & 1.0000 & 0.0000 \\
13.0 & 0.8235 & 0.0240 & $0.2377 \mathrm{E}-01$ & $0.2380 \mathrm{E}-03$ & 1.0000 & 0.0000 \\
14.0 & 0.8824 & 0.0196 & $0.7263 \mathrm{E}-02$ & $0.1070 \mathrm{E}-03$ & 1.0000 & 0.0000 \\
15.0 & 0.9412 & 0.0139 & $0.1129 \mathrm{E}-02$ & $0.2500 \mathrm{E}-04$ & 1.0000 & 0.0000 \\
\hline \hline
\end{tabular}

TABle 5.6: Table of $Q^{2}=1 \mathrm{GeV}^{2} F_{2}^{D}$ Bernstein Moment Values. The Resonance Region here refers to $x$ values for which Resonance Data points exist.

\begin{tabular}{|c|c|c|c|c|c|c|}
\hline \hline$k$ & $x$ & $\Delta x$ & $F_{N, k}^{(e x p)}\left(Q^{2}\right)$ & $\Delta F_{N, k}^{(e r r)}\left(Q^{2}\right)$ & Res & DIS \\
\hline \hline 0.00 & 0.0588 & 0.0555 & 0.3477 & $0.3577 \mathrm{E}-02$ & 0.0175 & 0.9797 \\
1.00 & 0.1176 & 0.0537 & 0.3482 & $0.3440 \mathrm{E}-02$ & 0.0915 & 0.8976 \\
2.00 & 0.1765 & 0.0519 & 0.3347 & $0.3070 \mathrm{E}-02$ & 0.2450 & 0.7338 \\
3.00 & 0.2353 & 0.0500 & 0.3082 & $0.2317 \mathrm{E}-02$ & 0.4557 & 0.5176 \\
4.00 & 0.2941 & 0.0480 & 0.2746 & $0.1446 \mathrm{E}-02$ & 0.6681 & 0.3079 \\
5.00 & 0.3529 & 0.0460 & 0.2396 & $0.7850 \mathrm{E}-03$ & 0.8321 & 0.1517 \\
6.00 & 0.4118 & 0.0438 & 0.2069 & $0.4720 \mathrm{E}-03$ & 0.9305 & 0.0611 \\
7.00 & 0.4706 & 0.0416 & 0.1783 & $0.3890 \mathrm{E}-03$ & 0.9768 & 0.0199 \\
8.00 & 0.5294 & 0.0392 & 0.1534 & $0.3800 \mathrm{E}-03$ & 0.9937 & 0.0052 \\
9.00 & 0.5882 & 0.0367 & 0.1297 & $0.3850 \mathrm{E}-03$ & 0.9986 & 0.0011 \\
10.0 & 0.6471 & 0.0340 & 0.1042 & $0.3690 \mathrm{E}-03$ & 0.9998 & 0.0002 \\
11.0 & 0.7059 & 0.0310 & $0.7570 \mathrm{E}-01$ & $0.3150 \mathrm{E}-03$ & 1.0000 & 0.0000 \\
12.0 & 0.7647 & 0.0277 & $0.4682 \mathrm{E}-01$ & $0.2380 \mathrm{E}-03$ & 1.0000 & 0.0000 \\
13.0 & 0.8235 & 0.0240 & $0.2284 \mathrm{E}-01$ & $0.1520 \mathrm{E}-03$ & 1.0000 & 0.0000 \\
14.0 & 0.8824 & 0.0196 & $0.7747 \mathrm{E}-02$ & $0.7000 \mathrm{E}-04$ & 1.0000 & 0.0000 \\
15.0 & 0.9412 & 0.0139 & $0.1361 \mathrm{E}-02$ & $0.1700 \mathrm{E}-04$ & 1.0000 & 0.0000 \\
\hline \hline
\end{tabular}


TABle 5.7: Table of $Q^{2}=1.8 \quad \mathrm{GeV}^{2} F_{2}^{P}$ Bernstein Moment Values. The Resonance Region here refers to $x$ values for which Resonance Data points exist.

\begin{tabular}{|c|c|c|c|c|c|c|}
\hline \hline$k$ & $x$ & $\Delta x$ & $F_{N, k}^{(e x p)}\left(Q^{2}\right)$ & $\Delta F_{N, k}^{(e r r)}\left(Q^{2}\right)$ & Res & DIS \\
\hline \hline 0.00 & 0.0588 & 0.0555 & 0.3664 & $0.3892 \mathrm{E}-02$ & 0.0009 & 0.9987 \\
1.00 & 0.1176 & 0.0537 & 0.3770 & $0.3763 \mathrm{E}-02$ & 0.0078 & 0.9887 \\
2.00 & 0.1765 & 0.0519 & 0.3745 & $0.3519 \mathrm{E}-02$ & 0.0348 & 0.9522 \\
3.00 & 0.2353 & 0.0500 & 0.3606 & $0.3188 \mathrm{E}-02$ & 0.1024 & 0.8671 \\
4.00 & 0.2941 & 0.0480 & 0.3376 & $0.2793 \mathrm{E}-02$ & 0.2239 & 0.7250 \\
5.00 & 0.3529 & 0.0460 & 0.3062 & $0.2239 \mathrm{E}-02$ & 0.3914 & 0.5438 \\
6.00 & 0.4118 & 0.0438 & 0.2682 & $0.1573 \mathrm{E}-02$ & 0.5765 & 0.3591 \\
7.00 & 0.4706 & 0.0416 & 0.2272 & $0.9900 \mathrm{E}-03$ & 0.7442 & 0.2049 \\
8.00 & 0.5294 & 0.0392 & 0.1871 & $0.6280 \mathrm{E}-03$ & 0.8687 & 0.0989 \\
9.00 & 0.5882 & 0.0367 & 0.1504 & $0.4530 \mathrm{E}-03$ & 0.9440 & 0.0396 \\
10.0 & 0.6471 & 0.0340 & 0.1178 & $0.3640 \mathrm{E}-03$ & 0.9805 & 0.0130 \\
11.0 & 0.7059 & 0.0310 & $0.8790 \mathrm{E}-01$ & $0.3070 \mathrm{E}-03$ & 0.9945 & 0.0034 \\
12.0 & 0.7647 & 0.0277 & $0.5918 \mathrm{E}-01$ & $0.2490 \mathrm{E}-03$ & 0.9987 & 0.0008 \\
13.0 & 0.8235 & 0.0240 & $0.3273 \mathrm{E}-01$ & $0.1700 \mathrm{E}-03$ & 0.9998 & 0.0002 \\
14.0 & 0.8824 & 0.0196 & $0.1282 \mathrm{E}-01$ & $0.8400 \mathrm{E}-04$ & 1.0000 & 0.0000 \\
15.0 & 0.9412 & 0.0139 & $0.2609 \mathrm{E}-02$ & $0.2200 \mathrm{E}-04$ & 1.0000 & 0.0000 \\
\hline \hline
\end{tabular}

TABle 5.8: Table of $Q^{2}=1.8 \mathrm{GeV}^{2} F_{2}^{D}$ Bernstein Moment Values. The Resonance Region here refers to $x$ values for which Resonance Data points exist.

\begin{tabular}{|c|c|c|c|c|c|c|}
\hline \hline$k$ & $x$ & $\Delta x$ & $F_{N, k}^{(e x p)}\left(Q^{2}\right)$ & $\Delta F_{N, k}^{(e r r)}\left(Q^{2}\right)$ & Res & DIS \\
\hline \hline 0.00 & 0.0588 & 0.0555 & 0.2772 & $0.3358 \mathrm{E}-02$ & 0.0073 & 0.9927 \\
1.00 & 0.1176 & 0.0537 & 0.1727 & $0.2346 \mathrm{E}-02$ & 0.0933 & 0.9067 \\
2.00 & 0.1765 & 0.0519 & 0.1248 & $0.1403 \mathrm{E}-02$ & 0.4864 & 0.5136 \\
3.00 & 0.2353 & 0.0500 & 0.1626 & $0.1817 \mathrm{E}-02$ & 0.8809 & 0.1191 \\
4.00 & 0.2941 & 0.0480 & 0.2427 & $0.2776 \mathrm{E}-02$ & 0.9817 & 0.0183 \\
5.00 & 0.3529 & 0.0460 & 0.3000 & $0.3191 \mathrm{E}-02$ & 0.9974 & 0.0026 \\
6.00 & 0.4118 & 0.0438 & 0.2992 & $0.2787 \mathrm{E}-02$ & 0.9996 & 0.0004 \\
7.00 & 0.4706 & 0.0416 & 0.2505 & $0.1895 \mathrm{E}-02$ & 1.0000 & 0.0000 \\
8.00 & 0.5294 & 0.0392 & 0.1866 & $0.1037 \mathrm{E}-02$ & 1.0000 & 0.0000 \\
9.00 & 0.5882 & 0.0367 & 0.1322 & $0.5120 \mathrm{E}-03$ & 1.0000 & 0.0000 \\
10.0 & 0.6471 & 0.0340 & $0.9350 \mathrm{E}-01$ & $0.2980 \mathrm{E}-03$ & 1.0000 & 0.0000 \\
11.0 & 0.7059 & 0.0310 & $0.6659 \mathrm{E}-01$ & $0.2180 \mathrm{E}-03$ & 1.0000 & 0.0000 \\
12.0 & 0.7647 & 0.0277 & $0.4642 \mathrm{E}-01$ & $0.1650 \mathrm{E}-03$ & 1.0000 & 0.0000 \\
13.0 & 0.8235 & 0.0240 & $0.3132 \mathrm{E}-01$ & $0.1270 \mathrm{E}-03$ & 1.0000 & 0.0000 \\
14.0 & 0.8824 & 0.0196 & $0.2306 \mathrm{E}-01$ & $0.1090 \mathrm{E}-03$ & 1.0000 & 0.0000 \\
15.0 & 0.9412 & 0.0139 & $0.2327 \mathrm{E}-01$ & $0.1510 \mathrm{E}-03$ & 1.0000 & 0.0000 \\
\hline \hline
\end{tabular}




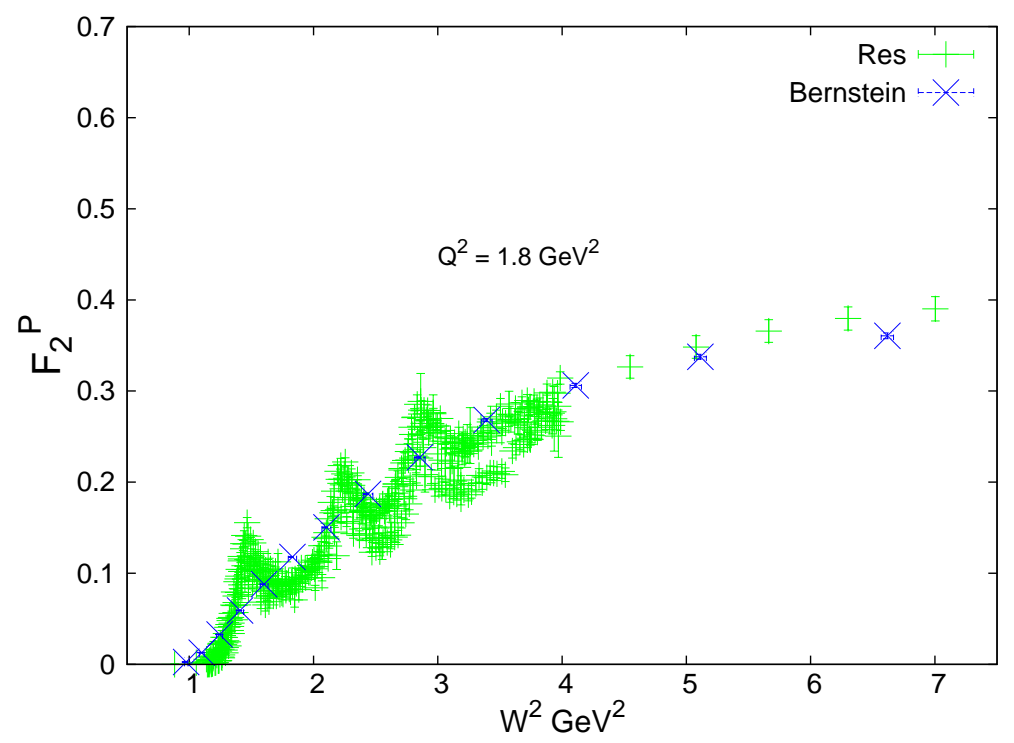

FiguRE 5.14: The $F_{2}^{P}$ values for the resonance region and the Bernstein moments are shown here. The green points are all of the $F_{2}^{P}$ resonance points in the $Q^{2}$ interval $(1.5: 2.2)$ and blue points are the Bernstein averages for those points in the $W^{2}$ range that constitutes the resonance region for $Q^{2}=1.8 \mathrm{GeV}^{2}$. 


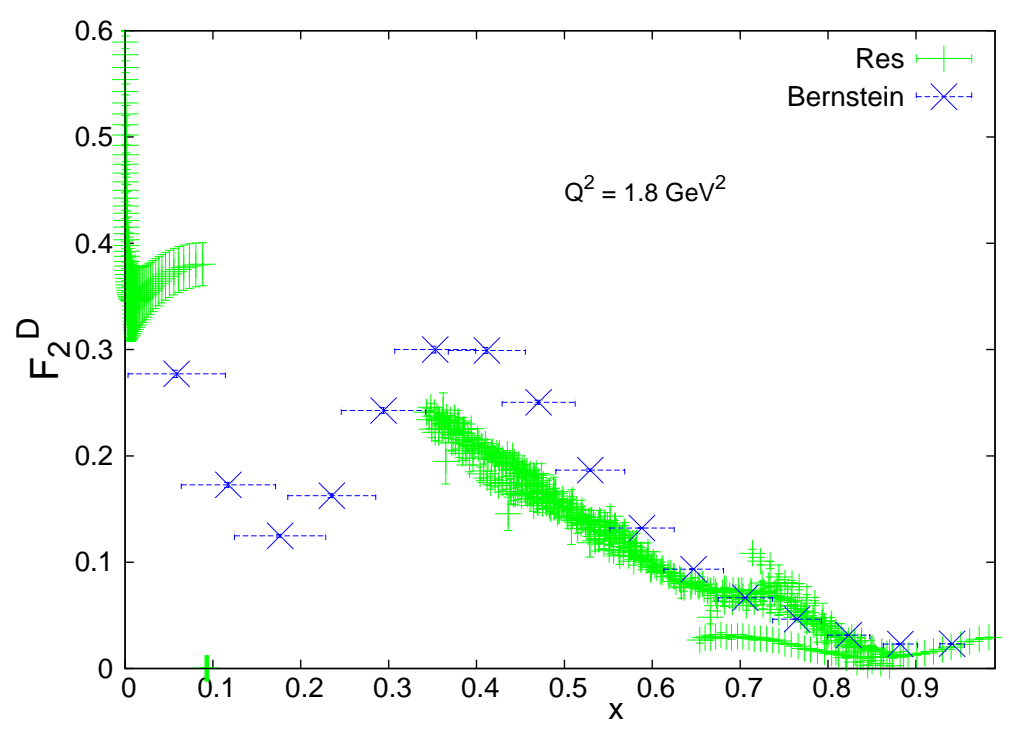

Figure 5.15: The $F_{2}^{D}$ values for the resonance region and the Bernstein moments are shown here. The green points are all of the $F_{2}^{D}$ resonance points in the $Q^{2}$ interval $(1.5: 2.2)$ and blue points are the Bernstein averages for those points in the $x$ range that constitutes the resonance region for $Q^{2}=1.8 \mathrm{GeV}^{2}$. 


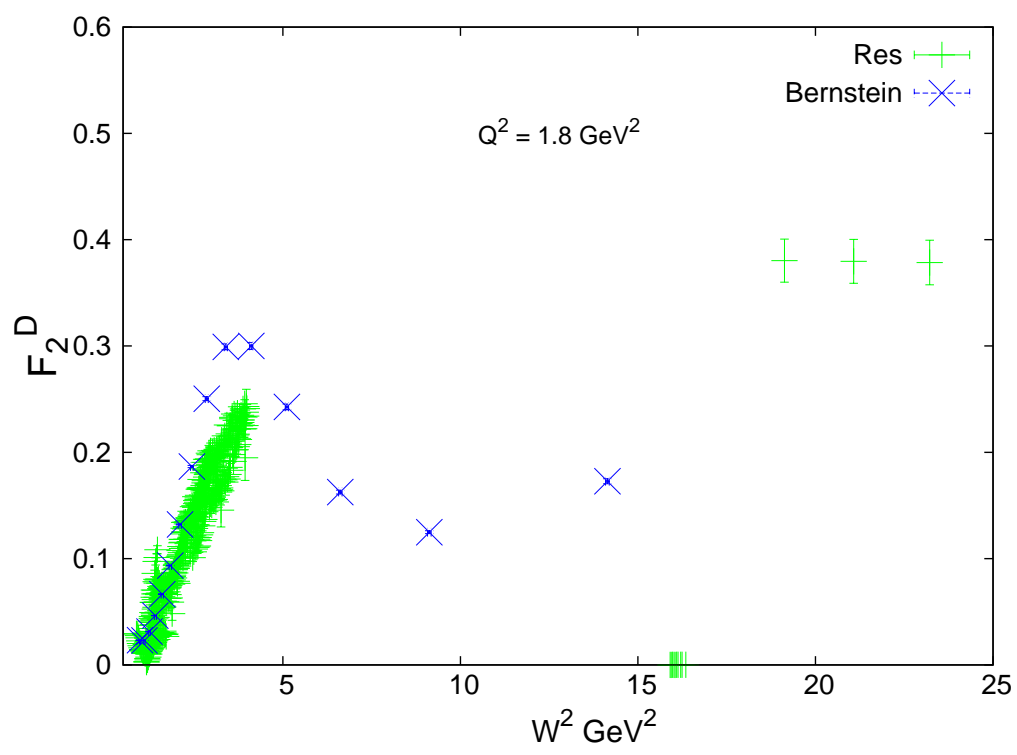

Figure 5.16: The $F_{2}^{D}$ values for the resonance region and the Bernstein moments are shown here. The green points are all of the $F_{2}^{D}$ resonance points in the $Q^{2}$ interval $(1.5: 2.2)$ and blue points are the Bernstein averages for those points in the $W^{2}$ range that constitutes the resonance region for $Q^{2}=1.8 \mathrm{GeV}^{2}$.

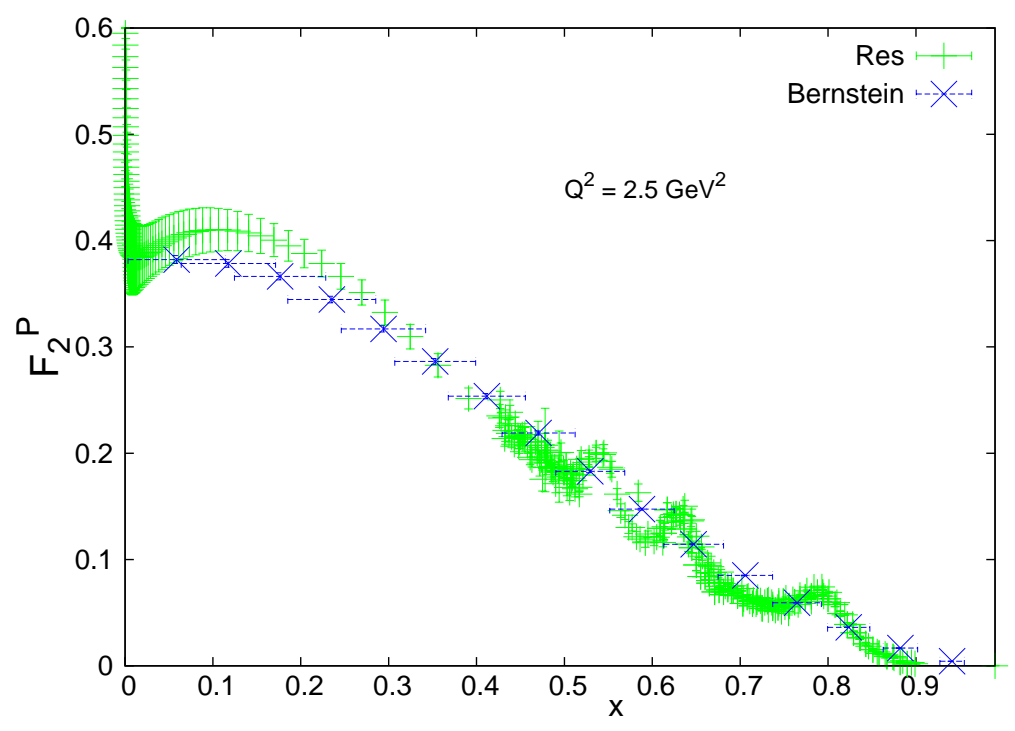

FIGURE 5.17: The $F_{2}^{P}$ values for the resonance region and the Bernstein moments are shown here. The green points are all of the $F_{2}^{P}$ resonance points in the $Q^{2}$ interval $(2.3: 2.5)$ and blue points are the Bernstein averages for those points in the $x$ range that constitutes the resonance region for $Q^{2}=2.5 \mathrm{GeV}^{2}$. 


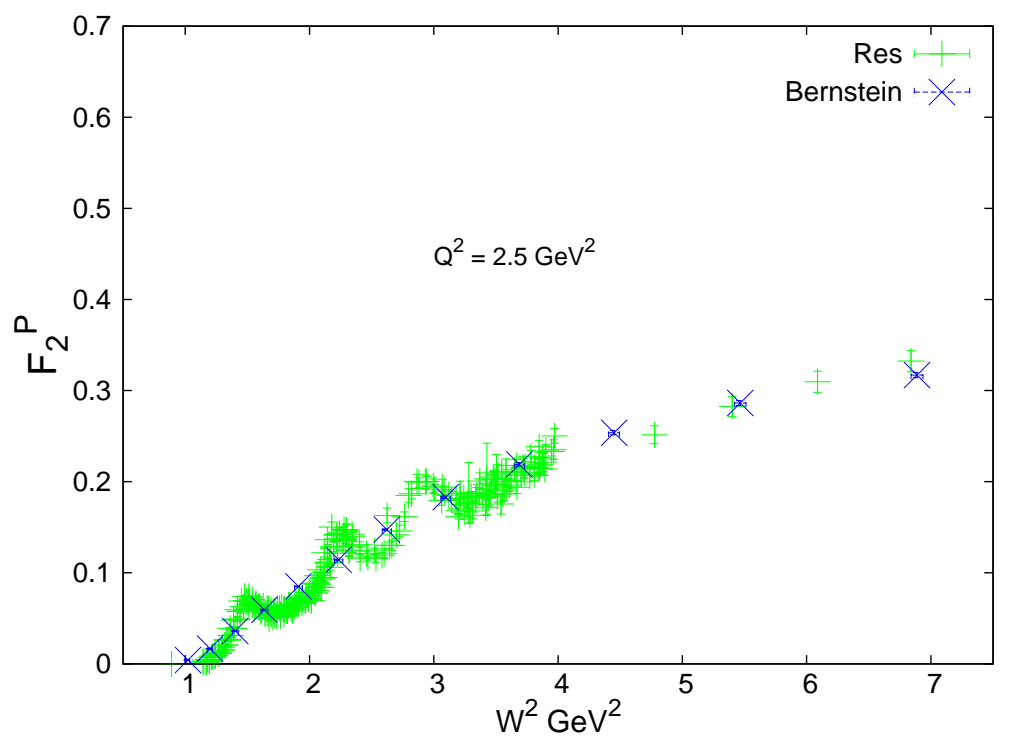

Figure 5.18: The $F_{2}^{P}$ values for the resonance region and the Bernstein moments are shown here. The green points are all of the $F_{2}^{P}$ resonance points in the $Q^{2}$ interval $(2.3: 2.5)$ and blue points are the Bernstein averages for those points in the $W^{2}$ range that constitutes the resonance region for $Q^{2}=2.5 \mathrm{GeV}^{2}$.

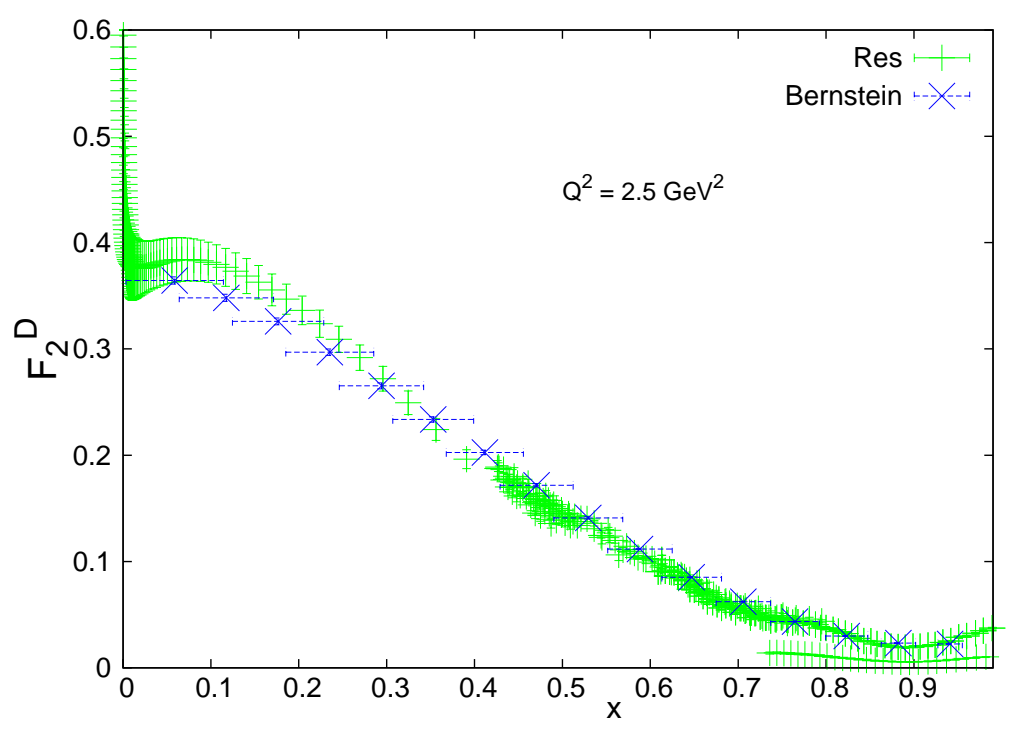

FIGURE 5.19: The $F_{2}^{D}$ values for the resonance region and the Bernstein moments are shown here. The green points are all of the $F_{2}^{D}$ resonance points in the $Q^{2}$ interval $(2.3: 2.5)$ and blue points are the Bernstein averages for those points in the $x$ range that constitutes the resonance region for $Q^{2}=2.5 \mathrm{GeV}^{2}$. 
TABle 5.9: Table of $Q^{2}=2.5 \quad \mathrm{GeV}^{2} F_{2}^{P}$ Bernstein Moment Values. The Resonance Region here refers to $x$ values for which Resonance Data points exist.

\begin{tabular}{|c|c|c|c|c|c|c|}
\hline \hline$k$ & $x$ & $\Delta x$ & $F_{N, k}^{(e x p)}\left(Q^{2}\right)$ & $\Delta F_{N, k}^{(e r r)}\left(Q^{2}\right)$ & Res & DIS \\
\hline \hline 0.00 & 0.0588 & 0.0555 & 0.3822 & $0.3736 \mathrm{E}-02$ & 0.0001 & 0.9998 \\
1.00 & 0.1176 & 0.0537 & 0.3785 & $0.3594 \mathrm{E}-02$ & 0.0009 & 0.9981 \\
2.00 & 0.1765 & 0.0519 & 0.3664 & $0.3346 \mathrm{E}-02$ & 0.0059 & 0.9891 \\
3.00 & 0.2353 & 0.0500 & 0.3446 & $0.3092 \mathrm{E}-02$ & 0.0241 & 0.9586 \\
4.00 & 0.2941 & 0.0480 & 0.3170 & $0.2939 \mathrm{E}-02$ & 0.0717 & 0.8866 \\
5.00 & 0.3529 & 0.0460 & 0.2864 & $0.2780 \mathrm{E}-02$ & 0.1650 & 0.7596 \\
6.00 & 0.4118 & 0.0438 & 0.2537 & $0.2461 \mathrm{E}-02$ & 0.3073 & 0.5874 \\
7.00 & 0.4706 & 0.0416 & 0.2190 & $0.1948 \mathrm{E}-02$ & 0.4815 & 0.4021 \\
8.00 & 0.5294 & 0.0392 & 0.1830 & $0.1354 \mathrm{E}-02$ & 0.6570 & 0.2396 \\
9.00 & 0.5882 & 0.0367 & 0.1474 & $0.8480 \mathrm{E}-03$ & 0.8038 & 0.1221 \\
10.0 & 0.6471 & 0.0340 & 0.1143 & $0.5160 \mathrm{E}-03$ & 0.9055 & 0.0520 \\
11.0 & 0.7059 & 0.0310 & $0.8508 \mathrm{E}-01$ & $0.3280 \mathrm{E}-03$ & 0.9627 & 0.0180 \\
12.0 & 0.7647 & 0.0277 & $0.5933 \mathrm{E}-01$ & $0.2250 \mathrm{E}-03$ & 0.9881 & 0.0050 \\
13.0 & 0.8235 & 0.0240 & $0.3621 \mathrm{E}-01$ & $0.1640 \mathrm{E}-03$ & 0.9970 & 0.0011 \\
14.0 & 0.8824 & 0.0196 & $0.1674 \mathrm{E}-01$ & $0.1060 \mathrm{E}-03$ & 0.9993 & 0.0002 \\
15.0 & 0.9412 & 0.0139 & $0.4250 \mathrm{E}-02$ & $0.4100 \mathrm{E}-04$ & 1.0000 & 0.0000 \\
\hline \hline
\end{tabular}

TABle 5.10: Table of $Q^{2}=2.5 \quad \mathrm{GeV}^{2} F_{2}^{D}$ Bernstein Moment Values. The Resonance Region here refers to $x$ values for which Resonance Data points exist.

\begin{tabular}{|c|c|c|c|c|c|c|}
\hline \hline$k$ & $x$ & $\Delta x$ & $F_{N, k}^{(e x p)}\left(Q^{2}\right)$ & $\Delta F_{N, k}^{(e r r)}\left(Q^{2}\right)$ & Res & DIS \\
\hline \hline 0.00 & 0.0588 & 0.0555 & 0.3644 & $0.3418 \mathrm{E}-02$ & 0.0001 & 0.9999 \\
1.00 & 0.1176 & 0.0537 & 0.3481 & $0.3315 \mathrm{E}-02$ & 0.0008 & 0.9984 \\
2.00 & 0.1765 & 0.0519 & 0.3259 & $0.3188 \mathrm{E}-02$ & 0.0052 & 0.9903 \\
3.00 & 0.2353 & 0.0500 & 0.2969 & $0.3018 \mathrm{E}-02$ & 0.0218 & 0.9623 \\
4.00 & 0.2941 & 0.0480 & 0.2653 & $0.2843 \mathrm{E}-02$ & 0.0664 & 0.8938 \\
5.00 & 0.3529 & 0.0460 & 0.2336 & $0.2577 \mathrm{E}-02$ & 0.1560 & 0.7702 \\
6.00 & 0.4118 & 0.0438 & 0.2026 & $0.2144 \mathrm{E}-02$ & 0.2952 & 0.5996 \\
7.00 & 0.4706 & 0.0416 & 0.1717 & $0.1578 \mathrm{E}-02$ & 0.4680 & 0.4136 \\
8.00 & 0.5294 & 0.0392 & 0.1411 & $0.1010 \mathrm{E}-02$ & 0.6443 & 0.2487 \\
9.00 & 0.5882 & 0.0367 & 0.1118 & $0.5780 \mathrm{E}-03$ & 0.7940 & 0.1281 \\
10.0 & 0.6471 & 0.0340 & $0.8515 \mathrm{E}-01$ & $0.3300 \mathrm{E}-03$ & 0.8991 & 0.0553 \\
11.0 & 0.7059 & 0.0310 & $0.6215 \mathrm{E}-01$ & $0.2080 \mathrm{E}-03$ & 0.9594 & 0.0195 \\
12.0 & 0.7647 & 0.0277 & $0.4346 \mathrm{E}-01$ & $0.1420 \mathrm{E}-03$ & 0.9872 & 0.0054 \\
13.0 & 0.8235 & 0.0240 & $0.2998 \mathrm{E}-01$ & $0.1000 \mathrm{E}-03$ & 0.9971 & 0.0011 \\
14.0 & 0.8824 & 0.0196 & $0.2329 \mathrm{E}-01$ & $0.8800 \mathrm{E}-04$ & 0.9996 & 0.0001 \\
15.0 & 0.9412 & 0.0139 & $0.2247 \mathrm{E}-01$ & $0.1260 \mathrm{E}-03$ & 1.0000 & 0.0000 \\
\hline \hline
\end{tabular}




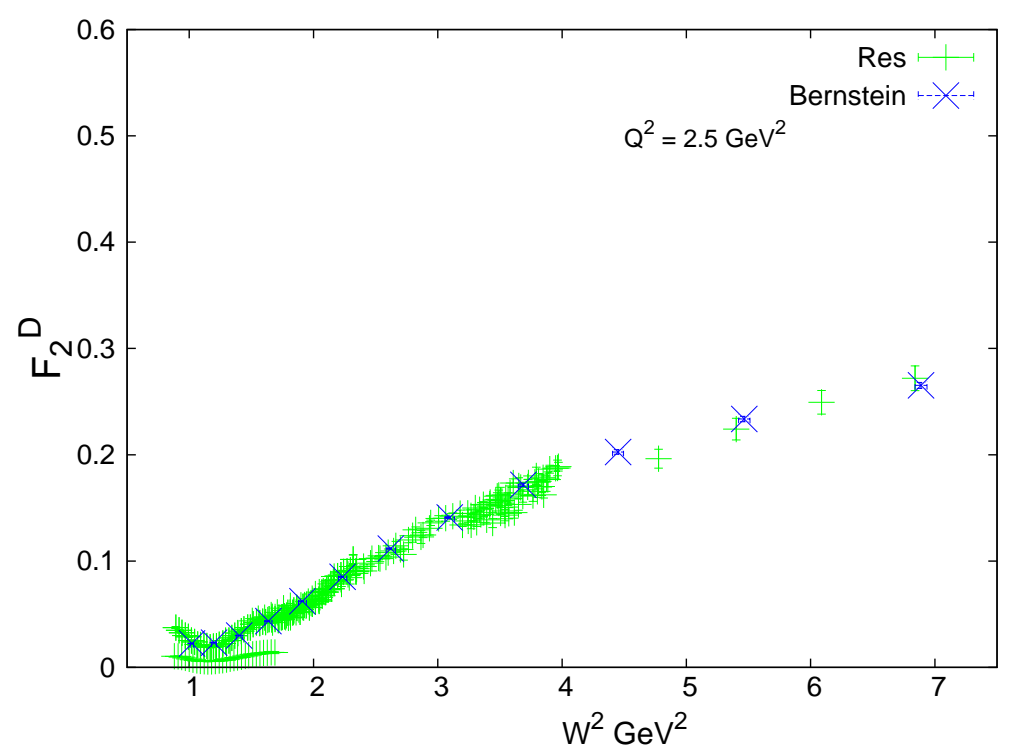

FiguRE 5.20: The $F_{2}^{D}$ values for the resonance region and the Bernstein moments are shown here. The green points are all of the $F_{2}^{D}$ resonance points in the $Q^{2}$ interval $(2.3: 2.5)$ and blue points are the Bernstein averages for those points in the $W^{2}$ range that constitutes the resonance region for $Q^{2}=2.5 \mathrm{GeV}^{2}$. 
TABle 5.11: Table of $Q^{2}=3.4 \mathrm{GeV}^{2} F_{2}^{P}$ Bernstein Moment Values. The Resonance Region here refers to $x$ values for which Resonance Data points exist.

\begin{tabular}{|c|c|c|c|c|c|c|}
\hline \hline$k$ & $x$ & $\Delta x$ & $F_{N, k}^{(e x p)}\left(Q^{2}\right)$ & $\Delta F_{N, k}^{(e r r)}\left(Q^{2}\right)$ & Res & DIS \\
\hline \hline 0.00 & 0.0588 & 0.0555 & 0.3949 & $0.3624 \mathrm{E}-02$ & 0.0000 & 1.0000 \\
1.00 & 0.1176 & 0.0537 & 0.3799 & $0.3468 \mathrm{E}-02$ & 0.0000 & 0.9999 \\
2.00 & 0.1765 & 0.0519 & 0.3611 & $0.3209 \mathrm{E}-02$ & 0.0004 & 0.9995 \\
3.00 & 0.2353 & 0.0500 & 0.3332 & $0.2953 \mathrm{E}-02$ & 0.0026 & 0.9970 \\
4.00 & 0.2941 & 0.0480 & 0.2987 & $0.2792 \mathrm{E}-02$ & 0.0112 & 0.9875 \\
5.00 & 0.3529 & 0.0460 & 0.2613 & $0.2640 \mathrm{E}-02$ & 0.0369 & 0.9593 \\
6.00 & 0.4118 & 0.0438 & 0.2247 & $0.2397 \mathrm{E}-02$ & 0.0969 & 0.8953 \\
7.00 & 0.4706 & 0.0416 & 0.1911 & $0.2041 \mathrm{E}-02$ & 0.2060 & 0.7810 \\
8.00 & 0.5294 & 0.0392 & 0.1604 & $0.1591 \mathrm{E}-02$ & 0.3627 & 0.6204 \\
9.00 & 0.5882 & 0.0367 & 0.1315 & $0.1101 \mathrm{E}-02$ & 0.5434 & 0.4391 \\
10.0 & 0.6471 & 0.0340 & 0.1037 & $0.6680 \mathrm{E}-03$ & 0.7140 & 0.2715 \\
11.0 & 0.7059 & 0.0310 & $0.7750 \mathrm{E}-01$ & $0.3750 \mathrm{E}-03$ & 0.8472 & 0.1431 \\
12.0 & 0.7647 & 0.0277 & $0.5372 \mathrm{E}-01$ & $0.2350 \mathrm{E}-03$ & 0.9324 & 0.0626 \\
13.0 & 0.8235 & 0.0240 & $0.3305 \mathrm{E}-01$ & $0.1830 \mathrm{E}-03$ & 0.9758 & 0.0221 \\
14.0 & 0.8824 & 0.0196 & $0.1614 \mathrm{E}-01$ & $0.1410 \mathrm{E}-03$ & 0.9931 & 0.0063 \\
15.0 & 0.9412 & 0.0139 & $0.4626 \mathrm{E}-02$ & $0.6600 \mathrm{E}-04$ & 0.9985 & 0.0015 \\
\hline \hline
\end{tabular}

TABle 5.12: Table of $Q^{2}=3.4 \mathrm{GeV}^{2} F_{2}^{D}$ Bernstein Moment Values. The Resonance Region here refers to $x$ values for which Resonance Data points exist.

\begin{tabular}{|c|c|c|c|c|c|c|}
\hline \hline$k$ & $x$ & $\Delta x$ & $F_{N, k}^{(e x p)}\left(Q^{2}\right)$ & $\Delta F_{N, k}^{(e r r)}\left(Q^{2}\right)$ & Res & DIS \\
\hline \hline 0.00 & 0.0588 & 0.0555 & 0.3766 & $0.3316 \mathrm{E}-02$ & 0.0000 & 1.0000 \\
1.00 & 0.1176 & 0.0537 & 0.3491 & $0.3201 \mathrm{E}-02$ & 0.0000 & 1.0000 \\
2.00 & 0.1765 & 0.0519 & 0.3209 & $0.3061 \mathrm{E}-02$ & 0.0003 & 0.9996 \\
3.00 & 0.2353 & 0.0500 & 0.2868 & $0.2888 \mathrm{E}-02$ & 0.0021 & 0.9975 \\
4.00 & 0.2941 & 0.0480 & 0.2494 & $0.2722 \mathrm{E}-02$ & 0.0092 & 0.9894 \\
5.00 & 0.3529 & 0.0460 & 0.2119 & $0.2511 \mathrm{E}-02$ & 0.0309 & 0.9649 \\
6.00 & 0.4118 & 0.0438 & 0.1769 & $0.2217 \mathrm{E}-02$ & 0.0828 & 0.9081 \\
7.00 & 0.4706 & 0.0416 & 0.1457 & $0.1847 \mathrm{E}-02$ & 0.1800 & 0.8044 \\
8.00 & 0.5294 & 0.0392 & 0.1180 & $0.1417 \mathrm{E}-02$ & 0.3251 & 0.6539 \\
9.00 & 0.5882 & 0.0367 & $0.9314 \mathrm{E}-01$ & $0.9650 \mathrm{E}-03$ & 0.5009 & 0.4764 \\
10.0 & 0.6471 & 0.0340 & $0.7080 \mathrm{E}-01$ & $0.5620 \mathrm{E}-03$ & 0.6767 & 0.3036 \\
11.0 & 0.7059 & 0.0310 & $0.5135 \mathrm{E}-01$ & $0.2810 \mathrm{E}-03$ & 0.8224 & 0.1641 \\
12.0 & 0.7647 & 0.0277 & $0.3524 \mathrm{E}-01$ & $0.1370 \mathrm{E}-03$ & 0.9206 & 0.0722 \\
13.0 & 0.8235 & 0.0240 & $0.2256 \mathrm{E}-01$ & $0.8800 \mathrm{E}-04$ & 0.9727 & 0.0245 \\
14.0 & 0.8824 & 0.0196 & $0.1324 \mathrm{E}-01$ & $0.8700 \mathrm{E}-04$ & 0.9935 & 0.0057 \\
15.0 & 0.9412 & 0.0139 & $0.7927 \mathrm{E}-02$ & $0.1930 \mathrm{E}-03$ & 0.9992 & 0.0006 \\
\hline \hline
\end{tabular}




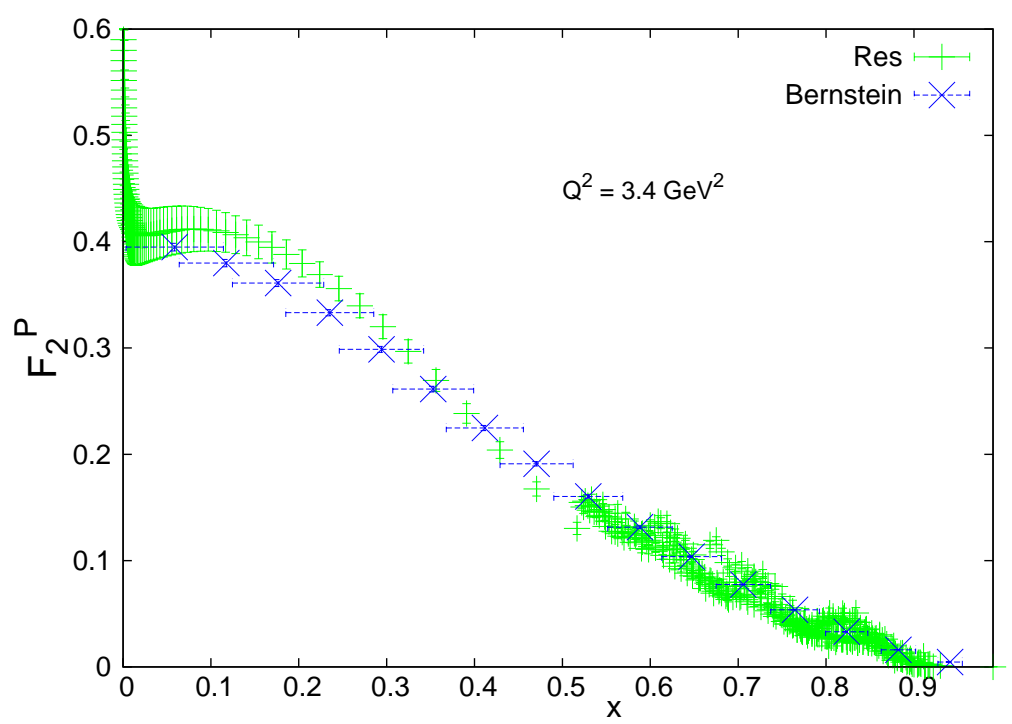

Figure 5.21: The $F_{2}^{P}$ values for the resonance region and the Bernstein moments are shown here. The green points are all of the $F_{2}^{P}$ resonance points in the $Q^{2}$ interval $(2.9: 3.9)$ and blue points are the Bernstein averages for those points in the $x$ range that constitutes the resonance region for $Q^{2}=3.4 \mathrm{GeV}^{2}$.

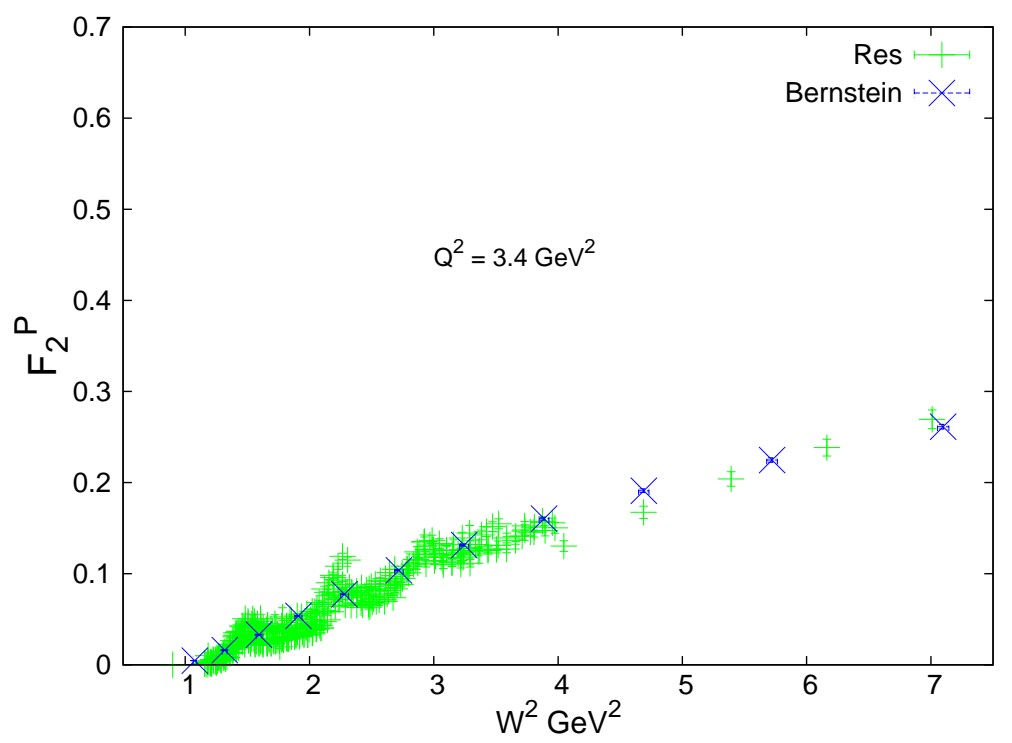

FiguRE 5.22: The $F_{2}^{P}$ values for the resonance region and the Bernstein moments are shown here. The green points are all of the $F_{2}^{P}$ resonance points in the $Q^{2}$ interval $(2.9: 3.9)$ and blue points are the Bernstein averages for those points in the $W^{2}$ range that constitutes the resonance region for $Q^{2}=3.4 \mathrm{GeV}^{2}$. 


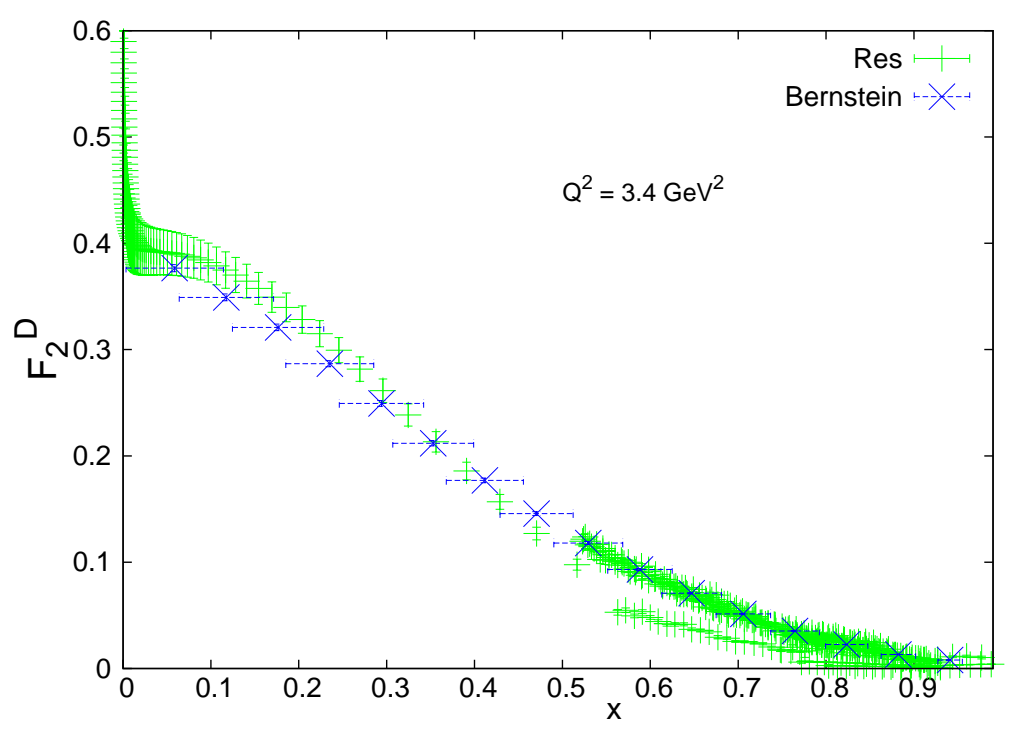

FiguRE 5.23: The $F_{2}^{D}$ values for the resonance region and the Bernstein moments are shown here. The green points are all of the $F_{2}^{D}$ resonance points in the $Q^{2}$ interval $(2.9: 3.9)$ and blue points are the Bernstein averages for those points in the $x$ range that constitutes the resonance region for $Q^{2}=3.4 \mathrm{GeV}^{2}$. 


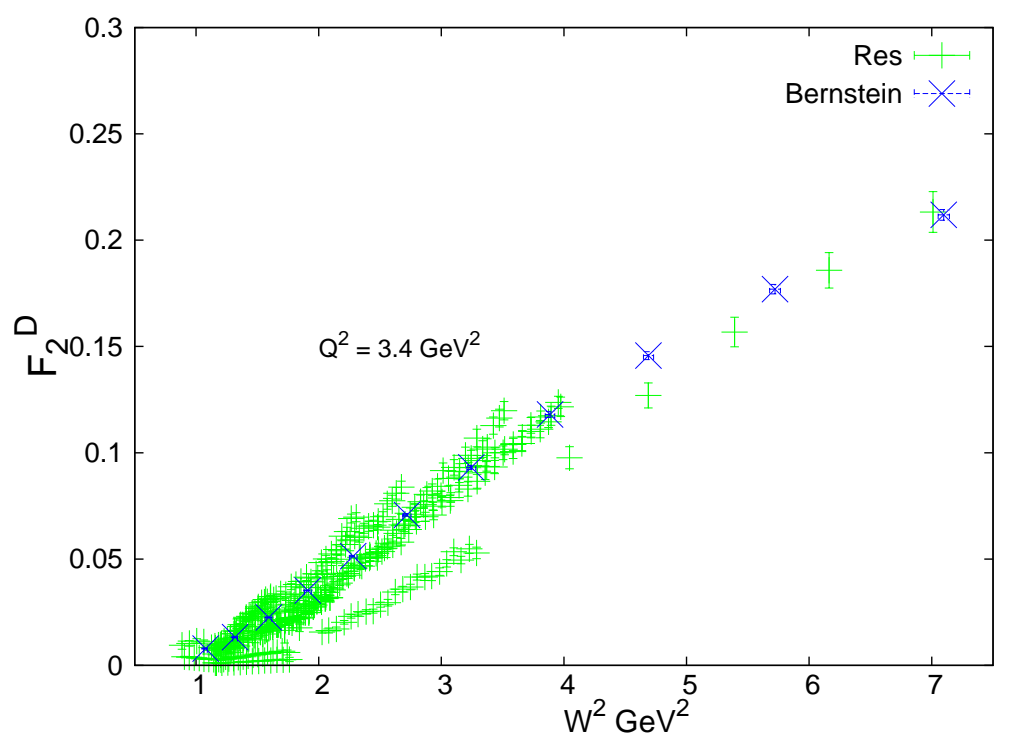

FiguRE 5.24: The $F_{2}^{D}$ values for the resonance region and the Bernstein moments are shown here. The green points are all of the $F_{2}^{D}$ resonance points in the $Q^{2}$ interval $(2.9: 3.9)$ and blue points are the Bernstein averages for those points in the $W^{2}$ range that constitutes the resonance region for $Q^{2}=3.4 \mathrm{GeV}^{2}$. 
TABle 5.13: Table of $Q^{2}=5.7 \quad \mathrm{GeV}^{2} F_{2}^{P}$ Bernstein Moment Values. The Resonance Region here refers to $x$ values for which Resonance Data points exist.

\begin{tabular}{|c|c|c|c|c|c|c|}
\hline \hline$k$ & $x$ & $\Delta x$ & $F_{N, k}^{(e x p)}\left(Q^{2}\right)$ & $\Delta F_{N, k}^{(e r r)}\left(Q^{2}\right)$ & Res & DIS \\
\hline \hline 0.00 & 0.0588 & 0.0555 & 0.4148 & $0.3466 \mathrm{E}-02$ & 0.0000 & 1.0000 \\
1.00 & 0.1176 & 0.0537 & 0.3815 & $0.3286 \mathrm{E}-02$ & 0.0000 & 1.0000 \\
2.00 & 0.1765 & 0.0519 & 0.3541 & $0.3011 \mathrm{E}-02$ & 0.0000 & 1.0000 \\
3.00 & 0.2353 & 0.0500 & 0.3207 & $0.2756 \mathrm{E}-02$ & 0.0000 & 0.9999 \\
4.00 & 0.2941 & 0.0480 & 0.2818 & $0.2593 \mathrm{E}-02$ & 0.0002 & 0.9995 \\
5.00 & 0.3529 & 0.0460 & 0.2396 & $0.2438 \mathrm{E}-02$ & 0.0013 & 0.9974 \\
6.00 & 0.4118 & 0.0438 & 0.1972 & $0.2220 \mathrm{E}-02$ & 0.0058 & 0.9893 \\
7.00 & 0.4706 & 0.0416 & 0.1578 & $0.1964 \mathrm{E}-02$ & 0.0212 & 0.9639 \\
8.00 & 0.5294 & 0.0392 & 0.1240 & $0.1726 \mathrm{E}-02$ & 0.0628 & 0.9014 \\
9.00 & 0.5882 & 0.0367 & $0.9681 \mathrm{E}-01$ & $0.1523 \mathrm{E}-02$ & 0.1505 & 0.7823 \\
10.0 & 0.6471 & 0.0340 & $0.7504 \mathrm{E}-01$ & $0.1298 \mathrm{E}-02$ & 0.2925 & 0.6096 \\
11.0 & 0.7059 & 0.0310 & $0.5647 \mathrm{E}-01$ & $0.9890 \mathrm{E}-03$ & 0.4716 & 0.4171 \\
12.0 & 0.7647 & 0.0277 & $0.3960 \mathrm{E}-01$ & $0.6270 \mathrm{E}-03$ & 0.6541 & 0.2463 \\
13.0 & 0.8235 & 0.0240 & $0.2457 \mathrm{E}-01$ & $0.3430 \mathrm{E}-03$ & 0.8082 & 0.1221 \\
14.0 & 0.8824 & 0.0196 & $0.1239 \mathrm{E}-01$ & $0.2160 \mathrm{E}-03$ & 0.9143 & 0.0486 \\
15.0 & 0.9412 & 0.0139 & $0.3950 \mathrm{E}-02$ & $0.1140 \mathrm{E}-03$ & 0.9706 & 0.0149 \\
\hline \hline
\end{tabular}

TABle 5.14: Table of $Q^{2}=5.7 \mathrm{GeV}^{2} F_{2}^{D}$ Bernstein Moment Values. The Resonance Region here refers to $x$ values for which Resonance Data points exist.

\begin{tabular}{|c|c|c|c|c|c|c|}
\hline \hline$k$ & $x$ & $\Delta x$ & $F_{N, k}^{(e x p)}\left(Q^{2}\right)$ & $\Delta F_{N, k}^{(e r r)}\left(Q^{2}\right)$ & Res & DIS \\
\hline \hline 0.00 & 0.0588 & 0.0555 & 0.3958 & $0.3173 \mathrm{E}-02$ & 0.0000 & 1.0000 \\
1.00 & 0.1176 & 0.0537 & 0.3503 & $0.3036 \mathrm{E}-02$ & 0.0000 & 1.0000 \\
2.00 & 0.1765 & 0.0519 & 0.3141 & $0.2878 \mathrm{E}-02$ & 0.0000 & 1.0000 \\
3.00 & 0.2353 & 0.0500 & 0.2756 & $0.2697 \mathrm{E}-02$ & 0.0000 & 0.9999 \\
4.00 & 0.2941 & 0.0480 & 0.2351 & $0.2525 \mathrm{E}-02$ & 0.0002 & 0.9996 \\
5.00 & 0.3529 & 0.0460 & 0.1948 & $0.2315 \mathrm{E}-02$ & 0.0011 & 0.9977 \\
6.00 & 0.4118 & 0.0438 & 0.1566 & $0.2054 \mathrm{E}-02$ & 0.0052 & 0.9903 \\
7.00 & 0.4706 & 0.0416 & 0.1227 & $0.1791 \mathrm{E}-02$ & 0.0189 & 0.9672 \\
8.00 & 0.5294 & 0.0392 & $0.9427 \mathrm{E}-01$ & $0.1583 \mathrm{E}-02$ & 0.0558 & 0.9104 \\
9.00 & 0.5882 & 0.0367 & $0.7161 \mathrm{E}-01$ & $0.1424 \mathrm{E}-02$ & 0.1336 & 0.8017 \\
10.0 & 0.6471 & 0.0340 & $0.5355 \mathrm{E}-01$ & $0.1226 \mathrm{E}-02$ & 0.2617 & 0.6414 \\
11.0 & 0.7059 & 0.0310 & $0.3852 \mathrm{E}-01$ & $0.9200 \mathrm{E}-03$ & 0.4300 & 0.4558 \\
12.0 & 0.7647 & 0.0277 & $0.2583 \mathrm{E}-01$ & $0.5570 \mathrm{E}-03$ & 0.6140 & 0.2801 \\
13.0 & 0.8235 & 0.0240 & $0.1580 \mathrm{E}-01$ & $0.2680 \mathrm{E}-03$ & 0.7851 & 0.1404 \\
14.0 & 0.8824 & 0.0196 & $0.8917 \mathrm{E}-02$ & $0.1260 \mathrm{E}-03$ & 0.9151 & 0.0498 \\
15.0 & 0.9412 & 0.0139 & $0.5163 \mathrm{E}-02$ & $0.6300 \mathrm{E}-04$ & 0.9841 & 0.0083 \\
\hline \hline
\end{tabular}




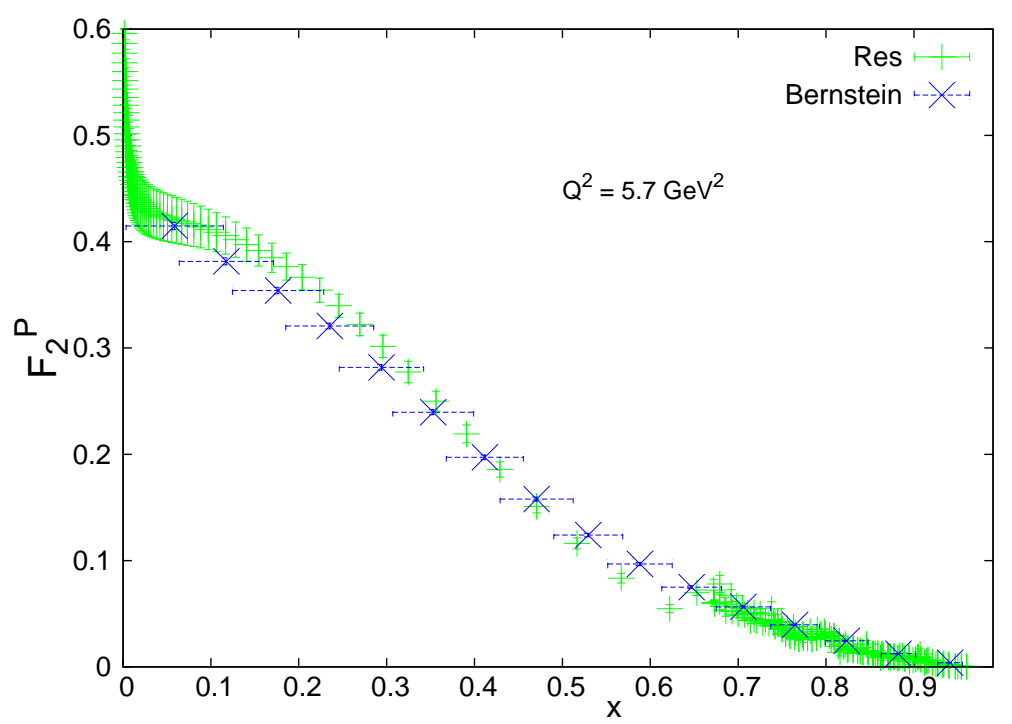

Figure 5.25: The $F_{2}^{P}$ values for the resonance region and the Bernstein moments are shown here. The green points are all of the $F_{2}^{P}$ resonance points in the $Q^{2}$ interval $(5.3: 6.2)$ and blue points are the Bernstein averages for those points in the $x$ range that constitutes the resonance region for $Q^{2}=5.7 \mathrm{GeV}^{2}$.

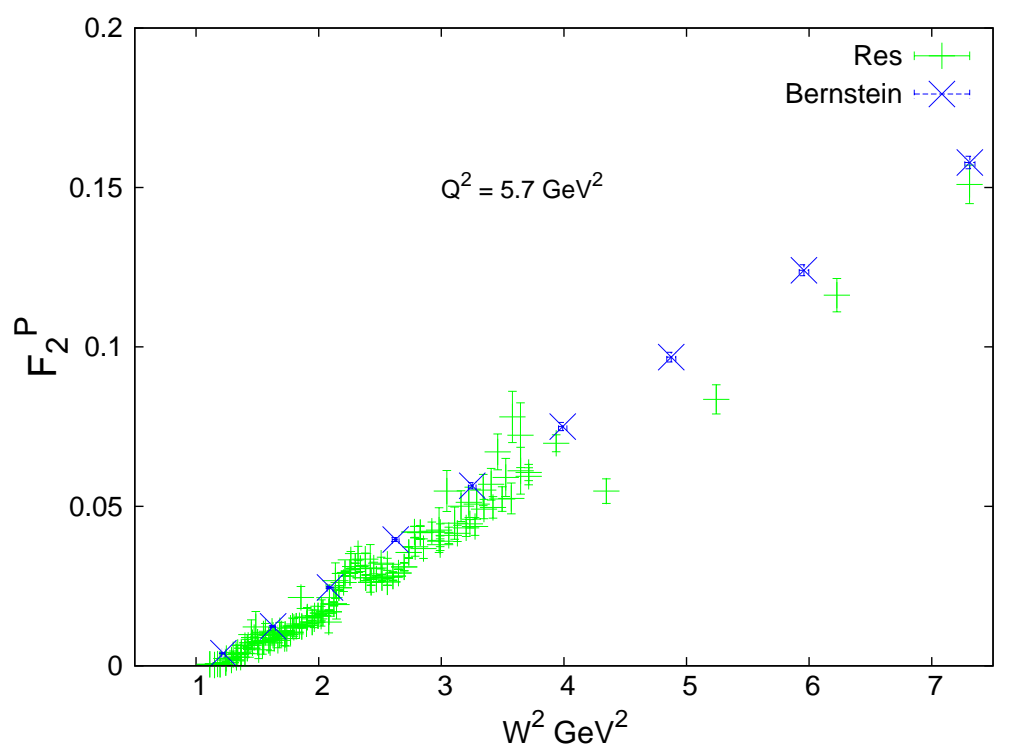

FIGURE 5.26: The $F_{2}^{P}$ values for the resonance region and the Bernstein moments are shown here. The green points are all of the $F_{2}^{P}$ resonance points in the $Q^{2}$ interval $(5.3: 6.2)$ and blue points are the Bernstein averages for those points in the $W^{2}$ range that constitutes the resonance region for $Q^{2}=5.7 \mathrm{GeV}^{2}$. 


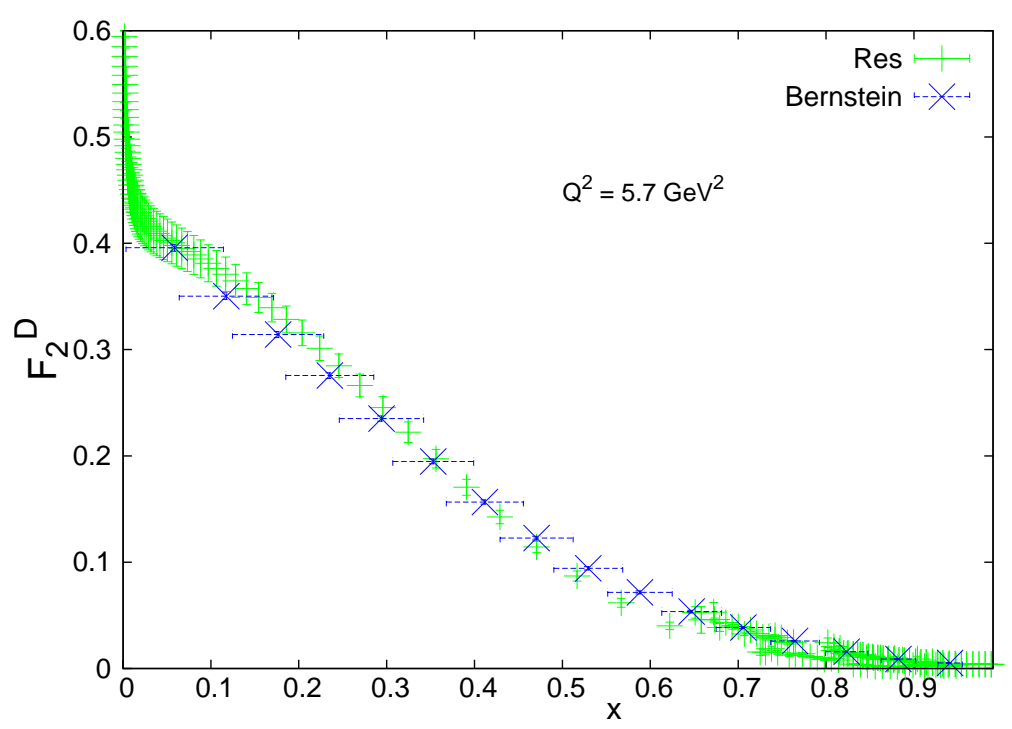

Figure 5.27: The $F_{2}^{D}$ values for the resonance region and the Bernstein moments are shown here. The green points are all of the $F_{2}^{D}$ resonance points in the $Q^{2}$ interval $(5.3: 6.2)$ and blue points are the Bernstein averages for those points in the $x$ range that constitutes the resonance region for $Q^{2}=5.7 \mathrm{GeV}^{2}$. 


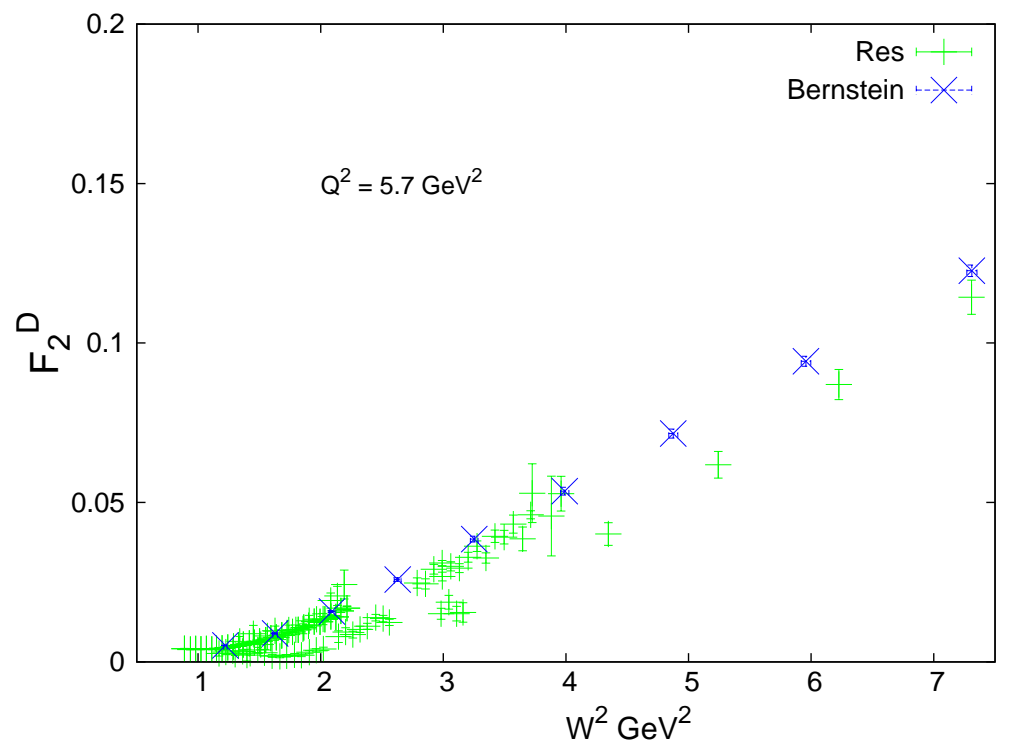

Figure 5.28: The $F_{2}^{D}$ values for the resonance region and the Bernstein moments are shown here. The green points are all of the $F_{2}^{D}$ resonance points in the $Q^{2}$ interval $(5.3: 6.2)$ and blue points are the Bernstein averages for those points in the $W^{2}$ range that constitutes the resonance region for $Q^{2}=5.7 \mathrm{GeV}^{2}$.

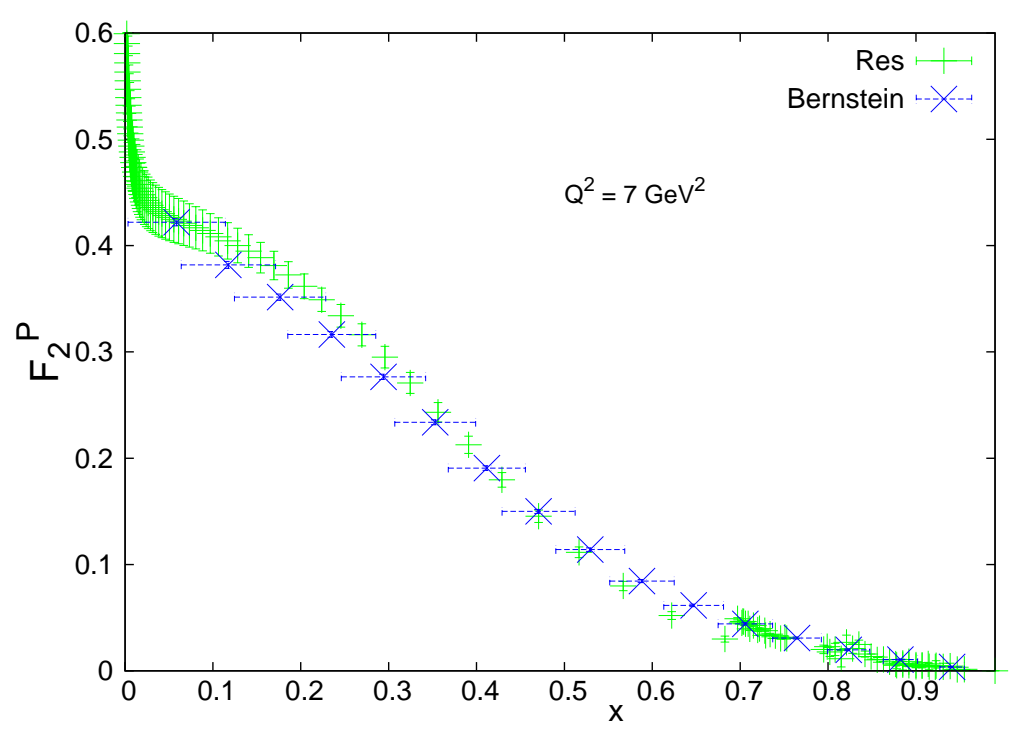

FIgURE 5.29: The $F_{2}^{P}$ values for the resonance region and the Bernstein moments are shown here. The green points are all of the $F_{2}^{P}$ resonance points in the $Q^{2}$ interval $(7.0: 7.35)$ and blue points are the Bernstein averages for those points in the $x$ range that constitutes the resonance region for $Q^{2}=7 \mathrm{GeV}^{2}$. 
TABle 5.15: Table of $Q^{2}=7 \mathrm{GeV}^{2} F_{2}^{P}$ Bernstein Moment Values. The Resonance Region here refers to $x$ values for which Resonance Data points exist.

\begin{tabular}{|c|c|c|c|c|c|c|}
\hline \hline$k$ & $x$ & $\Delta x$ & $F_{N, k}^{(e x p)}\left(Q^{2}\right)$ & $\Delta F_{N, k}^{(e r r)}\left(Q^{2}\right)$ & Res & DIS \\
\hline \hline 0.00 & 0.0588 & 0.0555 & 0.4220 & $0.3414 \mathrm{E}-02$ & 0.0000 & 1.0000 \\
1.00 & 0.1176 & 0.0537 & 0.3819 & $0.3224 \mathrm{E}-02$ & 0.0000 & 1.0000 \\
2.00 & 0.1765 & 0.0519 & 0.3515 & $0.2945 \mathrm{E}-02$ & 0.0000 & 1.0000 \\
3.00 & 0.2353 & 0.0500 & 0.3164 & $0.2690 \mathrm{E}-02$ & 0.0000 & 1.0000 \\
4.00 & 0.2941 & 0.0480 & 0.2765 & $0.2526 \mathrm{E}-02$ & 0.0000 & 0.9999 \\
5.00 & 0.3529 & 0.0460 & 0.2338 & $0.2368 \mathrm{E}-02$ & 0.0002 & 0.9996 \\
6.00 & 0.4118 & 0.0438 & 0.1907 & $0.2150 \mathrm{E}-02$ & 0.0013 & 0.9980 \\
7.00 & 0.4706 & 0.0416 & 0.1500 & $0.1893 \mathrm{E}-02$ & 0.0057 & 0.9916 \\
8.00 & 0.5294 & 0.0392 & 0.1141 & $0.1644 \mathrm{E}-02$ & 0.0204 & 0.9713 \\
9.00 & 0.5882 & 0.0367 & $0.8445 \mathrm{E}-01$ & $0.1418 \mathrm{E}-02$ & 0.0607 & 0.9192 \\
10.0 & 0.6471 & 0.0340 & $0.6152 \mathrm{E}-01$ & $0.1203 \mathrm{E}-02$ & 0.1488 & 0.8131 \\
11.0 & 0.7059 & 0.0310 & $0.4422 \mathrm{E}-01$ & $0.1029 \mathrm{E}-02$ & 0.2990 & 0.6456 \\
12.0 & 0.7647 & 0.0277 & $0.3080 \mathrm{E}-01$ & $0.9280 \mathrm{E}-03$ & 0.4966 & 0.4424 \\
13.0 & 0.8235 & 0.0240 & $0.1978 \mathrm{E}-01$ & $0.7860 \mathrm{E}-03$ & 0.6968 & 0.2528 \\
14.0 & 0.8824 & 0.0196 & $0.1060 \mathrm{E}-01$ & $0.5460 \mathrm{E}-03$ & 0.8531 & 0.1160 \\
15.0 & 0.9412 & 0.0139 & $0.3682 \mathrm{E}-02$ & $0.3140 \mathrm{E}-03$ & 0.9457 & 0.0407 \\
\hline \hline
\end{tabular}

TABle 5.16: Table of $Q^{2}=7 \quad \mathrm{GeV}^{2} F_{2}^{D}$ Bernstein Moment Values. The Resonance Region here refers to $x$ values for which Resonance Data points exist.

\begin{tabular}{|c|c|c|c|c|c|c|}
\hline \hline$k$ & $x$ & $\Delta x$ & $F_{N, k}^{(e x p)}\left(Q^{2}\right)$ & $\Delta F_{N, k}^{(e r r)}\left(Q^{2}\right)$ & Res & DIS \\
\hline \hline 0.00 & 0.0588 & 0.0555 & 0.4027 & $0.3125 \mathrm{E}-02$ & 0.0000 & 1.0000 \\
1.00 & 0.1176 & 0.0537 & 0.3505 & $0.2980 \mathrm{E}-02$ & 0.0000 & 1.0000 \\
2.00 & 0.1765 & 0.0519 & 0.3117 & $0.2815 \mathrm{E}-02$ & 0.0000 & 1.0000 \\
3.00 & 0.2353 & 0.0500 & 0.2717 & $0.2632 \mathrm{E}-02$ & 0.0000 & 1.0000 \\
4.00 & 0.2941 & 0.0480 & 0.2306 & $0.2459 \mathrm{E}-02$ & 0.0000 & 0.9999 \\
5.00 & 0.3529 & 0.0460 & 0.1900 & $0.2248 \mathrm{E}-02$ & 0.0002 & 0.9996 \\
6.00 & 0.4118 & 0.0438 & 0.1515 & $0.1988 \mathrm{E}-02$ & 0.0012 & 0.9981 \\
7.00 & 0.4706 & 0.0416 & 0.1168 & $0.1722 \mathrm{E}-02$ & 0.0053 & 0.9921 \\
8.00 & 0.5294 & 0.0392 & $0.8730 \mathrm{E}-01$ & $0.1491 \mathrm{E}-02$ & 0.0197 & 0.9724 \\
9.00 & 0.5882 & 0.0367 & $0.6377 \mathrm{E}-01$ & $0.1292 \mathrm{E}-02$ & 0.0604 & 0.9202 \\
10.0 & 0.6471 & 0.0340 & $0.4611 \mathrm{E}-01$ & $0.1090 \mathrm{E}-02$ & 0.1515 & 0.8114 \\
11.0 & 0.7059 & 0.0310 & $0.3322 \mathrm{E}-01$ & $0.8860 \mathrm{E}-03$ & 0.3090 & 0.6373 \\
12.0 & 0.7647 & 0.0277 & $0.2349 \mathrm{E}-01$ & $0.7160 \mathrm{E}-03$ & 0.5143 & 0.4275 \\
13.0 & 0.8235 & 0.0240 & $0.1552 \mathrm{E}-01$ & $0.5410 \mathrm{E}-03$ & 0.7168 & 0.2364 \\
14.0 & 0.8824 & 0.0196 & $0.8891 \mathrm{E}-02$ & $0.3180 \mathrm{E}-03$ & 0.8719 & 0.1012 \\
15.0 & 0.9412 & 0.0139 & $0.4347 \mathrm{E}-02$ & $0.1410 \mathrm{E}-03$ & 0.9664 & 0.0253 \\
\hline \hline
\end{tabular}




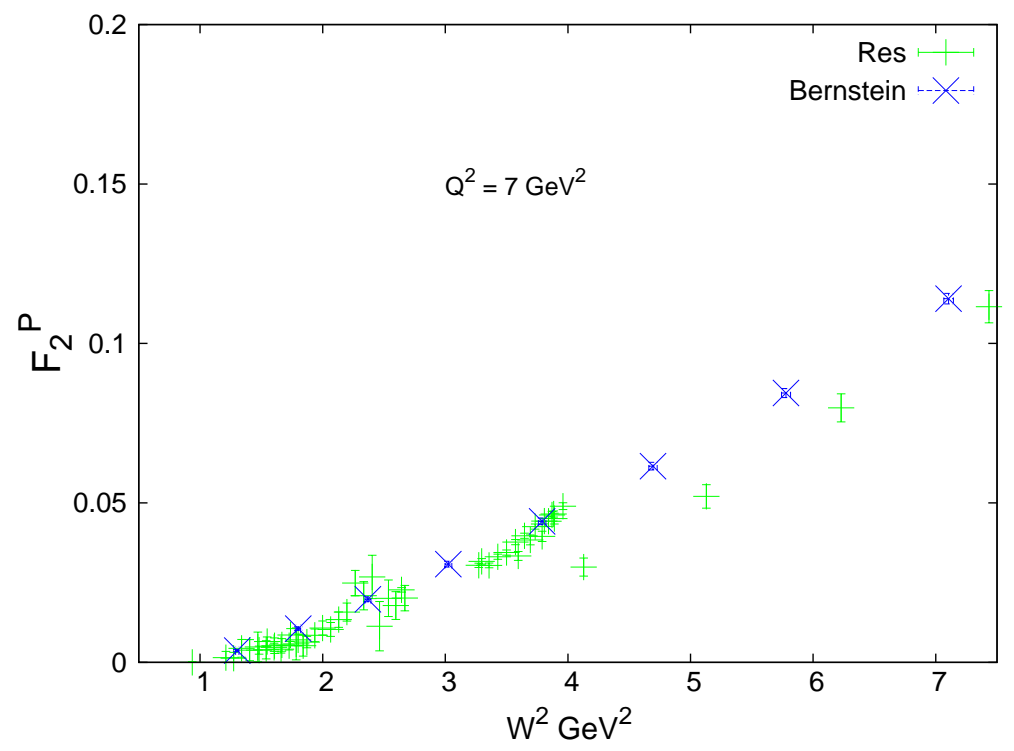

FiguRE 5.30: The $F_{2}^{P}$ values for the resonance region and the Bernstein moments are shown here. The green points are all of the $F_{2}^{P}$ resonance points in the $Q^{2}$ interval $(7.0: 7.35)$ and blue points are the Bernstein averages for those points in the $W^{2}$ range that constitutes the resonance region for $Q^{2}=7 \mathrm{GeV}^{2}$. 


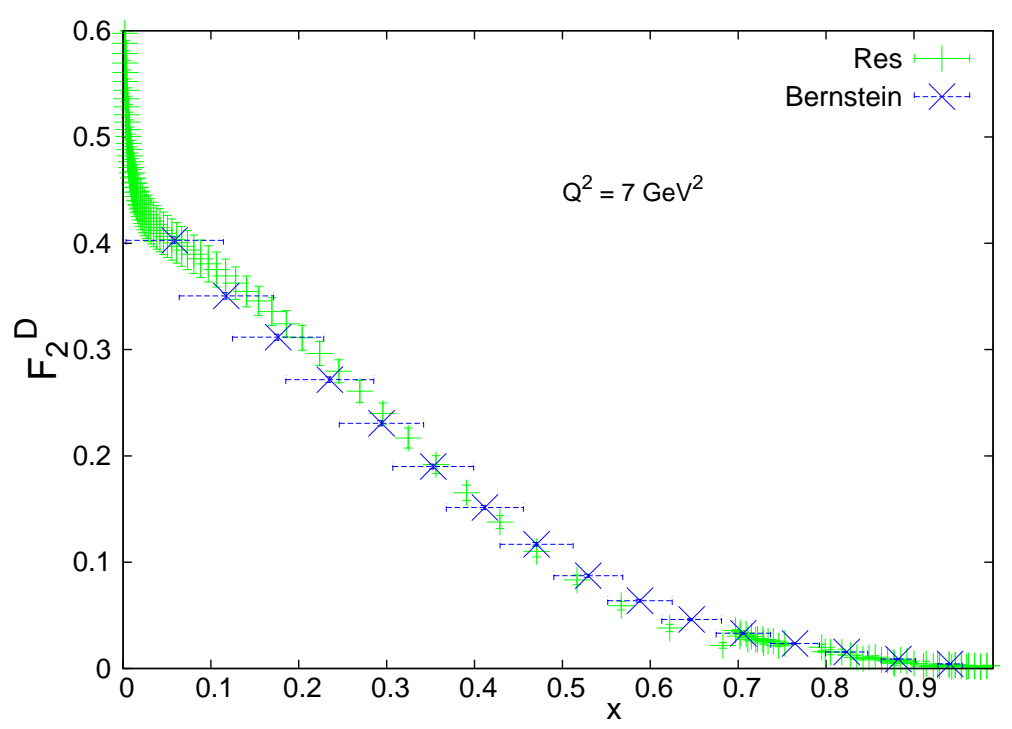

FiguRE 5.31: The $F_{2}^{D}$ values for the resonance region and the Bernstein moments are shown here. The green points are all of the $F_{2}^{D}$ resonance points in the $Q^{2}$ interval $(7.0: 7.35)$ and blue points are the Bernstein averages for those points in the $x$ range that constitutes the resonance region for $Q^{2}=7 \mathrm{GeV}^{2}$. 


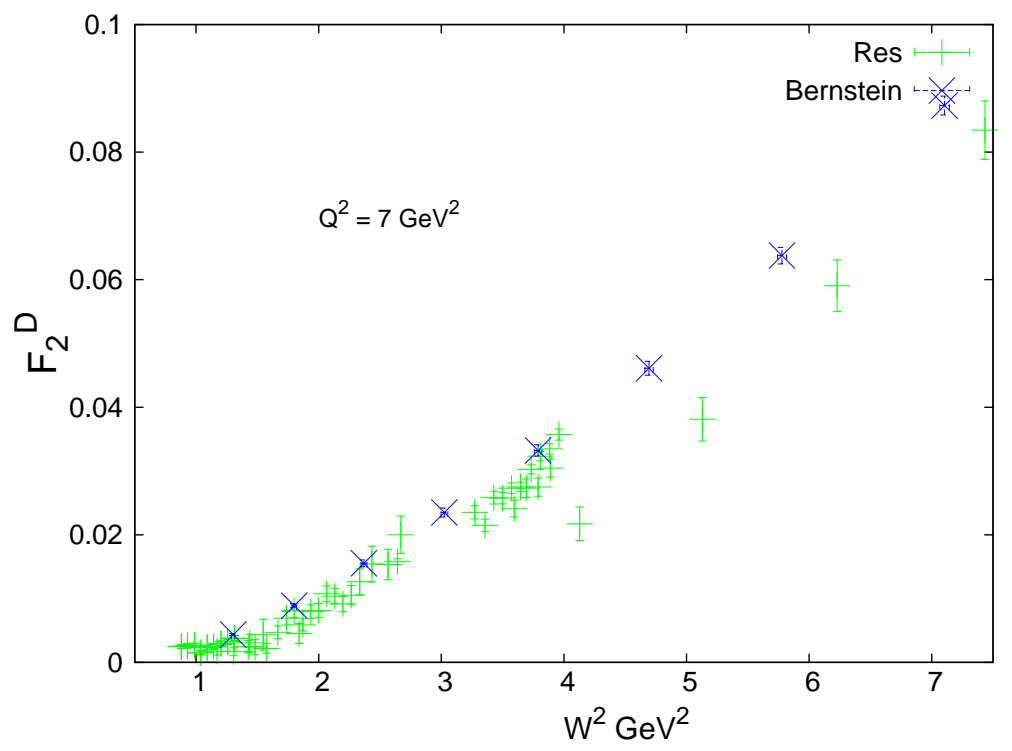

FiguRE 5.32: The $F_{2}^{D}$ values for the resonance region and the Bernstein moments are shown here. The green points are all of the $F_{2}^{D}$ resonance points in the $Q^{2}$ interval $(7.0: 7.35)$ and blue points are the Bernstein averages for those points in the $W^{2}$ range that constitutes the resonance region for $Q^{2}=7 \mathrm{GeV}^{2}$. 
TABle 5.17: Table of $Q^{2}=8.2 \quad \mathrm{GeV}^{2} F_{2}^{P}$ Bernstein Moment Values. The Resonance Region here refers to $x$ values for which Resonance Data points exist.

\begin{tabular}{|c|c|c|c|c|c|c|}
\hline \hline$k$ & $x$ & $\Delta x$ & $F_{N, k}^{(e x p)}\left(Q^{2}\right)$ & $\Delta F_{N, k}^{(e r r)}\left(Q^{2}\right)$ & Res & DIS \\
\hline \hline 0.00 & 0.0588 & 0.0555 & 0.4272 & $0.3378 \mathrm{E}-02$ & 0.0000 & 1.0000 \\
1.00 & 0.1176 & 0.0537 & 0.3821 & $0.3181 \mathrm{E}-02$ & 0.0000 & 1.0000 \\
2.00 & 0.1765 & 0.0519 & 0.3496 & $0.2898 \mathrm{E}-02$ & 0.0000 & 1.0000 \\
3.00 & 0.2353 & 0.0500 & 0.3133 & $0.2643 \mathrm{E}-02$ & 0.0000 & 1.0000 \\
4.00 & 0.2941 & 0.0480 & 0.2728 & $0.2479 \mathrm{E}-02$ & 0.0000 & 1.0000 \\
5.00 & 0.3529 & 0.0460 & 0.2299 & $0.2319 \mathrm{E}-02$ & 0.0001 & 0.9997 \\
6.00 & 0.4118 & 0.0438 & 0.1868 & $0.2102 \mathrm{E}-02$ & 0.0006 & 0.9986 \\
7.00 & 0.4706 & 0.0416 & 0.1463 & $0.1848 \mathrm{E}-02$ & 0.0031 & 0.9937 \\
8.00 & 0.5294 & 0.0392 & 0.1105 & $0.1606 \mathrm{E}-02$ & 0.0123 & 0.9766 \\
9.00 & 0.5882 & 0.0367 & $0.8116 \mathrm{E}-01$ & $0.1392 \mathrm{E}-02$ & 0.0407 & 0.9294 \\
10.0 & 0.6471 & 0.0340 & $0.5860 \mathrm{E}-01$ & $0.1181 \mathrm{E}-02$ & 0.1094 & 0.8273 \\
11.0 & 0.7059 & 0.0310 & $0.4179 \mathrm{E}-01$ & $0.9260 \mathrm{E}-03$ & 0.2368 & 0.6605 \\
12.0 & 0.7647 & 0.0277 & $0.2878 \mathrm{E}-01$ & $0.6180 \mathrm{E}-03$ & 0.4165 & 0.4570 \\
13.0 & 0.8235 & 0.0240 & $0.1802 \mathrm{E}-01$ & $0.3230 \mathrm{E}-03$ & 0.6139 & 0.2674 \\
14.0 & 0.8824 & 0.0196 & $0.9233 \mathrm{E}-02$ & $0.1340 \mathrm{E}-03$ & 0.7875 & 0.1283 \\
15.0 & 0.9412 & 0.0139 & $0.3000 \mathrm{E}-02$ & $0.5200 \mathrm{E}-04$ & 0.9080 & 0.0480 \\
\hline \hline
\end{tabular}

TABle 5.18: Table of $Q^{2}=8.2 \quad \mathrm{GeV}^{2} F_{2}^{D}$ Bernstein Moment Values. The Resonance Region here refers to $x$ values for which Resonance Data points exist.

\begin{tabular}{|c|c|c|c|c|c|c|}
\hline \hline$k$ & $x$ & $\Delta x$ & $F_{N, k}^{(e x p)}\left(Q^{2}\right)$ & $\Delta F_{N, k}^{(e r r)}\left(Q^{2}\right)$ & Res & DIS \\
\hline \hline 0.00 & 0.0588 & 0.0555 & 0.4078 & $0.3092 \mathrm{E}-02$ & 0.0000 & 1.0000 \\
1.00 & 0.1176 & 0.0537 & 0.3507 & $0.2940 \mathrm{E}-02$ & 0.0000 & 1.0000 \\
2.00 & 0.1765 & 0.0519 & 0.3099 & $0.2771 \mathrm{E}-02$ & 0.0000 & 1.0000 \\
3.00 & 0.2353 & 0.0500 & 0.2690 & $0.2587 \mathrm{E}-02$ & 0.0000 & 1.0000 \\
4.00 & 0.2941 & 0.0480 & 0.2274 & $0.2412 \mathrm{E}-02$ & 0.0000 & 1.0000 \\
5.00 & 0.3529 & 0.0460 & 0.1867 & $0.2200 \mathrm{E}-02$ & 0.0001 & 0.9997 \\
6.00 & 0.4118 & 0.0438 & 0.1484 & $0.1942 \mathrm{E}-02$ & 0.0006 & 0.9986 \\
7.00 & 0.4706 & 0.0416 & 0.1140 & $0.1681 \mathrm{E}-02$ & 0.0029 & 0.9935 \\
8.00 & 0.5294 & 0.0392 & $0.8475 \mathrm{E}-01$ & $0.1456 \mathrm{E}-02$ & 0.0116 & 0.9758 \\
9.00 & 0.5882 & 0.0367 & $0.6150 \mathrm{E}-01$ & $0.1265 \mathrm{E}-02$ & 0.0387 & 0.9271 \\
10.0 & 0.6471 & 0.0340 & $0.4406 \mathrm{E}-01$ & $0.1071 \mathrm{E}-02$ & 0.1041 & 0.8229 \\
11.0 & 0.7059 & 0.0310 & $0.3123 \mathrm{E}-01$ & $0.8340 \mathrm{E}-03$ & 0.2255 & 0.6555 \\
12.0 & 0.7647 & 0.0277 & $0.2136 \mathrm{E}-01$ & $0.5600 \mathrm{E}-03$ & 0.3985 & 0.4537 \\
13.0 & 0.8235 & 0.0240 & $0.1336 \mathrm{E}-01$ & $0.3070 \mathrm{E}-03$ & 0.5963 & 0.2648 \\
14.0 & 0.8824 & 0.0196 & $0.7197 \mathrm{E}-02$ & $0.1380 \mathrm{E}-03$ & 0.7857 & 0.1205 \\
15.0 & 0.9412 & 0.0139 & $0.3287 \mathrm{E}-02$ & $0.4900 \mathrm{E}-04$ & 0.9331 & 0.0319 \\
\hline \hline
\end{tabular}




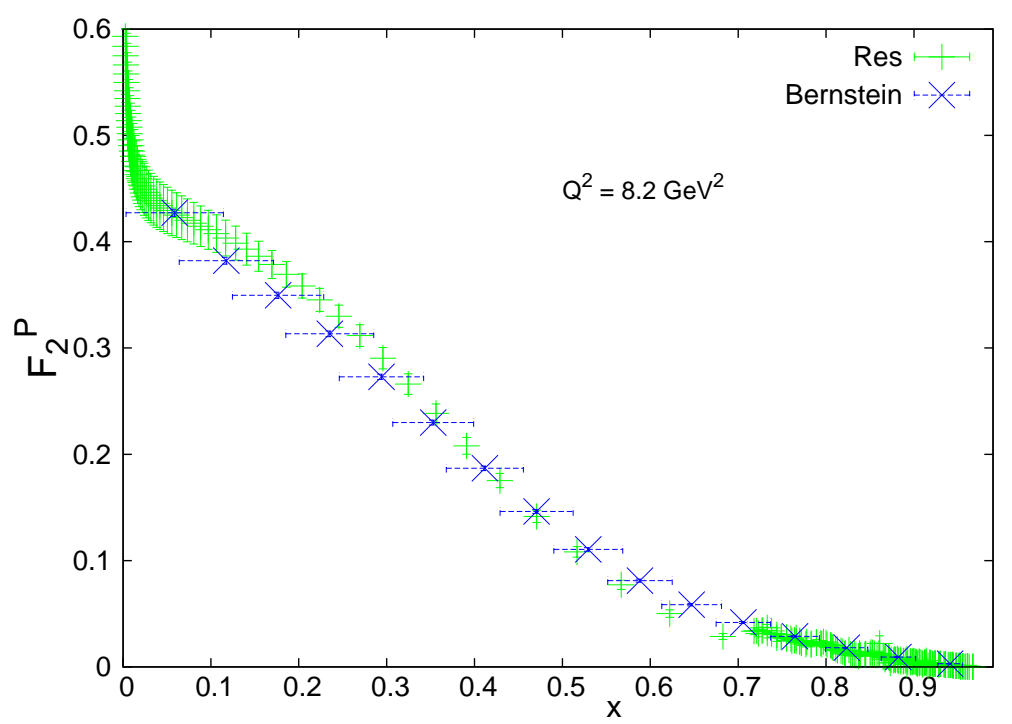

Figure 5.33: The $F_{2}^{P}$ values for the resonance region and the Bernstein moments are shown here. The green points are all of the $F_{2}^{P}$ resonance points in the $Q^{2}$ interval $(7.8: 8.8)$ and blue points are the Bernstein averages for those points in the $x$ range that constitutes the resonance region for $Q^{2}=8.2 \mathrm{GeV}^{2}$.

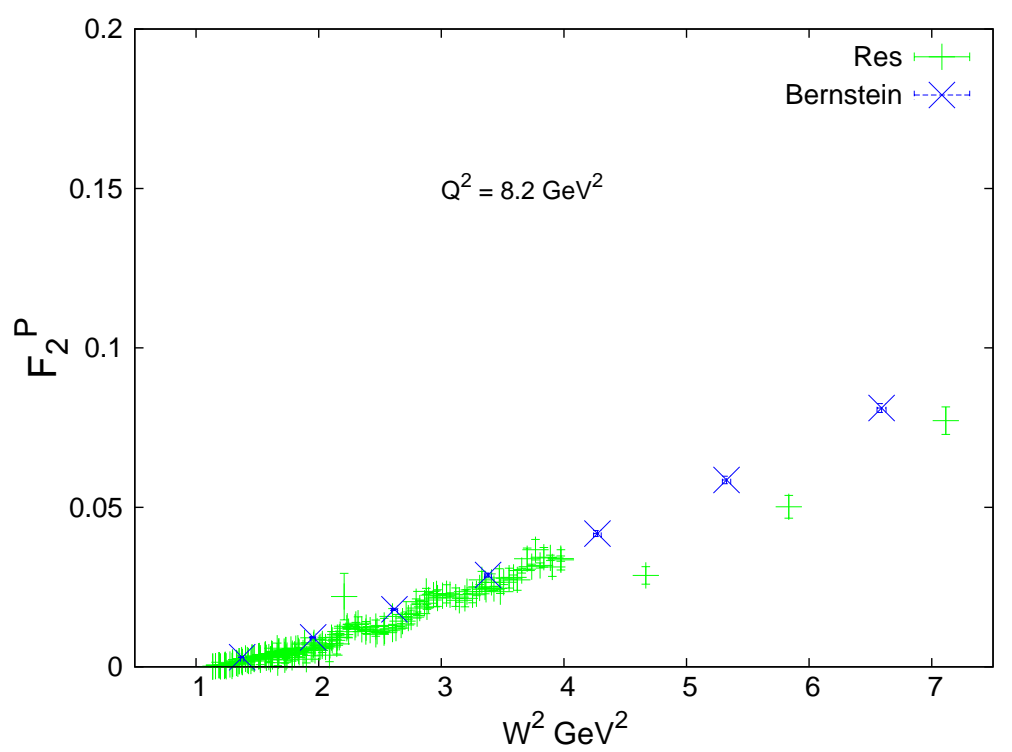

FIgURE 5.34: The $F_{2}^{P}$ values for the resonance region and the Bernstein moments are shown here. The green points are all of the $F_{2}^{P}$ resonance points in the $Q^{2}$ interval $(7.8: 8.8)$ and blue points are the Bernstein averages for those points in the $W^{2}$ range that constitutes the resonance region for $Q^{2}=8.2 \mathrm{GeV}^{2}$. 


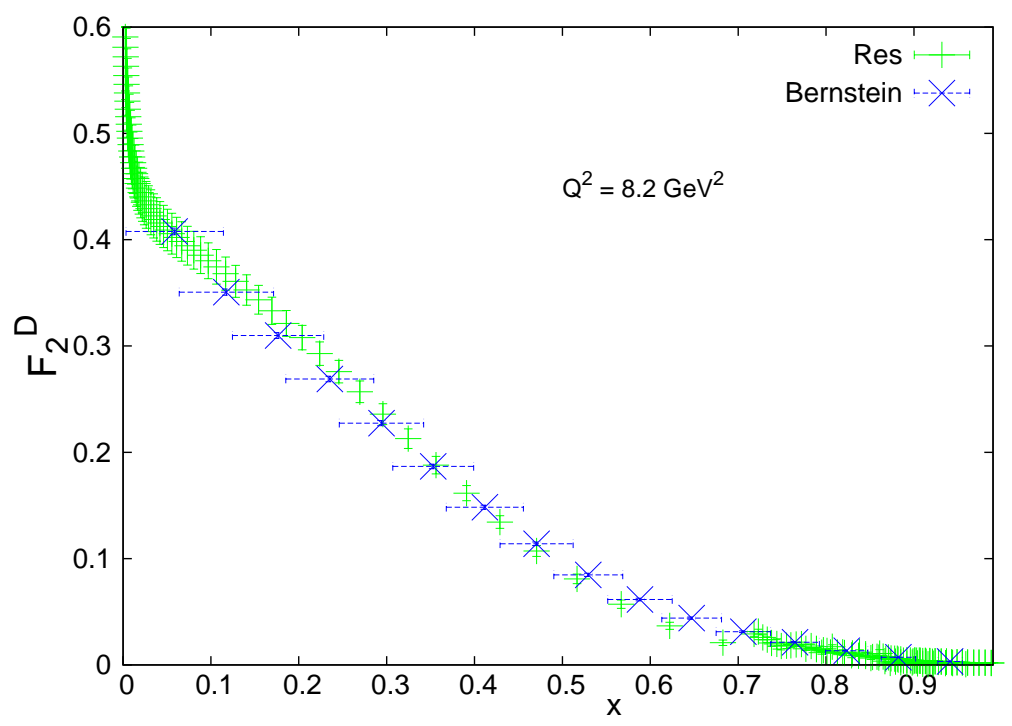

FigURE 5.35: The $F_{2}^{D}$ values for the resonance region and the Bernstein moments are shown here. The green points are all of the $F_{2}^{D}$ resonance points in the $Q^{2}$ interval $(7.8: 8.8)$ and blue points are the Bernstein averages for those points in the $x$ range that constitutes the resonance region for $Q^{2}=8.2 \mathrm{GeV}^{2}$.

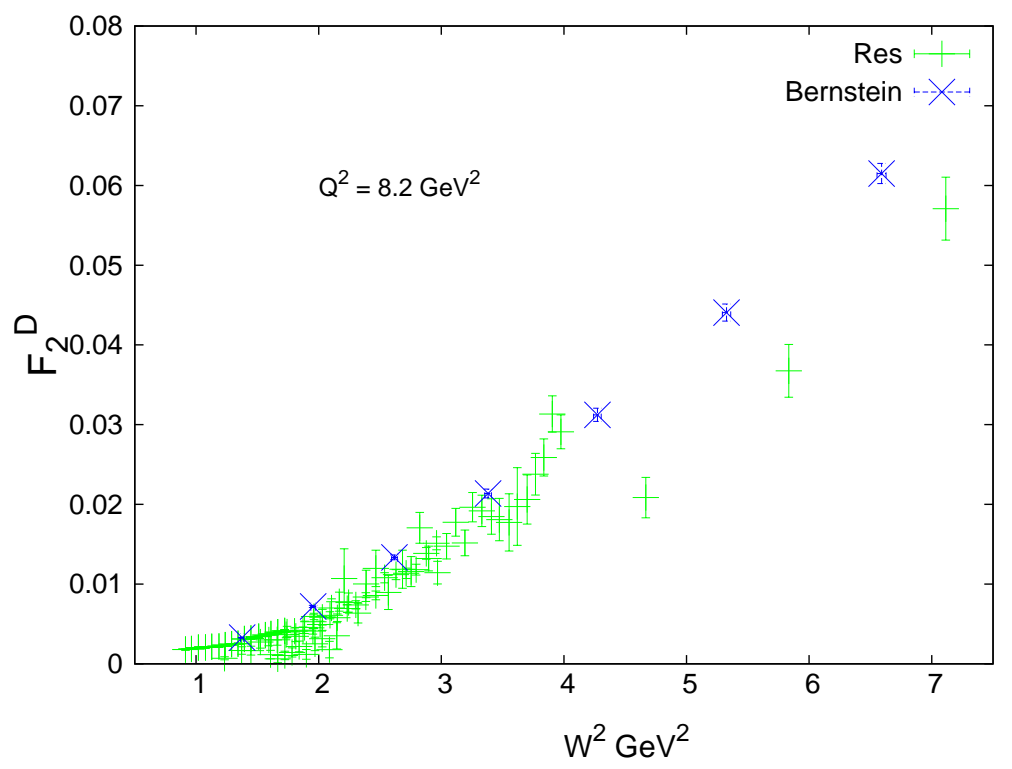

FiguRE 5.36: The $F_{2}^{D}$ values for the resonance region and the Bernstein moments are shown here. The green points are all of the $F_{2}^{D}$ resonance points in the $Q^{2}$ interval $(7.8: 8.8)$ and blue points are the Bernstein averages for those points in the $W^{2}$ range that constitutes the resonance region for $Q^{2}=8.2 \mathrm{GeV}^{2}$. 


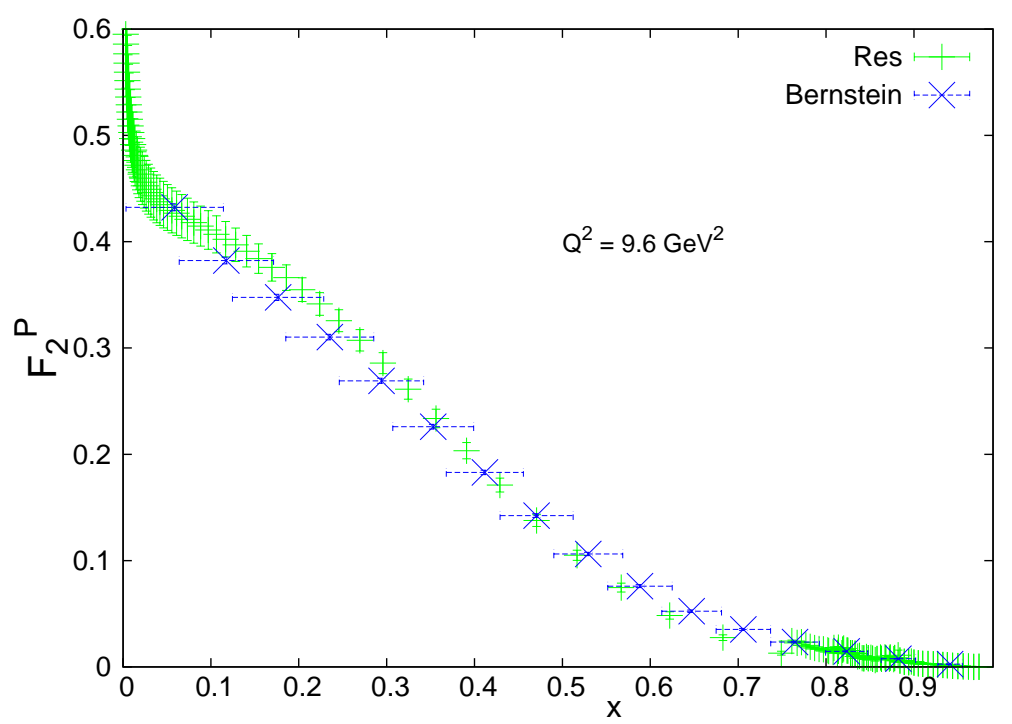

Figure 5.37: The $F_{2}^{P}$ values for the resonance region and the Bernstein moments are shown here. The green points are all of the $F_{2}^{P}$ resonance points in the $Q^{2}$ interval $(9.2: 10.2)$ and blue points are the Bernstein averages for those points in the $x$ range that constitutes the resonance region for $Q^{2}=9.6 \mathrm{GeV}^{2}$.

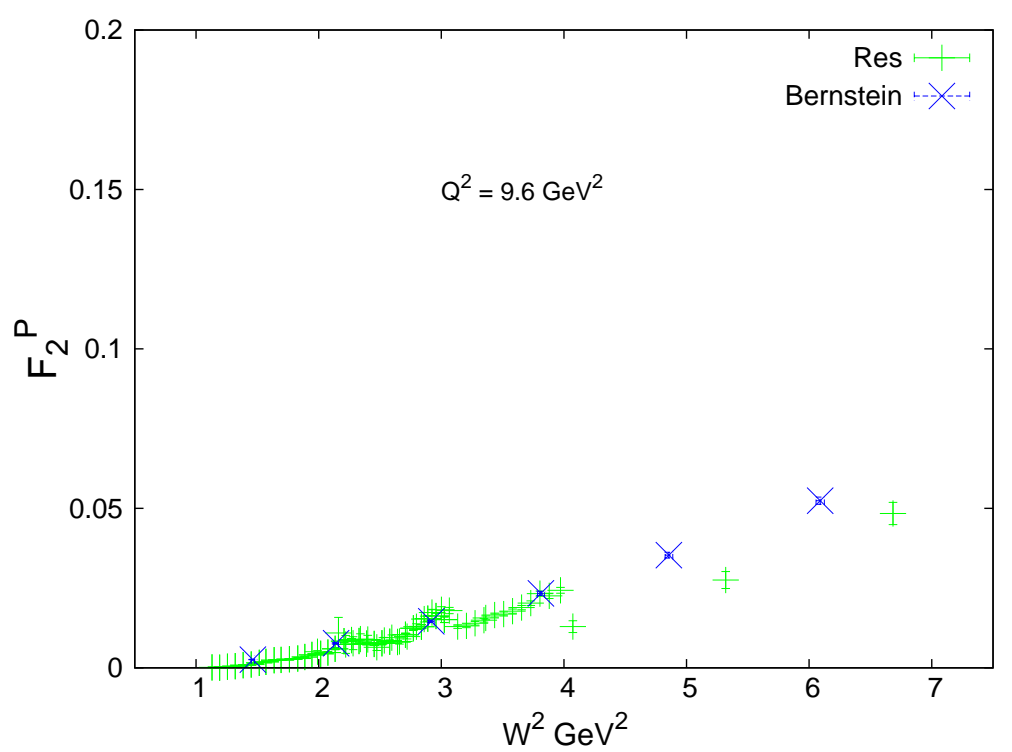

FIGURE 5.38: The $F_{2}^{P}$ values for the resonance region and the Bernstein moments are shown here. The green points are all of the $F_{2}^{P}$ resonance points in the $Q^{2}$ interval $(9.2: 10.2)$ and blue points are the Bernstein averages for those points in the $W^{2}$ range that constitutes the resonance region for $Q^{2}=9.6 \mathrm{GeV}^{2}$. 
TABle 5.19: Table of $Q^{2}=9.6 \mathrm{GeV}^{2} F_{2}^{P}$ Bernstein Moment Values. The Resonance Region here refers to $x$ values for which Resonance Data points exist.

\begin{tabular}{|c|c|c|c|c|c|c|}
\hline \hline$k$ & $x$ & $\Delta x$ & $F_{N, k}^{(e x p)}\left(Q^{2}\right)$ & $\Delta F_{N, k}^{(e r r)}\left(Q^{2}\right)$ & Res & DIS \\
\hline \hline 0.00 & 0.0588 & 0.0555 & 0.4324 & $0.3342 \mathrm{E}-02$ & 0.0000 & 1.0000 \\
1.00 & 0.1176 & 0.0537 & 0.3822 & $0.3138 \mathrm{E}-02$ & 0.0000 & 1.0000 \\
2.00 & 0.1765 & 0.0519 & 0.3476 & $0.2852 \mathrm{E}-02$ & 0.0000 & 1.0000 \\
3.00 & 0.2353 & 0.0500 & 0.3103 & $0.2598 \mathrm{E}-02$ & 0.0000 & 1.0000 \\
4.00 & 0.2941 & 0.0480 & 0.2692 & $0.2433 \mathrm{E}-02$ & 0.0000 & 1.0000 \\
5.00 & 0.3529 & 0.0460 & 0.2260 & $0.2271 \mathrm{E}-02$ & 0.0000 & 1.0000 \\
6.00 & 0.4118 & 0.0438 & 0.1829 & $0.2054 \mathrm{E}-02$ & 0.0001 & 0.9998 \\
7.00 & 0.4706 & 0.0416 & 0.1424 & $0.1803 \mathrm{E}-02$ & 0.0006 & 0.9990 \\
8.00 & 0.5294 & 0.0392 & 0.1062 & $0.1563 \mathrm{E}-02$ & 0.0032 & 0.9954 \\
9.00 & 0.5882 & 0.0367 & $0.7596 \mathrm{E}-01$ & $0.1348 \mathrm{E}-02$ & 0.0135 & 0.9817 \\
10.0 & 0.6471 & 0.0340 & $0.5240 \mathrm{E}-01$ & $0.1142 \mathrm{E}-02$ & 0.0461 & 0.9404 \\
11.0 & 0.7059 & 0.0310 & $0.3532 \mathrm{E}-01$ & $0.9180 \mathrm{E}-03$ & 0.1274 & 0.8437 \\
12.0 & 0.7647 & 0.0277 & $0.2336 \mathrm{E}-01$ & $0.6620 \mathrm{E}-03$ & 0.2779 & 0.6757 \\
13.0 & 0.8235 & 0.0240 & $0.1459 \mathrm{E}-01$ & $0.3960 \mathrm{E}-03$ & 0.4813 & 0.4641 \\
14.0 & 0.8824 & 0.0196 & $0.7691 \mathrm{E}-02$ & $0.1920 \mathrm{E}-03$ & 0.6866 & 0.2663 \\
15.0 & 0.9412 & 0.0139 & $0.2587 \mathrm{E}-02$ & $0.6800 \mathrm{E}-04$ & 0.8469 & 0.1233 \\
\hline \hline
\end{tabular}

TABle 5.20: Table of $Q^{2}=9.6 \mathrm{GeV}^{2} F_{2}^{D}$ Bernstein Moment Values. The Resonance Region here refers to $x$ values for which Resonance Data points exist.

\begin{tabular}{|c|c|c|c|c|c|c|}
\hline \hline$k$ & $x$ & $\Delta x$ & $F_{N, k}^{(e x p)}\left(Q^{2}\right)$ & $\Delta F_{N, k}^{(e r r)}\left(Q^{2}\right)$ & Res & DIS \\
\hline \hline 0.00 & 0.0588 & 0.0555 & 0.4128 & $0.3059 \mathrm{E}-02$ & 0.0000 & 1.0000 \\
1.00 & 0.1176 & 0.0537 & 0.3507 & $0.2901 \mathrm{E}-02$ & 0.0000 & 1.0000 \\
2.00 & 0.1765 & 0.0519 & 0.3080 & $0.2728 \mathrm{E}-02$ & 0.0000 & 1.0000 \\
3.00 & 0.2353 & 0.0500 & 0.2662 & $0.2542 \mathrm{E}-02$ & 0.0000 & 1.0000 \\
4.00 & 0.2941 & 0.0480 & 0.2243 & $0.2366 \mathrm{E}-02$ & 0.0000 & 1.0000 \\
5.00 & 0.3529 & 0.0460 & 0.1835 & $0.2154 \mathrm{E}-02$ & 0.0000 & 1.0000 \\
6.00 & 0.4118 & 0.0438 & 0.1452 & $0.1898 \mathrm{E}-02$ & 0.0001 & 0.9998 \\
7.00 & 0.4706 & 0.0416 & 0.1108 & $0.1641 \mathrm{E}-02$ & 0.0006 & 0.9991 \\
8.00 & 0.5294 & 0.0392 & $0.8132 \mathrm{E}-01$ & $0.1419 \mathrm{E}-02$ & 0.0031 & 0.9956 \\
9.00 & 0.5882 & 0.0367 & $0.5732 \mathrm{E}-01$ & $0.1232 \mathrm{E}-02$ & 0.0130 & 0.9825 \\
10.0 & 0.6471 & 0.0340 & $0.3905 \mathrm{E}-01$ & $0.1048 \mathrm{E}-02$ & 0.0451 & 0.9424 \\
11.0 & 0.7059 & 0.0310 & $0.2605 \mathrm{E}-01$ & $0.8430 \mathrm{E}-03$ & 0.1262 & 0.8467 \\
12.0 & 0.7647 & 0.0277 & $0.1713 \mathrm{E}-01$ & $0.6010 \mathrm{E}-03$ & 0.2788 & 0.6774 \\
13.0 & 0.8235 & 0.0240 & $0.1081 \mathrm{E}-01$ & $0.3440 \mathrm{E}-03$ & 0.4902 & 0.4587 \\
14.0 & 0.8824 & 0.0196 & $0.6078 \mathrm{E}-02$ & $0.1370 \mathrm{E}-03$ & 0.7127 & 0.2460 \\
15.0 & 0.9412 & 0.0139 & $0.2784 \mathrm{E}-02$ & $0.3800 \mathrm{E}-04$ & 0.8973 & 0.0837 \\
\hline \hline
\end{tabular}




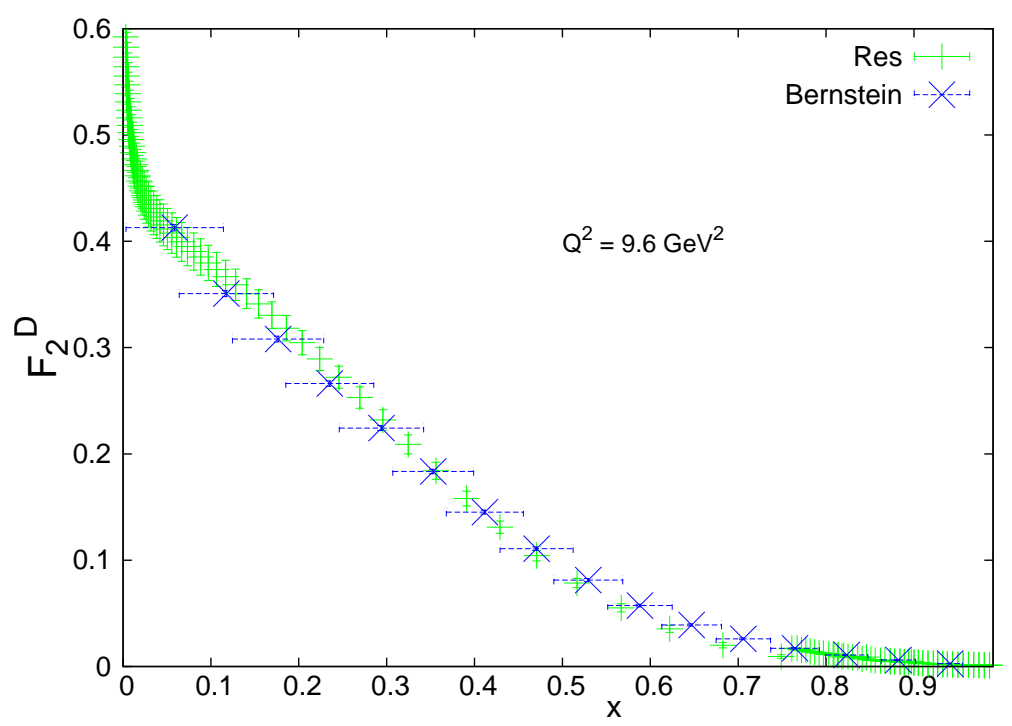

FiguRE 5.39: The $F_{2}^{D}$ values for the resonance region and the Bernstein moments are shown here. The green points are all of the $F_{2}^{D}$ resonance points in the $Q^{2}$ interval $(9.2: 10.2)$ and blue points are the Bernstein averages for those points in the $x$ range that constitutes the resonance region for $Q^{2}=9.6 \mathrm{GeV}^{2}$.

\subsection{Large x Fit SOM}

The SOM is particularly useful for extracting $\frac{d}{u}$ values from the ratio of the Deuteron Structure Functions to the Proton Structure Functions in the large $x$ limit, where $d$ is the total down quark distribution $d=d_{v}+2 \bar{d}$ and $u$ is the total up quark distribution $u=u_{v}+2 \bar{u} . \frac{d}{u}$ values, particularly at large $x$, are a notable example of how QCD methods, including Generalized Parton Distributions, and low energy non perturbative QCD models for nucleon spin structure and flavor can provide clear predictions [76]. The $u$ quark behavior at large $x$ is taken primarily from proton data and so therefore is largely independent of nuclear corrections; this allows us to use $\frac{d}{u}$ to extract $d$ quark values. Currently, the overall behavior of $\frac{d}{u}$ for $x \geq 0.2$ is not well known despite the theoretical models that have been made for this function as $x \rightarrow 1$. In addition, measurements of the $d$ quark and gluon PDFs at large $x$, the former of which the $\frac{d}{u}$ determinations can assist in, are also needed to compute QCD cross sections in collider experiments. The large $x$ effects discussed previously, the TMC, LxR and smearing factors, further complicate the extraction of the $\frac{d}{u}$ behavior at large $x$ from the structure functions. In addition, nuclear corrections in the extraction of neutron structure functions lead to uncertainties 


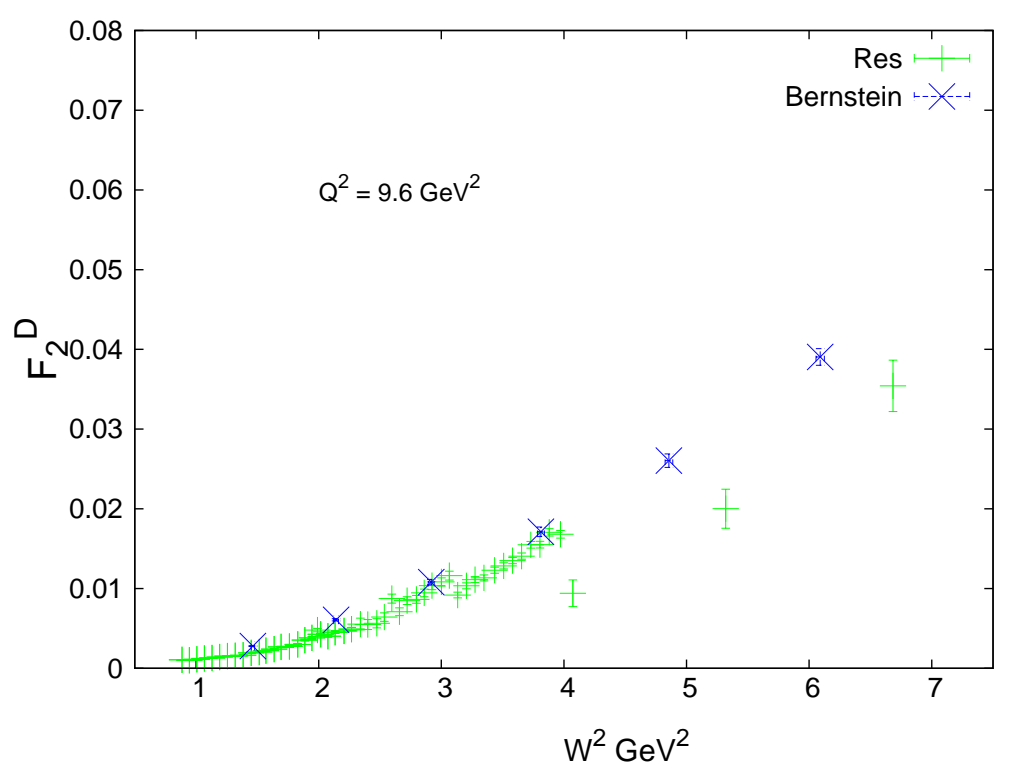

FiguRE 5.40: The $F_{2}^{D}$ values for the resonance region and the Bernstein moments are shown here. The green points are all of the $F_{2}^{D}$ resonance points in the $Q^{2}$ interval $(9.2: 10.2)$ and blue points are the Bernstein averages for those points in the $W^{2}$ range that constitutes the resonance region for $Q^{2}=9.6 \mathrm{GeV}^{2}$.

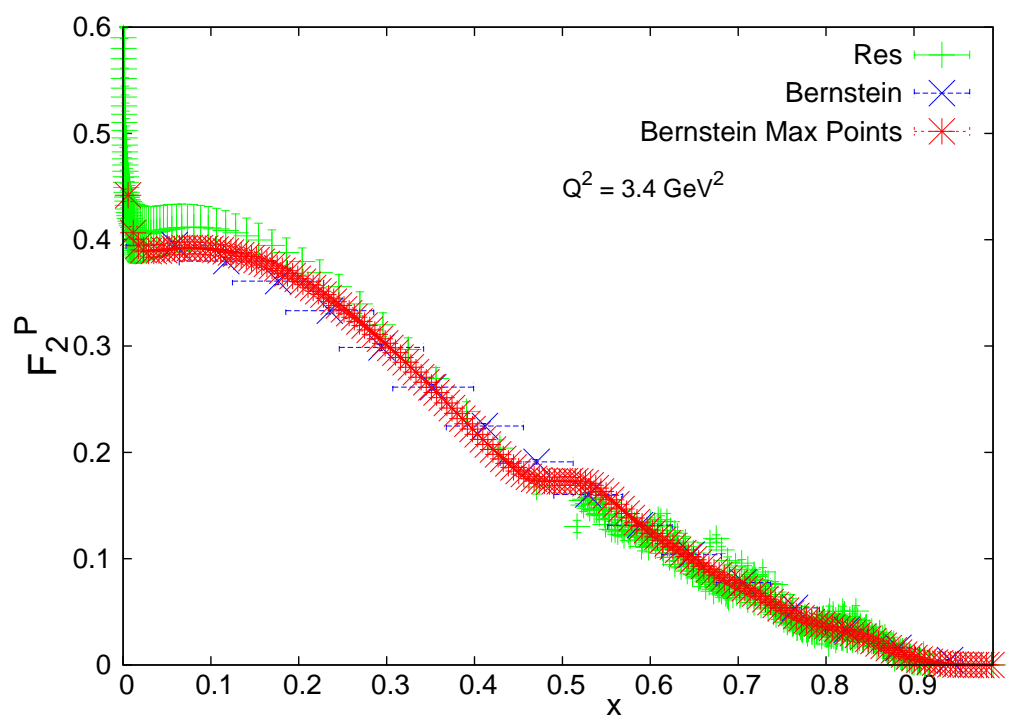

Figure 5.41: The $F_{2}^{P}$ values for the resonance region and the Bernstein moments are shown here along with a computation of 169 Bernstein resonance points to show how the Bernstein functions behave for larger numbers of chosen resonance points. 


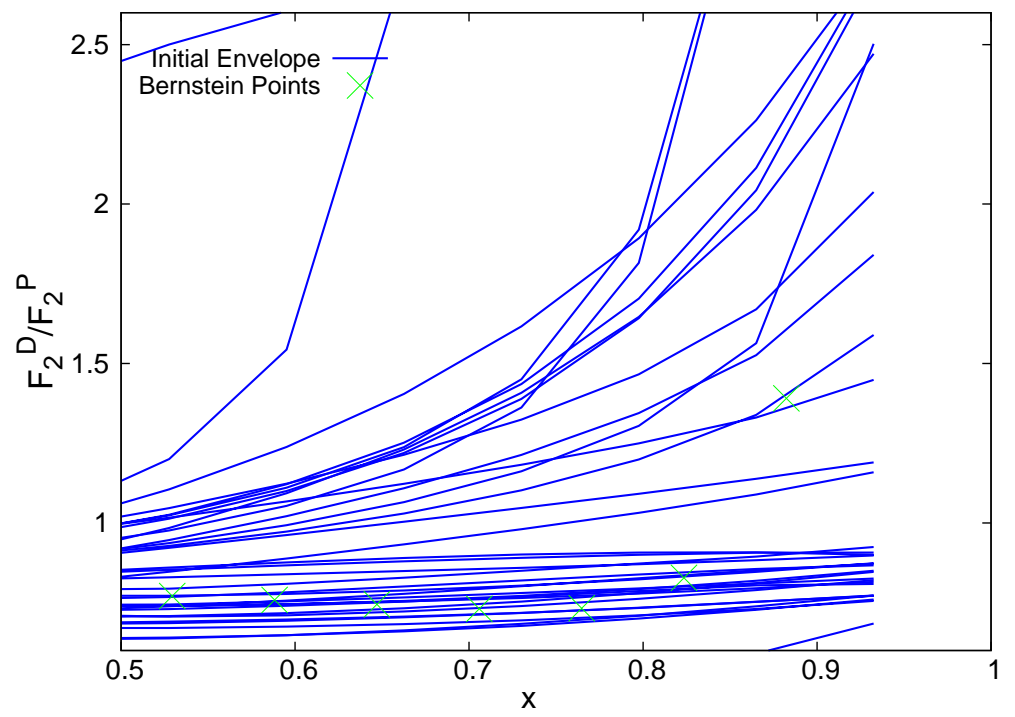

Figure 5.42: The initial generated envelope of $\frac{F_{2}^{D}}{F_{2}^{P}}$ theoretical curves for $Q^{2}=2.5$ $\mathrm{GeV}^{2}$ relative to the Bernstein moment points for the same $Q^{2}$ value. The blue curves are the initial envelope of theoretical generated $\frac{F_{2}^{D}}{F_{2}^{P}}$ curves. The green points are the computed $\frac{F_{2}^{D}}{F_{2}^{P}}$ values taken from the Bernstein moment integrals of resonance $\frac{F_{2}^{D}}{\text { and }}$ $\underline{F_{2}^{P}}$

data. This shows the ability of the SOMPDF procedure to generate unbiased theoretical fits to experimental scattering sets when the ratios of Deuteron to Proton structure functions are plotted as opposed to the Deuteron and Proton structure functions separately.

in $d$ quark calculations [77]. The BONUS collaboration has used tagging measurements to reduce the uncertainty due to these corrections for $x \leq 0.7$ [78]; however more work is needed to understand the uncertainty within the resonance region. The SOM is ideal for using pattern recognition among clusters that form from mapping the PDFs in order to identify the physics behind the large $x \frac{d}{u}$ behavior. These SOM $\chi^{2}$ and $\frac{d}{u}$ values are shown on a lego plot in Figure 5.45 - 5.46 .

The SOM for the ratio of the total up and down quarks has been done with and without the combined TMC and LxR. The map of $\chi^{2}$ values without large $x$ Corrections is in Figure 5.44 and the $\chi^{2}$ with these large $x$ corrections is in Figure 5.45. For the Maps of $\frac{d}{u}$ values, Figure 5.46 shows them without the large $x$ Corrections and Figure 5.47 shows this map with the large $x$ Corrections. The results can be analyzed in terms of the size and the location of the clusters for which, in the limit $x \rightarrow 1.0, \frac{d}{u} \rightarrow 0$ or $\frac{d}{u}$ 


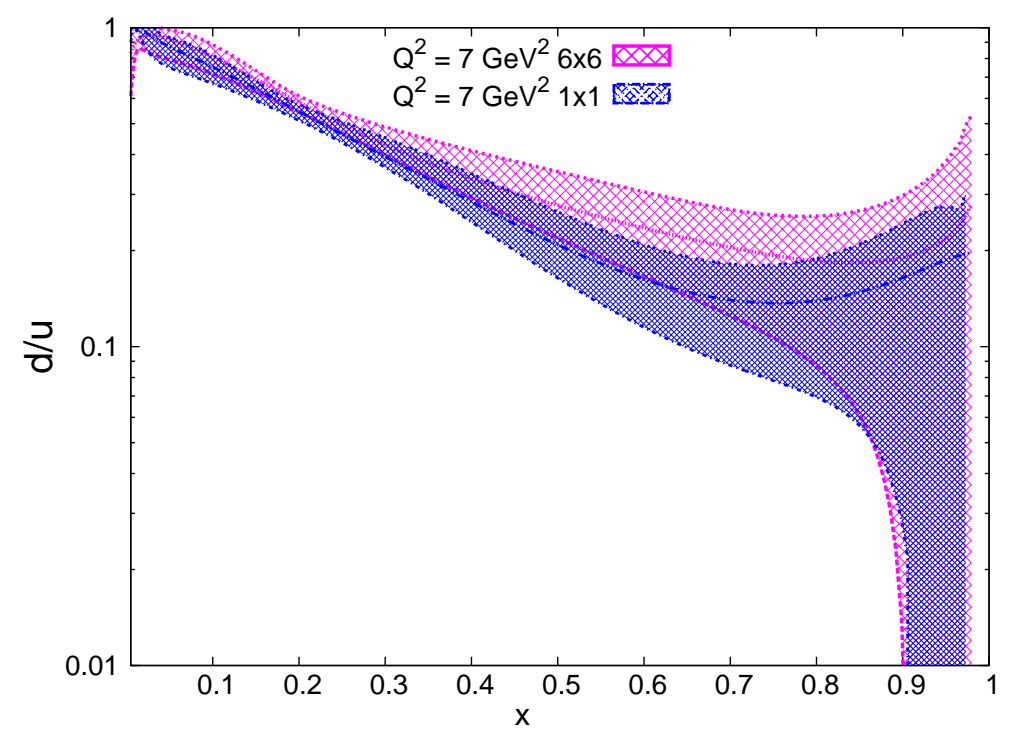

Figure 5.43: This is a generated best fitting $\frac{d}{u}$ curve fitting generated $\frac{F_{2}^{D}}{F_{2}^{P}}$ values to the large $x$ data sets.

is in the range $(0.05: 0.5)$ and additionally for the clusters for which $\frac{d}{u}$ becomes close to one. For quarks in a proton or neutron, $S U(6)$ symmetry predicts that the quarks will exhibit flavor, or $S U(3)$ and spin, or $S U(2)$, symmetry in terms of the interactions among the quarks. For the large $x$ region, $S U(6)$ symmetry can be broken; if the spin of the quark pairs in the hadron is zero this refers to the $S=0$ qq model and if the spin projection of quark pairs is zero then this refers to the $S_{z}=0$ qq model. For a proton, $S U(6)$ symmetry predicts two up quarks for one down quark and so the $\frac{d}{u}$ limit is predicted to be 0.5 . The $S=0$ qq model predicts a limit of zero and the $S_{z}=0$ qq model predicts a limit of 0.2. The quark hadron duality model predicts a limit of roughly 0.42 and if $\frac{d}{u}>>1$ for large $x$ that corresponds to a heavy prevalence of down quarks over up quarks. The clusters for $\frac{d}{u} \rightarrow 0$ for one or both dimensions correspond to the $S=0$ qq dominated limit for large $x$.

The comparison of these clusters can inform us about the strength of the effects of the TMC and LxR. Without the large $x$ corrections, large clusters of data units where $\frac{d}{u}$ is close to zero form throughout the map. For the $\frac{d}{u}$ data unit clusters, with the large $x$ corrections the clusters become more distinct in the upper left corner for $\frac{d}{u}$ values in the range $(0.05: 0.5)$ and in the center for $\frac{d}{u}$ values greater than one. Essentially, clusters of data units for which the $\frac{d}{u}$ values are greater than zero but less than one 


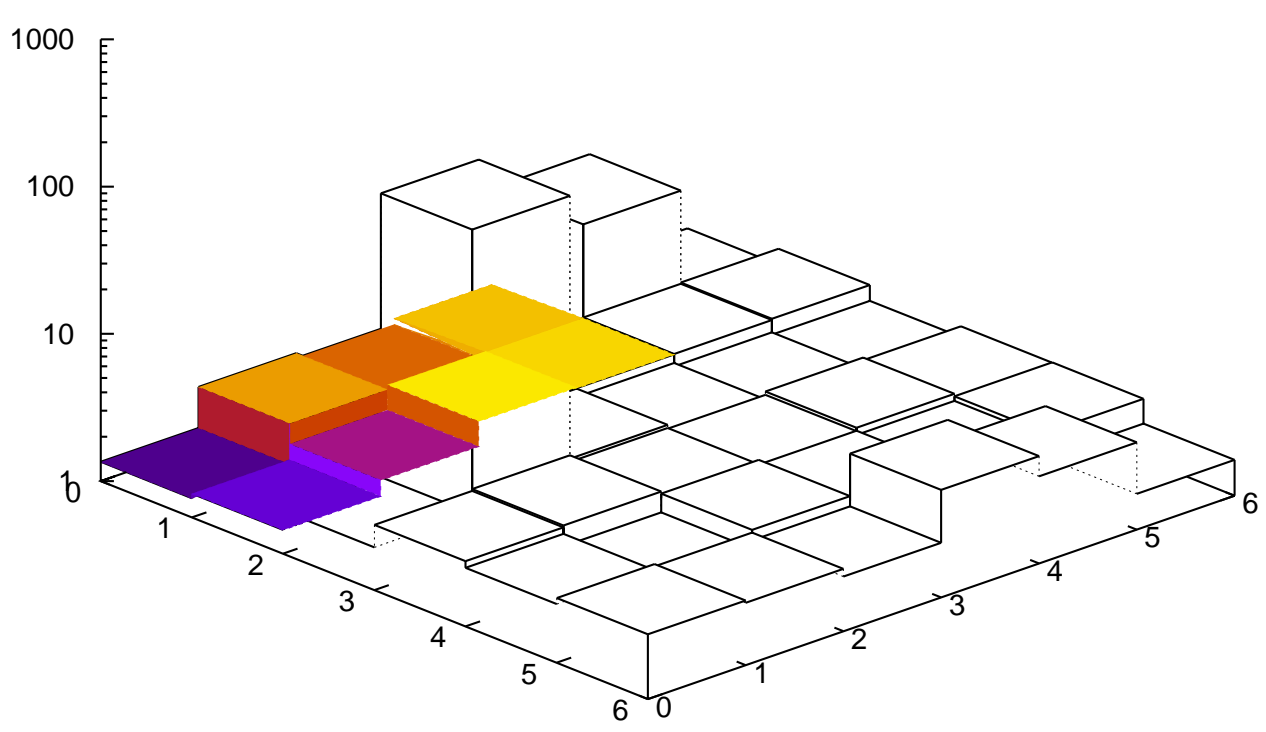

Figure 5.44: A $\chi^{2}$ lego plot for a $6 \times 6$ map is shown without Target Mass Corrections and large $x$ Resummations added for $Q^{2}=2.5 \mathrm{GeV}^{2}$. An example of a cluster of neighboring $\chi^{2}$ units is shown in the colored region.

form to an extent that does not occur without large $x$ Corrections. Therefore, the large $x$ Corrections leading to the PDF fitting procedure divide the $\frac{d}{u}$ neural network units into new patterns of fundamentally different values as opposed to the more uniform map of very low $\frac{d}{u}$ values without the large $x$ corrections. This shows that the large $x$ corrections lead to distinct sets of possible values for the prevalence of up and down quarks with large hadron momentum fractions that are not present when the corrections are not applied.

The SOM can also be used to show correlations between the individual PDFs and the Structure Functions they comprise. Previous attempts to show the correlations among the different PDFs are in [79]. This can be done by observing the relationships between the $\chi^{2}$ clusters and any clusters that form for any of the individual PDFs during the training process. Without the TMC and LxR, the $\chi^{2}$ map shows clustering of the 


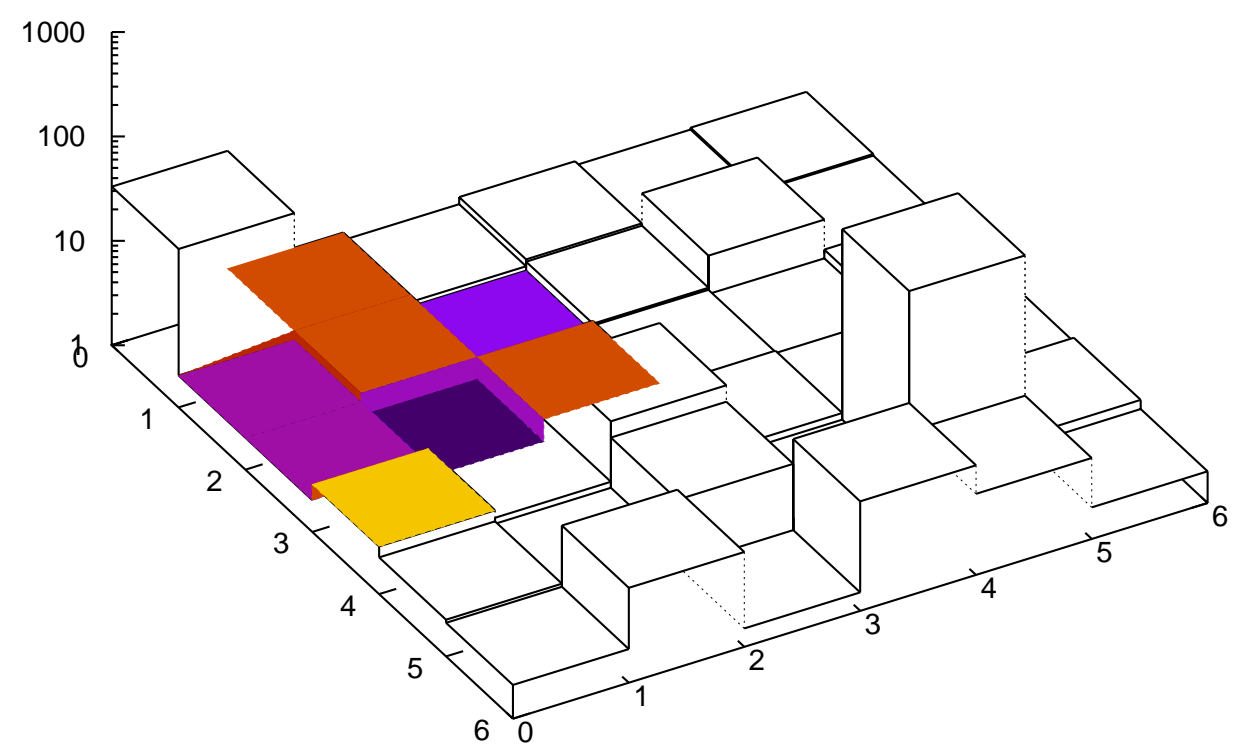

Figure 5.45: A $\chi^{2}$ lego plot for a $6 \times 6$ map is shown with Target Mass Corrections and large $x$ Resummations added for $Q^{2}=2.5 \mathrm{GeV}^{2}$. An example of a cluster of neighboring $\chi^{2}$ units is shown in the colored region.

Structure functions with the lowest $\chi^{2}$ value in the lower left hand corner, around the map vector with the lowest $\chi^{2}$ value. With the TMC and LxR added, the $\chi^{2}$ map illustrates a cluster of functions around the map vector with the smallest $\chi^{2}$ value, which is located in the first entry in the fourth row. Therefore, cluster formation around the map vector unit with the lowest $\chi^{2}$ value occurred regardless of the large $x$ conditions implemented. With the large $x$ corrections, however, more distinct clusters around the larger $\chi^{2}$ values form in the lower left and right hand corners and in the upper right hand corner. 


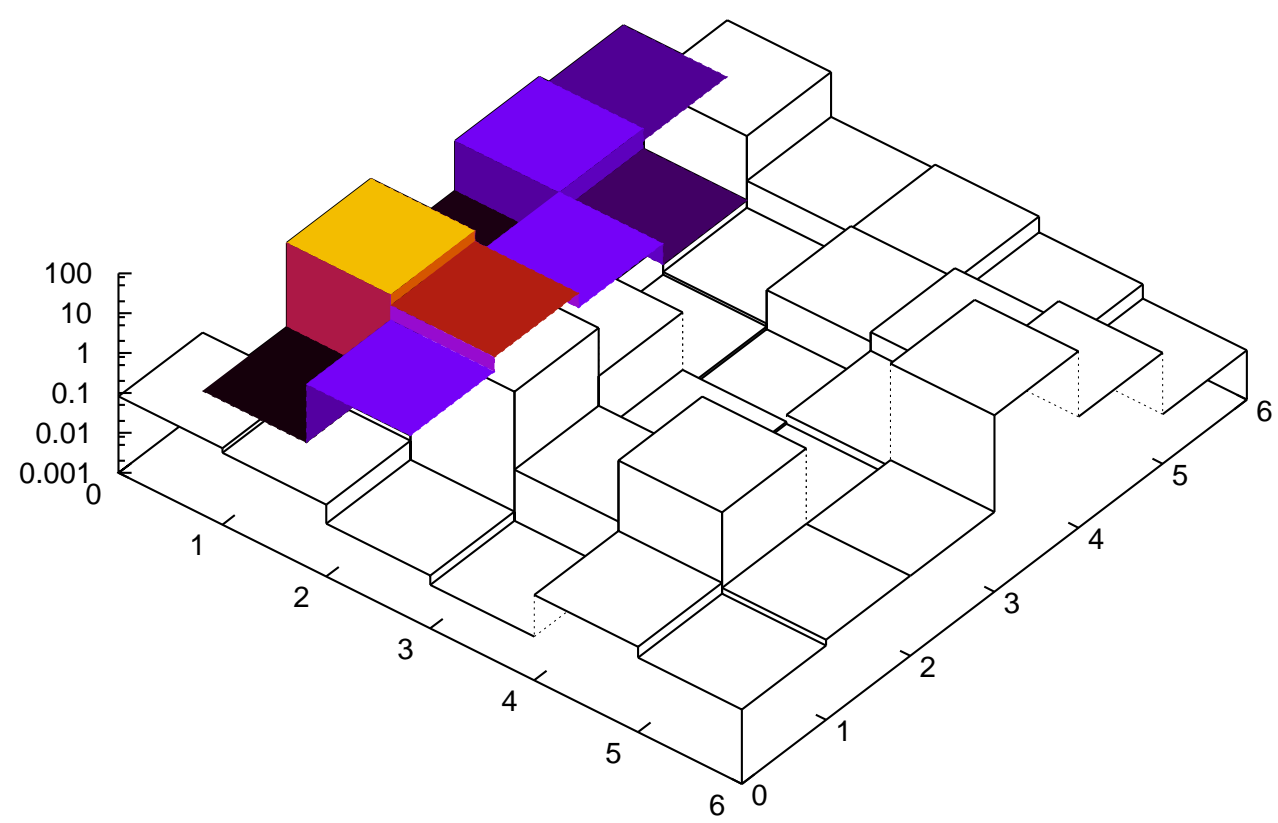

Figure 5.46: A $\frac{d}{u}$ lego plot for a $6 \times 6$ map is shown without Target Mass Corrections and large $x$ Resummations added for $Q^{2}=2.5 \mathrm{GeV}^{2}$. An example of a cluster of neighboring $\frac{d}{u}$ units is shown in the colored region.

\subsection{Large $\mathrm{x} \frac{d}{u}$ Cluster Analysis}

The clusters for the full plots of $\frac{d}{u}$ are shown in Figure 5.48 - 5.49. The data units, representing plots of $\frac{d}{u}$, can be classified according to the quark models the represent in the limit $x \rightarrow 1$. This enables us to take full advantage of the ability of the SOM to group neural network units into easily visualized two dimensional representations and identify key common futures of PDFs in these data network units. The clusters for $\frac{d}{u}$ are organized according to the possible limits predicted by various conditions outlined previously.

Without TMC and LxR added, the range of $d / u$ values is approximately the same as the range with the corrections added. As a result of this, the clustering and classification of the data units based on appropriate quark models occurs with the same possible values 


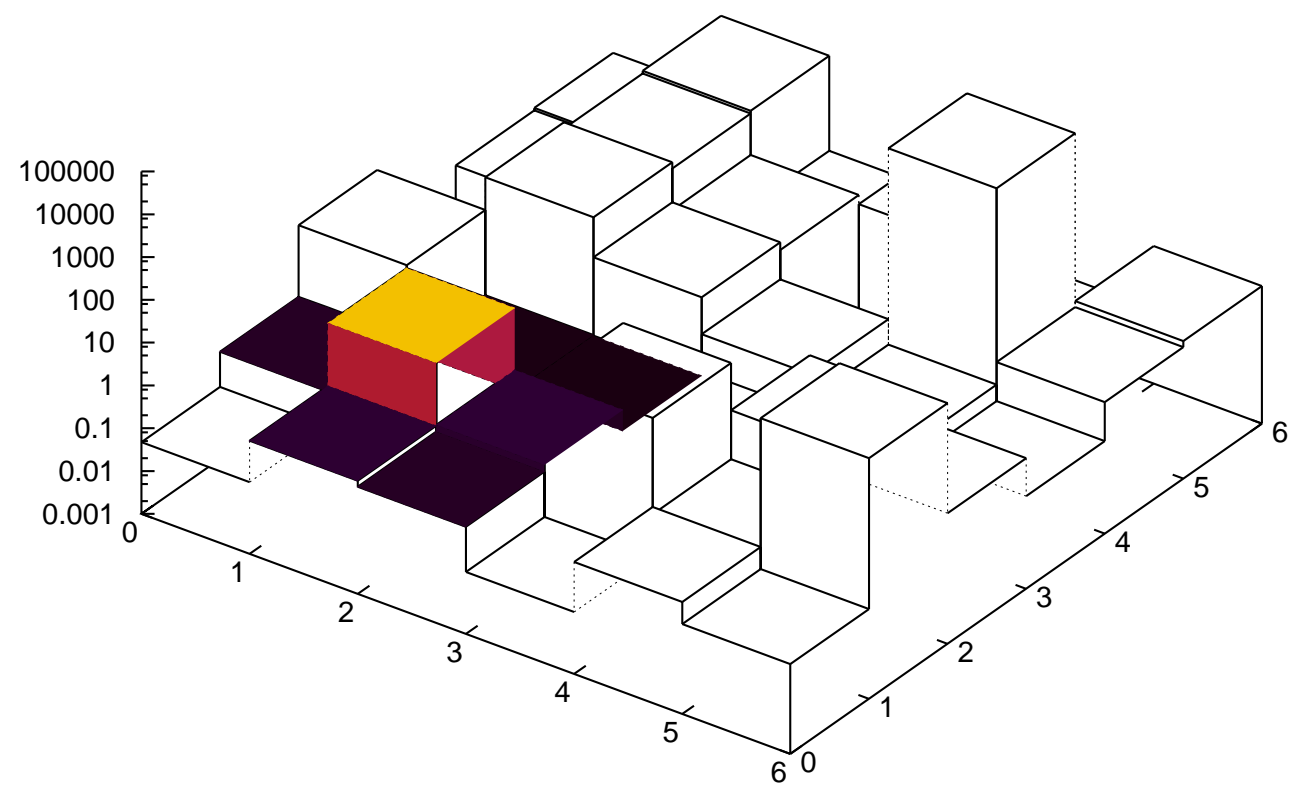

Figure 5.47: A $\frac{d}{u}$ lego plot for a $6 \times 6$ map is shown with Target Mass Corrections and large $x$ Resummations added for $Q^{2}=2.5 \mathrm{GeV}^{2}$. An example of a cluster of neighboring $\frac{d}{u}$ units is shown in the colored region.

of data units for both conditions. For the map vectors without TMC and LxR added, shown in Figure 5.48, the $d / u$ value map shows clustering of the upper left and right corners as well as in the center for the lowest $d / u$ values. For the map vectors with TMC and LxR added, shown in Figure 5.49 , clusters of $d / u>>1$ data units form along the upper right hand corner. In the left side of the map, clusters data units corresponding to the $S=0 \mathrm{qq}, S_{z}=0 \mathrm{qq}$ and quark hadron duality conditions form around data units with a $d / u>>1$ limit. With large $x$ corrections added, the number of PDF units corresponding to the $S_{z}=0 \mathrm{qq}$ and quark hadron duality conditions also increases; there are $14 \mathrm{PDF}$ data units corresponding to one of these two quark models when large $x$ corrections are added and only 8 PDF data units corresponding to these conditions without large $x$ Corrections. This reveals the capability of the SOM and the GA, in conjunction with the large $x$ corrections, to produce neighborhood regions of PDFs that 

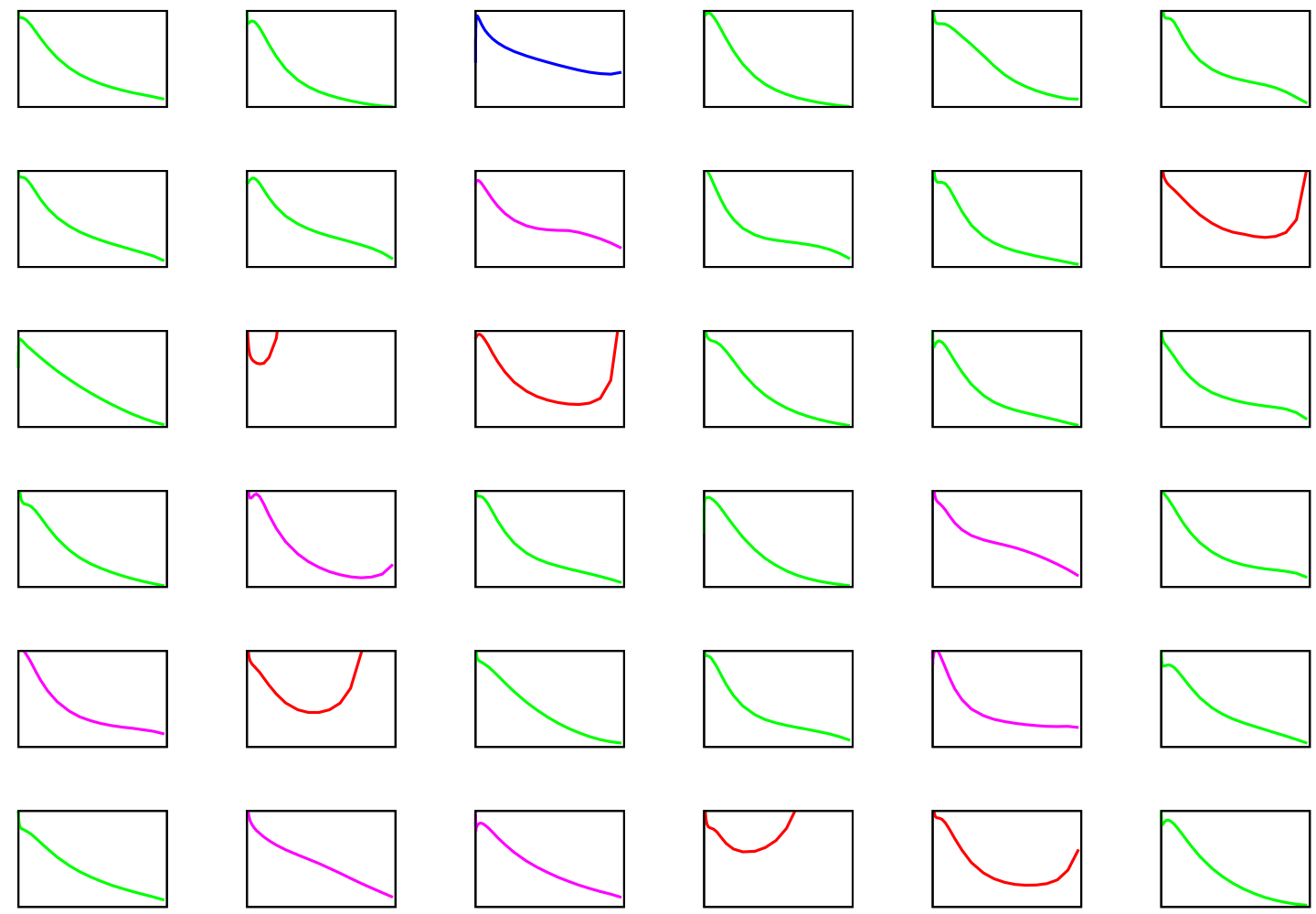

Figure 5.48: Shown is a plot of $\frac{d}{u}$ functions, without Target Mass Corrections and large $x$ Resummations added for $Q^{2}=2.5 \mathrm{GeV}^{2}$, for the Bjorken $x$ range (the $\mathrm{x}$ axis) $(0: 0.95)$ and the $\frac{d}{u}$ range (y axis) $(0: 1.0)$. The curves are colored according to the $\frac{d}{u}$ values in the limit $x \rightarrow 1$. The red curves represent $\frac{d}{u}$ functions such that $\frac{d}{u} \rightarrow \frac{1}{2}$, which approximates to $S U(6)$ symmetry, or $\frac{d}{u}>0.5$. The green curves represent $\frac{d}{u}$ functions such that $\frac{d}{u} \rightarrow 0$, which approximates to $S=0$ qq model. The purple curves represent $\frac{d}{u}$ functions such that $\frac{d}{u} \rightarrow \frac{1}{5}$, which approximates to $S_{z}=0$ qq model. The blue curves represent $\frac{d}{u}$ functions such that $\frac{d}{u} \rightarrow 0.42$, which approximates to quark hadron duality.

conform to specific physical models.

The clustering for the full $d / u \mathrm{PDF}$ ratios illustrates how the fitting procedure influences their formation; the size of these clusters with and without TMC and LxR can also aid in visualizing the strength of the large $x$ corrections in PDF formation. The size of the cluster of $d / u$ values less than 0.2 with TMC and LxR is zero map units. Without the TMC and LxR, the largest cluster for the lowest $d / u$ values is 15 map vector units in size. Therefore, for data units $d / u$ values less than 0.2 , adding large $x$ corrections eliminates clusters of this data type, although clusters corresponding to $d / u$ values less than 0.5 

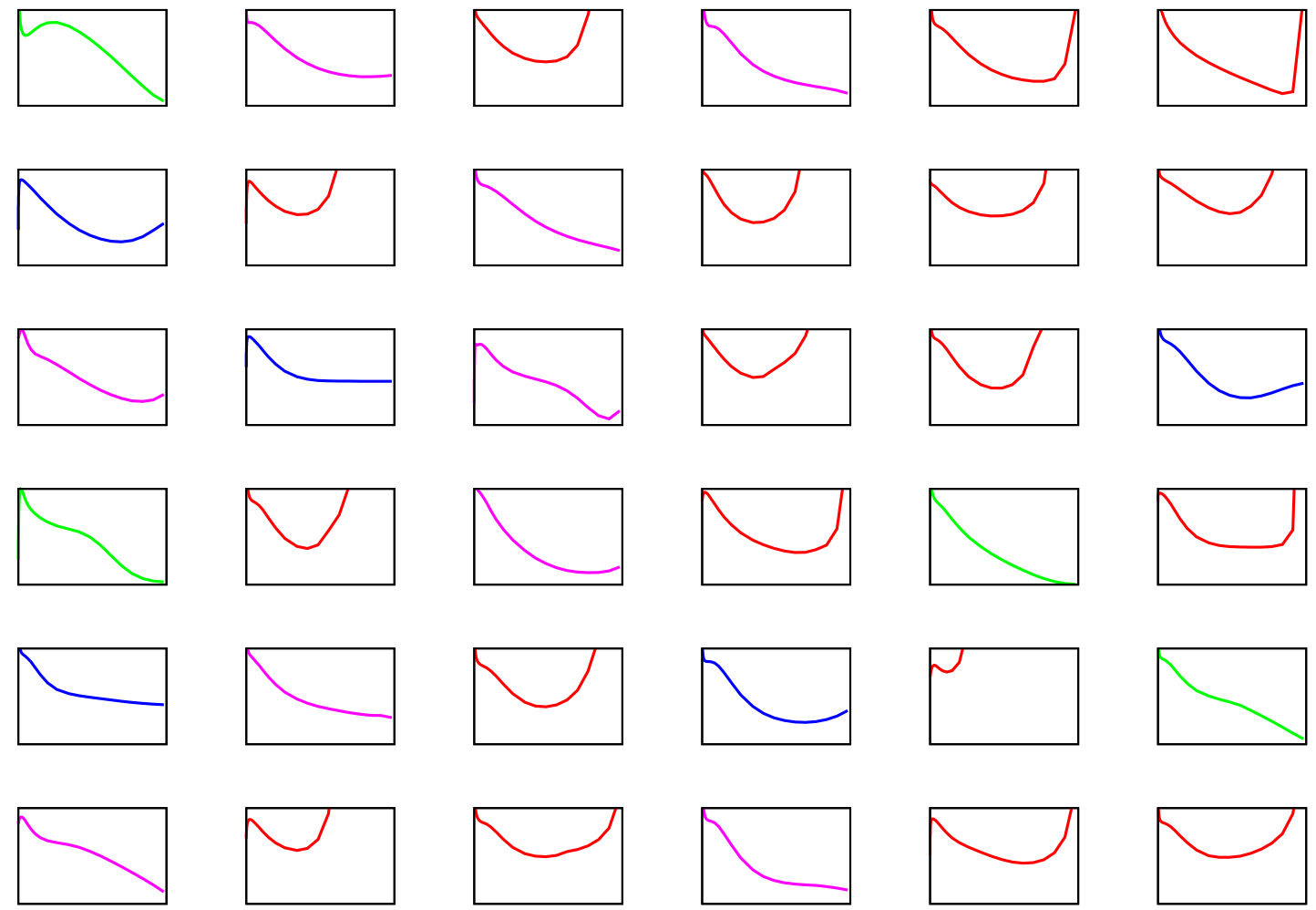

Figure 5.49: Shown is a plot of $\frac{d}{u}$ functions, with Target Mass Corrections and large $x$ Resummations added for $Q^{2}=2.5 \mathrm{GeV}^{2}$, for the Bjorken $x$ range (the x axis) $(0: 0.95)$ and the $\frac{d}{u}$ range (y axis) $(0: 1.0)$. The curves are colored according to the $\frac{d}{u}$ values in the limit $x \rightarrow 1$. The red curves represent $\frac{d}{u}$ functions such that $\frac{d}{u} \rightarrow \frac{1}{2}$, which approximates to $S U(6)$ symmetry, or $\frac{d}{u}>0.5$. The green curves represent $\frac{d}{u}$ functions such that $\frac{d}{u} \rightarrow 0$, which approximates to the $S=0$ qq model. The purple curves represent $\frac{d}{u}$ functions such that $\frac{d}{u} \rightarrow \frac{1}{5}$, which approximates to the $S_{z}=0$ qq model. The blue curves represent $\frac{d}{u}$ functions such that $\frac{d}{u} \rightarrow 0.42$, which approximates to quark hadron duality.

are still visible. In addition, in terms of the total number of map vector units with a $d / u$ value greater than 0.47 , regardless of cluster formation, there are 6 such units on the $d / u$ grid without large $x$ corrections added where as there are 18 such units on the grid with the large $x$ corrections added. This helps to determine the effects of the TMC and LxR on the ranges of $d / u$ ratios. Furthermore, the size of the clusters for various $d / u$ values with the large $x$ corrections relative to the size of the clusters without such large $x$ corrections enables us to visualize how adding the large $x$ corrections directly affects PDF formation throughout the GA procedure. In particular, it reveals that when large $x$ 

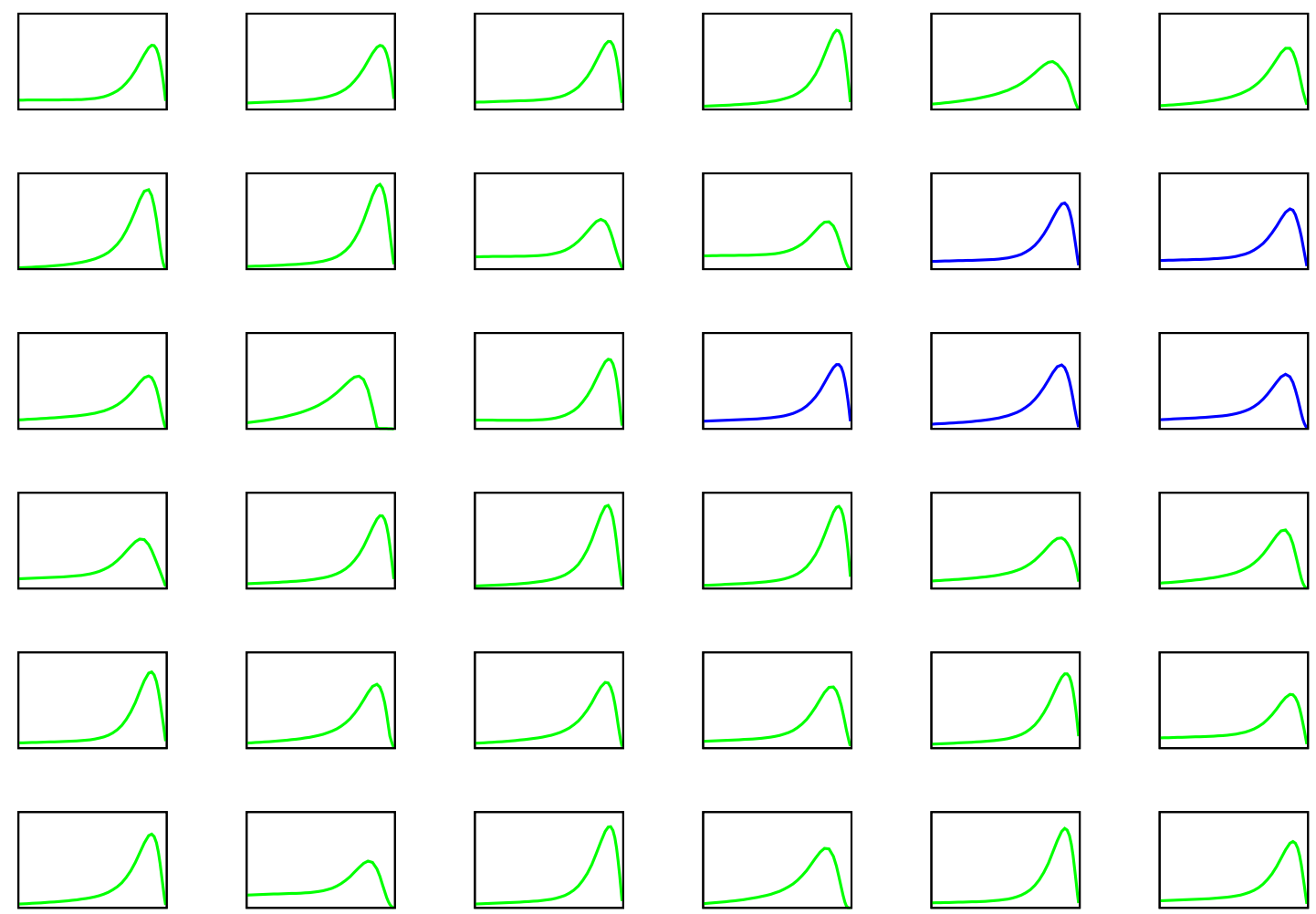

FiguRe 5.50: Shown are plots of $u_{v}$ PDFs without large $x$ Corrections added for $Q^{2}=2.5 \mathrm{GeV}^{2}$. The Bjorken $x$ range (the $\mathrm{x}$ axis) is $(0.0001: 1)$ and the $u_{v}$ range (the $\mathrm{y}$ axis $)$ is $(0: 1)$. The PDF curves are in green with the clusters, identified based on visualization of PDFs with common behaviors for large and small $x$ regions, shown as blue curves.

corrections are added, $d / u$ data units are split between those corresponding to $S=0 \mathrm{qq}$, $S_{z}=0$ qq and quark hadron duality conditions and units where down quarks with large momentum fractions are far more prevalent than up quarks. $d / u$ values do not form large clusters corresponding to the $S=0$ qq condition the way they do without large $x$ corrections. This suggests that when large $x$ corrections are added, multiple types of physics conditions, including a heavy prevalence of down quarks over up quarks, are possible when the PDFs are run through the SOM network to fit scattering data. Adding the large $x$ corrections also results in the creation of patterns of data units following the $S_{z}=0 \mathrm{qq}$ and quark hadron duality models that are not present when these corrections are not added. Subsequently, the maps show the necessity of adding TMC and LxR along with nuclear corrections in order to maximize the ability of the SOMPDF code to 

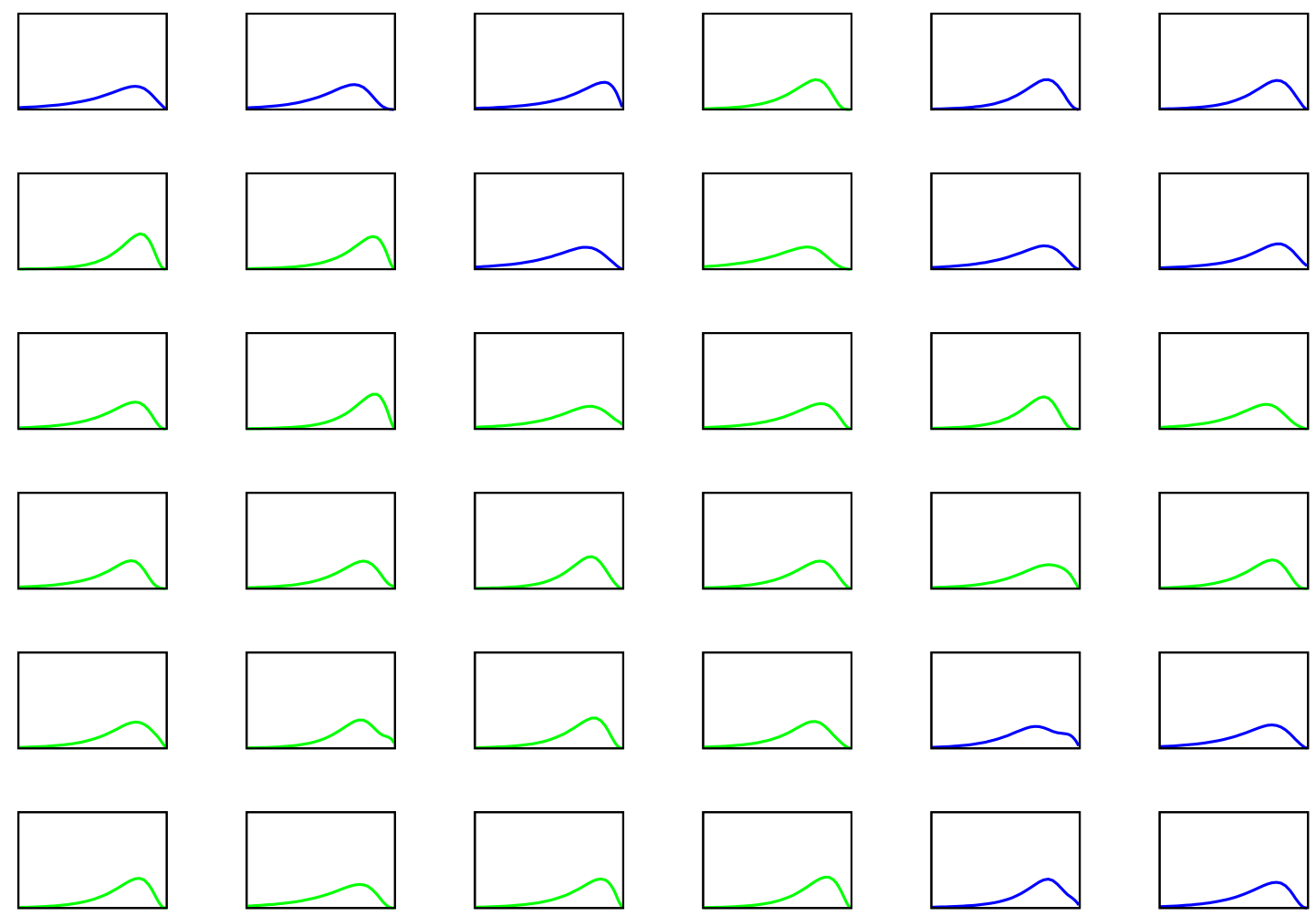

Figure 5.51: Shown are plots of $d_{v}$ PDFs without large $x$ Corrections added for $Q^{2}=2.5 \mathrm{GeV}^{2}$. The Bjorken $x$ range (the $\mathrm{x}$ axis) is $(0.0001: 1)$ and the $d_{v}$ range (the $\mathrm{y}$ axis $)$ is $(0: 1)$. The PDF curves are in green with the clusters, identified based on visualization of PDFs with common behaviors for large and small $x$ regions, shown as blue curves.

achieve these models.

\subsection{Large x quark and gluon Cluster Analysis}

The map vector grids for the valence quark, sea quark and gluon PDFs are shown without large $x$ Corrections in Figure 5.50 - 5.56. Without the large $x$ Corrections, the $u_{v}$ map is in Figure 5.50, the $d_{v}$ map is in Figure 5.51, the $u$ quark map is in Figure 5.52, the $d$ quark map is in Figure 5.53, the gluon Map is in Figure 5.54, the strange map is in Figure 5.55 and the charm map is in Figure 5.56. When there is no TMC or LxR factored in, The $u_{v}$ vectors form clusters based on their similar behavior for small and large $x$ values in the upper right hand corner. The $d_{v}$ vectors form clusters based 

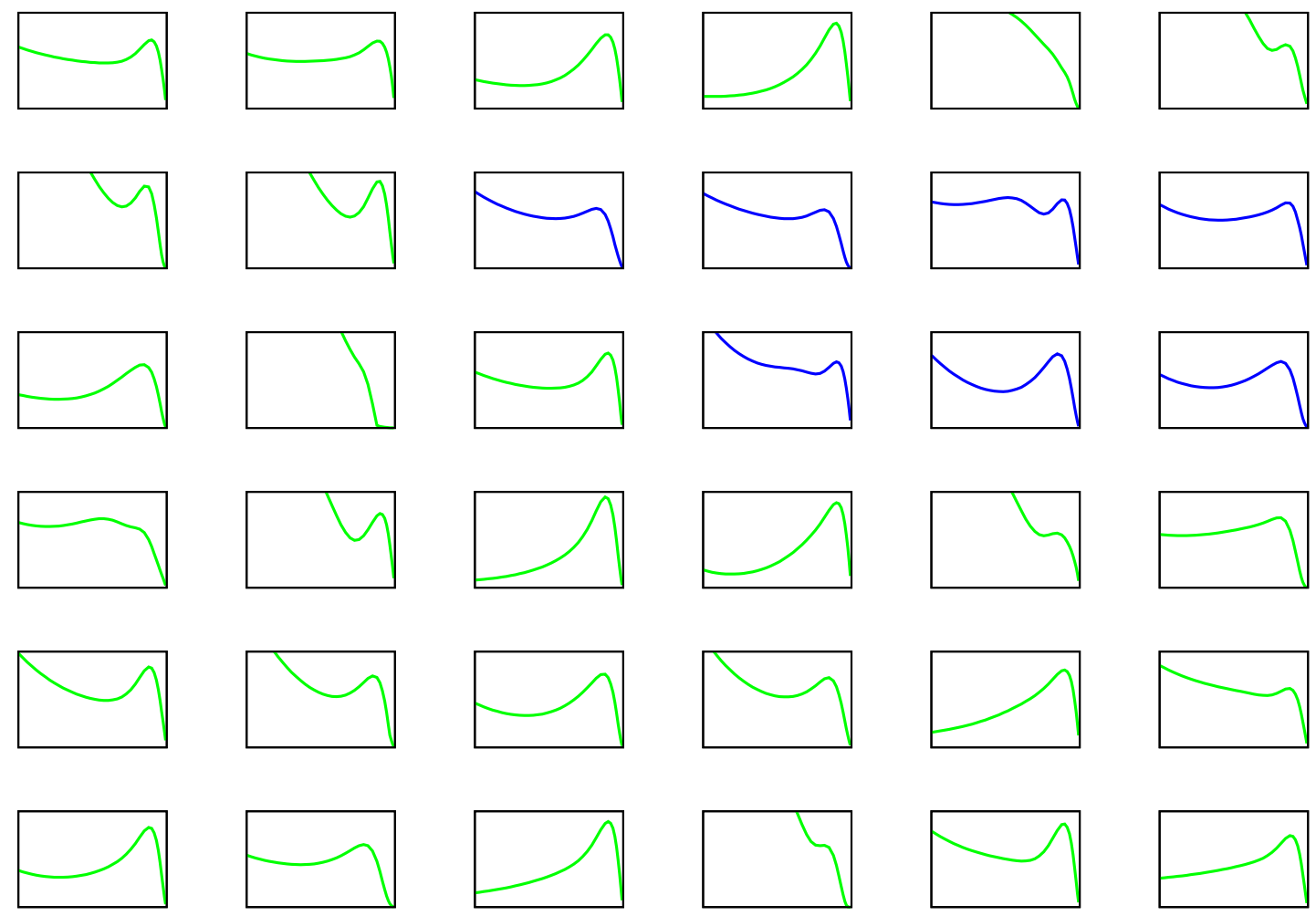

FiguRe 5.52: Shown are plots of $u=u_{v}+2 \bar{u}$, or up quark, PDFs without large $x$ Corrections added for $Q^{2}=2.5 \mathrm{GeV}^{2}$. The Bjorken $x$ range (the x axis) is $(0.0001: 1)$ and the $u$ range (the y axis) is $(0: 1)$. The PDF curves are in green with the clusters, identified based on visualization of PDFs with common behaviors for large and small $x$ regions, shown as blue curves.

on similar relations in $x$ behavior in the upper and lower right corner and upper left corner. The $u$ quark map vector grid shows clusters of PDFs based on similar behaviors for small and large $x$ in the upper half of the map. The $d$ quark map vector grid showed a clustering of the vectors in the upper right corner for small and large $x$ values as well as a clustering in the lower left corner based on PDF behavior for various $x$ values. The relationship between clusters of the gluon map vector grid and the $d$ quark vector grid can be seen as well. The gluon PDFs in fact cluster in the same regions as the $d$ quarks. The strange quark map vector grid shows clusters based on the PDF behavior in the upper right and lower left corner as well. The charm quark map vector grid additionally shows clustering in the same regions as the strange and gluon PDFs. However the range of charm quark values is substantially smaller than for the map vectors of the other 

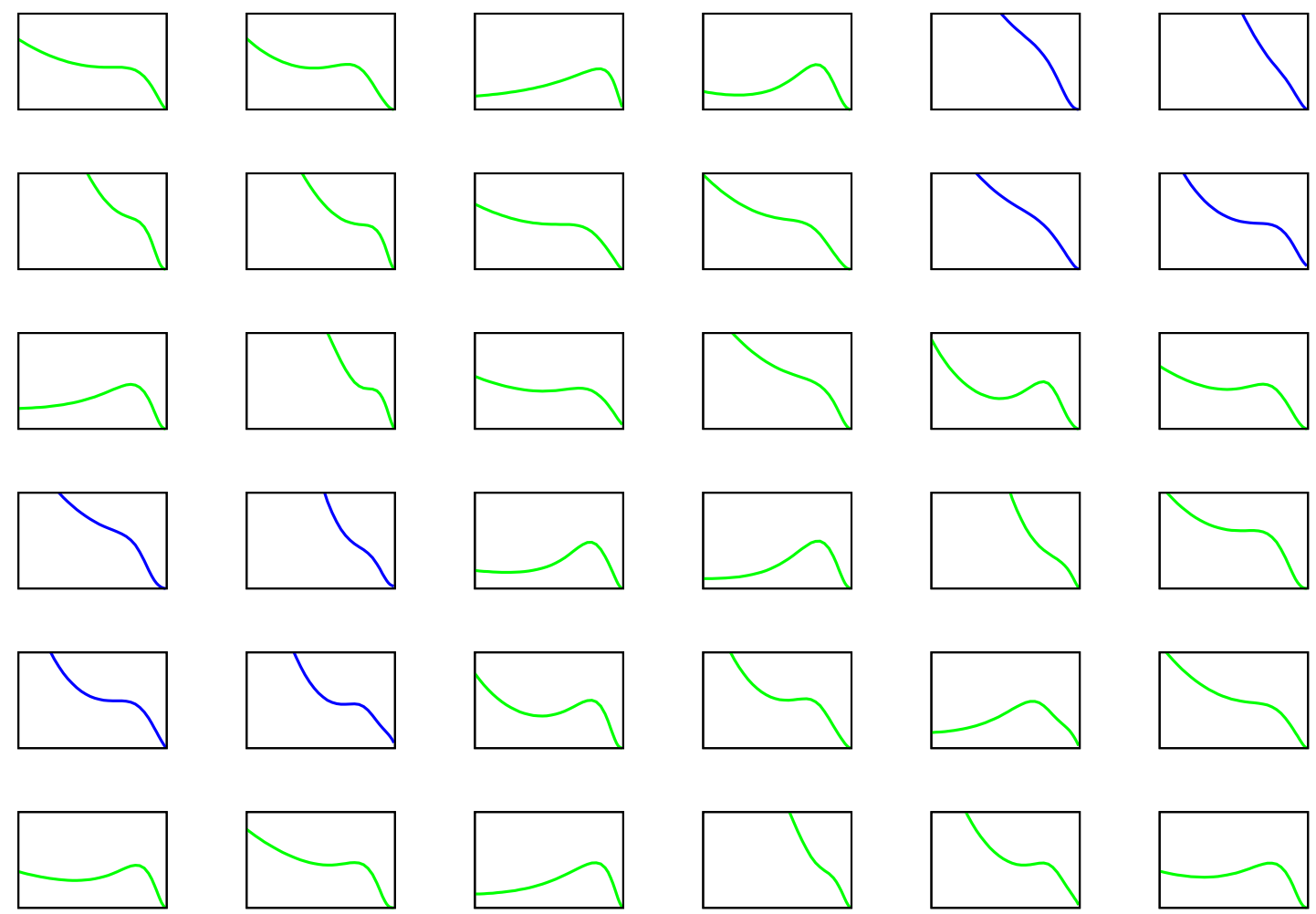

Figure 5.53: Shown are plots of $d=d_{v}+2 \bar{d}$, or down quark, PDFs without large $x$ Corrections added for $Q^{2}=2.5 \mathrm{GeV}^{2}$. The Bjorken $x$ range (the x axis) is $(0.0001: 1)$ and the $d$ range (the y axis) is $(0: 1)$. The PDF curves are in green with the clusters, identified based on visualization of PDFs with common behaviors for large and small $x$ regions, shown as blue curves.

PDFs. The maps of the PDFs for each parton type effectively showed how the possible behaviors of each PDF type can create the most ideal fit for the generated structure functions.

We can also observe the effects of the PDF clusters when TMC and LxR are added; the maps with these effects added are in Figure 5.57 - 5.63. With the large $x$ Corrections, the $u_{v}$ map is in Figure 5.57, the $d_{v}$ map is in Figure 5.58, the $u$ quark map is in Figure 5.59, the $d$ quark map is in Figure 5.60, the gluon Map is in Figure 5.61, the strange map is in Figure 5.62 and the charm map is in Figure 5.63. The $u_{v}$ map vectors show the clustering in the upper left and lower right hand corners. The $d_{v}$ map vectors show clustering in the upper right corner and the lower left corner. The $u$ quark map vector grid shows clustering in the lower left corner and in the right side of the map. The $d$ 

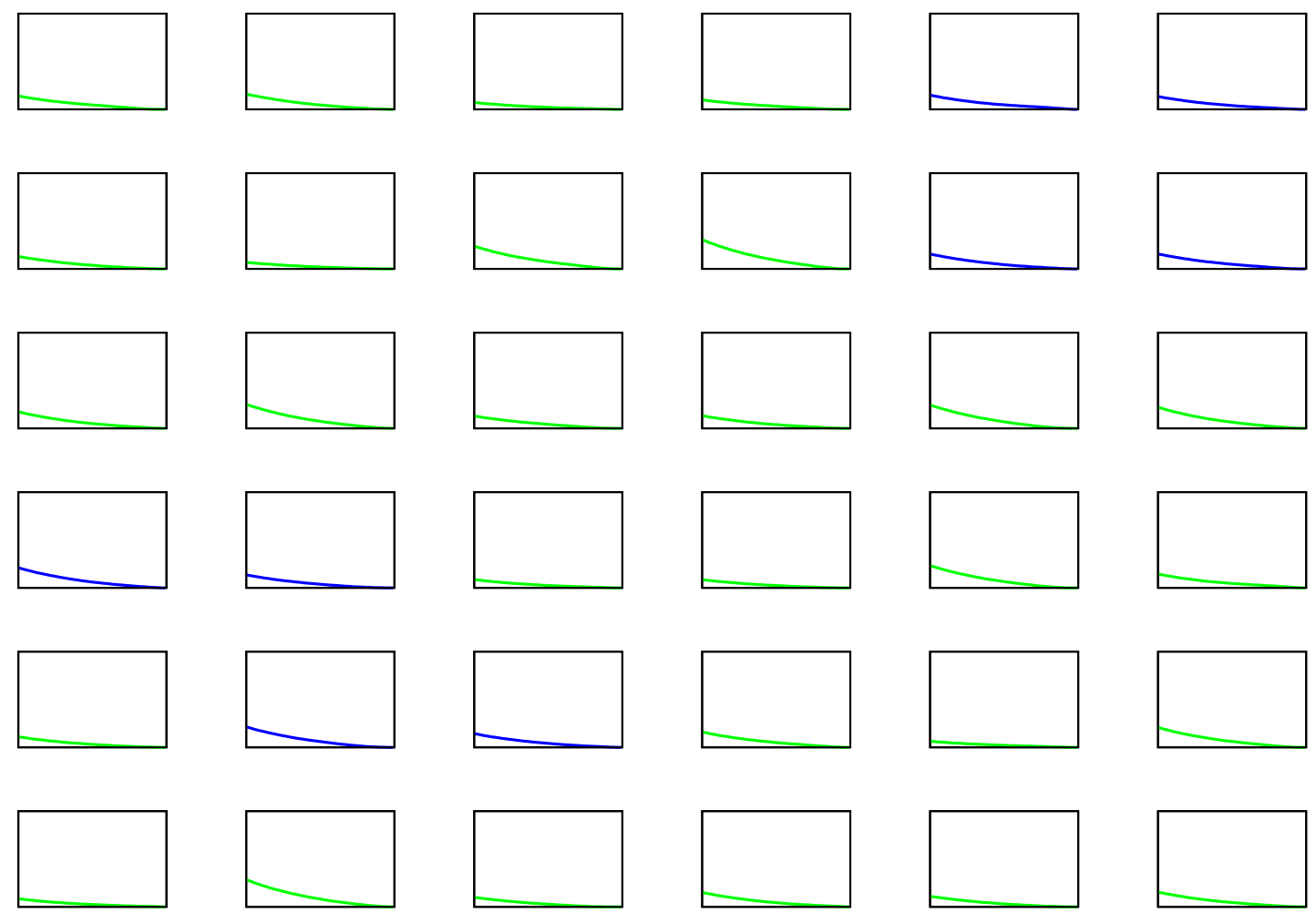

Figure 5.54: Shown are plots of gluon PDFs without large $x$ Corrections added for $Q^{2}=2.5 \mathrm{GeV}^{2}$. The Bjorken $x$ range (the $\mathrm{x}$ axis) is $(0.0001: 1)$ and the gluon range (the y axis) is $(0: 50)$. The PDF curves are in green with the clusters, identified based on visualization of PDFs with common behaviors for large and small $x$ regions, shown as blue curves.

quark map vector grid shows clustering in the lower left and right corners. The gluon map vector grid reveals clusters of gluon vectors in the upper left section and lower left section, in a similar vein to how the $d$ quark PDFs form clusters, in the map. The strange quark map shows clustering in the upper right and lower left corners of the map and the charm quark map shows clustering in the upper left corner. These PDF maps illustrate how the large $x$ corrections affect the relationship between the quality of fit of the PDF clusters and the PDF behavior over various $x$ ranges. 

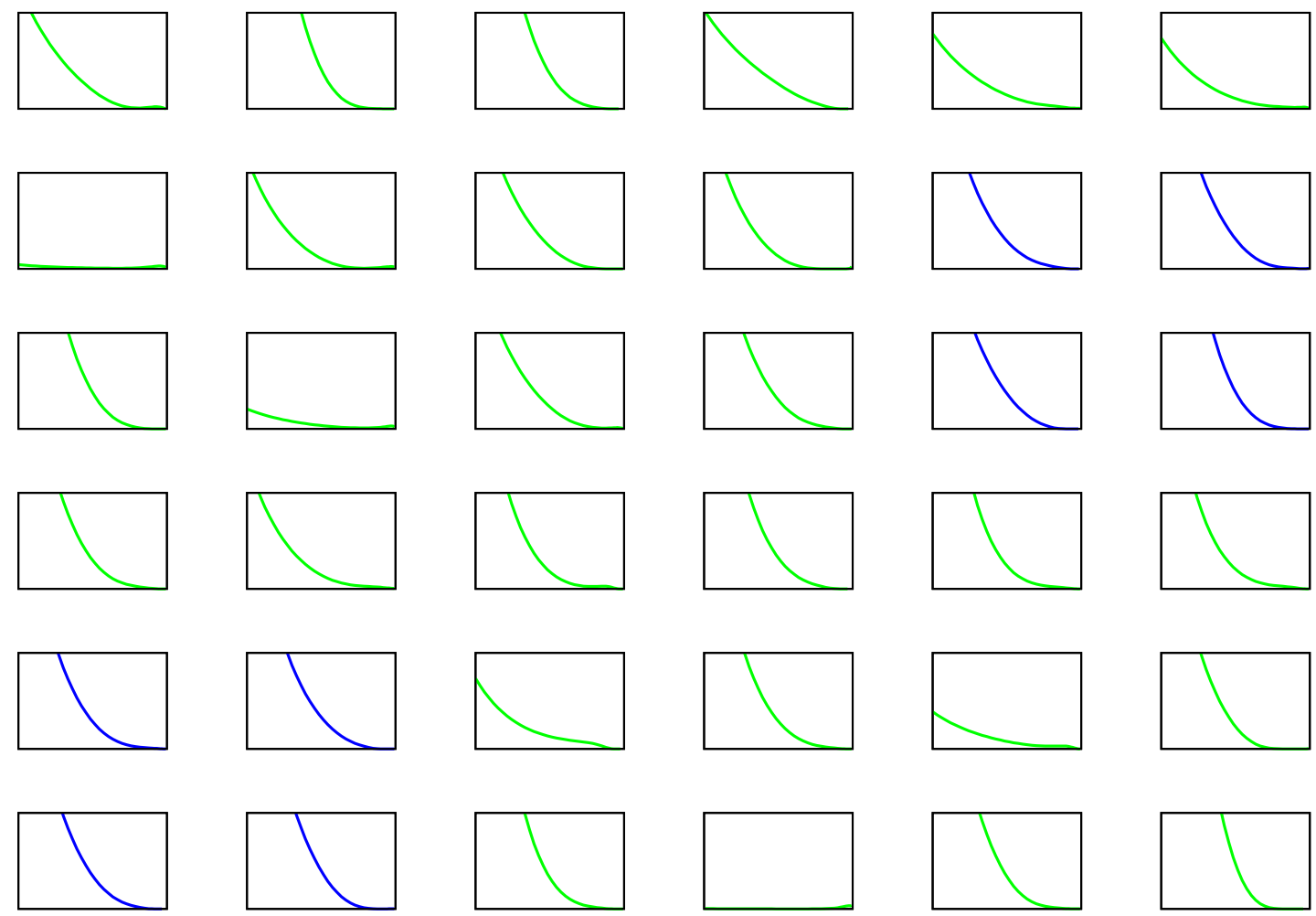

FiguRE 5.55: Shown are plots of $s$, or strange quark, PDFs without large $x$ Corrections added for $Q^{2}=2.5 \mathrm{GeV}^{2}$. The Bjorken $x$ range (the $\mathrm{x}$ axis) is $(0.0001: 1)$ and the $s$ range (the y axis) is $(0: 1)$. The PDF curves are in green with the clusters, identified based on visualization of PDFs with common behaviors for large and small $x$ regions, shown as blue curves.

\section{$5.7 \frac{d}{u}$ Cluster Quantification}

The sizes of the clusters with and without the large $x$ corrections can be analyzed by using the maps of the $\frac{d}{u}$ values with and without these corrections. Clusters for the purpose of this formulaic analysis are defined as groups of map vectors that contain a uniting element and connected to each other by neighboring units to the left or right or upwards or downwards. The cluster sizes can be analyzed for the plot of $\frac{d}{u}$ values for $x$ in the range $(0.0001: 1.0)$. Without the large $x$ corrections, the largest cluster in Figure 5.48 consists of 15 map vector units conforming to the physical $S=0 \mathrm{qq}$ model, located in the upper left side and the center of the map vector grid. With the large $x$ corrections added, the largest cluster in Figure 5.49 consists of 8 map vectors, 

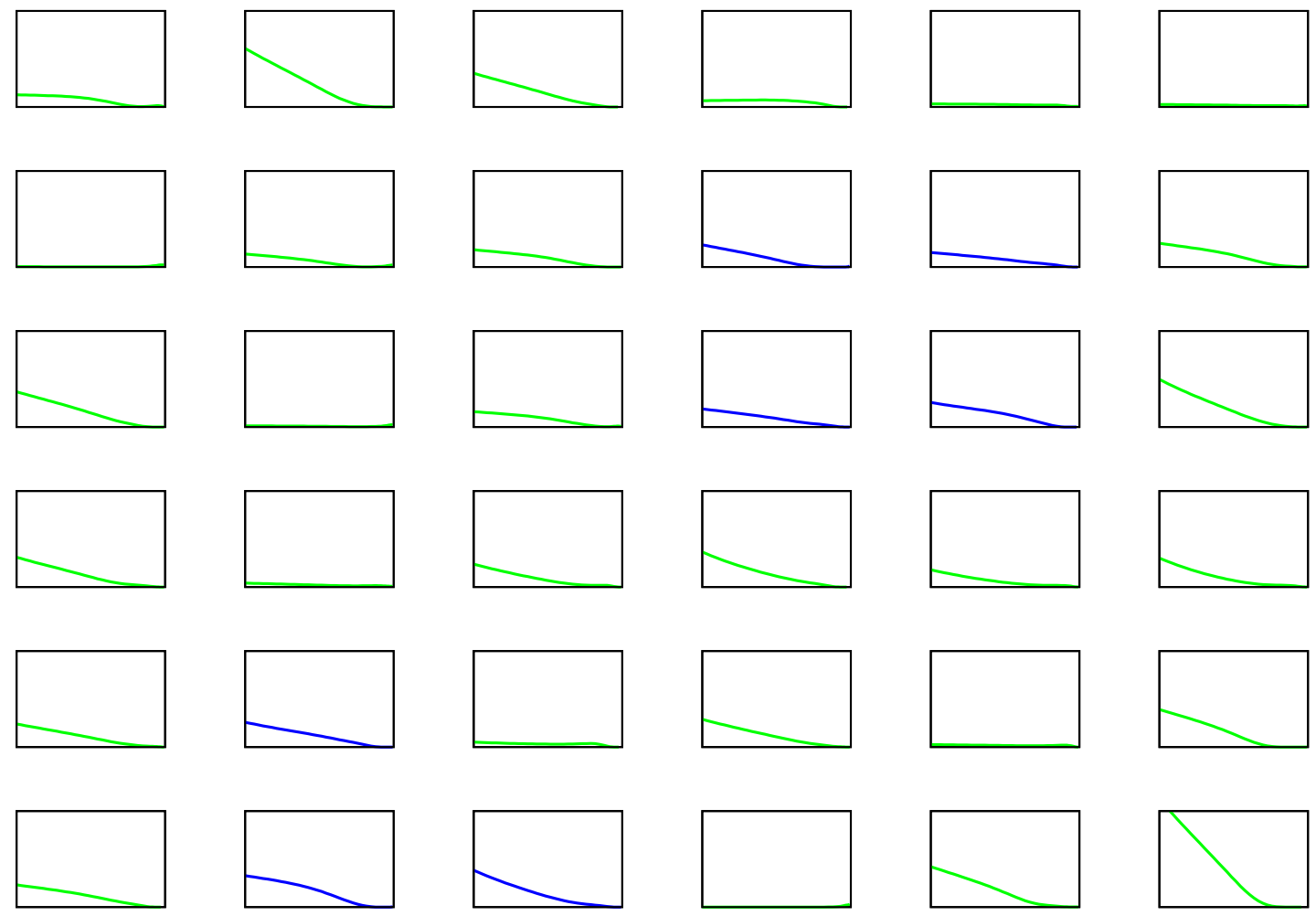

FiguRE 5.56: Shown are plots of $c$, or charm quark, PDFs without large $x$ Corrections added for $Q^{2}=2.5 \mathrm{GeV}^{2}$. The Bjorken $x$ range (the $\mathrm{x}$ axis) is $(0.0001: 1)$ and the $c$ range (the y axis) is $(0: 1)$. The PDF curves are in green with the clusters, identified based on visualization of PDFs with common behaviors for large and small $x$ regions, shown as blue curves.

consisting of data units where for large $x \frac{d}{u} \geq 1$. So the rate of change $R$ of clustering due to the corrections, a measurement of the large $x$ correction effects, can be written equation 5.39.

$$
R=\frac{C_{c}}{C_{n}}
$$

In this formula, $C_{n}$ is the size of the largest continuous cluster without the corrections and $C_{c}$ is the largest size with corrections. In this case, we have $C_{c}=8.0$ and $C_{n}=15.0$. $C_{c}$ also defines the cluster size for the largest cluster with the large $x$ corrections added with either $\frac{d}{u} \rightarrow 1$ or $\frac{d}{u}>>1$. Without the large $x$ corrections added, $C_{n}$ is also the size of the clusters for which $\frac{d}{u} \rightarrow 0$. Both of these clusters contain data units with PDFs that 

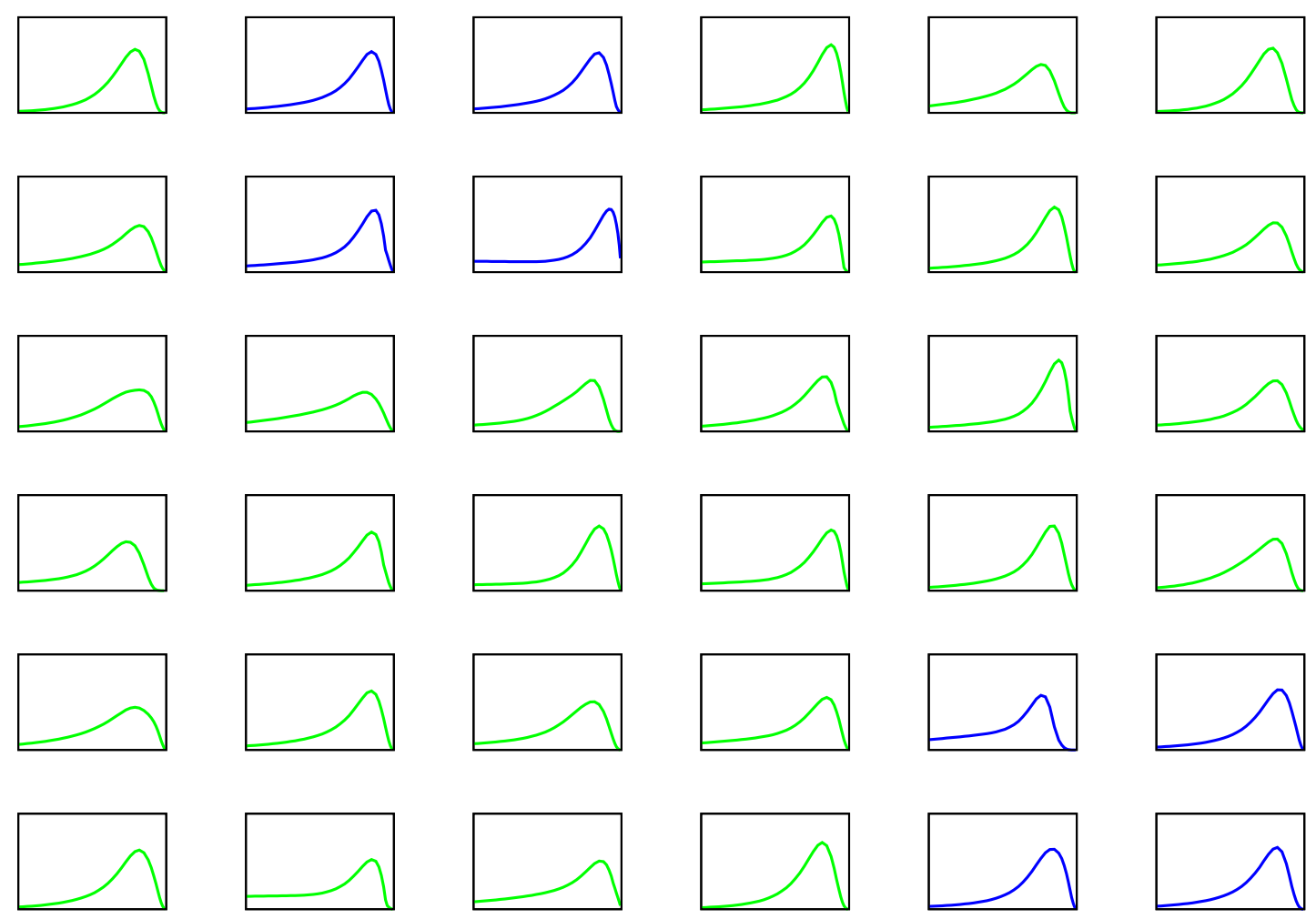

Figure 5.57: Shown are plots of $u_{v}$ PDFs with large $x$ Corrections added for $Q^{2}=2.5$ $\mathrm{GeV}^{2}$. The Bjorken $x$ range (the $\mathrm{x}$ axis) is $(0.0001: 1)$ and the $u_{v}$ range (the $\mathrm{y}$ axis) is $(0: 1)$. The PDF curves are in green with the clusters, identified based on visualization of PDFs with common behaviors for large and small $x$ regions, shown as blue curves.

break $S U(6)$ symmetry, therefore they can be classified as symmetry breaking clusters. These cluster sizes give us $\frac{C_{c}}{C_{n}}=0.533$ for the effects of TMC and LxR on the broken symmetry clustering. Relative to the size of the clusters without TMC and LXR, the percent error can be determined to be $\frac{C_{n}-C_{c}}{C_{n}} \times 100=87.5 \%$ for the cluster change. Essentially, the cluster sizes and types enable us to analyze the extent to which the addition of large $x$ Corrections effectively broke up clusters of $S=0$ qq data units and led to formation of clusters with new types of physical models for the data units.

\section{$5.8 \frac{d}{u}$ Dimensional Clusters and Error Extraction}

The SOM PDFs can be clustered in two dimensional plots where each of the two dimensions represents an observable value under a specific set of conditions. The two 

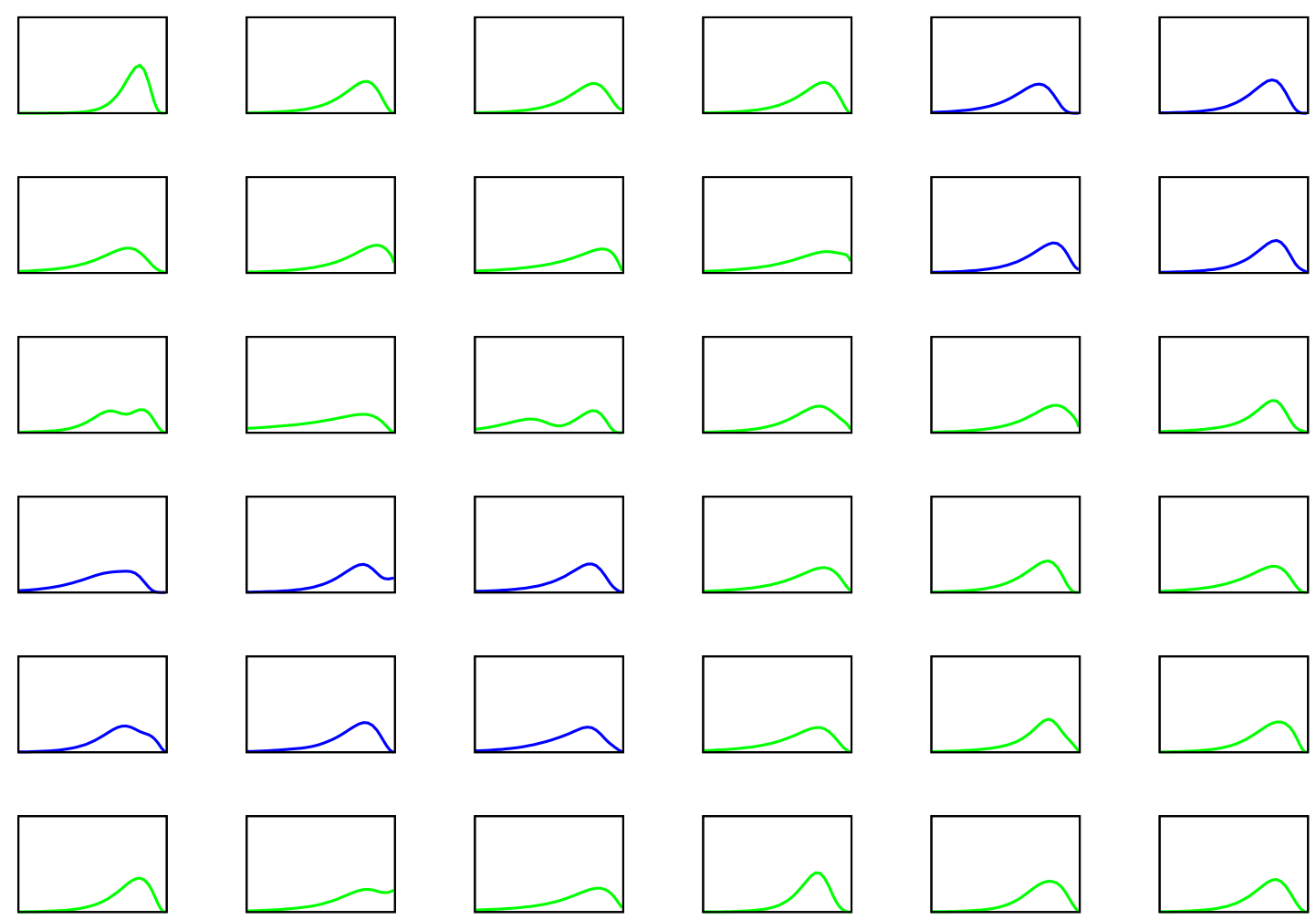

Figure 5.58: Shown are plots of $d_{v}$ PDFs with large $x$ Corrections added for $Q^{2}=2.5$ $\mathrm{GeV}^{2}$. The Bjorken $x$ range (the $\mathrm{x}$ axis) is $(0.0001: 1)$ and the $d_{v}$ range (the $\mathrm{y}$ axis) is $(0: 1)$. The PDF curves are in green with the clusters, identified based on visualization of PDFs with common behaviors for large and small $x$ regions, shown as blue curves.

dimensions can be cluster plots of $\frac{d}{u}$ or $\chi^{2}$ with various combinations of large $x$ corrections. In Figure 5.64, the $\frac{d}{u}$ values with TMC and large $x$ corrections, which are displayed in dimension two, are shown relative to $\frac{d}{u}$ values with no corrections, which is designated dimension one. In Figure 5.65, the two dimensions are $\frac{d}{u}$ with large $x$ Resummations for dimension two and $\frac{d}{u}$ with TMC for dimension one. Figure 5.66 - 5.67 show the same results with the $25^{\text {th }}$ and $50^{\text {th }}$ iterations omitted for clarity so that the iterations with better fit values can be displayed in the cluster regions. The clusters are grouped based on the possible large $x$ conditions outlined previously: $S=0$ qq, $S_{z}=0$ qq, quark hadron duality and $S U(6)$ symmetry. The grouping of the SOM values into $\frac{d}{u}$ clusters based on physical limits enables us to visualize how the large $x$ corrections directly influenced PDF formation during the Self Organizing and fitting procedures. A black line equivalent to $y=x$ on the $x$ and $y$ axis is drawn in all the dimensional plots 

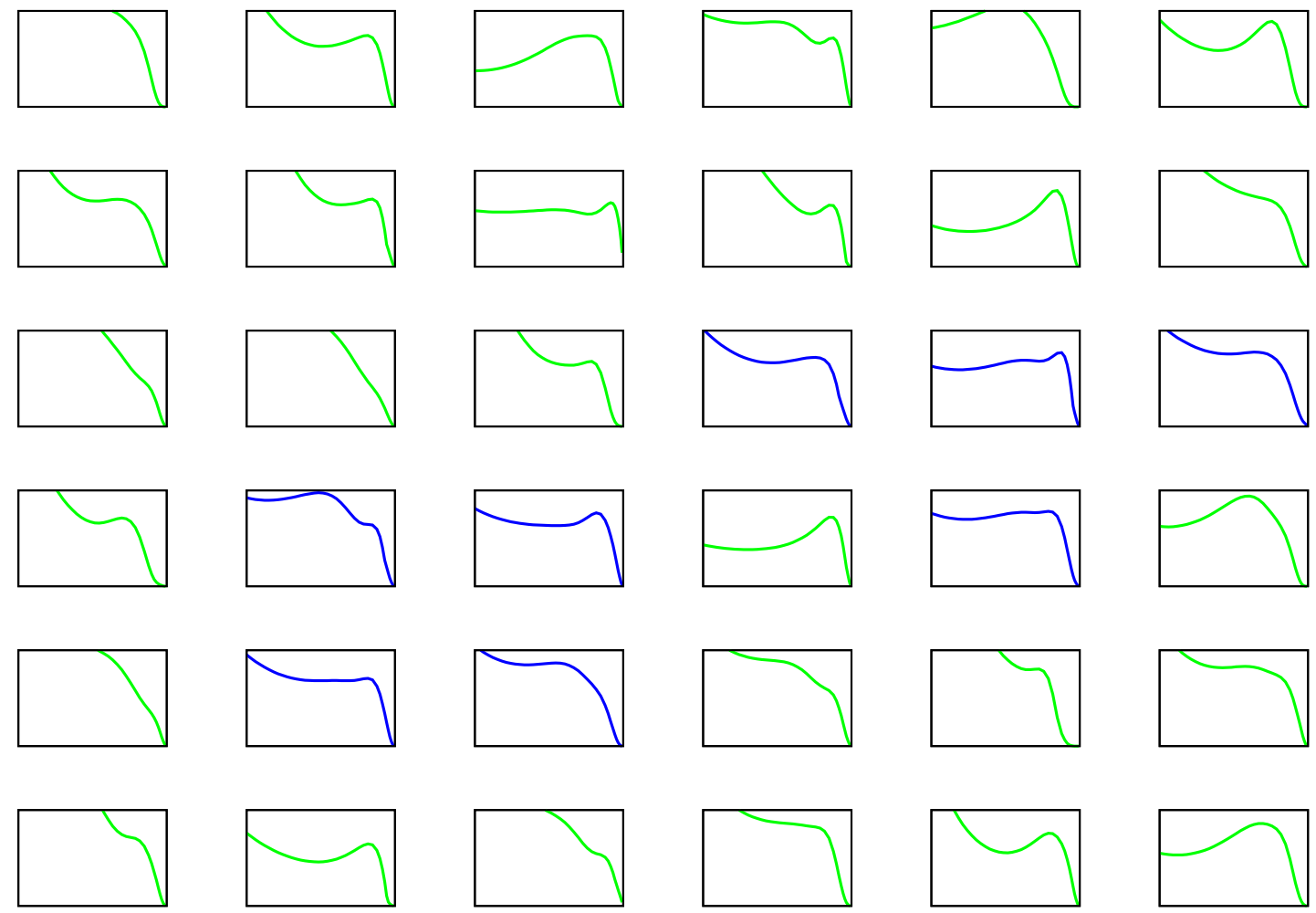

FIgURE 5.59: Shown are plots of $u=u_{v}+2 \bar{u}$, or up quark, PDFs with large $x$ Corrections added for $Q^{2}=2.5 \mathrm{GeV}^{2}$. The Bjorken $x$ range (the x axis) is $(0.0001: 1)$ and the $u$ range (the y axis) is $(0: 1)$. The PDF curves are in green with the clusters, identified based on visualization of PDFs with common behaviors for large and small $x$ regions, shown as blue curves.

in order to enable us to visualize the extent to which the clusters show linear and non linear correlations.

The relationship between the $\frac{d}{u}$ values in a cluster one dimension and the corresponding $\frac{d}{u}$ values in a second dimension reveal the strength of the effects of large $x$ corrections in terms of altering the $x \rightarrow 1$ limit for a given dimensional cluster. The number of data points present in dimension two relative to dimension one follows linear as well as non linear trends which illustrate how large $x$ corrections affect the resulting nuclear models for the up and down quarks when they are carrying close to the full hadron momentum. For a given value in dimension two, as the value of dimension one increases there is no uniform pattern or trend for the number of data units that exist in the dimension two value. In Figure 5.64, the clusters such that $\frac{d}{u} \rightarrow 0$ for dimension one show that a 

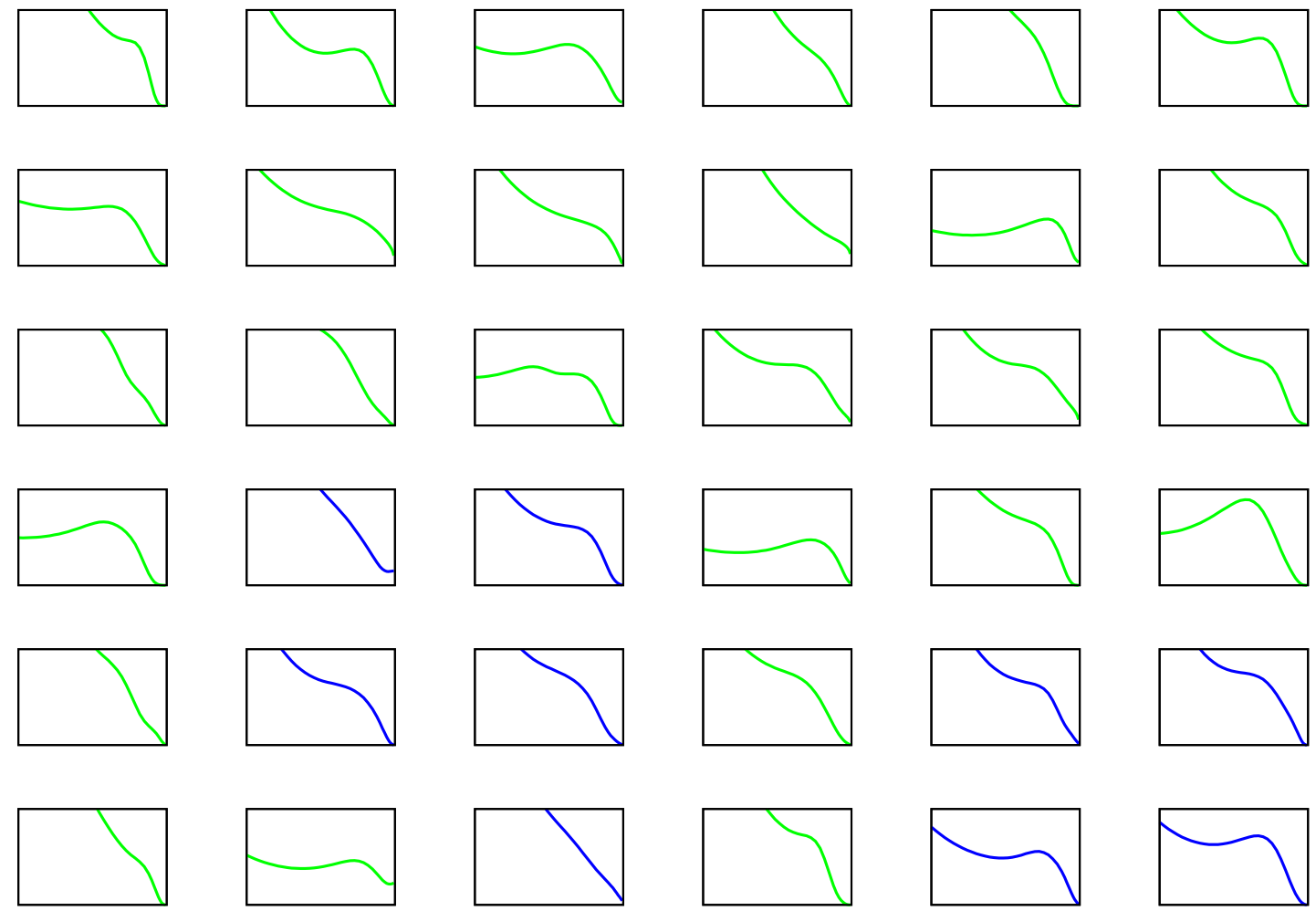

FiguRe 5.60: Shown are plots of $d=d_{v}+2 \bar{d}$, or down quark, PDFs with large $x$ Corrections added for $Q^{2}=2.5 \mathrm{GeV}^{2}$. The Bjorken $x$ range (the x axis) is $(0.0001: 1)$ and the $d$ range (the y axis) is $(0: 1)$. The PDF curves are in green with the clusters, identified based on visualization of PDFs with common behaviors for large and small $x$ regions, shown as blue curves.

significant of the cluster points are also in the region $\frac{d}{u} \rightarrow 0$ for dimension two and that the majority of points exist in clusters for the other limit ranges. For $\frac{d}{u} \rightarrow \frac{1}{5}, 0.42$ and for $\frac{d}{u}$ limits greater than 0.47 there are also more points in the clusters where dimension one and dimension two are not equal. Furthermore, this difference is small for the data units where $\frac{d}{u}>>1$ showing that the large $x$ corrections make less of an impact on the data units when down quarks are more prevalent for large momentum fractions. Therefore, the two dimensional plot for $\frac{d}{u}$ values under the conditions in Figure 5.64 shows significant linear as well as non linear clustering. To measure this effect, we can define $N_{s}$ as the number of points on the two dimension plot that are in the same $\frac{d}{u}$ range for dimension two as they are for dimension one and $N_{d}$ as the number of points that are in one of three possible different ranges. We can then quantify the strength of 

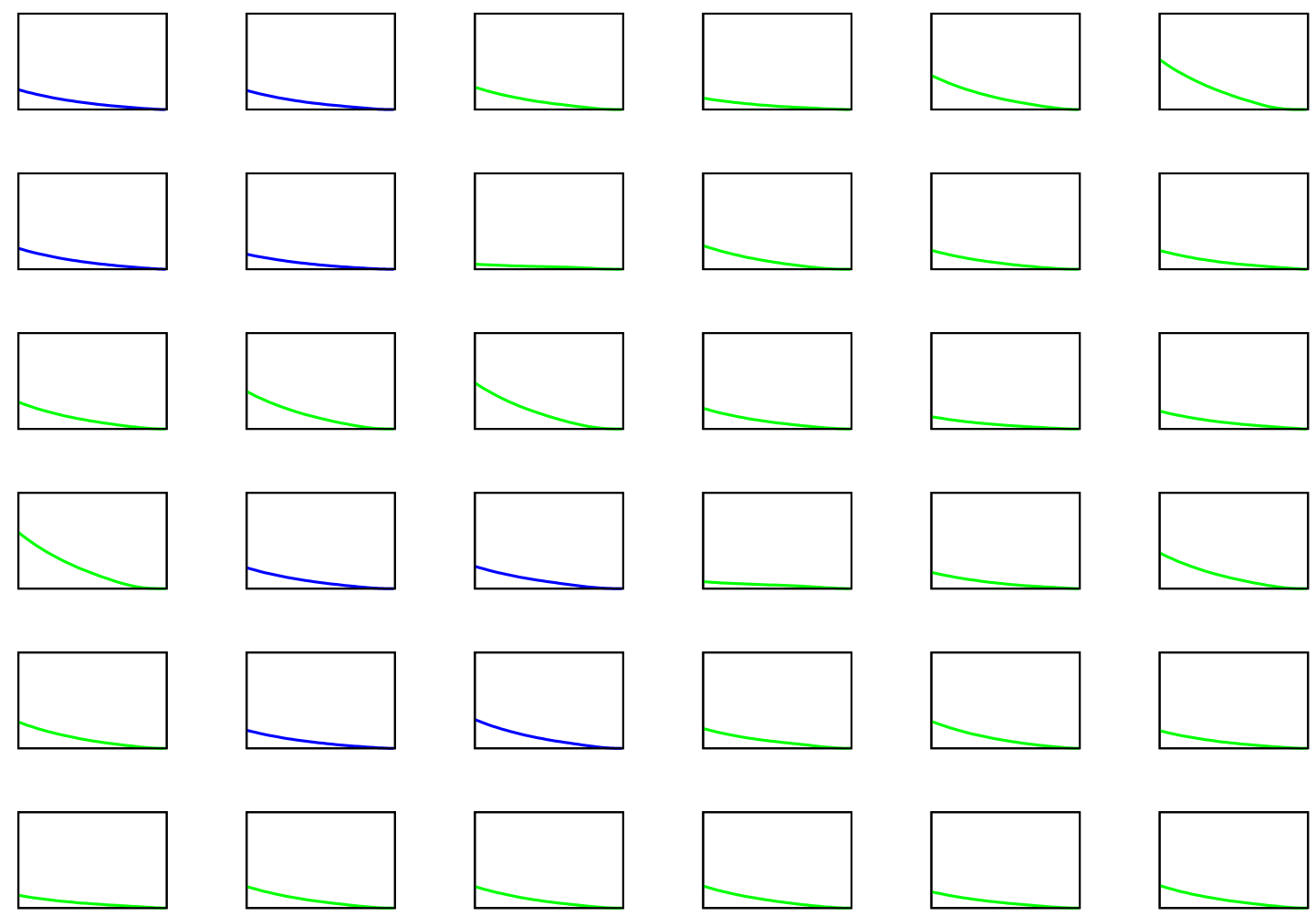

Figure 5.61: Shown are plots of gluon PDFs with large $x$ Corrections added for $Q^{2}=2.5 \mathrm{GeV}^{2}$. The Bjorken $x$ range (the $\mathrm{x}$ axis) is $(0.0001: 1)$ and the gluon range (the y axis) is $(0: 50)$. The PDF curves are in green with the clusters, identified based on visualization of PDFs with common behaviors for large and small $x$ regions, shown as blue curves.

the large $x$ corrections, $S_{c}$ in breaking up the clusters of $\frac{d}{u}$ values that formed without these corrections with equation 5.40 .

$$
S_{c}=\frac{N_{d}}{N_{s}}
$$

In the $\frac{d}{u} \rightarrow 0$ range, there are 36 points in dimension two in the $\frac{d}{u} \rightarrow 0$ range and 81 points in dimension two located in one of the other three regions, so we have $N_{s}=36$ and $N_{d}=81$ giving us $S_{c}=\frac{N_{d}}{N_{s}}=\frac{81}{36}=2.25$ For dimension one in the $\frac{d}{u} \rightarrow \frac{1}{5}$ range, there are $N_{s}=2$ points in dimension two that are in the same range and $N_{d}=13$ points in dimension two in the other three ranges, so $S_{c}=\frac{N_{d}}{N_{s}}=\frac{13}{2}=6.5$. For dimension one in $\frac{d}{u} \rightarrow 0.42$ range, there are $N_{s}=7$ points in the same range for dimension two and 

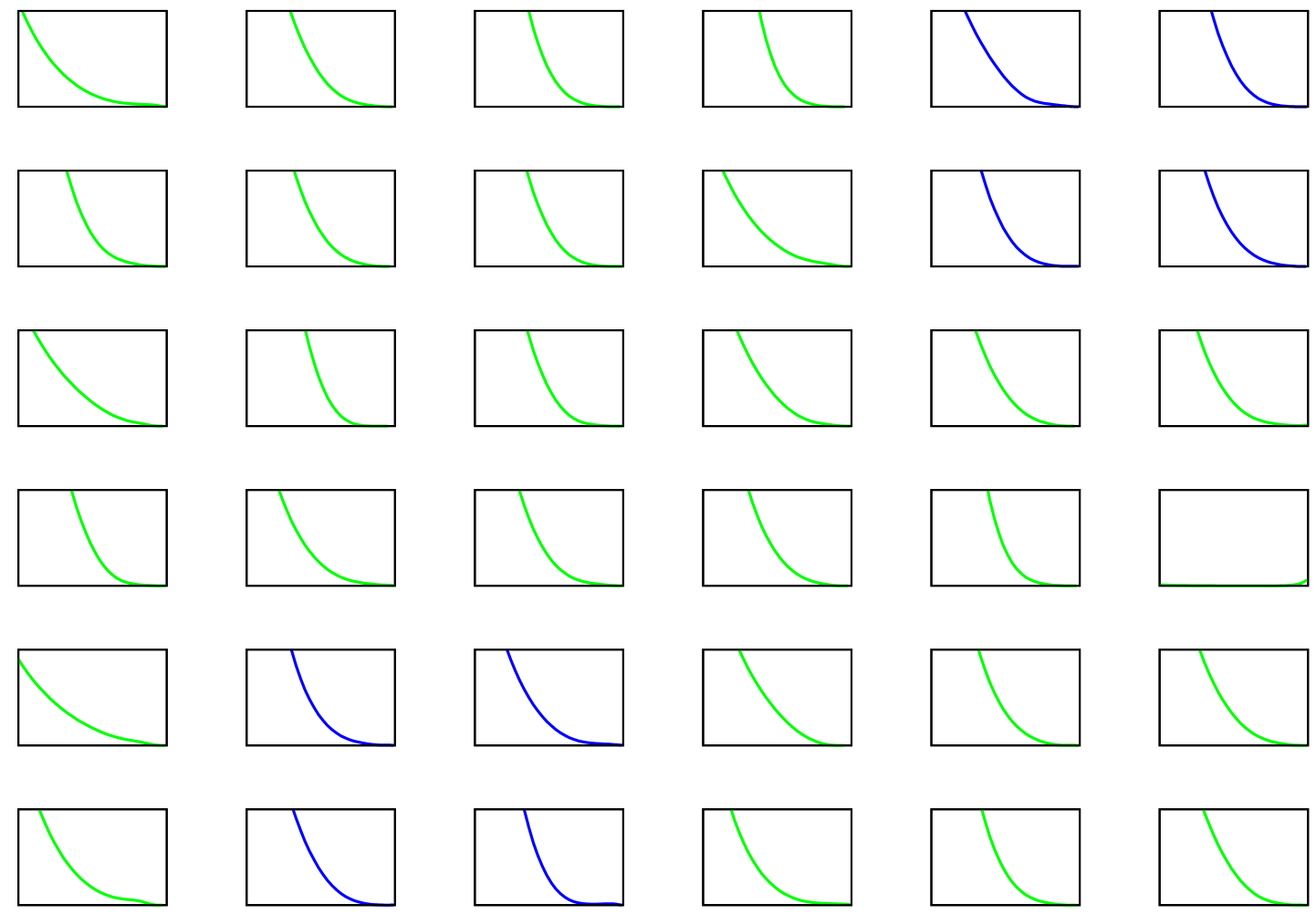

FIGURE 5.62: Shown are plots of $s$, or strange quark, PDFs with large $x$ Corrections added for $Q^{2}=2.5 \mathrm{GeV}^{2}$. The Bjorken $x$ range (the $\mathrm{x}$ axis) is $(0.0001: 1)$ and the $s$ range (the y axis) is $(0: 1)$. The PDF curves are in green with the clusters, identified based on visualization of PDFs with common behaviors for large and small $x$ regions, shown as blue curves.

$N_{d}=13$ points in one of the other three ranges giving a ratio of $S_{c}=\frac{N_{d}}{N_{s}}=\frac{13}{7}=1.86$. For dimension one in the $\frac{d}{u}$ range such that $\frac{d}{u}$ is greater than or equal to 0.47 , there are $N_{s}=22$ points in dimension two in the same range and $N_{d}=42$ points in dimension two in different ranges giving a value of 1.09 for $\frac{N_{d}}{N_{s}}$. This illustrates that the large $x$ corrections used together have a level of influence on cluster formation for all possible $\frac{d}{u}$ limits, an effect which is weaker for higher $\frac{d}{u}$ limits and stronger for the quark hadron duality, $S=0 \mathrm{qq}, S_{z}=0 \mathrm{qq}$ and $S U(6)$ symmetry models. In Figure 5.65, the number of points in dimension two for a given dimension one show similar results. In this figure, for dimension one in the $\frac{d}{u} \rightarrow 0$ range, there are $N_{s}=36$ points in the same range for dimension two and $N_{d}=108$ points in one of three different ranges giving $S_{c}=\frac{N_{d}}{N_{s}}=3$. In the $\frac{d}{u}$ range greater than or equal to 0.47 for dimension one, there are $N_{s}=19$ 

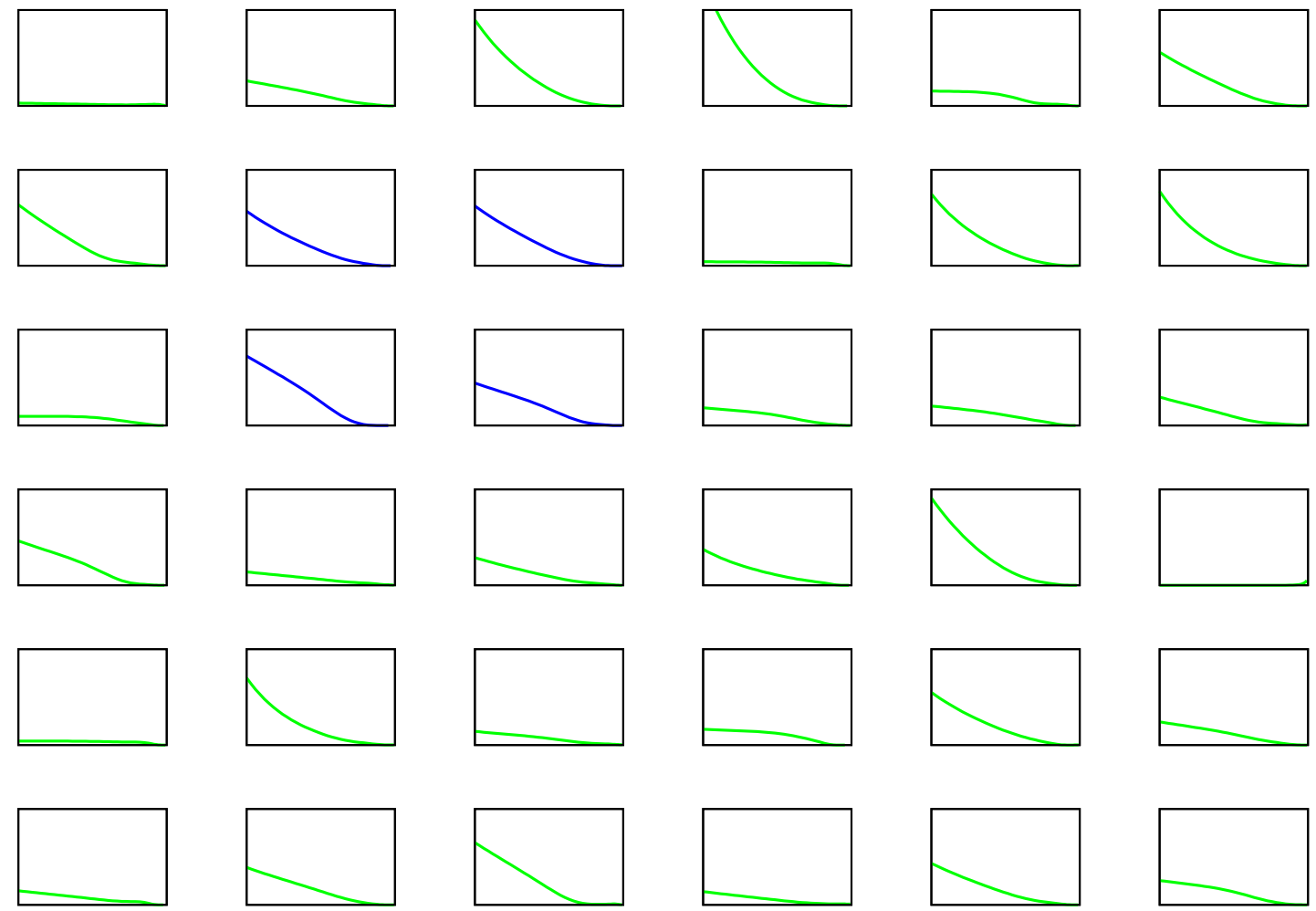

Figure 5.63: Shown are plots of $c$, or charm quark, PDFs with large $x$ Corrections added for $Q^{2}=2.5 \mathrm{GeV}^{2}$. The Bjorken $x$ range (the $\mathrm{x}$ axis) is $(0.0001: 1)$ and the $c$ range (the y axis) is $(0: 1)$. The PDF curves are in green with the clusters, identified based on visualization of PDFs with common behaviors for large and small $x$ regions, shown as blue curves.

points in the same region for dimension two and $N_{d}=13$ points in different regions; the strength is now $S_{c}=\frac{N_{d}}{N_{s}}=0.68$. Therefore, the clusters in a physical sense reveal the effects of various conditions imposed for specific quark types when they are carrying various fractions of the hadron's momentum in inelastic scattering. $\frac{d}{u}$ in the large $x$ limit is equivalent to the prevalence of up valence quarks relative to down valence quarks with nearly all of the hadron's momentum. Therefore, the clusters for this SOM also reveal that the large $x$ corrections have a smaller effect on the distribution of the valence quarks when down valence quarks are more prevalent at large momentum fractions relative to up valence quarks such that the $x \rightarrow 1$ limit for $\frac{d}{u}$ is significantly larger than zero. The two dimensional groupings of $\frac{d}{u}$ provide an in depth analysis of how the theoretical PDFs are formed that could not have been achieved with the previously utilized supervised 
networks or with PCA.

Figure 5.64 and Figure 5.65 also show how the $x \rightarrow 1$ limit for $\frac{d}{u}$ for the generated theoretical curves increasingly approach the physical limits as the number of SOM and GA iterations increases as well. For the curves in Figure 5.64, for example, from the $25^{t h}$ and $50^{t h}$ iteration, for instance, roughly half of the total of 36 in the map have the generated curves had a $\frac{d}{u}$ limit within zero and one. When the SOMPDF process hits the $100^{\text {th }}$ and afterwards the $250^{\text {th }}$ iteration, there are still roughly half of the map curves with a $\frac{d}{u}$ limit between one and zero. Similar trends are observed for Figure 5.65 , when the effects of the LxR and TMC are isolated in the dimensional SOM plot. Therefore, non linear correlations of $\frac{d}{u}$ values among data units are preserved as the neighborhood radius for the fitting procedure is implemented for the PDFs in the data units. In Figure 5.68 and Figure 5.69 the dimensional plots are shown with each section of $\frac{d}{u}$ values given a separate color to highlight the formation of dimensional clusters.

The PDF errors can also be extracted from the SOMs using clusters of closely related $\frac{d}{u}$ and $\chi^{2}$ values that form from the fitting procedure and the neighborhood radius function. For the $250^{\text {th }}$ iteration without large $x$ corrections, the cluster from 5.44 with $\chi^{2}$ a range of $1.35: 2.75:$ and a $\frac{d}{u}$ range of $(0.05: 0.35)$ was taken. For the $250^{\text {th }}$ iteration with large $x$ corrections, the cluster from 5.45 with a $\chi^{2}$ range of $(1.5: 2.5)$ and a $\frac{d}{u}$ range of $(0.1: 0.5)$ was used. The cluster of data units for the generated map of theoretical curves without large $x$ corrections was taken from the first and third data units in the first row and the first four data units in the second row. The cluster of data units with large $x$ corrections was taken from the second element of the first row, the first and third element of the second row and the first three elements of the third row. The error bands for each of the two theoretical PDF sets was taken from the standard deviation of the curves. This resulted in an error uniquely based on the clustering of the curves based on the neighborhood radius function in the Self Organizing procedure. These curves bundles with errors for the best fitting curves are in Figure 5.70 without large $x$ corrections and in Figure 5.71 with large $x$ corrections. The error band is shown along with the curve among the generated bundle with the lowest $\chi^{2}$ value. Without the large $x$ corrections, the relative error at $x=0.93$, the largest $x$ value for the generated curve bundles, was 1.25; when the large $x$ corrections were added the relative error at $x=0.93$ was 0.27 . For the previous $6 \times 6 \frac{d}{u}$ curve in 5.43 , for the $6 \times 6$ map the relative error was 0.67 for the upper band and 1.31 for the lower band. For the previous $1 \times 1$ curve 


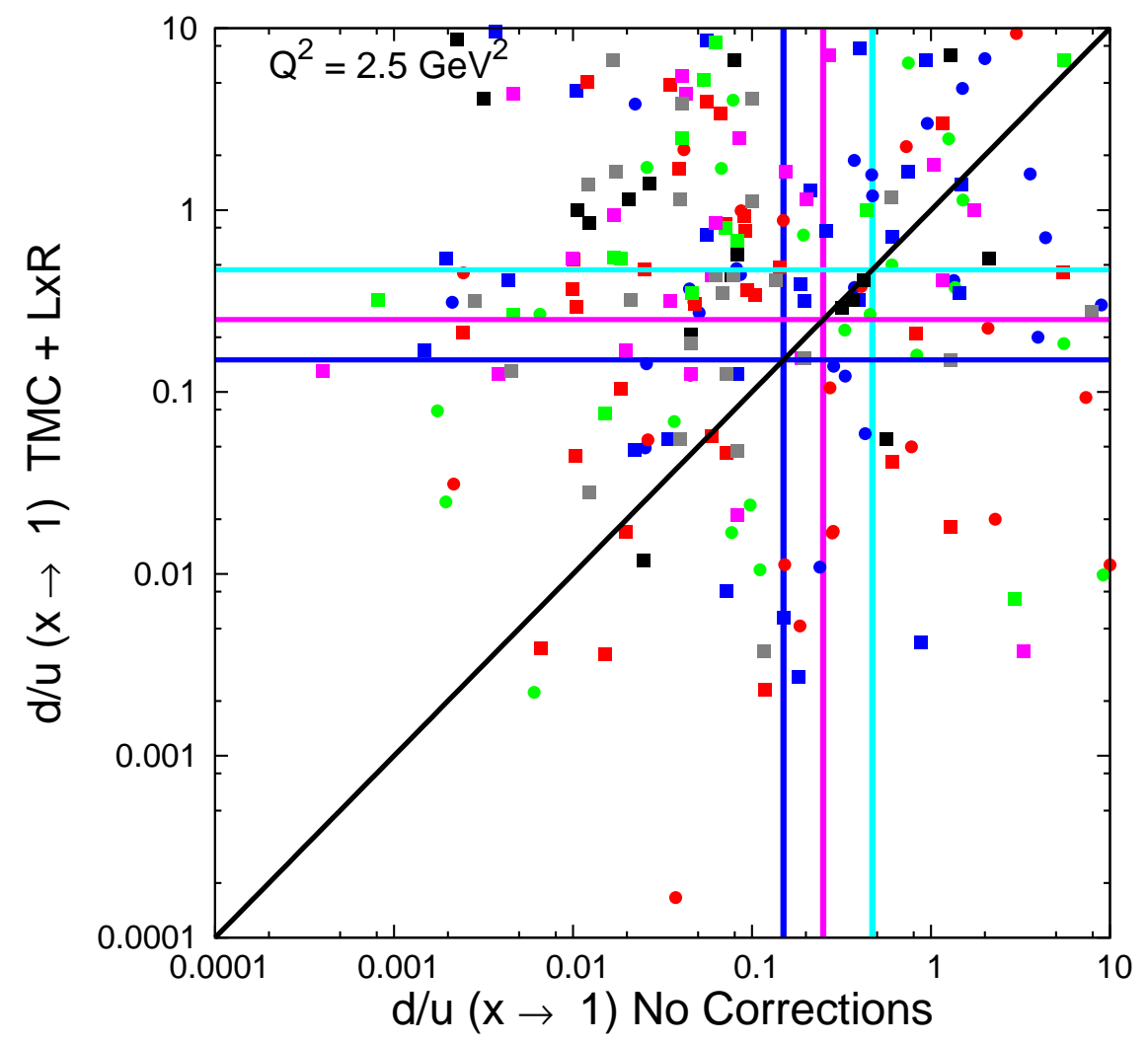

Iteration 25

Iteration 50

Iteration 100

Iteration 150

Iteration 200

Iteration 215

Iteration 225

Iteration 240

Iteration 250

Figure 5.64: The ratios $\frac{d}{u}$ for $Q^{2}=2.5 \mathrm{GeV}^{2}$, where $d$ is the distribution of down quarks (sea and valence) and $u$ is the distribution of up quarks (sea and valence), are shown on a two dimensional plot. $\frac{d}{u}$ is computed for 36 PDFs per iteration, corresponding to the number of PDFs produced on a $6 \times 6$ map, with large $x$ Resummations and Target Mass Corrections for a given number of iterations. $\frac{d}{u}$ is then computed without these corrections for the same number of iterations. Dimension one is the $\frac{d}{u}$ values when neither large $x$ Resummations or Target Mass Corrections are added. Dimension two is the $\frac{d}{u}$ values when these large $x$ corrections are added. The $x$ axis is dimension one and the $y$ axis is dimension two. The black line corresponsing to $y=x$ along the $y$ and $x$ axis is drawn to highlight the extent of the linear and non linear correlations. The cluster points in different dimension one and two regions represent non linear correlations. 


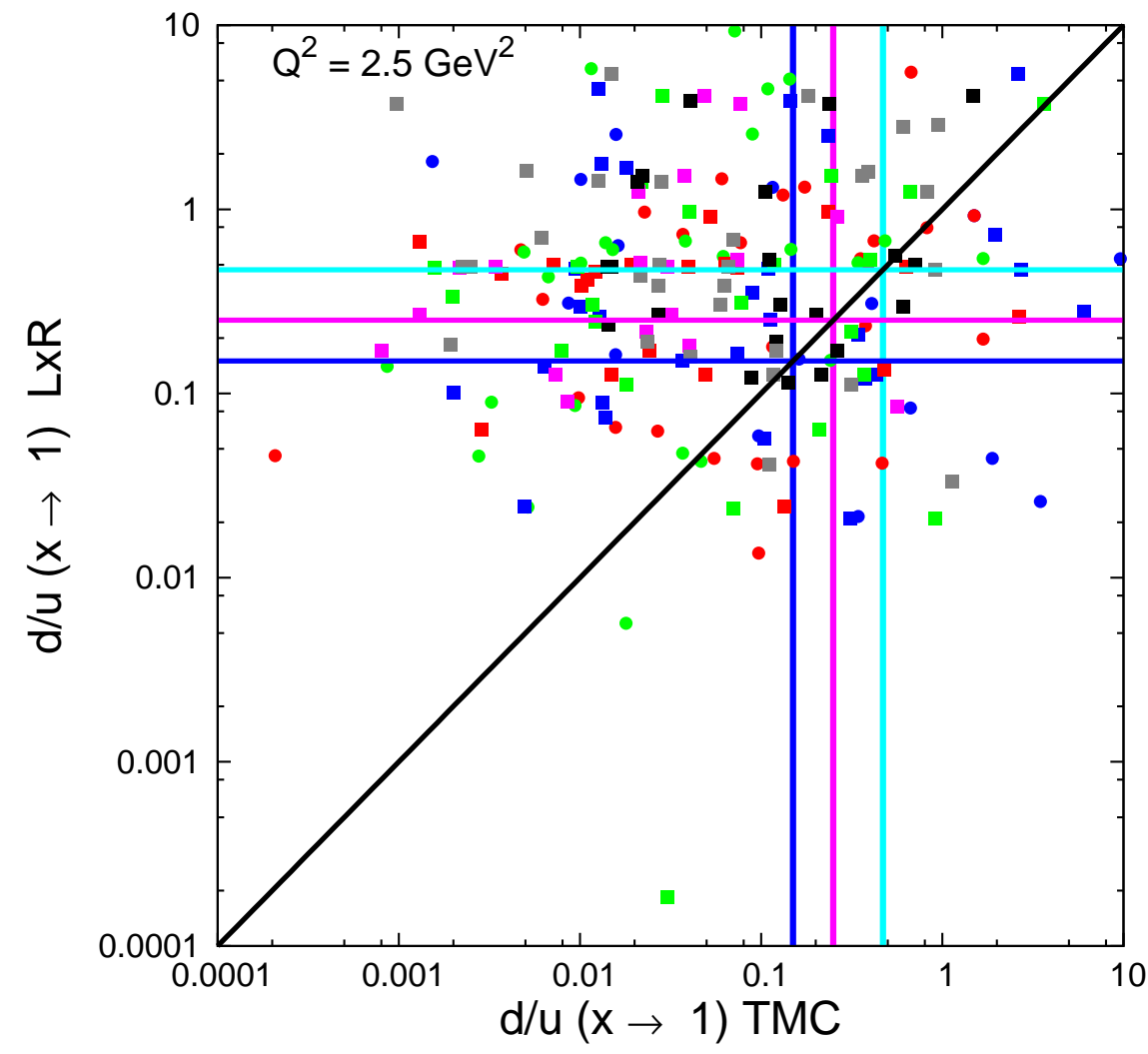

Iteration 25 •

Iteration 50 •

Iteration 100 •

Iteration 150

Iteration 200

Iteration 215

Iteration 225

Iteration 240

Iteration 250

Figure 5.65: The ratios $\frac{d}{u}$ for $Q^{2}=2.5 \mathrm{GeV}^{2}$, where $d$ is the distribution of down quarks (sea and valence) and $u$ is the distribution of up quarks (sea and valence), are shown on a two dimensional plot. $\frac{d}{u}$ is computed for 36 PDFs per iteration, corresponding to the number of PDFs produced on a $6 \times 6$ map, with only large $x$ Resummations for a given number of iterations. $\frac{d}{u}$ is then computed with only Target Mass Corrections for the same number of iterations. Dimension one is the $\frac{d}{u}$ values when Target Mass Corrections are added. Dimension two is the $\frac{d}{u}$ values when large $x$ Resummations are added. The $x$ axis is dimension one and the $y$ axis is dimension two. The black line corresponsing to $y=x$ along the $y$ and $x$ axis is drawn to highlight the extent of the linear and non linear correlations. The cluster points in different dimension one and two regions represent non linear correlations. 


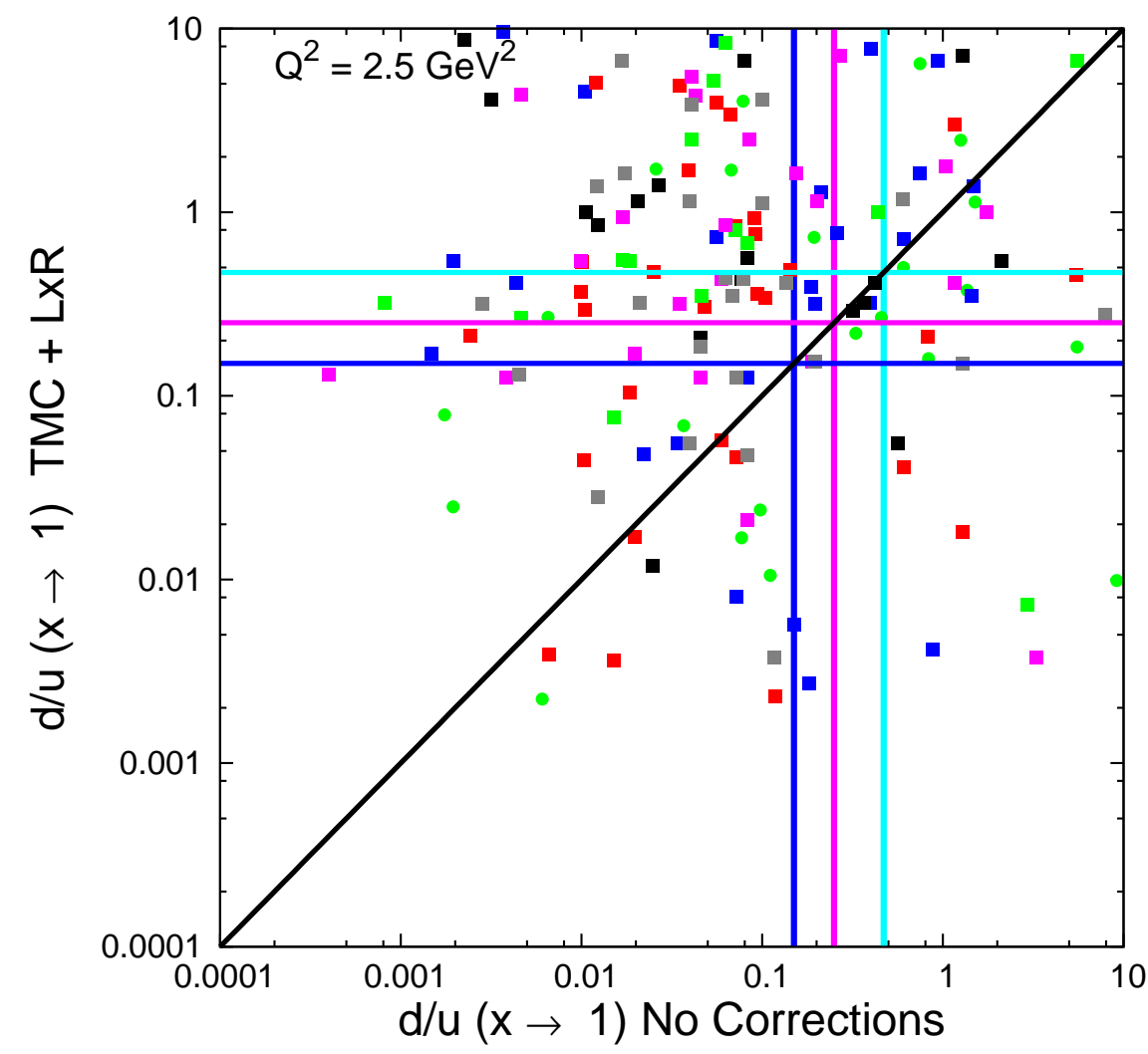

Iteration 100

Iteration 150

Iteration 200

Iteration 215

Iteration 225

Iteration 240

Iteration 250

Figure 5.66: The ratios $\frac{d}{u}$ for $Q^{2}=2.5 \mathrm{GeV}^{2}$, where $d$ is the distribution of down quarks (sea and valence) and $u$ is the distribution of up quarks (sea and valence), are shown on a two dimensional plot. The $25^{\text {th }}$ and $50^{\text {th }}$ iterations are omitted for clarity. $\frac{d}{u}$ is computed for 36 PDFs per iteration, corresponding to the number of PDFs produced on a $6 \times 6$ map, with large $x$ Resummations and Target Mass Corrections for a given number of iterations. $\frac{d}{u}$ is then computed without these corrections for the same number of iterations. Dimension one is the $\frac{d}{u}$ values when neither large $x$ Resummations or Target Mass Corrections are added. Dimension two is the $\frac{d}{u}$ values when these large $x$ corrections are added. The $x$ axis is dimension one and the $y$ axis is dimension two. The black line corresponsing to $y=x$ along the $y$ and $x$ axis is drawn to highlight the extent of the linear and non linear correlations. The cluster points on the black line represent strictly linear correlations. The cluster points in different dimension one and two regions represent non linear correlations. 


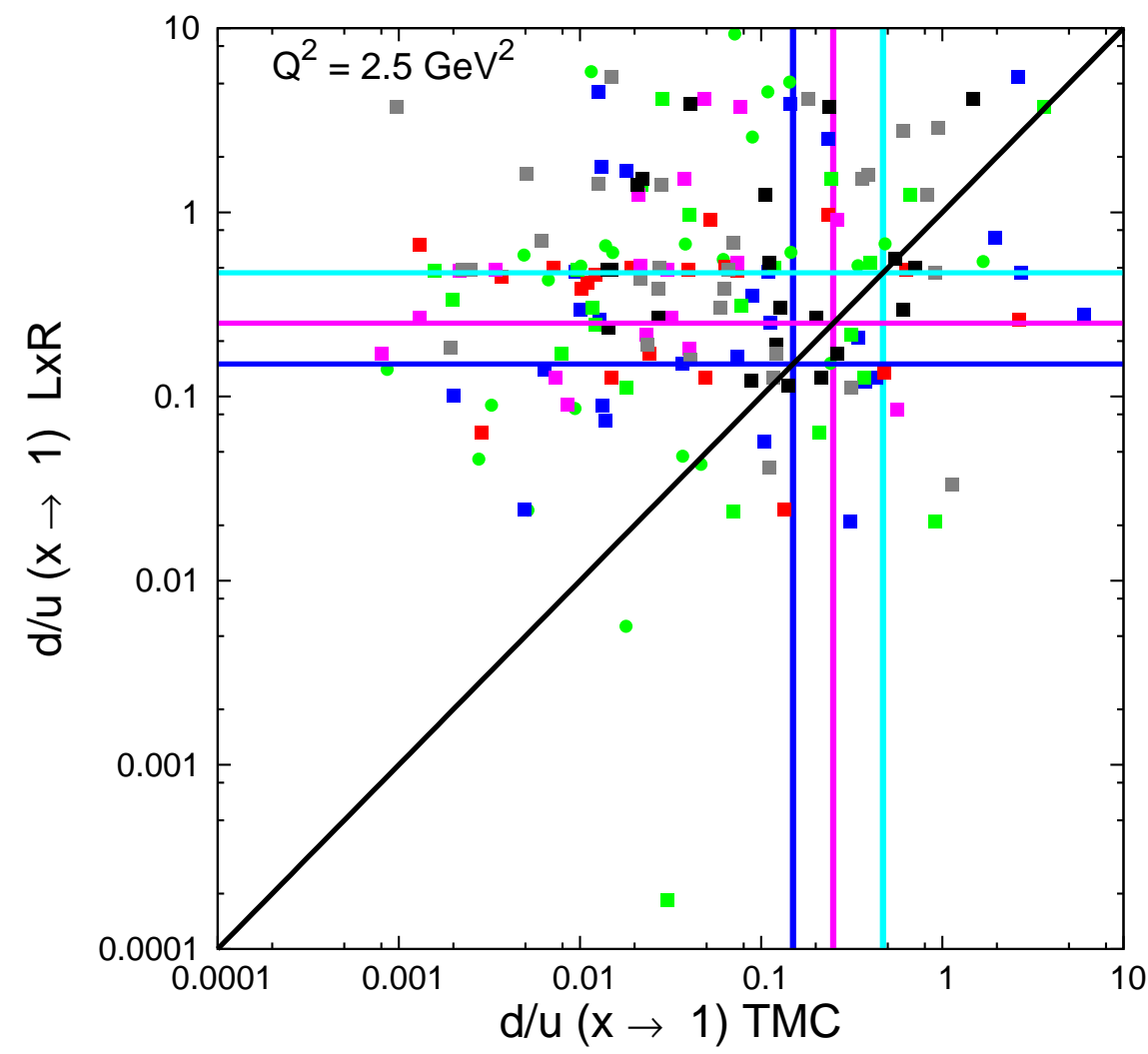

Iteration 100

Iteration 150

Iteration 200

Iteration 215

Iteration 225

Iteration 240

Iteration 250

Figure 5.67: The ratios $\frac{d}{u}$ for $Q^{2}=2.5 \mathrm{GeV}^{2}$, where $d$ is the distribution of down quarks (sea and valence) and $u$ is the distribution of up quarks (sea and valence), are shown on a two dimensional plot. The $25^{\text {th }}$ and $50^{t h}$ iterations are omitted for clarity. $\frac{d}{u}$ is computed for 36 PDFs per iteration, corresponding to the number of PDFs produced on a $6 \times 6$ map, with only large $x$ Resummations for a given number of iterations. $\frac{d}{u}$ is then computed with only Target Mass Corrections for the same number of iterations. Dimension one is the $\frac{d}{u}$ values when Target Mass Corrections are added. Dimension two is the $\frac{d}{u}$ values when large $x$ Resummations are added. The $x$ axis is dimension one and the $y$ axis is dimension two. The black line corresponsing to $y=x$ along the $y$ and $x$ axis is drawn to highlight the extent of the linear and non linear correlations. The cluster points in different dimension one and two regions represent non linear correlations.

in 5.43, the relative errors were 0.50 for the upper band and 1.07 for the lower band. So the relative error based on clustering of the PDF units was successfully reduced. For a comparison to the statistical error when all $36 \mathrm{PDFs}$ are generated in a $6 \times 6$ map iteration without looking for specific clusters, the PDF curve bundles with an error band formed from their standard deviation are in Figure 5.72 without large $x$ corrections and in Figure 5.73 with large $x$ corrections. 


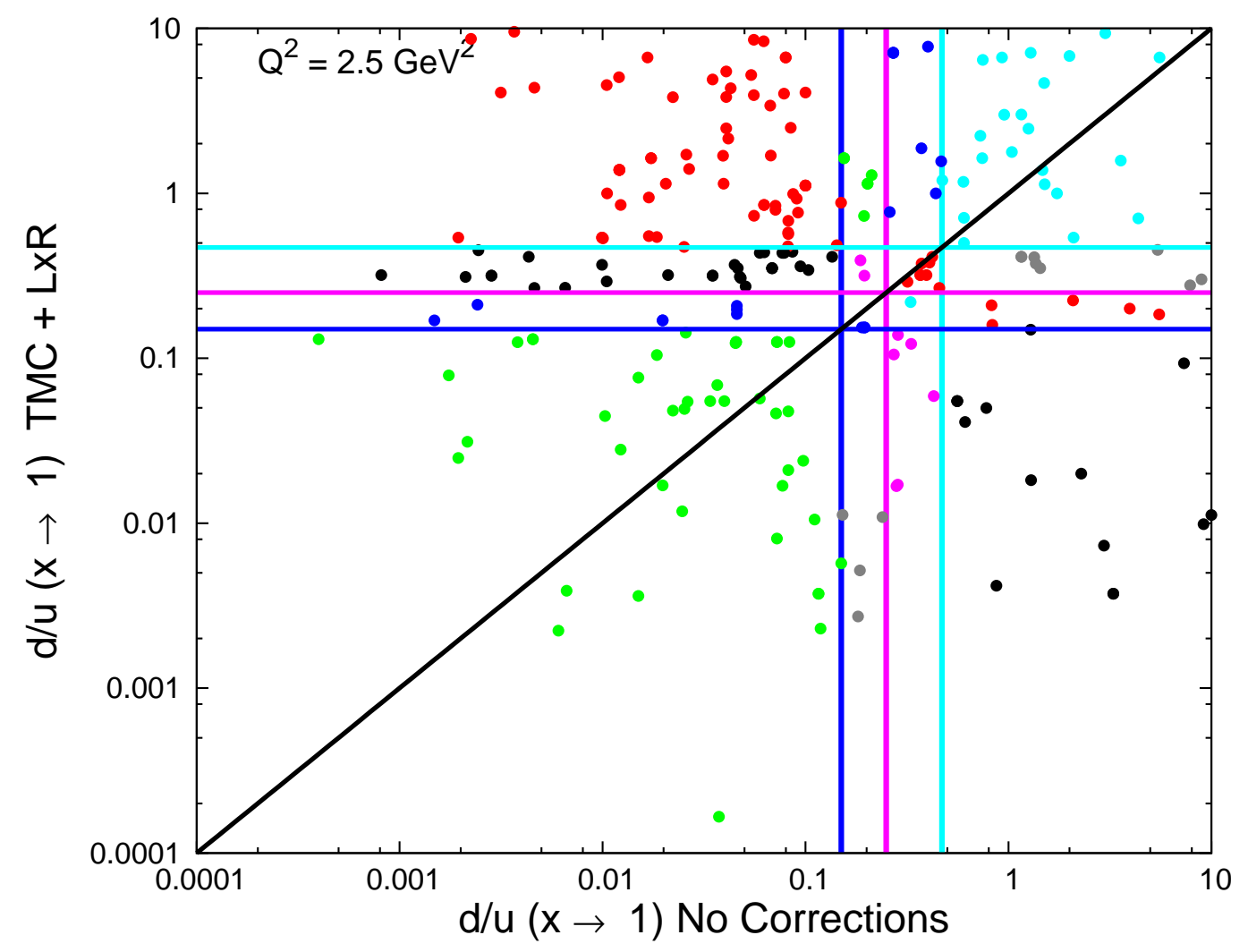

Figure 5.68: Dimensional plots of $\frac{d}{u}$ values are shown with each of the possible $\frac{d}{u}$ regions color coded to represent different $\frac{d}{u}$ sections. The ratios $\frac{d}{u}$ for $Q^{2}=2.5 \mathrm{GeV}^{2}$, where $d$ is the distribution of down quarks (sea and valence) and $u$ is the distribution of up quarks (sea and valence), are shown on a two dimensional plot. $\frac{d}{u}$ is computed for 36 PDFs per iteration, corresponding to the number of PDFs produced on a $6 \times 6$ map, with large $x$ Resummations and Target Mass Corrections for a given number of iterations. $\frac{d}{u}$ is then computed without these corrections for the same number of iterations. Dimension one is the $\frac{d}{u}$ values when neither large $x$ Resummations or Target Mass Corrections are added. Dimension two is the $\frac{d}{u}$ values when these large $x$ corrections are added. The $x$ axis is dimension one and the $y$ axis is dimension two. The black line corresponsing to $y=x$ along the $y$ and $x$ axis is drawn to highlight the extent of the linear and non linear correlations. The cluster points on the black line represent strictly linear correlations. The cluster points in different dimension one and two regions represent non linear correlations. 


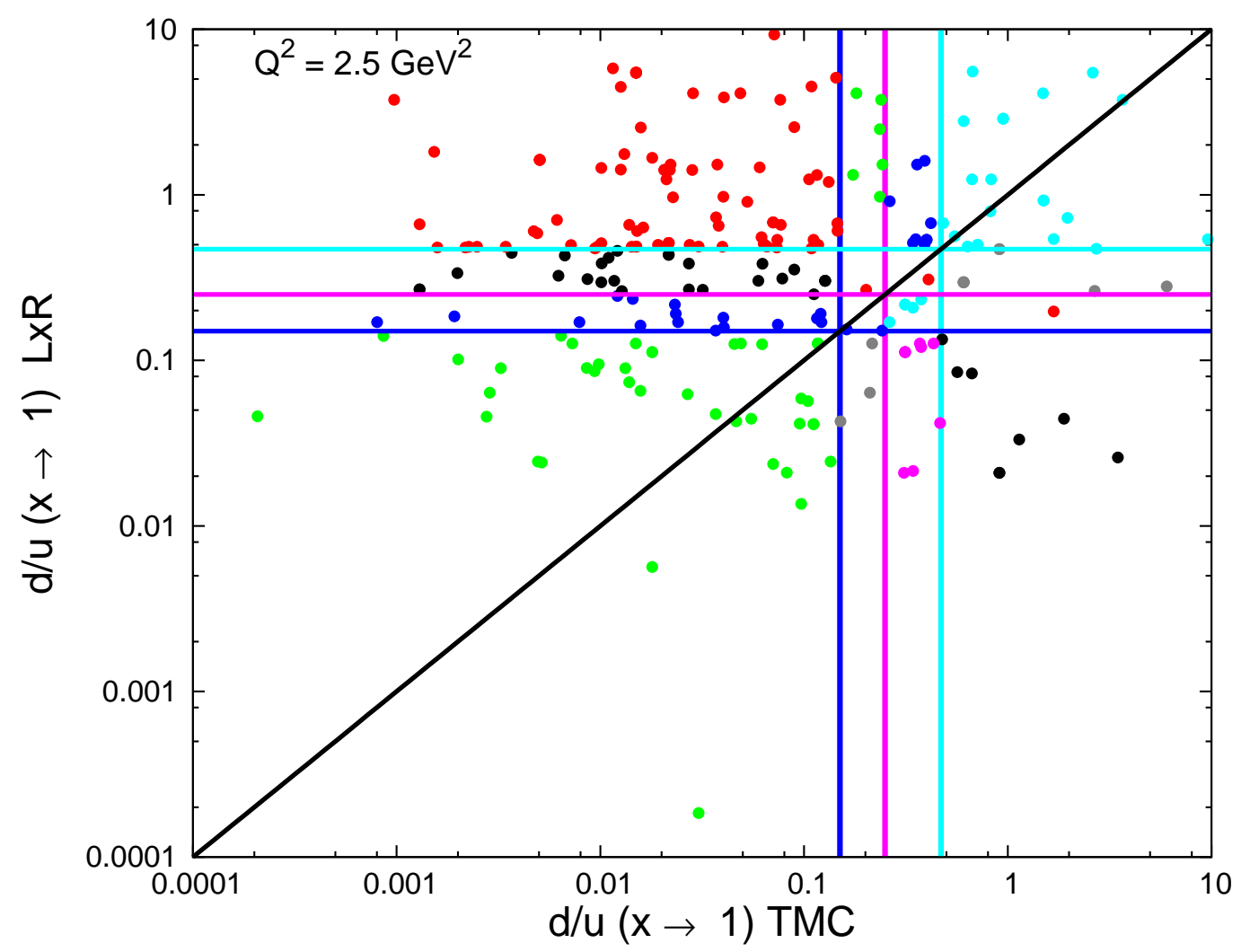

Figure 5.69: Dimensional plots of $\frac{d}{u}$ values are shown with each of the possible $\frac{d}{u}$ regions color coded to represent different $\frac{d}{u}$ sections. The ratios $\frac{d}{u}$ for $Q^{2}=2.5 \mathrm{GeV}^{2}$, where $d$ is the distribution of down quarks (sea and valence) and $u$ is the distribution of up quarks (sea and valence), are shown on a two dimensional plot. $\frac{d}{u}$ is computed for 36 PDFs per iteration, corresponding to the number of PDFs produced on a $6 \times 6$ map, with only large $x$ Resummations for a given number of iterations. $\frac{d}{u}$ is then computed with only Target Mass Corrections for the same number of iterations. Dimension one is the $\frac{d}{u}$ values when Target Mass Corrections are added. Dimension two is the $\frac{d}{u}$ values when large $x$ Resummations are added. The $x$ axis is dimension one and the $y$ axis is dimension two. The black line corresponsing to $y=x$ along the $y$ and $x$ axis is drawn to highlight the extent of the linear and non linear correlations. The cluster points in different dimension one and two regions represent non linear correlations. 


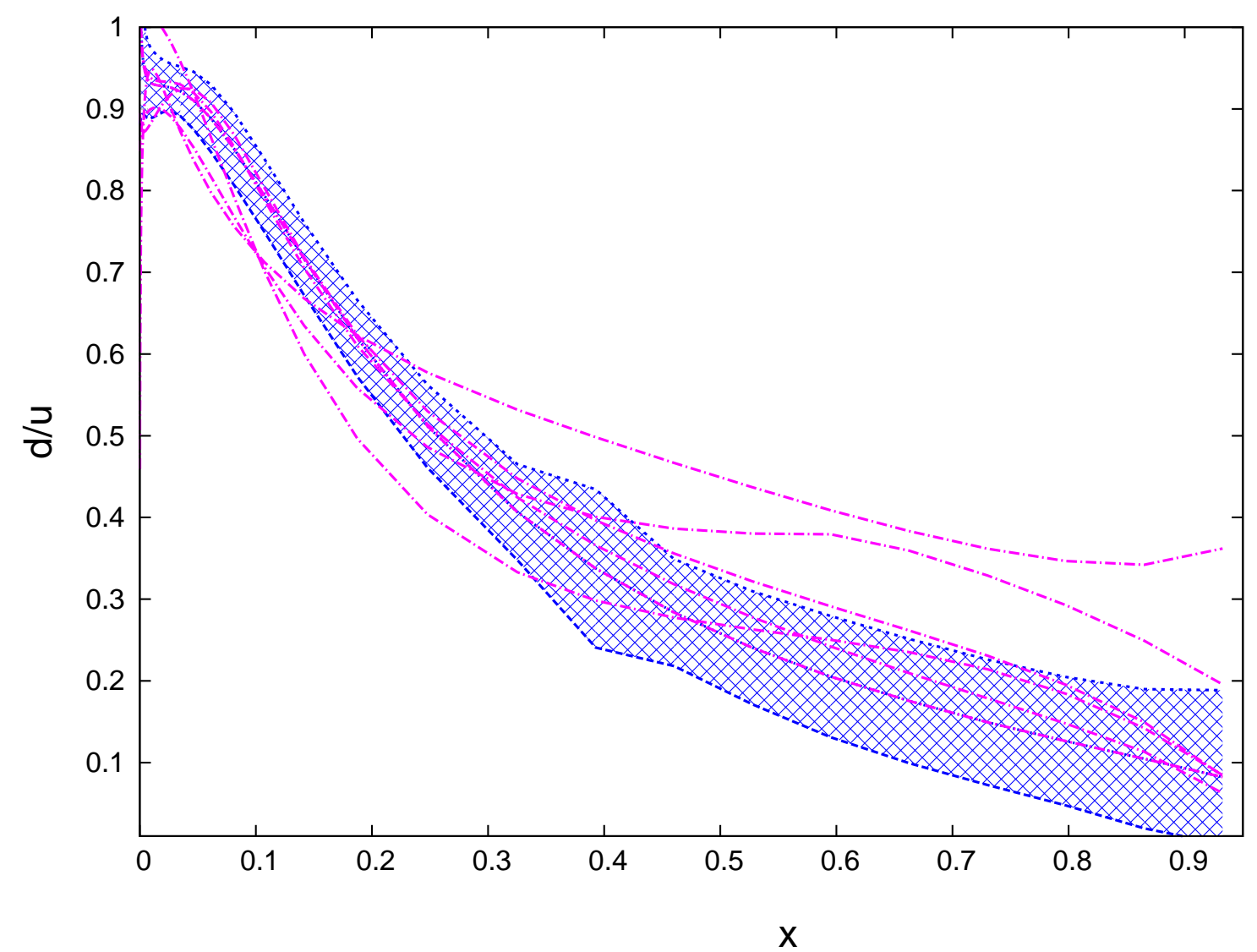

Figure 5.70: The bundle of theoretical $\frac{d}{u}$ curves, taken from a cluster of six PDF units with similarly $\chi^{2}$ and $\frac{d}{u}$ values, at $Q^{2}=2.5 \mathrm{GeV}^{\text {th }}$ generated from the $6 \times 6$ map is shown. Large $x$ Resummations and Target Mass Corrections are not included in these computations. The curve with the lowest $\chi^{2}$ value among this bundle is shown with an error band calculated using the standard deviation of the curve bundle.

\section{Conclusion}

The cross sections of inelastic nuclear scattering reactions have hard components, calculable by perturbative expansions around the strong coupling constant, and "soft" components for which there is a need to create reliable theoretical models. These models come from composite parton models, dependent on parton momentum fraction $x$, which contain a set of $Q^{2}$ dependent parameters. Previous attempts to create these theoretical models have not used the type of networks that we have used. There has not previously been a method to generate these nuclear theoretical models that eliminates bias in fitting them to scattering data and allows for visualization, analysis and classification of the subsequent generated theoretical models. Therefore, the use of unsupervised Artificial 


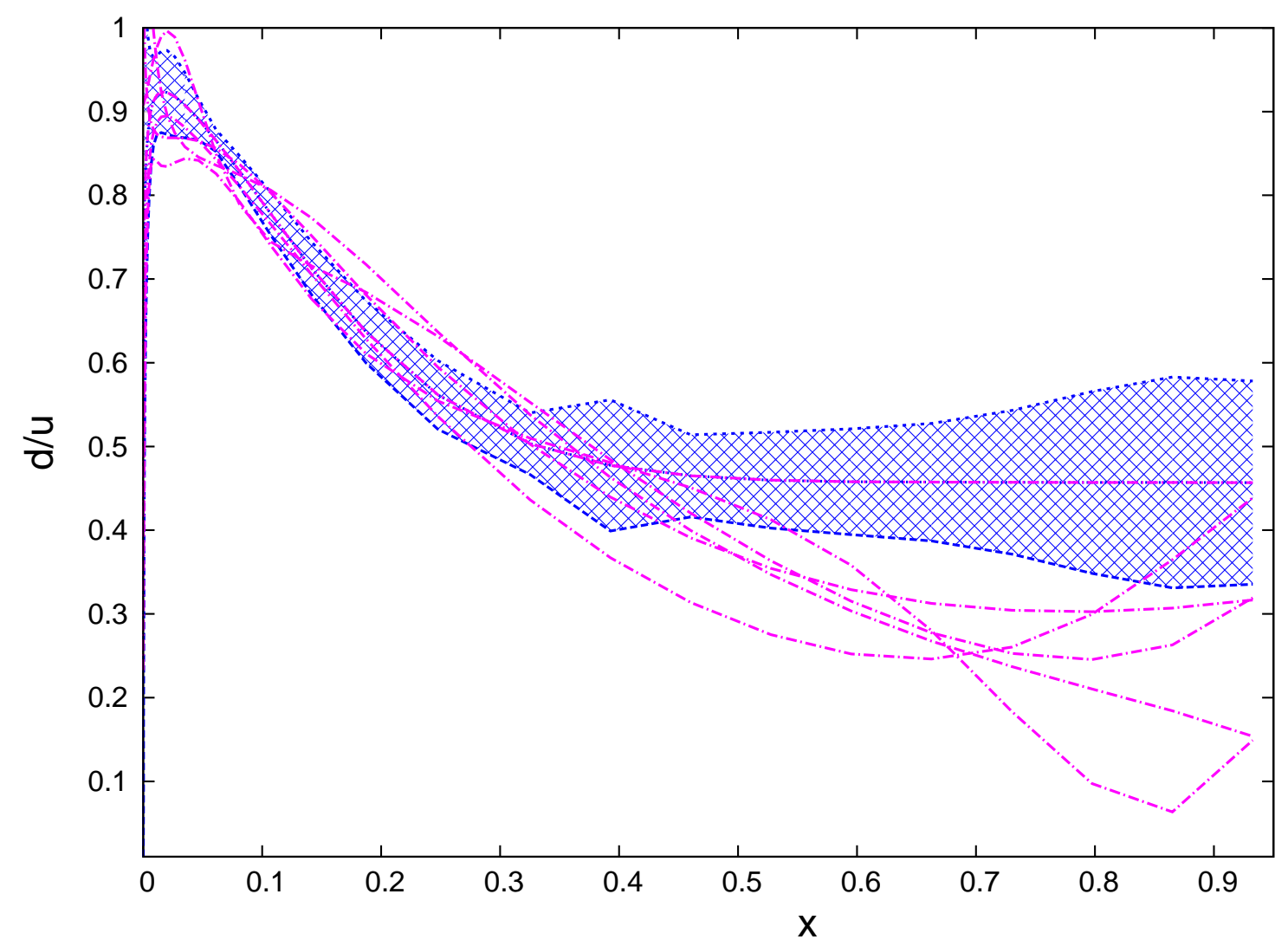

FIGURE 5.71: The bundle of theoretical $\frac{d}{u}$ curves, taken from a cluster of six PDF units with similarly $\chi^{2}$ and $\frac{d}{u}$ values, at $Q^{2}=2.5 \mathrm{GeV}^{\text {th }}$ generated from the $6 \times 6$ map is shown. Large $x$ Resummations and Target Mass Corrections are included in these computations. As a result, the effects of adding the large $x$ corrections on SOM error size is shown with the error band here relative to that of Figure 5.70. The curve with the lowest $\chi^{2}$ value among this bundle is shown with an error band calculated using the standard deviation of the curve bundle.

Neural Networks represented an unexplored procedure for probing inelastic scattering reactions and creating reliably generated theoretical parton models. This method of creating the parton models and attempt to maximize the structure functions they comprise to scattering data sets represented a practical and useful way to create unbiased parton models and structure functions.

The SOM and the GA were also proven to be uniquely successful in creating an unbiased set of theoretical PDF curves whose composite structure functions are capable of fitting experimental data. Therefore, this Artificial Neural Network was capable of achieving the same fundamental task as the supervised NNPDF network. The SOM and 


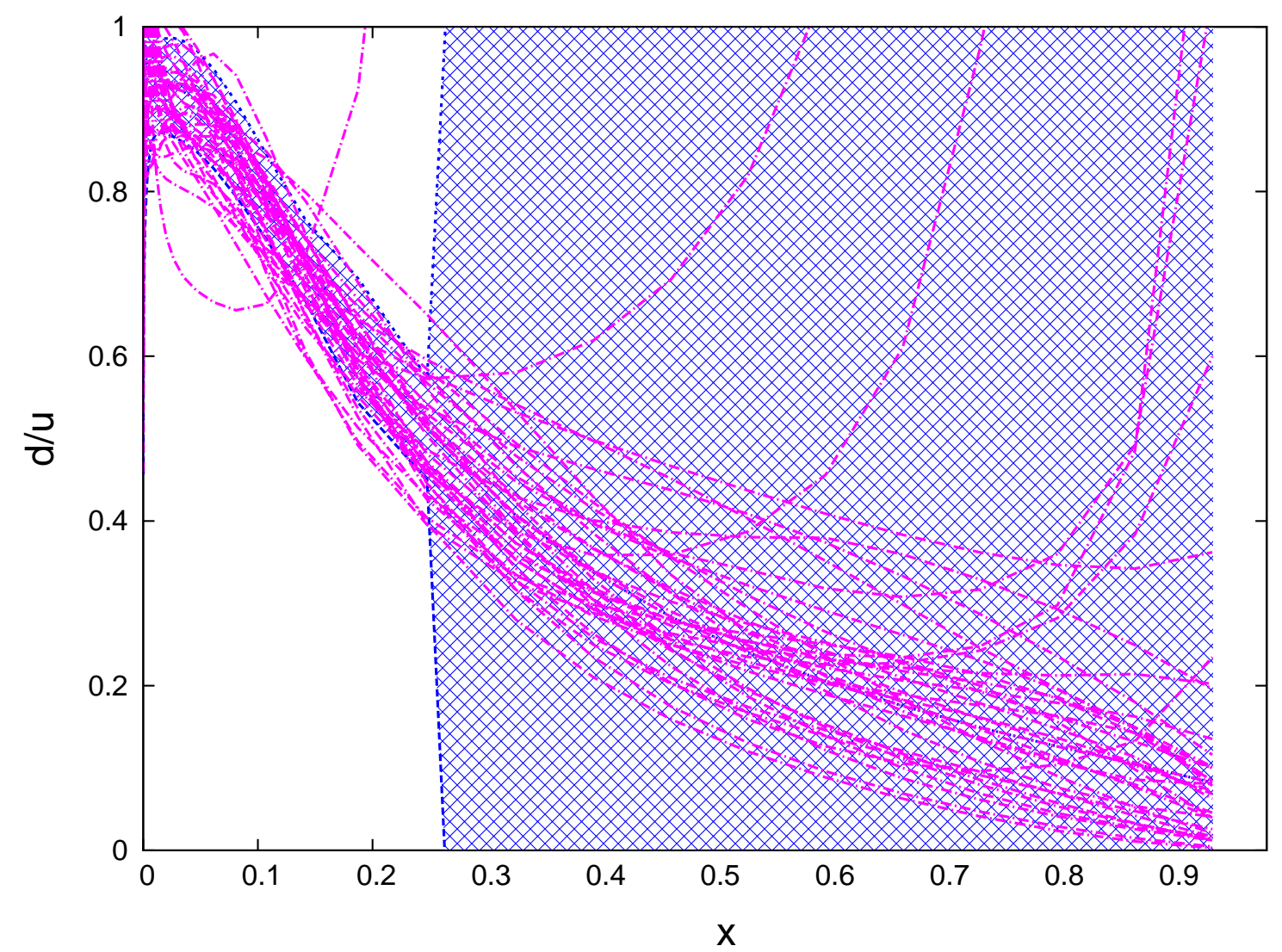

Figure 5.72: The bundle of theoretical $\frac{d}{u}$ curves, without Large $x$ Resummations and Target Mass Corrections added, for $Q^{2} \stackrel{u}{=} 2.5 \mathrm{GeV}^{\text {th }}$ generated from the $6 \times 6$ map is shown for all of the PDFs the $6 \times 6$ map generates. The curve with the lowest $\chi^{2}$ value among this bundle is shown with an error band calculated using the standard deviation of the curve bundle. The large error band, which results from using all the generated PDF curves regardless of how well they fit the data, reveals the necesseity of using clusters to generate a viable statistical error band.

GA method could also be applied simultaneously with the Lagrange Multiplier method to get an error determination that depended uniquely on the formation of the best fitting PDFs from semi random free parameter variation. In addition, the SOM network enabled us to identify clusters based on critical features that the generated PDFs had in common with each other. The values of the PDFs, for various kinematical ranges, that most effectively illustrated the effects of physical corrections for specific kinematics formed clusters according to how well they fit the scattering data and their behaviors in these kinematic ranges of interest. Due to the large number of varying free parameters and possible behaviors of the PDFs at various kinematics, the theoretical PDFs, when generated and assigned to map units, represented data with substantially more 


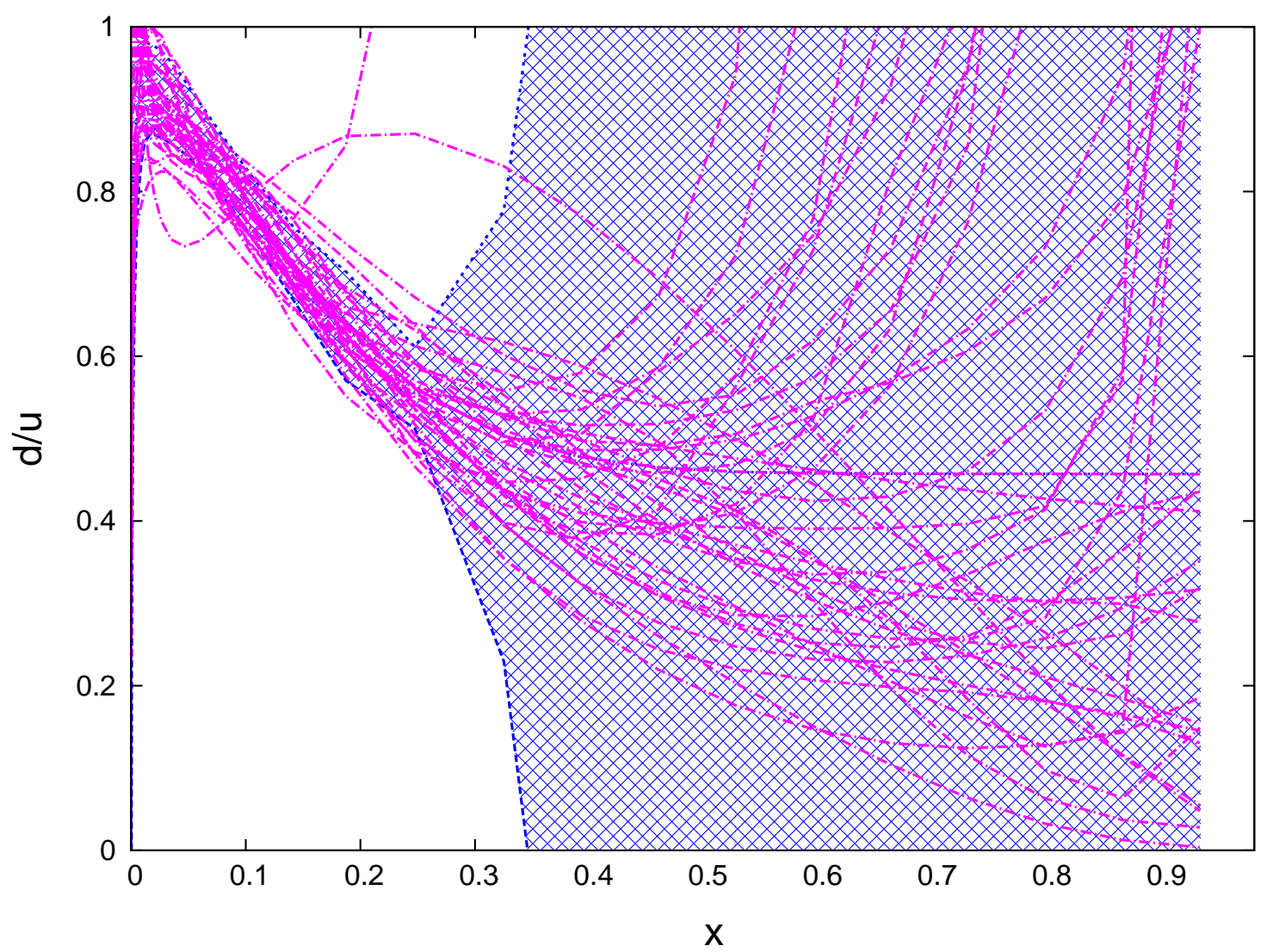

Figure 5.73: The bundle of theoretical $\frac{d}{u}$ curves, with Large $x$ Resummations and Target Mass Corrections added, for $Q^{2}=2.5 \mathrm{GeV}^{\text {th }}$ generated from the $6 \times 6$ map is shown for all of the PDFs the $6 \times 6$ map generates. Therefore, the effects of adding the large $x$ corrections on statistical error size is shown with the error band here relative to that of Figure 5.72. The curve with the lowest $\chi^{2}$ value among this bundle is shown with an error band calculated using the standard deviation of the curve bundle. The large error band, which results from using all the generated PDF curves regardless of how well they fit the data, reveals the necesseity of using clusters to generate a viable statistical error band.

than two dimensions. The SOM enabled us to reduce these PDFs to two dimensional representations with non linear relationships among data units which grouped together in map clusters.

These clusters could be identified based on the quality of fit of resulting structure functions to experimental data results, behaviors of PDF types for smaller values of $x$ or for their values in the limit $x \rightarrow 1$. When PDF clusters based around the quality of their fit to data were formed, this enabled us to identify multiple local minima in the PDF fitting procedure that would not have been made apparent without using the SOM to group 
the fit values into two dimensional arrays of data units. The PDFs at an $x$ range from zero to one could also be placed as data units on a SOM to see how the fitting procedure and use the neighborhood radius function forms non linear PDF clusters based on their behaviors over small $x$ and large $x$ values. The formation of clusters as a result of the fitting algorithm and neighborhood function could be observed for every type of quark and gluon PDF.

The large $x$ physics in particular, as a result of the SOMs, could be quantified based on groups of large $x$ PDF values under various conditions. When the ratios of down valence and sea quarks to up valence and sea quarks were plotted, they could be grouped into clusters of data units based on physical quark models that correspond to these ratios in the large $x$ limit. This map could be set up with large $x$ PDF data units corresponding to the conditions placed on the quarks at large $x$ and without these corrections added. When the physics effects on quarks with large momentum fractions were added in, the SOM provided a way to determine how the structure function fitting procedure and the large $x$ corrections applied together resulted in the formation of PDFs with fundamentally different quark models. The SOM also allowed us quantify how the cluster formations based on physical models were effected as a result of adding large $x$ corrections. The result was a visualization and determination of how adding the physics corrections for quarks with large parton momentum fractions led to the best fitting PDFs conforming to various physical models. With the addition of large $x$ Corrections, clusters of $\frac{d}{u}$ data units for which $\frac{d}{u} \rightarrow 0$ or $\frac{d}{u} \rightarrow 0.2$ without the Corrections added were broken up at a much higher rate than $\frac{d}{u}$ data units with higher $\frac{d}{u}$ values before the Corrections were added. This showed how adding the physics Corrections for large quark momentum fractions lead to a particularly strong breakup of clusters for the $S=0$ qq and $S_{z}=0$ qq conditions. This essentially showed the capability of the SOMPDF fitting procedure, using various large $x$ conditions, to obtain desired physical models. In addition, the Self Organizing Process and GA could be used to extract errors of the PDFs, which resulted in a reduced relative error when used in tandem with large $x$ corrections.

Since the PDFs and their fit to experimental scattering points represented data units with many different dimensions, the SOM provided a way to reduce these data units into two dimensional map representations so they could be clustered and subsequently classified in a manner that would be impossible with previously used supervised networks or with PCA. Therefore, the SOM and the GA combined represented a novel method to 
probe complex theoretical models of nuclear scattering components for which complete theoretical solutions do not yet exist.

This method has shown to be an invaluable method for probing more complicated theoretical nuclear models, in particular the Generalized Parton Distributions, or GPDs, in the future. This is largely because of how the SOM and GA allowed for extrapolation of the models to kinematical regions where experimental data are scarce. The GPDs will depend on a greater number of kinematics than the PDFs that were analyzed with this SOM. Subsequently, these distributions will have an even higher number of possible dimensions and parameters to analyze then the PDFs that have currently been probed with the SOM and GA methods. There will be an even greater need with the GPDs for a procedure that generates unbiased theoretical models of these curves that can be analyzed in terms of their quality of fit and the GPD behavior for various kinematics. Therefore, the use of the Self Organizing Process and the GA in tandem to reduce their dimensions, cluster and classify their properties and extract GPD errors will be of greater interest in future computations. 


\section{Appendix A}

The following is the gpdeval.f90 code used for evaluating the $\chi^{2}$ values of the structure functions after the theoretical PDFs are generated.

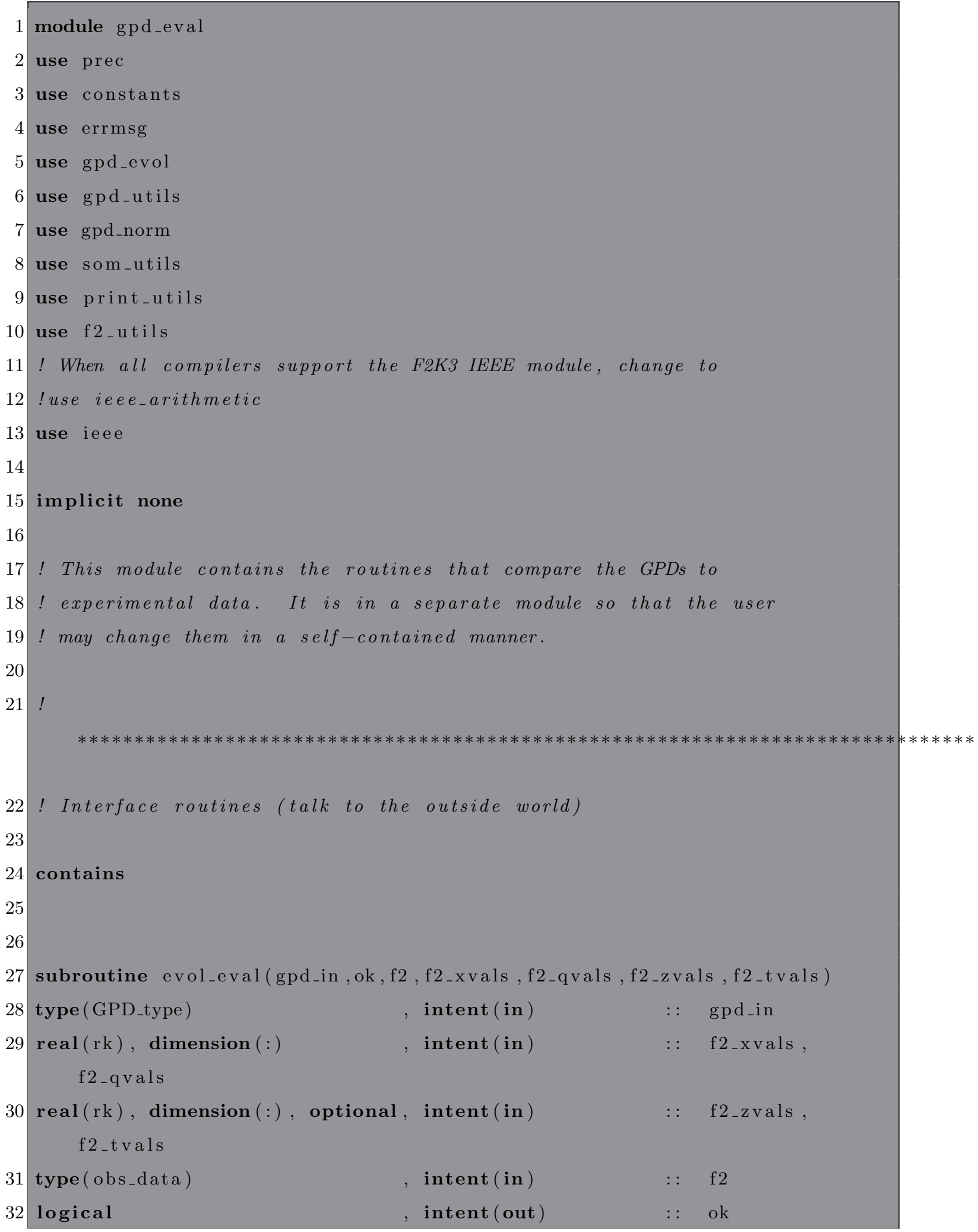




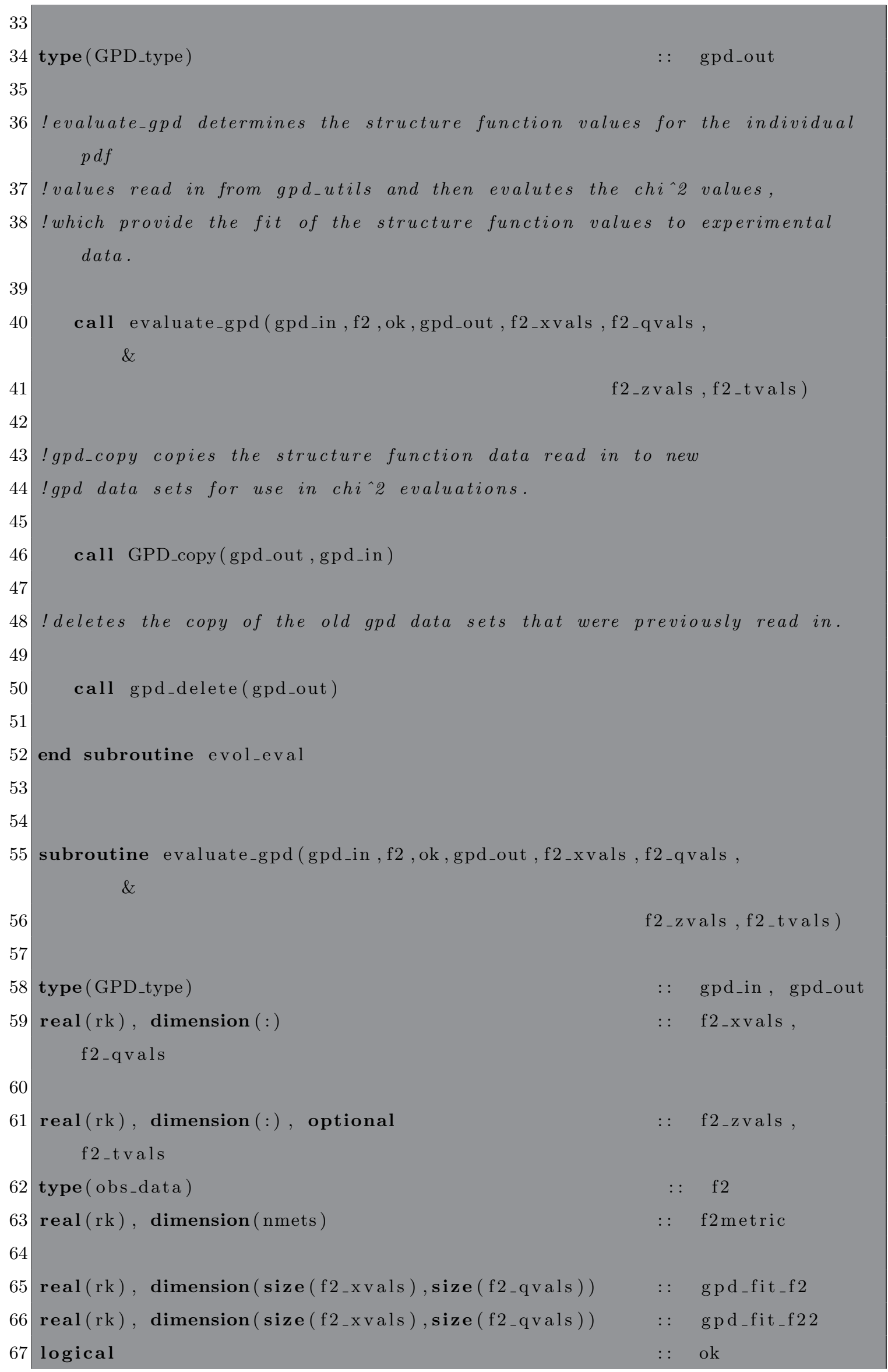




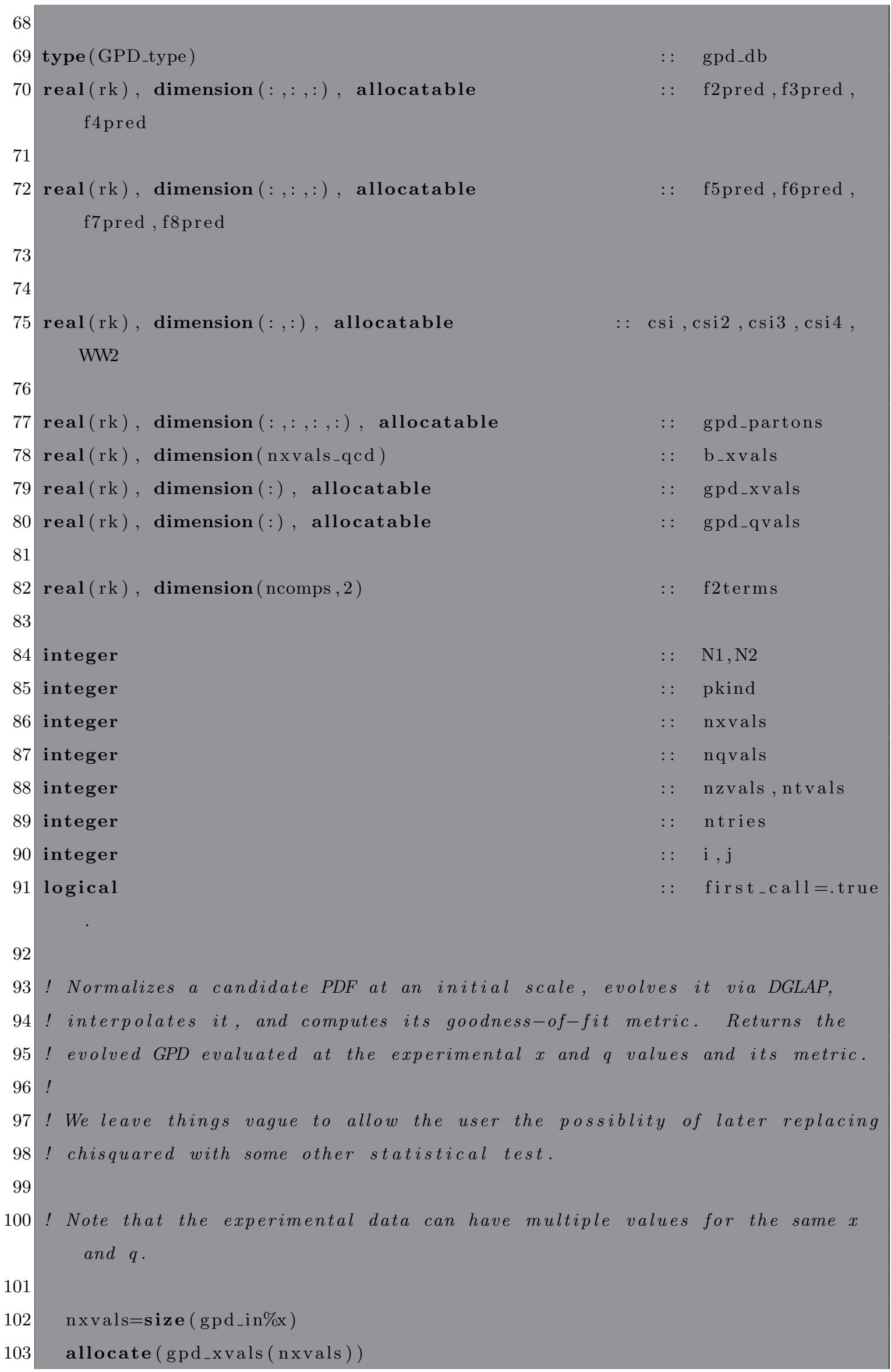




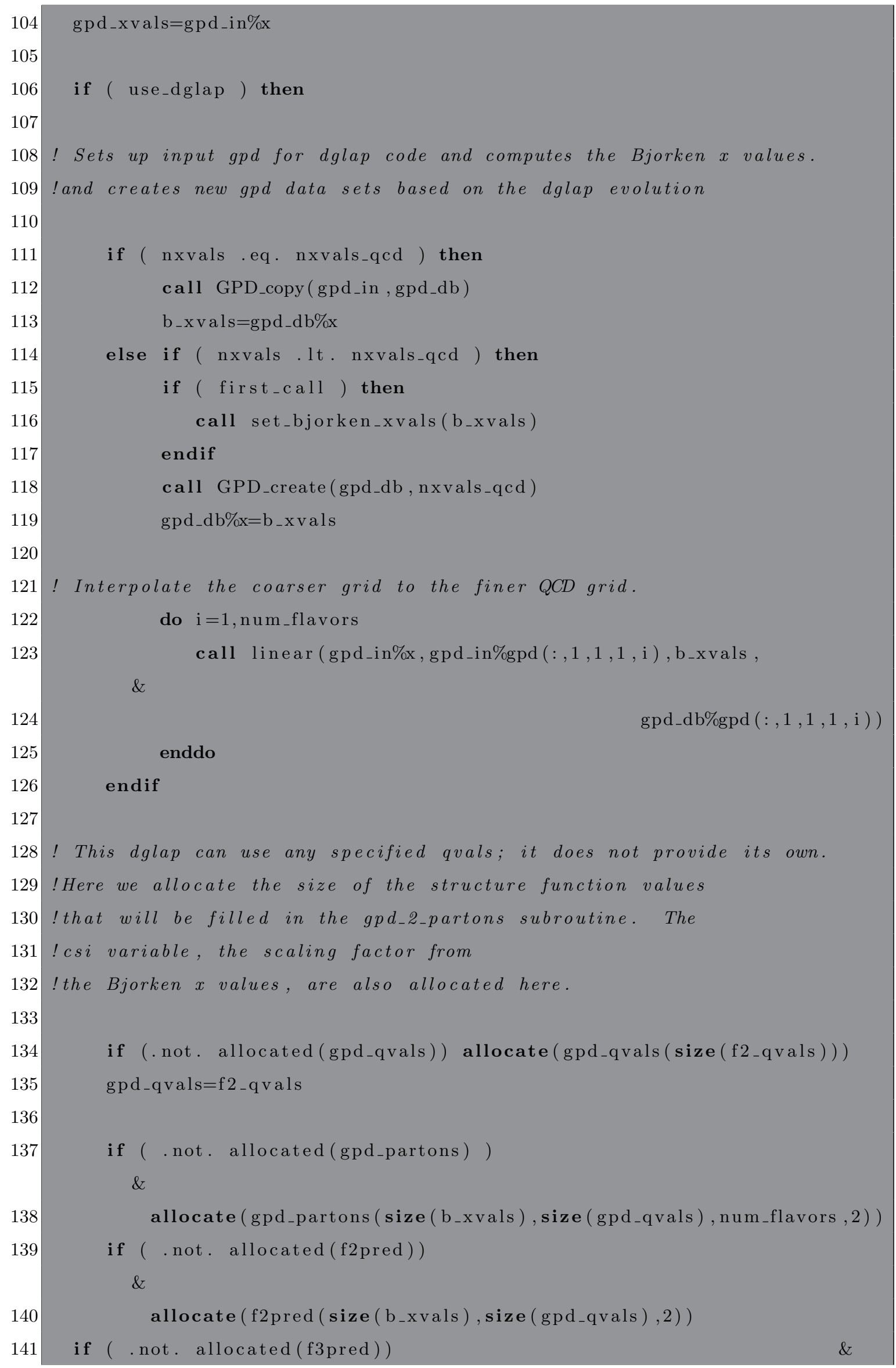




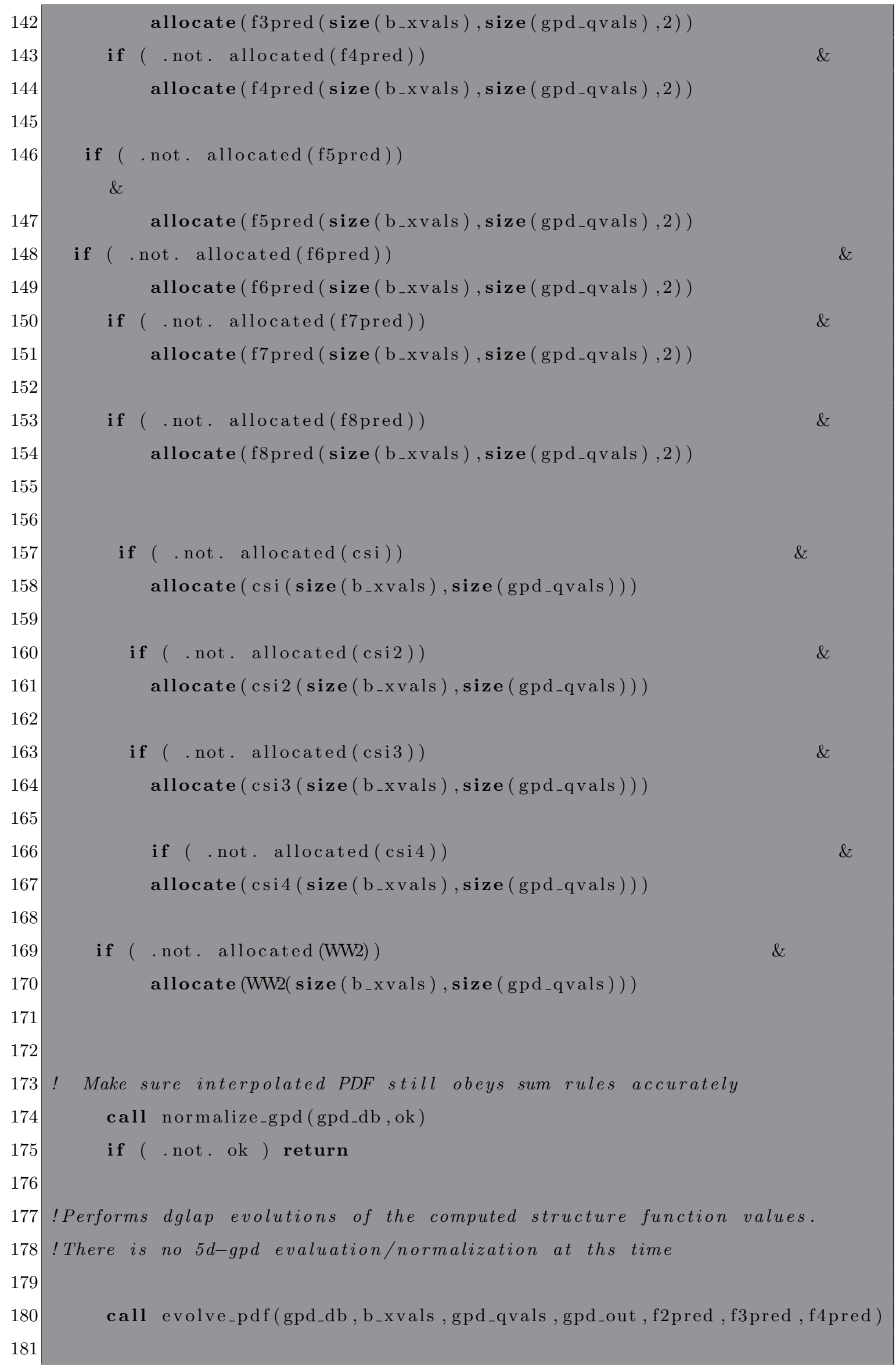




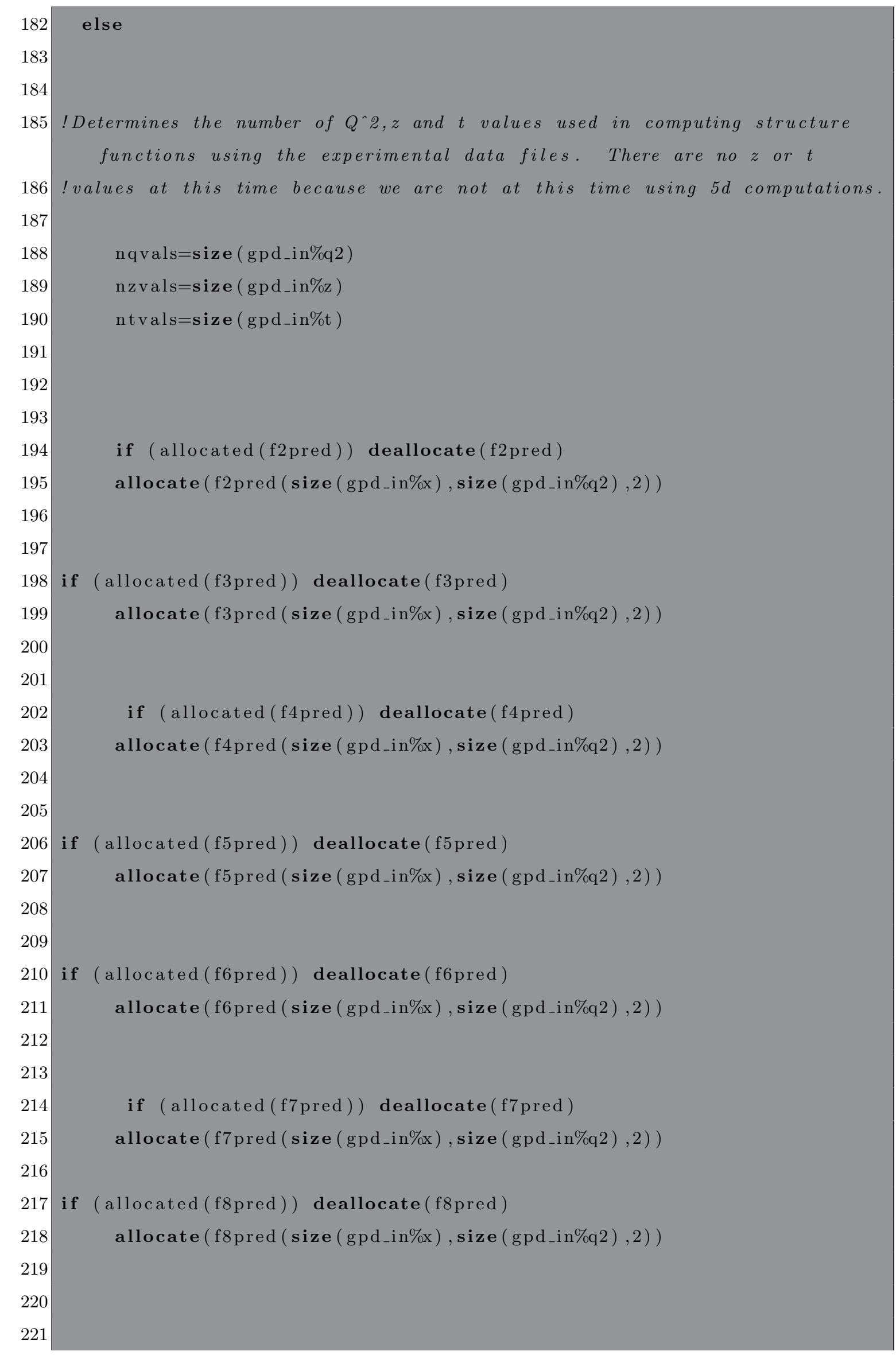




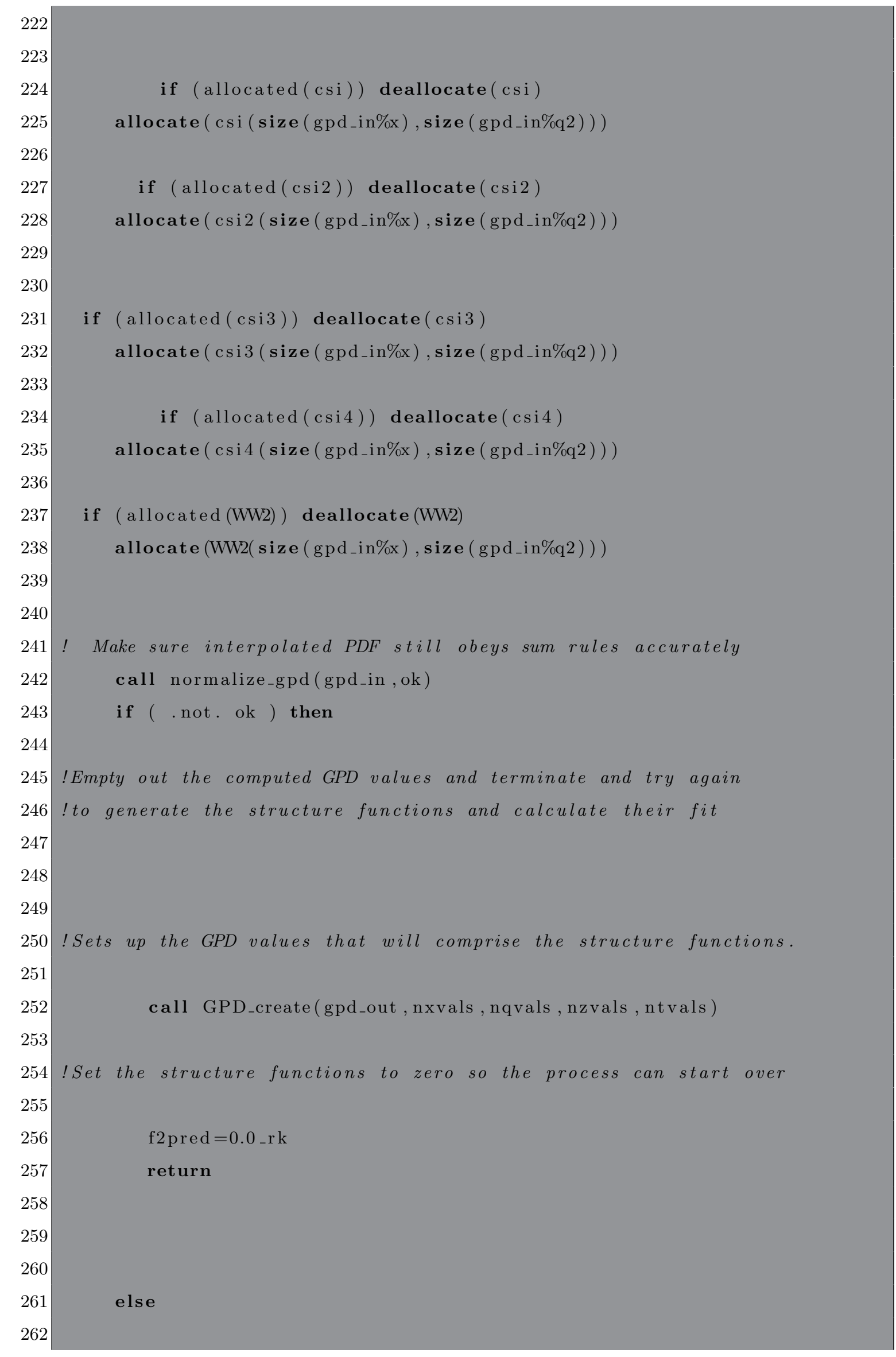




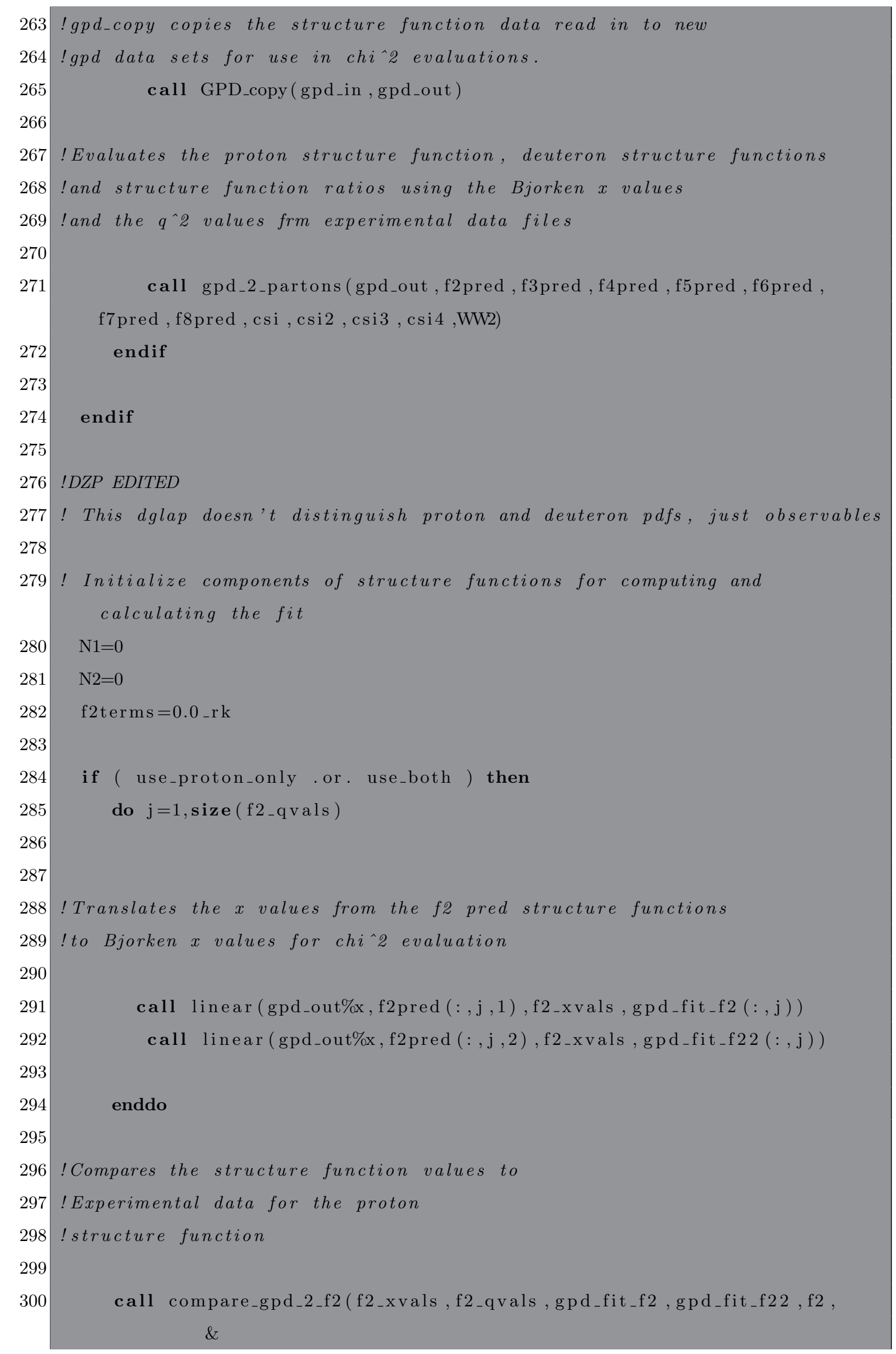




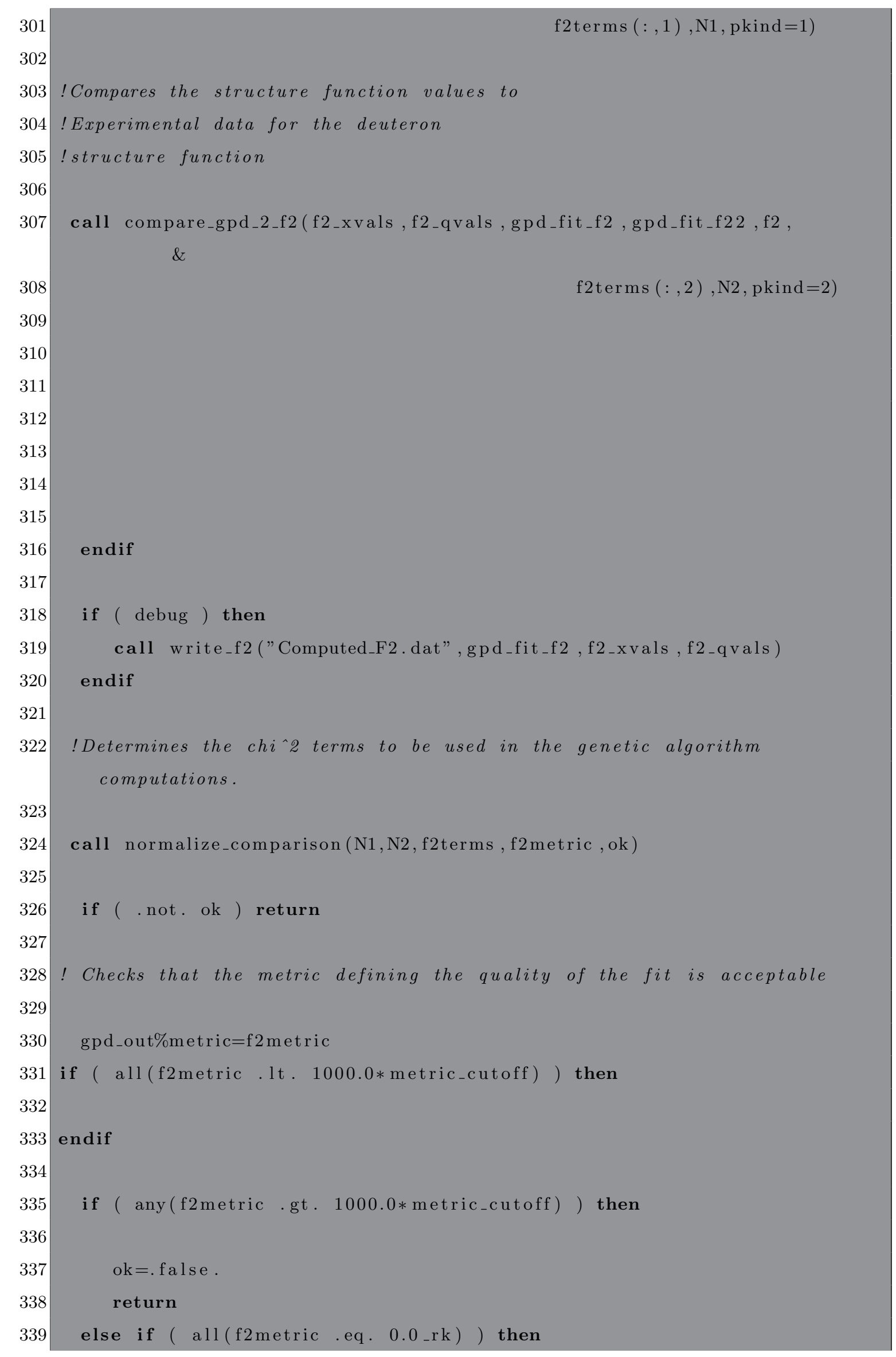




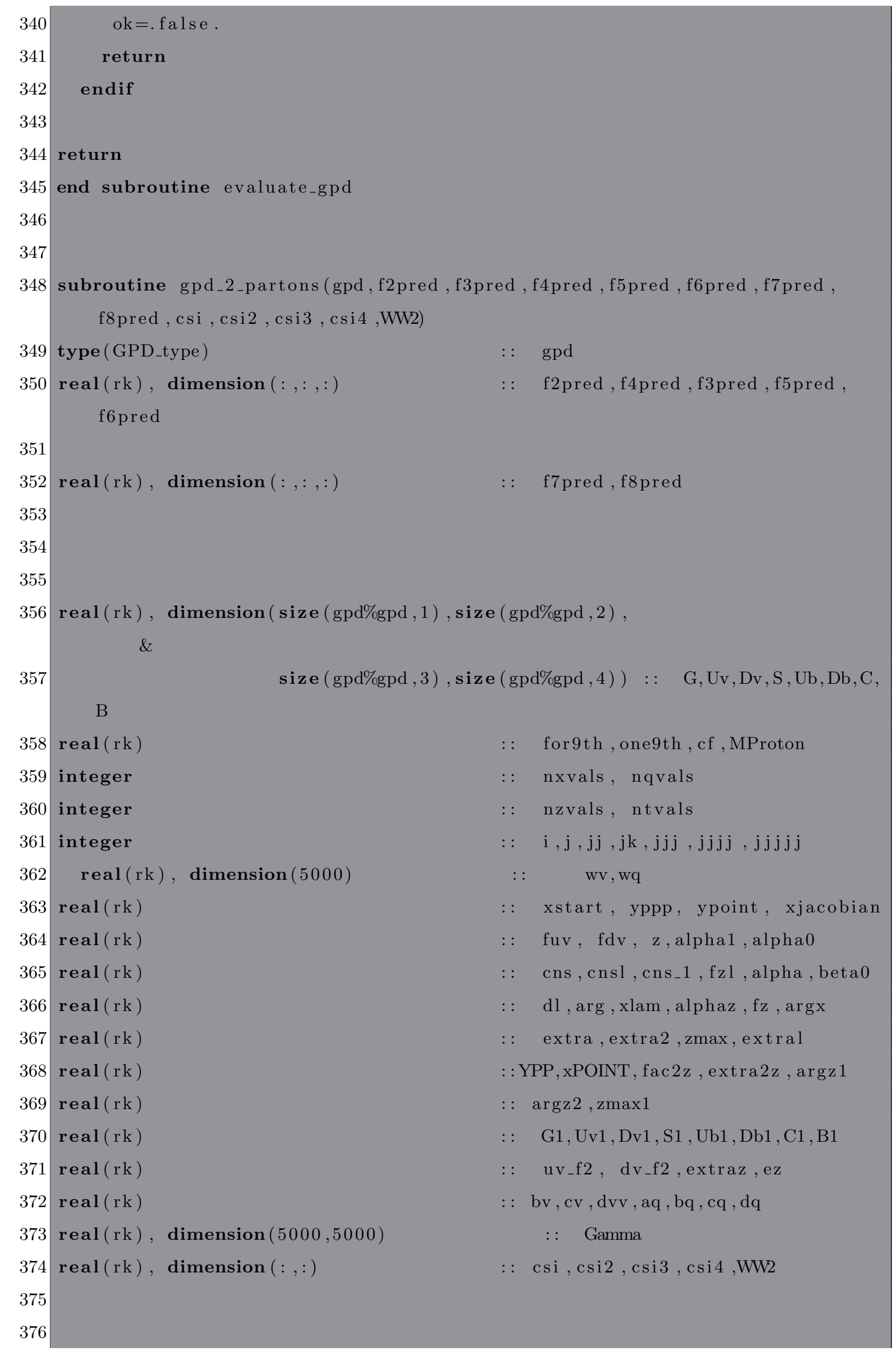




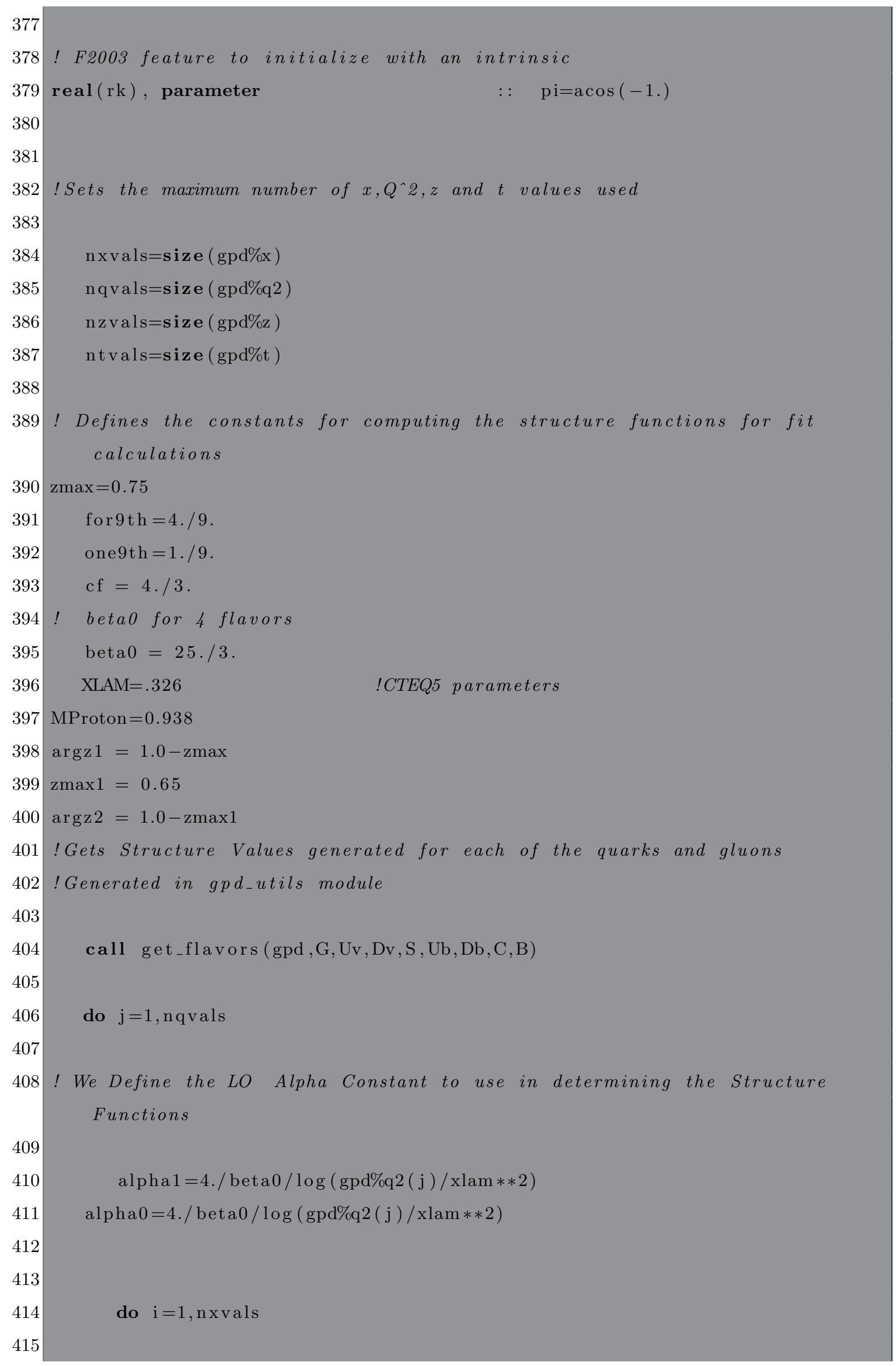




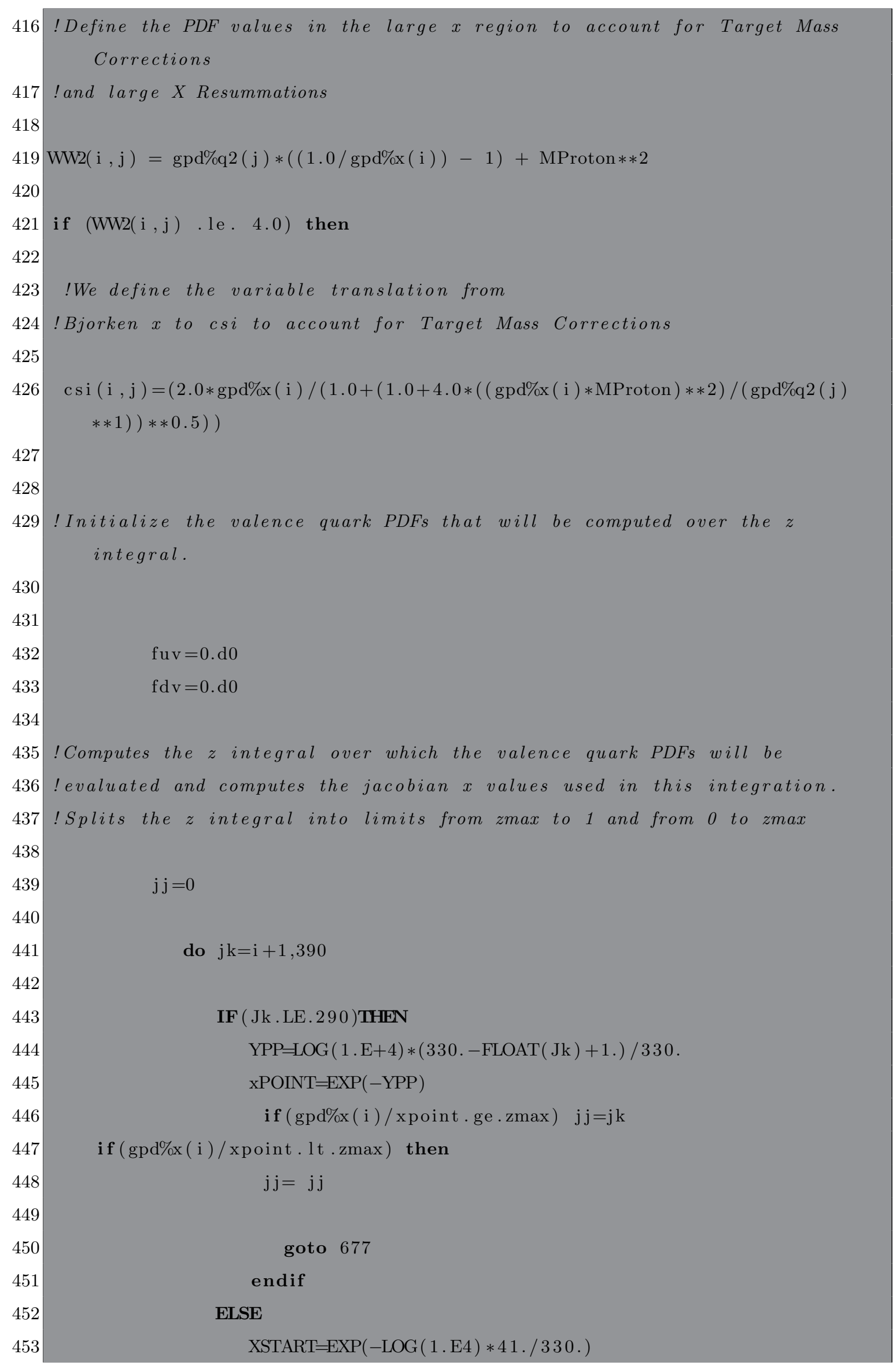




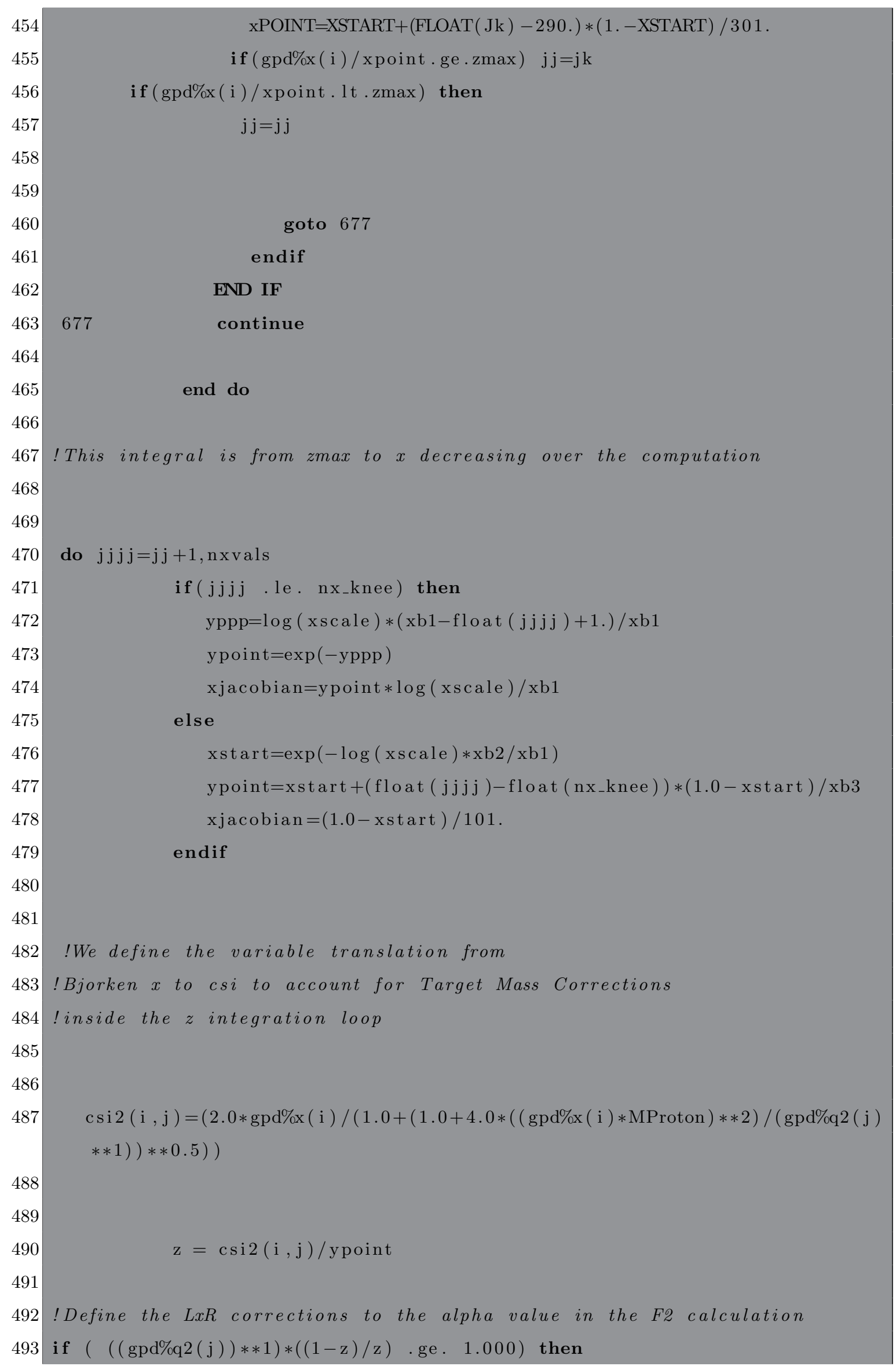




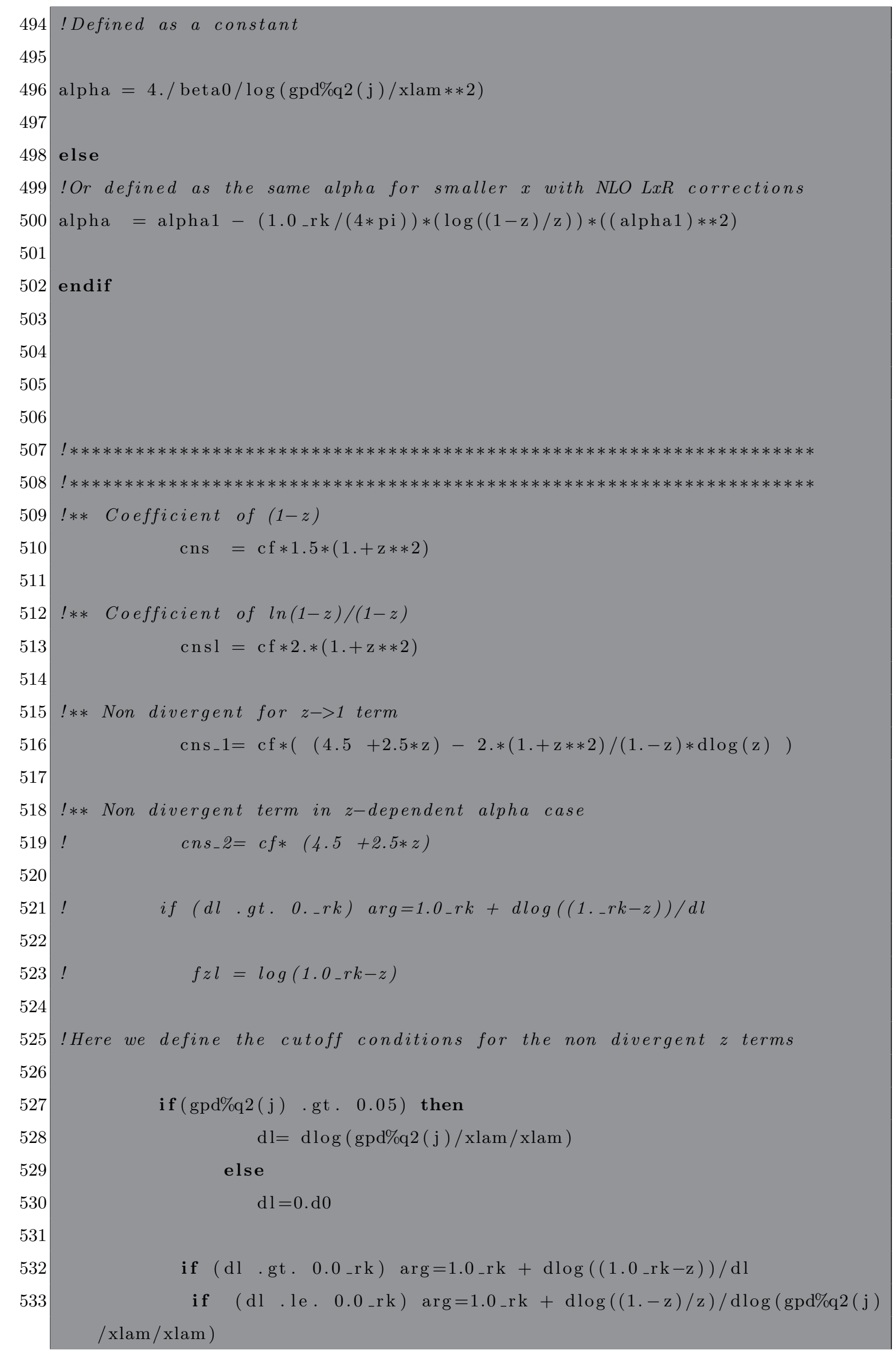




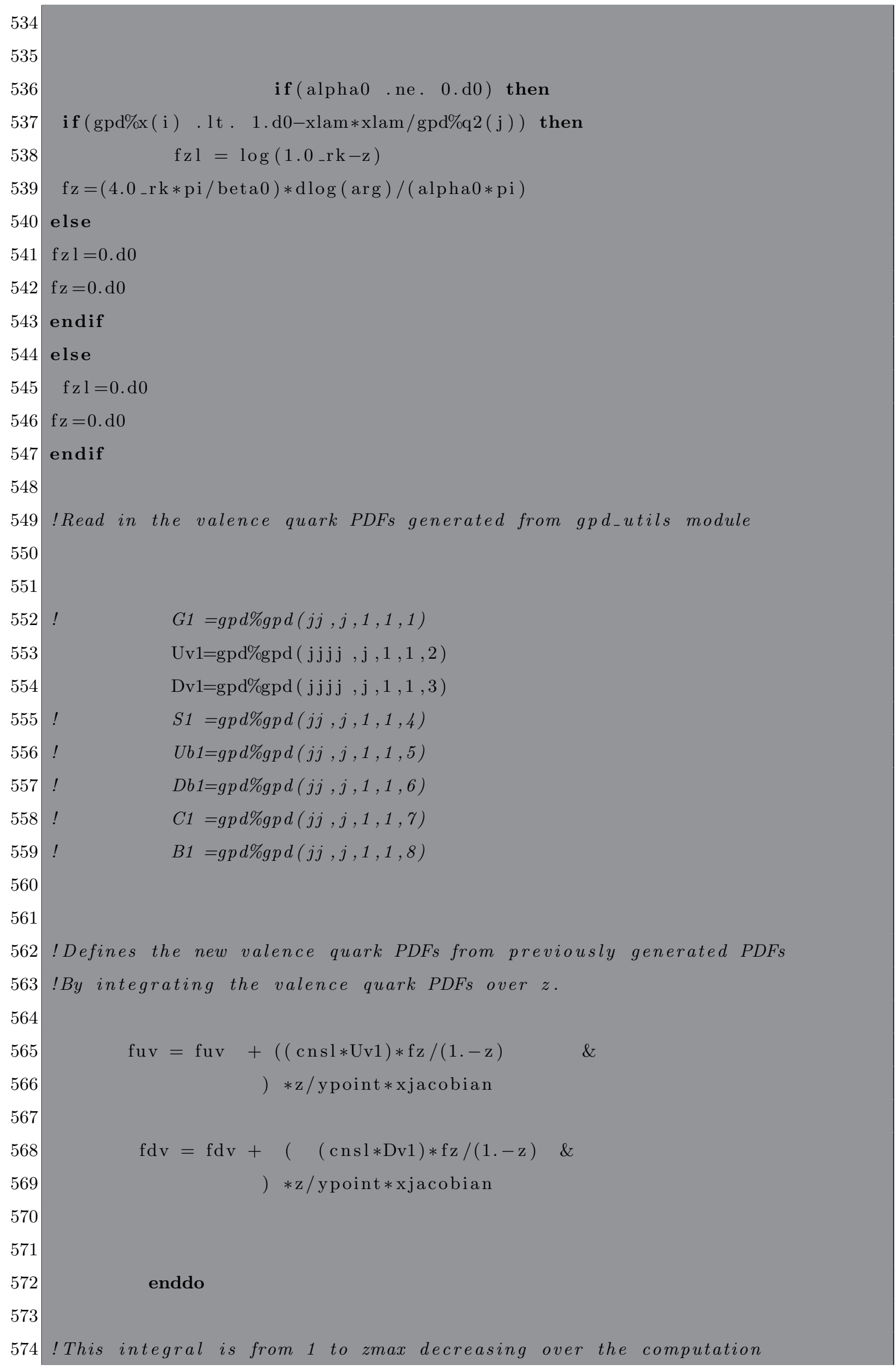




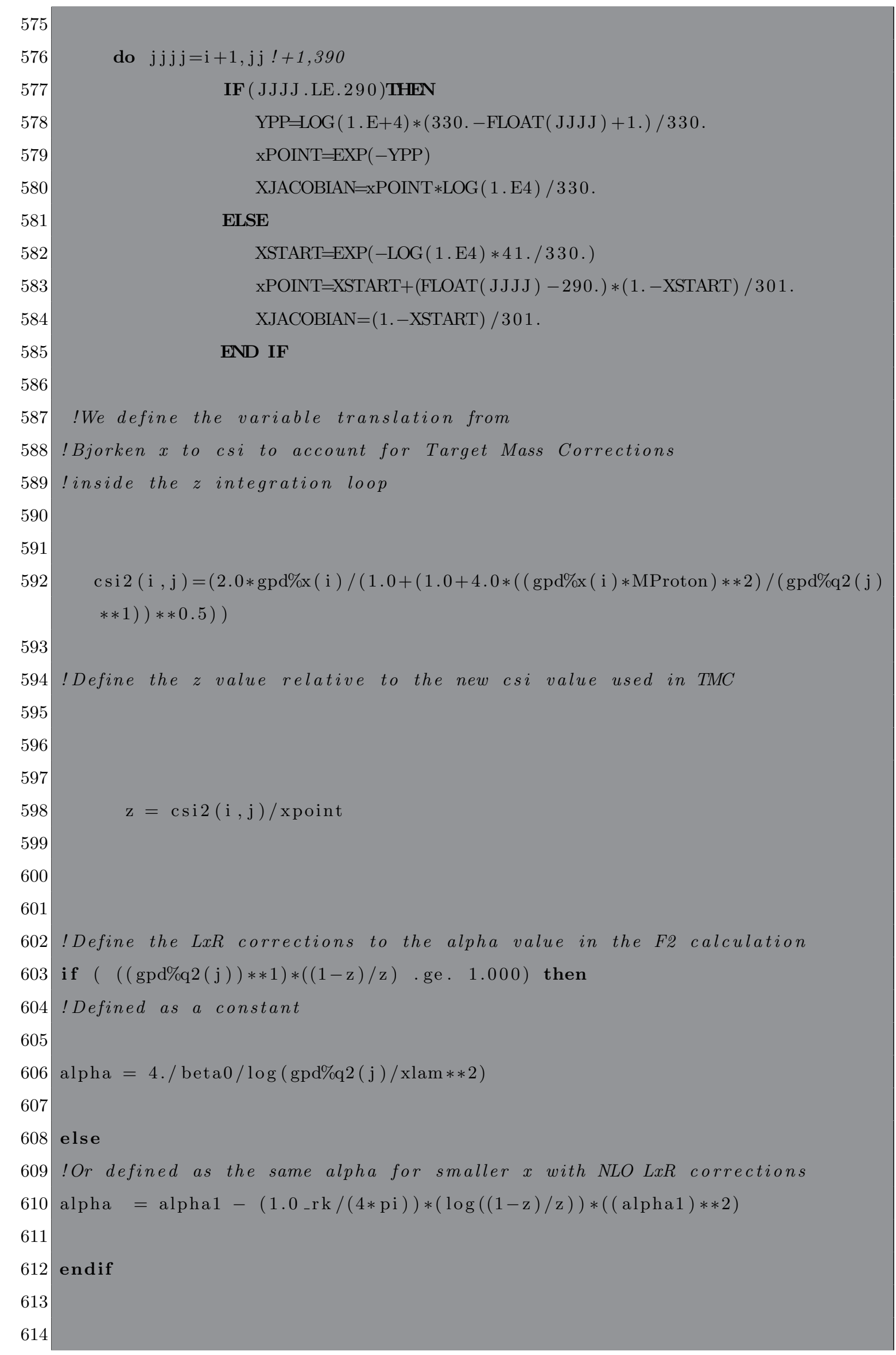




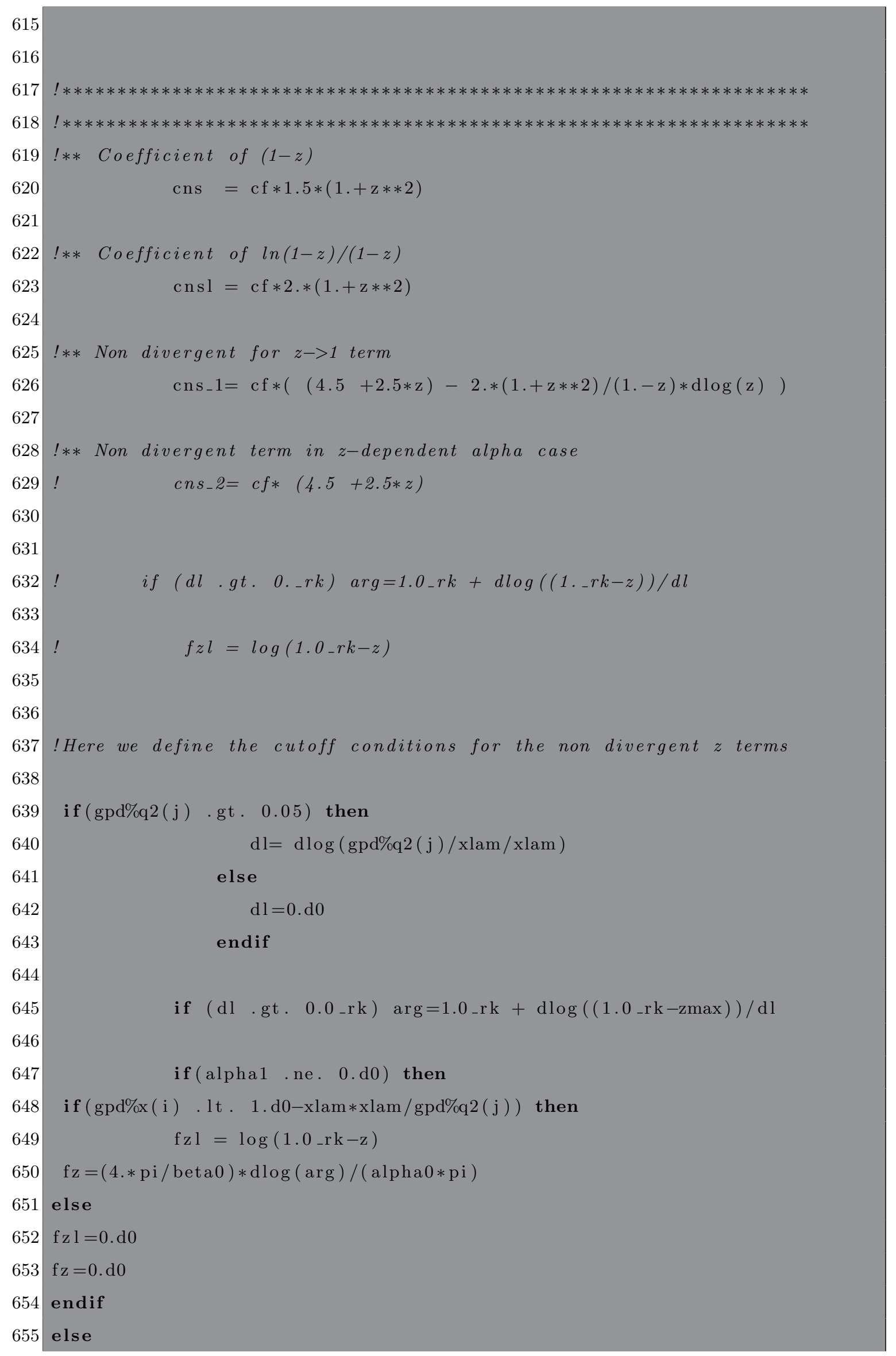




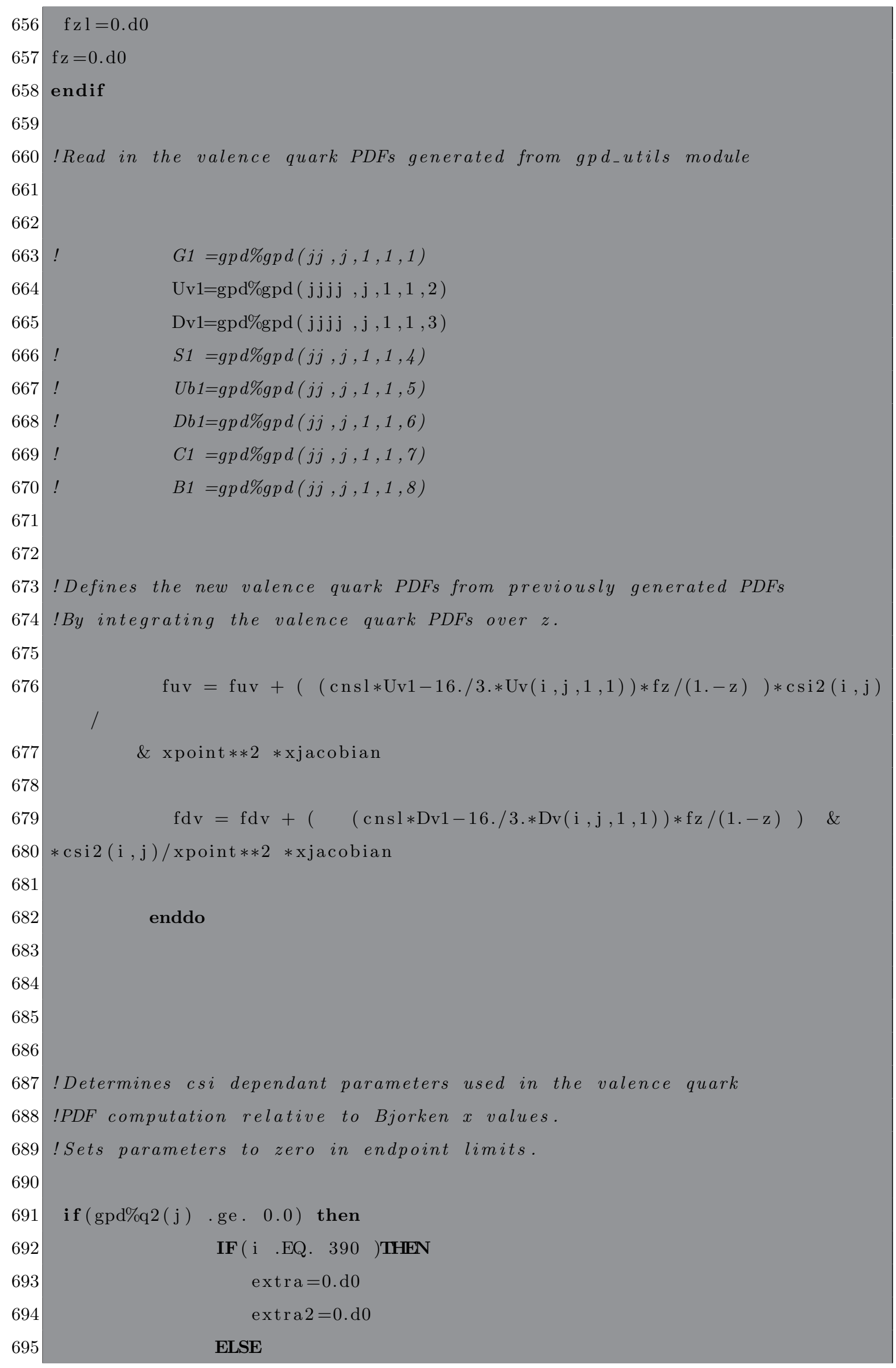




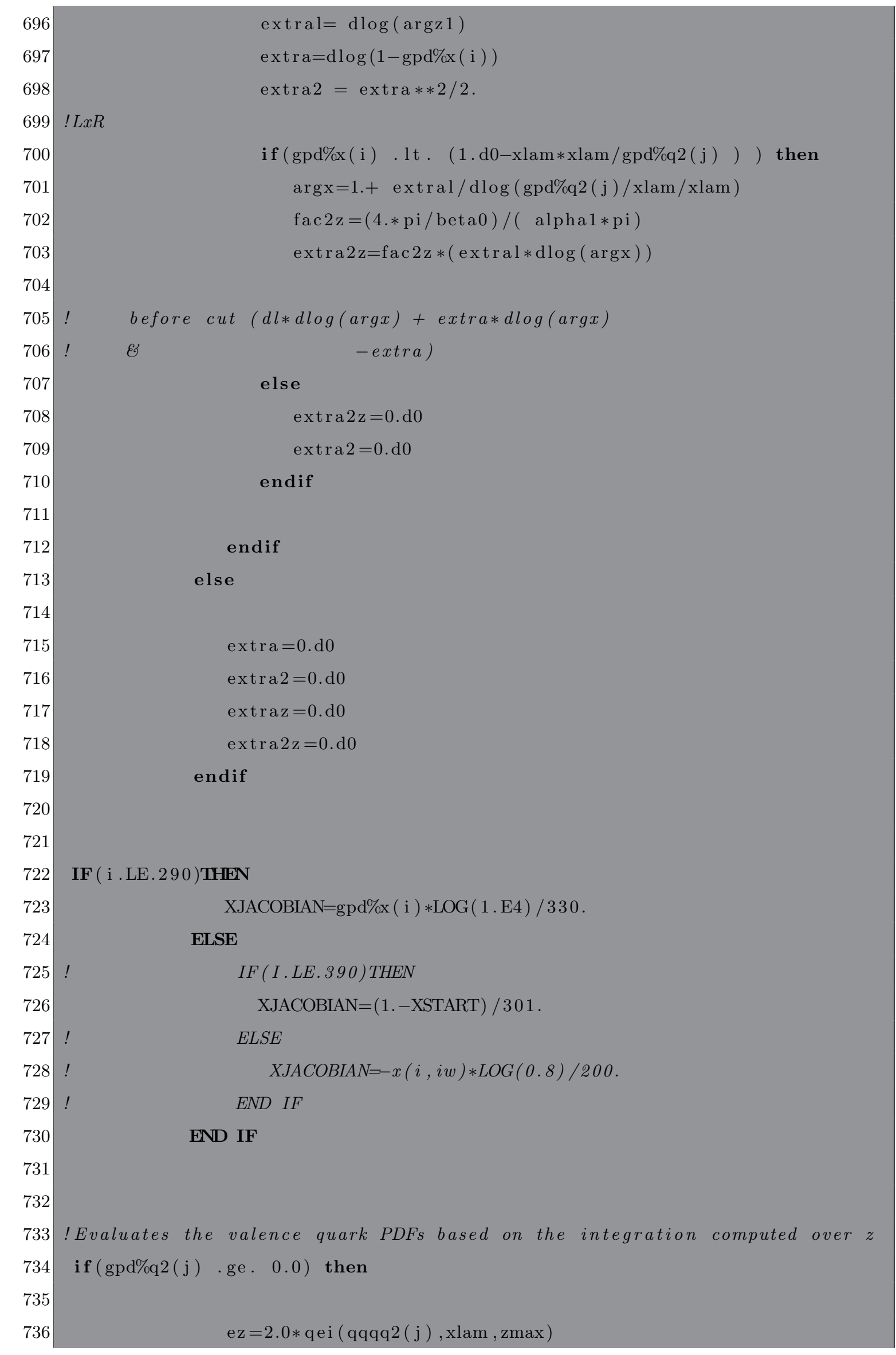




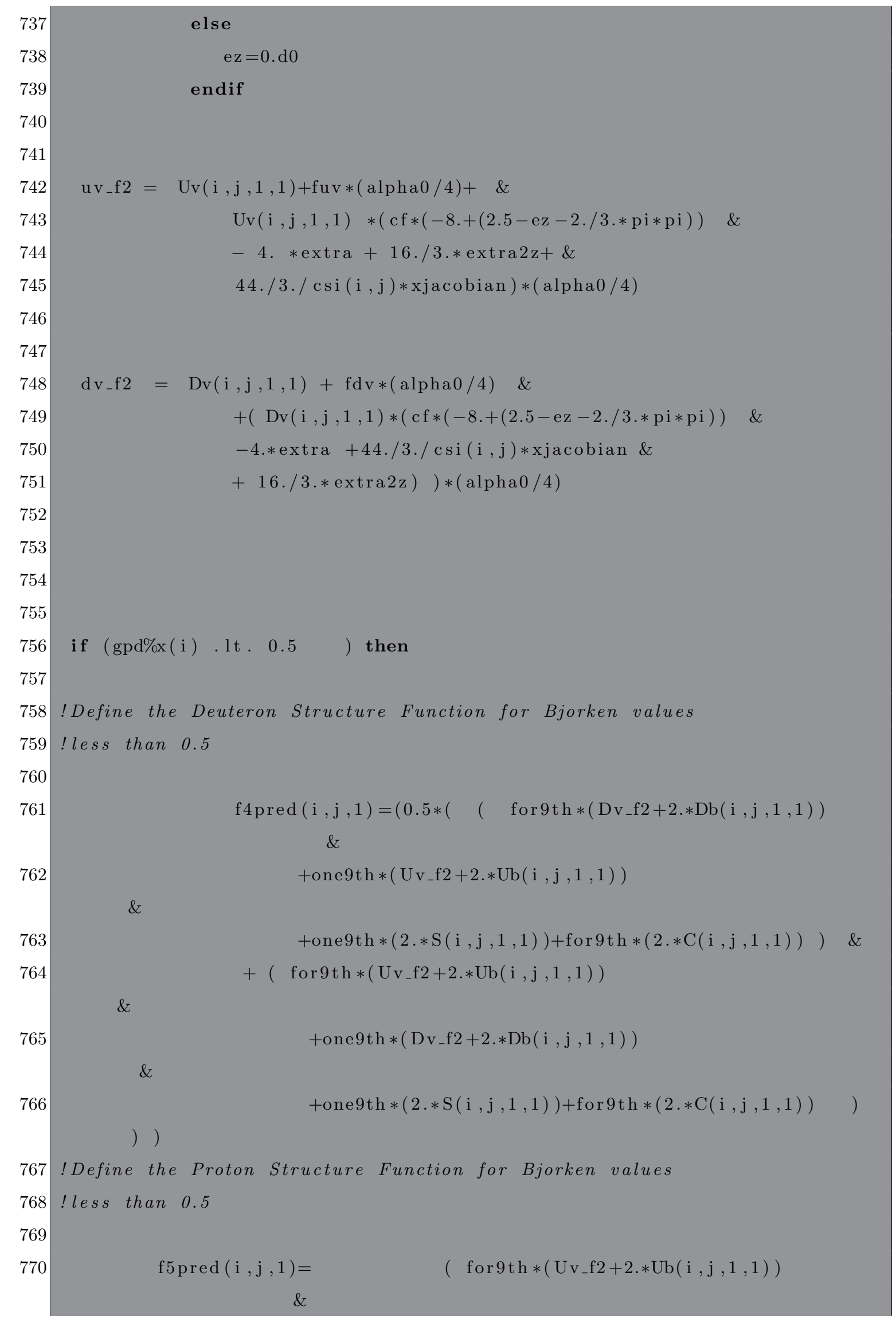




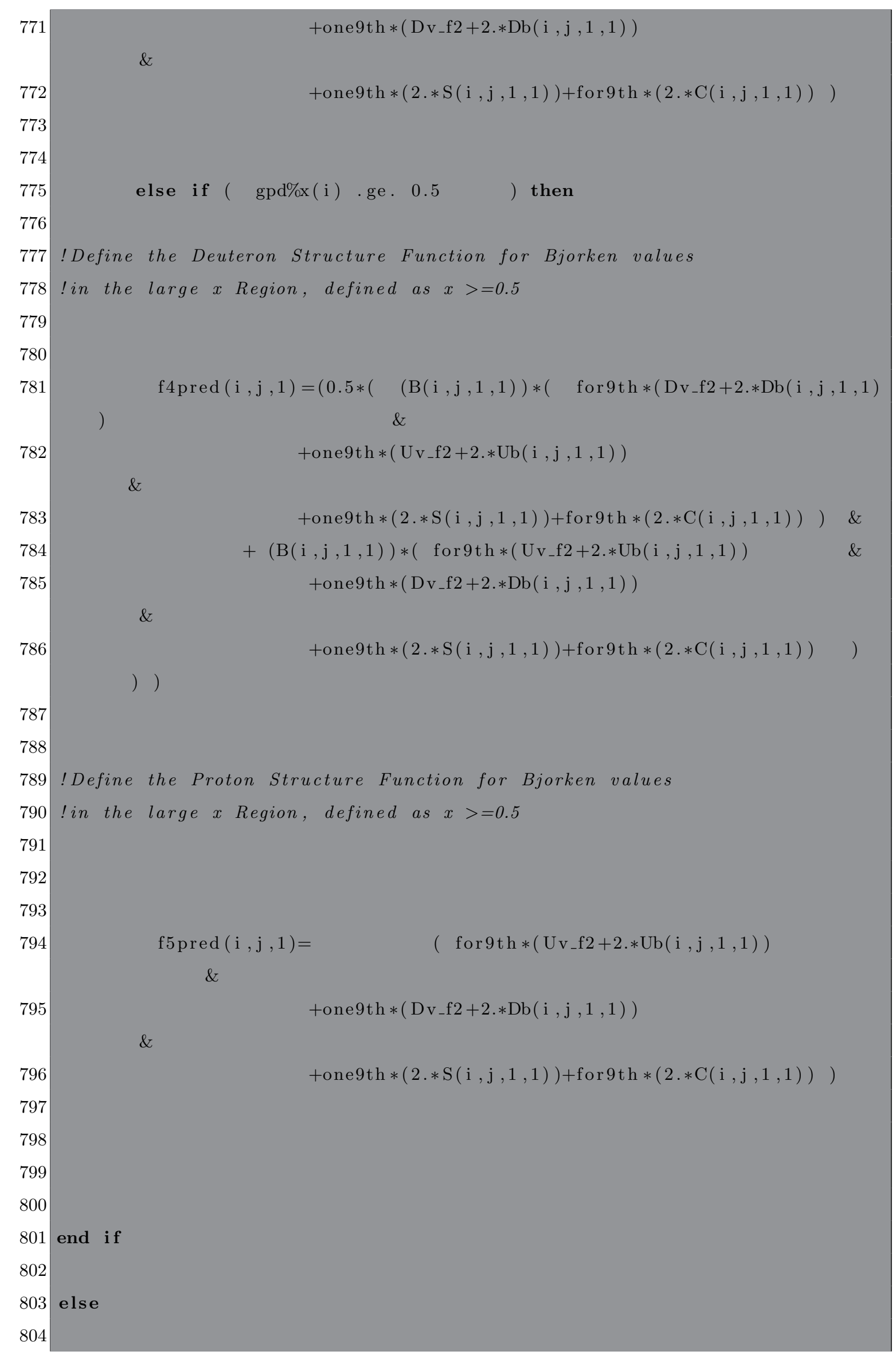




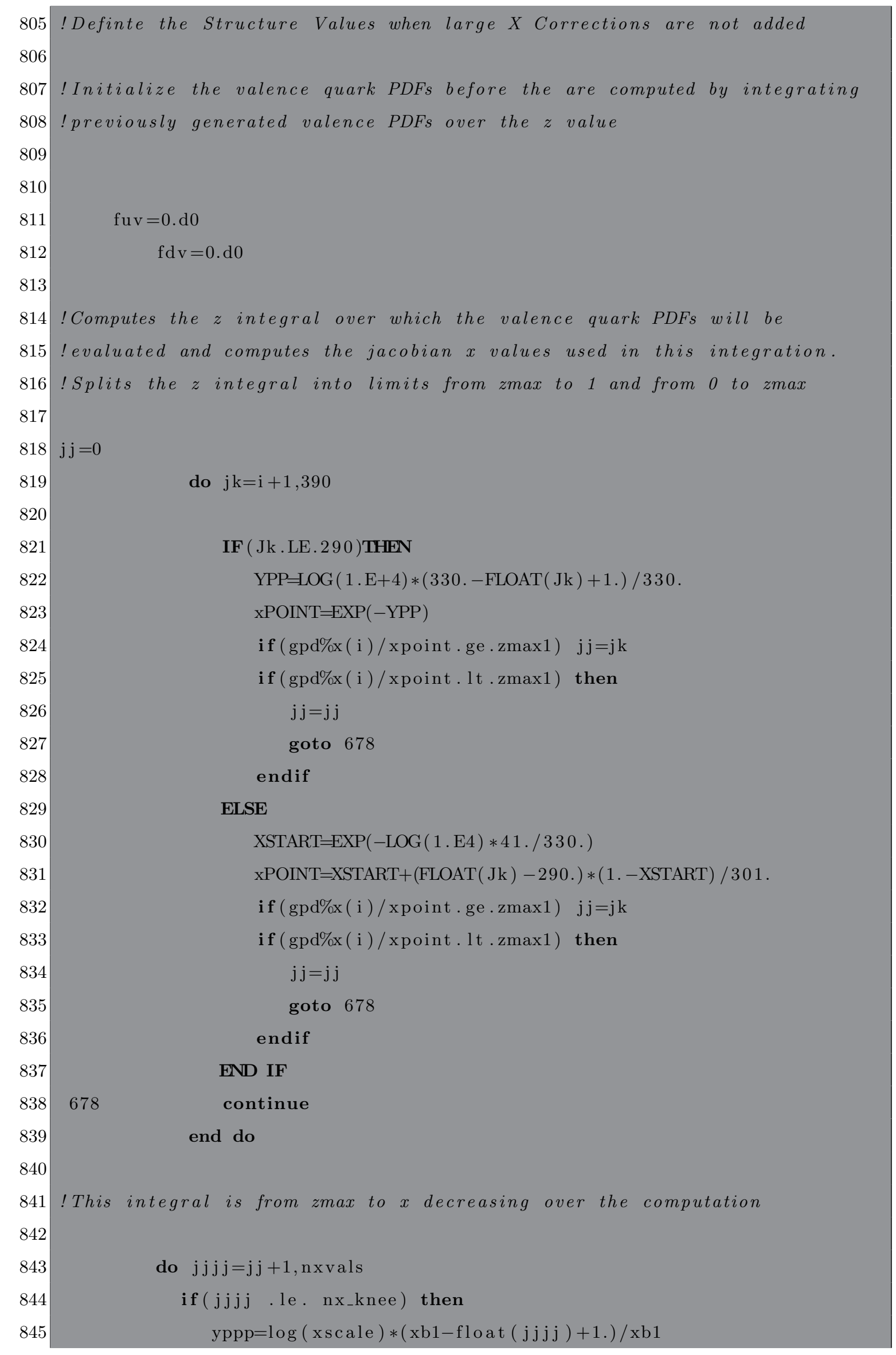




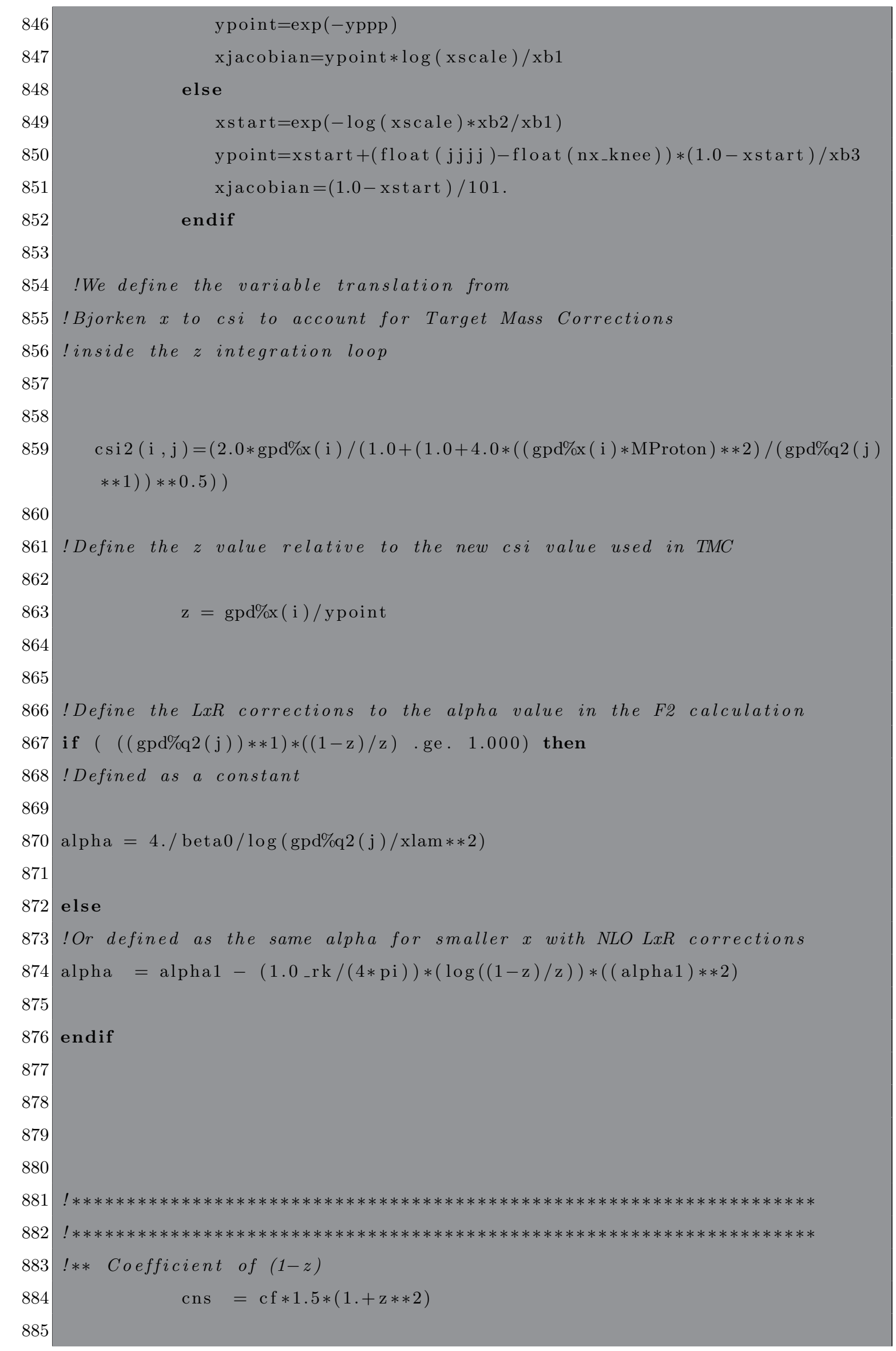




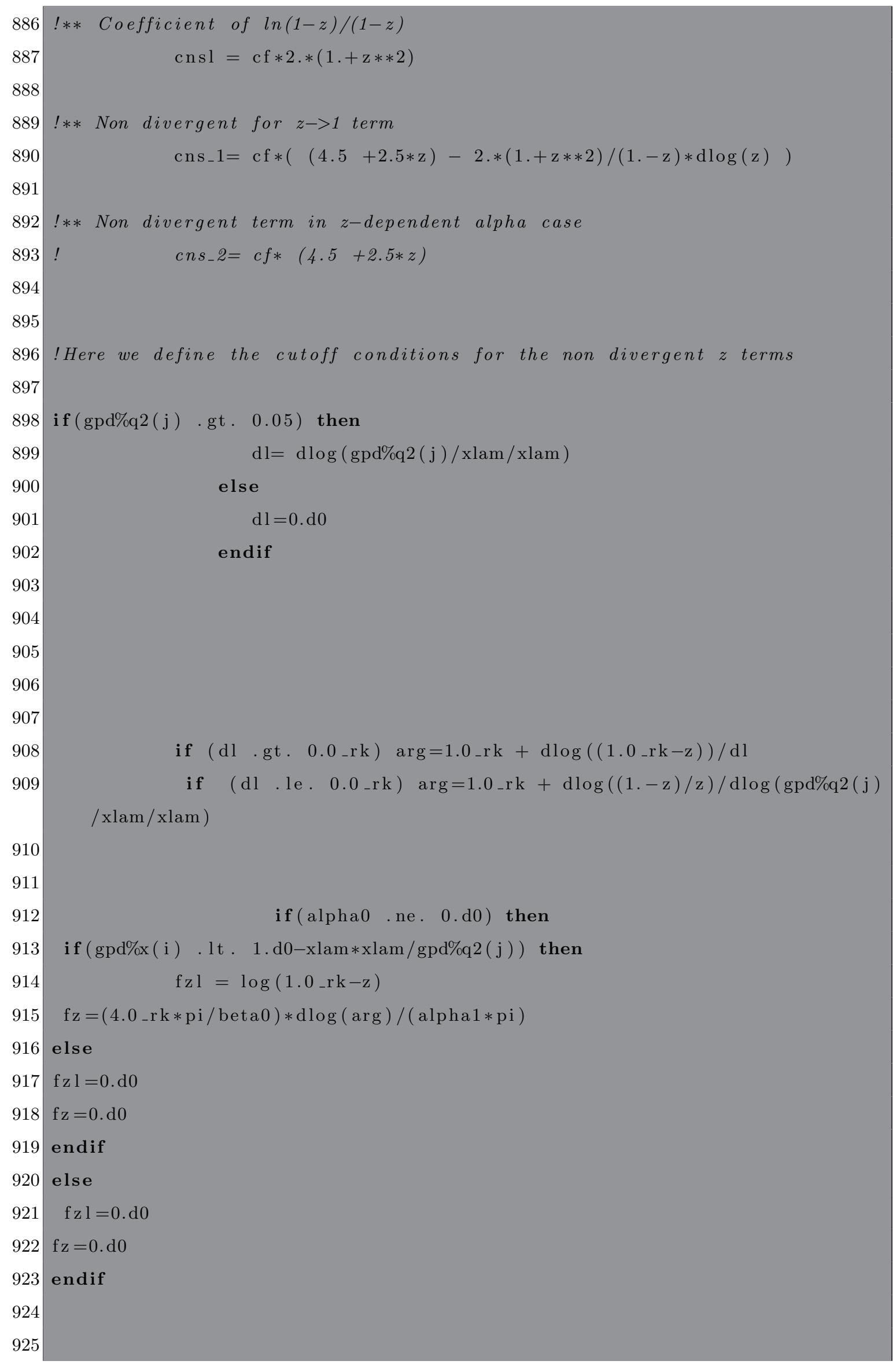




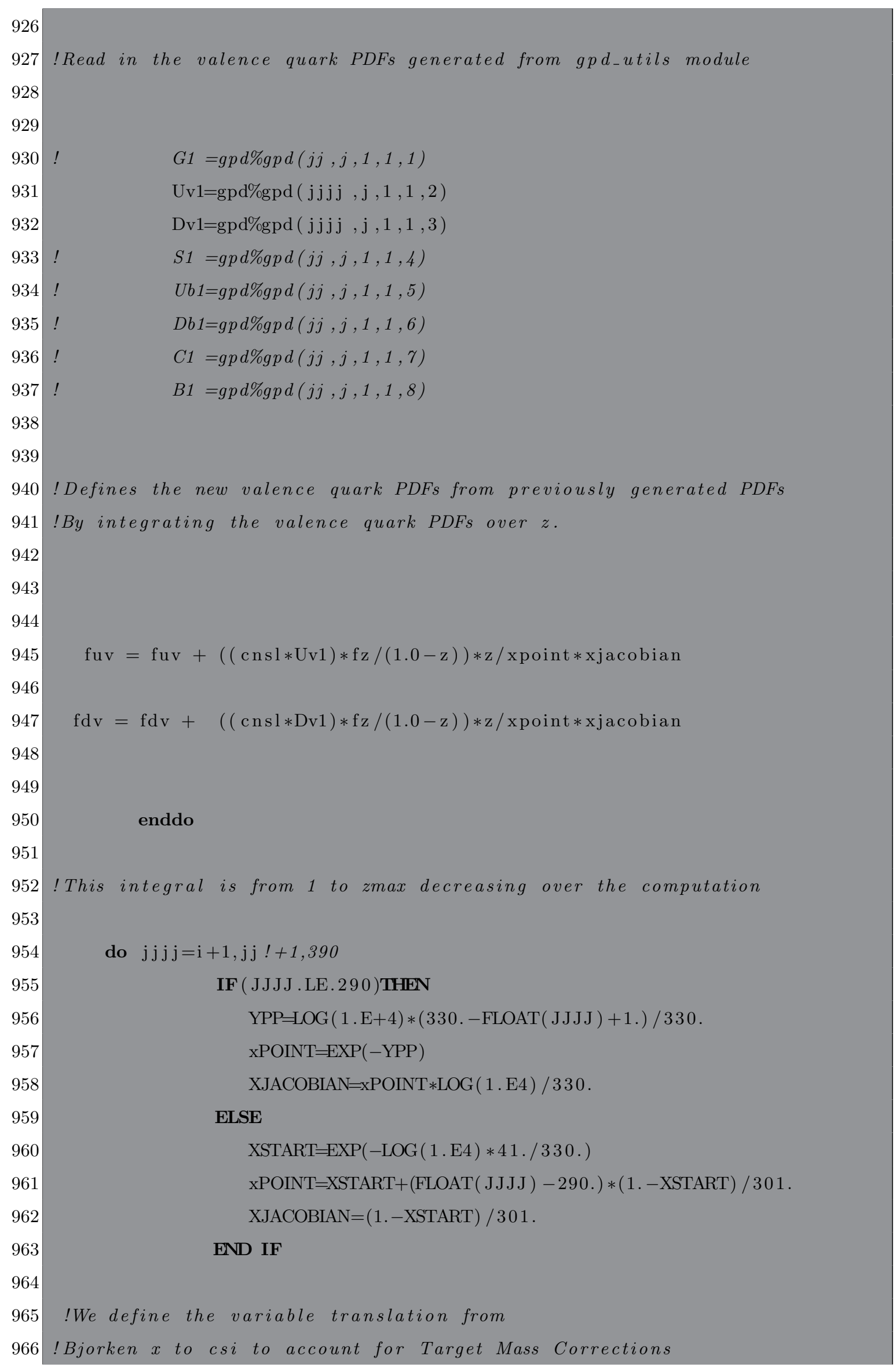




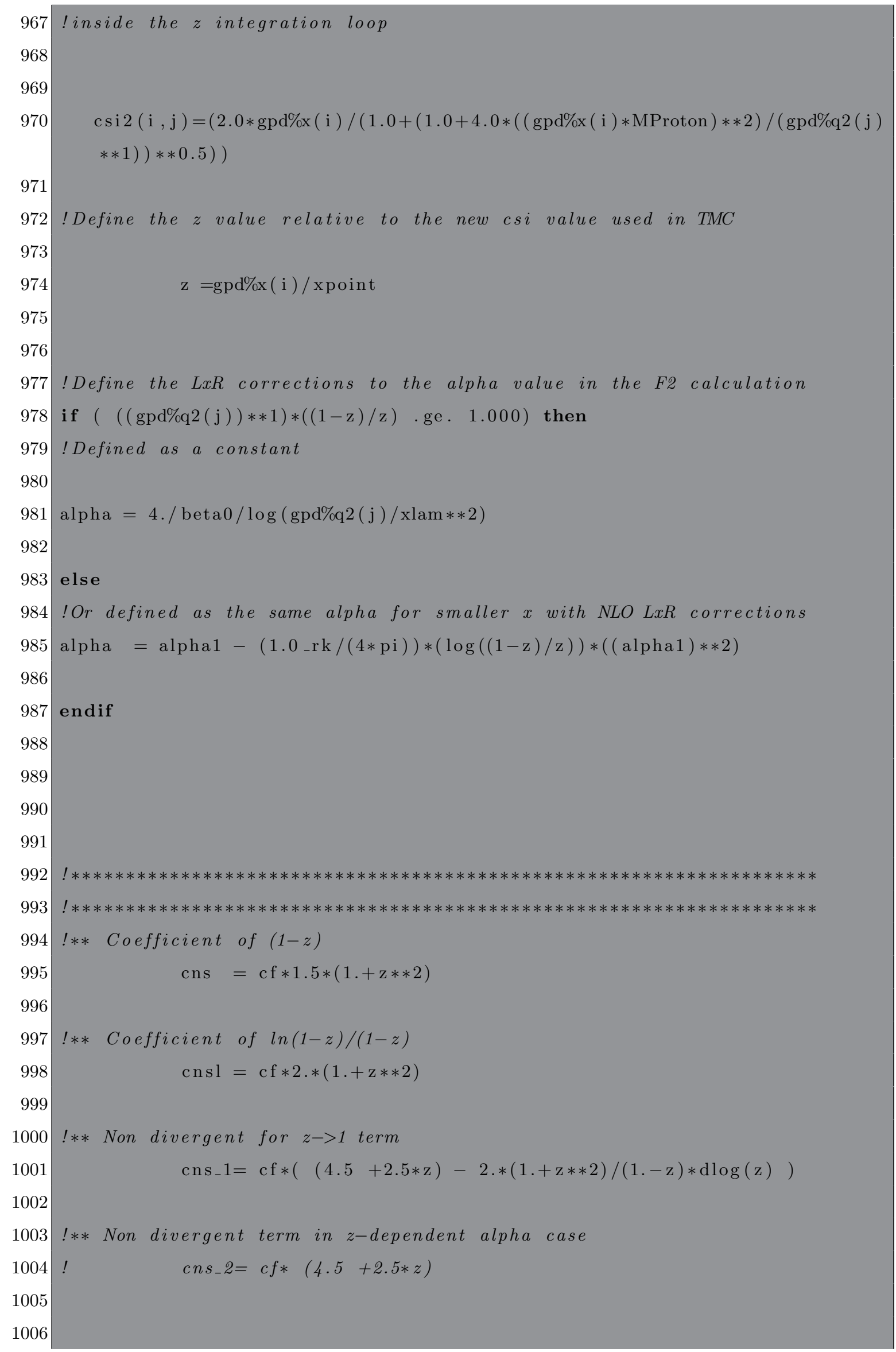




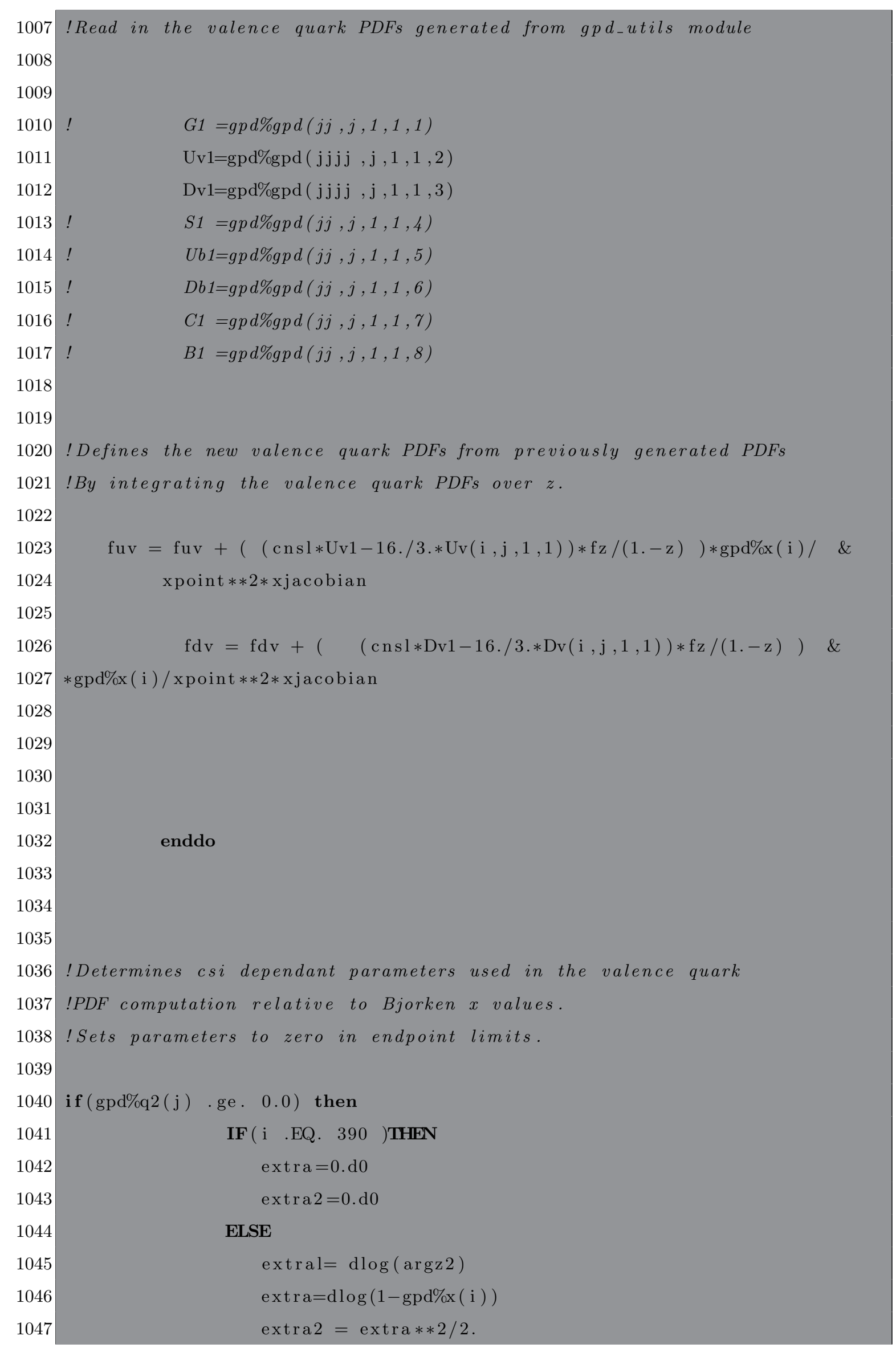




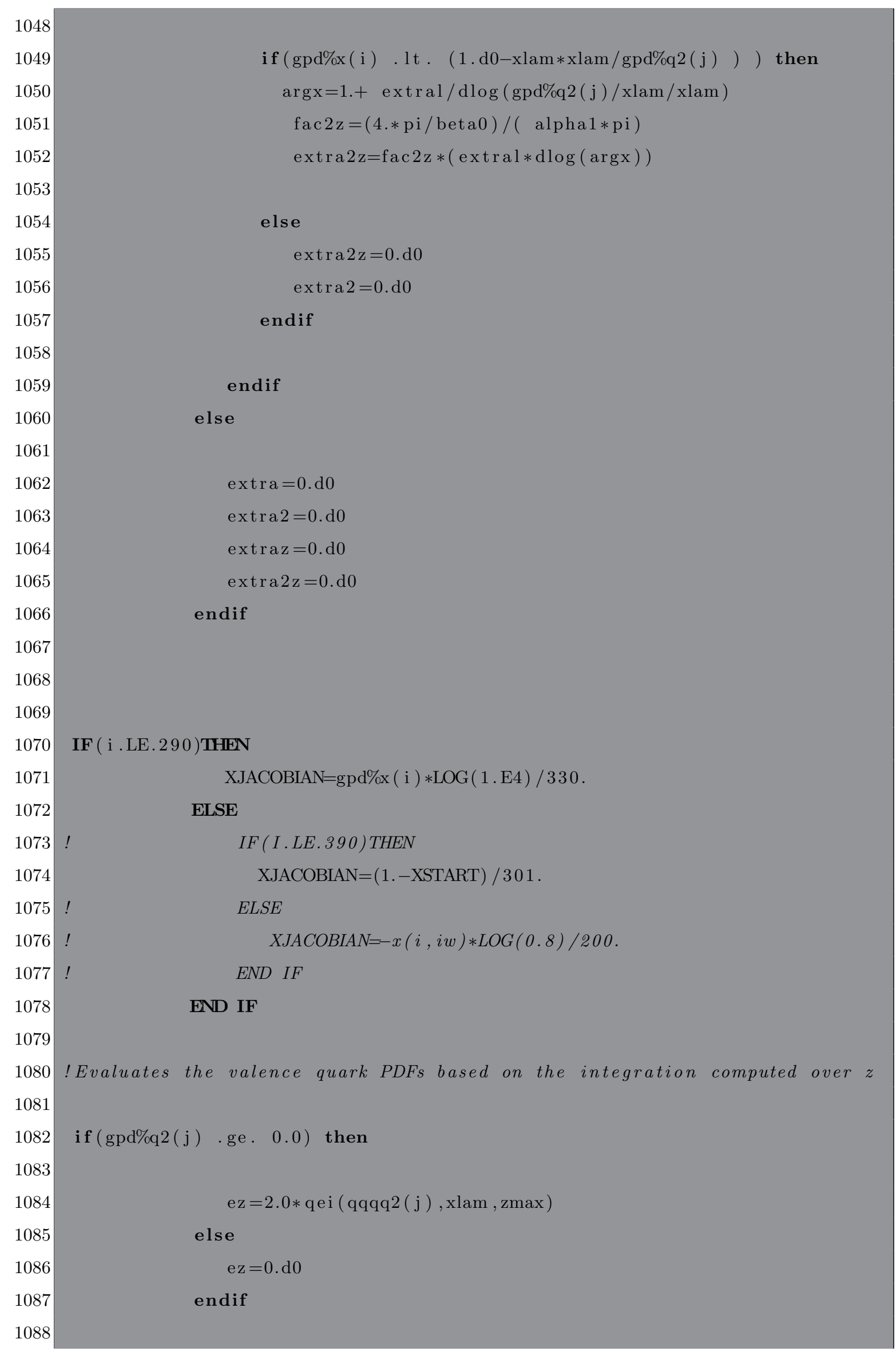




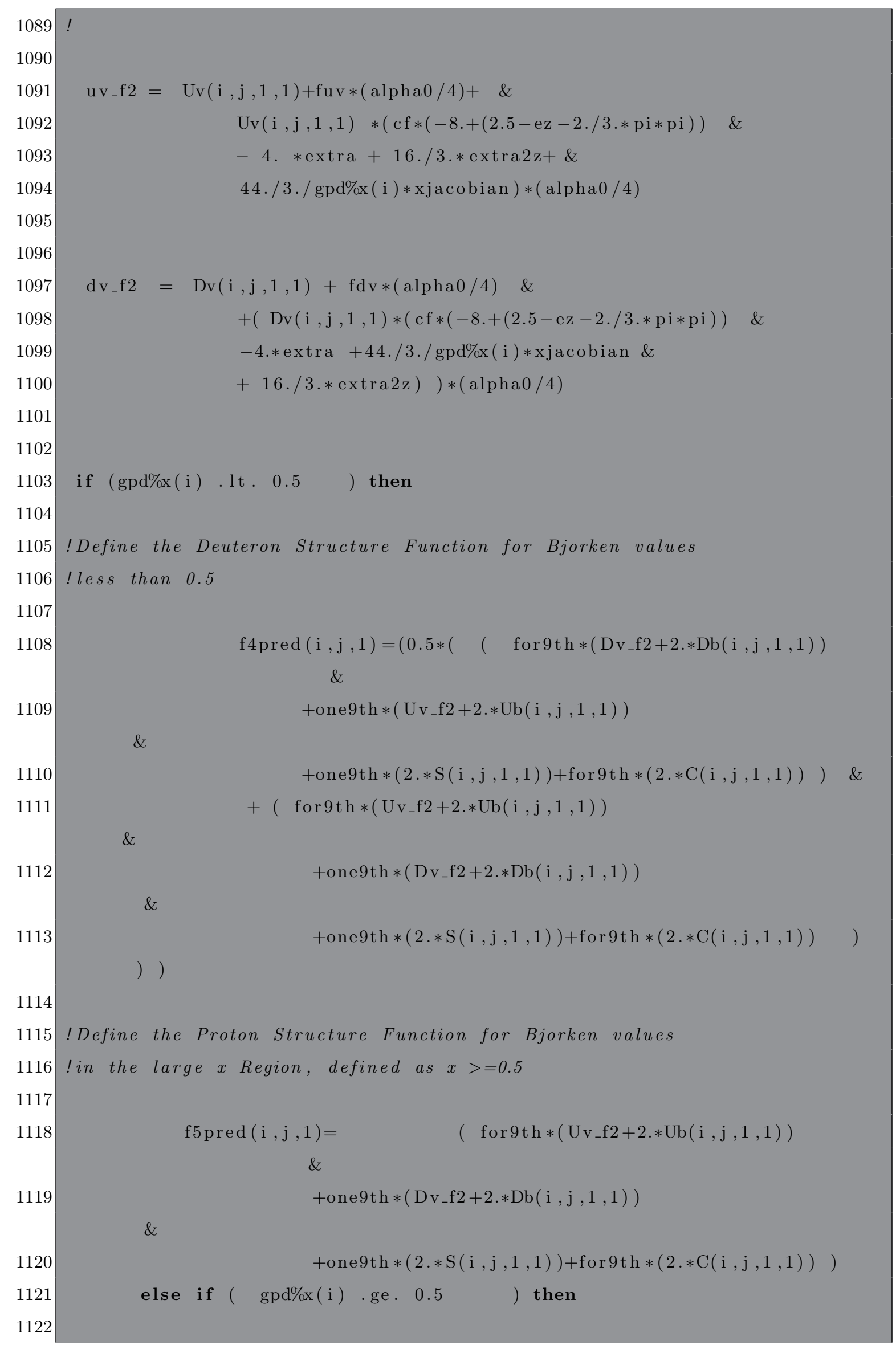




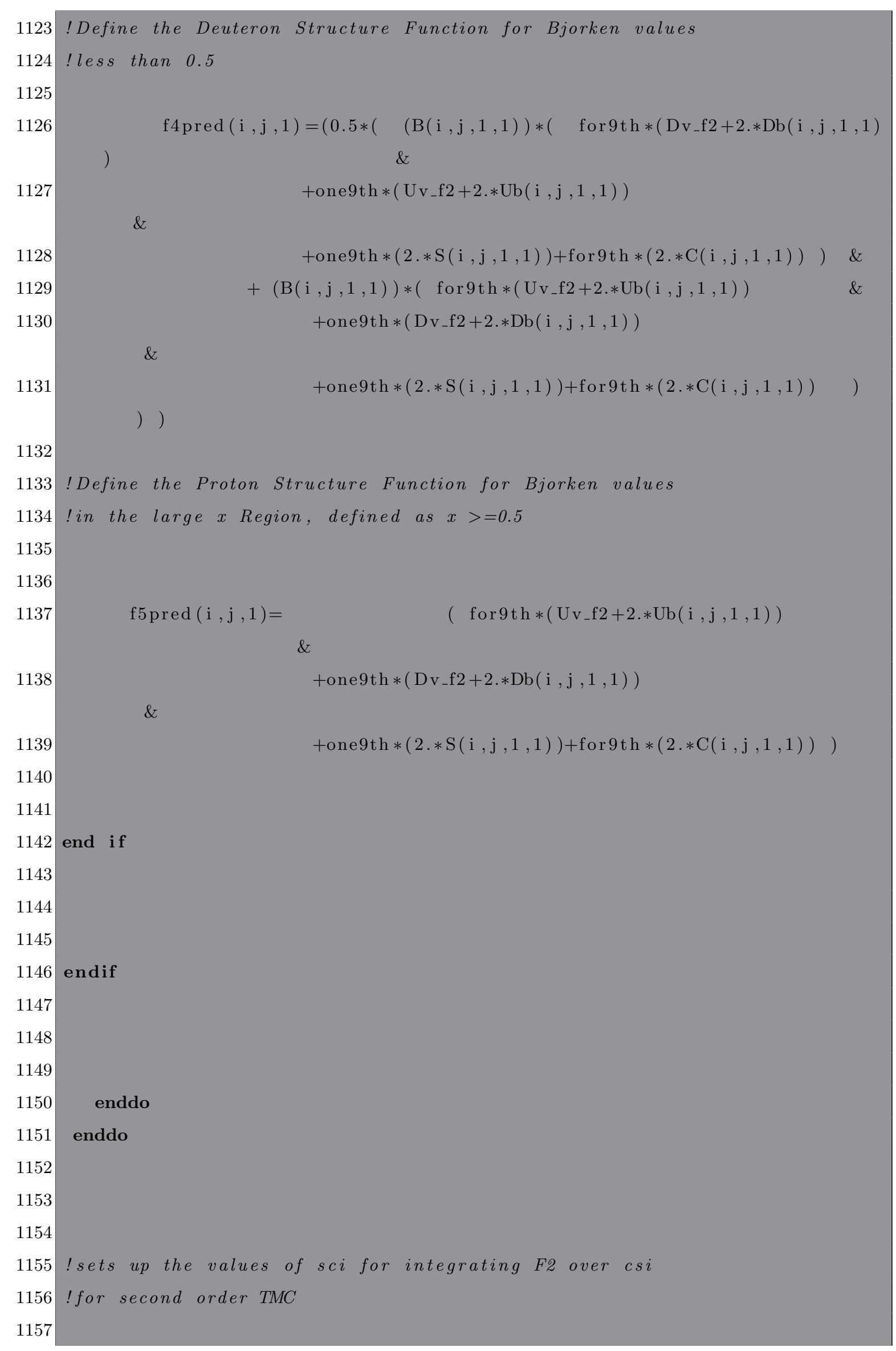




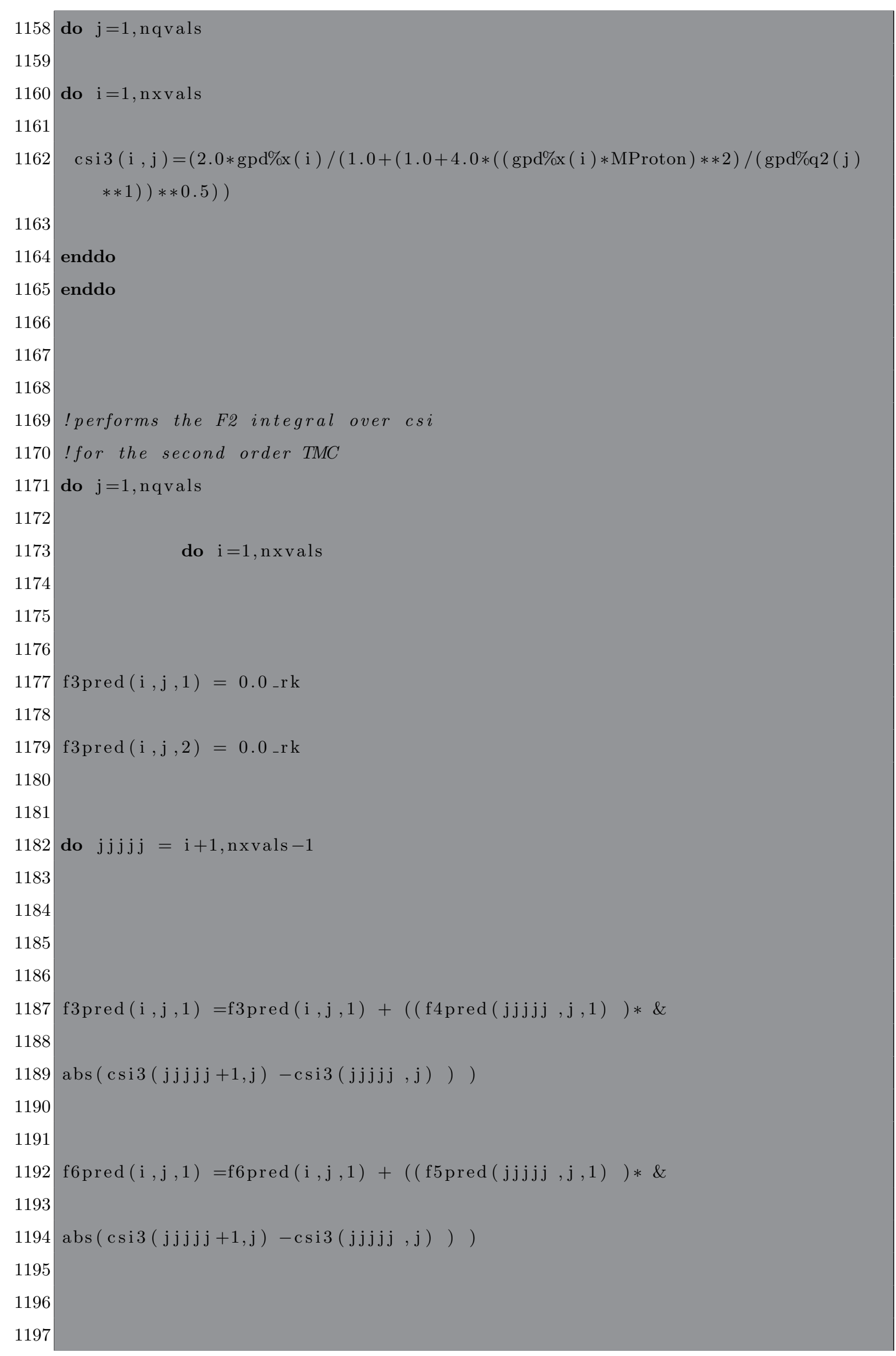




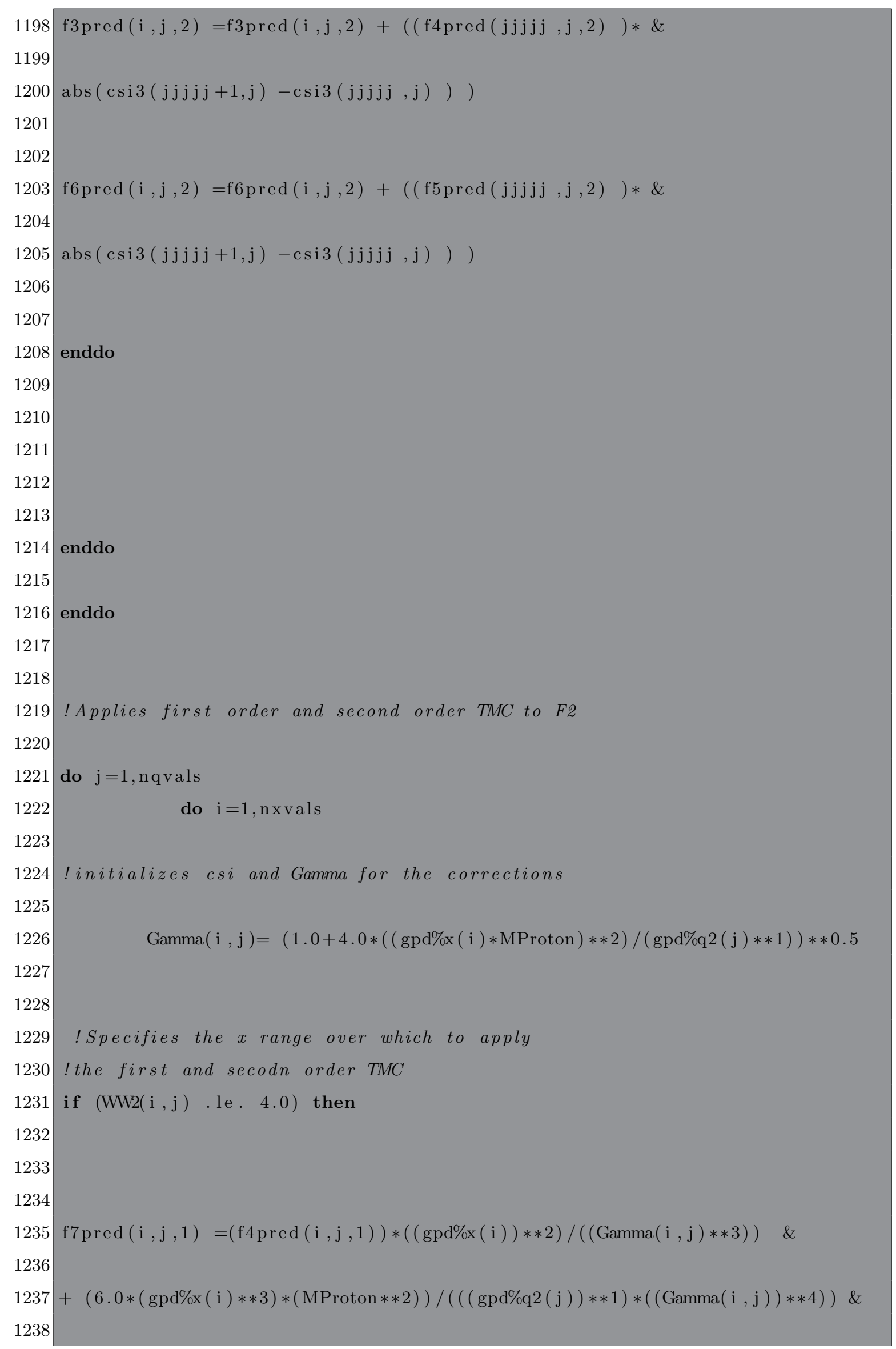




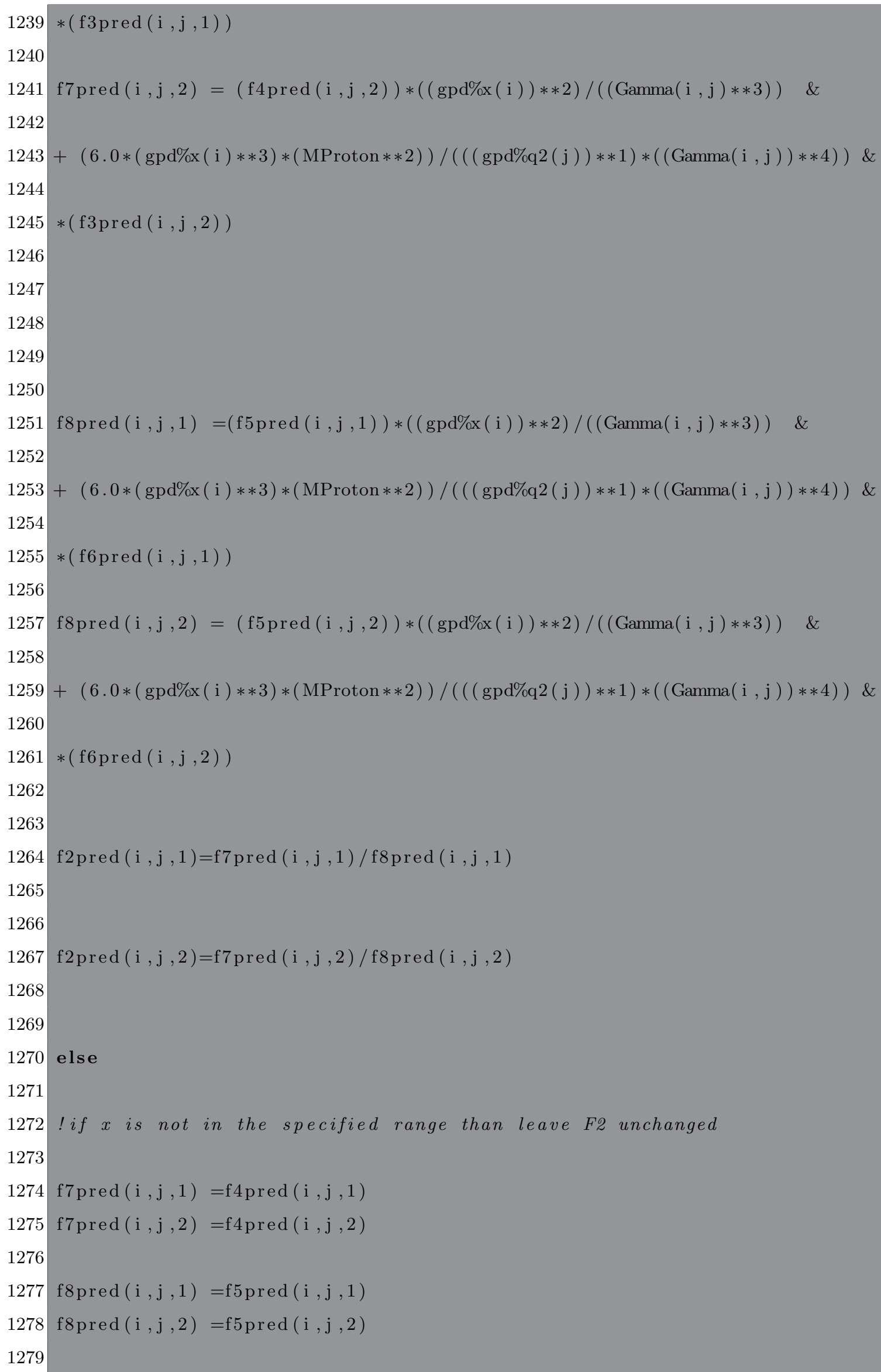




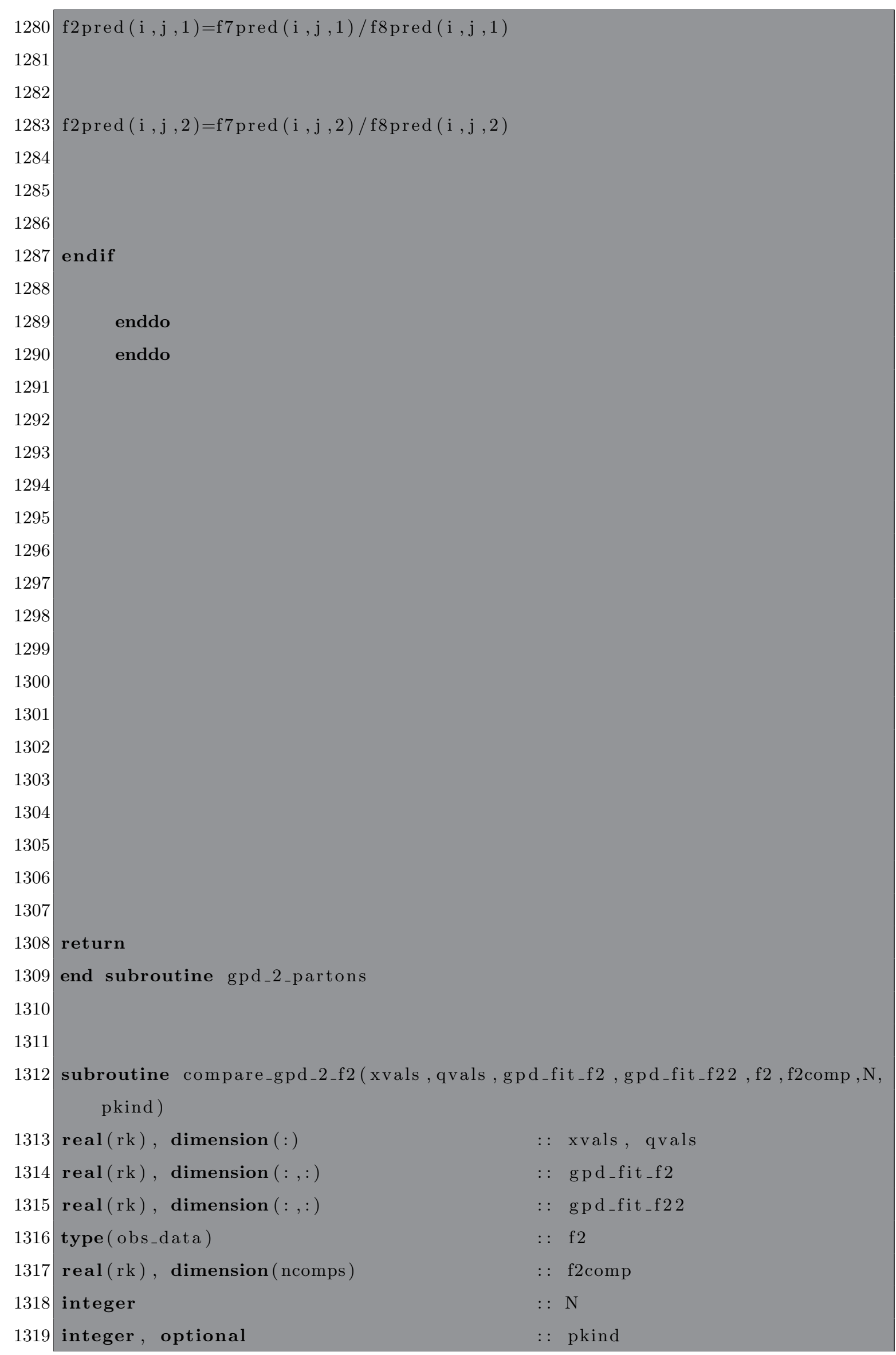




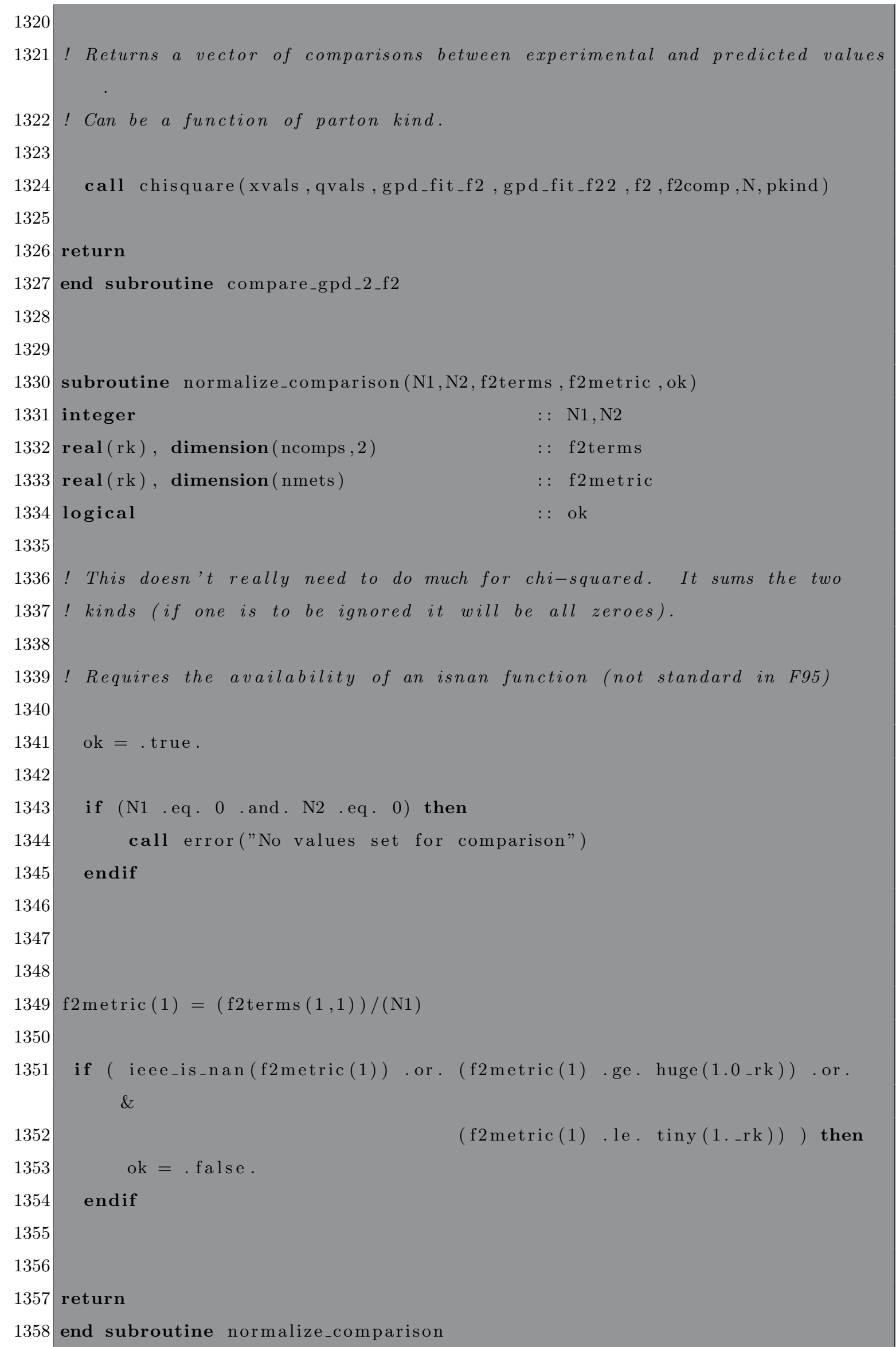




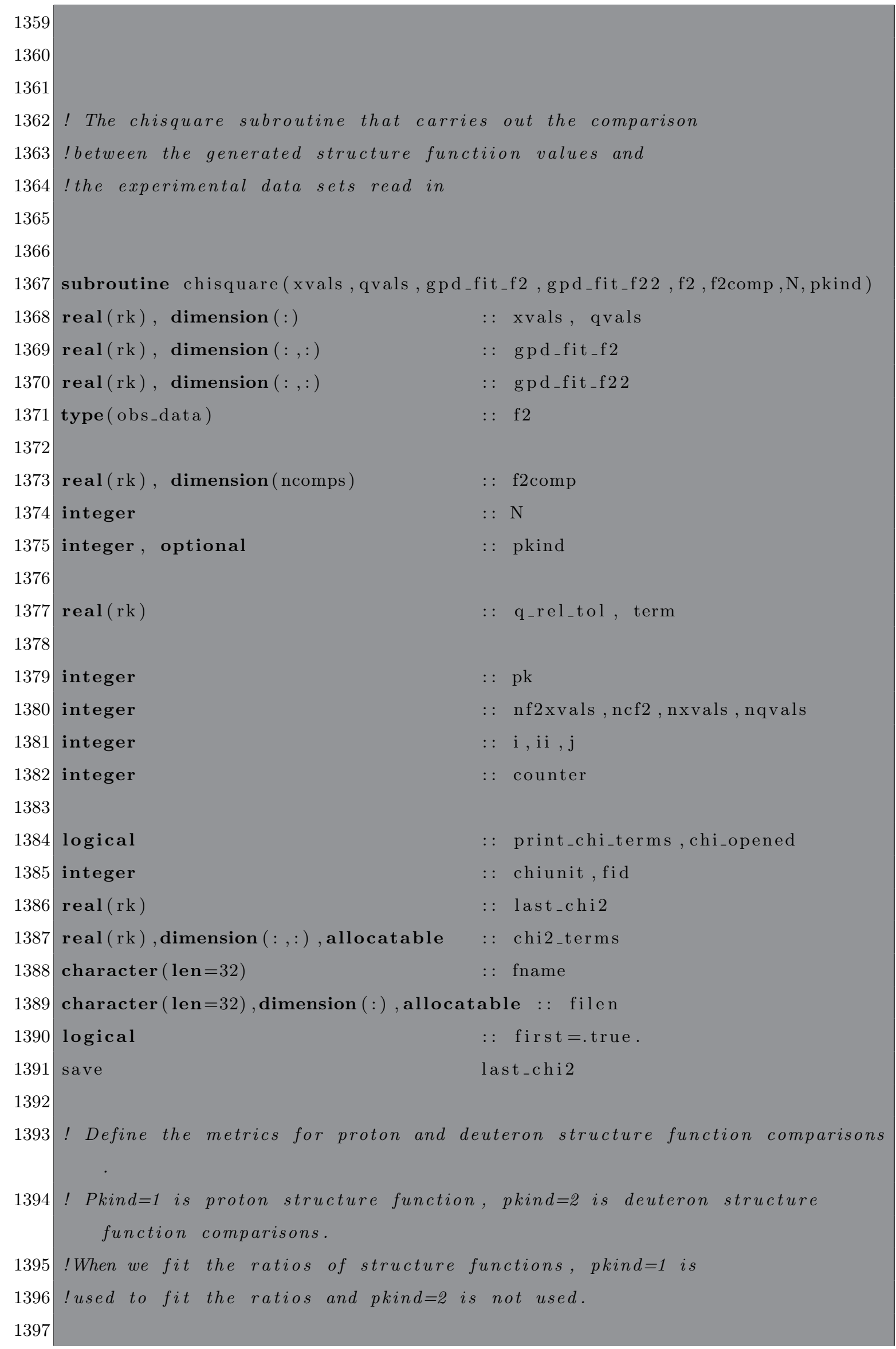




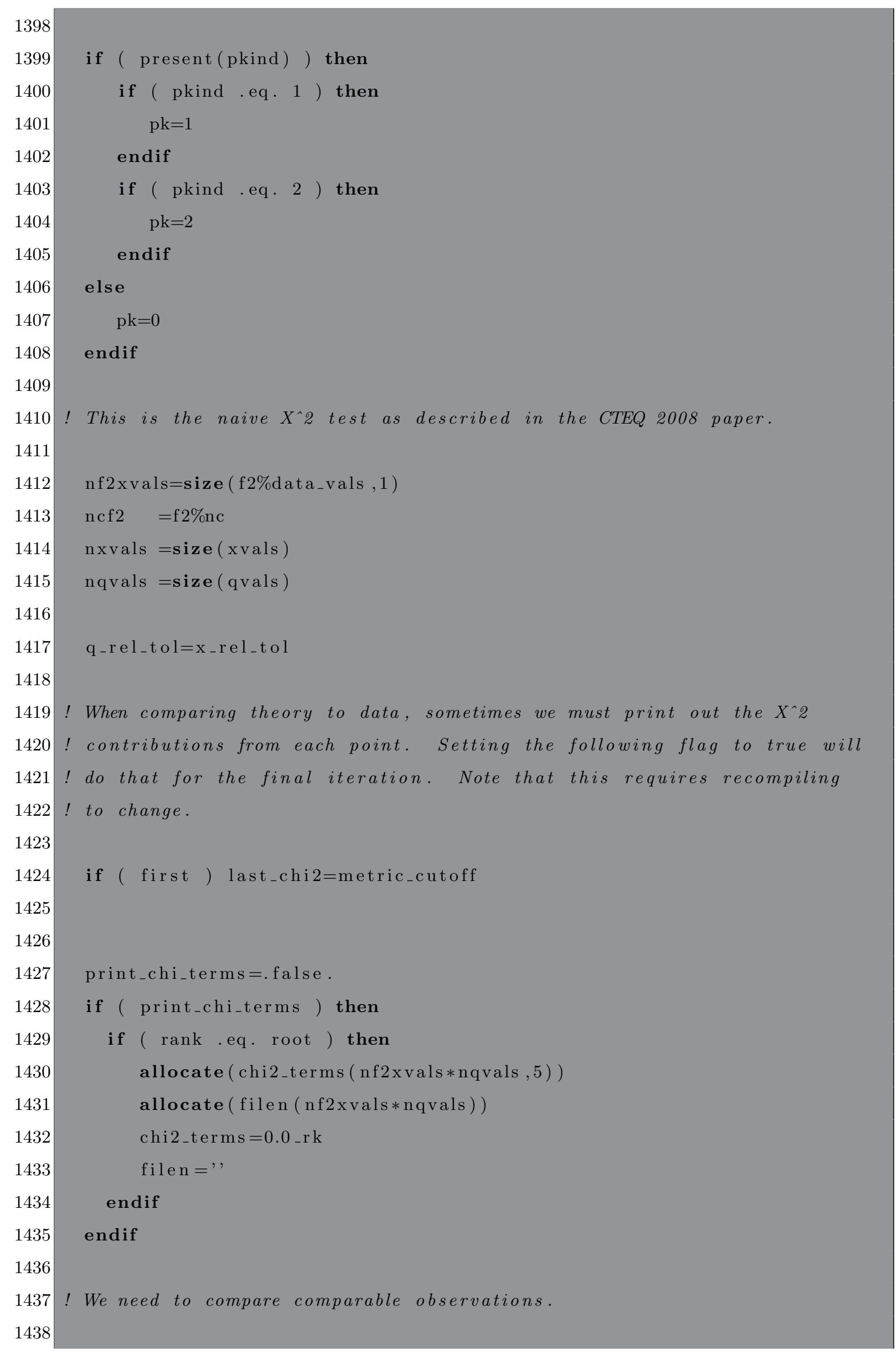




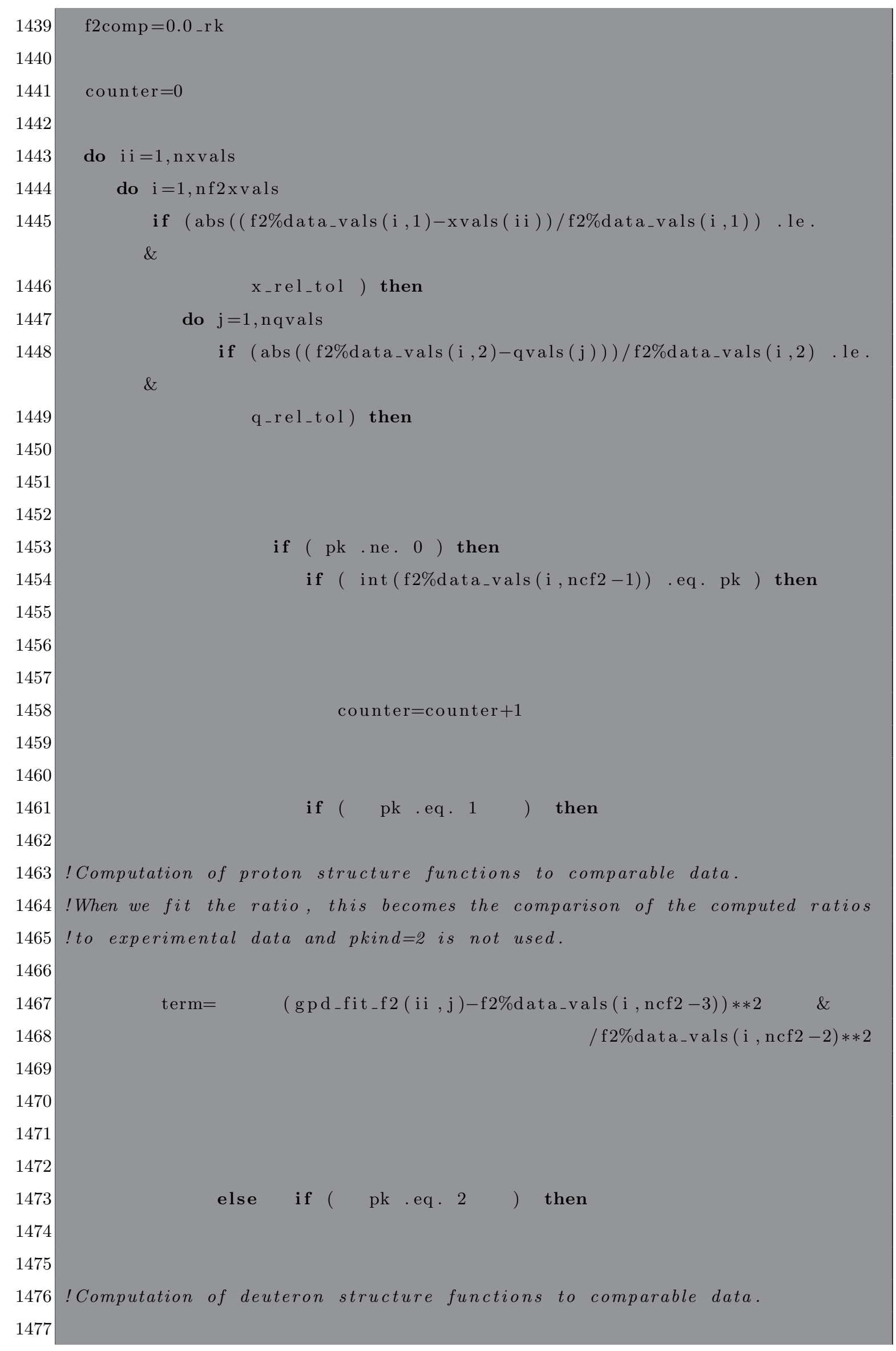




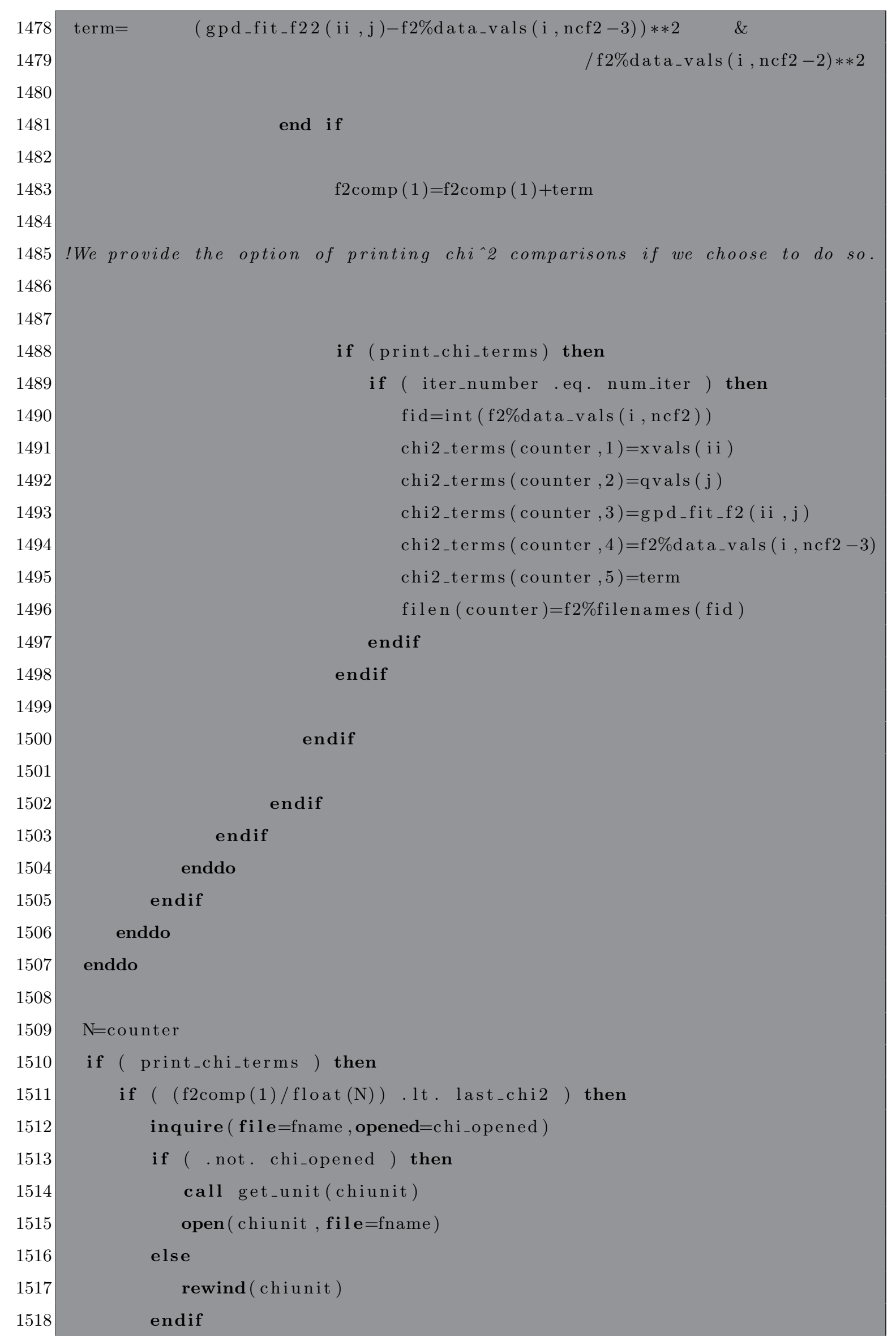




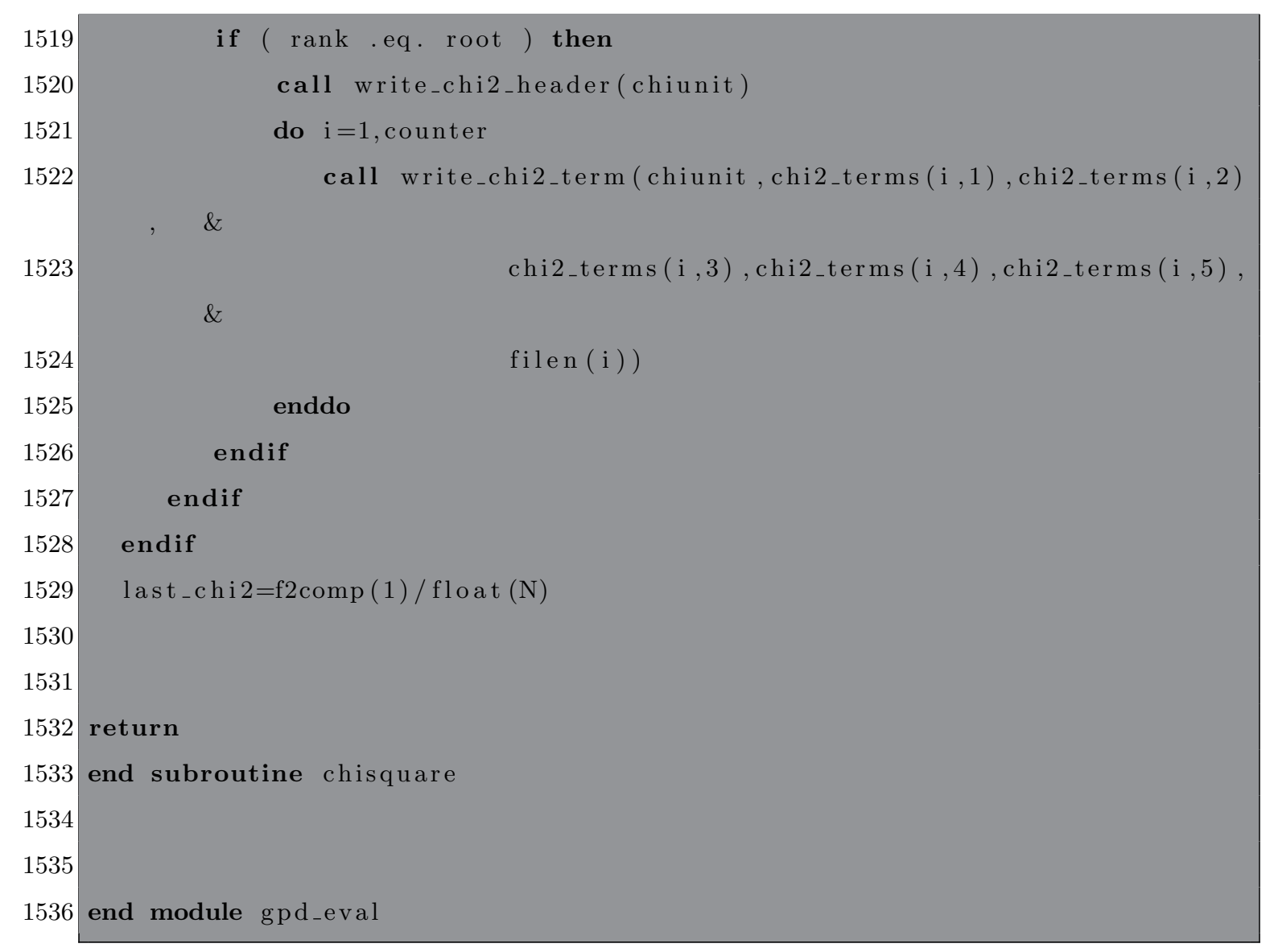

Listing 2: Code1

The following is the gpdnorm. $f 90$ code used for normalizing the theoretical PDFs after they are generated.

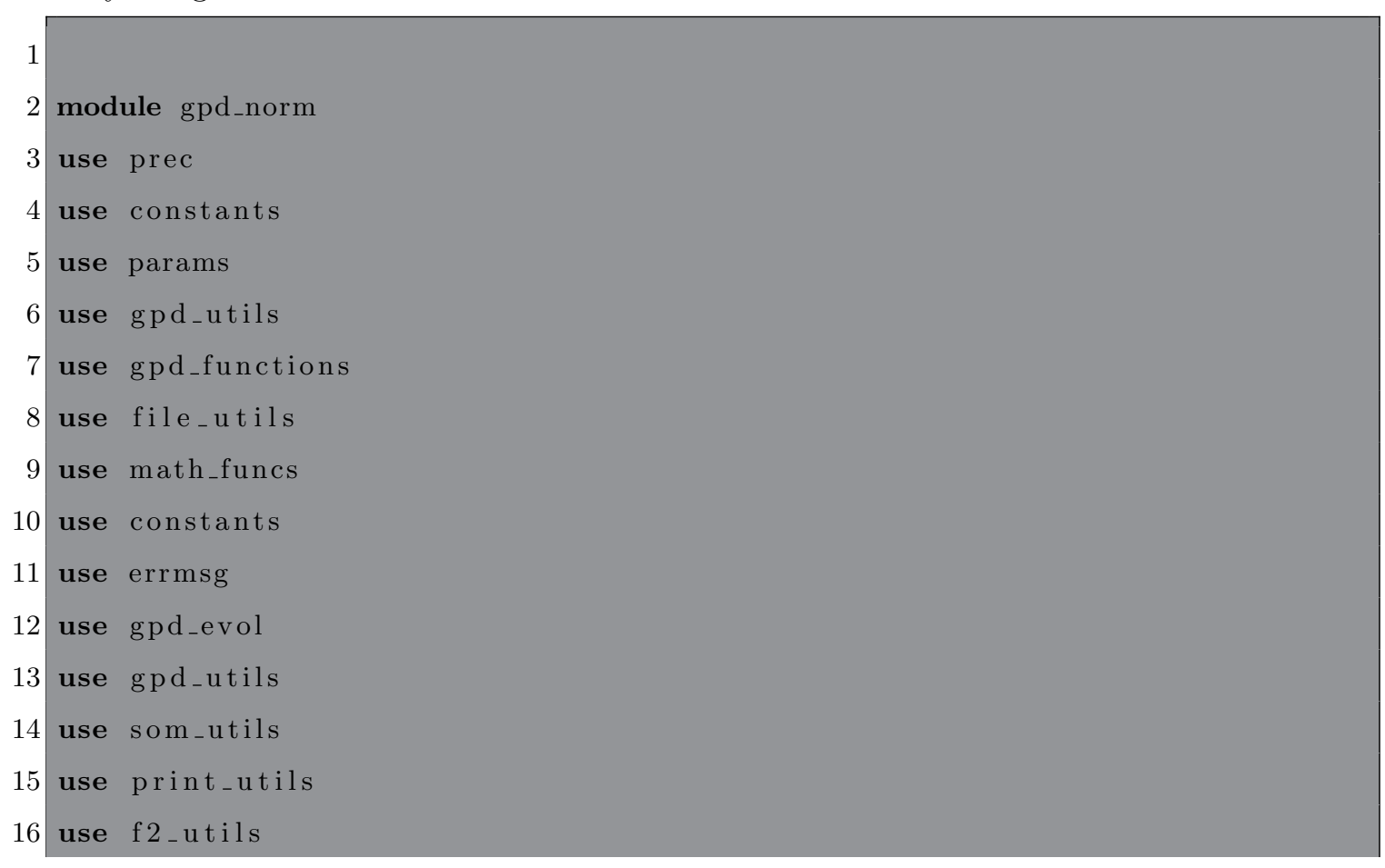




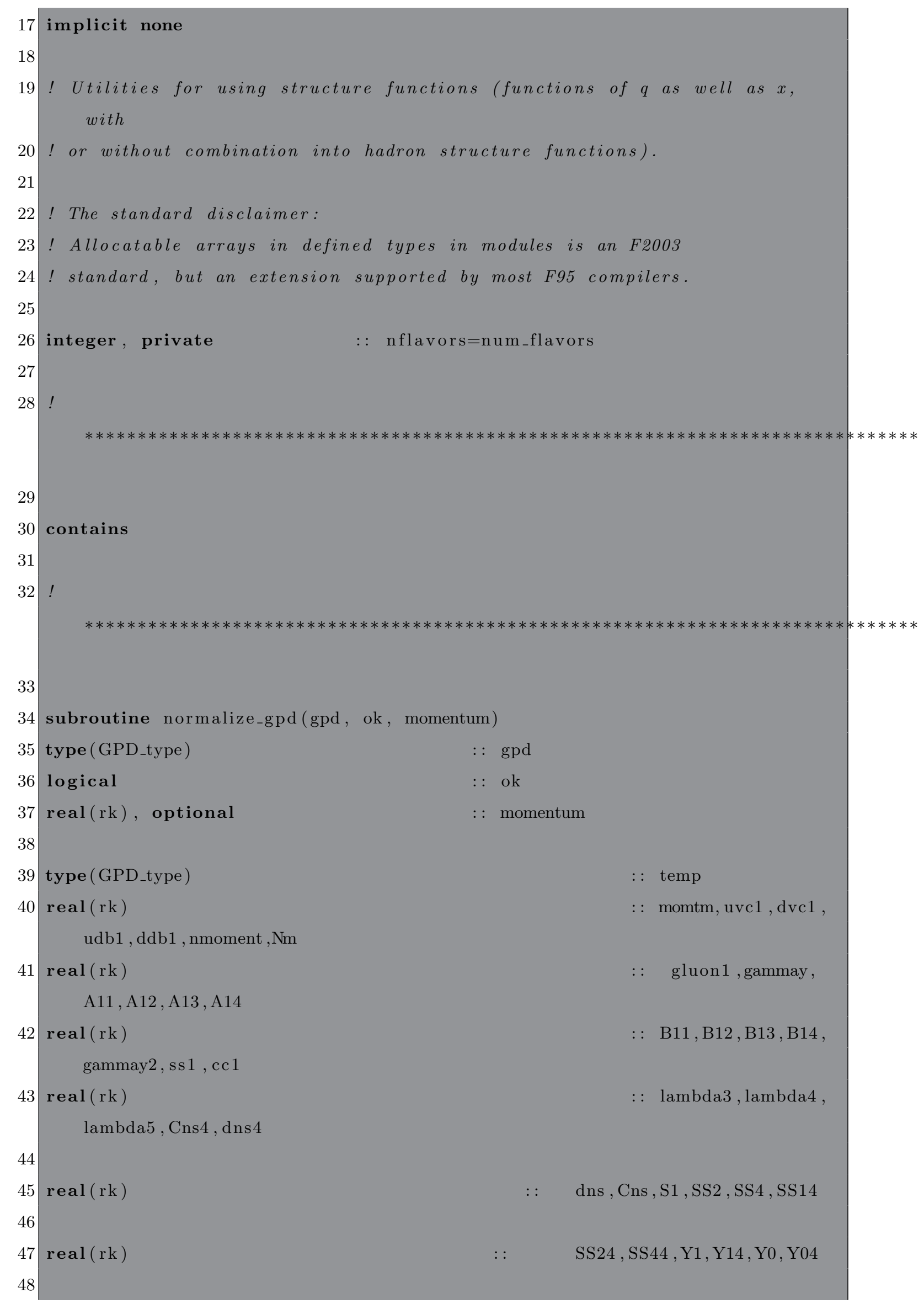




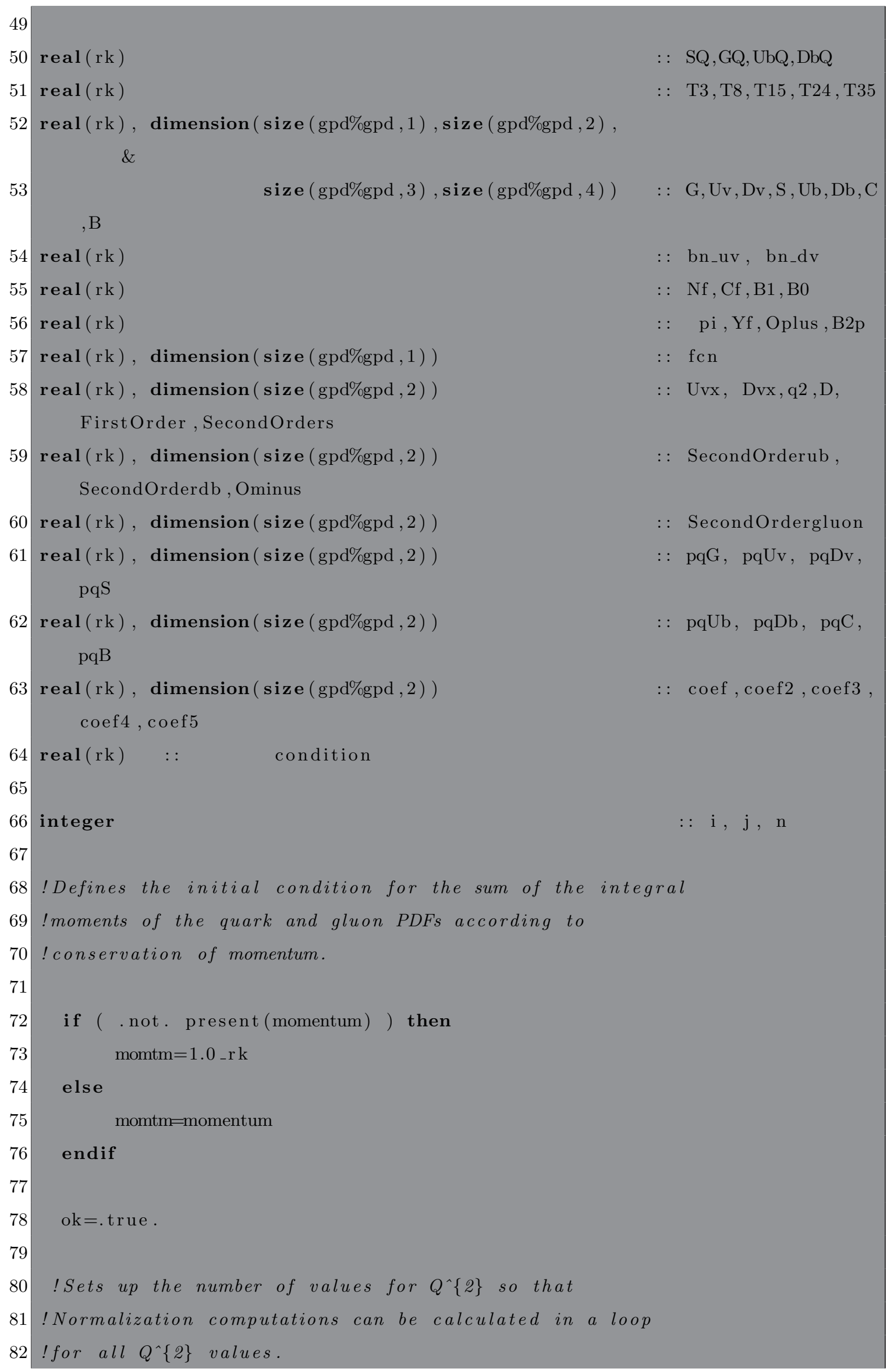




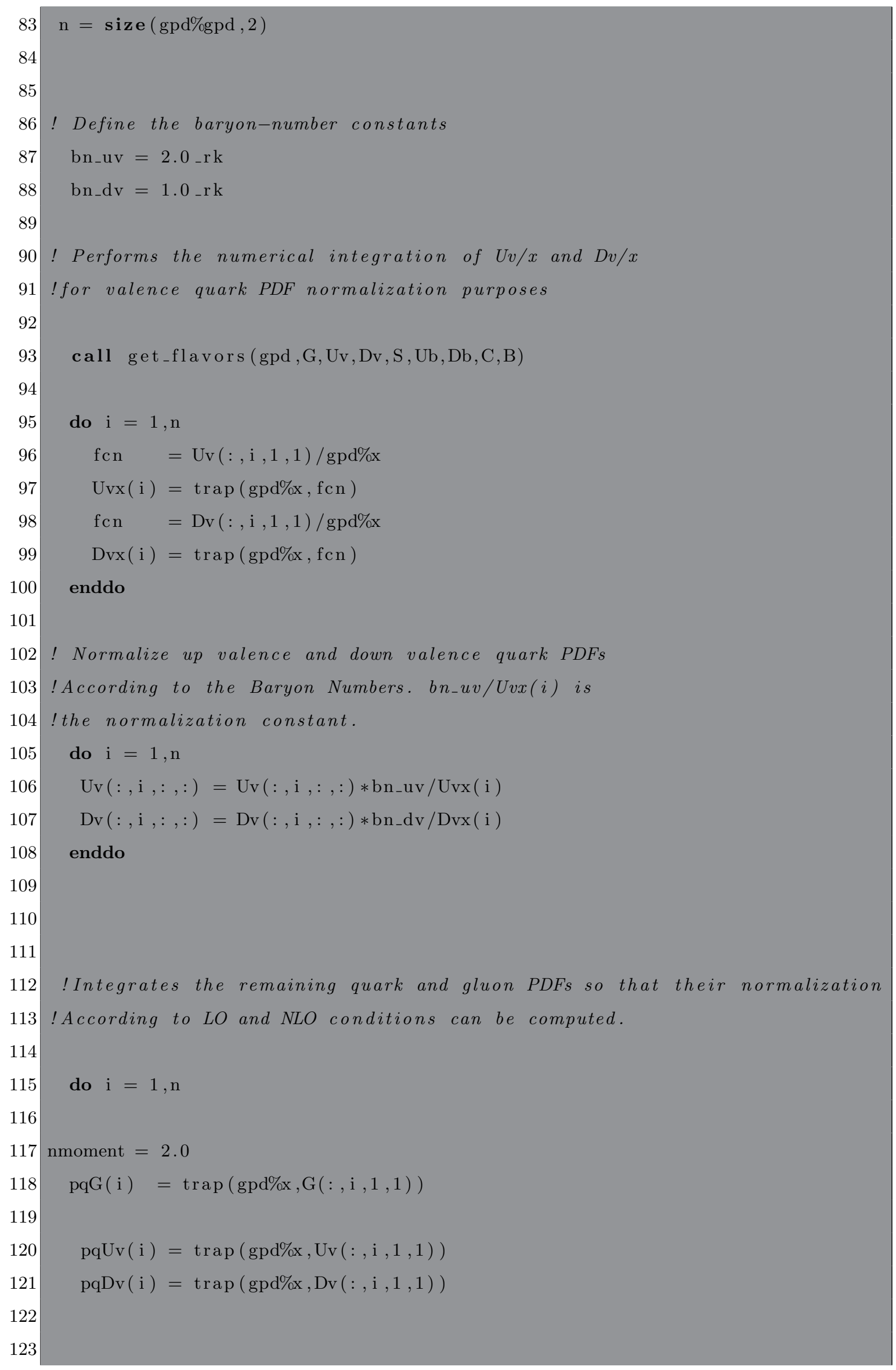




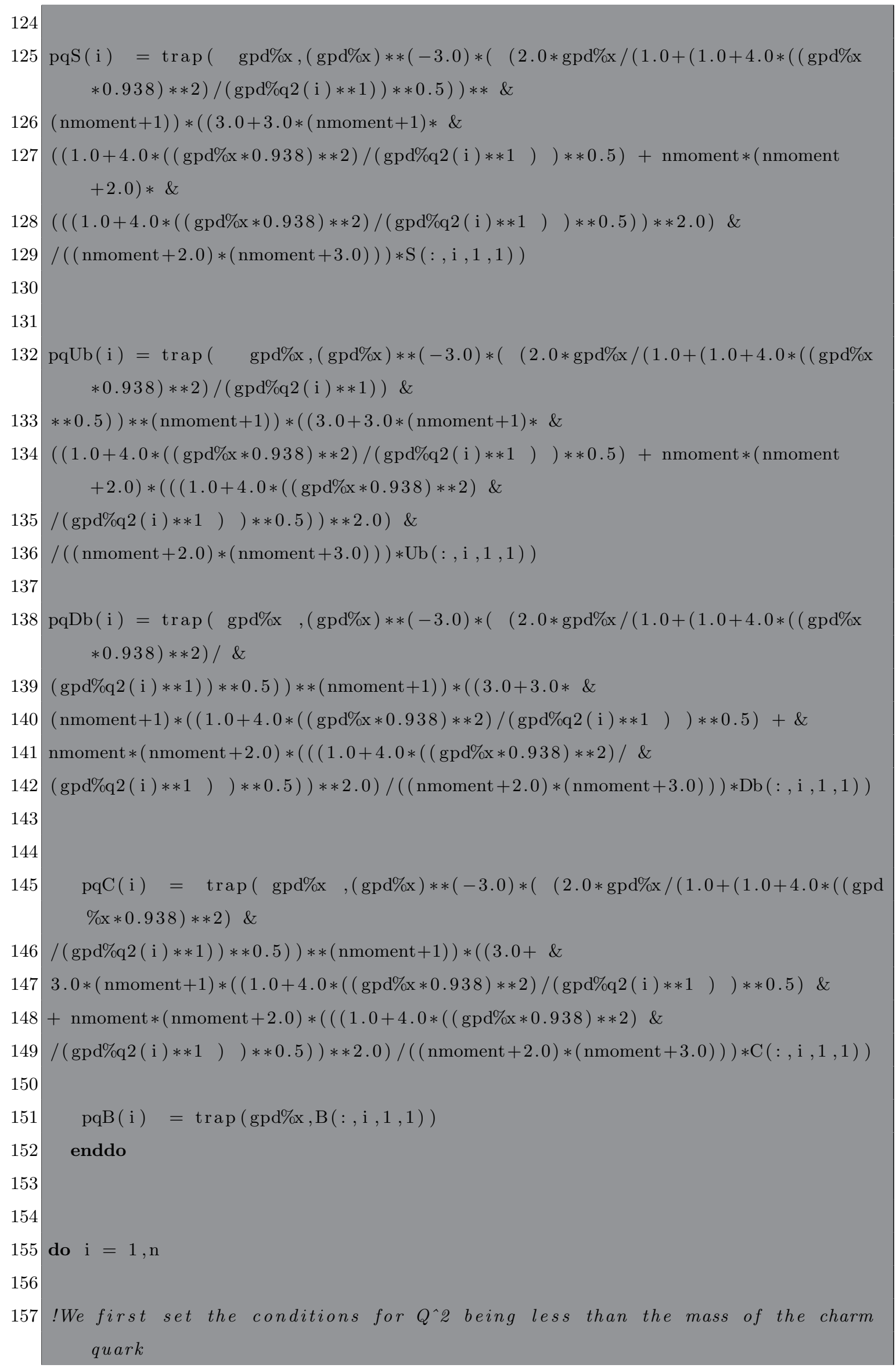




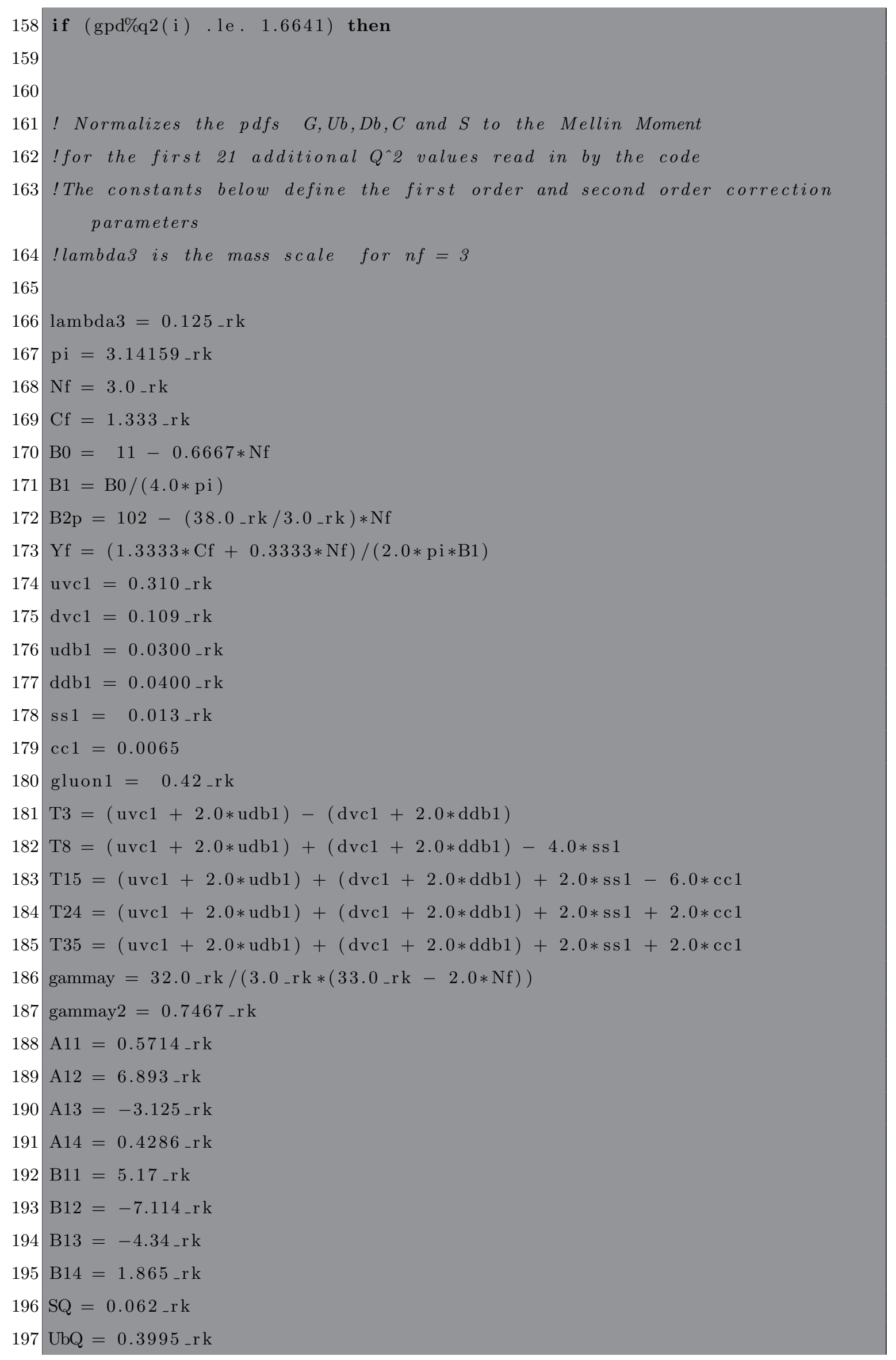




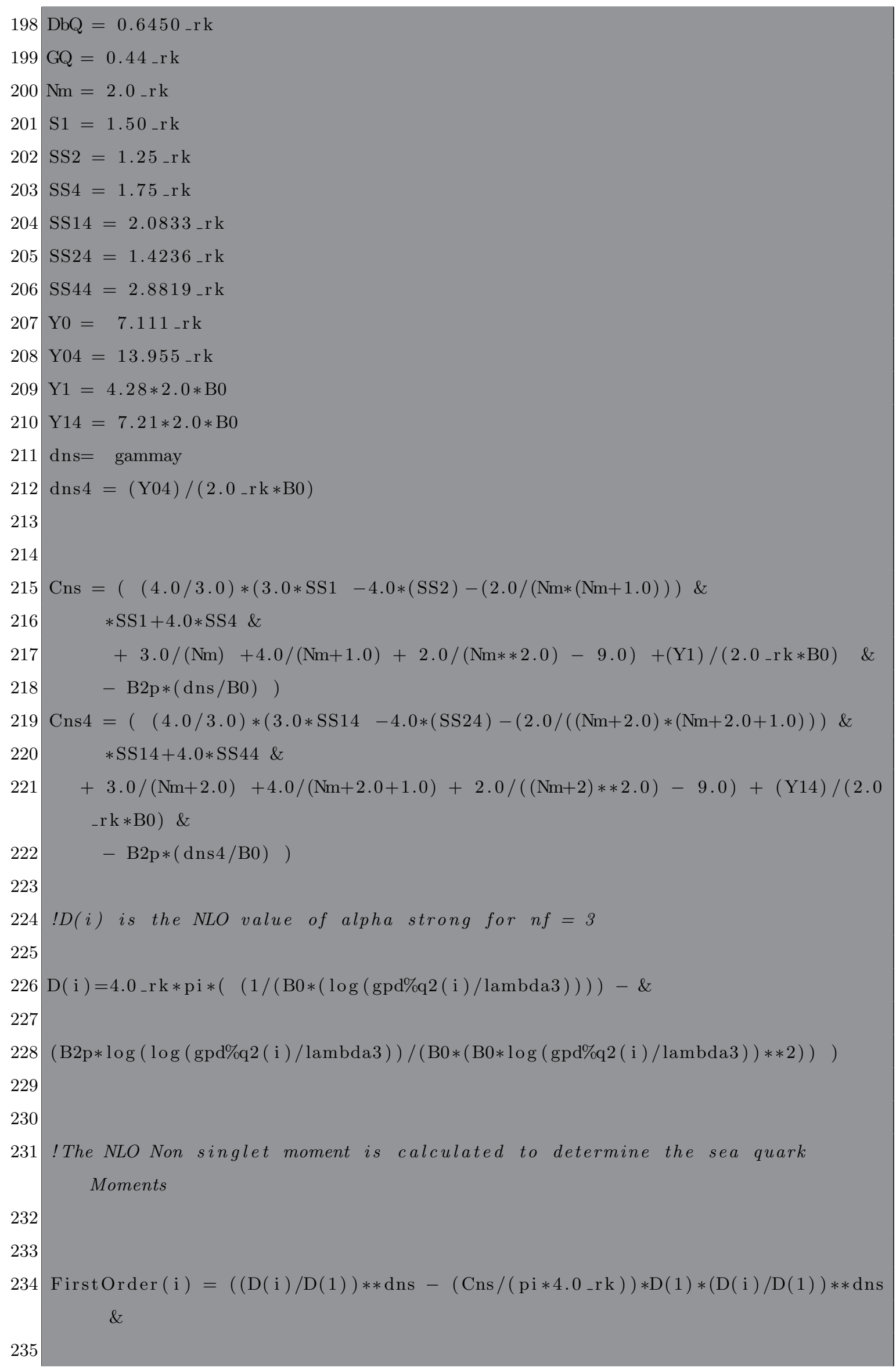




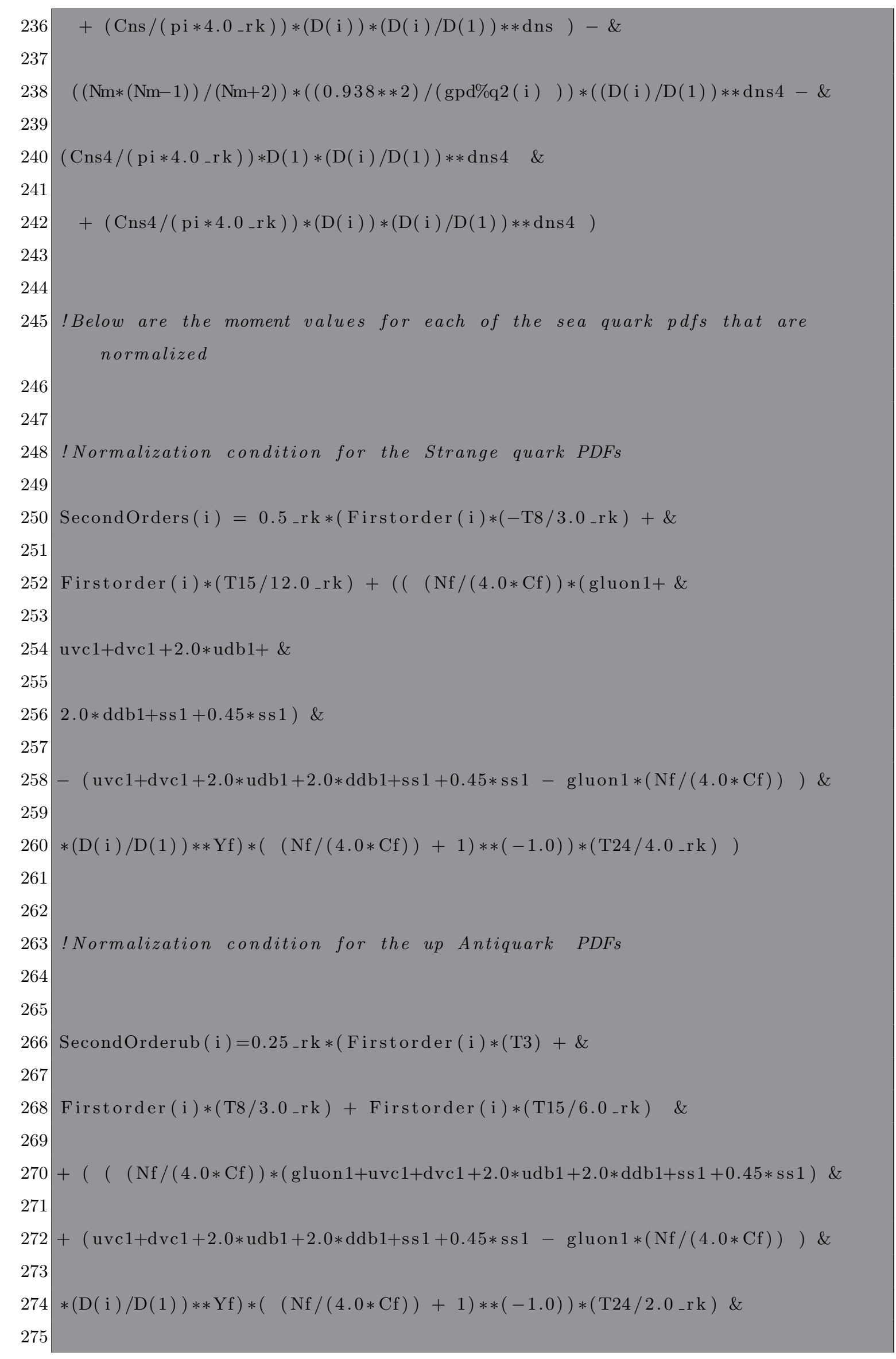




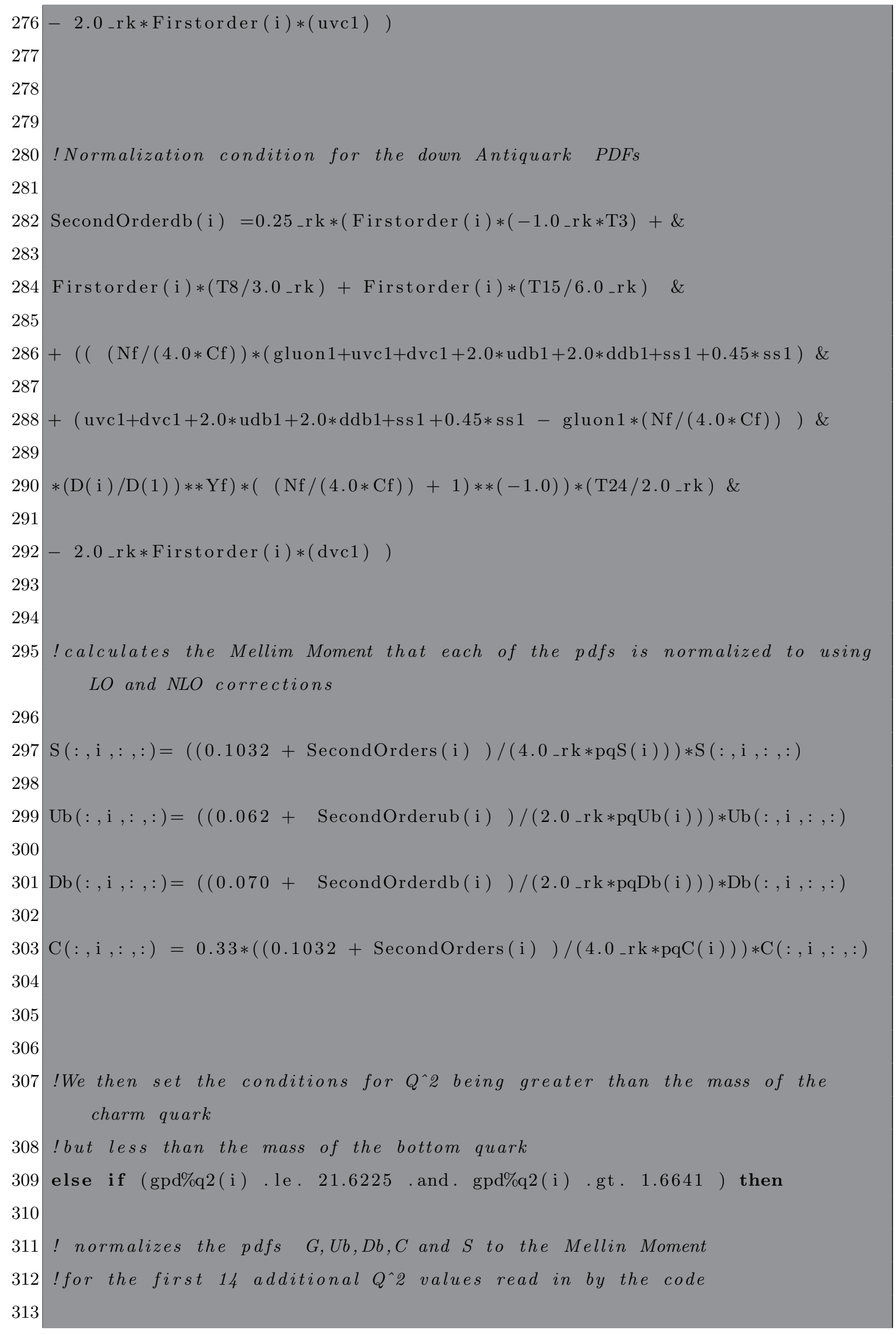




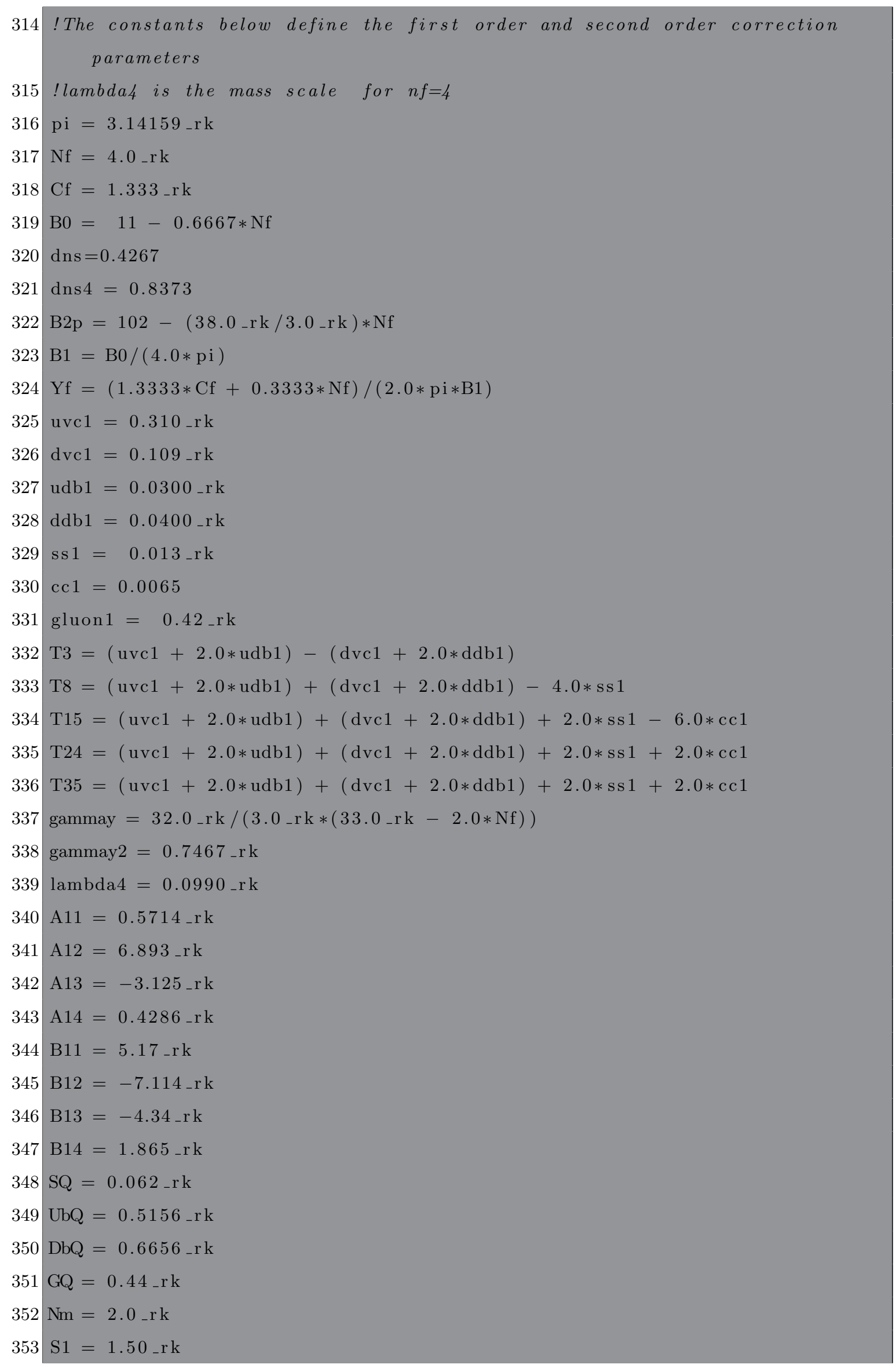




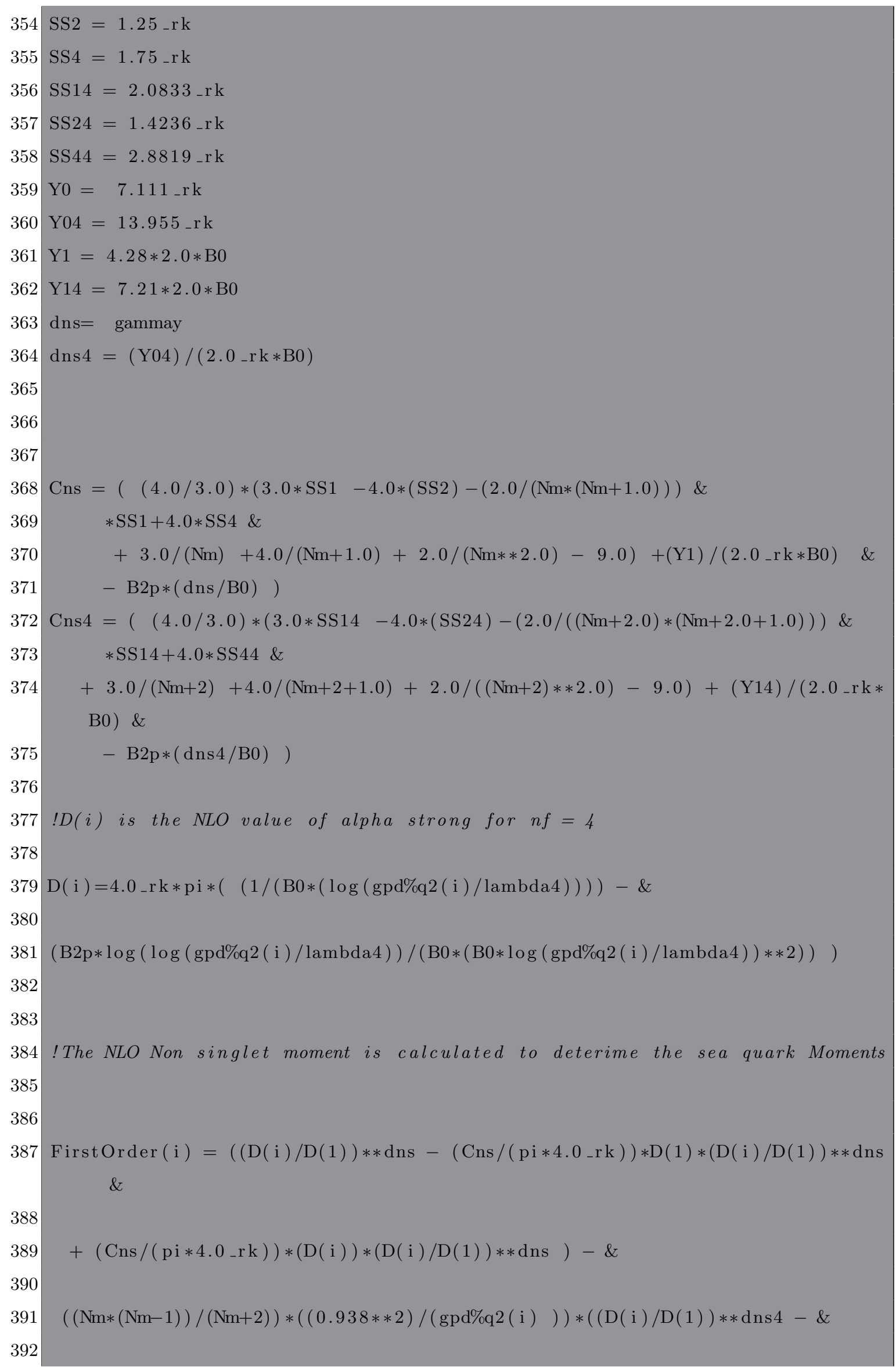




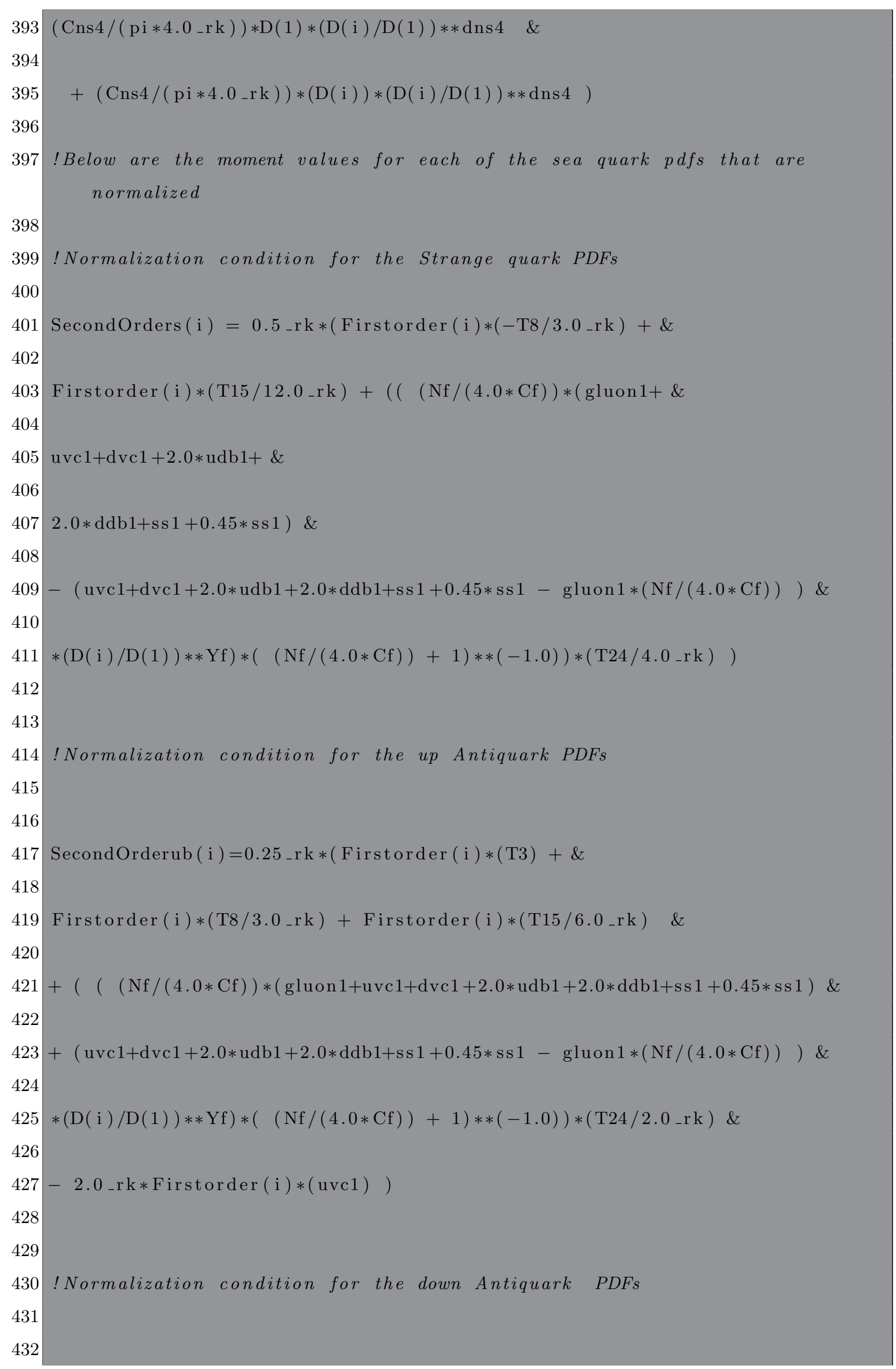




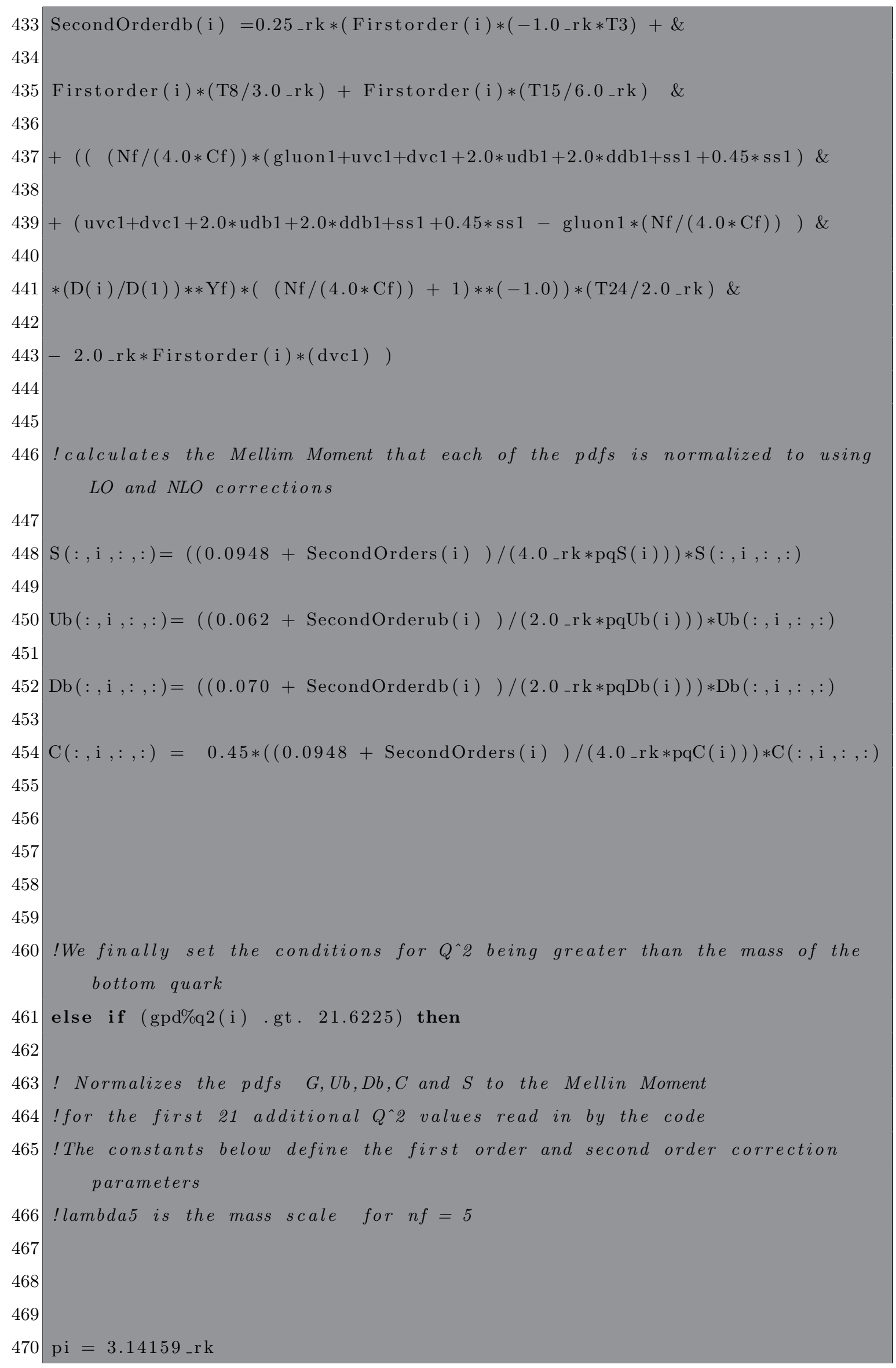




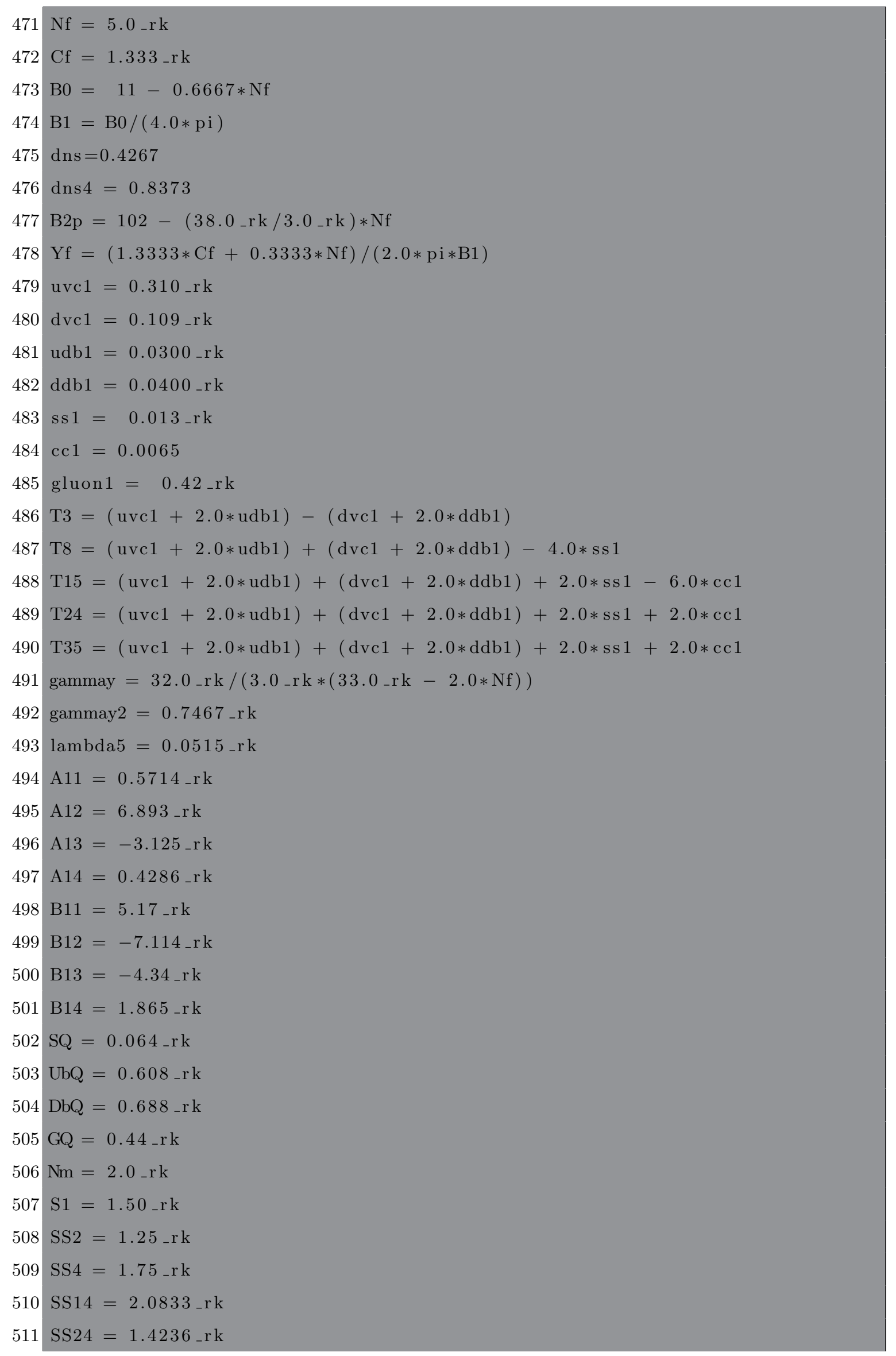




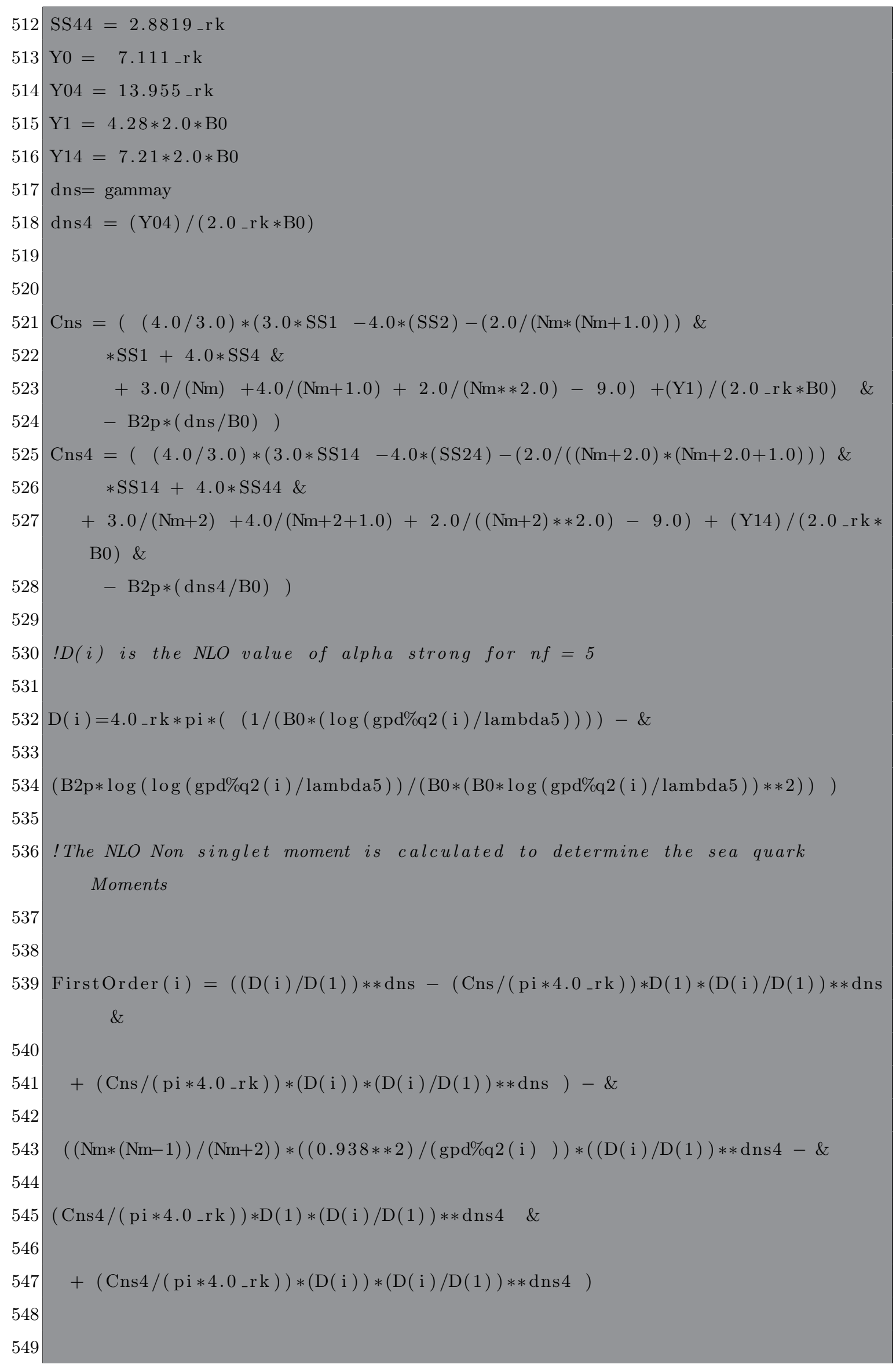




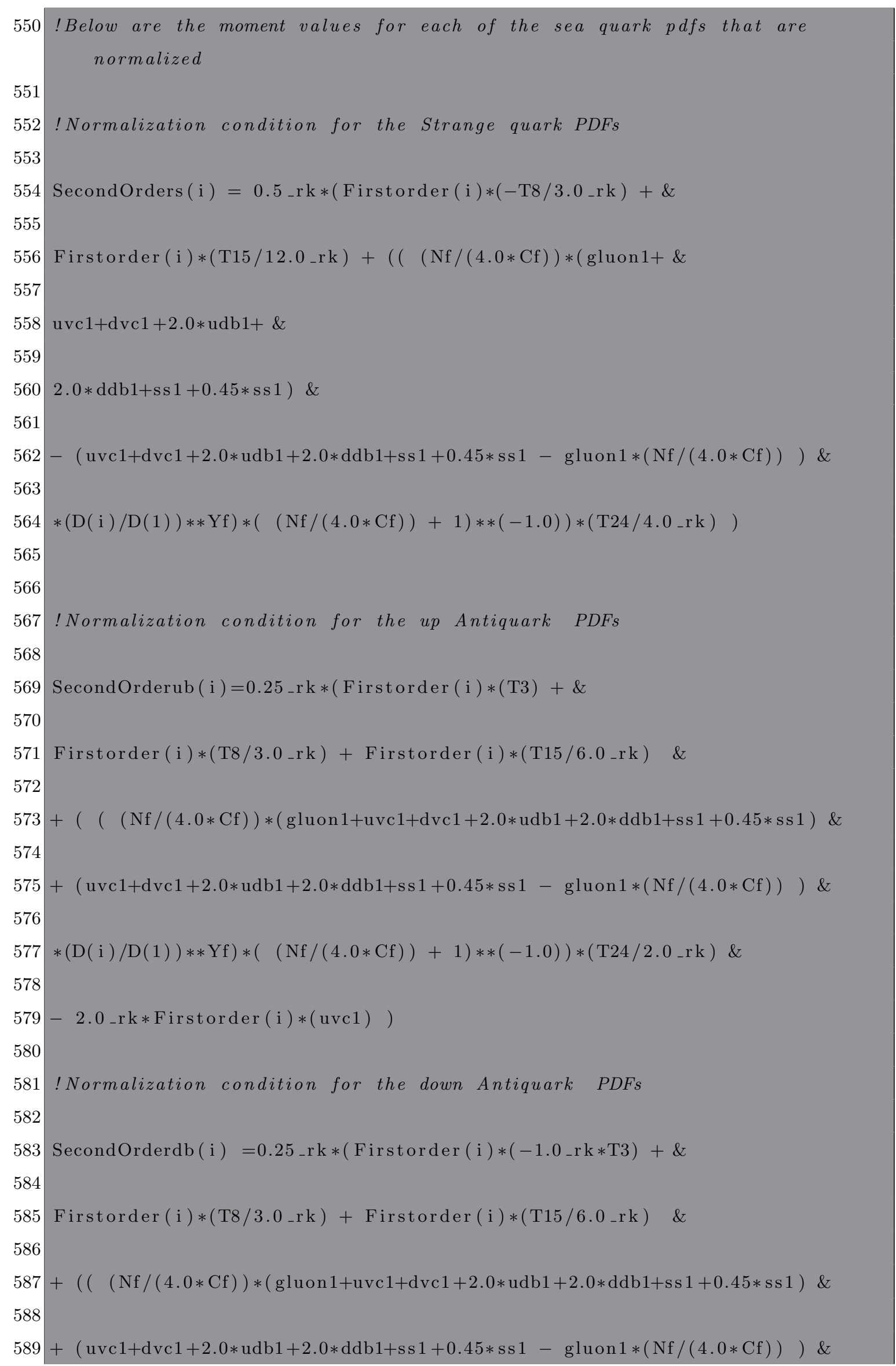




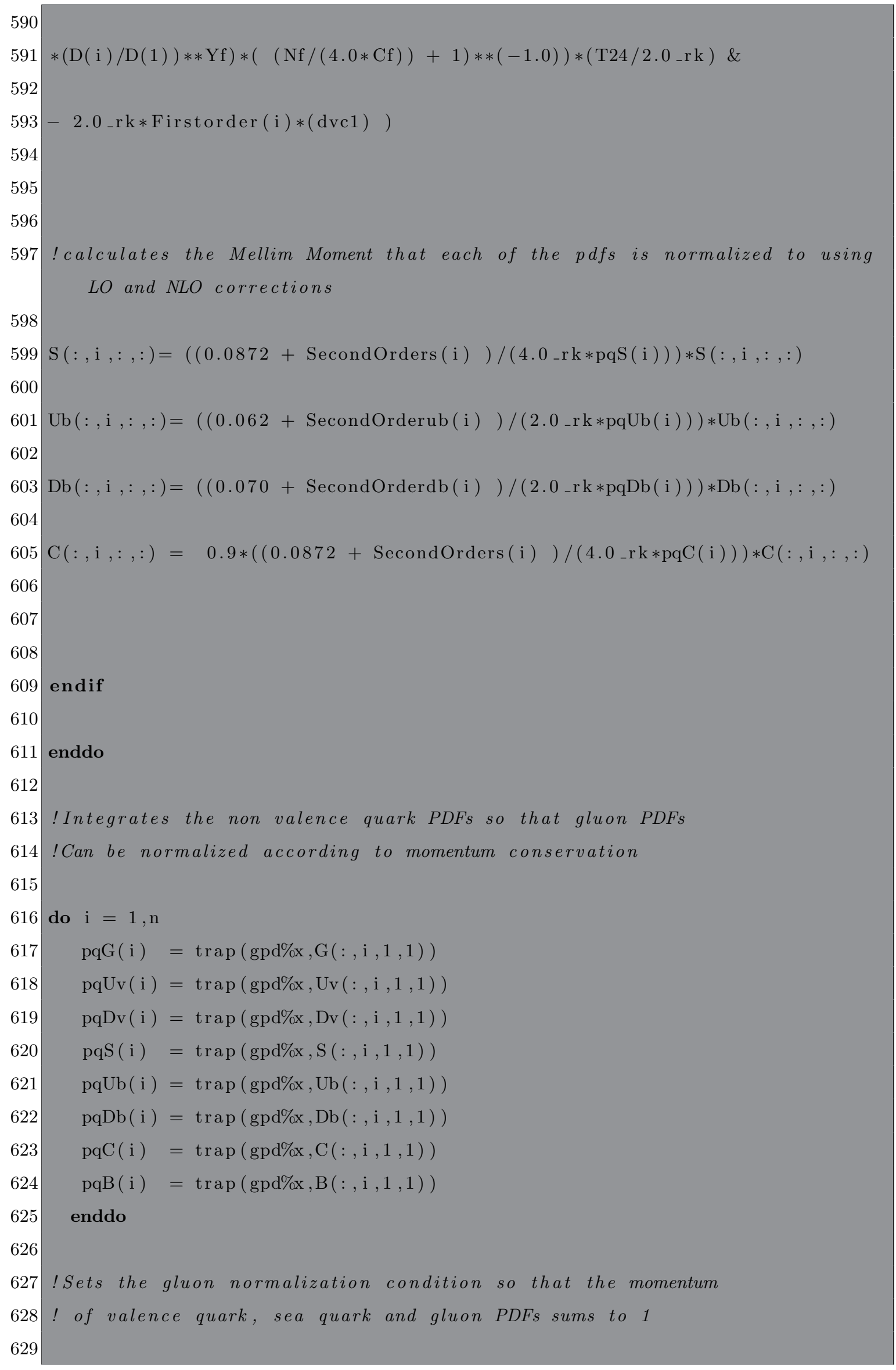




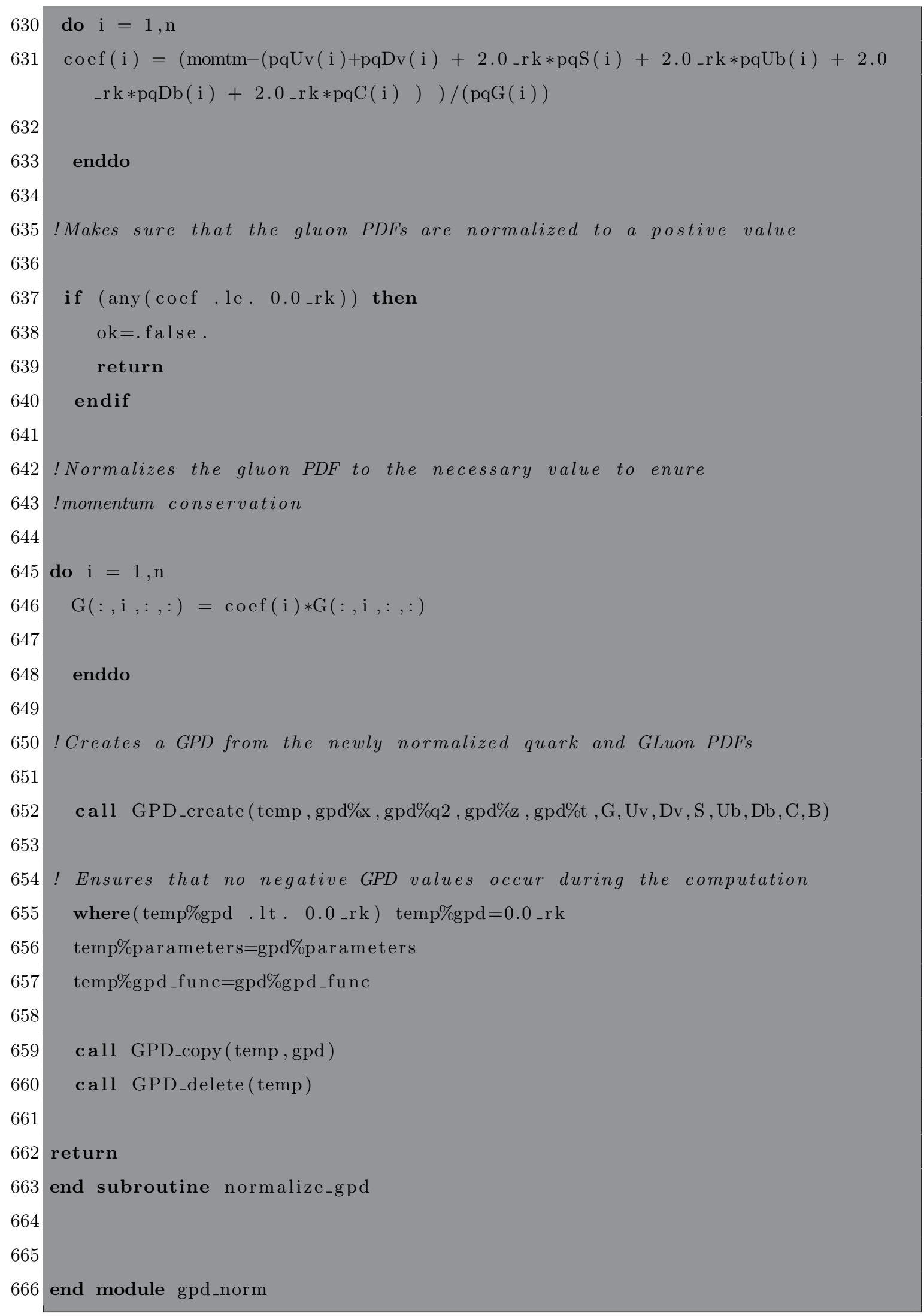

\section{Listing 3: Code2}




\section{Bibliography}

[1] L. Del Debbio, S. Forte, J.I. Latorre, A. Piccione and J. Rojo [NNPDF Collaboration], JHEP 0703, 039 (2007); ibid JHEP 0503, 080, (2005)

[2] R. D. Ball, L. Del Debbio, S. Forte, A. Guffanti, J. I. Latorre, J. Rojo and M. Ubiali, Nucl. Phys. B 838 (2010) 136; JHEP 1005, 075 (2010); Nucl. Phys. B 823, 195 (2009); Nucl. Phys. B809, 1 (2009), Erratum-ibid. B816, 293 (2009).

[3] S.Forte, L.Garrido, J.I.Latorre and A. Piccione, JHEP 0205, 062 (2002).

[4] M. Diehl, Phys. Rept. 388, 41 (2003)

[5] A.V. Belitsky and A. V. Radyushkin, Phys. Rept. 418, 1 (2005)

[6] L. W. Whitlow, E. M. Riordan, S. Dasu, S. Rock and A. Bodek, Phys. Lett. B 282, 475 (1992).

[7] A. C. Benvenuti et al. [BCDMS Collaboration], Phys. Lett. B 223, 485 (1989); ibid Phys. Lett. B237, 592 (1990).

[8] M. Arneodo et al. [New Muon Collaboration], Nucl. Phys. B 483, 3 (1997).

[9] Adams et al. , Phys.Rev.D54, 3006 (1996).

[10] T. Ahmed et al. [H1 Collaboration], Nucl. Phys. B439, 471 (1995)

[11] M. Derrick et al. [ZEUS Collaboration], Z. Phys. C65, 379 (1995).

[12] S. Malace et al., Phys.Rev.C80, 035207 (2009).

[13] L. Lonnblad, C. Peterson, H. Pi, T. Rognvaldsson, Comput. Phys. Commun. 67, 193-209 (1991).

[14] H. Honkanen, S. Liuti, J. Carnahan, Y. Loitiere, P. R. Reynolds, Phys. Rev. D 79, $034022(2009)$ 
[15] D. Z. Perry, K. Holcomb and S. Liuti, PoS DIS 2010 , 242 (2010) [arXiv:1008.2137 [hep-ph]].

[16] K. Holcomb, S. Liuti and D. Z. Perry, arXiv:1008.4197 [hep-ph]

[17] C. Peterson, T. Rognvaldsson, L. Lonnblad, Comput. Phys. Commun. 81, 185-220 (1994); C. Peterson, in Proceedings of Workshop on "New Computing Techniques in Physics Research II ", Edited by D. Perret-Gallix. River Edge, N.J., World Scientific, 1992.

[18] McCulloch, Warren; Walter Pitts (1943). "A Logical Calculus of Ideas Immanent in Nervous Activity". Bulletin of Mathematical Biophysics 5 (4): 115

[19] Hebb, Donald (1949). The Organization of Behavior. New York: Wiley.

[20] http://www.kurzweilai.net/how-bio-inspired-deep-learning-keeps-winningcompetitions 2012 Kurzweil AI Interview with Jurgen Schmidhuber on the eight competitions won by his Deep Learning team 2009-2012

[21] T. Kohonen, Self-organizing Maps (Springer, New York, 2001), 3rd. ed.

[22] Panovsky, W.K.H. (1968) Rapporteurs talk, in International Conference on High Energy Physics, Vienna, ed. J. Prentki and J. Steinberger (CERN, Geneva).

[23] Richard P. Feynmann, "Very high-energy collisions of hadrons", Phys. Rev. Lett. vol. 23. pp. 1415-1417.

[24] Bloom. E.D. et al (1969).,Phys.Rev.Lett. 23,930

[25] Breidenbach,M et al. (1969).,Phys. Rev.Lett. 23,935.

[26] Gross, J.D. and Wilczek, F.A. (1973).,Phys. Rev. Lett. 30, 1343

[27] t'Hooft, G. (1972). unpublished.

[28] Politzer, H.D. (1973),.Phys. Rev. Lett. 30, 1346

[29] Richard P. Feynman, "Very high-energy collisions of hadrons", Phys. Rev. Lett. vol. 23 , pp. $1415-1417,1969$

[30] J.D. Bjorken and Emmanuel A. Paschos, "Inelastic Electron Proton and gamma Proton Scattering, and the Structure of the Nucleon", Phys. Rev., vol. 185, pp. 1975-1982, 1969. 
[31] M. Klein and R. Yoshida, "Collider Physics at HERA", 2008.

[32] H. Fritsch, Murray Gell-Mann, and H. Leutwyler, "Advantages of the Color Octet Gluon Picture",Phys. Lett.,vol B47, pp. 265-368, 1973

[33] D. J.Gross and Frank Wilczek, "Asymptotically Free Gauge Theories. 1",Phys. Rev., vol. D8, pp. 3633-3652, 1973.

[34] Steven Weinberg, "Nonabelian Gauge Theories of the Strong Interactions",Phys. Rev. Lett., vol. 31, pp. 494-497, 1973.

[35] Murray Gell-Mann, "Symmetries of baryons and mesons",Phys. Lett., vol.125, pp. 1067-1084, 1962.

[36] Murray Gell-Mann, "Schematic Model of Baryons and Mesons",Phys. Rev., vol.8, pp. 214-215, 1964.

[37] G. Zweig, "An SU(3) Model For Strong Interaction Symmetry and its Breaking. 2", CERN-TH-412.

[38] Y. Dothan, Murray Gell-Mann, and Yuval Neeman, "Series of Hadron Energy Levels as Representations of Noncompact Groups",Phys. Lett., vol. 17, pp. 148-151, 1965.

[39] Callan, C.G. and Gross, J.D. (1969).,Phys.Rev. Lett. 22, 156.

[40] Bjorken, J.D. (1969)., Phys. Rev. 179, 1547.

[41] Brodsky, S.J. and Farrar,G. (1973)., Phys. Rev. Lett. 31,1153

[42] Matveev, V.A., Murddyan, R.M. and Tavkheldize, A.N. (1973)., Lett. Nuo. Cim. 7,719

[43] Pennington, M.R., Rep. Prog. Phys. 46, 393.

[44] J. Pumplin, D. R. Stump, J. Huston, H. L. Lai, P. M. Nadolsky and W. K. Tung, JHEP 0207, 012 (2002)

[45] D Stump, J Pumplin, R Brock, D Casey, J Huston, J Kalk, HL Lai, WK Tung Physical Review D 65 (1), 014012 (2001)

[46] H. Abdi and L.J. Williams, L.J., Wiley Interdisciplinary Reviews: Computational Statistics 2, 433 (2010). 
[47] S. Haykin, Neural Networks and Learning Machines, (Pearson, New Jersey, 2009), 3rd ed.

[48] D. G. Ireland, Phys. Rev. C82, 025204 (2010)

[49] M. Gluck, P. JimenezDelgado, E. Reya, Eur. Phys. J. C 53, 355 (2008)

[50] P. Jimenez-Delgado and E. Reya, Phys. Rev. D80, 114011 (2009); ibid Phys. Rev. D 79, 074023 (2009).

[51] A. D. Martin, W. J. Stirling, R. S. Thorne and G. Watt, Eur. Phys. J. C63, 189 (2009)

[52] S. Alekhin, J. Blumlein, S. Klein and S. Moch, Phys. Rev. D81, 014032 (2010)

[53] A. Accardi, W. Melnitchouk, J. F. Owens, M. E. Christy, C. E. Keppel, L. Zhu, J. G. Morn Phys. Rev. D 84, 014008 (2011)

[54] J. F. Owens, A. Accardi and W. Melnitchouk, Phys. Rev. D87, 094012 (2013)

[55] A. Courtoy and S. Liuti, arXiv:1302.4439 [hep-ph],to be published in Phys. Lett.B ;ibid arXiv:1307.4211 [hep-ph].

[56] N. Bianchi, A. Fantoni and S. Liuti, Phys. Rev. D69, 014505 (2004)

[57] R. D. Ball, S. Carrazza, L. Del Debbio, S. Forte, J. Gao, N. Hartland, J. Huston and P. Nadolsky et al., JHEP 1304, 125 (2013)

[58] H. -L. Lai, M. Guzzi, J. Huston, Z. Li, P. M. Nadolsky, J. Pumplin and C. -P. Yuan, Phys. Rev. D82, 074024 (2010)

[59] A. Accardi, W. Melnitchouk, J. F. Owens, M. E. Christy, C. E. Keppel, L. Zhu, J. G. Morn Phys. Rev. D 84014008 (2011), arXiv:1102.3686 [hep-ph

[60] J. F. Owens, A. Accardi and W. Melnitchouk, Phys. Rev. D87, 094012 (2013)

[61] S. Alekhin, J. Blumlein and S. Moch, Phys. Rev. D86, 054009 (2012)

[62] M. Diehl, Phys. Rept.388, 41 (2003)

[63] H. Honkanen, S. Liuti, J. Carnahan, Y. Loitiere, P. R. Reynolds, Phys. Rev. D 79, $034022(2009)$

[64] M. Gluck, E. Reya and A. Vogt, Z. Phys. C48, 471 (1990). 
[65] Roberts, R.G. The Structure of the proton, (Cambridge: University Press 1990)

[66] Gross, J.D. and Wilczek, F.A. (1974).,Phys. Rev. D9, 980

[67] Georgi, H. and Politzer, H.D. (1974)., Phys. Rev. D9, 416

[68] Altarelli, G. and Parisi, G. (1977)., Nucl. Phys. B126, 298

[69] Floratos, E.G.,Ross, D.A. and Sachrajda, C.T. (1977)., Nucl. Phys., B129, 66: $\mathrm{B} 139,545(\mathrm{E})$

[70] Bardeen, W.A., Buras, A.J., Duke, D.W. and Muta, T. (1978)., Phys. Rev. D18, 3998

[71] Nachtmann, O. (1973)., Nucl. Phys. B63, 237.

[72] H. Georgi and H. D. Politzer, Phys.Rev. D14, 1829 (1976).

[73] A. Courtoy, and S. Liuti, Phys. Lett. B 726, 320 (2013)

[74] https://hallcweb.jlab.org/resdata/database/

[75] M. Arneodo et al. [New Muon Collaboration], Nucl. Phys. B 487, 3 (1997).

[76] R. J. Holt and C. D. Roberts, Rev. Mod. Phys. 82, 2991 (2010)

[77] J. Arrington, J. G. Rubin and W. Melnitchouk, Phys. Rev. Lett.108, 252001 (2012)

[78] S. Tkachenkoet al. [CLAS Collaboration], Phys. Rev. C89, 045206 (2014)

[79] S. I. Alekhin, S. A. Kulagin and S. Liuti, Phys. Rev. D69, 114009 (2004) 